\title{
Modulation and adaption in the normal and obstructed urinary bladder : a morphological and functional assessment
}

Citation for published version (APA):

de Jongh, R. (2009). Modulation and adaption in the normal and obstructed urinary bladder : a morphological and functional assessment. [Doctoral Thesis, Maastricht University]. Maastricht University. https://doi.org/10.26481/dis.20091218rj

Document status and date:

Published: 01/01/2009

DOI:

10.26481/dis.20091218rj

Document Version:

Publisher's PDF, also known as Version of record

\section{Please check the document version of this publication:}

- A submitted manuscript is the version of the article upon submission and before peer-review. There can be important differences between the submitted version and the official published version of record.

People interested in the research are advised to contact the author for the final version of the publication, or visit the DOI to the publisher's website.

- The final author version and the galley proof are versions of the publication after peer review.

- The final published version features the final layout of the paper including the volume, issue and page numbers.

Link to publication

\footnotetext{
General rights rights.

- You may freely distribute the URL identifying the publication in the public portal. please follow below link for the End User Agreement:

www.umlib.nl/taverne-license

Take down policy

If you believe that this document breaches copyright please contact us at:

repository@maastrichtuniversity.nl

providing details and we will investigate your claim.
}

Copyright and moral rights for the publications made accessible in the public portal are retained by the authors and/or other copyright owners and it is a condition of accessing publications that users recognise and abide by the legal requirements associated with these

- Users may download and print one copy of any publication from the public portal for the purpose of private study or research.

- You may not further distribute the material or use it for any profit-making activity or commercial gain

If the publication is distributed under the terms of Article $25 \mathrm{fa}$ of the Dutch Copyright Act, indicated by the "Taverne" license above, 


\section{Modulation and adaptation in the normal and obstructed urinary bladder}

a morphological and functional assessment 
Modulation and adaptation in the normal and obstructed urinary bladder a morphological and functional assessment

\section{Rik de Jongh}

18 december, 2009

Thesis with summary in Dutch

ISBN no: $978-90-76014-25-8$

NUR no: 883

Lay-out: Henk en Hannie Dinnissen

Printed by: Verhagen Grafische Media, Veldhoven, The Netherlands

The publication of this thesis was financially supported by:

Abbott, American Medical Systems, Astellas, AstraZeneca, Bayer Health Care, Boerhinger Ingelheim, Coloplast, Eli Lilly, Eurocept B.V., Ferring Pharmaceuticals, GlaxoSmithKline, Hoogland Medical B.V., Johnson \& Johnson, LASERVISION INSTRUMENTS B.V., Medtronic, Novartis, Olympus Nederland B.V., Pfizer, Pohl Boskamp, WAMU and Wetenschappelijke Fonds Catharina-Ziekenhuis. 


\title{
Modulation and adaptation in the normal and obstructed urinary bladder
}

\author{
a morphological and functional assessment
}

\author{
PROEFSCHRIFT \\ ter verkrijging van de graad van doctor aan de Universiteit Maastricht, \\ op gezag van de Rector Magnificus, Prof. mr. G.P.M.F. Mols \\ volgens het besluit van het College van Decanen, \\ in het openbaar te verdedigen op \\ vrijdag 18 december om 10.00 uur
}

door

Rik de Jongh

Geboren op 19 juni 1978 te Gouda 
Promotor:

Co-promotores:

Dr. G.A. van Koeveringe

Prof. dr. J.I. Gillespie

(Universiteit van Newcastle, Groot Brittannië)

Beoordelingscommissie: Prof. dr. A. Bast

Prof. dr. J.G.R. de Mey

Prof. dr. M.C. Michel

(Universiteit van Amsterdam)

Prof. dr. H.W.M. Steinbush

Prof. dr. J.J. Wyndaele

(Universiteit van Antwerpen, België) 


\section{Contents}

Chapter 1 General introduction and outline of the thesis

Chapter 2 The effects of exogenous prostaglandins and the identification of constitutive cyclooxygenase $I$ and II immunoreactivity in the normal guinea pig bladder.

BJU Int. 2007; 100(2):419-29.

Chapter 3 The localisation of cyclo-oxygenase immunoreactivity (COX I-IR) to the urothelium and to interstitial cells in the bladder wall.

J Cell Mol Med in press.

Chapter 4 Damage to the bladder neck alters autonomous activity and its sensitivity to cholinergic agonists.

BJU Int. 2007; 100(4):919-29.

Chapter 5 Alterations to network of NO/CGMP-responsive interstitial cells induced by outlet obstruction in guinea-pig bladder. Cell Tissue Res. 2007; 330(1):147-60.

Chapter 6 Partial bladder outlet obstruction reduces the tissue antioxidant capacity and muscle nerve density of the guinea pig bladder.

Neurourol Urodyn 2009; 28(5):461-7.

Chapter 7 Oxidative stress reduces the muscarinic receptor function in the urinary bladder.

Neurourol Urodyn. 2007; 26(2):302-8.

Chapter 8 Lipid peroxidation product 4-hydroxynonenal contributes to bladder smooth muscle damage.

Urology 2008; 71(5):974-8. 
Dankwoord

Curriculum vitae 


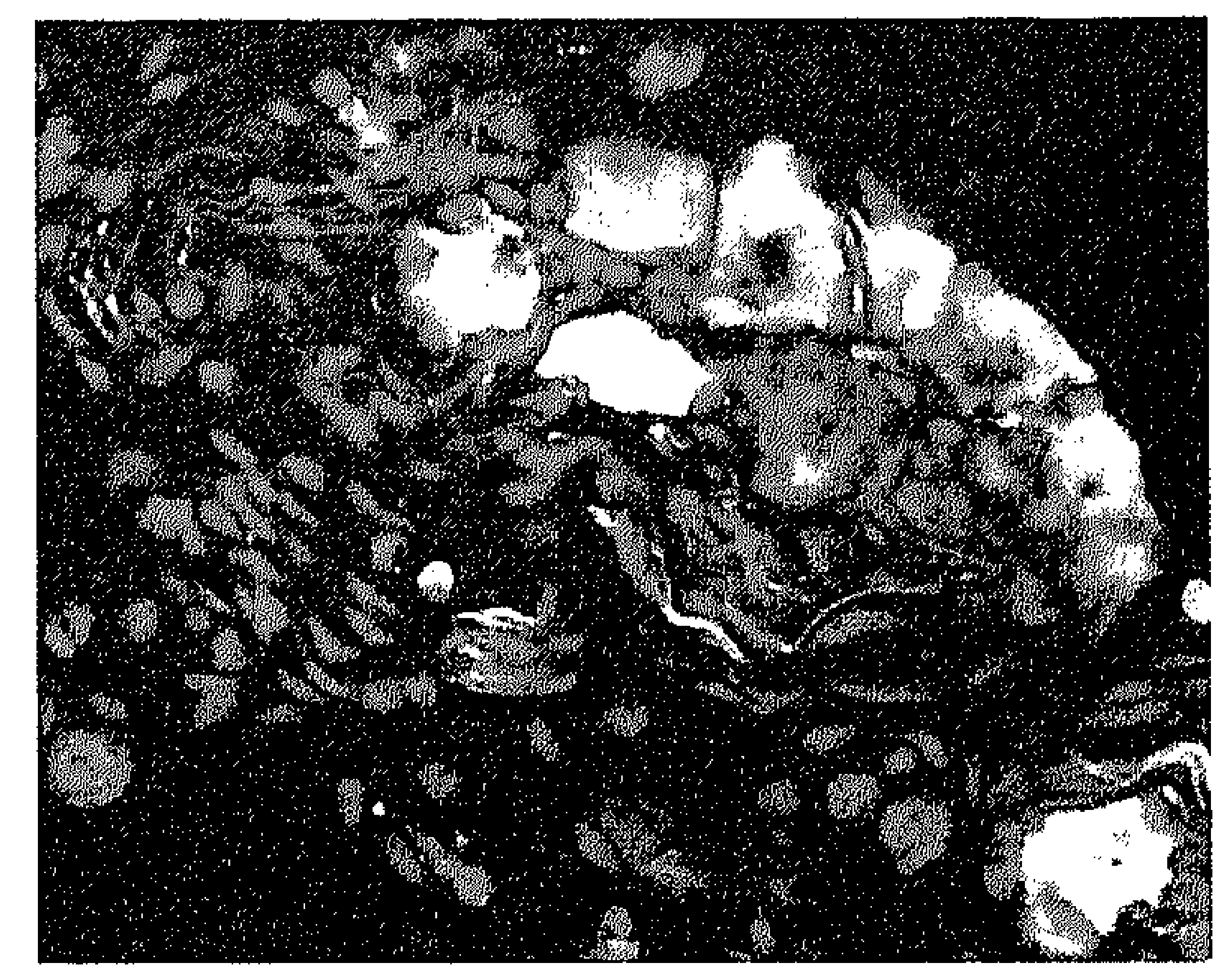

General introduction 


\section{Anatomy and physiology of the lower urinary tract}

The lower urinary tract is composed of bladder and urethra. The smooth muscle lining the bladder neck and urethra form the internal sphincter, this is surrounded by striated muscle called the rhabdosphincter. The two major functions of the lower urinary tract are to store urine and, periodically and at an appropriate moment, to empty the stored urine. The bladder and urethra function reciprocally. As the bladder fills during the urine storage phase, the detrusor remains relatively quiescent and the urethra outlet is activated. During the micturition phase, the detrusor muscles contract and the urethra outlet relaxes. This involves a complex pattern of efferent (motor) and afferent (sensory) signalling in the autonomic and somatic nervous systems. The nerves involved form part of a reflex pathway, with an incorporated conscious control component. The different anatomical components involved during the storage and micturition phase will be discussed briefly.

\section{Central nervous system}

The normal micturition reflex is mediated by a spinal-brainstem-spinal pathway, which passes through relay centres in the brain. Micturition occurs in response to afferent signals from the lower urinary tract. During bladder filling, once threshold tension is achieved, afferent impulses reach centres in the central nervous system. Afferent neurons send information to the periaqueductal gray (PAG), which in turn communicates with the pontine tegmentum. In the brainstem, two distinct regions are involved in micturition control. One is located in the dorsomedial pontine tegmentum and is called the pontine micturition center ( $P M C), M$ (= medial) region or Barrington nucleus. A cell group located in the lateral part of the pontine tegmentum is called the $L$ (lateral) region. ${ }^{1}$ The $L$-region could serve as a pontine storage centre. It has been suggested that this storage centre suppresses bladder contraction and regulates the activity of the striated musculature of the bladder outlet. The PMC receives direct projections from the PAG. ${ }^{2}$ The PAG receives many afferents from brain regions, such as the amygdala, the bed nucleus of stria terminalis, parts of the hypothalamus and from the orbital and prefrontal cortex. ${ }^{2-3}$ The PAG plays a crucial part in the micturition reflex. The decision to initiate micturition is made in the PAG, based on afferent information from the sacral cord and from brain structures belonging to or associated with the limbic system and the prefrontal cortex. In healthy people the signal from the limbic system and prefrontal cortex can prevent the PAG from exciting the PMC neurons, even when other parts of the PAG receive strong impulses from a full bladder. The micturition reflexes involve several transmitters which can manipulate central micturition circuitry. These transmitters include dopamine, glutamate, $y$-aminobutyric acid, serotonin and enkephalins. ${ }^{4}$ 


\section{Peripheral nervous system and bladder muscle}

The lower urinary tract is innervated by three sets of peripheral nerves involving the parasympathetic (pelvic), sympathetic (hypogastric) and somatic (pudendal) systems. These nerves contain afferent as well as efferent axons. Parasympathetic preganglionic neurons are located in the lateral part of the sacral intermediate gray matter (sacral parasympathetic nucleus). Parasympathetic preganglionic neurons send axons via the ventral roots to peripheral ganglia. In humans, parasympathetic postganglionic neurons are located in the detrusor wall and the pelvic plexus. The sympathetic nerves pass the sympathetic chain ganglia, the inferior mesenteric ganglia and through the hypogastric nerves they form connections with the pelvic and intramural ganglia. The somatic neurons are located along the lateral border of the ventral horn (Onuf's nucleus).

\section{Sensory afferent pathways}

Sensory (afferent) nerves. Afferent axons in the pudendal, pelvic and hypogastric nerves transmit information from the lower urinary tract to the lumbosacral spinal cord. Most of the sensory information of the bladder and urethra reaches the spinal cord via the pelvic nerve. In addition, some afferents travel through the hypogastric nerves. Pelvic nerve afferents consist of myelinated (A-delta) and unmyelinated $(C)$ fibers. A-delta fibres are low threshold mechanoreceptors whereas C-bladder afferents are normally mechano-insensitive. ${ }^{5-6}$ These fibres become mechanosensitive following the actions of various mediators. In both humans and animals, afferent nerves have been identified suburothelially as well as in the detrusor muscle. ${ }^{7}$ Just below the urothelium the nerves form a plexus. Some terminals are located in the basal parts of the urothelium. The suburothelial plexus is sparce in the dome of the bladder, becomes denser near the bladder neck and is prominent in the trigone. ${ }^{7}$

Immunohistochemical studies have demonstrated that bladder afferent nerves contain various neuropeptides, including substance $P$, neurokinin $A$, calcitonin gene related peptide (CGRP), vasoactive intestinal polypeptide (VIP), and enkephalins. ${ }^{8}$ These peptides are located in capsaicin-sensitive, C-fibre bladder afferents and are released in the bladder by noxious stimulation. ${ }^{9}$

Urothelium and lamina propria. The urothelium is more than a barrier that separates urine from extracellular fluid. The urothelium is recognized as having substantial sensory properties, ${ }^{10}$ especially the lamina propria is extensively innervated. ${ }^{11-12}$ Several "sensory molecules" associated with neurones have been identified in the urothelium. These include receptors for bradykinin, purines (P2X and P2Y) norepinephrine ( $\alpha$ and $\beta$ ), acetylcholine (nicotinic and muscarinic), and neurotrophins. ${ }^{13-18}$ Furthermore, a number of transient receptor potential (TRP) channels (TRPV1, 2 and 4 ) have been demonstrated in the urothelium. ${ }^{19-20}$ Painful sensations induced by capsaicin are caused by the stimulation of TPRV1 receptors. Several substances (e.g. ATP, NO, substance P, acetylcholine, prostanoids and 
cytokines) can be released from urothelial cells following physical and chemical stimulation. $^{21-25}$ This suggests the existence of reciprocal communication with neighbouring urothelial cells, nerves and other cell types such as interstitial cells. The urothelium may serve as a mechanosensor which by producing nitric oxide, ATP, acetylcholine and other mediators, can control the activity in afferent nerves.

Suburothelial interstitial cells. Suburothelial interstitial cells have been identified in human and animal bladders. The precise identification and definition of what is an interstitial cell in the bladder is, at present under discussion. Several terms in the field of the urinary tract have been applied, including myofibroblasts, interstitial cells of Cajal, and interstitial cells. The initial description of interstitial cells in the human and guinea pig bladder was based on their ability to show a rise in CGMP in response to nitric oxide donors. ${ }^{26-27}$ Different sub-types of interstitial cells, based on the location of the cells in the tissue, have been identified: interstitial cells lying in the sub-urothelial layer and in the outer muscle layer. ${ }^{26}$ Other markers, notably the c-Kit receptor and vimentin, have been used to identify interstitial cells. ${ }^{28-31}$ C-Kit and vimentin positive cells are found in the lamina propria and around the muscle bundles of both the inner and outer muscle layers. The connexin proteins of gap junctions are expressed by many interstitial cells. Within the bladder the suburothelial interstitial cells express connexion $43 .{ }^{11,32-33}$ Neither c-kit, vimentin, and cGMP labelling seem to be ideal in identifying interstitial cells. Lots of interstitial cells do not express the c-kit receptor. ${ }^{31}$ Vimentin identifies several interstitial cells types however also other cell types such as fibroblasts can express vimentin. ${ }^{31}$ Furthermore, cGMP staining identifies besides interstitial cells also other cell structures such as umbrella cells and some nerves. ${ }^{27}$ Identification of cells as interstitial cells requires investigation of their ultrastructural properties using transmission electron microscopy. The ultrastructural features include the presence of abundant intermediate filaments, numerous mitochondria, moderately developed Golgi apparatus, smooth endoplasmatic reticulum, contacts with nerves and formation of gap junction with each other and with smooth muscle cells. ${ }^{34}$ The suburothelial interstitial cells found in the bladder form close association with afferent nerves, and it has been suggested that these cells play a role in modulating activity of the suburothelial sensory nerves. ${ }^{30}$ For example, isolated C-Kit positive cells from the suburothelial layer, can respond to stimuli such as caffeine, muscarinic and purinergic agonists with a rise in intracellular calcium concentration. ${ }^{35-36}$ Furthermore, vanilloid receptors have been identified on interstitial cells. ${ }^{37}$

\section{Motoric efferent pathway}

Motoric (efferent) nerves. Contraction of the detrusor smooth muscle and relaxation of the outflow region result from activation of parasympathetic neurons located in the sacral parasympathetic nucleus in the spinal cord at the level S2-4. ${ }^{38}$ The axons pass through the pelvic nerve and synapse with the postganglionic nerve in the pelvic plexus, the serosal ganglia or the suburothelial ganglia. The preganglionic neurotransmission is mediated by acetylcholine acting on nicotinic receptors. The 
neurotransmission can be modified by adrenergic, muscarinic, purinergic and peptidergic presynaptic receptors. ${ }^{38}$ The postganglionic neurons in the pelvic nerve induce excitatory input by releasing acetylcholine, acting on muscarinic receptors. A separate nonadrenergic, noncholinergic component (probably ATP) participates in activating the bladder. The pelvic nerve sends parasympathetic fibres to the outflow region and the urethra. These fibres exert an inhibitory effect and thus relax the outflow region, partly mediated by $\mathrm{NO}^{39}$ The sympathetic innervation of the bladder and urethra originates from the thoraco-lumbar region ( $T 10-L 2)$ of the spinal cord. The axons travel either through the inferior mesenteric ganglia and the hypogastric nerve, or they pass through the paravertebral chain and enter the pelvic nerve. The effect of sympathetic innervation of the lower urinary tract is inhibition of parasympathetic pathways at spinal and ganglion levels and mediation of contraction of the bladder base and urethra. The response of the bladder body to noradrenaline exposure is relaxation.

\section{Muscle and receptors}

Cholinergic system. Muscarinic receptors are found throughout the bladder. The bladder contains a heterogeneous population of muscarinic receptors: $M_{1}$ through $M_{5}$ receptors have been demonstrated in the human bladder with a predominance of $M_{2}$ and $M_{3}$ receptors. ${ }^{40}$ The density of $M_{2}$ receptors is 3- to 10-fold greater than the density of $M_{3}$ receptors. ${ }^{41}$ The $M_{3}$ receptors are most important for detrusor contraction. In the positive inotropic response to muscarinic receptor agonist in the bladder a cascade of biochemical reactions is involved. During $M_{3}$ receptor activation, the receptor-agonist complex couples to a G-protein that subsequently activates phopholipase $C$ and leads to mobilization of extra-and intracellular calcium ions, eventually leading to a detrusor contraction. ${ }^{42}$ The $M_{2}$ receptors contribute to bladder contraction by preventing $\beta$-adrenergic-induced cyclic adenosine monophosphate (CAMP) formation, which mediates detrusor relaxation. Muscarinic receptors are also located in presynaptic nerve terminals. Prejunctional facilitatory $M_{1}$ and inhibitory $M_{2}$ receptors are demonstrated in the bladder. ${ }^{43-44}$

Adrenergic system. The bladder body of the human detrusor contains a high density of $\beta$-adrenergic receptors and contains only a few $\alpha_{1}$-adrenergic receptors. In contrast, the bladder neck and urethra contain a high density of $\alpha$-adrenergic receptors. $60-70 \%$ of the $\alpha_{1}$-adrenergic receptors expressed in human bladder are of the $\alpha_{1 d}$-subtype and the remainder is the $\alpha_{1 a}$-subtype. ${ }^{45}$ Stimulation of $\alpha$-adrenergic receptors results in muscle contraction. Prejunctional $\alpha_{1}$ - and $\alpha_{2}$ adrenergic receptors are found in the bladder, where they modulate release of acetylcholine and norepinephrine. ${ }^{46}$ The detrusor muscle contains 3 subtypes of $\beta$-adrenergic receptors $\left(\beta_{1}, \beta_{2}\right.$, and $\left.\beta_{3}\right)$. Recent studies have revealed a predominant expression of $\beta_{3}$-adrenergic mRNA in human detrusor. ${ }^{47}$ Stimulation of $\beta_{3}$-adrenergic receptors results in relaxation of bladder smooth muscle.

Purinergic system. P2X1-receptor immunoreactivity is associated with bladder smooth muscle membranes ${ }^{48}$ and contractile responses of bladder strips to ATP are 
probably mediated by $\mathrm{P} 2 \times 1$-receptors. ${ }^{49}$ P2Y1-receptor mRNA is expressed in detrusor muscle cells ${ }^{50}$ and precontracted bladder strips respond to stimulation of P2Y-receptors with relaxation. ${ }^{49}$

Muscle interstitial cel/s. In the guinea pig three types of muscle interstitial cells have been described: cells in the outer muscle coat (muscle coat interstitial cells: mc-ics), cells on the surface of the muscle bundles (surface muscle interstitial cells: sm-ics) and cells within the muscle bundles (intra-muscle interstitial cells: im-ics). ${ }^{12,27,51} \mathrm{~A}$ sub-population of cells may be identified from their expression of choline acetyltransferase. ${ }^{52}$ The muscle interstitial cells are similar to interstitial cells of Cajal in the gut that generate peristalsis contraction. ${ }^{53-54}$ Therefore, a pacemaking role has been suggested for these cells in the bladder. Interstitial cells have close contacts with intramural nerves, indicating that these cells might be under influence of nerves.

\section{Overactive Bladder (OAB)}

Pathological conditions of the brain, central nervous system, peripheral nerves and the bladder can result in lower urinary tract dysfunctions, which can be classified as disturbances of storage and emptying. Failure to store urine can result in various forms of incontinence (urgency and stress incontinence) and failure to empty can lead to urinary retention. The Overactive bladder syndrome (OAB) compromises storage symptoms. Overactive bladder is a symptom complex that includes urinary urgency with or without urgency incontinence, urinary frequency (voiding eight or more times in a 24-hour period), and nocturia (awaking two or more times at night to void). The International Continence Society classifies overactive bladder as a syndrome for which no precise cause has been identified, with local abnormalities ruled out by diagnostic evaluation..$^{55}$ Because $O A B$ syndrome is a clinical diagnosis it is important that patients are assessed appropriately. Basic assessment necessary in the evaluation of $O A B$ syndrome includes taking a thorough history, physical examination and appropriate investigation. Investigations should include urine analysis (exclude infection, haematuria and glucosuria), measurement of postvoid residual, a frequency / volume chart and a quality of life ( $Q \circ$ L) questionnaire.

\section{Prevalence}

Functional problems of the lower urinary tract are a significant problem. In a European study an overall prevalence of the OAB syndrome was $16.6 \% .{ }^{56}$ Especially the older population suffers from this disorder, where $40 \%$ over the age of 70 years are affected. The overall prevalence of OAB syndrome in the USA was $16.6 \%$ and it was higher in women $(16.9 \%)$ than in men $(16.0 \%) .{ }^{57} \mathrm{OAB}$ syndrome is probably more prevalent than asthma and heart disease. Although the $O A B$ syndrome is not life threatening it seriously affects the quality of life and the ability to work. Patients with symptoms of overactive bladder tend to curtail their participation in social 
activities and to isolate themselves and are predisposed to depression. Nocturia is associated with sleep disruption, which decreases the quality of life. ${ }^{58-61}$ Postmenopausal women with urge incontinence have a substantially higher risk of falling and sustaining a fracture than women without urge incontinence. ${ }^{62}$

\section{Costs}

The total economic costs of overactive bladder are high. In 2002 the costs in the US were approximately $\$ 12.7$ billion. ${ }^{63}$ Approximately $25 \%$ of this expenditure is spent on treatment (drug therapy, clinical consultation and surgery). Overall, about 49 million people in Europe are probably affected, of whom only $60 \%$ have consulted a medical practitioner and $27 \%$ received treatment. Less than $3 \%$ regain long lasting normal control. The above costs are an underestimation and the problem is large. Therefore, $O A B$ syndrome causes major problems to individuals, health professions and society. Importantly, as people generally living longer, the numbers affected by $O A B$ syndrome are increasing.

\section{Physiologic and pathologic sensations of bladder filling}

Several normal and abnormal bladder sensations associated with bladder filling have been described. ${ }^{64}$ These sensations are based on reports of patients during urodynamic testing although similar sensations are felt in ordinary life. Normal bladder sensations progress in a continuum of gradually increasing intensity and unpleasantness. Immediately after micturition, when the bladder is empty, no sensation is perceived. When the bladder is filled, a first sensation of filling (FSF) occurs. This sensation is not constant, lasts for a few seconds and then disappears. The sensation is probably related to increased attention toward bladder sensation during artificial filling. As filling continues, a first desire to void is perceived (FDV). This desire is recognized as a familiar, constant sensation that normally persuades a person to seek a place to void. It is felt in the lower abdomen, and can easily be displaced from mind. As filling progresses, this sensation gradually becomes stronger and can only be ignored with increasing difficulty and only for a short period of time. If micturition is further postponed, a strong desire to void (SDV) is felt. This corresponds with a constant almost uncomfortable sensation in the perineal region or urethra. At this point, contraction of the pelvic floor can avert impeding micturition. The volumes at which the different filling sensations are reported vary widely, but have some sort of pattern with FSF occurring at a mean of $40 \%$ of full bladder and FDV at a mean of $59 \%$ of cystometric bladder capacity. ${ }^{65}$ Therefore, it has been suggested that these sensations correspond with specific physiological mechanisms. ${ }^{64}$ Micturition is usually free of sensation, although passing urine can be felt. Urgency is the complaint of a sudden compelling desire to pass urine which is difficult to defer. ${ }^{55}$ The sensation of urgency seems to be abnormal. It is probably not part of a normal continuum but is instead the primary symptom of the $O A B$ syndrome. Urgency can be felt in many conditions such as the presence of stones, infections, tumours and after radiotherapy. During urodynamic testing, it is often 
observed that urgency is accompanied by involuntary detrusor contraction (detrusor overactivity) and or by involuntary decrease in urethral pressure. Digesu et al found that $54 \%$ of the patients complaining of symptoms belonging to the overactive bladder have detrusor overactivity during urodynamics. ${ }^{66}$ Overactivity of the detrusor muscle can result in urgency and or urge incontinence, depending on the response of the urinary sphincter. In $46 \%$ of patients with symptoms of an overactive bladder, sensation is not accompanied by pressure changes and the condition can be described as bladder overactivity (BO), previously described as sensory urgency. Interestingly, many people have detrusor overactivity on cystometry but do not have symptoms of an overactive bladder. ${ }^{67}$

\section{Pathophysiology of an overactive bladder: different theories}

The aetiology and pathophysiology of symptoms of the overactive bladder have not yet been identified. The symptoms of an overactive bladder have many potential causes and contributing factors. For example, brain disorders such as stroke, Alzheimer's disease, Parkinson's disease are all related to the occurrence of an overactive bladder. Spinal cord lesions such as multiple sclerosis and spinal cord injuries can also result in an overactive bladder. Several bladder abnormalities, e.g. bladder outlet obstruction, inflammation and bladder cancer are related to symptoms of an overactive bladder. In lots of patients no focus can be identified, this condition is described as idiopathic detrusor overactivity.

Three main theories concerning the pathophysiology of the $O A B$ and detrusor overactivity have been proposed:

1) The myogenic theory suggests that smooth muscle changes are necessary for the development of an overactive bladder. ${ }^{68}$ Brading proposed that partial denervation, regardless of aetiology, alter the properties of the smooth muscle, leading to increased excitability and electrical coupling between muscle cells. A local contraction occurring in any part of the detrusor will spread throughout the bladder wall, resulting in coordinated myogenic contraction of the entire bladder. Changes in ultrastructure, such as ultra close abutments between muscle cells and increased numbers of gap junctions facilitate propagation of the contraction.

2) The neurogenic theory suggests that damage to central inhibitory pathways in the brain or spinal cord or sensitization of peripheral afferent nerves in the bladder can unmask primitive voiding reflexes that trigger detrusor overactivity. ${ }^{69}$

3) A relatively new theory is the autonomous bladder theory. ${ }^{70}$ This theory compromises that during normal bladder filling there are non micturition contractions (autonomous activity). Non micturition activity has been observed in humans and animals. ${ }^{70-74}$ In ambulatory urodynamics, non-micturition activity can be seen in approximately $70 \%$ of asymptomatic 'normal' individuals and can be regarded as a normal physiological process. ${ }^{75}$ Spontaneous phasic contractions have been 
investigated extensively in isolated whole guinea pig and rat bladders. ${ }^{70,76}$ The activity is intrinsic to the bladder wall. This autonomous activity is associated with propagating waves of contractions spreading over the bladder surface accompanied by local areas of stretch. The frequency and amplitude of the transients in guinea pig bladders can be augmented by nerve stimulation or direct application of muscarinic agonists. Furthermore, the phasic activity can be increased by ATP, substance $P$ and NO and decreased by noradrenaline, CAMP, $^{75}$ and calcitonin gene related peptide (cGRP). ${ }^{77}$ Increased intravesical volume activates the spontaneous activity. The initiation, coordination and control of this activity appears to be different from the micturition contraction. ${ }^{70}$ The question remains what the physiological function of the non-micturition activity is. In the cat, phasic non micturition activity accompanies bursts of afferent activity. ${ }^{78}$ Coolsaet and Gillespie speculated that small local contractions in the isolated (guinea) pig bladder generates local stretches eventually activating afferent nerves. ${ }^{70,79}$ It is reasonable to suggest that the non-micturition activity plays a role in generating and modulating bladder sensations. The cells responsible for the generation and propagation of non micturition activity are not known yet. It is well known that smooth muscle cells are spontaneously active. However, local propagating waves of contraction cannot be due to the direct spread of excitation from smooth muscle cell to smooth muscle cell, since the trabecular organization of the muscle bundles does not allow a direct coupling between adjacent areas of muscle. Therefore, it has been postulated that there must be structures involving specialized cells to generate and distribute activity over the bladder surface. A recently identified population of cells known as interstitial cells or myofibroblasts may be responsible for the non micturition activity. They are similar to interstitial cells of Cajal in the gut that generate peristalsis contraction. ${ }^{53-54}$ In summary, the autonomous bladder theory proposes that non-micturition activity can activate afferent nerves eventually resulting in bladder sensations. Thus, autonomous activity is a central part of a motor/sensory system in the bladder wall which might be responsible for generating and modulating sensation during filling.

In conclusion, the pathophysiology of $O A B$ syndrome and detrusor overactivity may include one or more of the above mentioned theories. It is clear that the sensory and motor pathways are modified in an $O A B$ condition. Theoretically, several factors can contribute to the development of an overactive bladder, including decreased suprapontine inhibition, decreased capacity of the central nervous system to handle afferent activity, increased afferent activity and increased sensitivity to efferent stimulation of the detrusor smooth muscle. ${ }^{80}$ The pathological condition underlying symptoms of the overactive bladder and/or detrusor overactivity is multifactorial and it is probably different in the various disorders. For example, in brain disorders such as stroke, Alzheimer's disease, Parkinson's disease, impairment in the cortical inhibition of the pontine micturition center might be the main pathological condition resulting in an overactive bladder. In spinal cord lesions (multiple sclerosis and spinal cord injuries) a decreased capacity to handle bladder sensations might be the main 
factor resulting in symptoms of an overactive bladder. On the other hand, several bladder conditions, such as bladder outlet obstruction, result in structural and functional bladder changes which might be the main determinant giving rise to overactive bladder symptoms. These symptoms may relay to an increased afferent activity and or increased muscular sensitivity to neurotransmitters. In this thesis we will focus on the bladder and study the structural and functional changes in the bladder induced by a bladder outlet obstruction possibly contributing to the $O A B$ syndrome.

\section{Bladder Outlet Obstruction}

Bladder outlet obstruction is associated with bladder dysfunction eventually leading to Lower Urinary Tract Symptoms (LUTS). LUTS compromise storage symptoms (daytime urinary frequency, nocturia, urgency, urinary incontinence) voiding symptoms (slow stream, splitting or spraying, intermittency, hestitancy, straining, terminal dribble) and postmicturition symptoms (sensation of incomplete emptying, postmicturition dribble). ${ }^{55}$ Storage LUTS include symptoms of the overactive bladder $(O A B)$ syndrome. LUTS have historically been linked to benign prostatic hyperplasia (BPH). Benign prostatic enlargement (BPE) and consequent bladder outflow obstruction were believed to be the basis for LUTS in male. However, several lines of evidence have questioned if these mechanisms can indeed explain LUTS in men. It was thought that the therapeutic effect of $\alpha_{1}$-adrenergic blocker was based on relaxing the prostatic smooth muscle and thereby reducing bladder outlet resistance. However, the mechanism for $\alpha_{1}$-adrenergic mediated symptom improvement appears to be independent of reducing bladder outlet resistance. ${ }^{81-82}$ Transurethral resection of the prostate (TURP) results in significant improvements in LUTS, however the effect is independent of changes in peak flow rate. ${ }^{83}$ Furthermore, LUTS also occur in women. It is suggested that there are common pathophysiological mechanisms in the pathophysiology of the $O A B$ independent of the prostate. Changes to the bladder and the bladder neck are obvious candidates.

\section{Animal model of bladder outlet obstruction}

Experiments in humans and human material raise legal, moral and ethical dilemmas. These studies are limited and it is unlikely they will alone result in a better understanding of changes to the bladder (neck), which in turn may lead to the development of $O A B$ syndrome. Therefore animal models of bladder outlet obstruction have been developed using several species, including rat, rabbit, guinea pig, and pig. Since, morphologically the guinea pig bladder has many similarities with the human bladder ${ }^{26,84-86}$ and pathologies produced by partial bladder outlet obstruction in guinea pigs are similar to those seen in $\operatorname{man}_{r}{ }^{87}$ we used for this thesis a guinea pig model for gradual bladder outlet obstruction. Under sterile conditions, the bladder neck and urethra were exposed via a vertical midline abdominal incision 
(Figs. 1A-B). A silver jeweller's jump ring (1.8 mm internal diameter) was placed loosely around the proximal urethra (Fig. 1C). Bladder outlet obstruction occurred during subsequent growth of the animal. The guinea pig model of partial bladder outlet obstruction will give more insight in the changes to the bladder structure and function probably resulting in an overactive bladder.

\section{Pathophysiology of bladder outlet obstruction}

Bladder outlet obstruction in humans and animals results in functional and structural bladder changes giving rise to LUTS. These functional and structural changes will be described briefly in the next sections.
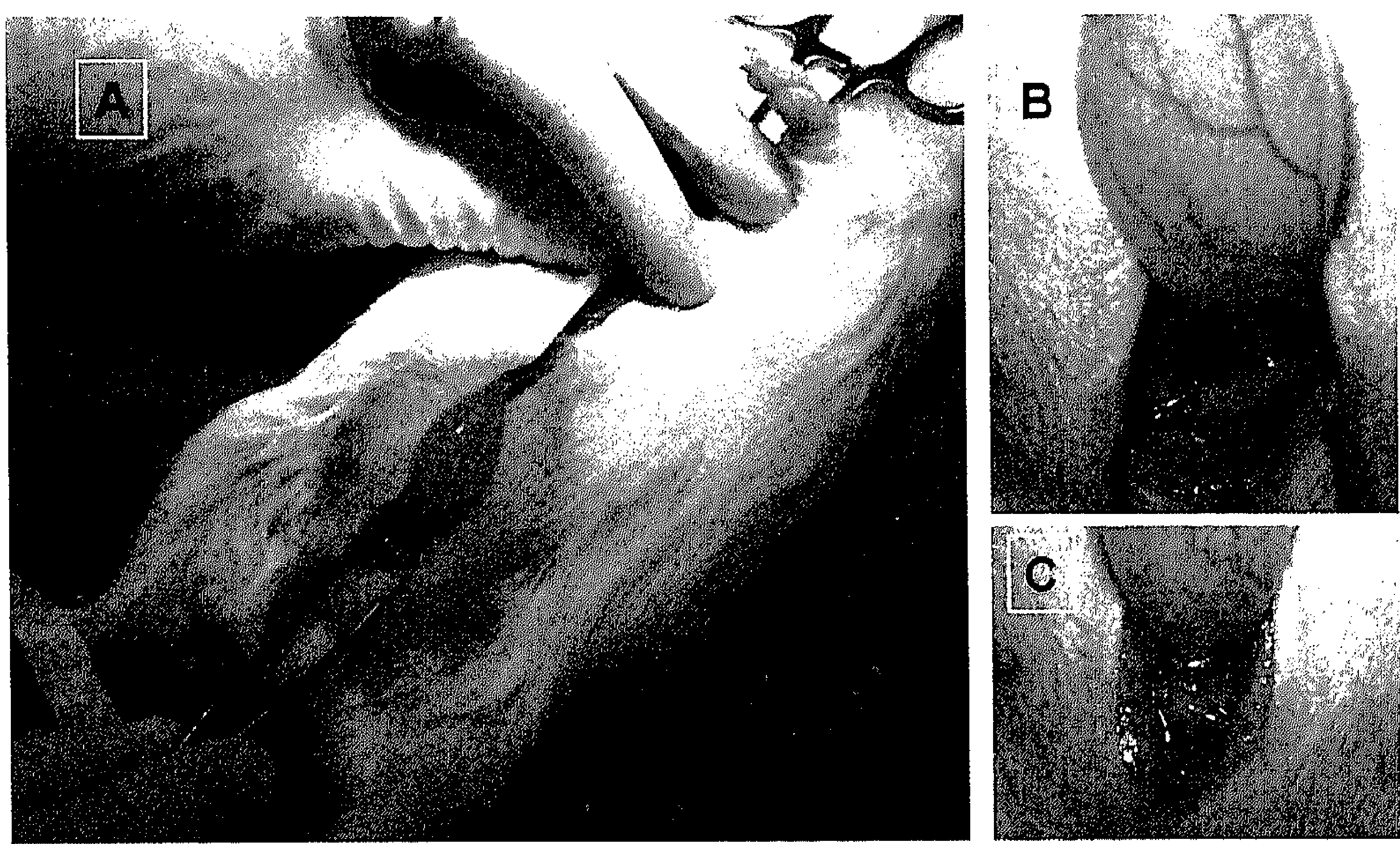

Figure 1. Surgical technique for bladder outlet obstruction in the guinea pig. (coloured image page 193) The bladder neck and urethra of the guinea pig were exposed via a vertical midline abdominal incision (Fig. 1A-B). A silver jeweller's jump ring was placed loosely around the proximal urethra (Fig. 1C). 
Chapter 1

\section{Functional changes}

Spontaneous activity and detrusor contractility in obstructed bladders

Smooth muscle strips dissected from obstructive overactive bladders often show increase in spontaneous contractile activity. ${ }^{88}$ These strips show fused tetanic contractions indicating that the cells in the strips are well coupled. ${ }^{88-89}$ In addition, bladders from rats exposed to an outlet obstruction show increased and more coordinated non micturition contractions (autonomous activity). ${ }^{90}$ Pathological modification to the autonomous activity in obstructed bladders might be related to the increased bladder sensations in patients with a bladder outlet obstruction.

Lots of studies examined alterations in detrusor contractility in obstructed bladders. Electrical field stimulation induced contractions are mainly based on nerve stimulation. The smooth muscle contraction of the urinary bladder in response to nerve stimulation is predominantly mediated by the release of the neurotransmitters acetylcholine and ATP. Several pharmacological studies performed on detrusor strips from obstructed bladders have shown a reduction in nerve mediated responses, compared to strips from normal bladders. ${ }^{91}$ This reduction is mainly based on denervation of the obstructed detrusor muscle. However, post-junctional alterations can also contribute to reduced field stimulation responses. Some studies have indeed reported reduced contractile responses to cholinergic agonists. ${ }^{92-93}$ In contrast, other studies reported unchanged responses ${ }^{94}$ or a relative supersensitivity to cholinergic agonists. ${ }^{68}$ Since potassium induces a receptor-independent contractile response via depolarization of the cell membrane, it allows examination of the intrinsic contractile properties of the bladder smooth muscle. Similar to the studies on muscarinic receptor agonists, various studies in obstructed bladders reported decreased contractile responses to potassium ${ }^{92}$ while others reported increased responses. ${ }^{68}$ The conflicting data on contractile responses to cholinergic agonists and potassium are probably related to the severity and/ or duration of outlet obstruction. Increased responses are observed in mild forms of bladder outlet obstruction whereas decreased responses are found in more severely obstructed bladders. ${ }^{95}$

\section{Bladder outlet obstruction and oxidative stress}

Levin and co-workers demonstrated that after a partial bladder outlet obstruction, the bladder function changes and mainly two stages develop. A compensated stage is characterized by an increase in bladder mass, a nearly normal muscle function and the appearance of detrusor overactivity. At some point, bladder function destabilizes and the organ enters a decompensated state characterized by a further increase in bladder mass, decreased contractile responses to pharmacological stimulation and an increase in post-void residual volume. ${ }^{96}$ The shift from a compensated to a decompensated state might be related to cyclical periods of ischemia-reperfusion during bladder contraction and emptying. ${ }^{97-98}$ Especially the increase in bladder wall thickness causes vascular compression and contributes to the cyclical periods of ischemia-reperfusion during bladder filling and micturition. During ischemic periods there is a release of intracellular $\mathrm{Ca}^{2+}$ from the sarcoplasmatic reticulum which can 
stimulate $\mathrm{Ca}^{2+}$ activated hydrolytic enzymes such as calpain and phospholipase. ${ }^{99}$ During the reperfusion period reactive nitrogen and oxygen species (RNOS) are formed. Reactive oxygen species (ROS) is a collective term that includes both oxygen radicals and certain nonradicals that are oxidizing agents and are easily converted into radicals. Reactive nitrogen species (RNS) is a collective term including nitric oxide and nitrogen dioxide radicals as well as non radicals such as nitrous acid $\left(\mathrm{HNO}_{2}\right)$. Free radicals are defined as molecules having an unpaired electron in the outer orbit. ${ }^{100}$ They are generally unstable and very reactive. Examples of oxygen free radicals are superoxide $\left(\mathrm{O}_{2}{ }^{-}\right)$, hydroxyl $\left(\mathrm{OH}^{\bullet}\right)$, peroxyl $\left(\mathrm{RO}_{2}{ }_{2}\right)$, alkoxyl $\left.(\mathrm{RO})^{*}\right)$ and hydroperoxyl $\left(\mathrm{HO}_{2}{ }_{2}\right)$ radicals. Nitric oxide $(\mathrm{NO} \cdot)$ and nitrogen dioxide $\left(\mathrm{NO}_{2}{ }_{2}\right)$ are two nitrogen free radicals. Oxygen and nitrogen free radicals can be converted to other non-radical reactive species, such as hydrogen peroxide $\left(\mathrm{H}_{2} \mathrm{O}_{2}\right)$, hypochlorous acid $(\mathrm{HOCl})$ and peroxynitrite (ONOO-). Free radicals have several crucial physiological functions such as smooth muscle relaxation, metabolism of xenobiotics and the respiratory burst to kill invading micro-organism. ${ }^{101}$ On the other hand, free radicals can have a damaging effect on practically all bio-molecules, including DNA, RNA, proteins, carbohydrates and lipids. The reaction with these molecules often starts with the subtraction of a hydrogen atom from the attacked molecule, thereby converting the unpaired electron into a more stable electron-pair. Alternatively, an electron instead of a hydrogen atom might be transferred. The hydrogen or electron donating molecule is oxidized.

Fortunately, the human body compromises an elaborate antioxidant defence system to protect cellular compounds from damage induced by free radicals. The antioxidants can be subdivided into enzymatic and non enzymatic antioxidants. Enzymatic antioxidants react with reactive species and are subsequently efficiently recycled. Important antioxidant enzymes are superoxide dismutases, catalase, glutathione peroxidases and glutathione reductases. Non enzymatic antioxidants can be divided into hydrophilic and hydrophobic antioxidants. Hydrophobic antioxidants include vitamin $E$, carotenoids, and ubiquinol-10 and are mostly present in lipoproteins and membranes. Hydrophilic scavengers include gluthathione, vitamin $C$ and uric acid. They can predominantly be found in cytosolic, mitochondrial and nuclear aqueous compartments. The most important endogenous hydrophilic antioxidants that contribute to the total antioxidant defence are gluthathione, vitamin $C$ and uric acid. In normal situation, the endogenous antioxidant network provides sufficient protection against free radicals. However, when an imbalance between the production of and the protection against reactive species occurs in favour of the production, a situation called oxidative stress arises. Oxidative stress may result in increased oxidative damage and can be caused either by an overproduction of free radicals or by an impairment of the endogenous antioxidant defence system. In situations such as ischemia-reperfusion and inflammation, free radicals may overwhelm the antioxidant defence system and oxidative damage to various molecules including proteins, DNA and lipids may occur. An important target of oxidation by ROS and RNS are the polyunsaturated fatty acids present in the cell 
membrane. Initiation of this reaction involves the subtraction of a hydrogen atom from the attacked fatty acid, thereby leaving an unpaired electron on the lipid. This newly-formed lipid radical undergoes molecular rearrangement to increase its stability and will then rapidly react with oxygen, thereby creating a peroxyl radical. Subsequently, this peroxyl radical will create a lipid hydroperoxide as well as a new lipid radical by subtracting a hydrogen atom from a second fatty acid and so on. This chain reaction is called lipid peroxidation. Peroxidation of fatty acids gives rise to various reactive aldehydes such as malondialdehyde (MDA) and 4-hydroxynonenal (HNE). HNE is highly reactive and produced in relative large amounts during lipid peroxidation. This reactive aldehyde is an important mediator of free radical damage. Bladder outlet obstruction is associated with increased levels of markers for oxidative stress. The levels of MDA, HNE and nitrotyrosine (end product of oxidative damage to proteins) are increased in bladders exposed to a partial bladder outlet obstruction. ${ }^{102-104}$ One hypothesis has been put forward that the denervation and muscle dysfunction observed after partial bladder outlet obstruction is partly mediated by oxidative stress. ${ }^{96}$ Several studies revealed that dietary antioxidant therapy, protect the bladder against functional and structural damage due to bladder outlet obstruction. ${ }^{105-107}$ In addition, therapies based on the relief of ischemia/reperfusion by increasing blood flow to the bladder ( $\alpha_{1}$-adrenoceptor antagonist) can reduce the severity of bladder dysfunction secondary to partial outlet obstruction. ${ }^{108}$

\section{Structural changes}

Muscle, connective tissue

Histological studies demonstrated that there are major structural changes in the bladder wall associated with bladder outlet obstruction. Smooth muscle cells show hypertrophy. In the bladder wall there is a patchy denervation; several muscle bundles are completely denervated, while in other bundles sparce innervation is seen. Bundles of smooth muscle become infiltrated by elastin and collagen. ${ }^{109-110}$ Electron microscopy studies in bladders from patients with an outlet obstruction revealed moderately widened intercellular spaces, abundance of distinctive protrusion junctions and close cell abutments. ${ }^{111}$ As discussed before interstitial cells have been identified in human and animal bladders. ${ }^{26}$ No data are yet available concerning changes to the network of interstitial cells in obstructed and or overactive bladders.

Afferent mechanism (nerves and urothelium)

Hyperexcitability of bladder afferent pathways, especially the C-fibre population, and/or increased number of afferent nerves, likely contributes to the emergence of overactive bladder symptoms. Vanilloid receptors (TRPV1) are present in the membrane of type $C$ primary afferent nerve fibres innervating the bladder wall and the periurethral zone. ${ }^{112-113}$ This receptor may be upregulated by nerve growth factor (NGF), a molecule detected in high concentrations in patients with a bladder outlet obstruction. ${ }^{114}$ The increased expression of NGF is accompanied by hypertrophy of 
afferent as well as efferent neurons. ${ }^{115-116}$ The increase in NGF content precedes the enlargement of bladder neurones and the development of urinary frequency. Blockade of the NGF in obstructed animals using auto-antibodies prevents neural plasticity and urinary frequency. ${ }^{117}$

The ice water test triggers a capsaicin-sensitive spinal micturition reflex mediated by unmyelinated $C$ fibres in the bladder and urethra. ${ }^{18-119} \mathrm{~A}$ positive ice water test has been demonstrated in $71 \%$ of the patients with a bladder outlet obstruction compared to $7 \%$ in patients without a bladder obstruction. ${ }^{119}$ This suggests that a bladder outlet obstruction in humans can lead to facilitation of the spinal micturition reflex. ${ }^{115-116,120}$ Desensitizing $C$ fibres with intravesical vanilloid or resiniferatoxin (RTX) would be an interesting treatment approach. The C-fibre afferents have endings in the suburothelial layer and can release tachykinins (substance $P$, cGRP) and other peptides. ${ }^{121}$ The peptides mediate several effects such as bladder contraction, facilitation of neural transmission and increased vascular permeability. ${ }^{121}$ It has been suggested in rats with bladder outlet obstruction that tachykinins can influence, via neurokinin receptors, both the spinal and supraspinal control of bladders. ${ }^{122-123}$ Smet et al have shown that the density of nerve fibres immunoreactive for substance $P$ and CGRP was significantly higher in patients with idiopathic detrusor overactivity compared to normal age-matched controls. ${ }^{124}$ In contrast another study demonstrated that a bladder outlet obstruction reduced the density of some neuropeptide containing nerves in the bladder muscle. ${ }^{125}$

The urothelium can release ATP, prostanoids, nitric oxide and acetylcholine during bladder distension..$^{21,125-126}$ Receptors for ATP, prostanoids have been demonstrated on sensory afferent nerves. It is likely that a cascade of stimulatory (ATP, prostanoids, acetylcholine, tachykinins) and inhibitory (NO) mediators are involved in the transduction mechanisms underlying the activation of sensory afferent fibres during bladder filling. Upregulation of this urothelial afferent transduction process may generate symptoms of overactive bladder. Several specific alterations in urothelial function and ultrastucture have been demonstrated in $O A B$. The expression of the mechanosensitive epithelium sodium channel $\mathrm{ENaC}$ is increased in human obstructed bladders compared to controls and correlates with storage LUTS. ${ }^{128}$ Increased expression of mechanosensitive channels in the urothelium may enhance substance release upon bladder stretch. Enhanced ATP release from urothelium is associated with aging and patients with LUTS secondary to bladder outlet obstruction. ${ }^{129-130}$ Release of acetylcholine is increased from urothelial and suburothelial sites with age and bladder stretch. ${ }^{25}$ Prostanoids are synthesized in detrusor and urothelium. The level of urinary prostaglandin $E 2$ is increased in women and men with $O A B$ symptoms compared to controls. ${ }^{131-132}$ Despite the above mentioned functional and structural changes, little is yet known what happens with the afferent nerves and urothelium/lamina propria in obstructed bladders.

\section{Therapeutic options}

Medical treatment of LUTS has been based primarily on three types of agents: 
$\alpha_{1}$ - adrenergic antagonists, $5 \alpha$-reductase inhibitors and phytotherapy. Since the pathophysiology of LUTS is multifactorial, there are many potential therapeutically targets. Some of these targets related to this dissertation will be briefly discussed.

$\alpha_{1}$ - adrenergic antagonist and $\beta_{3^{-}}$adrenergic agonist

Currently used $\alpha_{1}$-adrenergic receptor antagonists are effective for the treatment of LUTS associated with BPH. As discussed before, several lines of evidence have questioned the therapeutic effect of $\alpha_{1}$-adrenergic blocker based on relaxing the prostatic smooth muscle and subsequently reducing bladder outlet resistance. Studies with $\alpha_{1}$-adrenergic blockers have demonstrated symptom reduction assessed by IPSS (international prostate symptom score) or similar scores independent of maximum urinary flow rate. ${ }^{81-82}$ Pressure flow studies in males with LUTS have not consistently shown reductions of bladder outlet resistance upon treatment with $\alpha$ blockers. ${ }^{133}$ If the beneficial effects of $\alpha$-blockers are not due to relaxation of the prostatic smooth muscle then the question arises whether these drugs act on extraprostatic $\alpha_{1}$-adrenergic receptors. The bladder body in contrast to the bladder neck of the human detrusor contains only a few $\alpha_{1}$-adrenergic receptors. $\alpha_{1}$-adrenergic receptors are expressed on urothelium ${ }^{134}$ and human spinal cord..$^{135}$ These structures could be the site of action of $\alpha$-blockers. It could also be that the therapeutic effects are based on the relief of ischemia/reperfusion in the bladder wall. For instance, the smooth muscle of vessels contains $\alpha_{1}$-adrenergic receptors, and $\alpha_{1}$-adrenoceptor antagonists can induce vessel relaxation. $\alpha_{1}$-adrenoceptor antagonists already demonstrated to reduce the severity of bladder dysfunction in obstructed bladders probably via increasing the blood flow to the bladder. ${ }^{108}$

The bladder body of the human detrusor contains a high density of $\beta$-adrenergic receptors. Three subtypes of $\beta$-adrenergic receptors $\left(\beta_{1}, \beta_{2}\right.$, and $\left.\beta_{3}\right)$ are described. Recent studies revealed a predominant expression of $\beta_{3}$-adrenergic mRNA in human detrusor. ${ }^{47}$ Stimulation of $\beta_{3}$-adrenergic receptors results in relaxation of bladder smooth muscle. Animal studies have shown that $\beta_{3}$-adrenergic agonists increase bladder capacity with no change in micturition pressure and residual volume. ${ }^{136}$ Studies on safety and efficacy of $\beta_{3}$-adrenergic agonists in patients with bladder outlet obstruction are needed to assess their usefulness.

\section{Muscarinic receptor antagonist}

Muscarinic receptor antagonists show improvement of storage LUTS in many cases, but are limited by significant side effects such as dry mouth, blurred vision and constipation. ${ }^{137}$ As discussed before, conditions of bladder outlet obstruction can result in storage LUTS. Surprisingly, anticholinergic agents have historically not been studied in patients with a bladder outlet obstruction. It was thought that antimuscarinic agents in obstructed bladders would lead to urinary retention by inhibiting bladder contractions. Several studies of $M_{3}$ specific anticholinergics however, demonstrated that, at therapeutic effective doses, these drugs do not affect either the non-voiding contractions or the voiding contraction. ${ }^{138-139}$. In addition, 
anticholinergic drugs seem to be effective for the treatment of patients with LUTS, without the occurrence of urinary retention. ${ }^{140}$ Therefore, it can be implicated that anticholinergic drugs act on other structures instead of the detrusor muscle. Indeed, the effects of antimuscaric drugs have been linked to suppression of afferent nerve activity in animal models. ${ }^{141-142}$ Furthermore, immunohistochemical techniques in humans and animals showed that $M_{2}$ and $M_{3}$ receptors are located to the urothelium and to interstitial cells in the suburothelial and detrusor layer of the bladder. ${ }^{143-144}$ Thus, it can be suggested that anticholinergic drugs act on the urothelium, afferent nerves and interstitial cells instead of the detrusor muscle. ${ }^{75,145-146}$

\section{Cyclo-oxygenase inhibitors and prostanoid receptors antagonist}

Prostanoids (prostaglandin and thromboxanes) are generated in the detrusor muscle and urothelium. ${ }^{126,147-150}$ Two enzymes are responsible for prostaglandin production: cyclo-oxygenase 1 (COX I) and cyclo-oxygenase 2 (COX II). COX I is generally described as a constitutive form. COX II is also expressed constitutively in the brain and spinal cord but is more often described as an inducible form. Both forms of COX have been detected in the normal bladder. ${ }^{151}$

Several studies showed that exogenous prostaglandins can result in the appearance of non-voiding contractions and increase voiding frequency in different species (e.g. humans, rats, guinea pigs, rabbits and monkeys). ${ }^{152-163}$ Prostaglandins can act directly on smooth muscle ${ }^{156,162-163}$ and enhance myogenic activity. ${ }^{164}$ Furthermore, prostaglandins can alter sensory afferent nerve activity. ${ }^{126,165}$ It has been suggested by Maggi and co-workers that the prostaglandin induced myogenic activity plays a key role in the generation of bladder sensations via activation of afferent nerves. ${ }^{126,165}$ This concept is similar to the autonomous bladder theory where the non-micturition contractions are linked to the generation of bladder sensations. Prostaglandin production is initiated in the bladder by several stimuli such as stretching of the detrusor muscle, ${ }^{151}$ injuries of the mucosa, nerve stimulation and by agents such as ATP and mediators of inflammation ${ }^{24,126}$ Bladder outlet obstruction is associated with increased levels of urinary prostaglandins ${ }^{131-132}$ and increased expression of COX II in bladder tissue. ${ }^{167}$ Theoretically, the increased production of prostaglandins in obstructed bladders could play a role in the pathophysiology of storage LUTS. Controlled clinical trials with COX inhibitors for the treatment of the overactive bladder are scarce. Flurbiprofen and indomethacin treatment in patients with bladder instability ${ }^{168-169}$ results in symptomatic relief, however the incidence of side effects is high. Since prostanoid actions are mediated by specific receptors on cell membranes, prostanoid receptor antagonists could be helpful in treating storage LUTS. Prostanoid receptors include DP, EP, FP, IP and TP receptors that respond to $\mathrm{PGD}_{2}, \mathrm{PGE}_{2}, \mathrm{PGF}_{2 \alpha}, \mathrm{PGI}_{2}$ and $\mathrm{TXA}_{2}$, respectively. EP receptors are divided into $\mathrm{EP}_{1}$, $\mathrm{EP}_{2}, \mathrm{EP}_{3}$ and $\mathrm{EP}_{4}$ receptors. Preliminary animal studies revealed that $\mathrm{EP}_{1}$ receptor antagonists may have potential in the treatment of detrusor overactivity related to bladder outlet obstruction. ${ }^{170}$ 
Chapter 1

Vanilloid receptors

Vanilloid receptors (TRPV1) are present in the membrane of type C primary afferent nerve fibres innervating the bladder wall and the periurethral zone. ${ }^{112-113}$ Vanilloid receptors are also present on urinary bladder epithelial cells. ${ }^{20}$ Capsaicin and resiniferatoxin (RTX) can bind to specific vanilloid receptors on the peripheral terminal of nociceptive neurons. This initiates intracellular events which can be divided into 3 separate phenomena: excitation, desensitization and neurotoxity. ${ }^{171}$ Excitation of sensory neurons by vanilloids is followed by a refractory state. In this state the neurons do not respond to a subsequent vanilloid challenge and neurons are resistant to various stimuli. Desensitization of the afferent fibres in the bladder could contribute to the therapeutic effects of capsaicin and RTX in patients with a bladder outlet obstruction. Two preliminary studies with intravesical RTX treatment in patients with a bladder outlet obstruction showed improvement of storage LUTS whereas improvement of voiding LUTS was marginal. Urodynamic parameters such as maximum flow rate or postvoiding residual volume were not changed after treatment. ${ }^{172-173}$ These studies implicate that increased sensitivity of bladder afferent nerves, especially the $\mathrm{C}$-fibre population, plays a role in the emergence of overactive bladder symptoms in obstructed bladders.

\section{Phytotherapy}

Phytotherapeutic preparations (plant extracts) are used for the treatment of LUTS associated with benign prostatic hyperplasia (BPH). These plant preparations likely have antioxidative effects. The two most popular phytotherapeutic agents that are widely prescribed are Pygeum africanum (Tadenan), an extract from the bark of the African plum tree, and Serenoa repens (Permixon), a lipido-sterol extract of dwarf palm. Pygeum africanum provides a moderately large improvement in LUTS compared to placebo in the treatment of men with $\mathrm{BPH} .{ }^{174}$ The effects of serenoa repens on urinary symptoms related to $\mathrm{BPH}$ are conflicting. Initially a Cochrane review reported mild to moderate improvement in urinary symptoms. ${ }^{175}$ However, the latest placebo-controlled double blind randomized trial demonstrated that serenoa repens did not improve symptoms or objective measures of $\mathrm{BPH} .{ }^{176}$

\section{Outline of the thesis}

This dissertation focuses on abnormalities of the bladder and the bladder neck possibly contributing to the $O A B$ syndrome. After a general introduction on the different anatomical components involved in the storage and micturition phase of the lower urinary tract in Chapter 1, we discuss the autonomous bladder theory in more detail. Our hypothesis is that an increased autonomous activity plays a role in the occurrence of OAB symptoms. Patients with a bladder inflammation or a bladder outlet obstruction can develop symptoms of an overactive bladder. In order to test our hypothesis we describe the autonomous activity in pathological bladder 
conditions, such as a bladder outlet obstruction (BOO) and increased levels of prostaglandins in the bladder. In Chapter 2 we present the role of prostaglandins in modifying autonomous activity. The study showed that the autonomous activity was increased by prostaglandins. The mechanism(s) responsible for the origin of autonomous activity and how this activity is modulated by prostaglandins has not been elucidated yet. It has been postulated that autonomous activity is generated and distributed by a network of interstitial cells. Broadly, two subtypes of interstitial cells have been described lying in the suburothelial and muscle layer. Different immunohistochemical markers such as vimentin, c-Kit and cGMP have been used to identify interstitial cells. Since prostaglandins modify autonomous activity and this activity is possibly generated and distributed by interstitial cells, the structural relationship between cell types synthesizing endogenous prostaglandins and networks of interstitial cells was presented. Immunohistochemical techniques to localise cyclo-oxygenase 1 (COX I) and cyclo-oxygenase 2 (COX II) were used in combination with antibodies for interstitial cells (Chapter 2 and 3). In Chapter 4 we describe that damage to the bladder neck and or outlet obstruction in guinea pig bladders alters the autonomous activity and changes the sensitivity to cholinergic agonists. Since interstitial cells are thought to be responsible for the generation and modulation of autonomous activity, we present in Chapter $\mathbf{5}$ the effect of a bladder outlet obstruction on the network of interstitial cells. In addition, structural changes to the urothelium in obstructed bladders are briefly discussed.

Bladder outlet obstruction is associated with tissue denervation and muscle dysfunction which seem to be partly due to ischemia/reperfusion and inflammation in the bladder wall. Ischemia-reperfusion and inflammation are known to induce the production of reactive nitrogen and oxygen species (RNOS). The human body has its own endogenous protection against RNOS consisting of antioxidant enzymes and various antioxidants. In situations such as ischemia-reperfusion and inflammation, free radicals may overwhelm the antioxidant defense system; a situation denoted as oxidative stress. Oxidative stress is regarded as noxious because RNOS are able to destroy virtually any molecule, including proteins, DNA and lipids. Nerves and muscle are especially susceptible to oxidative stress induced damage. Antioxidants protect the obstructed bladder against functional and structural damage, suggesting that tissue of obstructed bladders have a reduced antioxidative capacity. Therefore, in Chapter 6 the effect of a partial bladder outlet obstruction on the bladder antioxidant capacity was described and correlated to bladder structure and function. If oxidative stress plays a significant role in the tissue injury of obstructed bladders, then direct application of free radicals over a relevant time period to bladder tissue at concentrations within the range found in vivo, could reproduce some of the tissue injury observed in obstructed bladders. In Chapter $\mathbf{7}$ and $\mathbf{8}$ the effects of oxidative stress in vitro on muscle contractility of pig urinary bladder are presented. The most sensitive component in the stimulation pathway of detrusor smooth muscle (cholinergic nerves, membrane-bound receptors, intracellular contractile mechanisms) to oxidative stress was determined. In the general discussion 
(Chapter 9), the results of the studies and the future aims are discussed and this dissertation ends with a summary (Chapter 10).

\section{References}

1. Holstege G, Griffiths D, de Wall H, Dalm E. Anatomical and physiological observations on supraspinal control of bladder and urethral sphincter muscles in the cat. J Comp Neurol. 1986; 250(4):449-61.

2. Kuipers R, Mouton $L$, Holstege G. Afferent projections to the pontine micturition center in the cat. J Comp Neurol. 2006; 494(1):36-53.

3. Holstege $G$. Descending motor pathways and the spinal motor system: limbic and non-limbic components. Prog Brain Res. 1991; 87:307-421.

4. de Groat WC, Yoshimura N. Pharmacology of the lower urinary tract. Annu Rev Pharmacol Toxicol. 2001; 41:691-721.

5. Habler $\mathrm{H} J$, Janig $\mathrm{W}$, Koltzenburg $M$. Activation of unmyelinated afferent fibres by mechanical stimuli and inflammation of the urinary bladder in the cat. J Physiol. 1990; 425:545-62.

6. Habler $\mathrm{HJ}$, Janig $W$, Koltzenburg $M$. Myelinated primary afferents of the sacral spinal cord responding to slow filling and distension of the cat urinary bladder. J Physiol. 1993; 463:44960.

7. Gabella G, Davis C. Distribution of afferent axons in the bladder of rats. I Neurocytol. 1998; 27(3):141-55.

8. Yoshimura N, de Groat WC. Neural control of the lower urinary tract. Int J Urol. 1997; 4(2):11125.

9. Lecci A, Giuliani S, Patacchini R, Maggi CA. Evidence against a peripheral role of tachykinins in the initiation of micturition reflex in rats. J Pharmacol Exp Ther. 1993; 264(3):1327-32.

10. Birder LA. More than just a barrier: urothelium as a drug target for urinary bladder pain. Am J Physiol Renal Physiol. 2005; 289(3):F489-95.

11. Wiseman OJ, Brady CM, Hussain IF, Dasgupta $P$, Watt $H$, Fowler $C$, et al. The ultrastructure of bladder lamina propria nerves in healthy subjects and patients with detrusor hyperreflexia. J Urol. 2002; 168(5):2040-5.

12. Gillespie JI, Markerink-van Ittersum M, de Vente J. Expression of neuronal nitric oxide synthase (nNOS) and nitric-oxide-induced changes in CGMP in the urothelial layer of the guinea pig bladder. Cell Tissue Res. 2005; 321(3):341-51.

13. Chopra B, Barrick SR, Meyers S, Beckel JM, Zeidel ML, Ford AP, et al. Expression and function of bradykinin B1 and B2 receptors in normal and inflamed rat urinary bladder urothelium. J Physiol. 2005; 562(Pt 3):859-71.

14. Birder LA, Ruan $H Z$, Chopra $B$, Xiang $Z$, Barrick $S$, Buffington $C A$, et al. Alterations in $P 2 X$ and $P 2 Y$ purinergic receptor expression in urinary bladder from normal cats and cats with interstitial cystitis. Am J Physiol Renal Physiol. 2004; 287(5):F1084-91.

15. Burnstock G, Knight GE. Cellular distribution and functions of P2 receptor subtypes in different systems. Int Rev Cytol. 2004; 240:31-304.

16. Birder LA, Nealen ML, Kiss S, de Groat WC, Caterina MJ, Wang E, et al. Beta-adrenoceptor agonists stimulate endothelial nitric oxide synthase in rat urinary bladder urothelial cells. J Neurosci. 2002; 22(18):8063-70.

17. Chess-Williams R. Muscarinic receptors of the urinary bladder: detrusor, urothelial and prejunctional. Auton Autacoid Pharmacol. 2002; 22(3):133-45.

18. Gotti C, Clementi F. Neuronal nicotinic receptors: from structure to pathology. Prog Neurobiol. 2004; 74(6):363-96.

19. Birder LA, Nakamura $Y$, Kiss $S$, Nealen ML, Barrick $S$, Kanai AJ, et al. Altered urinary bladder function in mice lacking the vanilloid receptor TRPV1. Nat Neurosci. 2002; 5(9):856-60. 
20. Birder LA, Kanai AJ, de Groat WC, Kiss S, Nealen ML, Burke NE, et al. Vanilloid receptor expression suggests a sensory role for urinary bladder epithelial cells. Proc Natl Acad Sci U S A. 2001; 98(23):13396-401.

21. Birder LA, Apodaca G, De Groat WC, Kanai AJ. Adrenergic- and capsaicin-evoked nitric oxide release from urothelium and afferent nerves in urinary bladder. Am J Physiol. 1998; 275(2 Pt 2):F226-9.

22. Burnstock G. Purine-mediated signalling in pain and visceral perception. Trends Pharmacol Sci. $2001 ; 22(4): 182-8$.

23. Ferguson DR, Kennedy I, Burton TJ. ATP is released from rabbit urinary bladder epithelial cells by hydrostatic pressure changes-a possible sensory mechanism? J Physiol. 1997; 505(Pt 2):503-11.

24. Mikhailidis DP, Jeremy JY, Dandona P. Urinary bladder prostanoids-their synthesis, function and possible role in the pathogenesis and treatment of disease. J Urol. 1987; 137(3):577-82.

25. Yoshida $M$, Inadome $A$, Maeda $Y$, Satoji $Y$, Masunaga $K$, Sugiyama $Y$, et al. Non-neuronal cholinergic system in human bladder urothelium. Urology. 2006; 67(2):425-30.

26. Smet PJ, Jonavicius J, Marshall VR, de Vente J. Distribution of nitric oxide synthaseimmunoreactive nerves and identification of the cellular targets of nitric oxide in guinea-pig and human urinary bladder by CGMP immunohistochemistry. Neuroscience. 1996; 71(2):337-48.

27. Gillespie JI, Markerink-van Ittersum M, de Vente J. cGMP-generating cells in the bladder wall: identification of distinct networks of interstitial cells. BJU Int. 2004; 94(7):1114-24.

28. MCCloskey KD, Gurney AM. Kit positive cells in the guinea pig bladder. J Urol. 2002; 168(2):8326.

29. Davidson RA, McCloskey KD. Morphology and localization of interstitial cells in the guinea pig bladder: structural relationships with smooth muscle and neurons. J Urol. 2005; 173(4):138590.

30. Brading AF, MCCloskey KD. Mechanisms of Disease: specialized interstitial cells of the urinary tract-an assessment of current knowledge. Nat Clin Pract Urol. 2005; 2(11):546-54.

31. Drake MJ, Fry CH, Eyden B. Structural characterization of myofibroblasts in the bladder. BJU Int. 2006; 97(1):29-32.

32. Sui GP, Rothery S, Dupont E, Fry CH, Severs NJ. Gap junctions and connexin expression in human suburothelial interstitial cells. BJU Int. 2002; 90(1):118-29.

33. Ikeda $Y$, Fry C, Hayashi $F$, Stolz D, Griffiths D, Kanai A. Role of gap junctions in spontaneous activity of the rat bladder. Am J Physiol Renal Physiol. 2007; 293(4):F1018-25.

34. Komuro T. Comparative morphology of interstitial cells of Cajal: ultrastructural characterization. Microsc Res Tech. 1999; 47(4):267-85.

35. MCCloskey KD. Calcium currents in interstitial cells from the guinea-pig bladder. BJU Int. 2006; 97(6):1338-43.

36. Wu $\mathrm{C}$, Sui GP, Fry $\mathrm{CH}$. Purinergic regulation of guinea pig suburothelial myofibroblasts. J Physiol. 2004; 559(Pt 1):231-43.

37. Ost $D$, Roskams T, Van Der Aa F, De Ridder $D$. Topography of the vanilloid receptor in the human bladder: more than just the nerve fibers. J Urol. 2002; 168(1):293-7.

38. de Groat WC. Anatomy and physiology of the lower urinary tract. Urol Clin North Am. 1993; 20(3):383-401.

39. Andersson KE, Persson K. The L-arginine/nitric oxide pathway and non-adrenergic, noncholinergic relaxation of the lower urinary tract. Gen Pharmacol. 1993; 24(4):833-9.

40. Sigala S, Mirabella G, Peroni A, Pezzotti G, Simeone C, Spano P, et al. Differential gene expression of cholinergic muscarinic receptor subtypes in male and female normal human urinary bladder. Urology. 2002; 60(4):719-25.

41. Wang $P$, Luthin GR, Ruggieri MR. Muscarinic acetylcholine receptor subtypes mediating urinary bladder contractility and coupling to GTP binding proteins. J Pharmacol Exp Ther. 1995; 273(2):959-66.

42. Andersson KE. Detrusor contraction-Focus on muscarinic receptors. Scand J Urol Nephrol Suppl 2004(215):54-7. 
43. D'Agostino G, Bolognesi ML, Lucchelli A, Vicini D, Balestra B, Spelta V, et al. Prejunctional muscarinic inhibitory control of acetylcholine release in the human isolated detrusor: involvement of the M4 receptor subtype. Br J Pharmacol. 2000; 129(3):493-500.

44. Inadome $A$, Yoshida $M$, Takahashi $W$, Yono $M$, Seshita $H$, Miyamoto $Y$, et al. Prejunctional muscarinic receptors modulating acetylcholine release in rabbit detrusor smooth muscles. Urol Int. $1998 ; 61(3): 135-41$.

45. Malloy BJ, Price DT, Price RR, Bienstock AM, Dole MK, Funk BL, et al. Alpha1-adrenergic receptor subtypes in human detrusor. J Urol. 1998; 160(3 Pt 1):937-43.

46. de Groat WC, Yoshiyama M, Ramage AG, Yamamoto T, Somogyi GT. Modulation of voiding and storage reflexes by activation of alpha1-adrenoceptors. Eur Urol. 1999; 36(Suppl 1):68-73.

47. Yamaguchi O. Beta3-adrenoceptors in human detrusor muscle. Urology. 2002; 59(5 Suppl 1):25-9.

48. Lee HY, Bardini M, Burnstock G. Distribution of $P 2 X$ receptors in the urinary bladder and the ureter of the rat. J Urol. 2000; 163(6):2002-7.

49. McMurray G, Dass N, Brading AF. Purinoceptor subtypes mediating contraction and relaxation of marmoset urinary bladder smooth muscle. Br J Pharmacol. 1998; 123(8):1579-86.

50. Obara K, Lepor $H$, Walden PD. Localization of P2Y1 purinoceptor transcripts in the rat penis and urinary bladder, J Urol. 1998; 160(2):587-91.

51. Lagou M, De Vente J, Kirkwood TB, Hedlund P, Andersson KE, Gillespie JI, et al. Location of interstitial cells and neurotransmitters in the mouse bladder. BJU Int. 2006; 97(6):1332-7.

52. Gillespie JI, Markerink-van Ittersum M, De Vente J. Interstitial cells and cholinergic signalling in the outer muscle layers of the guinea-pig bladder. BJU Int. 2006; 97(2):379-85.

53. Sanders KM. A case for interstitial cells of Cajal as pacemakers and mediators of neurotransmission in the gastrointestinal tract. Gastroenterology. 1996; 111(2):492-515.

54. Ward SM, Sanders KM, Hirst GD. Role of interstitial cells of Cajal in neural control of gastrointestinal smooth muscles. Neurogastroenterol Motil. 2004; 16(Suppl 1):112-7.

55. Abrams P, Cardozo L, Fall M, Griffiths D, Rosier P, Ulmsten U, et al. The standardisation of terminology in lower urinary tract function: report from the standardisation sub-committee of the International Continence Society. Urology. 2003; 61(1):37-49.

56. Milsom I, Abrams P, Cardozo L, Roberts RG, Thuroff J, Wein AJ. How widespread are the symptoms of an overactive bladder and how are they managed? A population-based prevalence study. BJU Int. 2001; 87(9):760-6.

57. Stewart WF, Van Rooyen JB, Cundiff GW, Abrams P, Herzog AR, Corey R, et al. Prevalence and burden of overactive bladder in the United States. World J Urol. 2003; 20(6):327-36.

58. Kobelt $G$. Economic considerations and outcome measurement in urge incontinence. Urology. 1997; 50(6A Suppl):100-7; discussion 108-10.

59. Brown JS, Posner SF, Stewart AL. Urge incontinence: new health-related quality of life measures. J Am Geriatr Soc. 1999; 47(8):980-8.

60. DuBeau CE, Kiely DK, Resnick NM. Quality of life impact of urge incontinence in older persons: a new measure and conceptual structure. J Am Geriatr Soc. 1999; 47(8):989-94.

61. Dugan E, Cohen SJ, Bland DR, Preisser JS, Davis CC, Suggs PK, et al. The association of depressive symptoms and urinary incontinence among older adults. J Am Geriatr Soc. 2000; 48(4):413-6.

62. Brown JS, Vittinghoff E, Wyman JF, Stone KL, Nevitt MC, Ensrud KE, et al. Urinary incontinence: does it increase risk for falls and fractures? Study of Osteoporotic Fractures Research Group. J Am Geriatr Soc. 2000; 48(7):721-5.

63. Hu TW, Wagner TH, Bentkover JD, Leblanc $K$, Zhou SZ, Hunt T. Costs of urinary incontinence and overactive bladder in the United States: a comparative study. Urology. $2004 ; 63(3): 461-5$.

64. Wyndaele JJ, De Wachter S. The sensory bladder (1): An update on the different sensations described in the lower urinary tract and the physiological mechanisms behind them. Neurourol Urodyn. 2008; 27(4):274-8.

65. Wyndaele J], De Wachter S. Cystometrical sensory data from a normal population: comparison of two groups of young healthy volunteers examined with 5 years interval. Eur Urol. 2002; 42(1):34-8. 
66. Digesu GA, Khullar V, Cardozo L, Salvatore S. Overactive bladder symptoms: do we need urodynamics? Neurourol Urodyn. 2003; 22(2):105-8.

67. Hashim $H$, Abrams $P$. Is the bladder a reliable witness for predicting detrusor overactivity? $]$ Urol. 2006; 175(1):191-4; discussion 194-5.

68. Brading AF. A myogenic basis for the overactive bladder. Urology. 1997; 50(6A Suppl):57-67; discussion 68-73.

69. de Groat WC. A neurologic basis for the overactive bladder. Urology. 1997; 50(6A Suppl):3652; discussion 53-6.

70. Gillespie JI. The autonomous bladder: a view of the origin of bladder overactivity and sensory urge. BJU Int. 2004; 93(4):478-83.

71. Sherrington. Notes on the arrangement of some motor fibres in the lumbo-sacral plexus. J Physiol. 1882(13):621-772.

72. Klevmark B. Motility of the urinary bladder in cats during filling at physiological rates. II. Effects of extrinsic bladder denervation on intramural tension and on intravesical pressure patterns. Acta Physiol Scand. 1977; 101(2):176-84.

73. Vaughan CW, Satchell PM. Role of sympathetic innervation in the feline continence process under natural filling conditions. J Neurophysiol. 1992; 68(5):1842-9.

74. Gillespie JI. A developing view of the origins of urgency: the importance of animal models. BJU Int. 2005; 96(Suppl 1):22-8.

75. Bristow SE, Neal DE. Ambulatory urodynamics. Br J Urol. 1996; 77(3):333-8.

76. Streng T, Hedlund P, Talo A, Andersson KE, Gillespie JI. Phasic non-micturition contractions in the bladder of the anaesthetized and awake rat. BJU Int. 2006; 97(5):1094-101.

77. Gillespie JI. Inhibitory actions of calcitonin gene-related peptide and capsaicin: evidence for local axonal reflexes in the bladder wall. BJU Int. 2005; 95(1):149-56.

78. Vaughan CW, Satchell PM. Urine storage mechanisms. Prog Neurobiol. 1995; 46(2-3):215-37.

79. Coolsaet BL, Van Duyl WA, Van Os-Bossagh P, De Bakker HV. New concepts in relation to urge and detrusor activity. Neurourol Urodyn. 1993; 12(5):463-71.

80. Andersson KE. Mechanisms of Disease: central nervous system involvement in overactive bladder syndrome. Nat Clin Pract Urol. 2004; 1(2):103-8.

81. Djavan B, Fong YK, Harik M, Milani S, Reissigl A, Chaudry A, et al. Longitudinal study of men with mild symptoms of bladder outlet obstruction treated with watchful waiting for four years. Urology. 2004; 64(6):1144-8.

82. Barendrecht MM, Abrams $P$, Schumacher $H$, de la Rosette J], Michel MC. Do alpha(1)adrenoceptor antagonists improve lower urinary tract symptoms by reducing bladder outlet resistance? Neurourol Urodyn. 2007; 27(3):226-230.

83. Lepor $H$, Rigaud $G$. The efficacy of transurethral resection of the prostate in men with moderate symptoms of prostatism. J Urol. 1990; 143(3):533-7.

84. Dixon JS, Gilpin SA, Gilpin CJ, Gosling JA. Intramural ganglia of the human urinary bladder. $\mathrm{Br}$ J Urol. 1983; 55(2):195-8.

85. Gabella G. Intramural neurons in the urinary bladder of the guinea-pig. Cell Tissue Res. 1990; 261(2):231-7.

86. Zhou $Y$, Ling EA. Colocalization of nitric oxide synthase and some neurotransmitters in the intramural ganglia of the guinea pig urinary bladder. J Comp Neurol. 1998; 394(4):496-505.

87. Mostwin JL, Karim OM, van Koeveringe G, Brooks EL. The guinea pig as a model of gradual urethral obstruction. J Urol. 1991; 145(4):854-8.

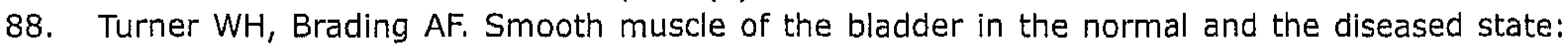
pathophysiology, diagnosis and treatment. Pharmacol Ther. 1997; 75(2):77-110.

89. Mills IW, Greenland JE, McMurray G, McCoy R, Ho KM, Noble JG, et al. Studies of the pathophysiology of idiopathic detrusor instability: the physiological properties of the detrusor smooth muscle and its pattern of innervation. J Urol. 2000; 163(2):646-51.

90. Drake MJ, Hedlund P, Harvey IJ, Pandita RK, Andersson KE, Gillespie JI. Partial outlet obstruction enhances modular autonomous activity in the isolated rat bladder. J Urol. 2003; 170(1):276-9.

91. Harrison SC, Hunnam GR, Farman P, Ferguson DR, Doyle PT. Bladder instability and denervation In patients with bladder outflow obstruction. $\mathrm{Br}$ J Urol. 1987; 60(6):519-22. 
92. Agartan CA, Whitbeck C, Chichester $P$, Kogan BA, Levin RM. Effect of age on rabbit bladder function and structure following partial outlet obstruction. J Urol. 2005; 173(4):1400-5.

93. Lluel $P$, Barras $M$, Palea 5. Cholinergic and purinergic contribution to the micturition reflex in conscious rats with long-term bladder outlet obstruction. Neurourol Urodyn. $2002 ; 21(2): 142$ 53.

94. Barendrecht MM, Chichester P, Michel MC, Levin RM. Effect of short-term outlet obstruction on rat bladder nerve density and contractility. Auton Autacoid Pharmacol. 2007; 27(1):47-53.

95. Michel MC, Barendrecht MM. Physiological and pathological regulation of the autonomic control of urinary bladder contractility. Pharmacol Ther. 2008; 117(3):297-312.

96. Levin RM, Levin SS, Zhao Y, Buttyan R. Cellular and molecular aspects of bladder hypertrophy Eur Urol. 1997; 32(Suppl 1):15-21.

97. Greenland JE, Hvistendahl JJ, Andersen H, Jorgensen TM, MCMurray G, Cortina-Borja M, et al The effect of bladder outlet obstruction on tissue oxygen tension and blood flow in the pig bladder. BJU Int, 2000; 85(9):1109-14.

98. Greenland JE, Brading AF. The effect of bladder outflow obstruction on detrusor blood flow changes during the voiding cycle in conscious pigs. J Urol. 2001; 165(1):245-8.

99. Zhao $Y$, Levin SS, Wein AJ, Levin RM. Correlation of ischemia/reperfusion or partial outlet obstruction-induced spectrin proteolysis by calpain with contractile dysfunction in rabbit bladder. Urology. 1997; 49(2):293-300.

100. Gilbert DL. Fifty years of radical ideas. Ann N Y Acad Sci. 2000; 899:1-14.

101. Bast A, Haenen GR, Doelman CJ. Oxidants and antioxidants: state of the art. Am J Med. 1991; 91(3C):2S-13S.

102. Mannikarottu $A$, Lin AD, Whitebeck $C$, Leggett $R$, Kogan $B$, Levin R. Effect of partial bladder outlet obstruction on nitrotyrosine levels and their correlation with contractile function. Neurourol Urodyn. 2006; 25(4):397-401.

103. Lin AT, Yang CH, Chen KK, Chang LS. Detrusor mitochondrial lipid peroxidation and superoxide dismutase activity in partial bladder outlet obstruction of rabbits. Neurourol Urodyn 2005; 24(3):282-7.

104. Saito M, Miyagawa I. Bladder dysfunction after acute urinary retention in rats. J Urol 2001; $165(5): 1745-7$

105. Conners $W$, Whitebeck $C$, Chicester $P$, Legget $R$, Lin AD, Johnson $A$, et al. L-NAME, a nitric oxide synthase inhibitor, diminishes oxidative damage in urinary bladder partial outlet obstruction. Am J Physiol Renal Physiol. 2006; 290(2):F357-63.

106. Levin RM, Kawashima Y, Leggett RE, Whitbeck C, Horan P, Mizutani K. Effect of oral Kohki tea on bladder dysfunction induced by severe partial outlet obstruction. J Urol $2002 ; 167(5): 2260$ 6.

107. Parekh $M H$, Lobel $R, O^{\prime}$ Connor $L J$, Leggett $R E$, Levin RM. Protective effect of vitamin $E$ on the response of the rabbit bladder to partial outlet obstruction. J Urol 2001; 166(1):341-6.

108. Das AK, Leggett RE, Whitbeck C, Eagen G, Levin RM. Effect of doxazosin on rat urinary bladder function after partial outlet obstruction. Neurourol Urodyn. 2002; 21(2):160-6.

109. Charlton RG, Morley AR, Chambers P, Gillespie JI. Focal changes in nerve, muscle and connective tissue in normal and unstable human bladder. BJU Int. 1999; 84(9):953-60.

110. Drake MJ, Hedlund P, Mills IW, McCoy R, McMurray G, Gardner BP, et al. Structural and functional denervation of human detrusor after spinal cord injury. Lab Invest. 2000; 80(10):1491-9.

111. Elbadawi A, Yalla SV, Resnick NM. Structural basis of geriatric voiding dysfunction. IV. Bladder outlet obstruction. ] Urol. 1993; 150(5 Pt 2):1681-95.

112. Yiangou $Y$, Facer $P$, Ford $A$, Brady $C$, Wiseman $O$, Fowler $C J$, et al. Capsaicin receptor VR 1 and ATP-gated ion channel P2X3 in human urinary bladder. BJU Int. $2001 ; 87(9): 774-9$.

113. Dinis P, Charrua A, Avelino A, Nagy I, Quintas 3, Ribau U, et al. The distribution of sensory fibers immunoreactive for the TRPV1 (capsaicin) receptor in the human prostate. Eur Urol. 2005; $48(1): 162-7$

114. Steers WD, Tuttle JB. Mechanisms of Disease: the role of nerve growth factor in the pathophysiology of bladder disorders. Nat Clin Pract Urol. 2006; 3(2):101-10. 
115. Steers WD, De Groat WC. Effect of bladder outlet obstruction on micturition reflex pathways in the rat. J Urol. $1988 ; 140(4): 864-71$.

116. Steers WD, Ciambotti J, Etzel B, Erdman S, de Groat WC. Alterations in afferent pathways from the urinary bladder of the rat in response to partial urethral obstruction. J Comp Neurol. 1991; 310(3):401-10.

117. Steers WD T]. Neurogenic inflammation and nerve growth factor: possible roles in interstitial cystitis. In: GR S, editor. Interstitial cystitis. Philadelphia: Lippincott-Raven; 1997. p. 67-75.

118. Geirsson $G$, Lindstrom $S$, Fall $M$. The bladder cooling reflex and the use of cooling as stimulus to the lower urinary tract. J Urol. 1999; 162(6):1890-6.

119. Chai TC, Gray ML, Steers WD. The incidence of a positive ice water test in bladder outlet obstructed patients: evidence for bladder neural plasticity. J Urol. 1998; 160(1):34-8.

120. Steers WD, Ciambotti J, Erdman S, de Groat WC. Morphological plasticity in efferent pathways to the urinary bladder of the rat following urethral obstruction. J Neurosci. 1990; 10(6):194351.

121. Maggi CA. Tachykinins and calcitonin gene-related peptide (CGRP) as co-transmitters released from peripheral endings of sensory nerves. Prog Neurobiol. 1995; 45(1):1-98.

122. Ishizuka $O$, Igawa $Y$, Lecci $A$, Maggi CA, Mattiasson A, Andersson KE. Role of intrathecal tachykinins for micturition in unanaesthetized rats with and without bladder outlet obstruction. Br J Pharmacol. 1994; 113(1):111-6.

123. Gu BJ, Ishizuka O, Igawa $Y$, Nishizawa $O$, Andersson KE. Role of supraspinal tachykinins for micturition in conscious rats with and without bladder outlet obstruction. Naunyn Schmiedebergs Arch Pharmacol. 2000; 361(5):543-8.

124. Smet PJ, Moore $\mathrm{KH}$, Jonavicius J. Distribution and colocalization of calcitonin gene-related peptide, tachykinins, and vasoactive intestinal peptide in normal and idiopathic unstable human urinary bladder. Lab Invest. 1997; 77(1):37-49.

125. Chapple CR, Milner $P$, Moss HE, Burnstock G. Loss of sensory neuropeptides in the obstructed human bladder. $\mathrm{Br}$ J Urol 1992; 70(4):373-81.

126. Maggi CA. Prostanoids as local modulators of reflex micturition. Pharmacol Res. $1992 ; 25(1)$ :1320.

127. Andersson $K E_{1}$, Wein $A J$. Pharmacology of the lower urinary tract: basis for current and future treatments of urinary incontinence. Pharmacol Rev. 2004; 56(4):581-631.

128. Araki I, Du S, Kamiyama M, Mikami $Y$, Matsushita K, Komuro M, et al. Overexpression of epithelial sodium channels in epithelium of human urinary bladder with outlet obstruction. Urology. 2004; 64(6):1255-60.

129. Sun Y, MaLossi J, Jacobs SC, Chai TC. Effect of doxazosin on stretch-activated adenosine triphosphate release in bladder urothelial cells from patients with benign prostatic hyperplasia. Urology. 2002; 60(2):351-6.

130. Yoshida M, Miyamae $K$, Iwashita $H$, Otani M, Inadome A. Management of detrusor dysfunction in the elderly: changes in acetylcholine and adenosine triphosphate release during aging. Urology. 2004; 63(3 Suppl 1):17-23.

131. Kim JC, Park EY, Seo SI, Park YH, Hwang TK. Nerve growth factor and prostaglandins in the urine of female patients with overactive bladder. J Urol. 2006; 175(5):1773-6; discussion 1776.

132. Kim JC, Park EY, Hong SH, Seo SI, Park YH, Hwang TK. Changes of urinary nerve growth factor and prostaglandins in male patients with overactive bladder symptom. Int J Urol. 2005; 12(10):875-80.

133. Kortmann BB, Floratos DL, Kiemeney LA, Wijkstra $H$, de la Rosette 3 J. Urodynamic effects of alpha-adrenoceptor blockers: a review of clinical trials. Urology. 2003; 62(1):1-9.

134. Ishihama H, Momota $Y$, Yanase H, Wang X, de Groat WC, Kawatani M. Activation of alpha1D adrenergic receptors in the rat urothelium facilitates the micturition reflex. J Urol. 2006; 175(1):358-64.

135. Smith MS, Schambra UB, Wilson KH, Page SO, Schwinn DA. Alpha1-adrenergic receptors in human spinal cord: specific localized expression of mRNA encoding alpha1-adrenergic receptor subtypes at four distinct levels. Brain Res Mol Brain Res. 1999; 63(2):254-61. 
136. Woods M, Carson N, Norton NW, Sheldon JH, Argentieri TM. Efficacy of the beta3-adrenergic receptor agonist CL-316243 on experimental bladder hyperreflexia and detrusor instability in the rat. $]$ Urol. 2001; 166(3):1142-7.

137. Dmochowski R. Antimuscarinic therapy in men with lower urinary tract symptoms: what is the evidence? Curr Urol Rep. 2006; 7(6):462-7.

138. Andersson KE, Yoshida M. Antimuscarinics and the overactive detrusor-which is the main mechanism of action? Eur Urol. 2003; 43(1):1-5.

139. Finney SM, Andersson KE, Gillespie JI, Stewart LH. Antimuscarinic drugs in detrusor overactivity and the overactive bladder syndrome: motor or sensory actions? BJU Int. 2006; 98(3):503-7.

140. Abrams P, Kaplan S, De Koning Gans HJ, Millard R. Safety and tolerability of tolterodine for the treatment of overactive bladder in men with bladder outlet obstruction. ] Urol. $2006 ; 175$ (3 Pt 1):999-1004; discussion 1004.

141. De Laet K, De Wachter S, Wyndaele J]. Systemic oxybutynin decreases afferent activity of the pelvic nerve of the rat: new insights into the working mechanism of antimuscarinics. Neurourol Urodyn. 2006; 25(2):156-61.

142. Yokoyama O, Yusup A, Miwa Y, Oyama N, Aoki $Y$, Akino H. Effects of tolterodine on an overactive bladder depend on suppression of C-fiber bladder afferent activity in rats. J Urol. 2005; 174(5):2032-6.

143. Mukerji G, Yiangou Y, Grogono J, Underwood J, Agarwal SK, Khullar V, et al. Localization of M2 and $\mathrm{M} 3$ muscarinic receptors in human bladder disorders and their clinical correlations. J Urol. 2006; 176(1):367-73.

144. Gillespie JI, Harvey IJ, Drake MJ. Agonist- and nerve-induced phasic activity in the isolated whole bladder of the guinea pig: evidence for two types of bladder activity. Exp Physiol. 2003; 88(3):343-57.

145. Fry $\mathrm{CH}$, Ikeda $\mathrm{Y}$, Harvey $\mathrm{R}, \mathrm{W} \cup \mathrm{C}$, Sui GP. Control of bladder function by peripheral nerves: avenues for novel drug targets. Urology. 2004; 63(3 Suppl 1):24-31.

146. de Groat WC. The urothelium in overactive bladder: passive bystander or active participant? Urology. 2004; 64(6 Suppl 1):7-11.

147. Brown WW, Zenser TV, Davis BB. Prostaglandin E2 production by rabbit urinary bladder. Am 3 Physiol. 1980; 239(5):F452-8.

148. Downie JW, Karmazyn M. Mechanical trauma to bladder epithelium liberates prostanoids which modulate neurotransmission in rabbit detrusor muscle. J Pharmacol Exp Ther. 1984; 230(2):445-9.

149. Jeremy JY, Tsang V, Mikhailidis DP, Rogers $H$, Morgan RJ, Dandona P. Eicosanoid synthesis by human urinary bladder mucosa: pathological implications. Br J Urol. 1987; 59(1):36-9.

150. Khan MA, Thompson CS, Mumtaz FH, Jeremy JY, Morgan RJ, Mikhailidis DP. Role of prostaglandins in the urinary bladder: an update. Prostaglandins Leukot Essent Fatty Acids. $1998 ; 59(6): 415-22$.

151. Park JM, Yang T, Arend LJ, Smart AM, Schnermann JB, Briggs JP, Cyclooxygenase-2 is expressed in bladder during fetal development and stimulated by outlet obstruction. Am J Physiol. 1997; 273(4 Pt 2):F538-44.

152. Bultitude MI, Hills NH, Shuttleworth KE. Clinical and experimental studies on the action of prostaglandins and their synthesis inhibitors on detrusor muscle in vitro and in vivo. $\mathrm{Br} \mathrm{J}$ Urol. $1976 ; 48(7): 631-7$.

153. Hills NH. Prostaglandins and tone in isolated strips of mammalian bladder [proceedings]. Br ] Pharmacol. 1976; 57(3):464P-465P.

154. Andersson KE, EK A, Persson CG. Effects of prostaglandins on the isolated human bladder and urethra. Acta Physiol Scand. 1977; 100(2):165-71.

155. Borda E, Contreras-Ortiz N, Gutnisky R, Gimeno MF. In vitro effect of acetylcholine and bethanechol on the contractions of the human detrusor muscle. Influence of prostaglandins. Arch Int Pharmacodyn Ther. 1982; 259(1):31-9.

156. Choo LK, Mitchelson F. The role of prostaglandins in the excitatory innervation of the rat urinary bladder. Prostaglandins. 1977; 13(5):917-26. 
157. Johns $A$. The effect of indomethacin and substance $P$ on the guinea pig urinary bladder. Life Sci. $1981 ; 29(17): 1803-9$.

158. Dean DM, Downie JW. Interaction of prostaglandins and adenosine $5^{\prime}$-triphosphate in the noncholinergic neurotransmission in rabbit detrusor. Prostaglandins. 1978; 16(2):245-51.

159. Husted S, Sjogren C, KE AN. Role of prostaglandins in the responses of rabbit detrusor to noncholinergic, non-adrenergic nerve stimulation and to ATP. Arch Int Pharmacodyn Ther. 1980; 246(1):84-97.

160. Andersson KE, Husted $S$, Sjogren C. Contribution of prostaglandins to the adenosine triphosphate-induced contraction of rabbit urinary bladder. Br J Pharmacol. 1980; 70(3):44352.

161. Downie JW, Larsson C. Prostaglandin involvement in contractions evoked in rabbit detrusor by field stimulation and by adenosine 5'-triphosphate. Can J Physiol Pharmacol. 1981; 59(3):25360.

162. Johns A, Paton DM. Evidence for a role of prostaglandins in atropine-resistant transmission in the mammalian urinary bladder. Prostaglandins. 1976; 11(3):595-7.

163. Johns A, Paton DM. Effect of indomethacin on atropine-resistant transmission in rabbit and monkey urinary bladder: evidence for involvement of prostaglandins in transmission. Prostaglandins, 1977; 13(2):245-54.

164. Andersson KE, Sjogren $C$. Aspects on the physiology and pharmacology of the bladder and urethra. Prog Neurobiol. 1982; 19(1-2):71-89.

165. Maggi CA, Giuliani $S$, Conte B, Furio $M$, Santicioli $P$, Meli $P$, et al. Prostanoids modulate reflex micturition by acting through capsaicin-sensitive afferents. Eur J Pharmacol. 1988; 145(2):10512.

166. Gilmore NJ, Vane JR. Hormones released into the circulation when the urinary bladder of the anaesthetized dog is distended. Clin Sci. 1971; 41(1):69-83.

167. Park JM, Yang T, Arend LJ, Schnermann JB, Peters CA, Freeman MR, et al. Obstruction stimulates COX-2 expression in bladder smooth muscle cells via increased mechanical stretch. Am J Physiol. 1999; 276(1 Pt 2):F129-36.

168. Cardozo LD, Stanton SL. A comparison between bromocriptine and indomethacin in the treatment of detrusor instability. J Urol. 1980; 123(3):399-401.

169. Cardozo LD, Stanton SL, Robinson H, Hole D. Evaluation of flurbiprofen in detrusor instability. $\mathrm{Br}$ Med J. 1980; 280(6210):281-2.

170. Lee $T$, Hedlund $P$, Newgreen $D$, Andersson KE. Urodynamic effects of a novel EP(1) receptor antagonist in normal rats and rats with bladder outlet obstruction. J Urol. 2007; 177(4):15627.

171. Szallasi A, Blumberg PM. Vanilloid (Capsaicin) receptors and mechanisms. Pharmacol Rev. 1999; 51(2):159-212.

172. Dinis $P$, Silva J, Ribeiro MJ, Avelino A, Reis M, Cruz F. Bladder C-fiber desensitization induces a long-lasting improvement of BPH-associated storage LUTS: a pilot study. Eur Urol. 2004; 46(1):88-93; discussion 93-4.

173. Kuo HC. Multiple intravesical instillation of low-dose resiniferatoxin is effective in the treatment of detrusor overactivity refractory to anticholinergics. BJU Int. 2005; 95(7):1023-7.

174. Wilt T, Ishani A, Mac Donald R, Rutks I, Stark G. Pygeum africanum for benign prostatic hyperplasia. Cochrane Database Syst Rev. 2002(1):CD001044.

175. Wilt T, Ishani A, Mac Donald R. Serenoa repens for benign prostatic hyperplasia. Cochrane Database Syst Rev. 2002(3):CD001423.

176. Bent 5, Kane C, Shinohara K, Neuhaus J, Hudes ES, Goldberg $H$, et al. Saw palmetto for benign prostatic hyperplasia. N Engl J Med. 2006; 354(6):557-66. 


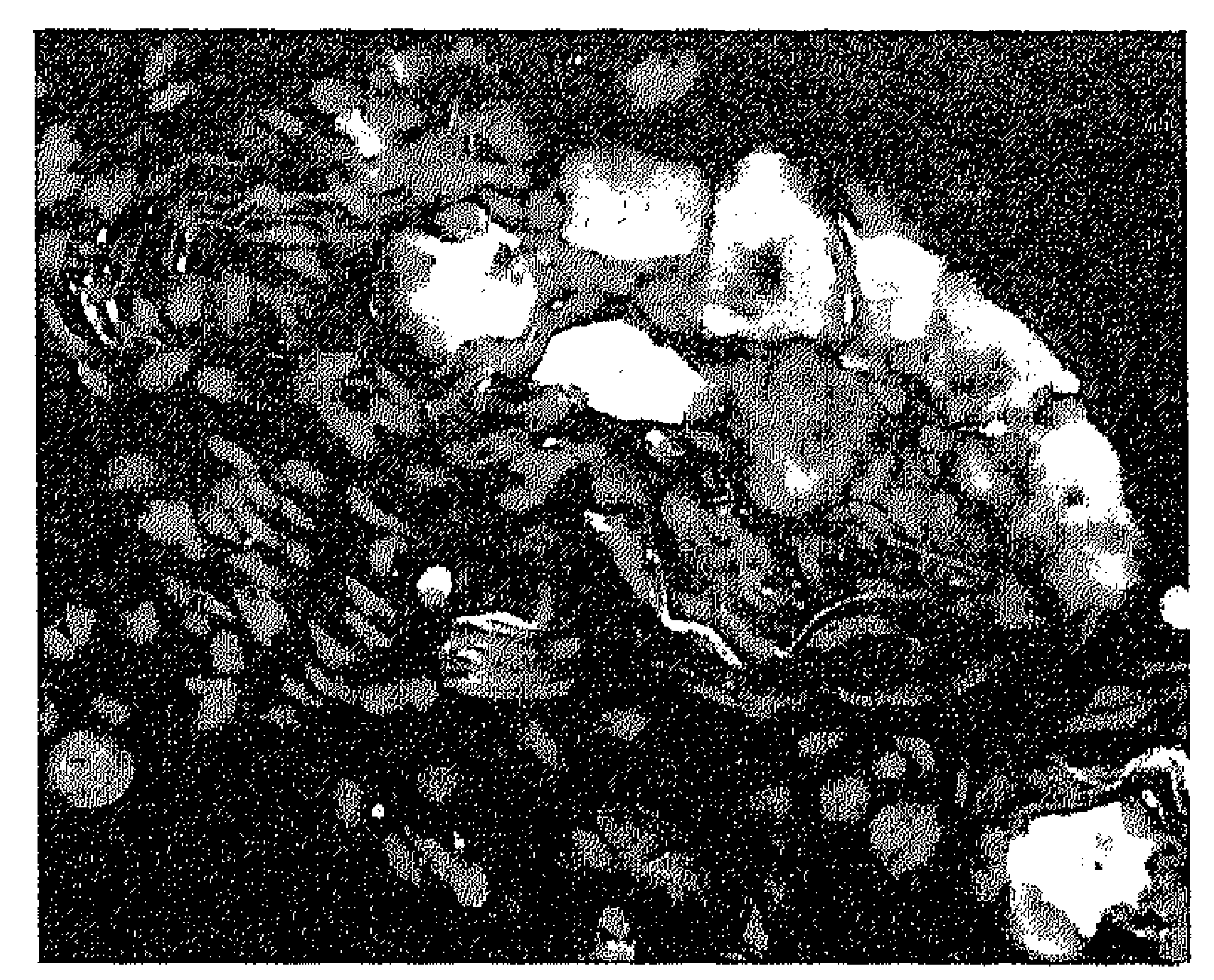

The effects of exogenous prostaglandins and the identification of constitutive cyclo-oxygenase I and II immunoreactivity in the normal guinea pig bladder

Rik de Jongh, Gommert A. van Koeveringe, Philip E.V. van Kerrebroeck, Marjanne Markerink-van Ittersum, Jan de Vente and James I Gillespie.

British Journal of Urology International 2007; 100 (2):419-29. 


\section{Abstract}

Aims: To establish the functional consequences of exposing the isolated whole bladder preparation to exogenous prostaglandins $\left(\mathrm{PGE}_{1}, \mathrm{PGE}_{2}, \mathrm{PGF}_{2 \alpha}\right)$ and to determine which cells express cyclo-oxygenase (COX) types I and II.

Materials and Methods: Fifteen female guinea pigs (270 - $350 \mathrm{~g})$ were used, i.e. seven for structural studies and eight for physiological measurement. For the structural study pieces of the lateral wall were incubated separately in Kreb's solution at $36{ }^{\circ} \mathrm{C}$, gassed with $95 \% \mathrm{O}_{2}$ and $5 \% \mathrm{CO}_{2}$ with $1 \mathrm{mM}$ isobutyl-methyl-xanthene (IBMX). Individual pieces were then exposed to $100 \mu \mathrm{M}$ of the nitric oxide (NO) donor NONOate for 10 minutes, control tissues remained in Kreb's solution. Tissues were then fixed in 4\% paraformaldehyde. For the physiological experiments bladders were isolated and a cannula inserted into the urethra to monitor intra-vesicle pressure. The bladders were suspended in a chamber containing carboxygenated physiological solution at $33-36^{\circ} \mathrm{C}$. All drugs were added to the abluminal bladder surface.

Results: In the resting bladder there were small spontaneous transient rises in pressure, i.e. autonomous activity. Exposure to $\mathrm{PGE}_{2}(3-300 \mathrm{nM})$ resulted in an increase in basal pressure on which were superimposed an autonomous activity, which was increased both in amplitude and frequency. The changes in the amplitude and frequency were dependent on the concentration of $P G E_{2}$. After a brief exposure (240 sec) to $\mathrm{PGE}_{2}$ the augmentation of the autonomous activity continued for over 60 minutes despite regular washing. The responses were similar with $P \mathrm{FE}_{1}$ but the responses to $\mathrm{PGF}_{2 \alpha}$ and arachadonic acid were reduced. The augmented activity was reduced by the EP1 / EP2 receptor blocking agent AH6809 (10 $\mu \mathrm{M})$. Using an antibody to the $70 \mathrm{kDa}$ constitutive form (COX I), COX I immuno-reactivity (COX IIR) was located to cells in the basal urothelium, in lamina propria and cells on the surface of the inner muscle bundles. There were few COX I-IR cells associated with the outer muscle bundles. The COX I-IR cells lying within the lamina propria were distinct from the sub-urothelial cells which respond to nitric oxide with a rise in CGMP. The lamina propria COX I-IR cells appeared to form a network surrounding muscle trabeculae within the inner muscle layer. COX II-IR was associated with the nuclei of cells in the urothelium, lamina propria and muscle.

Conclusions: These data show that prostaglandins regulate autonomous activity. Potential sources of endogenous prostaglandin are identified. It is unclear how the prostaglandins produced by these cells alter autonomous activity. There might be a direct activation of the muscle by prostaglandins released by the network of superficial muscle interstitial cells. It is also possible that prostaglandin released from the urothelium may influence phasic contractile activity via networks of COX I-IR interstitial cells. The possible roles and importance of this mechanism for bladder physiology and pathology are discussed. 


\section{Introduction}

It has been known for many decades that arachidonic metabolites, principally prostaglandins (PGs) are released into the general circulation in response to bladder distension. ${ }^{1}$ Both the mucosa and muscle layers of the bladder are reported to be capable of synthesising and releasing PGs in response to distension and to inflammation. ${ }^{1-4}$ It is now known that there are two enzymes responsible for PG production, i.e. cyclo-oxygenase 1 (COX I) and cyclo-oxygenase 2 (COX II), the former is generally described as a constitutive form. COX II is also expressed constitutively in the brain and spinal cord but is more often described as an inducible form, the expression of which is regulated by various stimuli including proinflammatory cytokines and growth factors. ${ }^{5}$ Both forms of COX have been detected in the normal bladder and there appears to be an increase in COX II expression immediately after experimentally induced bladder outflow obstruction. ${ }^{6}$ At present, there are few data to identify the precise physiological and patho-physiological role of $P G$ in the bladder and which cell types express the different COX enzymes.

Application of exogenous PGs can alter the bladder activity in different species, e.g. human, ${ }^{7-10}$ rat $^{1{ }^{1-13}}$ guinea pig, ${ }^{14-16}$ rabbit $^{17-21}$ and monkey ${ }^{21-22}$ suggesting that they might play key roles in both the normal and pathological bladder. Many ideas have been explored in order to identify the mode of action of the PGs. It was considered that they could exert their effects by acting directly or indirectly; e.g. direct actions on the bladder might involve PGs being co-released at efferent nerve endings to effect directly the smooth muscle ${ }^{21-22}$ or PGs may act pre-synaptically to affect the release of excitatory transmitter. ${ }^{12,13}$ Also, they might inhibit acetylcholine esterase ${ }^{10}$ or enhance myogenic bladder activity., ${ }^{7,23}$ These effects are complex and it has been difficult to identify which, if any, represents a major physiological mechanism. ${ }^{24}$

The present experiments were done to explore the possibility that PGs might be involved in the modulation of autonomous activity in the isolated whole bladder preparation. In addition, immuno-histochemical techniques were used to identify the cell types expressing COX I and COX II. The results show that PGs can modulate autonomous activity and the cyclo-oxygenase enzymes are located within a complex cellular network. The role of this system and the relevance of the structures expressing COX I and COX II are discussed in terms of basic bladder physiology and the origins of bladder pathology.

\section{Materials and Methods}

Guinea pigs ( $n=15$, weight range $270-350$ g) were killed by stunning and exsanguination. The urinary bladder was removed and placed in Kreb's solution: $(\mathrm{mM}): \mathrm{NaCl}, 121 . ; \mathrm{KCl} 1.87 ; \mathrm{CaCl}_{2} 1.2 ; \mathrm{MgSO}_{4} 1.15 ; \mathrm{NaHCO}_{3} 25 ; \mathrm{KH}_{2} \mathrm{PO}_{4}$ 1.17; glucose 11.0 and bubbled with $5 \% \mathrm{CO}_{2}$ and $95 \% \mathrm{O}_{2}(\mathrm{pH} 7.4)$.

The procedures for isolation, stimulation with $\mathrm{NO}$ and detection of CGMP have 
been described previously. ${ }^{25}$ The lateral wall bladder was dissected into sections, approximately $20 \mathrm{~mm}^{2}$, and maintained in Kreb's solution containing $1 \mathrm{mM}$ of the non-specific phosphodiesterase inhibitor isobutyl-methyl-xanthine (IBMX: SigmaAldrich) at $36^{\circ} \mathrm{C}$ for 30 minutes. Tissue pieces were maintained in $2 \mathrm{ml}$ of solution. Tissues were either stimulated with $100 \mu \mathrm{M}$ of the NO donor diethylamine-NONOate (DEANO) (Sigma-Aldrich) or left in control medium containing only IBMX. The NO donor was prepared immediately before use, added directly to the bathing solution and incubated for 10 minutes.

For physiological measurements, a flexible plastic cannula ( $2 \mathrm{~mm}$ diameter) was inserted into the urethra and secured at the bladder neck using a thread ligature. Residual urine was removed and replaced with Kreb's solution. The bladder was transferred to a heated organ bath (volume $30 \mathrm{ml}$, temperature $33-36{ }^{\circ} \mathrm{C}$ ) containing constantly-gassed Kreb's solution. The cannula was connected with the urethra uppermost via a fluid-filled tube and 3-way connector to a pressure transducer (Becton Dickinson DTX Plus) and a $1 \mathrm{ml}$ syringe to enable variation of the intra-vesicle volume. The transducer output was amplified, digitized (sampling rate $10 \mathrm{~Hz}$ ) and recorded using an Acqknowledge AD capture system. The transducer was calibrated before each experiment. At the outset each bladder was filled with Kreb's solution to give a baseline volume of $1000-1200 \mu \mathrm{l}$. These volumes were typically the amount of urine recovered from animals immediately after cervical dislocation. The time taken from killing the animal to beginning recording was typically 30 minutes. Pressure recording was begun immediately but the bladder was left to equilibrate for at least $20 \mathrm{~min}$ before any manipulations. Drugs used were from Tocris, UK, Bristol. Concentrated drug solutions were added directly to the bath to achieve the required final dilution. All drugs were added to the solution bathing the serosal surface of the isolated whole bladder.

Quantitative values are given as mean ( \pm 1 S.D) and differences between means were determined by a paired t-test and the level for significance set at 0.05 . The number of preparations used in the statistical analysis for each experiment and each condition are given in the appropriate figure legends.

The method for detecting CGMP in tissues sections has been described previously. ${ }^{6}$ Incubations were terminated by immersing bladder pieces in ice-cold fixative solution of $4 \%$ freshly prepared depolymerised paraformaldehyde for $120 \mathrm{~min}$ at $4{ }^{\circ} \mathrm{C}$. Then tissues were washed at $4^{\circ} \mathrm{C}$ in $0.1 \mathrm{M}$ phosphate buffer containing $10 \%$ sucrose ( 24 h), $20 \%$ sucrose $(24 \mathrm{~h})$ and $30 \%$ sucrose $(24 \mathrm{~h})$. The tissues from the lateral wall were then snap-frozen with $\mathrm{CO}_{2}$ in Tissue-Tek O.C.T. compound to form a single block. Cryostat sections $(10 \mu \mathrm{m})$ were cut, such that each section was perpendicular to the urothelial surface. Sections were then thawed on to chrome-alumn-gelatincoated slides and processed for immunocytochemistry. Sections were dried for 20 min at RT followed by three washes with Tris-buffered saline (TBS; pH 7.6), and thereafter incubated overnight with primary antibodies at $4^{\circ} \mathrm{C}$. To visualize cGMP we used rabbit anti-formaldehyde fixed-cGMP $(1: 150)$. Sections were co-stained with goat polyclonal antibody to COX I ( 1 : 2000) (Santa Cruz) and COX II 
(1:200) (Santa Cruz). After overnight incubation with the primary antibodies diluted in TBS containing $0.3 \%(\mathrm{~V} / \mathrm{v})$ Triton $X-100($ TBS-T), sections were washed in TBS, TBS-T and TBS; each wash step lasted $10 \mathrm{~min}$. Rabbit primary antibody was visualized using Alexa Fluor 488 donkey anti-rabbit IgG conjugate (Molecular Probes), diluted 1 : 100 in TBS-T. Goat primary antibodies were visualized with CY3 donkey anti-goat IgG conjugate (Jackson), diluted $1: 800$. Sections were incubated with the secondary antibodies for $60 \mathrm{~min}$ at room temperature in the dark. After washing with TBS-T, and TBS, sections were mounted with TBS-glycerol.

Typically, for each bladder and for each antibody combination staining was done in duplicate and repeated on at least 2 separate days. Observations were accumulated from the different slides and from the different bladders.

Sections were analysed and photographed using an Olympus AX70 microscope equipped with a cooled CCD Olympus Digital video camera F-view. For the detection of Alexa 488 fluorescence we used a narrow band-pass MNIBA-filter and for the detection of $\mathrm{CY} 3$ we used a filter with a narrow excitation band, the U-M41007A filter (both filters are from Chroma Technologies). Images were stored digitally by using the computer program analySIS ${ }^{\circledR}$ V.3.0. (Soft Imaging System, Münster, Germany) and arranged with the program Adobe Photoshop 5.5 or 7.01 (San Jose, CA, USA).

\section{Results}

In the isolated whole bladder, application of $\mathrm{PGE}_{2}$ resulted in distinct changes in bladder activity. Figure 1 illustrates an example from one bladder. Before applying $\mathrm{PGE}_{2}$ small transient rises in pressure were recorded, i.e. autonomous activity. A section of this control period is shown in figure $1 \mathrm{Ba}$ on an expanded time scale. On applying $\mathrm{PGE}_{2}$ there was a slow small rise in bladder pressure of approximately $1 \mathrm{~cm}$ $\mathrm{H}_{2} \mathrm{O}$. Superimposed upon this rise were rapid frequent transient pressure changes, the amplitude and frequency of these augmented transients was greater compared to the basal autonomous activity.

Figure 2 show data from a different bladder illustrating further details of the action of $P G E_{2}$. Panel A shows the result of a brief exposure $(240 \mathrm{sec})$ to $300 \mathrm{nM}$ $\mathrm{PGE}_{2}$. As in figure 1 there was a rise in basal pressure and an increase in the amplitude and frequency of the autonomous activity. This initial section of the records is shown on an expanded time scale in panel $B$. The dramatic increase in activity is clearly seen. After the exposure to $\mathrm{PGE}_{2}$ the bladder was washed every 15 minutes over the next hour. Despite this continual washing the response to PGE persisted only gradually declining over an hour, eventually approaching the control values. Panel $C$ show the effects of 250 second exposure to different concentrations of $P G E_{2}$. As the concentration of $P_{G E}$ is increased the effects on the magnitude of the basal change and the frequency and amplitude of the transients changed proportionately. Panel $D$ shows that the responses to $P G E_{2}$ were not inhibited by the non-specific muscarinic antagonist atropine. Thus, there is no cholinergic 
A

Figure 1.
B

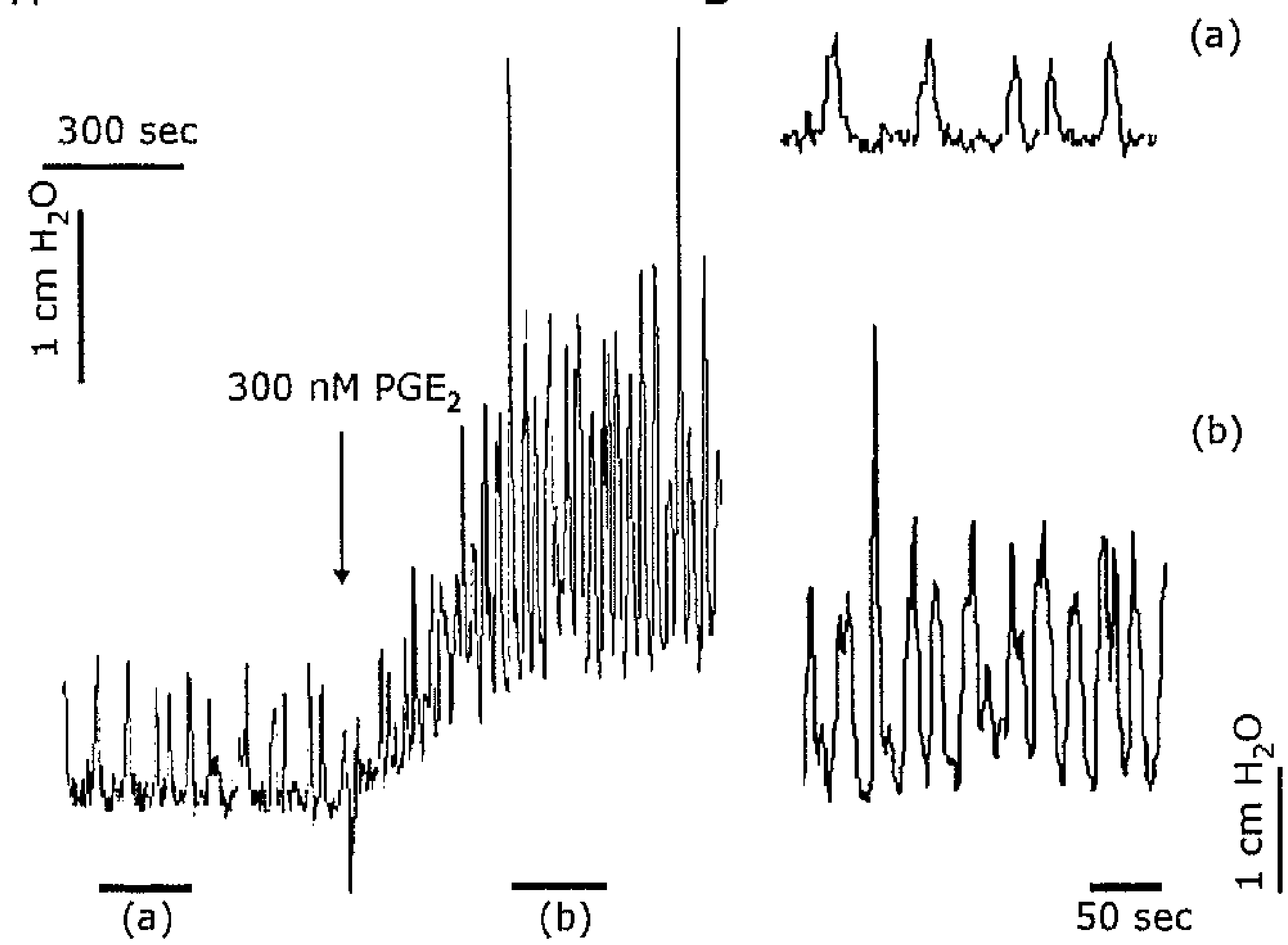

The effects of $\mathrm{PGE}_{2}$ on autonomous activity in the isolated whole bladder of the guinea pig. $\boldsymbol{A}$ illustrates the early part of an experimental run where a bladder was exposed to $300 \mathrm{nM} P G E_{2}$. PGE 2 was added where indicated by the arrow. Two sections of this record are shown in B. (a) is taken from the period before application of $P G E_{2}$, while (b) is during the application. Time and pressure calibration bars are as shown. All experiments were done at $34^{\circ} \mathrm{C}$.
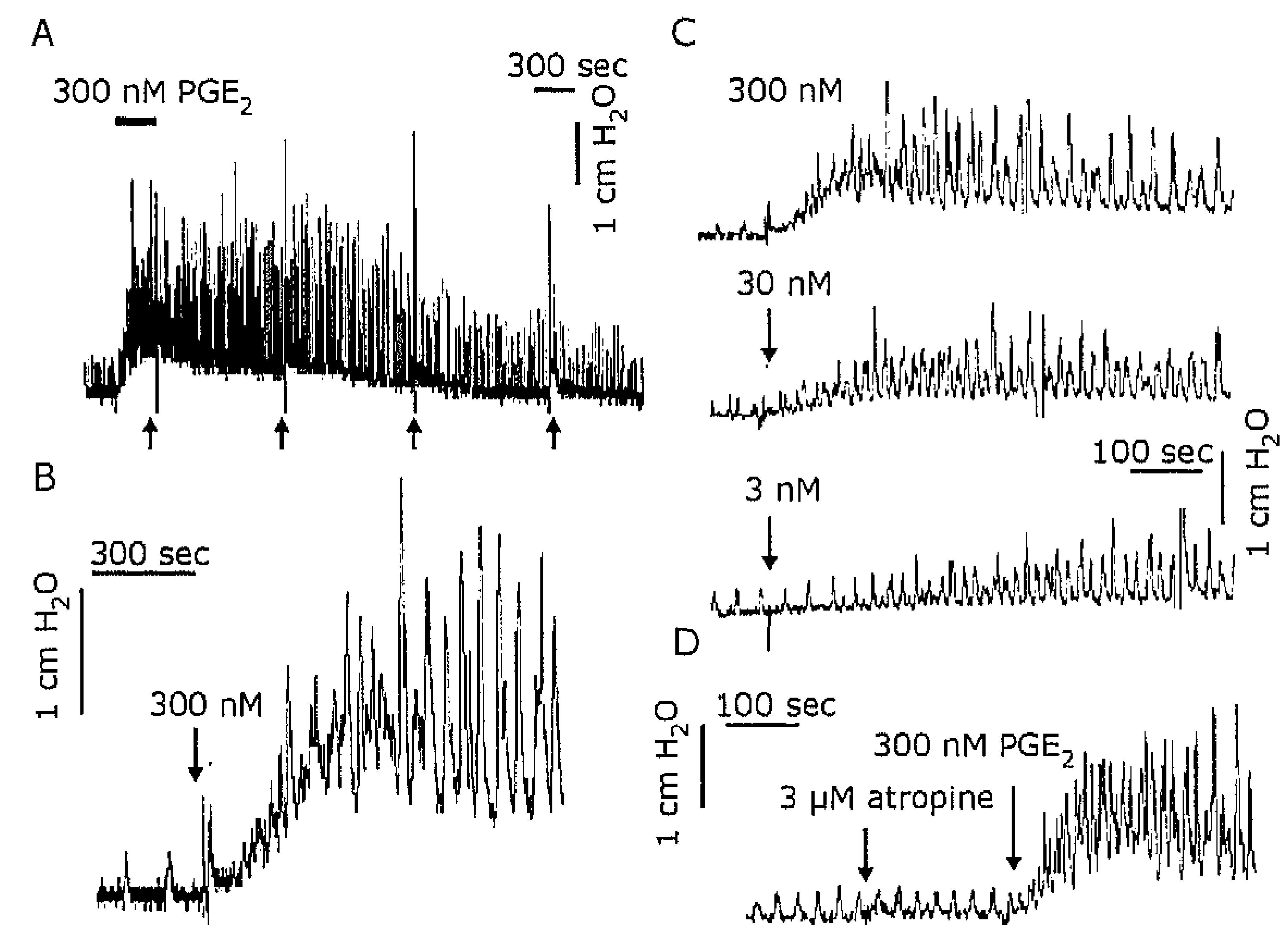

\section{Figure 2.}

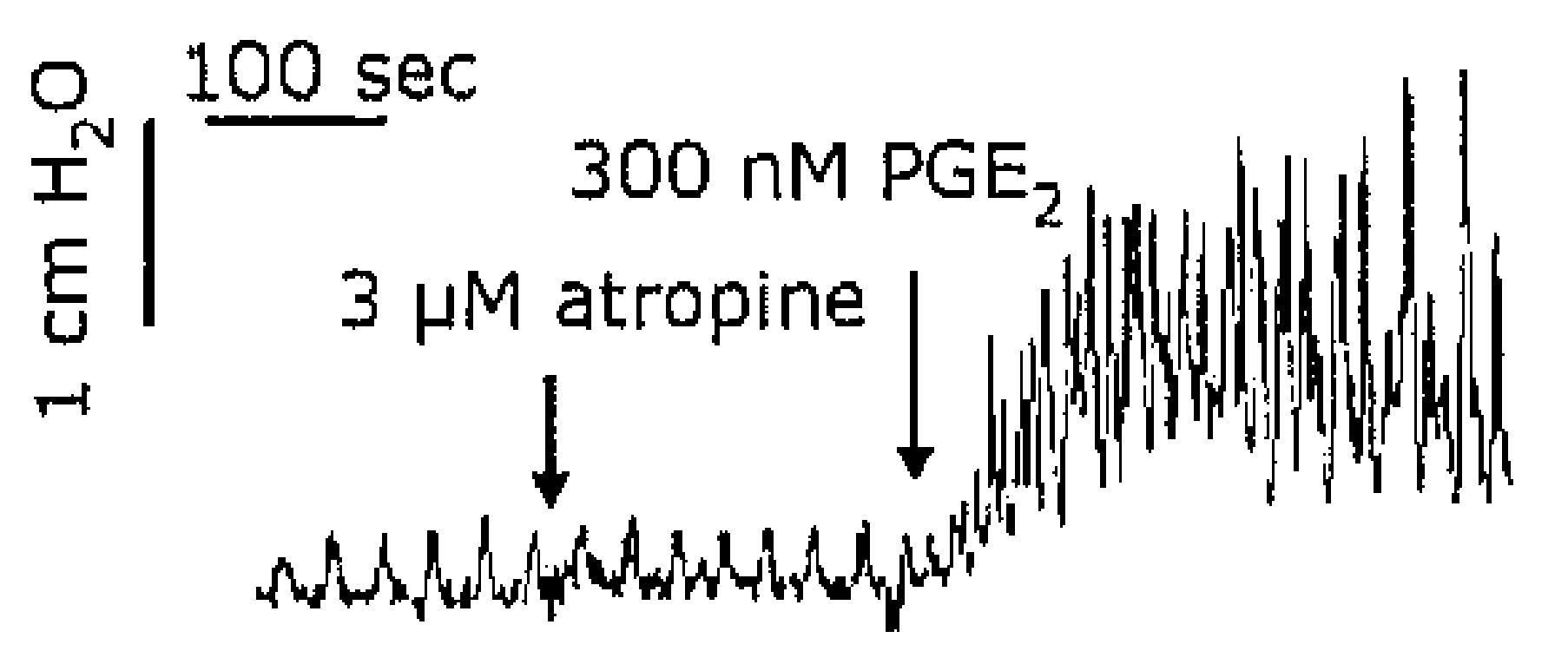

The effects of prostaglandin $E 2$ on autonomous activity in the isolated whole bladder of the guinea pig. A shows the effect of a brief exposure to $300 \Pi M P G E_{2}$ (240 seconds) on the autonomous activity in an isolated whole bladder preparation. A shows data from the entire experimental run lasting $>1 \mathrm{hr}$. PGE 2 was given where indicated by the horizontal bar. At each of the arrows the preparation was washed in normal Kreb's solution. B shows the first section of the record on an expanded timescale. $\boldsymbol{C}$ shows the effects of 300, 30 and $3 \cap M P G E_{2}$. D demonstrates that pre-treatment with the non-specific muscarinic antagonist atropine $(3 \mu M)$ has no effect of the response to $P_{G E_{2}}$. Time and pressure calibration bars are as shown. All experiments were done at $34^{\circ} \mathrm{C}$. 
A

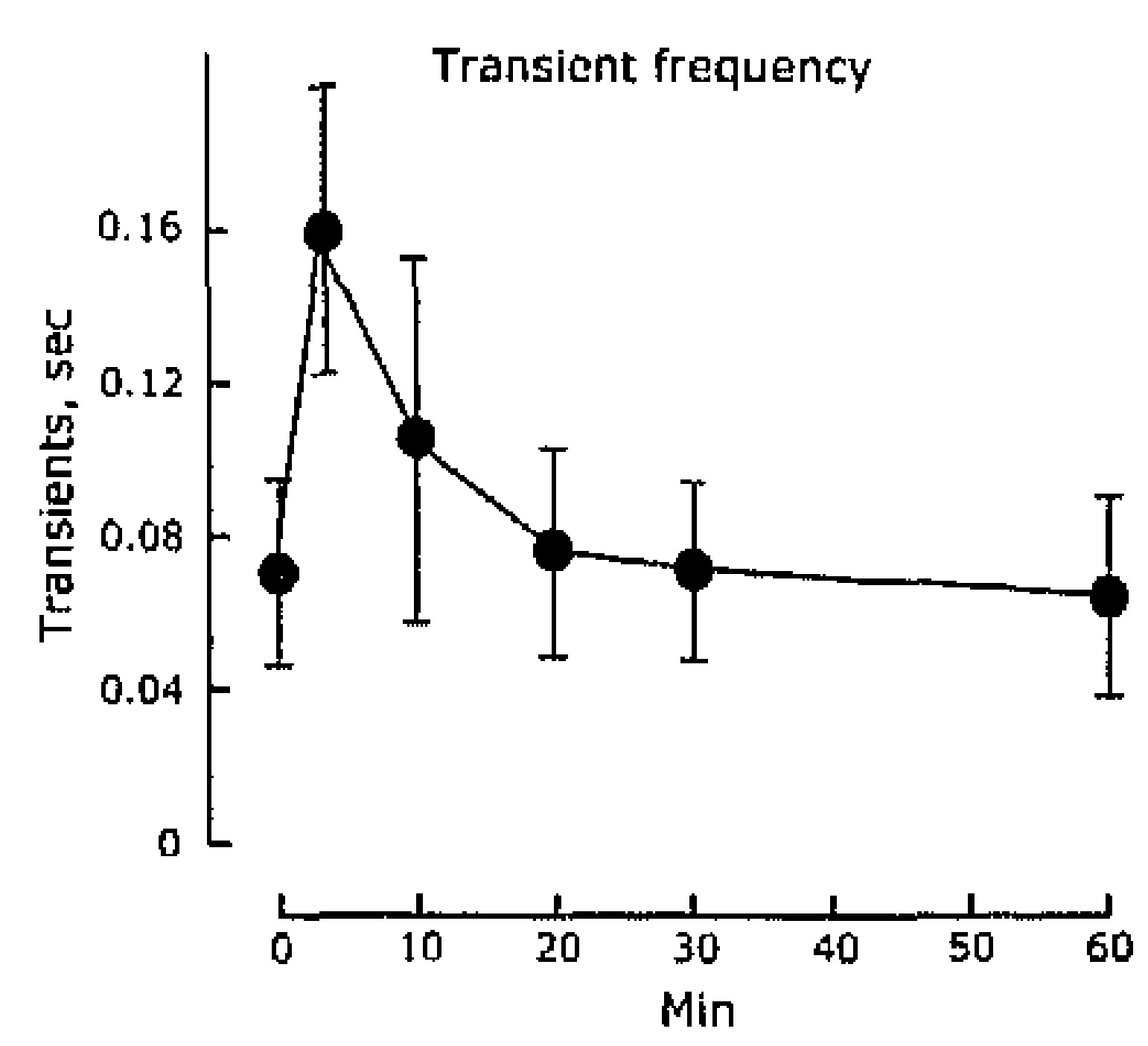

B

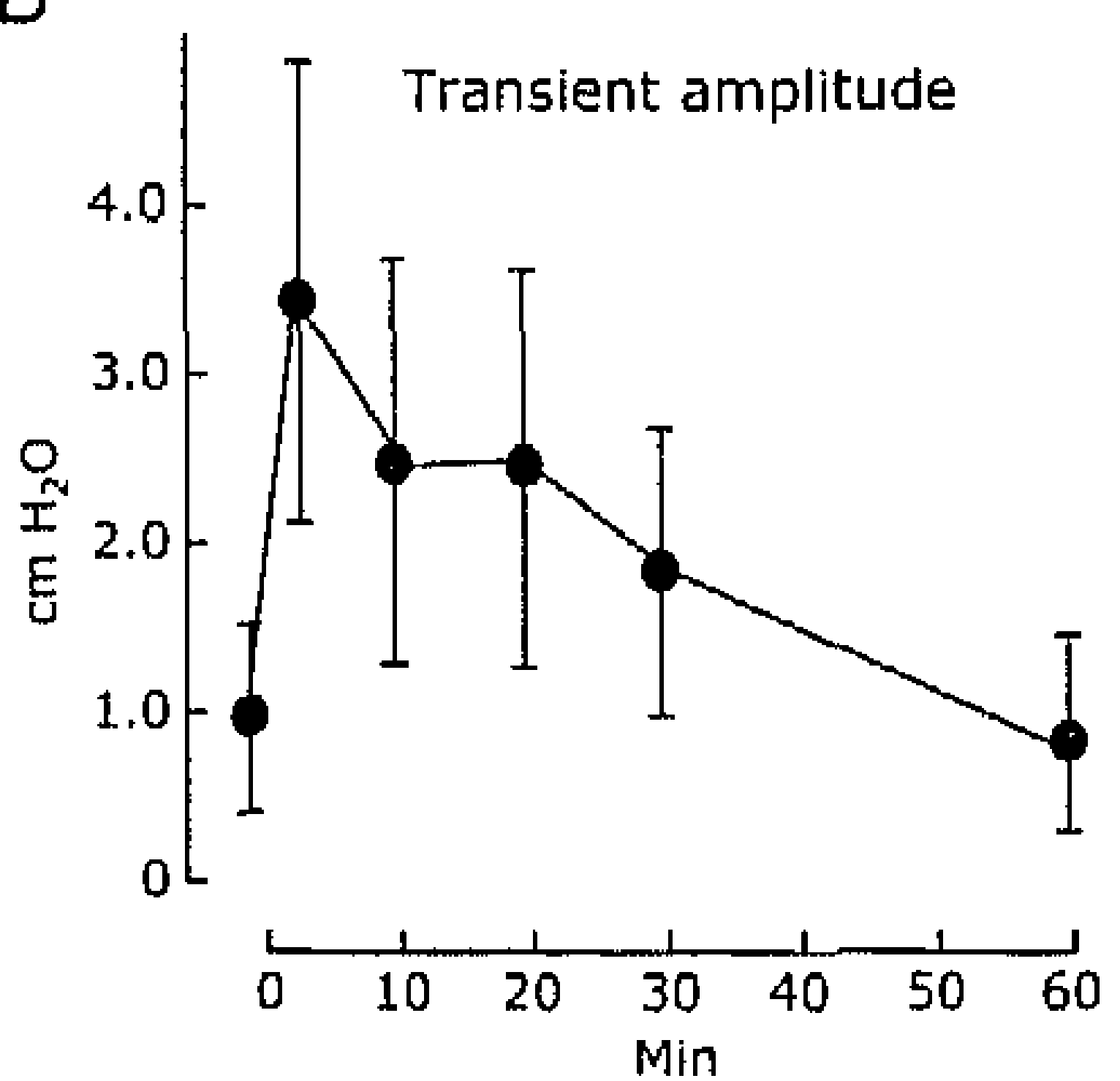

C

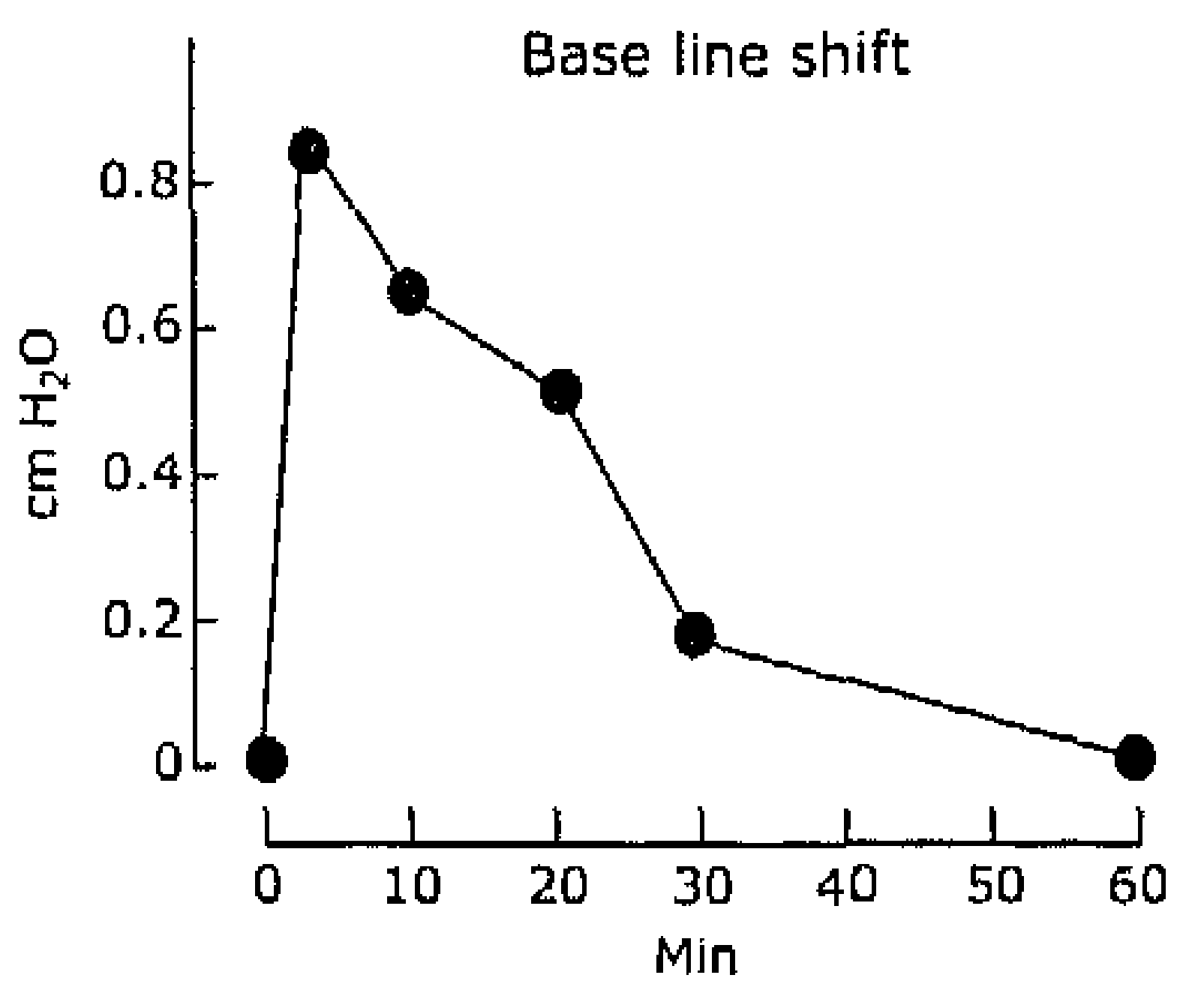

Figure 3.

Data collected from six different experiments showing the effects of $300 \mathrm{nM} \mathrm{PGE}_{2}$. A and B show the effects on the frequency and amplitude of the transients. Data are mean values \pm 1 S.D. $C$ shows the mean rise in baseline on application of agonist. Ordinates in $A$, transients/sec and in $B$ and $C$ pressure ( $c m$ $\mathrm{H}_{2} \mathrm{O}$ ). Abscissa, time in seconds. All experiments were done at $33-35^{\circ} \mathrm{C}$.

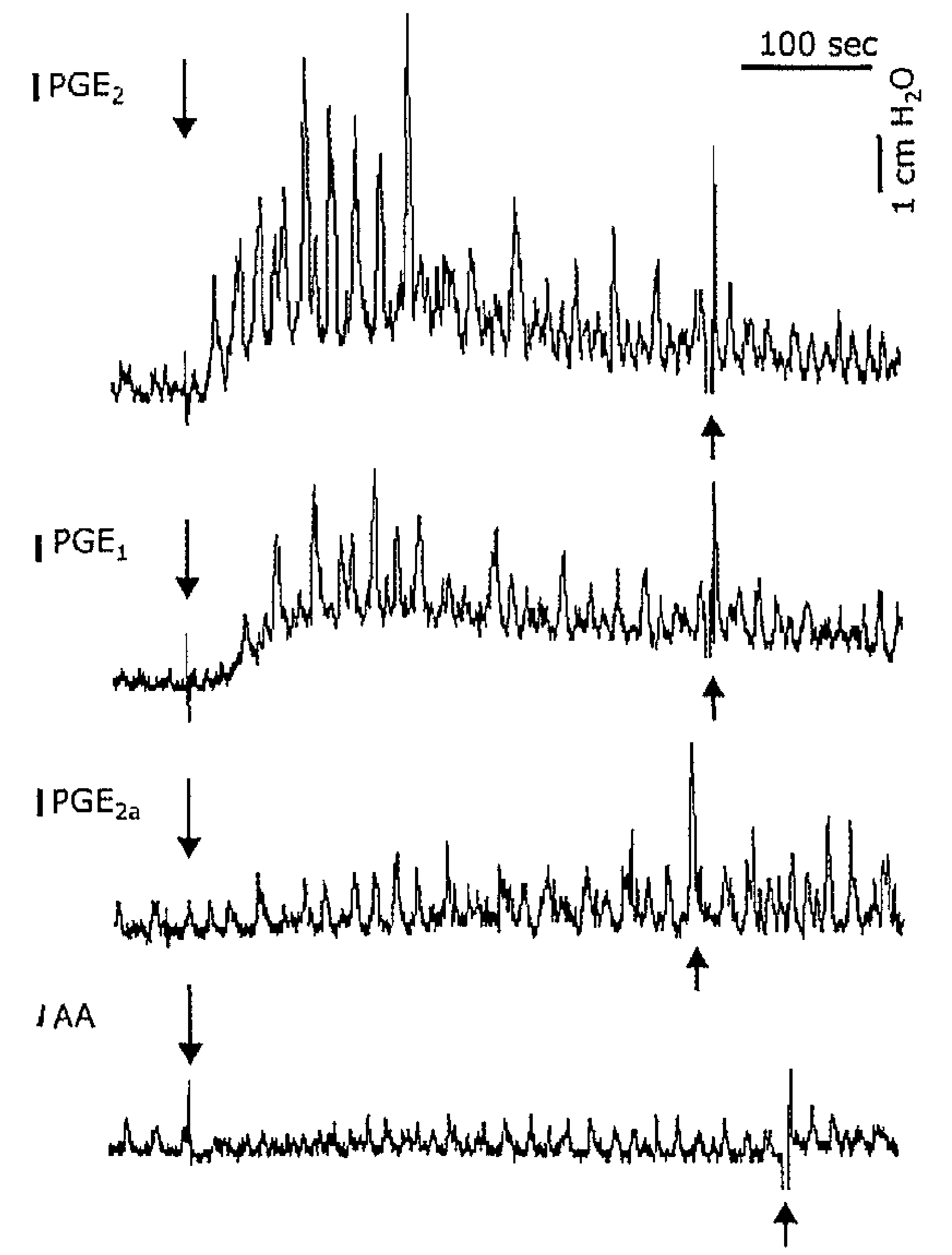

Figure 4.

Representative illustration of the effects of 300 nM PGE $E_{2}, P G E_{11}, P G F_{2 \alpha}$ and arachidonic acid on the autonomous activity of an isolated guinea pig bladder. Where indicated by the arrow $300 \mathrm{nM}$ of each drug was added to the bathing solution. Time and pressure calibration bars are as shown. Similar data was seen in a total of four separate bladders. All experiments were done at $34^{\circ} \mathrm{C}$. 
rating:

contribution to the generaton of this activty.

Data colketed from sik biadders are shown in figure 3. The increase in the frequency, ampltude of the transients and in baseline were significantly different from controls for the first 20 minutes after the exposure to PGE $_{2}$ (paired t-test: $p<0.03)$

Similar responses were induced by other prostaglandins (Fig. 4). Examples of the responses obtained to single doses $(300 \mathrm{nM}) \mathrm{PGE}_{1}, \mathrm{PGF}_{2}$, and arachadonic acid are shown in figure 4. Responses to $P G E_{1}$ were similar to those of $P G E_{2}$. There were clear effects of $P G F_{2}$, and arachidonic acid but these responses were generally smaller than $P G E_{2}$.

In an attempt to demonstrate that the action of $P G E_{2}$ was specific the effects of the EP1 and EP2 blocking agent AH6809 were examined on the augmented activity (Fig. 5). When AH6809 was added to the isolated bladder the responses to standard response to $300 \cap M \mathrm{PGE}_{2}$ was reduced in a dose dependent manner. Thus the $P \mathrm{PE}_{2}$ responses appear to be mediated through EP receptors.

It was concluded from these experiments that there are cells in the bladder wall that respond to exogenous prostaglandin. Activation of these cells results in complex motor activity. The two major questions are therefore; 'where is the endogenous prostaglandin produced' and 'where does it act'? We used immuno-histochemical techniques to localise the cyclo-oxygenase enzymes involved in the production of prostaglandins: the constitutive form COX I and the inducible form COX II.

Figure 6 shows images of tissue sections stained with a polyclonal antibody raised against the $70 \mathrm{kDa}$ constitutive cyclo-oxygenase enzyme COX I. Panel A shows COX I immuno-reactivity (COX I-IR) associated with the urothelium, the lamina propria and the inner muscle layer. Panel $B$ shows a high power image of the urothelium and immediate sub-urothelial layer of the lamina propria. The basal cells of the urothelium are strongly COX I-IR. Cells in the intermediate urothelial layer are also COX I-IR but the staining is less intense. Immediately below the urothelium there appears to be a network of spindle shaped cells which run in strands within the lamina propria. Panel $C$ shows the network of these sub-urothelial COX I-IR cells extending towards the inner-muscle layers where they form network like structures on the surface of the muscle bundles (panel D).

It is essential to establish that the staining seen is specific for COX I and that it is not non-specific binding of the antibody. To check this, the antibody was preabsorbed onto the protein to which it was raised. Figures 7A-B illustrates sections of urothelium and the muscle layer stained with the COX I antibody; figures 7C-D shows images, taken at the same intensity setting on the capture camera, of the urothelium and the muscle layer, but stained using the antibody / blocking peptide complex. The positive staining associated with the urothelium and the sub-urothelial layers and with the muscle bundles was eliminated by pre-absorption with the blocking peptide. The insets in panels $C$ and $D$ are digitally enhanced images of the images in $C$ and $D$ to show the underlying structures in the 'blocked images'.

NO donors affect PG biosynthesis in a variety of cell systems. ${ }^{26}{ }^{27}$ Therefore, we 
A

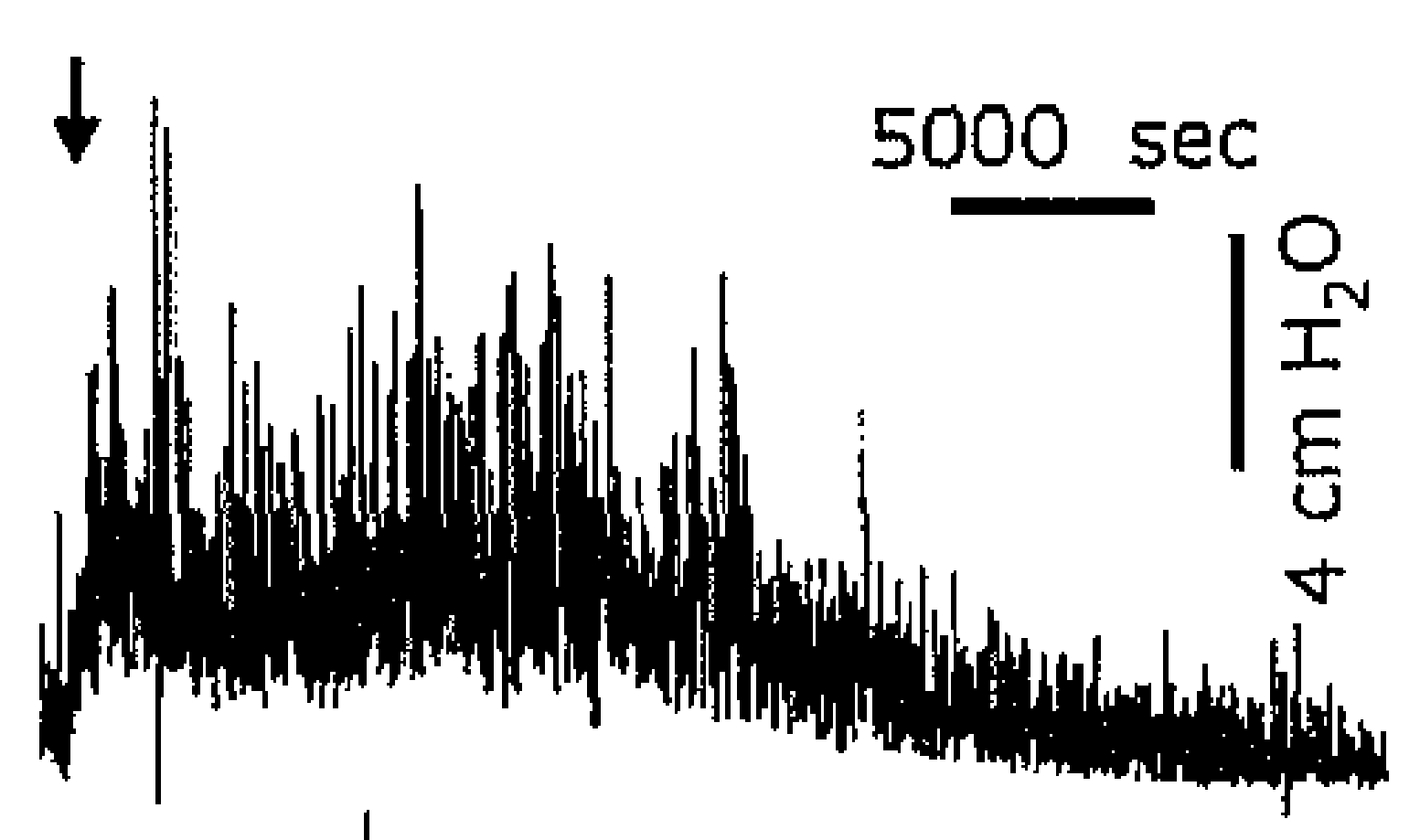

B

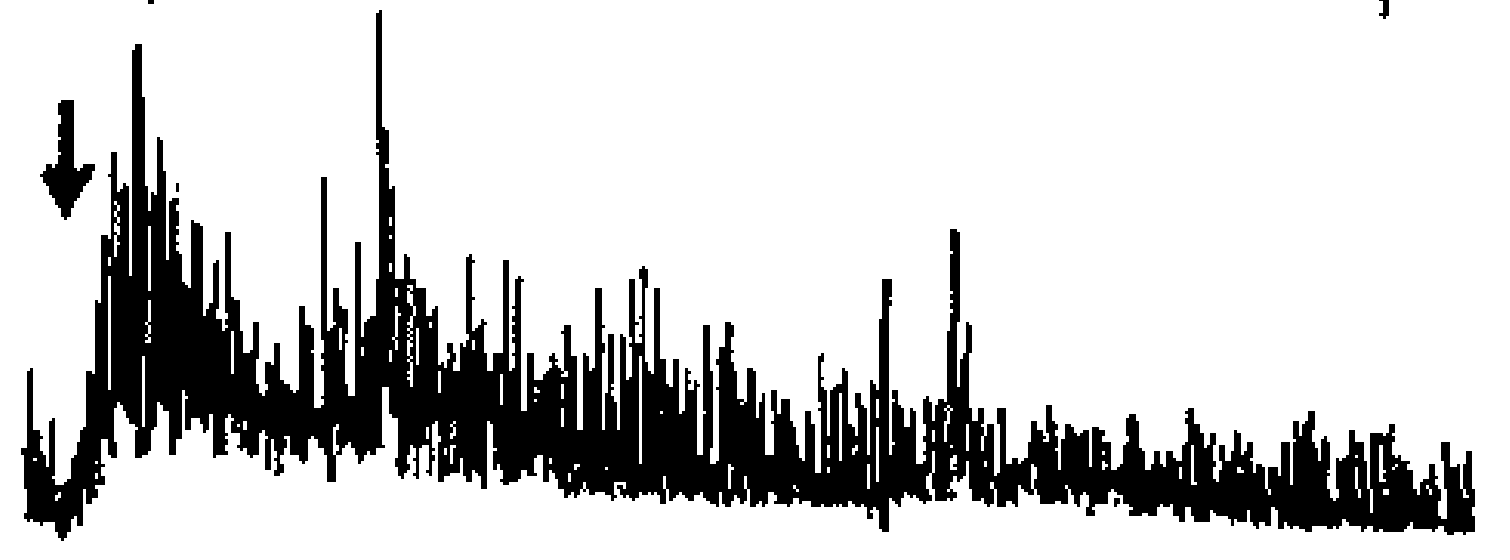

C

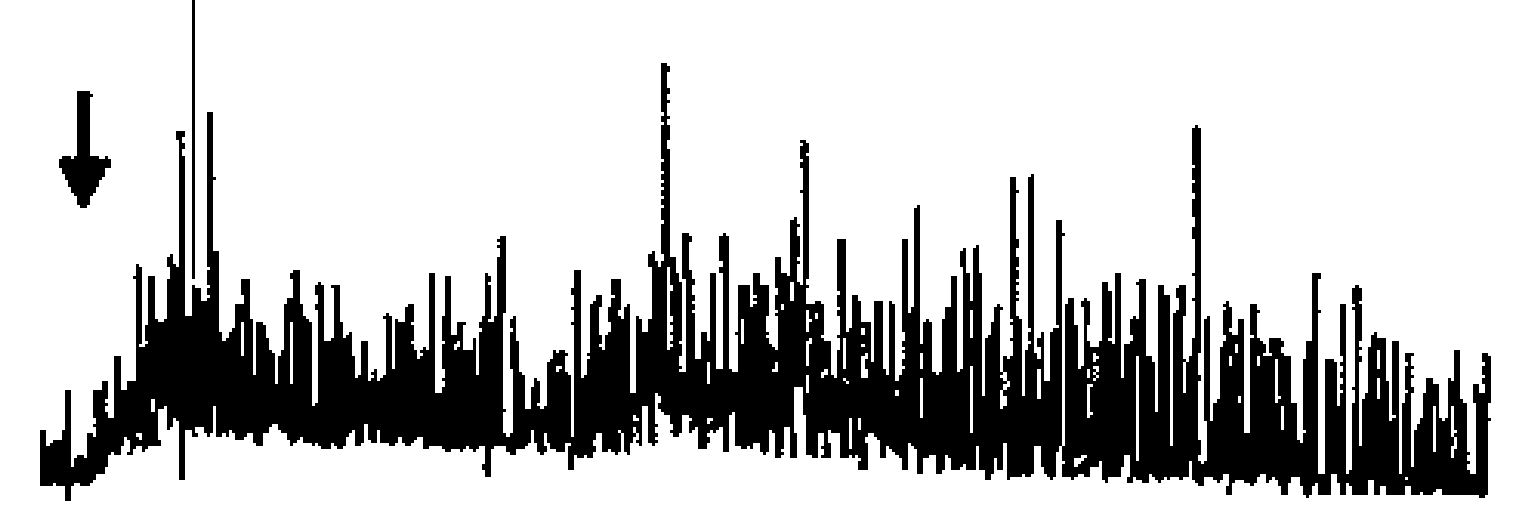

$D \downarrow$

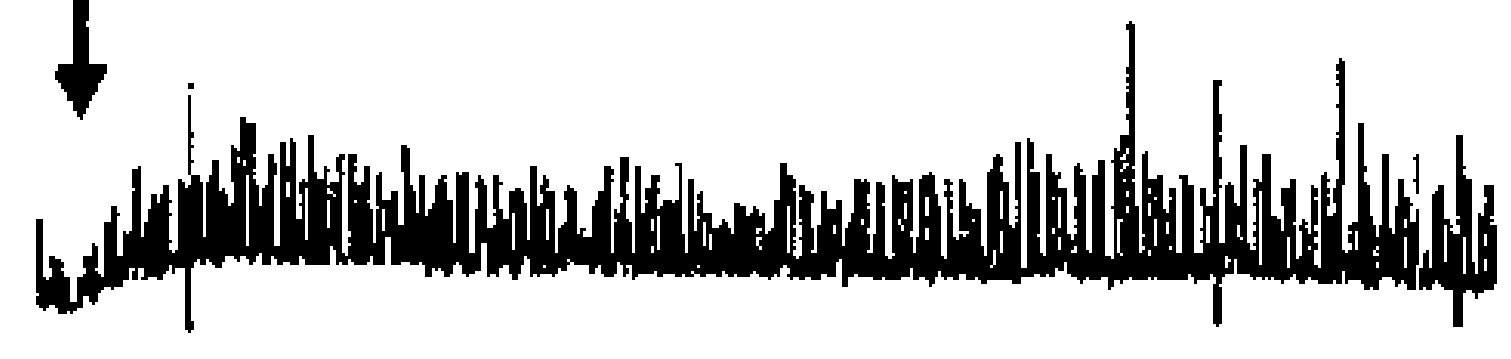

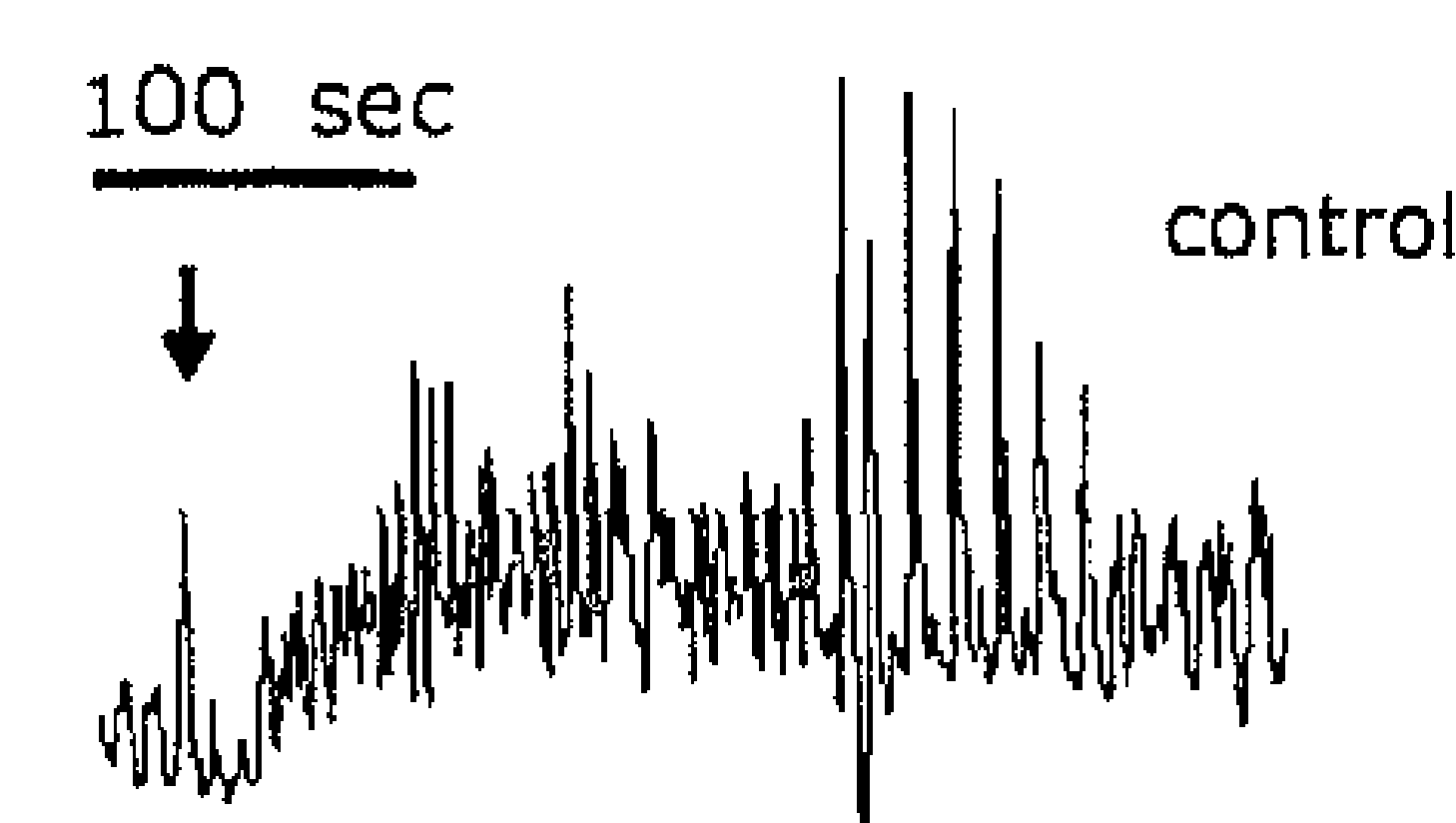

$14 \mathrm{MAH} 6809$

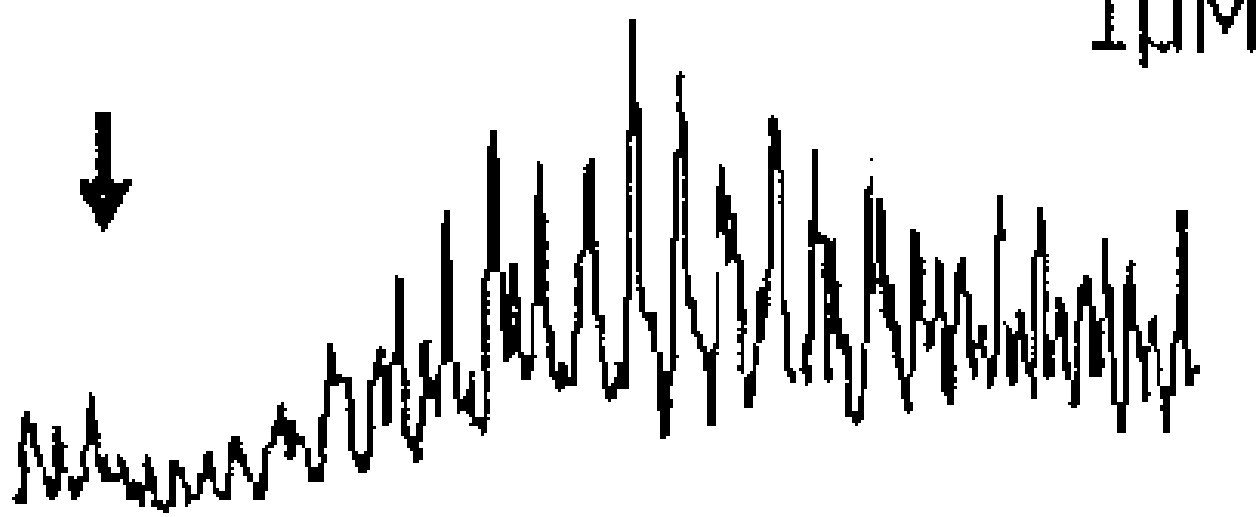

$\downarrow$

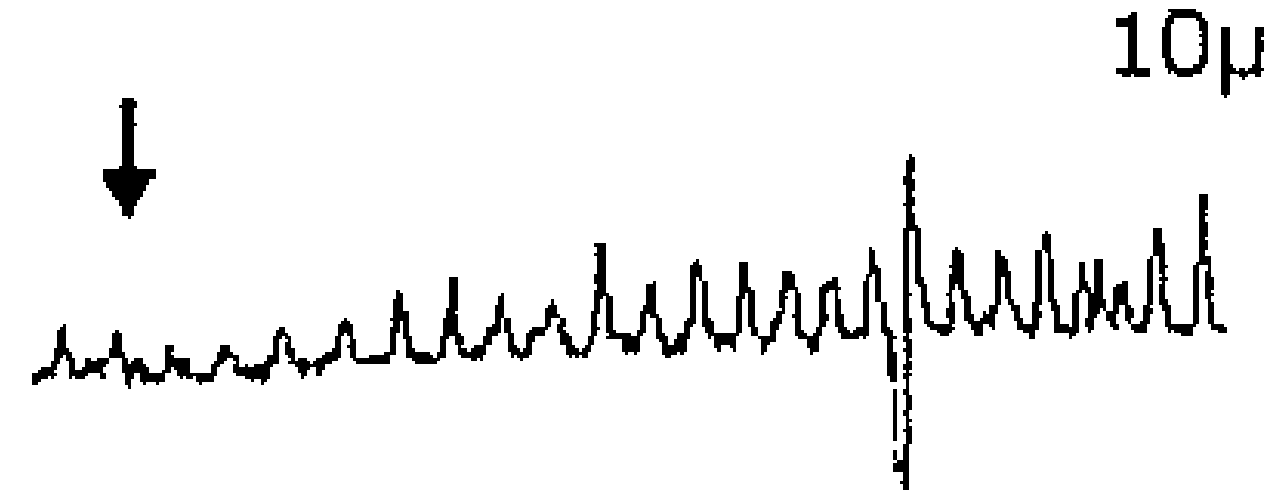

$10 \mu \mathrm{MAH} 6809$

Figure 5.

The effects of the EP1 and EP2 blocking agent AH6809 on the augmented phasic activity induced

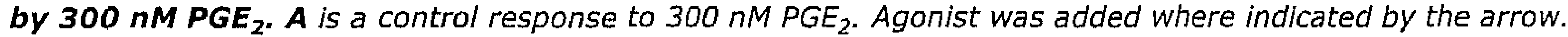
$\boldsymbol{B}-\boldsymbol{D}$ show responses to $300 \mathrm{nM} P \mathrm{PGE}_{2}$ with pre-incubation with different concentrations of $\mathrm{AH} 6809$ (1-10 $\left.\mu \mathrm{M}\right)$. The initial section of each record is also shown on an expanded time scale. Experiments done at a temperature of $33^{\circ} \mathrm{C}$.
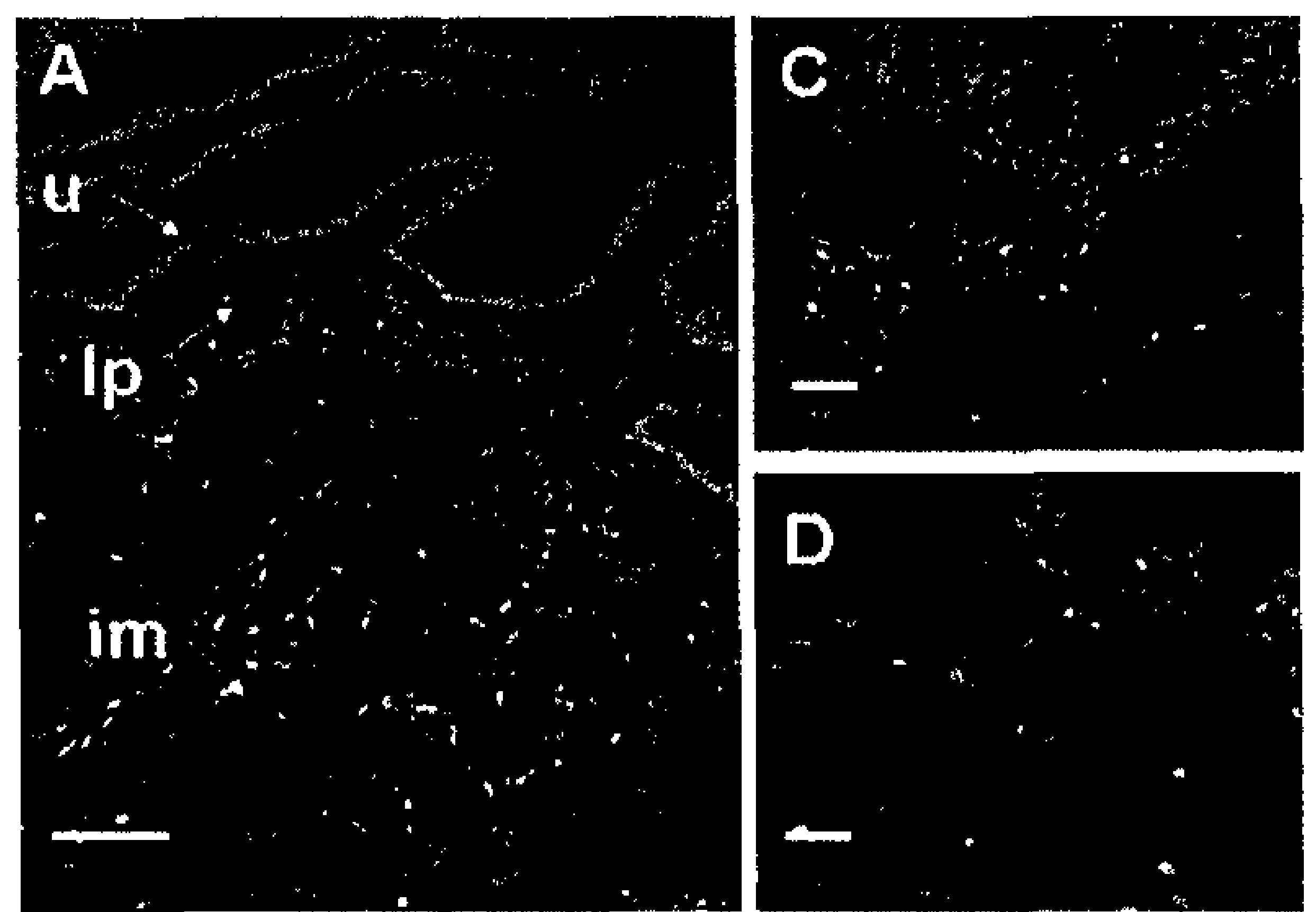

Figure 6.

COX I-IR in the normal guinea pig bladder. A shows a low power image of a section of the bladder wall stained with an antibody to COX I. The urothelium (u), lamina propria (l), and inner muscle layer are labelled as shown. B illustrates the structures seen within the urothelial layer $(u)$ and in the sub-urothelial space. In the sub-urothelial space a distinct network of interconnecting cells $(n)$ can be seen. $C$ and $D$ illustrate the COX I-IR cells in the lamina propria and inner muscle layer respectively. Calibration bars: $160 \mu \mathrm{m}$ in $\mathbf{A}, 60 \mu \mathrm{m}$ in $\boldsymbol{B}, 40 \mu \mathrm{m}$ in $\boldsymbol{C}$ and $\mathbf{D}$.

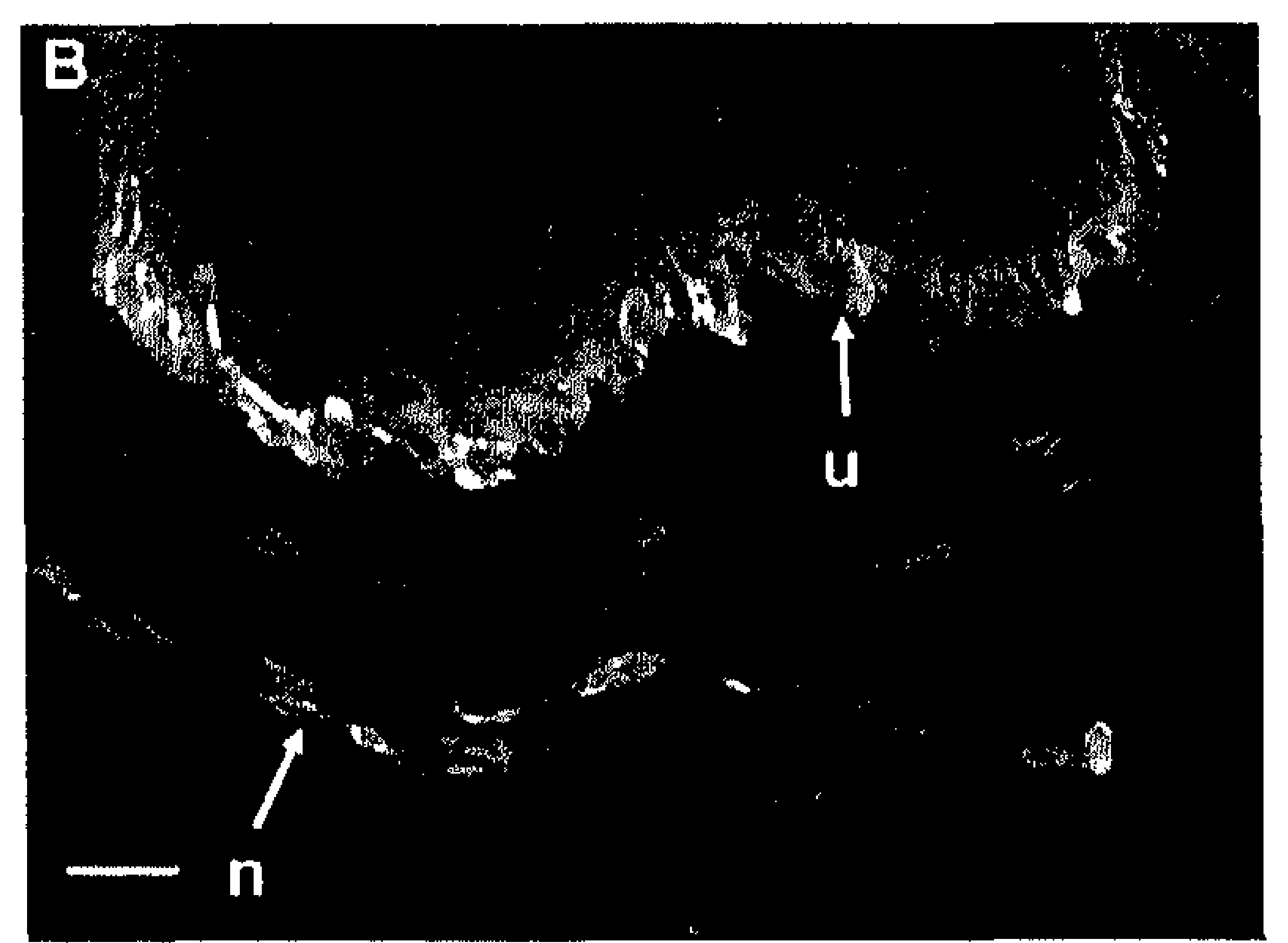



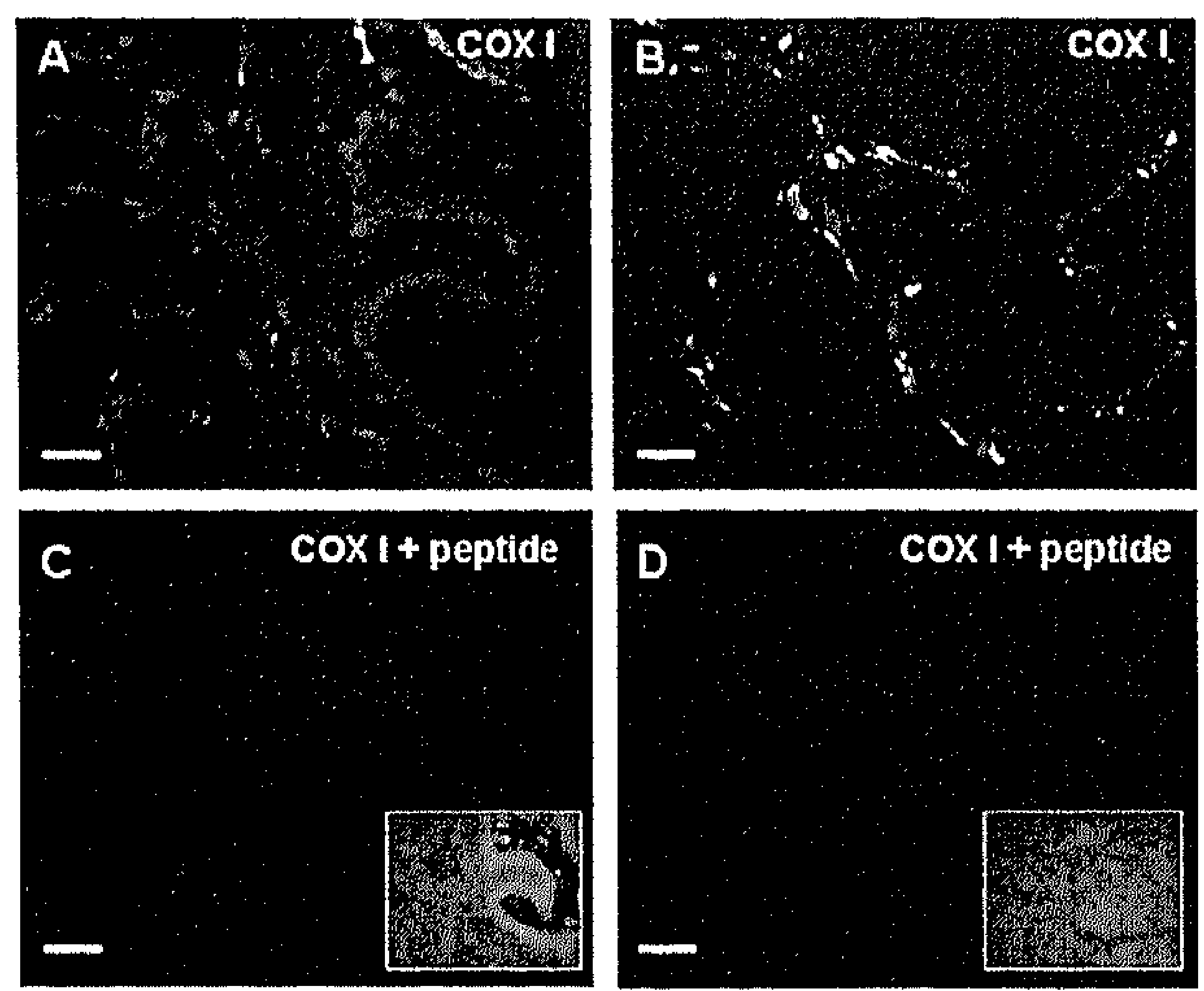

D
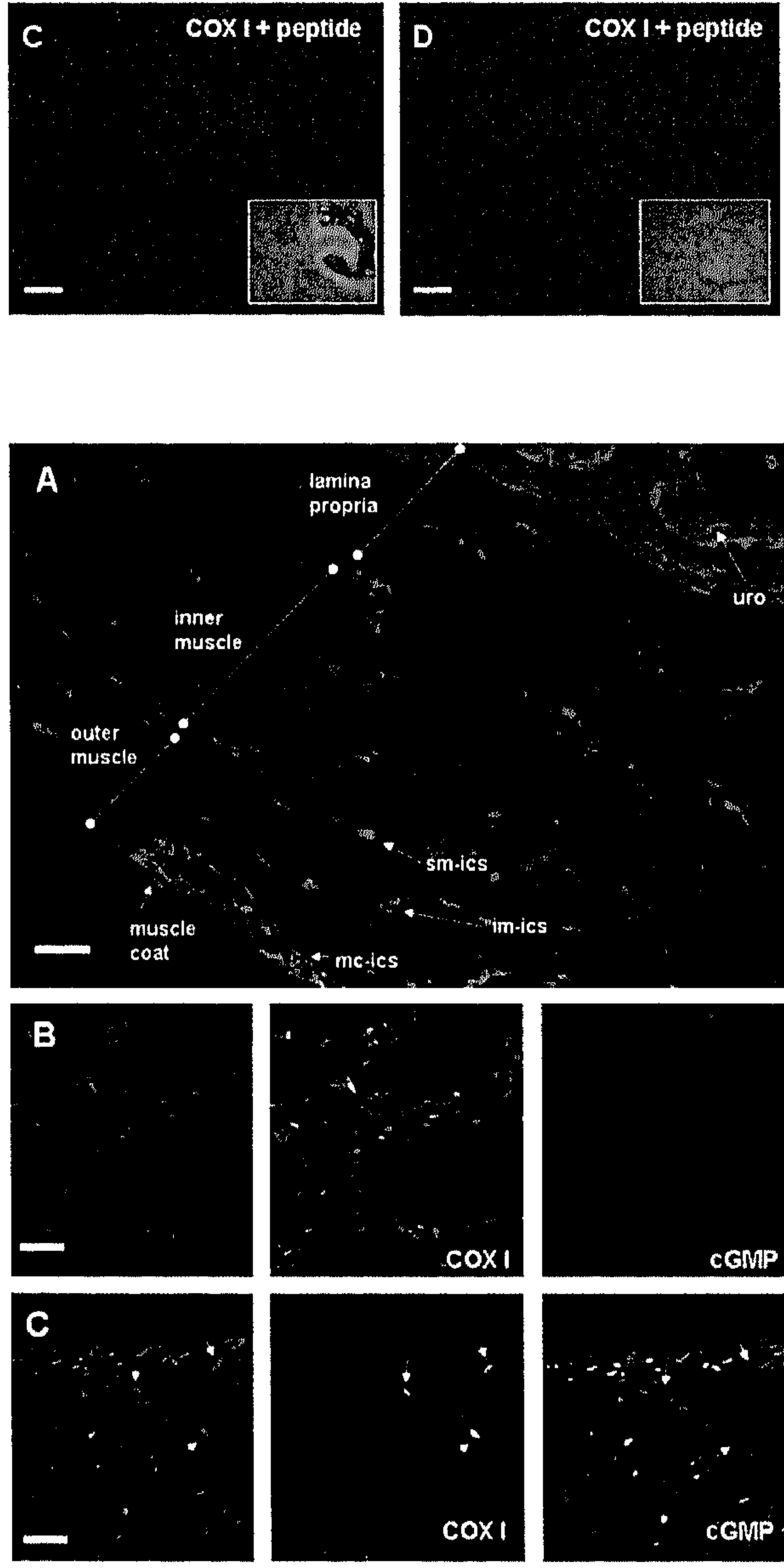

$\operatorname{cox} 1$
Figure 7.

The effects of blocking peptide on COX $I-I R$ on control guinea pig bladder. $\boldsymbol{A}$ and $\boldsymbol{B}$ show images of the urothelium and lamina propria (A) and the muscle layer stained using the antibody to COX $I$. Images were captured using a 1.5 second exposure. $\boldsymbol{C}$ and $\boldsymbol{D}$ show, on the urothelium and lamina propria respectively, the absence of staining with the same dilution of antibody but preabsorbed onto the blocking peptide. The images were captured using the same exposure (1.5 sec) as in $\boldsymbol{A}$ and $\boldsymbol{B}$. In each panel the inset show the image digitally enhanced to illustrate the structures that cannot be visualised with the camera gain used. Calibration bars: $100 \mu \mathrm{m}$ in $\boldsymbol{A}$ and $\boldsymbol{C}$ and $60 \mu \mathrm{m}$ in $\mathbf{B}$ and $\mathbf{D}$.

Figure 8.

The distribution of COX I-IR and NO sensitive CGMP interstitial cells in the wall of the normal guinea pig bladder (coloured image page 194).

A shows a section illustrating the entire thickness of the bladder wall from the urothelium (uro) to the muscle coat. The major layers are indicated: lamina propria, inner muscle and outer muscle. The three CGMP positive interstitial cell types in the outer muscle layer are also shown. B demonstrates the absence of CGMP positive cells in the inner muscle layer and that these COX I-IR cells do not respond to NO with a rise in CGMP. C illustrates a region of the outer muscle layer. The NO/CGMP responsive cells are readily identified (green) as are the COXI-IR cells (red). No co-localisation was noted. Arrows point to the COX I-IR cells. Calibration bar $180 \mu \mathrm{m}$ in $\mathbf{A}, 40 \mu \mathrm{m}$ in $\mathbf{B}$ and $50 \mu \mathrm{m}$ in $\mathbf{C}$. 

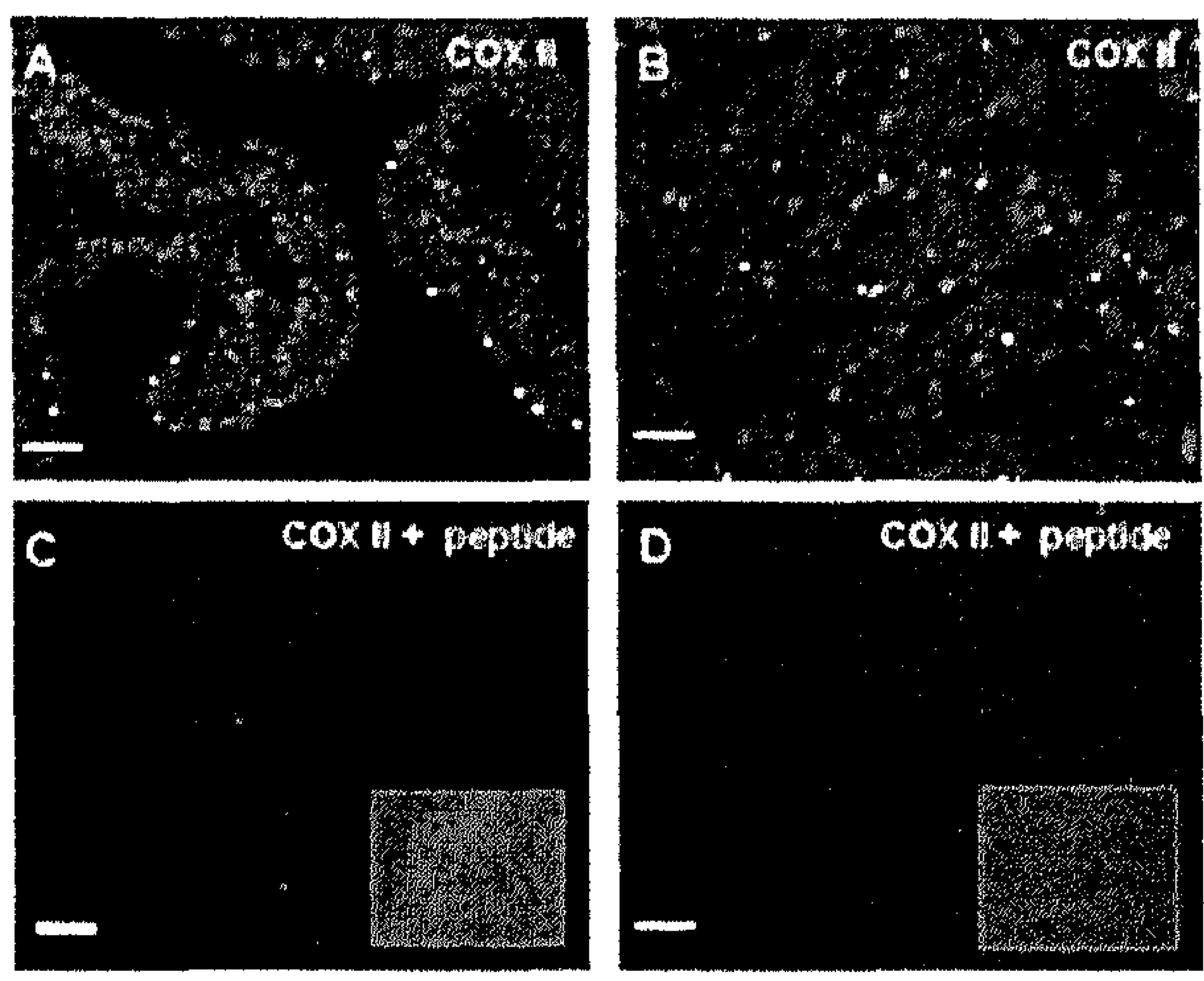

Figure 9.

The effects of blocking peptide on COX II-IR on guinea pig bladder. A and $B$

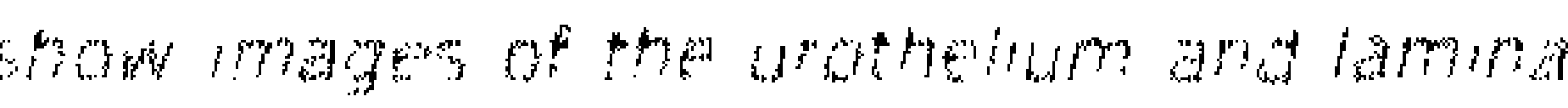

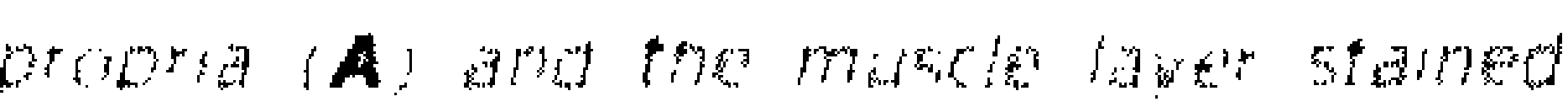

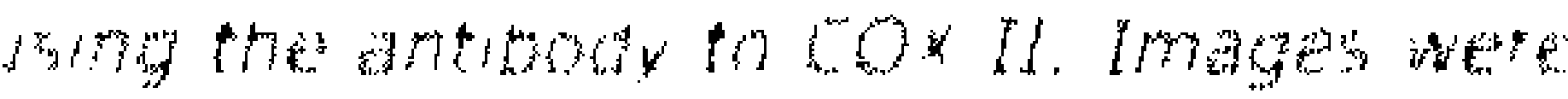
coptured uratg at 1.5 stoond exposure. $C$ and $D$ show. on the wethethom and lamma

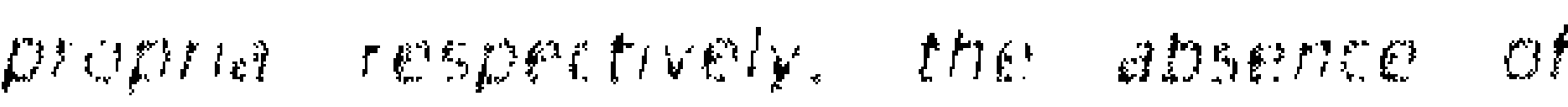
stearing with the same diuton antibody but preabsorbed onto the binckig paptide. The mages wer captured using the same

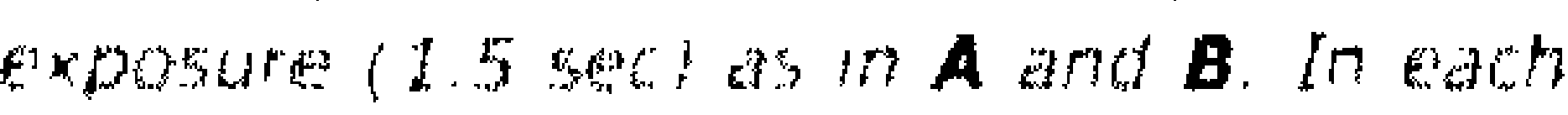
pune? the inset shows thas imate dighth ennanced to Hustrate the structures that cannot be visualisat with the cameta garn used. Calbraton bars: 100 um in $\mathbf{A}$ and $\boldsymbol{C}$ and $60 \mu \mathrm{m}$ in $\mathbf{B}$ and $\boldsymbol{D}$.
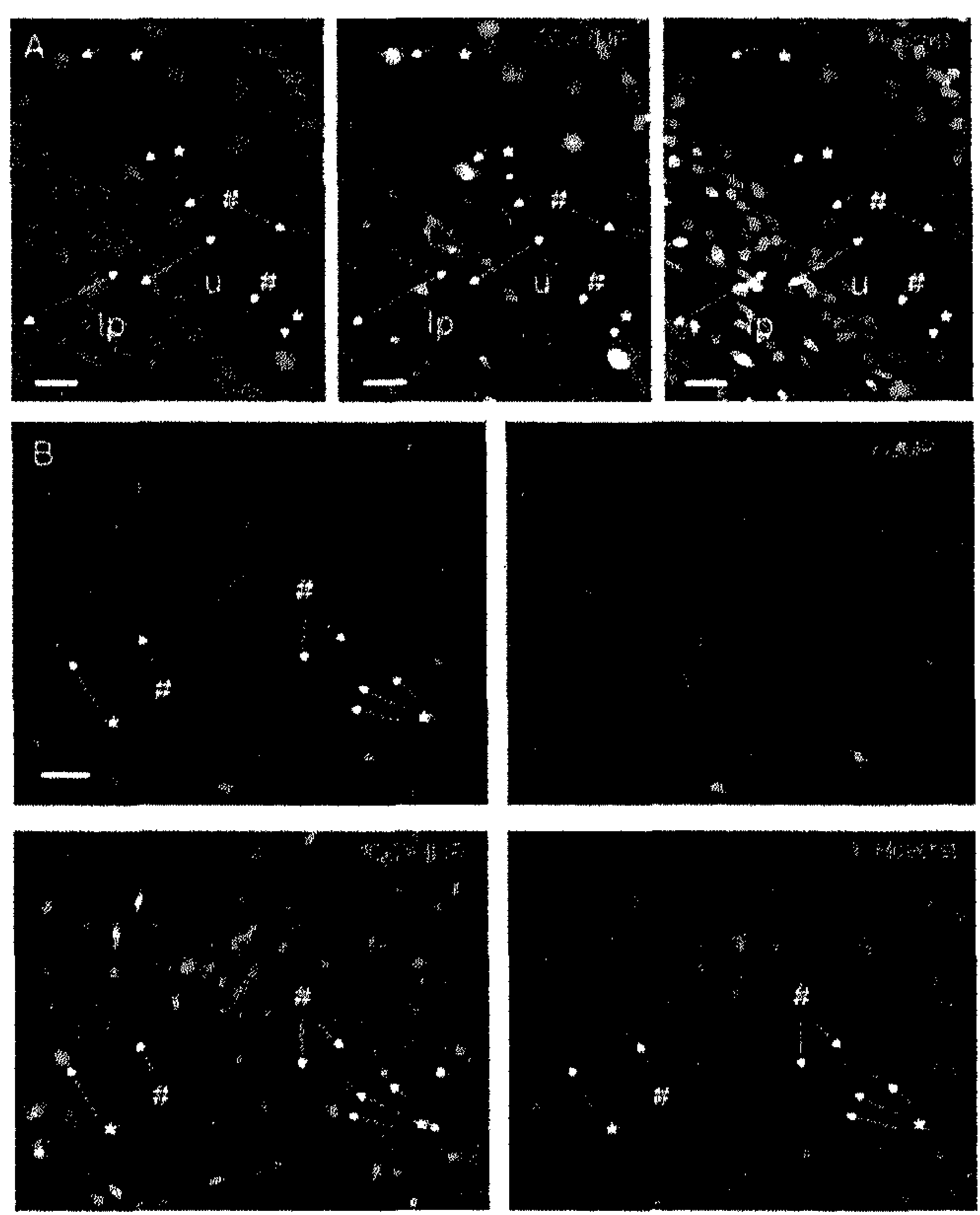

Figure 10.

The localisation of COX II-IR within the nucleus of cells in the urothelium and lamina propria (coloured image page 195).

A illustrates the co-localisation of $\mathrm{COX}$ II (red) and Hoechst (blue). The adjacent panels show COX II-IR and Hoechst staining. The urothelial layer and the lamina propria are shown by the arrows. Nuclei extibiting strong (*) and weak (" COX II-IR are shown by the arrows. Calibration bar $25 \mu \mathrm{m}$. B shows a region of the outer muscle layer. The section was triple labelled wth CGMP (green), COX II (red) and Hoechst (blue). 4 images are shown: a composite image showing the colour representation of all three antibodies and single images showing individual staining. The arrows show in each image, the cell nuclei and COX II-IR. Nuclei are seen which stain intensely with the COX II antibody (*) while other nuclei are found with less intense staining (\#).Calibration bar 40 $\mu m$. 


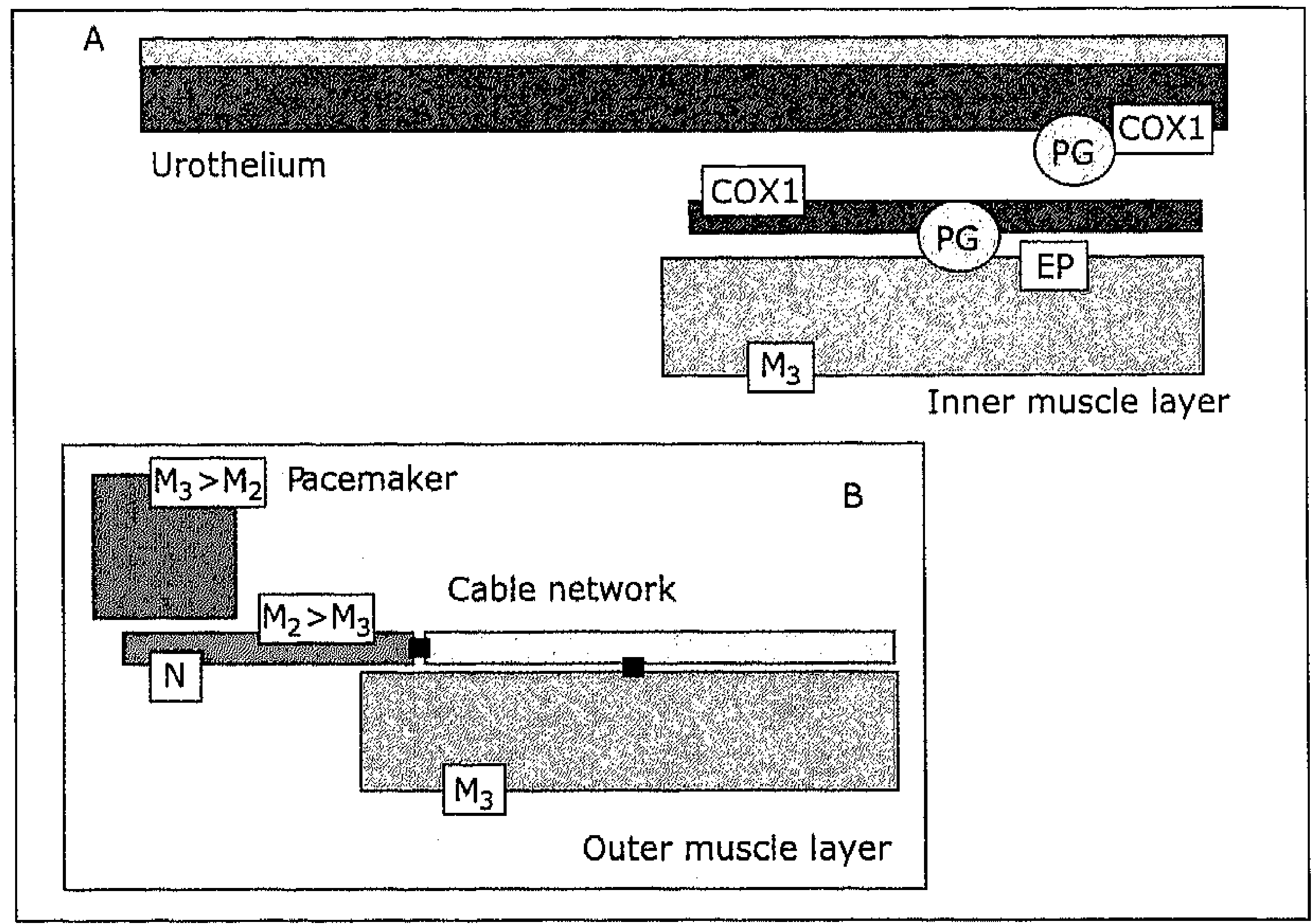

Figure 11.

Schematic diagram illustrating possible means of activation of the muscle layers of the detrusor. A shows the predominant cell types with COX I-IR which are likely to be synthesising prostaglandin: the urothelium and the network of cells lying on the surface of the muscle bundles of the inner muscle layer. It is proposed that these muscle bundles express the prostaglandin receptors and are activated to produce complex low amplitude phasic activity. The muscle cells here can also be activated by muscarinic stimulation via M3 receptors. B shows a proposed scheme to account for the effects of low concentrations of muscarinic agonist to produce large amplitude phasic activity. ${ }^{55}$ The idea is that there is a pacemaker mechanism which drives activity in a distributed network of interstitial cells resulting in co-ordinated waves of contractile activity. The suggestion is that there are distinct complex mechanisms activating the outer and inner muscle layers of the guinea pig bladder.

assessed the localization of COX enzymes in relation to the localization of NOresponsive, CGMP synthesizing cells. The distribution of COX I-IR cells in relation to CGMP-IR structures within the bladder wall is illustrated in figure 8 . In this section the tissues has been stained for COX I (red) and CGMP (green). CGMP staining identifies cells on and within the outer muscle layers: the muscle interstitial cells. The COX I-IR is seen predominantly within the lamina propria and associated with the inner muscle layers. There was no co-localization between CGMP-IR and COX I-IR. COX I-IR cells are seen in the outer muscle layers but the numbers are considerably low. Thus, there appears to be a regional distribution of COX I-IR cells associated with the muscle of the bladder wall.

The possibility that the inducible form of the cyclo-oxygenase enzyme COX II was also expressed in these normal tissues was explored. An antibody to COX II was used and COX II immuno-reactive (COX II-IR) structures were detected (Figs. 9A-B). As with the COX I antibody, the specificity of this staining was determined by incubating the COX II antibody with its blocking peptide. Figures 9C-D shows that the COX II- 
IR was abolished in sections exposed to the antibody pre-incubated with the blocking peptide suggesting that it is specific. Close examination of the images in figure 9 suggests that the COX II-IR is localised within the nucleus of the cells. Figure 10A shows, at high magnification, a region of the urothelium and lamina propria. The section is stained for COX II and the nuclear marker Hoechst. The COX II-IR appears to be associated with the cell nucleus. There were nuclei that were highly stained for COX II (*) while adjacent cells were less COX II-IR (\#). A similar pattern is seen in the muscle (Fig. 10B). The nuclei were positive for the COX II antibody and there were nuclei which were intensely COX II-IR $\left({ }^{*}\right)$ while nuclei close by were less so $(\#)$.

\section{Discussion}

It was demonstrated over 30 years ago that prostaglandins were released in response to stretch of the muscle and the urothelium. This suggested that there may be different sites of PG synthesis: the muscle and the urothelium, ${ }^{20,28,29}$ reviewed in. ${ }^{30}$ Since these initial findings it has been argued that prostaglandins play a central role in the physiology and patho-physiology of the bladder. ${ }^{1,4,31-34}$ This is exemplified by the growing interest in the possible use of cyclo-oxygenase inhibitors for the treatment of bladder overactivity. ${ }^{35}$ The present observations add to this body of knowledge, demonstrating an augmentation of autonomous activity in the isolated whole bladder and the localisation of the cyclo-oxygenase enzyme COX I to a network of cells lying within the lamina propria and on the surface of the muscle bundles of the inner muscle layer. These observations are important in the context of developing ideas relating to the possible roles of specialised cells in the bladder wall, interstitial cells. The current thinking suggests that one role of the interstitial cells is to drive activity in the muscle as a component part of a motor/sensory system involved in generation and modulation of bladder sensation.

Applying PG to isolated bladder strips or the bladder in vivo, results in a rise in basal tone and the appearance of small spontaneous contractions. Thus, there is a clear motor action of the PGs on the muscle, not influenced by the parasympathetic cholinergic transmission that does not involve the neuromuscular junction. The voiding contractions are not affected by PGs. ${ }^{36}$

It has long been recognised that small localised contractions of the bladder wall can activate afferent nerves. ${ }^{37-42}$ It was argued that the small contractions activated by prostaglandins would contribute to such a mechanism. ${ }^{31-32}$ Experiments showing that PG induced local contractions also showed an increased frequency of 'micturition like' contractions suggesting that the afferent discharge did indeed contribute to reflex activation of the detrusor. ${ }^{31-32}$ This concept was further supported by the observation that capsaicin, used to effectively remove the afferent contribution of $C$ fibres, inhibited the reflex activation of the detrusor by PG. ${ }^{33}$

In addition to these ideas of an indirect action on afferent firing it has been 
suggested that they may have a direct action on afferent nerves. In other tissues it is known that PGs stimulate afferent nerves either directly or by sensitising fibres to other stimuli. ${ }^{43}$ These mechanisms suggest a further possibility for the actions of PGs on the bladder. The action of PG on the afferent nerves to increase discharge would contribute to the activation of micturition reflexes. Thus, there are several possible sites of synthesis of PG in the bladder (the urothelium and the muscle) and several possible sites of action (the muscle and afferent nerves).

As a result of these observations the hypothesis was proposed that the prostanoids are endogenous amplifiers of the afferent input from the bladder to the central nervous system. ${ }^{32}$ This general concept is similar to that developed in relation to the cholinergic driven phasic contractions and their modulation by excitatory and inhibitory inputs: the autonomous bladder hypothesis. ${ }^{42-44}$ Augmentation of the autonomous activity by muscarinic stimulation, ${ }^{44}$ excitation by adenosine triphosphate (ATP) and substance $\mathrm{P}^{45}$ and inhibition by noradrenaline and calcitonin gene related peptide ${ }^{46-47}$ demonstrates that the motor/sensory system is highly regulated. Importantly, this implies that this motor/sensory system can be modulated and that sensation can be altered directly.

The general importance of PG input to such a sensory mechanism is demonstrated further by the infusion of PG into the bladder lumen where it results in an increase in micturition frequency. Indeed this approach has been considered as a model for bladder overactivity. ${ }^{48-49}$

The present experiments fit within this framework of local contractions contributing to a motor/sensory mechanism within the bladder. The data demonstrate that PGs are indeed an excitatory factor in modulating this motor/sensory system within the bladder wall.

Prostaglandin synthesis has been generally associated with the smooth muscle and the urothelium. ${ }^{20,28-30}$ The precise cell types and their location have not been considered in detail. The present observations put forward new ideas. First, the intense COX I-IR in the basal and intermediate layers of the urothelium suggest that it is these cell layers that are responsible for urothelial derived PG. Second, the presence of cells in the lamina propria but also on the surface of the muscle bundles suggest that these cells synthesize the PG involved in the activation of muscle. It must be pointed out that these conclusions rely entirely on the specificity of the antibodies used for the COX enzymes. In Western blots, the antibodies recognise the functional protein and the immuno-staining is inhibited if the antibody is preabsorbed with the appropriate blocking peptide. Thus, there is good circumstantial evidence to believe that the antibodies are locating functional COX enzyme.

The target cells for the PG synthesised by these different locations are not known. $P G$ released from the urothelium is in close proximity to sensory nerves which run adjacent to the basement membrane.$^{50}$ It is possible that this is the source of the PG and these are the nerves which are involved in the direct stimulation or modulation of afferent nerve activity. These sub-urothelial afferent nerves send collaterals to the intra-mural ganglia. It has been suggested that the ganglia send fibres to the muscle 
and so may contribute to contractile activation. ${ }^{50}$ Urothelial derived $P G$ and the activation of collateral or afferent fibres may provide a further means whereby PG can induce activity in the muscle.

A second source of PG appears to be associated with specialised cells lying on the surface of the muscle bundles of the inner muscle layer. It is hypothesised that PG synthesised and released from these cells would be responsible for activation of the underlying muscle bundles. These COX I-IR cells form a network which is very similar to the network of CGMP positive interstitial cells associated with the outer muscle bundles. ${ }^{25}$ Therefore, these COX I-IR cells may also be described as interstitial cells. In normal guinea pig bladders there are no CGMP positive interstitial cells associated with the inner muscle layers. In the outer layers CGMP positive interstitial cells are found within the muscle bundles. ${ }^{25}$ However, no COX-IR cells were found within the muscle bundles in the outer muscle layer. Thus, there is a clear indication of heterogeneity in interstitial cells in the bladder wall: a network of cGMP positive cells associated with the outer layers and a COX I-IR network associated with the inner layers.

It is not clear how the network of COX I-IR interstitial cells might be activated. They may be stimulated directly by stretching the wall as the bladder fills. Alternatively, since the COXI-IR network may be part of an extended network extending through the lamina propria to the sub-urothelial region it is possible that substances released by the urothelium would activate this network.

The detection of specific COX II-IR in the normal bladder was an unexpected finding, as this enzyme is more associated with inflammation and pathological changes. ${ }^{6,35}$ What is more curious is that the immuno-staining is associated with the nuclei. Furthermore, the nuclear staining seems to be variable with some nuclei appearing to have more COX II-IR than in adjacent cells. There are reports demonstrating COX II around the nucleus. ${ }^{51-54}$ It was suggested that this localised intracellular production of $P G$ acts on nuclear peroxisome proliferators-activated receptor $Y_{: " \text {. }}$ This receptor is a member of a diverse group of retinoid and thyroid nuclear receptors, all acting as transcription factors for genomic DNA. In some cells this system may be involved in the pre-cancerous progression of epithelial cells or in general expression control. ${ }^{5}$ Such a system regulating the genome of cells in the bladder may therefore play a similar role in gene regulation involved in the renewal of urothelial cells and tumorigenesis.

The presence of the different COX enzymes in the bladder is clear. However, it was argued that the detailed functions of these different enzymes in PG production and signalling are generally unknown. ${ }^{35}$ The present data supports the view of the different enzymes in different physiological systems in the bladder. These systems may also contribute to bladder pathology. It can be speculated that alterations to the COX I system in the urothelium/interstitial cells may be involved in alterations to the motor component of the motor/sensory system and urothelial activation of afferent nerves. The COX II system, in the guinea pig bladder at least, may be more involved in the regulation and modulation of cell proliferation and expression. PGs have been 
implicated in bladder pathologies, for example, inflammatory conditions such as interstitial cystitis and outflow obstruction. ${ }^{35}$ The different locations of the coX enzymes and their involvement in different bladder functions could be reflected in the contribution they make to bladder pathology.

In conclusion, it is now becoming clear that there is a motor/sensory system operating in the bladder. At least two systems now appear to contribute to this activity: a cholinergic driven system involving the outer muscle layer ${ }^{55}$ and a PG driven system likely acting on the inner muscle layers. In the guinea pig these different systems activate different responses. These two systems are illustrated in figure 11. The cholinergic system involves high pressure transient rises in pressure associated with propagating waves of contraction over the bladder surface..$^{56-57}$ This activity might occur in the outer muscle layers. Support for this possibility has come from studies in the mouse where there is direct evidence that the interstitial cells of the outer layer are involved in the generation of phasic activity. ${ }^{58}$ By contrast, the PG system activates small irregular micro-contractions probably acting on the inner muscle layer. The precise contribution of these systems to the overall physiological regulation of the bladder is not known. Furthermore, the contribution of these systems to bladder pathology remains to be established.

\section{References}

1. Gilmore NJ, Vane JR. Hormones released into the circulation when the urinary bladder of the anaesthetised dog is distended. Clin Sci. 1971; 41:69-83

2. Abrams $P H$, Sykes $3 A$, Rose AJ, Rogers $A F$. The synthesis and release of prostaglandins by human urinary bladder muscle in vitro. Invest Urol. 1979; 16:346-8

3. Jeremy JY, Tsang JJ, Mikhailidis DP, Rogers $H$, Morgan RJ et al. Ecosanoid synthesis by human urinary bladder mucosa: pathological implications. BJU. 1987; 59:36-9

4. Mikhailidis DP, Jeremy JY, Dandona P. Urinary bladder prostanoids-their synthesis, function and possible role in the pathogenesis and treatment of disease. J Urol. 1987; 137:577-582

5. Vane JR, Bakhle YS, Botting RM. Cycloxygenases 1 and 2. Ann Rev Pharmacol Toxicol, 1998; 38:97-120

6. Park $\mathrm{JM}$, Yang $\mathrm{T}$, Arend $\mathrm{LJ}$ et al. Cyclooxygenase 2 is expressed in bladder during fetal development and stimulated by outlet obstruction. Am J Physiol. 1997; 273:F538

7. Bultitude MI, Hills NH, Shuttleworth KEL. Clinical and experimental studies on the actions of prostaglandins and their synthesis inhibitors on detrusor muscle in vivo and vitro. BJU. 1976; 48:631-7

8. Hills $\mathrm{NH}$. Prostaglandins and tone in isolated strips of mammalian bladder. $\mathrm{Br}]$ Pharmacol. 1976; 57:464P-64P

9. Andersson K-E, Ek A, Perrson CGA. Effects of prostaglandins on the isolated human bladder and urethra. Acta Physiol Scand. 1977; 100:165-71

10. Borda E, Contreras-Ortiz N, Gutnisky R, Gimeno MF. In vitro effect of acetylcholine and bethanecol on the contractions of human detrusor muscle. Influence of prostaglandins. Arch Int Pharmacodyn Ther. 1982; 259:31-9

11. Brown C, Burnstock $G$, Cocks T. Effects of adenosine-5-triphosphate (ATP) and b-g-methelene ATP on rat urinary bladder. $\mathrm{Br} J$ Pharmacol. 1979; 65:97-102

12. Choo LK, Mitchelson F. The role of prostaglandins in the excitatory innervation of the rat bladder. Prostaglandins. 1977; 13:917-26 
13. Choo LK, Mitchelson $F$. The effects of indomethacin and ATP on the excitatory innervation of the rat urinary bladder. Can J Physiol Pharmacol. 1980; 58:1041-8

14. Burnstock $G$, Cocks $T$, Crowe R, Kasakov L. Purinergic innervation of the guinea pig urinary bladder. $\mathrm{Br}$ ] Pharmacol. 1978; 63:125-38

15. Burnstock G, Cocks T, Kasakov $L$, Wong HW. Direct evidence for ATP release from nonadrenergic (purinergic) nerves in the guinea pig tenae coli and bladder. Eur J Pharmacol. 1978; 49:145-9

16. Johns $A$. The effects of indomethacin and substance $P$ on the guinea pig urinary bladder. Life Sci. $1981 ; 29: 1003-9$

17. Dean DM, Downie JW. Innervation of prostaglandins and ATP in the noncholinergic neurotransmission in the rabbit detrusor. Prostaglandins. $1978 ; 16: 245-51$

18. Husted S, Sjorgren $C$, Andersson K-E. Role of prostaglandins in the responses of rabbit detrusor to non-cholinergic, non-adrenergic nerve stimulation and to ATP. Arch Int Pharmacodyn Ther. $1980 ; 246: 84-97$

19. Andersson $\mathrm{K}-\mathrm{E}$, Husted $\mathrm{S}$, Sjogren $\mathrm{C}$. Contribution of prostaglandins to the ATP induced contractions of rabbit urinary bladder. Br J Pharmacol. 1980; 70:443-52

20. Downie JW, Larson C. Prostaglandin involvement in contractions evoked in rabbit detrusor by field stimulation and by ATP. Can J Physiol Pharmacol. 1981; 59:253-60

21. Johns A, Paton MD. Evidence for a role for prostaglandins in atropine resistant transmission in rabbit and monkey urinary bladder. Prostaglandins. 1976; 11:595-7

22. Johns A, Paton MD. Effects of indomethacin on atropine resistant transmission in rabbit and monkey urinary bladder. Prostaglandins. $1977 ; 13: 245-54$

23. Andersson K-E, Sjorgen C. Aspects of the physiology and pharmacology of the bladder and urethra. Prog Neurol. 1982; 19:71-89

24. Andersson K-E. Current concepts in the treatment of disorders of micturition. Drugs. 1988; 35:477-94

25. Gillespie JI, Markerink-van Ittersum M, de Vente J. CGMP generating cells in the bladder wall: identification of distinct networks of interstitial cells. BJU Int. 2004; 94:1114-24

26. Salvemini D', Misko TP, Masferrer JL. Nitric oxide activates cyclooxygenase enzymes. Proc Natl Acad Sci USA. 1993; 90:7240-44

27. Salvemini D, Misko TP, Masferrer K. Role of nitric oxide in the regulation of cyclooxygenase. Biol Nitric Oxide. 1994; 4:304-9

28. Brown WW, Zenster TV, Davis BB. Prostaglandin E2 production by rabbit urinary bladder. Am J Physiol. 1980; 239:F452-8

29. Kasakov LM, Vlaskovska MV. Profile of prostaglandins generated in the detrusor muscle of rat urinary bladder. Eur J Pharmacol. 1985; 113:431-436

30. Andersson K-E. Pharmacology of the lower urinary tract. Pharmacol Rev $1993 ; 45: 253-66$.

31. Maggi CA. Prostanoids as local modulators of reflex micturition. Pharmacological Res. 1992; 25:13-20

32. Maggi CA, Evangelista S, Grimaldi G, Santicioli P, Giolitti A, Meli A. Evidence for the involvement of Arachadonic acid metabolites in Spontaneous and drug induced contractions of rat urinary bladder. J Pharm Exp Ther. 1984; 230:500-13

33. Maggi CA, Giuliani S, Conte B, Furio M, Santicioli P, Meli P, Grangnani L, Meli A. Prostanoids modulate reflex micturition by acting through capsaicin sensitive afferents. Eur J Pharmacol. $1988 ; 145: 105-12$

34. Palea S, Toson C, Pietra C, Trist DG, Artibani W, Romano O, Corsi M. Pharmacological characterisation of thromboxane and prostanoids receptors in human isolated urinary bladder. Br J Pharmacol. 1998; 124:865-72

35. Angelico P, Guarneri L, Velasco $C$, Cova $R$, Leonardi A, Clarke DE, Tessta R. Effect of cyclooxygenase inhibitors on the micturition reflex in rats: correlation with inhibition of cycooxygenase isozymes. BJU Int. 2006; 97:837-46

36. Morikawa $K$, Kakiuchi $M$, Yamaguchi $T$ et al. Pharmacological studies on the micturition reflex (2): effects of various drugs on ladder and urethral function in rats and dogs. Pharmacomimetics. 1989; 37:27-37 
37. Sherrington CS. Notes on the arrangement of some motor fibres in the lumbo-sacral plexus. J Physiol.1892; 13:621-772

38. Starling EH. Elements of Human Physiology.7th Edition. 1905. Churchill, London.

39. Iggo A. Tension receptors in the stomach and urinary bladder. J Physiol. 1955; 128:593-607

41. De Groat WC, Ryall RW. Reflexes to spinal parasympathetic neurones concerned with micturition in the cat. J Physiol. 1969; 200:87-108

41. Vaughan, CW, Satchell PM. Urine storage mechanisms. Progress in Neurobiology, 1995; 46:21537

42. Gillespie JI. Animal Models of Urge Incontinence: An evolving view of the origins of bladder overactivity and sensory urge. BJU Int. 2005; 96:22-8

43. Cohen RH, Perl ER. Chemical factors in the sensitisation of cutaneous nociceptors. Brain Res. $1988 ; 74: 201-6$

44. Gillespie JI. The Autonomous Bladder: A View of the Origin of Bladder Overactivity. BJU Int. 2004; 93:478-83

45. Gillespie JI. Modulation of Autonomous Contractile Activity in the Isolated Bladder of the GP. BJU Int. 2004; 93:393-400

46. Gillespie JI. Noradrenalin Inhibits Autonomous Activity in the Isolated Guinea Pig Bladder. BJU Int. $2004 ; 93: 401-9$

47. Gillesple JI. Inhibitory Actions of Calcitonin Gene Related Peptide and Capsaicin: Evidence for Local Axonal Reflexes in the Bladder Wall. BJU Int. 2005; 95:149-56

48. Ishizuka O, Mattiasson A, Andersson K-E. Prostaglandin induced bladder hyperactivity in normal conscious rats: involvement of tachykinins. J Urol. 1995; 153:2034

49. Takeda $H$, Yamazaki $Y$, Igawa $Y$, Kaidoh $K$, Akahane S, Miyata $H$, Nishizawa O, Akahane M, Andersson KE. Effects of beta(3)-adrenoceptor stimulation on prostaglandin $E(2)$-induced bladder hyperactivity and on the cardiovascular system in conscious rats. Neurourol Urodyn. 2002: 21:558-65

50. Gillespie JI, M Markerink-van Ittersum M, de Vente J. Sensory collaterals, intra-mural ganglia and motor nerves in the guinea pig bladder: evidence for intramural neural circuits Cell and Tissue Res. 2006; 325:33-45.

51. Ren Y, Walker C, Lose-Mitchell DS, Deng J, Ruan K-H. Topology of prostaglandin H synthase 1 in the endoplasmic reticulum membrane. Arch Biochem Biophys, 1995; 323:205-14

52. Regier MK, deWit DL,Schindler MS, Smith WI. Sub-cellular localisation of prostaglandin endperoxidase synthase 2 in murine 3T3 cells. Arch Biochem Biophys. 1993; 301:439-44

53. Morita I, Shindler MS, Reiger MK, Otto JC, Hori T et al. Different intracellular localisations for prostaglandin endoperoxidase $H$ synthase1 and 2. J Biol Chem. 1995; 270:10902-8

54. Otto JC, Smith WL. The orientation of prostaglandin enoperoxidases 1 and 2 in the endoplasmic reticulum. J Biol Chem. 1994; 269:19868-75

55. Finney SM, Stewart LH, Gillespie. Cholinergic activation of phasic activity in the isolated bladder; possible evidence for M3- and M2-dependent components of a motor/sensory system. BJU Int. 2007; 100(3):668-78.

56. Gillespie JI, Harvey IJ, Drake MJ. Agonist and Nerve Induced Phasic Activity in the Isolated Whole Bladder of the Guinea Pig: Evidence for Two Types of Bladder Activity. Exp Physiol. 2003; 88:343-57

57. Drake M], Harvey I], Gillespie JI. Autonomous activity in the isolated guinea pig bladder. Exp Physiol. 2003; 88:19-30

58. Lagou M, Drake MJ, de Vente J, Markerink-van Ittersum M and Gillespie JI.. The role of interstitial cells in the isolated mouse bladder. BJU Int. 2006; 98:643-50 


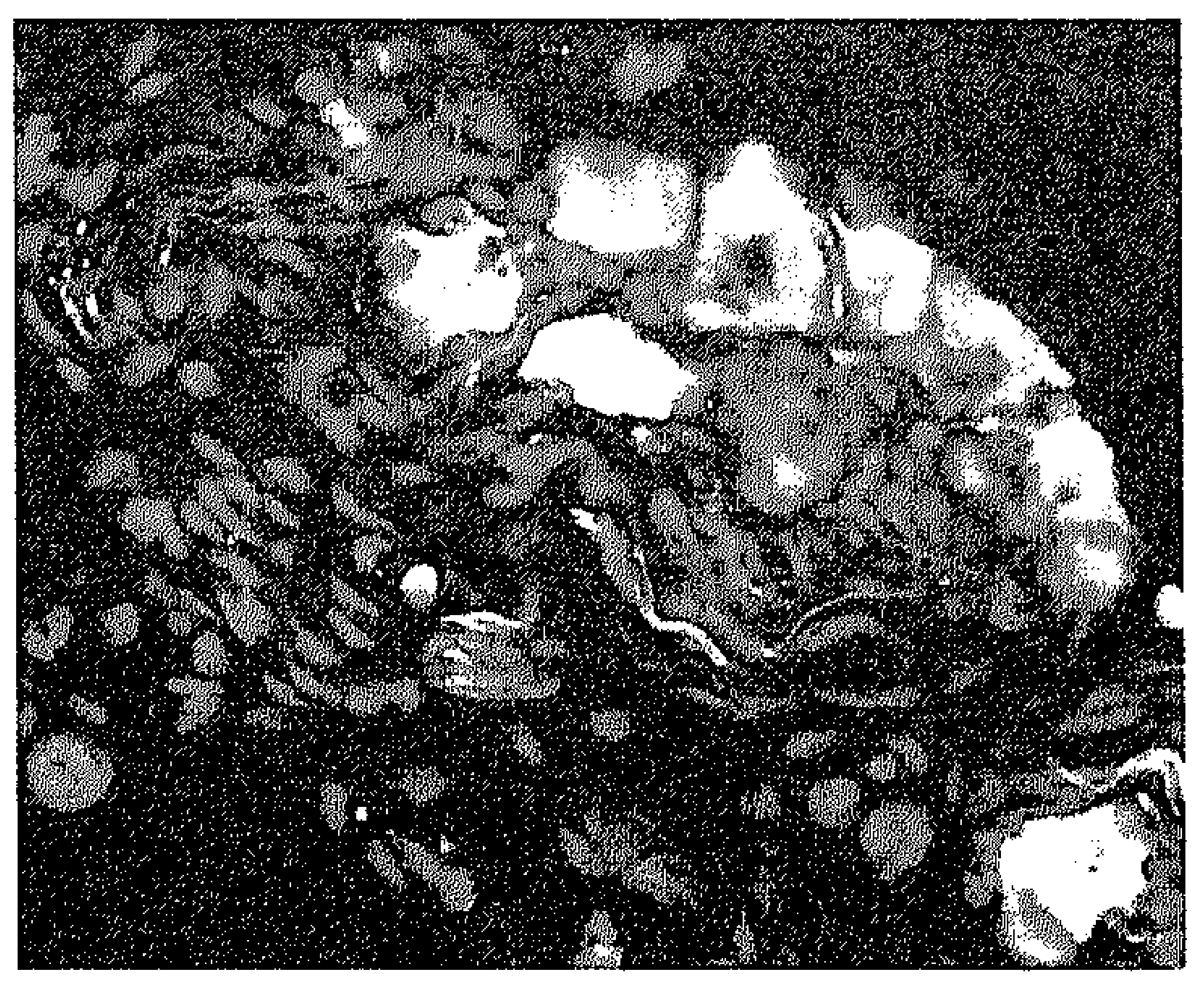

The localisation of cyclo-oxygenase immunoreactivity (COX I-IR) to the urothelium and to interstitial cells in the bladder wall

Rik de Jongh, Simone Grol, Gommert A. van Koeveringe,

Philip E.V. van Kerrebroeck, Jan de Vente and James I Gillespie.

Journal of Cellular and Molecular Medicine. 2008; 12(5):1-13. 


\begin{abstract}
Localised phasic contractions in the bladder wall (autonomous activity) have been hypothesized to be an integral part of a motor / sensory system contributing to bladder sensation. The sites responsible for generating this activity, the mechanisms involved in its propagation and modulation remain unknown. This phasic motor activity is modulated by exogenous prostaglandins. Therefore, analysis of the sites of prostaglandin production and action within the bladder wall may shed light on the mechanisms of generation and modulation of this phasic activity. In this paper, we report the localization of immuno-reactivity indicative of the expression of cyclooxygenase enzyme type I (COX I-IR) within the bladder wall. Basically, three types of COX I-IR cell were identified: epithelial cells in the basal and intermediate layers of the urothelium, complex vimentin-positive and COX I-IR cells in the lamina propria and vimentin-negative COX I-IR cells in the lamina propria and on the surface of the inner muscle bundles. These vimentin-negative/COX I-IR cells appear to be in close apposition to a continuous network of vimentin-positive cells which extends from the lamina propria into the inner muscle layers and subsequently into the outer muscle layers. However, the interstitial cells in this region might form a distinctly different sub-type. First, the interstitial cells in this region differ from those in the inner layer by their responsiveness to NO with a rise in CGMP. Two subtypes have been identified: cells on the surface of the muscle bundles and within the muscle bundles. Second, COX I-IR cells are not associated with the interstitial cells in the outer layers. The physiological significance for these apparent differences in the interstitial cell network is not clear. However, such differences are likely to reflect differences in the processes involved in their activation, modulation and control.
\end{abstract}




\section{Introduction}

Prostaglandins (PGs) are found in virtually all tissues where they exert a wide variety of functions including modulating smooth muscle activity, haemostasis and cytoprotection. ${ }^{1}$ It has been known for nearly half a century that, in the bladder, PGs are released into the general circulation by the bladder in response to distension. ${ }^{2}$ It has been established that the bladder PGs originate in both the urothelial and muscle layers. ${ }^{2.5}$ The precise role of this endogenous PG is not known but it is well documented that exogenous PGs alters bladder activity both in vivo and in vitro influencing voiding and smooth muscle contractility. This occurs in different species: human, ${ }^{6-9}$ rat, $^{10-12}$ guinea pig, ${ }^{13-15}$ rabbit $^{16-19}$ and monkey. ${ }^{20,21}$ The underlying mechanisms, which involve the PGs to alter voiding patterns and induce smooth muscle contraction, are not known. Regarding PG-induced changes in voiding frequency it was envisaged that they might act directly on the afferent nerves to modulate firing and so trigger micturition at lower bladder volumes. ${ }^{22-24}$ With regard to a direct action on the muscle, it was pointed out that they can be co-released with acetylcholine at efferent nerve endings and so directly contribute to muscle excitation. ${ }^{20,21}$ Alternatively, they might act indirectly on pre-synaptic motor terminals to affect the release of excitatory transmitters. ${ }^{11,12}$ It was considered they might also inhibit acetylcholine esterase ${ }^{9}$ or enhance myogenic bladder activity. ${ }^{6,22}$ In other organs, PG production in fibroblasts resulted in a decrease in collagen production. ${ }^{25}$ It has also been reported that prostaglandin production by fibroblasts plays a role in tumor necrosis. ${ }^{26}$ In the intestine, Powell et all. found that interstitial cells in the subepithelial space of the intestine are important in the organogenesis of the intestine, ${ }^{27}$ and secretion of prostaglandins by these interstitial cells is an important factor of this mechanism. ${ }^{27}$ Therefore, it must be taken into consideration that, next to effects on contractility, PGs might have additional functions in the bladder, e.g. a role in cell proliferation. ${ }^{25}$

Recently, a concept was proposed which attempts to integrate the effects of PG on the sensory elements of bladder control with its motor actions. Using the isolated bladder it was reported that exogenous PGs modulate the autonomous activity. ${ }^{28}$ It has been argued previously that autonomous activity is part of a motor/sensory system operating within the bladder wall whereby localised contractions of the bladder wall stimulate firing in afferent nerves contributing to sensation. ${ }^{29,30}$ PGinduced modulation of the autonomous activity would thus increase bladder sensation and so modify voiding activity. ${ }^{28}$

The origin of autonomous activity and how it is modulated by PGs is unknown. It has been hypothesized that it is generated within and distributed by a network of specialised cells in the bladder wall: interstitial cells. ${ }^{30,31}$ The precise identification and definition of what is an interstitial cell in the bladder is, at present under discussion. The initial description of interstitial cells was based on their ability to show a rise in CGMP in response to nitric oxide donors. ${ }^{31,32}$ Different sub-types of cGMP+ interstitial cell have been identified lying principally in the sub-urothelial layer and in the outer 
muscle layer. ${ }^{31}$ Finally, the stem cell factor receptor cKit, which identifies interstitial cells in the gut, has been reported to mark cells in the bladder. ${ }^{33}$ However, this cKit staining is proving difficult to reproduce. Direct evidence for the physiological role of interstitial cells is, so far, limited and comes from experiments which show that nitric oxide (NO), which generates a rise in CGMP in the interstitial cells, also abolishes autonomous activity. ${ }^{34}$

Since exogenous PG modulates autonomous activity ${ }^{28}$ further insights into the origin of this activity and possible links to interstitial cells may be achieved from a detailed understanding of where PGs are synthesised and where they act in the bladder. PGs are synthesised by the two distinct enzymes: cyclo-oxygenase type I (COX I) and cyclo-oxygenase type II (COX II). COX I is associated with cell somata in the urothelium and with a network of cells running over the muscle bundles. ${ }^{28} A$ detailed description of the different cell types expressing COX I is not yet available. Therefore, the present experiments were done to characterise the cells expressing COX I in the normal bladder and to determine the relationship between these cells to the networks of interstitial cells. The distribution and characteristics of COX II expression in the bladder is the subject of a separate paper. ${ }^{28}$

\section{Materials and Methods}

Guinea pigs (7 male, weight 270 - 350 g) were killed by stunning and exsanguinations. The urinary bladder was removed and placed in ice-cold Kreb's solution containing $121.1 \mathrm{mM} \mathrm{NaCl}, 1.87 \mathrm{mM} \mathrm{KCl}, 1.2 \mathrm{mM} \mathrm{CaCl}, 1.15 \mathrm{mM} \mathrm{MgSO}_{4}$, $25 \mathrm{mM} \mathrm{NaHCO}_{3}, 1.17 \mathrm{mM} \mathrm{KH}_{2} \mathrm{PO}_{4}, 11.0 \mathrm{mM}$ glucose, bubbled with $5 \% \mathrm{CO}_{2}$ and $95 \%$ $\mathrm{O}_{2}$. The bladder wall was cut into 3 or 4 sections from the base to the dome. The procedures for isolation, stimulation with the NO donor diethylamine-NONOate (DEANOI Sigma-ALdrich), and detection of CGMP-immunoreactivity were as described previously. ${ }^{28,31}$ Preparation of the cryostat sections and immunocytochemical procedures were as described before. ${ }^{28,31}$ Specificity studies on the sheep or rabbit anti-formaldehyde-fixed-cGMP antisera have been published before $^{31}$ and preabsorbtion studies to ascertain the specificity of the COX I antiserum have been published before also. ${ }^{28}$ Primary antibodies used were goat polyclonal antibody to COX I (1 : 2000; Santa Cruz Biotech, Santa Cruz, Ca, USA), rabbit antinNOS antiserum (1:3000, Diasorin), mouse anti-vimentin antiserum (1:5000; Sigma/Aldrich). Secondary antibodies were Alexa Fluor 488 donkey anti-sheep IgG $(\mathrm{H}+\mathrm{L})$ and donkey anti-mouse conjugate (Molecular Probes, 1 : 100); Alexa Fluor 594 donkey anti-rabbit IgG conjugate (Molecular probes, $1: 100)$; CY3 donkey antigoat IgG conjugate (Jackson Immunoresearch, Newmarket, UK; 1 : 800). Sections were analyzed using an Olympus AX70 fluorescence microscope, equipped with a narrow band-pass MNIBA-filter for the detection of Alexa 488, and for detection of $\mathrm{CY} 3$ and Alexa 594 we used a filter with a narrow excitation band, the U-M41007A filter (both from Chroma Technologies, Rockingham, Vt, USA). The microscope was 
equipped with a cooled charge-coupled device, the Olympus digital video camera Fview. Images were stored digitally as 16 bits images by using the computer program Cell^P (Soft Imaging Systems, Olympus, Germany). The number of grey values was reduced by using a linear function to 4095 . Colour images were produced by combination of the original grey values photographs using the Cell-P program. Images were arranged using the program Adobe Photoshop 7.0.1 (San Jose, Ca, USA) without further processing.
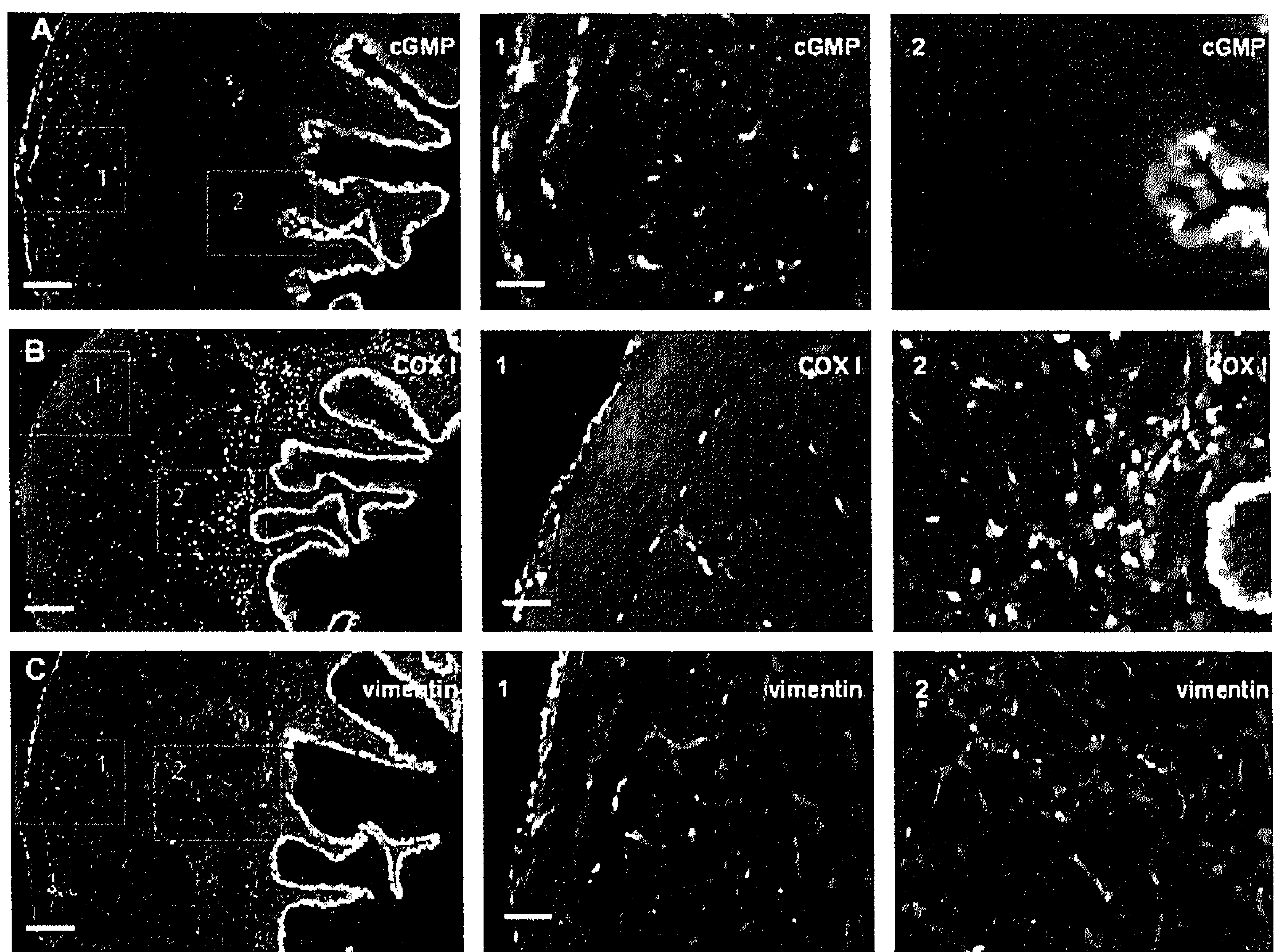

Figure 1.

The localisation of COX $I$-IR, vimentin+ cells, and NO-responsive CGMP+ cells in the guinea pig bladder. $\boldsymbol{A}, \boldsymbol{B}$ and $\boldsymbol{C}$ show respectively images stained with antibodies to CGMP, COX $I$, and vimentin. In each panel two regions are identified (1 and 2) located in the outer and inner regions of the bladder wall. For $\boldsymbol{A}-\boldsymbol{C}$ these identified regions are shown at greater magnification in the adjacent panels. In $\boldsymbol{A}$ the presence of cGMP+ cells is clearly seen. No CGMP cells are seen associated with the inner muscle layer but the umbrella cells and sub-urothelial cells are CGMP+. B illustrates COX I-IR. More COX I-IR cells are seen in the inner regions of the bladder. In $\mathbf{C}$ vimentin ${ }^{+}$cells are seen in both the outer and inner layers. Calibrations bars: $150 \mu \mathrm{m}$ and $40 \mu \mathrm{m}$. 


\section{Results}

The key basic observations regarding the distribution of COX I-IR in the bladder wall are illustrated in figure 1 . All animals showed the same results. Panel A shows a low power section of the entire bladder wall. COX I-IR cells are seen within the urothelium and also associated with a network of cells which extends from the lamina propria into the inner muscle layers. Note that there are fewer COX I-IR cells associated with the outer muscle layers. These observations are illustrated in greater detail in panels 1 and 2, which show the sub-urothelial region (1) and outer muscle layer (2) at higher magnification.

COX I-IR is therefore associated with two distinct structures: the urothelium and a network of cells in the lamina propria and around the muscle. The pattern of distribution of these network cells bears a strong resemblance to the distribution of vimentin positive $\left(\mathrm{vim}^{+}\right)$cells in the muscle layers bladder wall but not the distribution of NO-responsive CGMP+ interstitial cells (Figs. 1B-C). Note that the CGMP+ interstitial cells are found in the outer muscle layers where there is considerably less COX I-IR. These observations on COX I-IR in the urothelium and associated with the networks of interstitial cells are described in more detail below.

\section{COX I-IR in the urothelium and sub-urothelial layer}

Figure 2A illustrates a section showing the urothelium and sub-urothelial region,
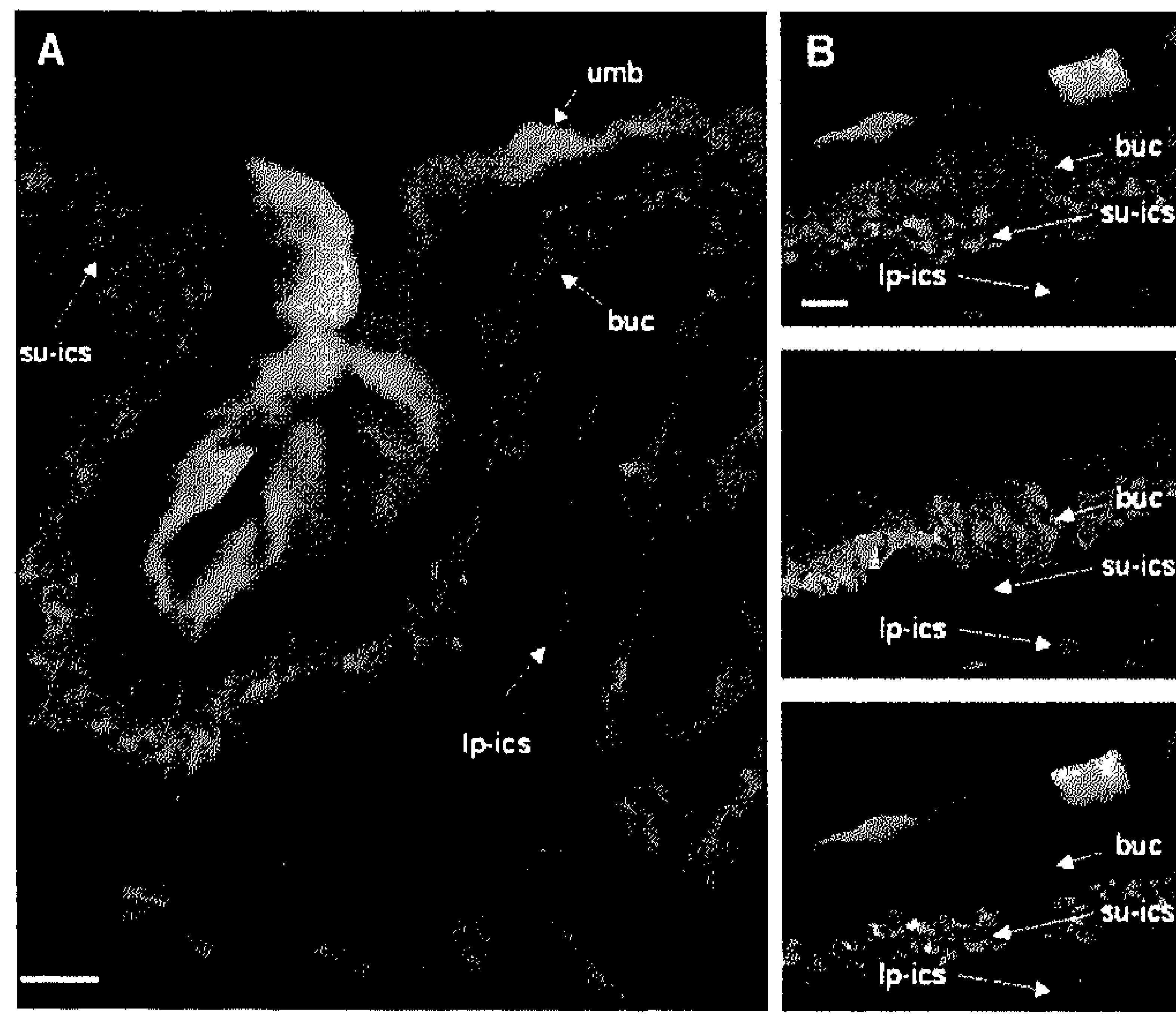

Figure 2.

CGMP- and COX I-IR associated with the urothelium in the guinea pig bladder (coloured image page 196). Panel A shows an image of the urothelium of the lateral wall stained for COX I-IR (red) and CGMP (green). The preparation was stimulated with a NO donor to elevate CGMP levels in responsive cells prior to fixation. The umbrella cells on the surface of the urothelium (umb) and cells in the sub-urothelial space, the suburothelial interstitial cells (su-ics) are cGMP+. Cells in the basal regions of the urothelium (basal urothelial cells: buc) are stained intensely with the COX I antibody. In addition, coX I-IR cells are also found in the lamina propria interstitial cells (lpics). B illustrates a section from a different bladder processed and stained in the same way as section $A$. The upper panel shows the combined colour image. The middle and lower panels show COX I-IR and CGMP-IR respectively. These two panels illustrate that the CGMP+ su-ics are negative for COX I-IR. They also show that the COX I-IR cells in the buc are separated from the COX I-IR Ip-ics. Calibration bars $50 \mu \mathrm{m}$ in $\boldsymbol{A}$ and $30 \mu \mathrm{m}$ in $\mathbf{B}$. 

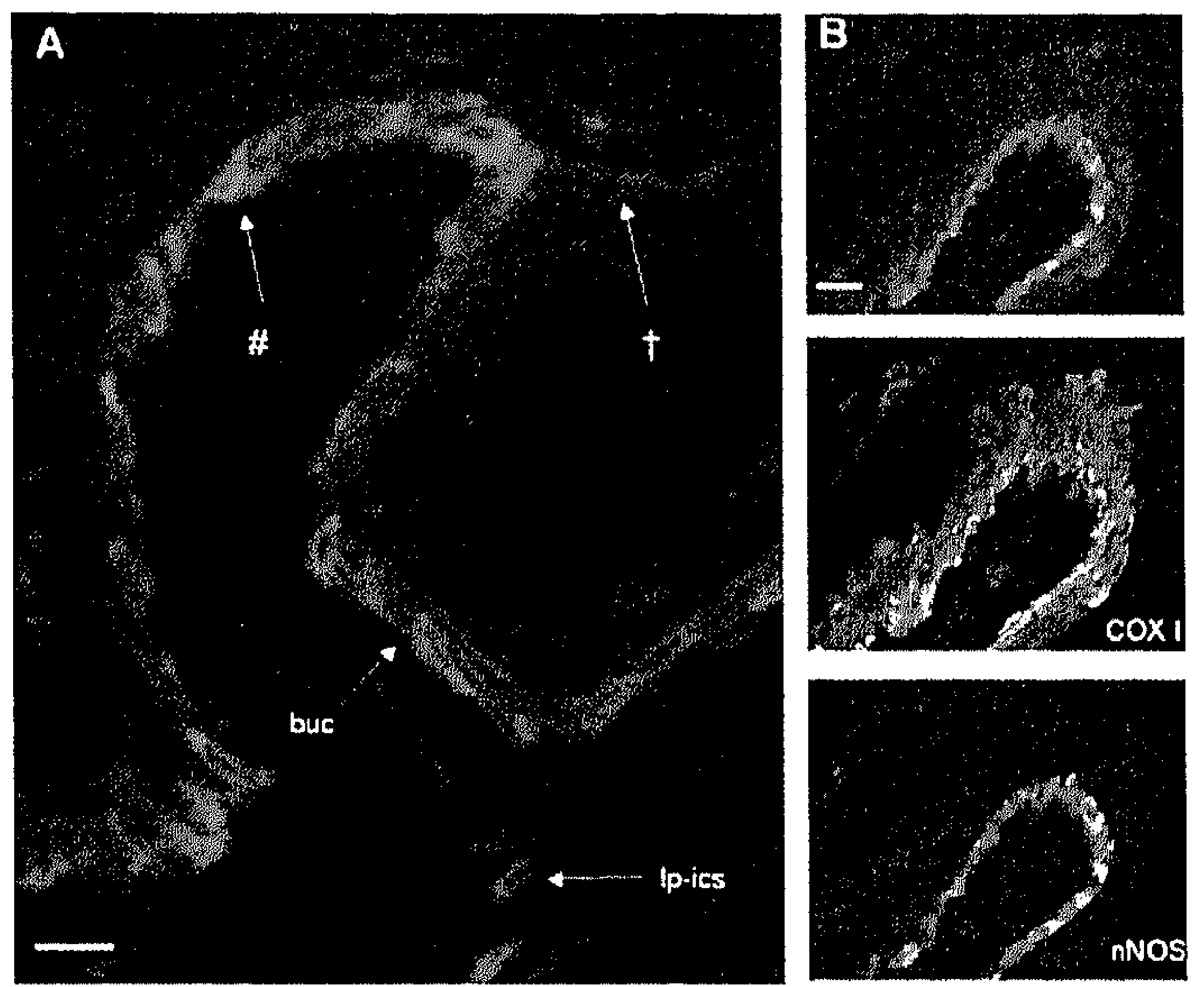

Figure 3.

The identification of cells expressing neuronal nitric oxide synthase (nNOS) and COX I-IR in the urothelial layer of the guinea pig bladder (coloured image page 196).

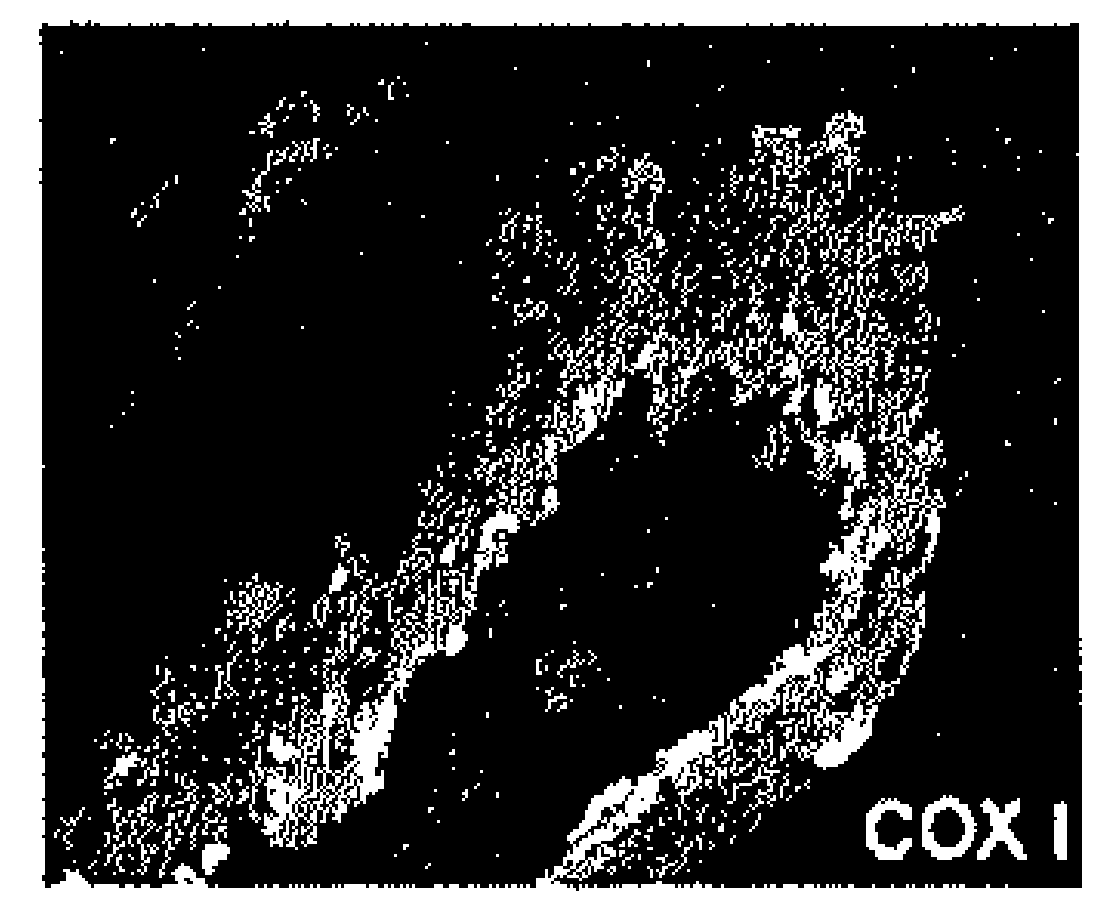

Sections were co-stained with antibodies to nNOS (green) and COXI (red). A shows an example of the staining pattern associated with the urothelium. nNOS+ cells (\#) are seen to be located within a single layer in the basal urothelium (buc). These cells also demonstrate coX I-

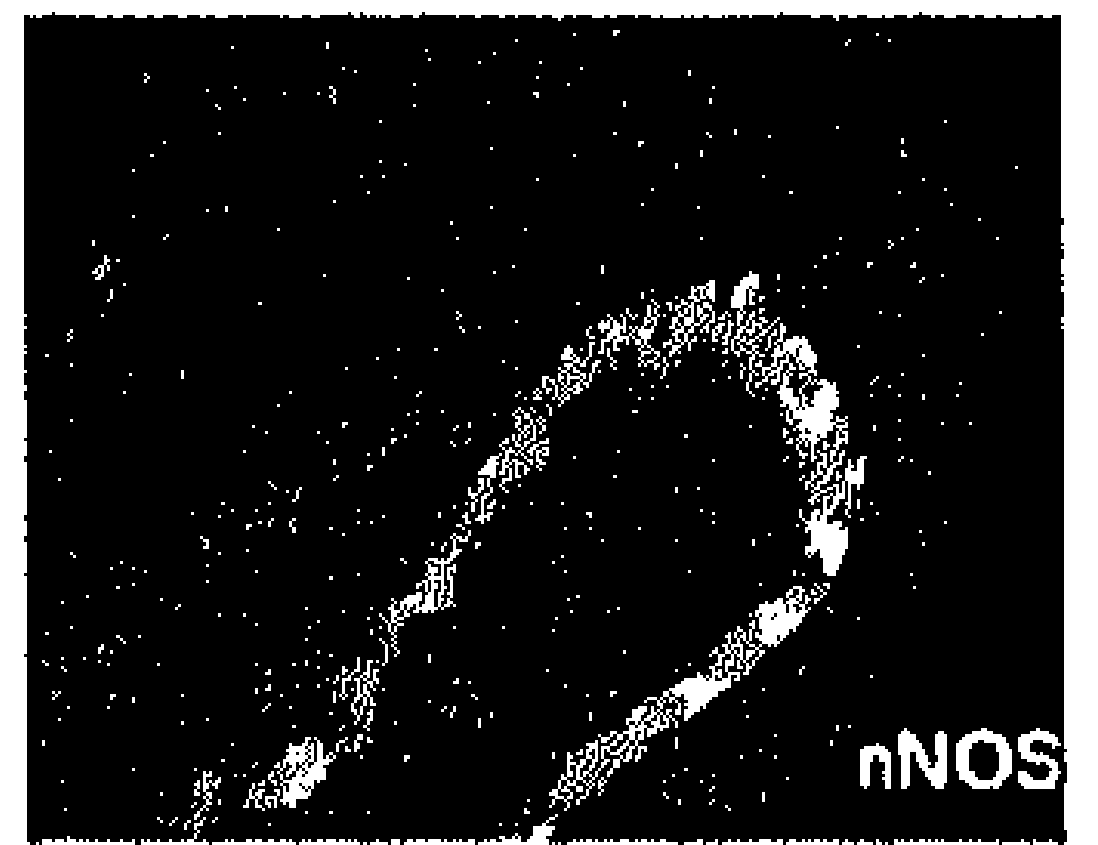
IR. Note that the COXI-IR extends into further cell layers within the urothelium $(t)$. B shows a further example from a different bladder. The combined image and the individually stained images are shown. The location of nNOS to a single layer in the basal urothelium is clearly seen while the more diffuse location of the COX I-IR is apparent. Calibration bars: $30 \mu \mathrm{m}$ in $\boldsymbol{A}$ and $\boldsymbol{B}$.

double stained for COX I (red) and CGMP (green). Figure 2B shows a similar section but from a different bladder illustrating the combined colour image (top) and the component COX I (middle) and CGMP (lower) images. As reported previously, the umbrella cells lying on the surface of the urothelium and the sub-urothelial interstitial cell layer close to the urothelium are sensitive to exogenous NO and demonstrate a rise in CGMP ( $\left(\mathrm{CGMP}^{+}\right)$. It is also clear that COX I-IR is associated with intense staining in the intermediate and basal layers of the urothelium (buc) and weekly in cells in the lamina propria (lamina propria interstitial cells (Ip-ics)). Figure 2B shows, in greater detail that, in this region of the bladder wall, the majority of the suburothelial CGMP+ interstitial cells do not have COX I-IR.

The cells of the basal layer of the urothelium express neuronal nitric oxide synthase (nNOS) ${ }^{31}$ The relationship between these cells and those with COX I-IR is shown in figure 3. In this experiment the sections were double stained with nNOS (green) and COX I (red). The nNOS positive (nNOS+) cells are easily seen in the basal layer. These cells are also COX I-IR (showing green / yellow in the combined sections). Note that in the intermediate urothelial layer the cells are COX I-IR but not nNOS+ (Fig. 3B). Thus, there are two types of COX I-IR cell in the urothelium: basal COX I-IR/nNOS+ and intermediate COX I-IR/nNOS-.

The layer of densely packed cells lying immediately below the urothelium, the sub-urothelial interstitial cells (su-ics) are responsive to NO with an elevation in cGMP ${ }^{31}$ Figure 4 illustrates a section double labelled to show these cGMP+ cells (green) and the vimentin+ cells (red). cGMP+ umbrella cells are seen on the surface of the urothelium but there were no vim+ cells in any cell type within the urothelium. In contrast, the cells immediately below the urothelium, the su-ics, were vim+ and 

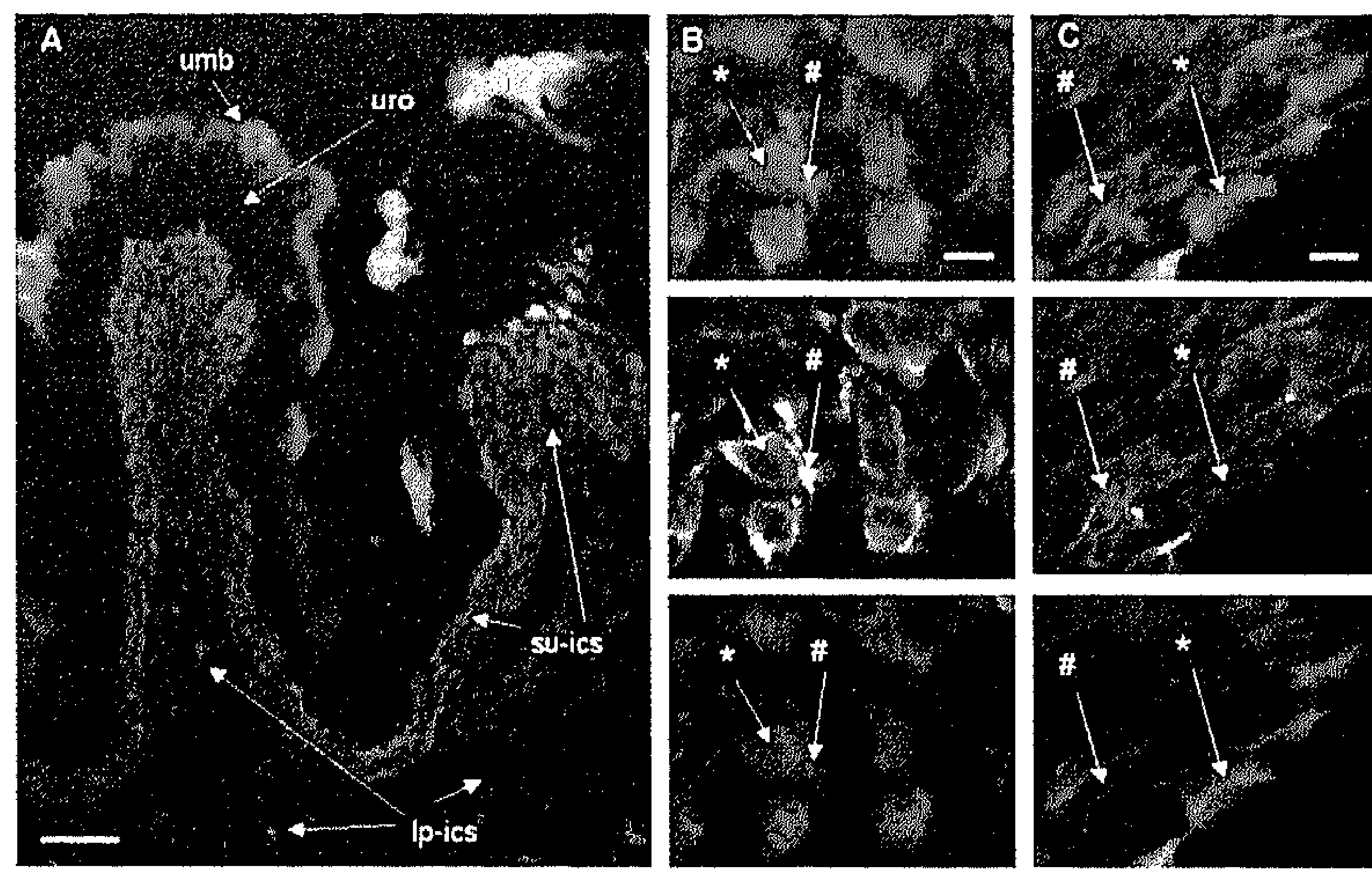

Figure 4.

The location of vimentin' structures in the urothelium and sub-urothelial space (coloured image page 197).

Sections were double labelled with antibodies to show CGMP (green) and vimentin (red). Panel A shows a section from the lateral wall of the bladder illustrating the urothelium and suburothelial space. The umbrella cel/s (umb) and sub-urothelial interstitial cells (su-ics) are $C G M P^{+}$, No vimentin+ structures are seen within the urothelium. Vimentin ${ }^{+}$structures are seen in the sub-urothelial space. Intense staining is seen associated with the cells immediately below the urothelium while structures lying deeper within the lamina propria are vimentin ${ }^{+}$but more defuse. $\boldsymbol{B}$ and $\boldsymbol{C}$ illustrates examples of the cells in the sub-urothelial layer at higher magnification, showing the combined colour image (upper panel: CGMP-green, vimentin-red), the CGMP image alone (middle panel) and vimentin image alone (lower panel). The CGMP+ sub-urothelial interstitial cells can readily be identified (*). Vimentin staining is associated with the cell bodies of these cells being highly concentrated around the cell nuclei (\#). $C$ Illustrates a further feature. Vimentin ${ }^{+}$but cGMP- fibres are seen (\# in C). Calibration bars $80 \mu \mathrm{m}$ in $\boldsymbol{A}$ and $20 \mu \mathrm{m}$ in $\mathrm{B}$ and $\boldsymbol{C}$.

CGMP+. Vimentin staining revealed a network of intracellular fibres, whereas the cGMP staining was most intense within the cell bodies (Fig. 4B). Processes of these cells were seen to contain vim+ fibres but these structures were often not strongly cGMP+ indicating that there are intracellular gradients of CGMP. Vim+ cells were seen in the layer of the lamina propria between the su-ic layer and the muscle layer (Fig. $4 A$ ). These cells did not demonstrate a cGMP signal and can be described as lamina propria interstitial cells (Ip-ics). Thus, there appear to be different types of interstitial cell within the lamina propria: a dense region of cGMP+ / vim+ and sparse cGMP- / vim+ cells.

An additional feature of the COX I / vimentin double staining becomes apparent when studying in detail the diffuse network of vim+ cells in the region of the lamina propria immediately below the su-ic and above the muscle layer (Fig. 5). These cells appear to form a distinct network of inter-connecting cells contiguous with the su-ic layer and with vim+ cells within the muscle layer (see below). In this section, no COX I-IR cells are visible within the su-ic layer. But, COX I-IR is seen within the cell bodies of the vim+ Ip-ics (Fig. 5B).

Regions of the lateral wall, particularly towards the bladder base, could be found in which the density of Ip-ics was high (Figs. 6-7). In these regions, two different cell types were readily identified based on the expression of vimentin and COX I-IR. Figure $6 \mathrm{~A}$ shows such a region at higher magnification. Small bipolar cells with round cell bodies, which were COX I-IR but did not stain strongly for vimentin (*) (Fig. 6B) 

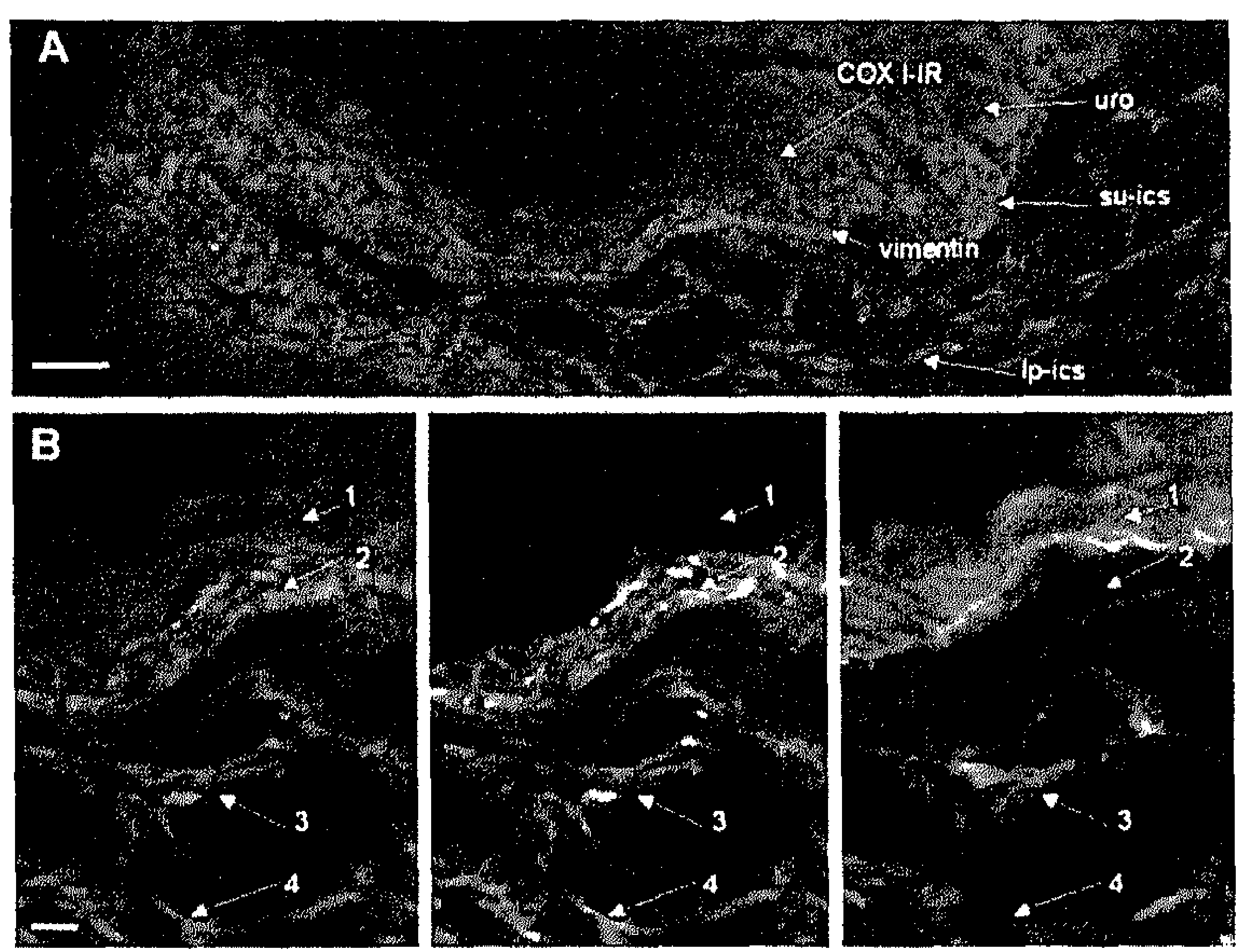

Figure 5.

Identification of COX I-IR and vimentin in the lamina propria (coloured image page 197).

Sections were double labelled with antibodies to COX I (red) and vimentin (green). A shows a low power image showing the urothelium, suburothelial layer, and the lamina propria. The COX I-IR cells are clearly visible within the urothelium (uro), the suburothelial interstitial cells (su-ics) and the lamina propria (Ip-ics). Below the suics there is a network of interstitial cells within the lamina propria (Ip-ics). Structures positive for vimentin and with COX I-IR are seen within this layer. B shows a section of the image in $A$ at higher magnification. The panel on the left shows the doubled labelled image (vimentin-green, COX I-red), the middle panel shows the vimentin image and the right panel shows the COX-I image. The basal urothelial layer (1), sub-urothelial layer (2) are identified. The staining pattern of the vimentin+ cells in the Ip is highly suggestive for a network of vimentin+ structures (4). In addition there appear to be COX I-IR cells (3). These cells are spindle shaped and are weakly positive for vimentin. They appear to lay on or between the vimentin ${ }^{+}$ structures. Calibration bars $40 \mu \mathrm{m}$ in $\mathbf{A}$ and $20 \mu \mathrm{m}$ in B.
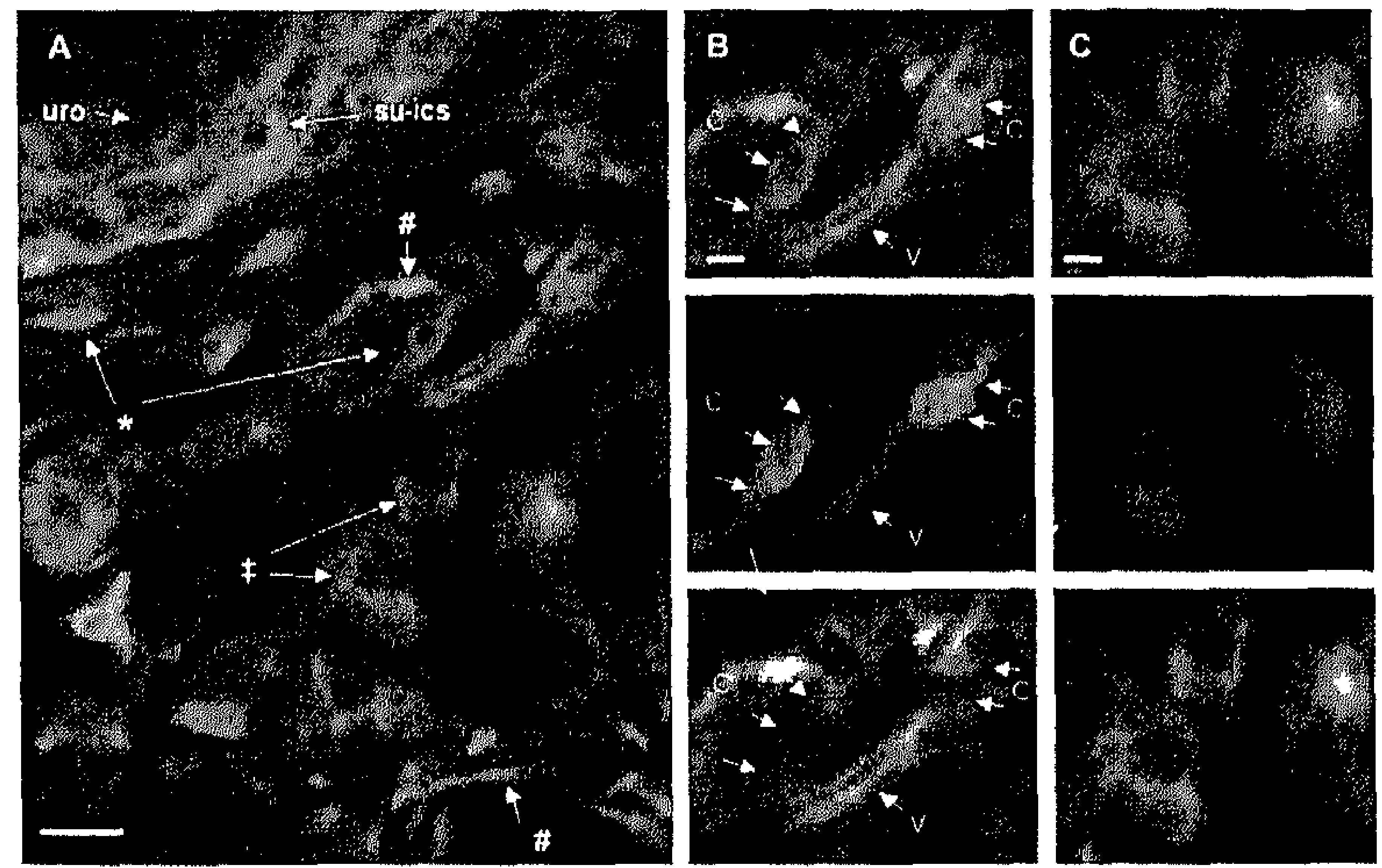

Figure 6.

Differentiation of COX IIR and vimentint structures in the lamina propria (coloured image page 198).
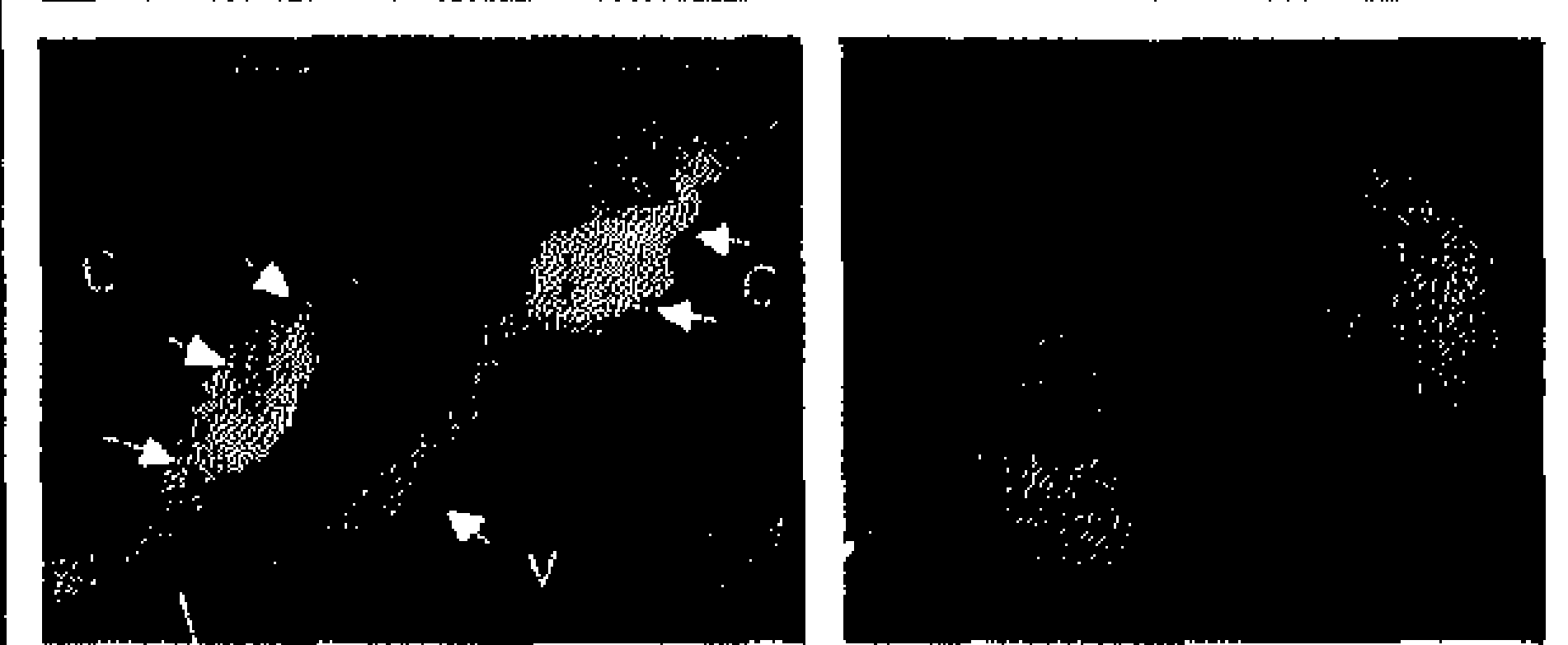

A shows a region of the lateral wall. The sections were stained with antibodies to COX I (red) and vimentin (green). $\boldsymbol{A}$ shows a region of the
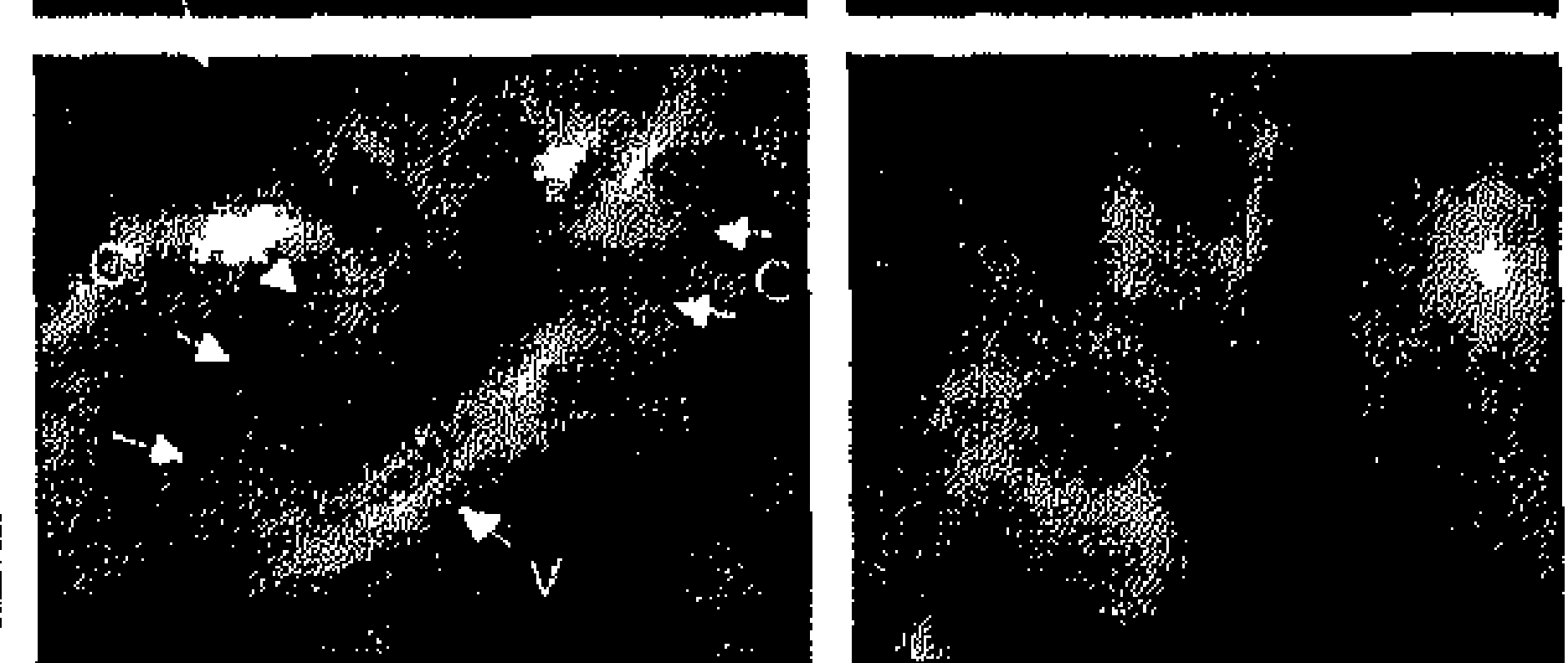
lamina propria immediately below the urothelium. The COX I-IR of the urothelium (uro) and spindle cells in the lamina propria are seen (*) (see also Fig. 5). Also, cell processes expressing vimentin are visible. Large irregular cells are also seen which are COX I-IR and which have an extensive diffuse network of vimentin fibres (₹). $\boldsymbol{B}$ and $\mathbf{C}$ show selected areas of the image in A with the individual images of COX I (middle panel) and vimentin (lower panel). In B the COX I-IR cells are seen to have little or no vimentin staining. The edges of the COX I-IR cells are indicated by the arrows and (c). Vimentin fibres are indicated (v). $C$ illustrates the larger cells with the diffuse vimentint network in the lamina propria. Calibration bars $20 \mu \mathrm{m}$ in $\boldsymbol{A}, \boldsymbol{B}$ and $\boldsymbol{C}$. 


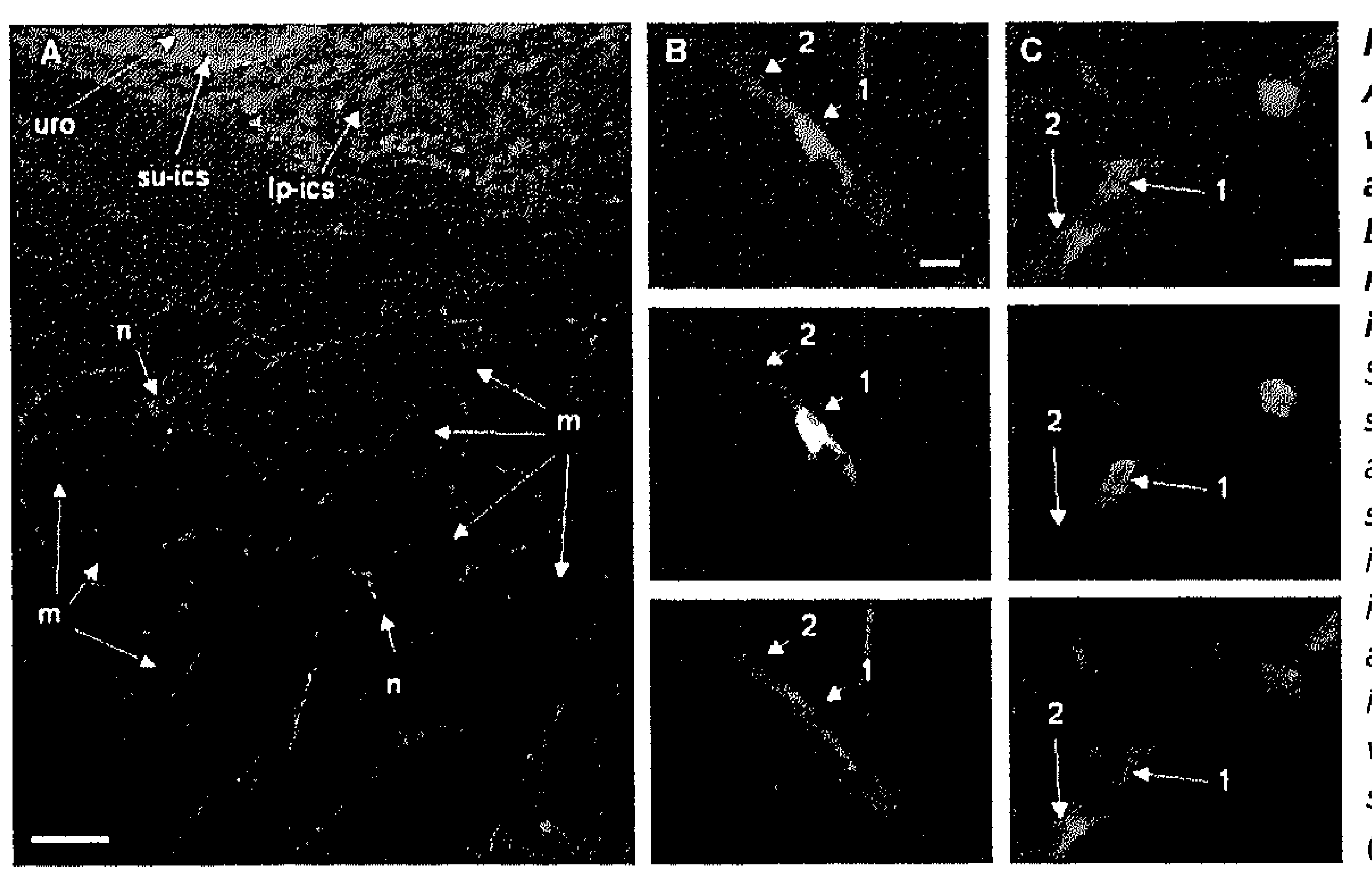

Figure 7.

A network of vimentint processes around the muscle bundles of the inner muscle layer (coloured image page 198).

Sections were double stained for COX I (red) and vimentin (green). A shows a low power image. The COX I-IR cells in the urothelium (uro) and lamina propria (Ipics) are seen. The vimentint fibres of the sub-urothelial cell layer (su-ics) and within the cell network in the lamina propria are also apparent. Muscle bundles in the inner muscle layer are shown ( $m$ ). A shows a network of vimentin fibres which are associated with the muscle bundles. Collections of COX IIR cells are seen between the muscle bundles $(n)$. These structures are described as 'nodes'. $\boldsymbol{B}$ and $\boldsymbol{C}$ show examples of the COX I-IR cells and their relationship to the vimentin' fibres. $B$ and $C$ show combined colour images: vimentin-green, COX I-red (upper panel), the COX I image alone (middle panel) and the vimentin image alone (lower panel). The COX I-IR cells (1) are weakly vimentint and are associated with the vimentin ${ }^{+}$processes (2) of other cells. Calibration bars $240 \mu \mathrm{m}$ in $\boldsymbol{A}$ and $10 \mu \mathrm{m}$ in $\boldsymbol{B}$ and $\boldsymbol{C}$.

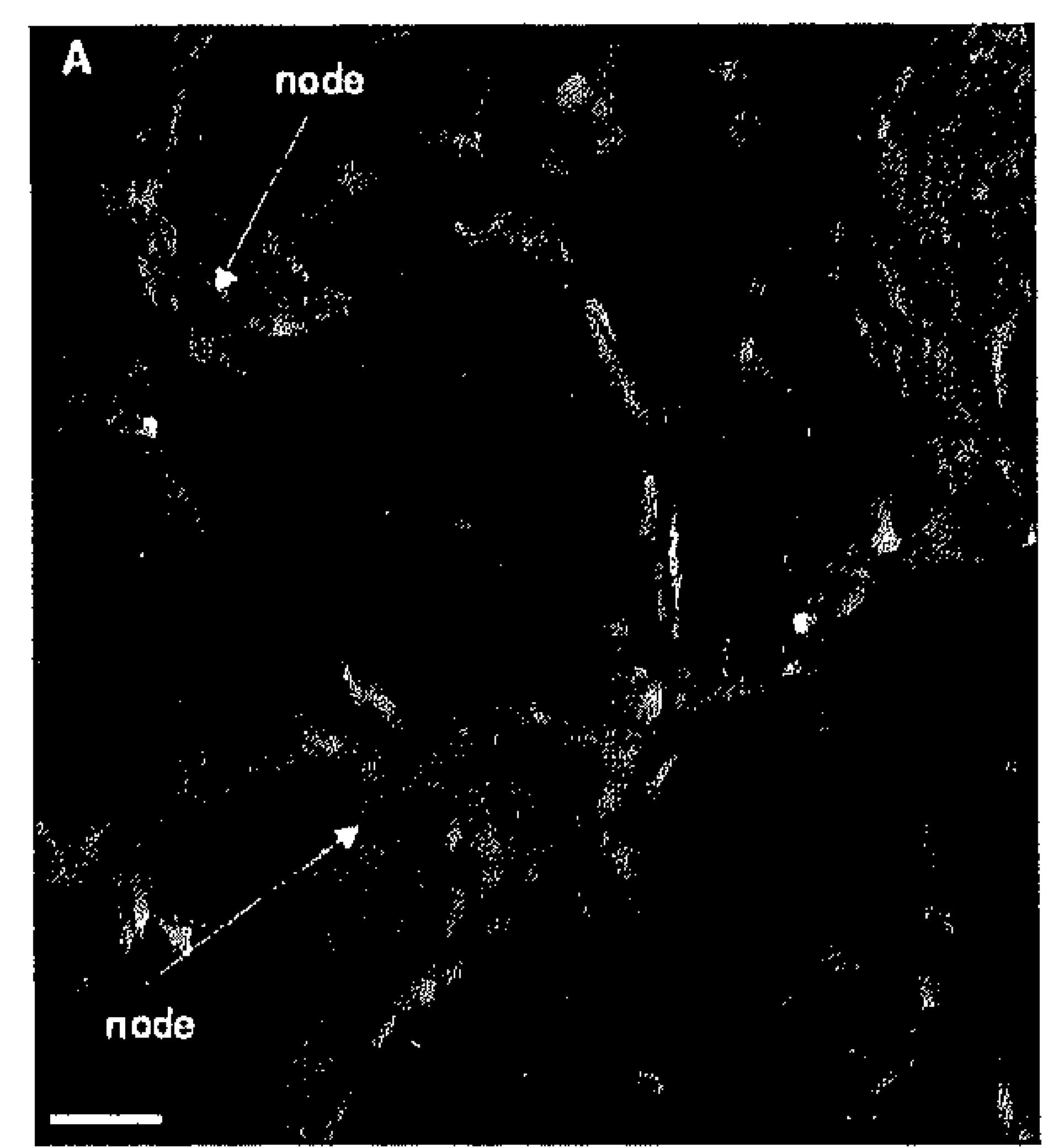

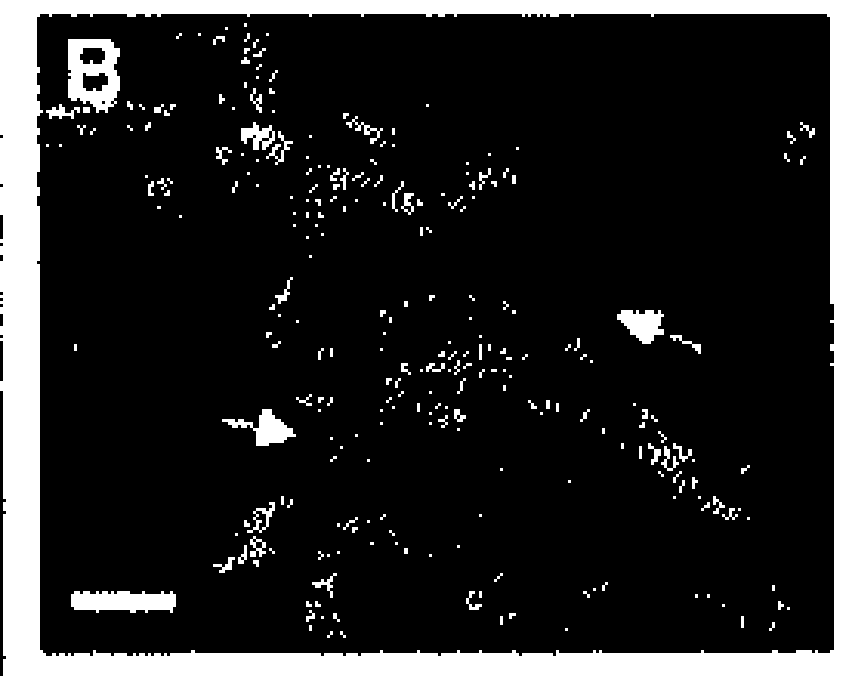
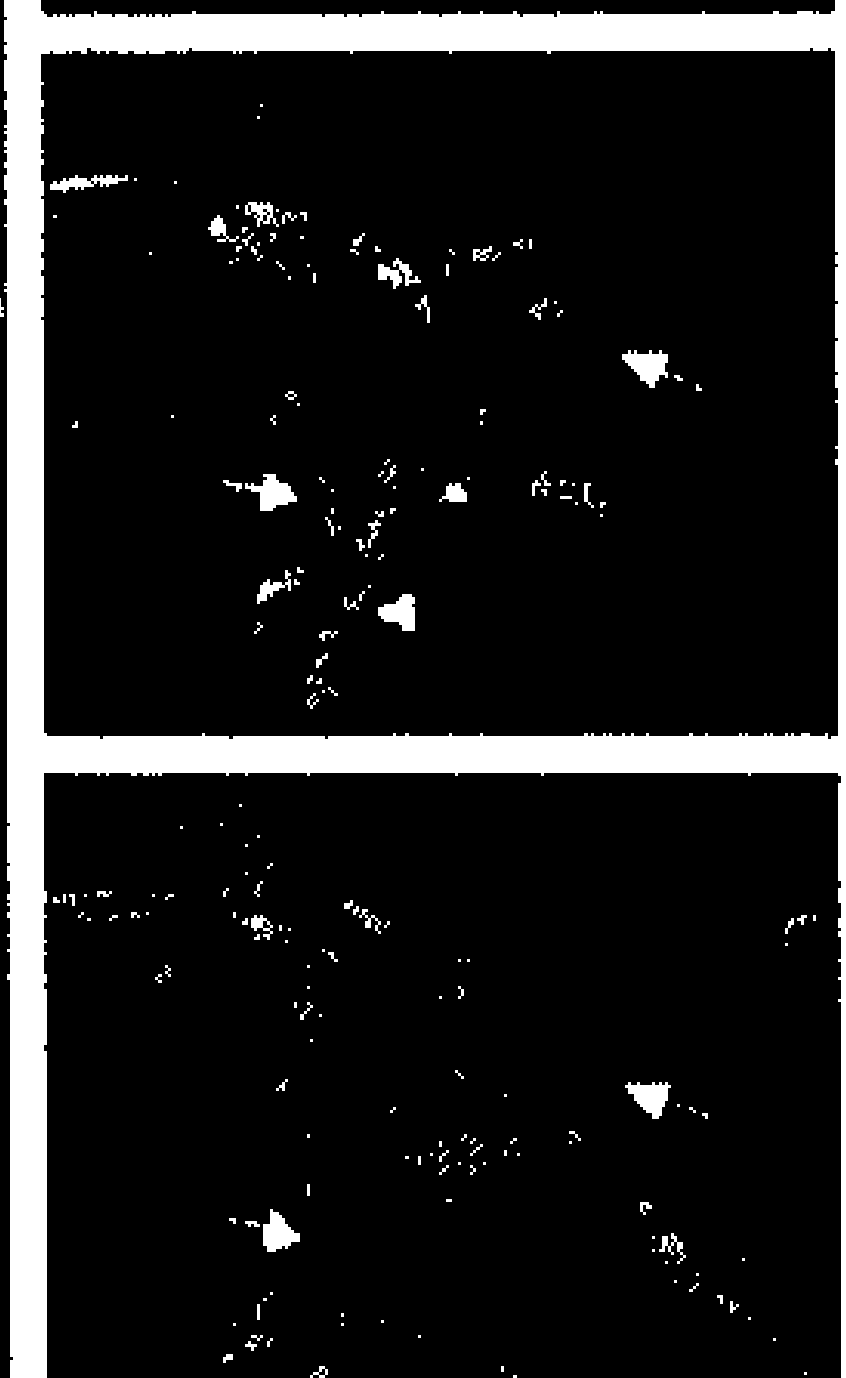
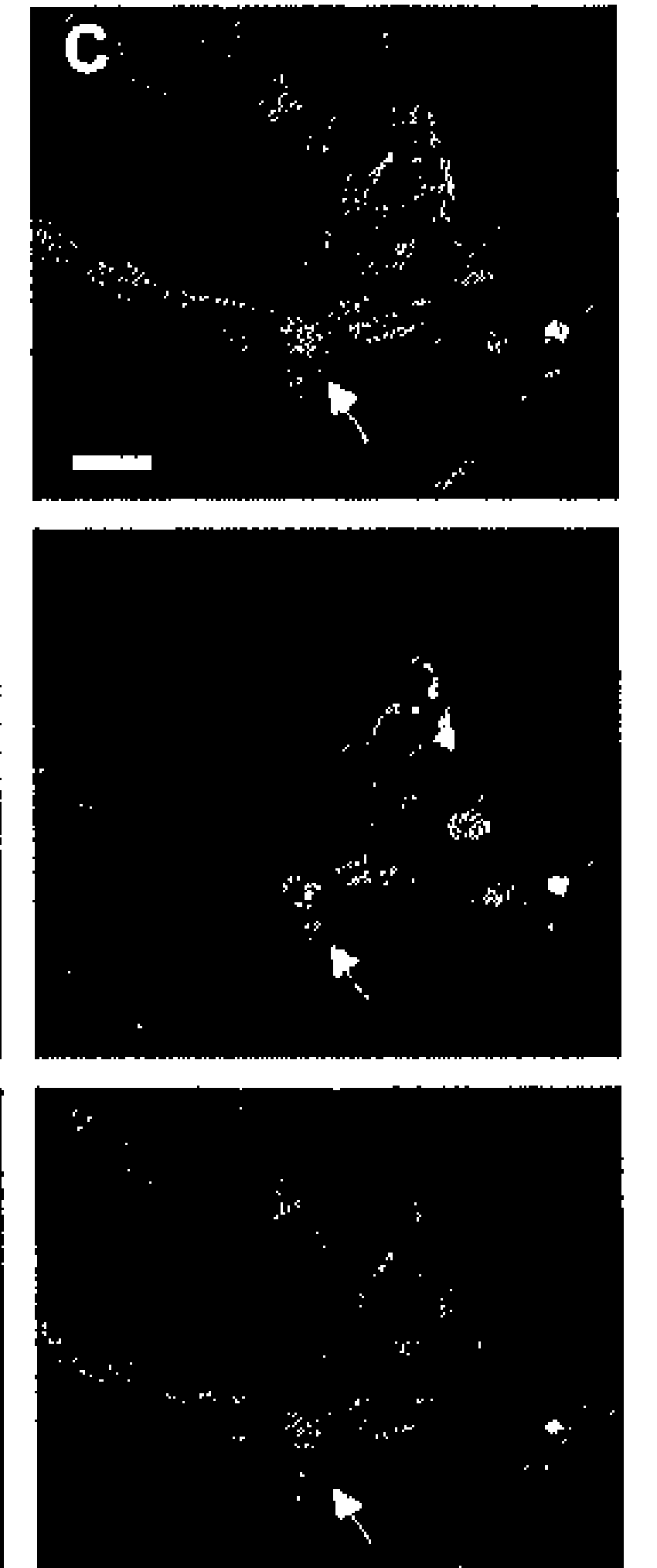

Figure 8.

Nodes associated with the interstitial cells within the lamina propria and inner muscle layer (coloured image page 199).

Sections were double stained with antibodies COX I (red) and vimentin (green). A shows a region of the lamina propria. Collections of COXIIR structures (nodes) are seen associated with collections of vimentint fibres. $\boldsymbol{B}$ and $\boldsymbol{C}$ show selected areas of the image in $A$ with the associated individual images to COX I (middle panel) and vimentin (lower panel). The COX I-IR structures are identified by the arrows. Little or no vimentin staining is associated with these COX I-IR structures. Calibration bars $50 \mu \mathrm{m}$ in $\mathbf{A}$ and $30 \mu \mathrm{m}$ in $\boldsymbol{B}$ and $\mathbf{C}$. 

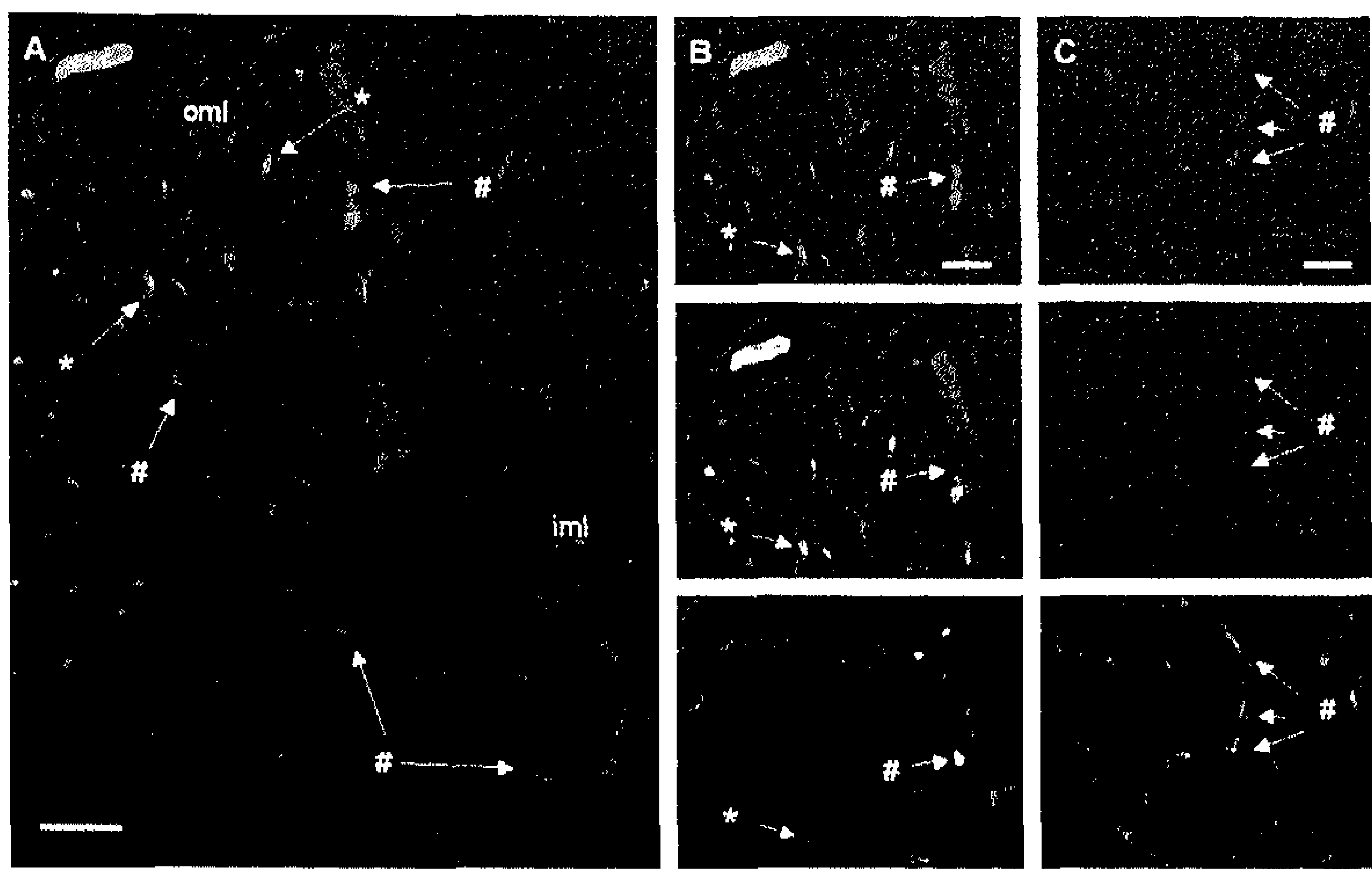

Figure 9.

The distribution of COX $I-I R$ and $C G M P^{+}$cells in the outer muscle layers (coloured image page 199).
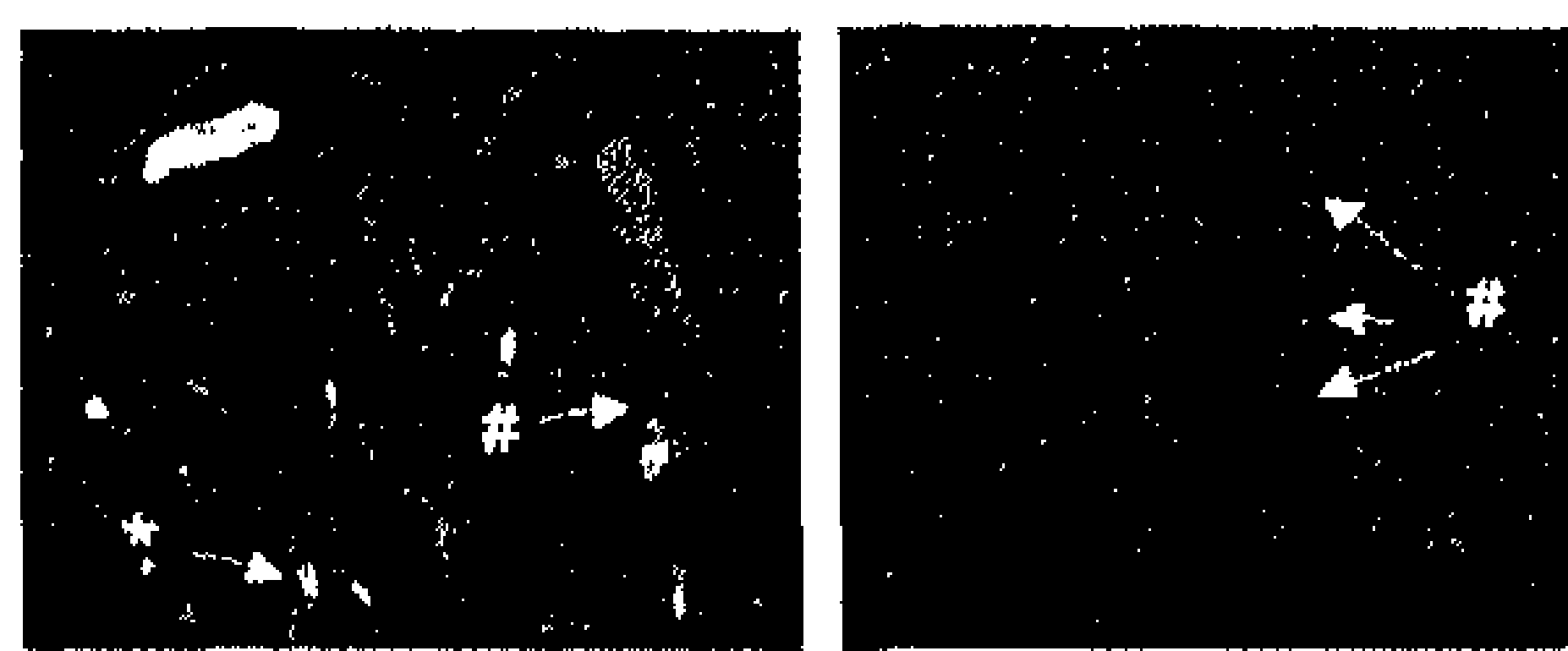

A shows an image double labelled with antibodies to CGMP (green) and COX I (red). CGMP+ cells are seen in the outer muscle layer (oml) while there are

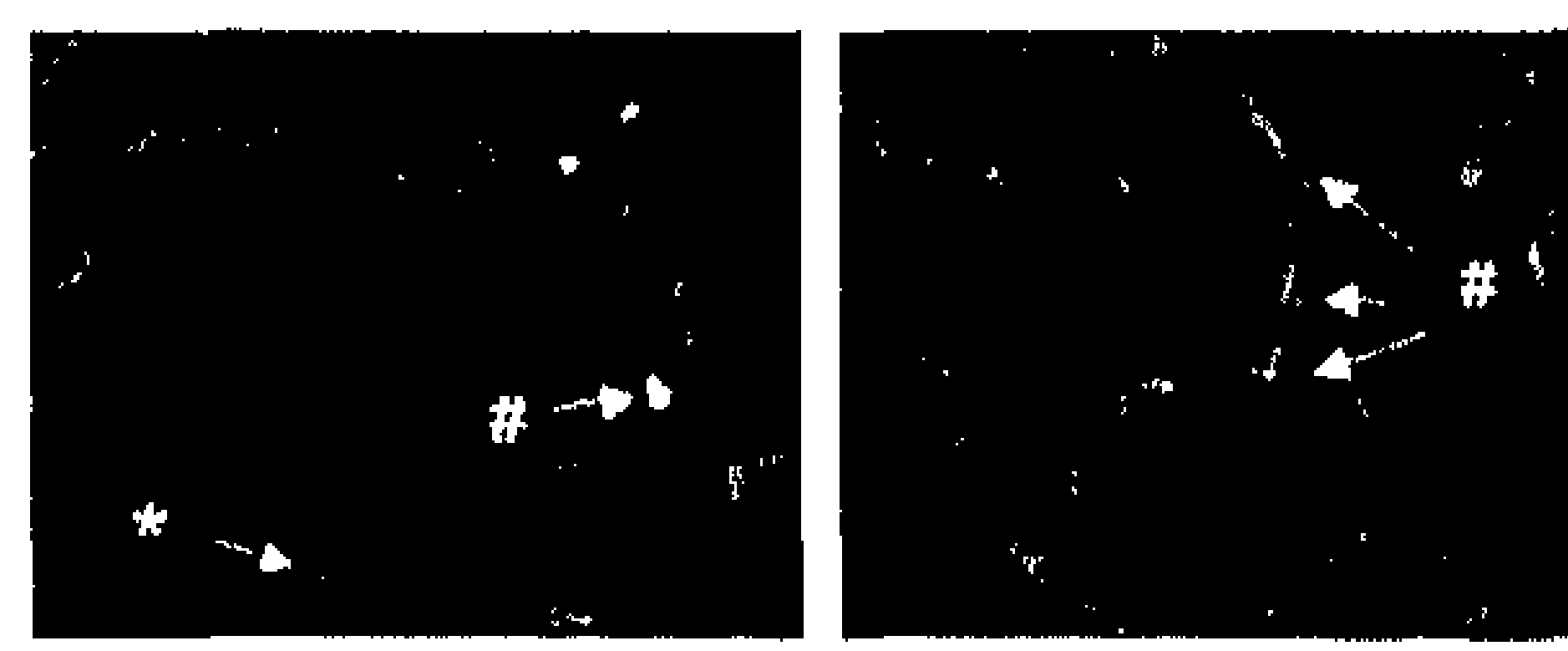
no cells associated with the inner muscle layer (im/). cGMP+ cells $\left(^{*}\right)$ and COX I-IR cells (\#) are identified. $B$ and $C$ show regions of the image in $A$ of the outer and inner layers respectively at higher magnification, showing the combined colour image (upper panel: CGMP-green, COX I-red), the CGMP image alone (middle panel) and COX I image alone (lower panel). In B cGMP+ cells are clearly seen (*) while COX I-IR cells are scarce (\#). The COX I-IR cells do not show any CGMP staining. $C$ shows the network of COX I-IR cells in the iml and the absence of CGMP staining. Calibration bars $120 \mu \mathrm{m}$ in $\boldsymbol{A}$ and $40 \mu \mathrm{m}$ in $\boldsymbol{B}$ and $\boldsymbol{C}$.
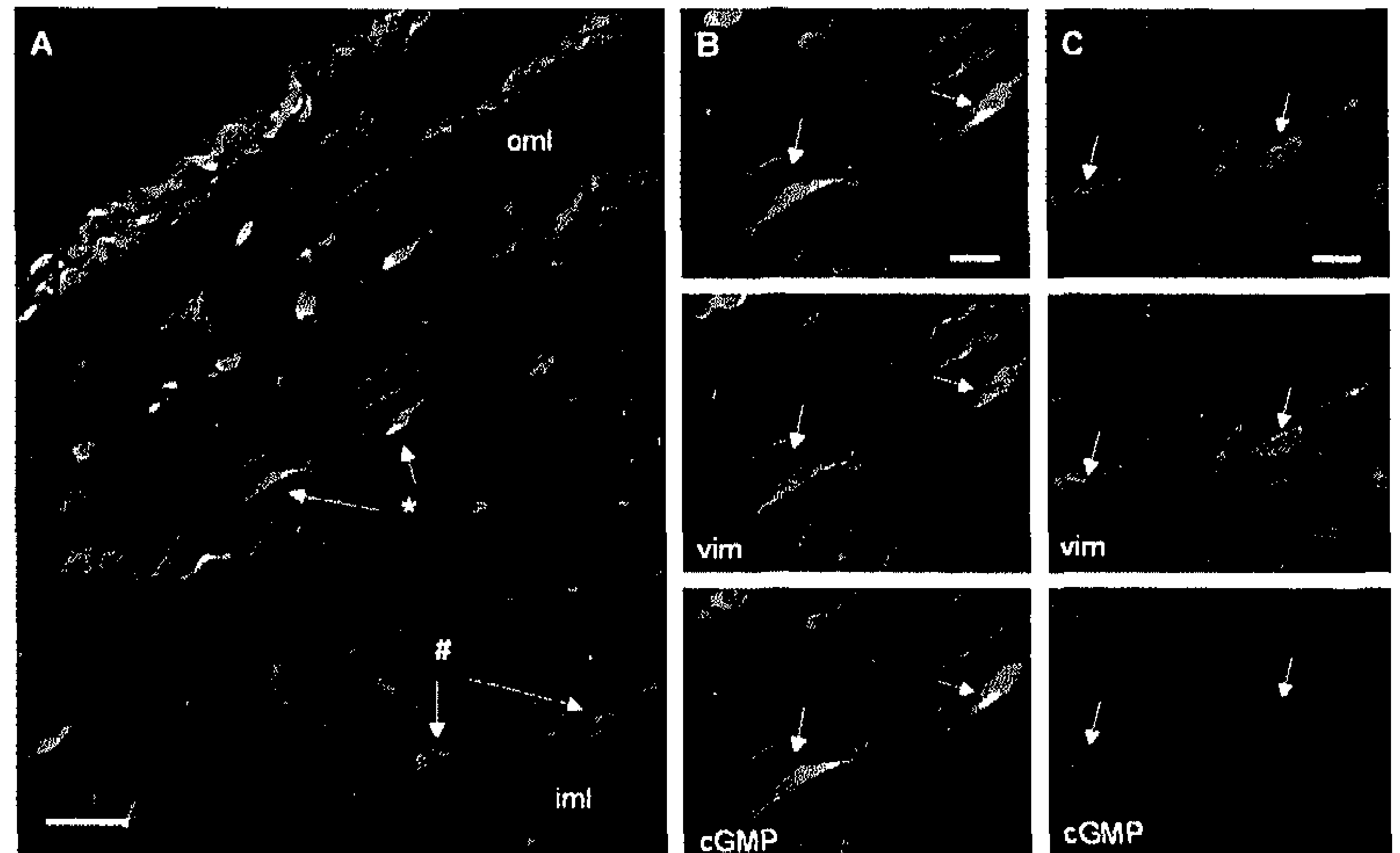

Figure 10.

Identification of

interstitial cells in the muscle layers of the

bladder (coloured image page 200).
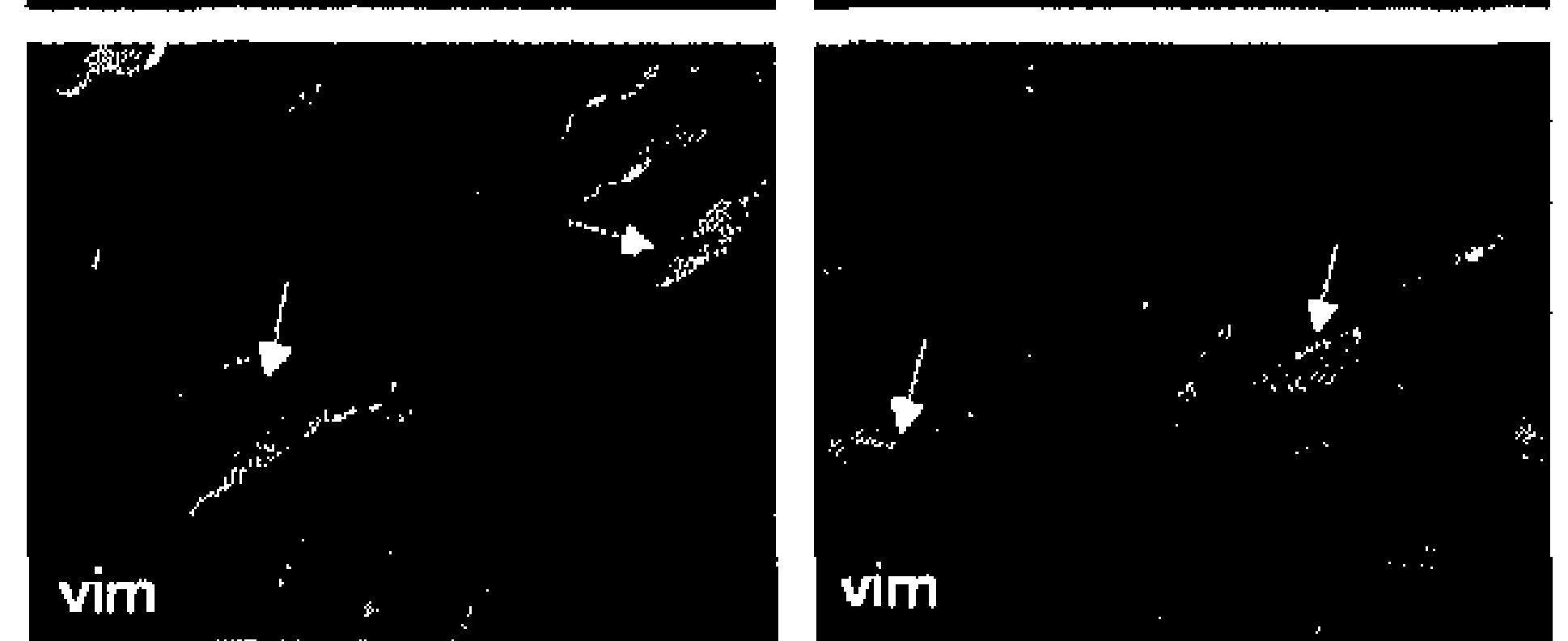

The sections are double labelled with antibodies to CGMP (green) and vimentin (red). A shows a low power image identifying the outer (oml)
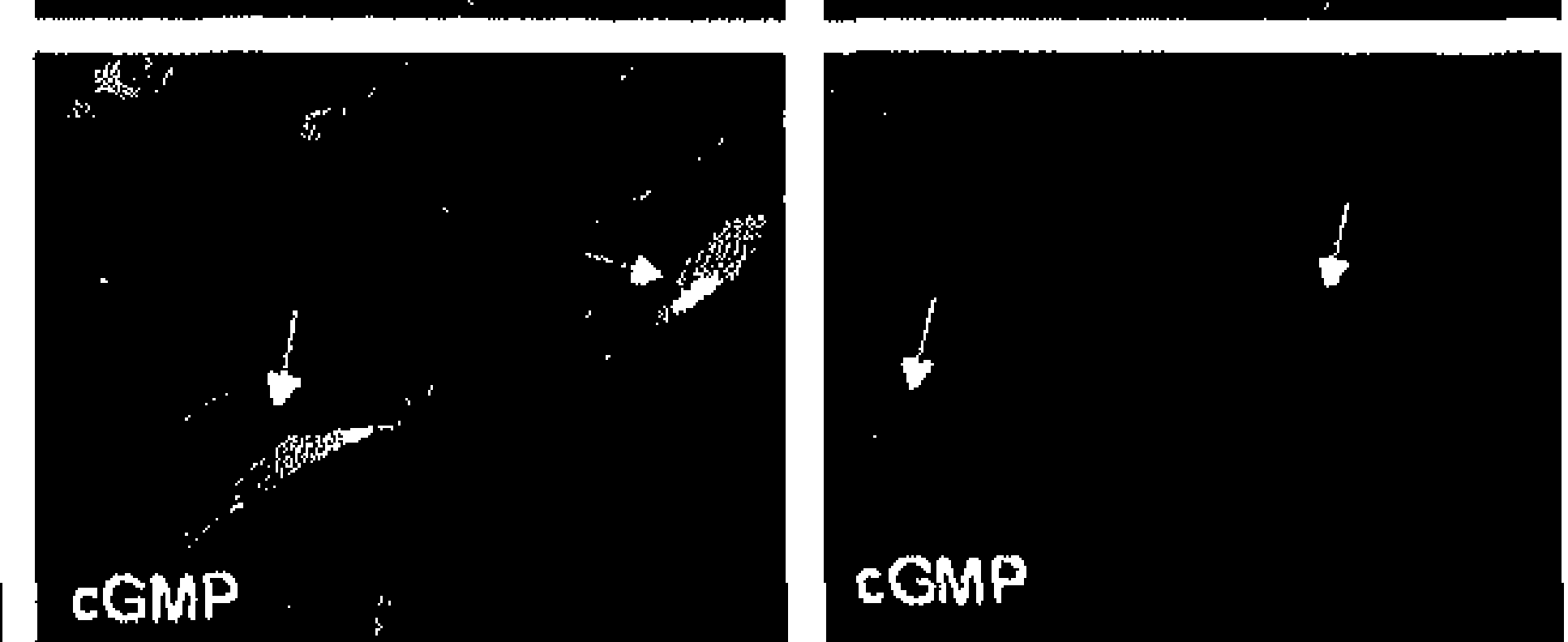
and inner muscle layers (iml). The network of CGMP+ ics associated with the outer layer and the network of vimentin+ cells in the inner $\left(^{*}\right)$ and outer layers are readily seen. The different types if CGMP+ ics are indicated by the arrows: muscle coat interstitial cells (mc-ics), surface muscle interstitial cells (sm-ics) and intra-muscular interstitial cells (im-ics). B and $\boldsymbol{C}$ illustrate regions of the image in $\boldsymbol{A}$ at higher magnification showing combined colour images and vimentin and CGMP images alone. B shows primarily the sm-ics. All CGMP+ structures are also vimentin ${ }^{+}$. $C$ focusses on the im-ics. $C$ illustrates that there is a variation on the intensity of CGMP and vimentin staining. All im-ics express vimentin. However, there are vimentin ${ }^{+}$cells which have little cGMP immunostaining. Calibration bars, $100 \mu \mathrm{m}$ in $\boldsymbol{A}, 20 \mu \mathrm{m}$ in $\boldsymbol{B}$ and $\boldsymbol{C}$ 
and larger complex cells with multiple processes which showed COX I-IR and a diffuse network of vimentin fibres (( $¥)$ and Fig. 6C), are now visible.

The network of vim+ fibres was observed to continue from the lamina propria into the inner smooth muscle layer where they run primarily on the surface of the muscle bundles (Fig. 7). Here, the vim+ cells appear on the surface of the muscle bundles and so can be described as surface muscle interstitial cell (sm-ics). Within this network, cell bodies were apparent which were COX I-IR but that did not stain strongly for vimentin. Examples of such cells are shown in figures 7B-C. At the junction of the lamina propria and inner smooth muscle cell layer, small clusters of COX I-IR cell bodies were often observed (Fig. 8). The cell bodies in these clusters did not stain strongly for vimentin but were in close proximity to vimentin positive cell processes (Figs. 8B-C). These collections of cell bodies, which have the appearance of nodes, were also apparent, although fewer in number and with fewer cells, lying between the muscle bundles of the inner muscle layer (Fig. 7A).

Table 1. Location of CGMP, vimentin, COX I and nNOS in the bladder.

\begin{tabular}{llcccc}
\hline Cell location & Cell type & \multicolumn{4}{c}{ Staining } \\
& & cGMP & Vimentin & COX I-IR & nNOS \\
\hline Urothelium & Umbrella & + & - & - & - \\
& Intermediate & - & - & + & - \\
& Basal & - & - & + & + \\
Lamina propria & Superficial su-ics & + & + & - & - \\
& Deep su-ics & - & + & - & - \\
& Lp-ics \#1 & - & + & - & - \\
& Ip-ics \#2 & - & - & + & - \\
Inner muscle & Ip-ics \#3 & - & + & + & - \\
& sm-ics \#1 & - & + & - & - \\
& sm-ics \#2 & - & - & + & - \\
Outer muscle & im-ics & - & + & - & - \\
Muscle coat & sm-ics & + & + & - & - \\
\hline
\end{tabular}

Abbreviations are:

Ip-ics : lamina propria interstitial cells

Ip-ics\#1: lamina propria interstitial cells type \#1 (only vimentin positive lp-ics)

Ip-ics\#2: lamina propria interstitial cells type \#2 (only COX I positive Ip-ics)

Ip-ics\#3: lamina propria interstitial cells type \#3 (Ip-ics that are both vimentin and COX I positive)

sm-ics: surface muscle interstitial cells

sm-ics\#1: surface muscle interstitial cells type \#1 (vimentin positive sm-ics)

sm-ics\#2: surface muscle interstitial cells type \#2 (COX I positive sm-ics)

im-ics: intramuscular interstitial cells

mc-ics: muscle coat interstitial cells 


\section{Outer muscle layers}

The network of vim ${ }^{+}$sm-ics was observed to extend into the outer muscle layers of the bladder wall (Figs. 1,9). However, in this outer region there are few cell bodies which are COX I-IR. This points out that there must be different types of sm-ic associated with the inner and outer muscle layers. This is supported in figure 10, which shows sections double labelled for CGMP (green) and vimentin (red). As has been reported previously, the sm-ics of the outer muscle layers respond to NO with a rise in CGMP. ${ }^{31}$ The cell bodies of these outer sm-ics are clearly seen but these are not COX I-IR. In contrast, there are few CGMP+ cells in the inner muscle layers (Fig. 1). NO-responsive cells producing CGMP are also found within the muscle bundles of the outer muscle layer: intramuscular interstitial cells (im-ics). Figure 10 illustrates that these im-ics are also vim+.

Thus, these data support the idea that there are different sub-types of interstitial cell associated with the lamina propria and inter-muscular spaces in the guinea pig bladder. The different cell types which have been identified and described above, based on the staining for CGMP, vimentin, COX I and nNOS, are summarised in Table 1.

\section{Discussion}

It has been known for over 30 years that PGs are released from the bladder in response to stretch, the PGs coming from both the urothelium and muscle layers. ${ }^{19,24,35,36}$ Sprem et al. found that intravesically administrated ketoprofen, a nonselective COX inhibitor, reduced detrusor instability. ${ }^{37}$ Based on these findings it has been argued that the PGs play central roles not only in bladder physiology but also in the generation of bladder patho-physiology, ${ }^{2-5,38-40}$ This led to the trial of cyclooxygenase inhibitors for the treatment of bladder over activity. ${ }^{37,41}$ One indication as to the possible role of PGs has come from experiments involving the infusion of PG into the bladder lumen. When this is done, it gives rise to an increase in micturition frequency and the incidence of non-voiding phasic contractions between voiding episodes ${ }^{42,43}$ Prostaglandin production in the bladder serves also other functions. Bachteeva et al. reported that PGs play a role in the osmotic water permeability of the frog urinary bladder. ${ }^{44}$ Several subtypes of prostaglandin receptors have been found in the bladder. It has been reported that the urothelium contains both the prostaglandin receptor subtype E2 (EP2), which plays a role in osmoregulation, ${ }^{45}$ as well as the EP1 receptor, which plays a role in the micturition reflex. ${ }^{46}$ Schröder et al. reported that the EP1 receptor has a role in the development of detrusor overactivity caused by PGE2 and outlet obstruction. ${ }^{47}$ The details of the mechanisms underlying this action of PGs on voiding frequency are not known. Several possible mechanisms have been put forward. One idea is that the PGs have a direct effect on bladder afferent nerve fibres. ${ }^{48}$ By increasing afferent nerve activity this would result in a more frequent activation of the micturition reflex. It is well documented that PGs 
cause a sensitisation of cutaneous nociceptors. ${ }^{49}$ This might also occur in the bladder. Support for the idea comes indirectly from experiments in which the bladder was treated with capsaicin to remove the afferent contribution of $\mathrm{C}$ fibres. After functionally removing the $C$ fibres, the $P G$ induced increase in frequency of micturition was reduced. ${ }^{40} \mathrm{~A}$ different mechanism involving an indirect action of PGs on afferent nerves has recently been proposed. It is known that small localised contractions occur in the bladder wall of many species. This complex activity is hypothesized to be the motor component of a motor/sensory system involved in the generation of afferent firing and bladder sensation. ${ }^{29,31}$ Using the isolated guinea pig bladder, it has been shown that PGs increase the frequency of this phasic motor activity. ${ }^{28}$ Thus, the increased phasic motor activity could result in an increase in afferent discharges and in so doing influence the point at which voiding is triggered. ${ }^{28}$

As discussed above, PGs are synthesised within the lamina propria and muscle layers. The present observations extend this broad observation and demonstrate specifically that the expression of COX I-IR predominates within two general cell systems in the bladder wall: (i) cells within the basal and intermediate layers of the urothelium and (ii) within a population of small cells which are closely associated with a network of vimentin positive cells. These vimentin positive cells are present through out the sub-urothelial space of the lamina propria and extend over the surface of the muscle bundles, which make up the inner layers. It is interesting and important to note that no COX I-IR was seen within the muscles indicating that, in the guinea pig, it is not the smooth muscle that is producing PG but the cells associated with the vimentin network.

These observations raise intriguing questions regarding the mechanisms of action of PGs in the guinea pig bladder. One hypothesis might be, that the COX I-IR cell in the basal urothelial cell layers are activated by bladder distension. The produced PGs could diffuse the relatively short distance to the sub-urothelial space in which sensory afferent nerve fibres are found. Thus, this arrangement would represent the site where there is a PG induced direct modulation of afferent nerves. However, the abundance of COX I-IR in the urothelium of the lateral wall and the relative paucity of afferent nerves indicates that this is not the only role for PGs produced by the urothelium in the lateral wall.

The present data also show that the COX I-IR cells in the base of the urothelium express nNOS. Like PG, NO is known to be produced by the urothelium in response to stretch..$^{50}$ It is also known, on other cell systems, that PG production is influenced by NO and, conversely, that NO production is influenced by PGs. ${ }^{51,52}$ Other signals also originate in the urothelium in response to stretch. Specifically ATP has been shown to be released ${ }^{53}$ and one of its actions is to influence afferent nerve firing. ${ }^{54}$ Also, there are reports that acetylcholine is released from the urothelium..$^{55}$ Thus, we conclude there is a complex and inter-related release of signalling substances from the urothelium in response to mechanical deformation.

To add to this complexity, the cell layer immediately below the urothelium, the sub-urothelial interstitial cell (su-ic) layer can be a possible location for further 
integration of urothelial derived signals. These su-ics, in the guinea pig and human, respond to both exogenous and endogenous NO demonstrating a rise in CGMP. ${ }^{30,32}$ These cells are also immuno-reactive for antibodies to the type 3 muscarinic receptor $\left(\mathrm{M}_{3}\right)$ (unpublished observation), purinergic receptor ${ }^{56}$ and the type 2 prostaglandin receptor (EP2) (unpublished observation). Thus, the integrated output of PG, NO, ATP and cholinergic stimuli from the urothelium can be further integrated and modulated on the su-ics. The specific function of the su-ics cells is, at present, not known.

Indeed, there is indirect support for such a possible integration on the suburothelial cells. The su-ics are in close relation with the network of interstitial cells of the lamina propria and on the surface of the inner muscles. The autonomous bladder hypothesis suggests that, based on the observations of Lagou et al..$^{34}$ that the muscle interstitial cells are involved in the activation and co-ordination of complex phasic activity (autonomous activity) within the smooth muscle ${ }^{57}$ and it is this activity that is the motor component of the motor sensory system. ${ }^{30}$ The amplitude and frequency of the autonomous activity is increased by cholinergic agonists ${ }^{58,59}$ and ATP. ${ }^{60}$ If this type of activity is generated within an interstitial cells network, this points out that there are $M_{3} /$ ATP activated pacemakers linked to a distributed network. ${ }^{59}$

It has also been shown that bladder distension alters autonomous activity: an increase in bladder volume increases autonomous activity while a decrease in bladder volume inhibits it. ${ }^{61}$ The complex scheme outlined above might provide an explanation for these volume related events. Mechanical deformation of the urothelium in the lateral wall triggers complex cascade of interacting signals within the urothelial epithelium resulting in the release of signals into the sub-urothelial space. There, these urothelial signals are further integrated on the su-ics. Activity in the su-ics is then distributed to the muscle via the vimentin positive interstitial cell network resulting in the excitatory and inhibitory effects on the phasic activity (Fig. 11).

Prostaglandin synthesis has been associated with the smooth muscle. ${ }^{24}$ The precise cell types and their location have not been considered in detail, it was simply assumed that it originated in smooth muscle cells. The present observations put forward a new idea that, at least in the guinea pig, it is the COX I-IR cells associated with the network of vimentin positive interstitial cells on the surface of the muscle bundles which are responsible for synthesising PG in this layer. As already discussed one of the possible roles of this network of muscle interstitial cells is to generate and distribute signals leading to phasic contractions in the bladder wall..$^{34,57,62}$ Thus the micro-anatomical arrangement of cells in the muscle layer appears to involve COX IIR (PG producing) cells contacting vimentin interstitial cells in close apposition to smooth muscle. Functionally, it can be hypothesized that this arrangement involves a PG regulation of activity in the vimentin network and consequently an input to the muscle. The actions of PG on the muscle would thus be indirect interceded by the network of interstitial cells (Fig. 11).

The present observations also demonstrate a difference in the interstitial cells in 


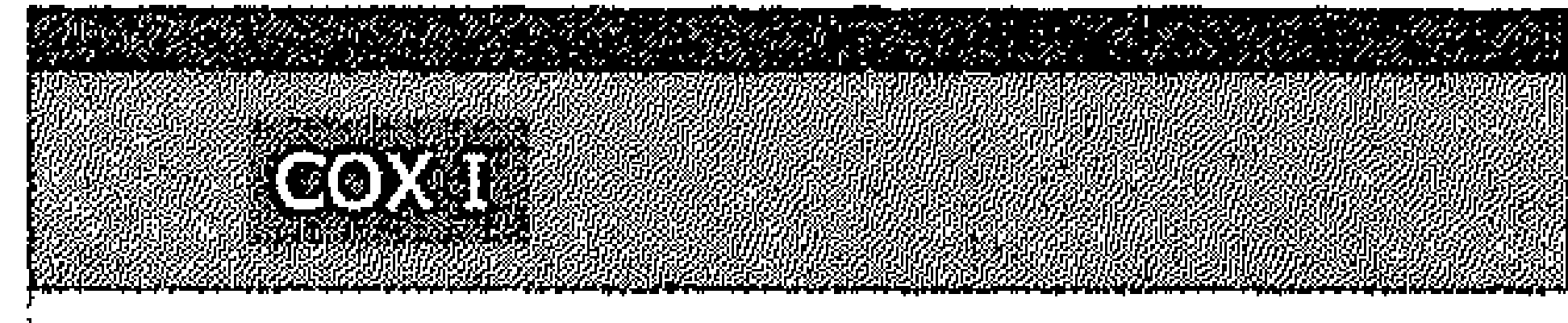

umbrella cells

intermediate urothelium

basal urothelium

urothelium
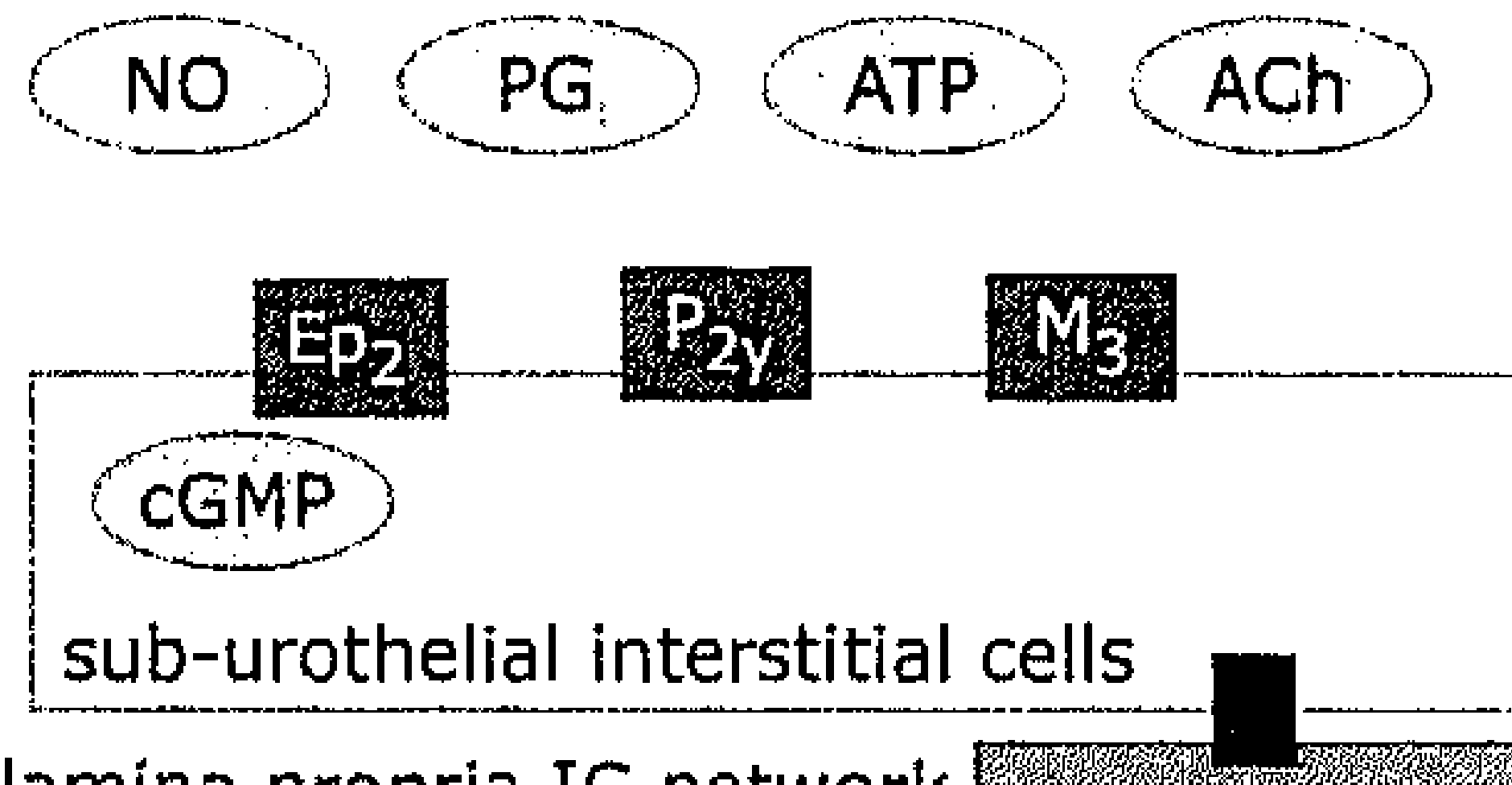

lamina propria IC network

inner muscle IC network
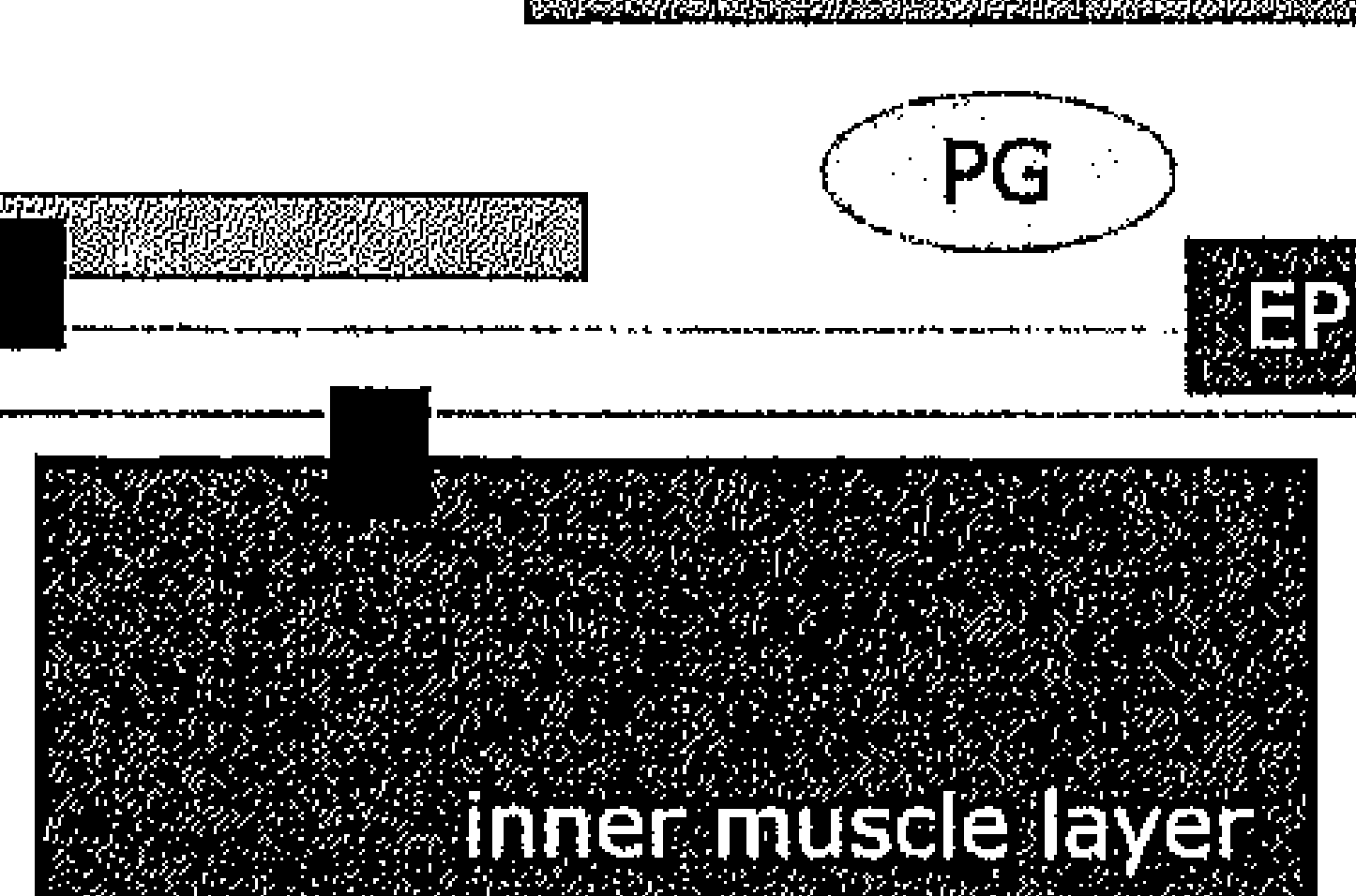

Figure 11.

Summary diagram illustrating the arrangement of cells found in the wall of the guinea pig bladder and their hypothesized interaction. The urothelium releases NO, PG, ATP and acetylcholine in response to stretch. The production of NO and $P G$ in the urothelium is interrelated. The substances released act on afferent nerves but may also act upon the sub-urothelial interstitial cells. This network is in direct contact with a network of vimentin positive interstitial cells which run in the sub-urothelial space of the lamina propria and extend over the surface of the inner muscle bundles. The mechanisms connecting cells in this network can be via gap junctions (black squares). COX I-IR cells (satellite cel/s) are associated with the vimentint interstitial cell network where they influence the activity of the network.

the outer and inner muscle layers of the bladder wall, specifically the reduction in number of COX I-IR cells in the outer layers. Differences have been noted before. ${ }^{31,34}$ However, the functional roles for these apparently different types of muscle interstitial cell are not known. What is clear is that the network of interstitial cells is complex and may sub-serve several different functions.

In conclusion, it is now quite clear that there are several complex signalling systems operating within the bladder wall. In addition, it is clear that these signals act within cells systems interacting between the urothelium, interstitial cells, muscle, sensory fibres and intra-mural ganglia. It is a major challenge for the future to unravel this complexity not only in relation to the physiology and pharmacology of the bladder but also in relation to the origins and treatment of bladder pathology. 


\section{References}

1. Khan MA, Thompson CS, Mumtaz FH, Jeremy JY, Morgan RJ, Mikhailidis DP. Role of prostaglandins in the urinary bladder: an update. Prostaglandins Leukot Essent Fatty Acids. $1998 ; 59: 415-22$.

2. Gilmore NJ, Vane JR. Hormones released into the circulation when the urinary bladder of the anaesthetized dog is distended. Clin Sci. 1971; 41:69-83.

3. Abrams PH, Sykes JA, Rose AJ, Rogers AF. The synthesis and release of prostaglandins by human urinary bladder muscle in vitro. Invest Urol. 1979; 16:346-8.

4. Jeremy JY, Tsang V, Mikhailidis DP, Rogers $H$, Morgan RJ, Dandona P. Eicosanoid synthesis by human urinary bladder mucosa: pathological implications. Br J Urol. 1987; 59:36-9.

5. Mikhailidis DP, Jeremy JY, Dandona P. Urinary bladder prostanoids-their synthesis, function and possible role in the pathogenesis and treatment of disease. J Urol. 1987; 137:577-82.

6. Bultitude MI, Hills NH, Shuttleworth KE. Clinical and experimental studies on the action of prostaglandins and their synthesis inhibitors on detrusor muscle in vitro and in vivo. $\mathrm{Br}]$ Urol. $1976 ; 48: 631-7$.

7. Hills $\mathrm{NH}$. Prostaglandins and tone in isolated strips of mammalian bladder. $\mathrm{Br} \mathrm{J}$ Pharmacol. 1976; 57:464P-5P.

8. Andersson KE, Ek A, Persson CG. Effects of prostaglandins on the isolated human bladder and urethra. Acta Physiol Scand. 1977; 100:165-71.

9. Borda E, Contreras-Ortiz N, Gutnisky R, Gimeno MF. In vitro effect of acetylcholine and bethanechol on the contractions of the human detrusor muscle. Influence of prostaglandins. Arch Int Pharmacodyn Ther. 1982; 259:31-9.

10. Brown C, Burnstock G, Cocks T. Effects of adenosine 5'-triphosphate (ATP) and beta-gammamethylene ATP on the rat urinary bladder. Br J Pharmacol. 1979; 65:97-102.

11. Choo LK, Mitchelson $\mathrm{F}$. The role of prostaglandins in the excitatory innervation of the rat urinary bladder. Prostaglandins. 1977; 13:917-26.

12. Choo LK, Mitchelson F. The effect of indomethacin and adenosine $5^{\prime}$ - triphosphate on the excitatory innervation of the rate urinary bladder. Can J Physiol Pharmacol. 1980; 58:1042-8.

13. Burnstock $G$, Cocks $T$, Crowe $R$, Kasakov $L$. Purinergic innervation of the guinea-pig urinary bladder. Br J Pharmacol. 1978; 63:125-38.

14. Burnstock G, Cocks T, Kasakov L, Wong HK. Direct evidence for ATP release from nonadrenergic, non-cholinergic ("purinergic") nerves in the guinea-pig taenia coli and bladder. Eur J Pharmacol. 1978; 1549:145-9.

15. Johns $A$. The effect of indomethacin and substance $P$ on the guinea pig urinary bladder. Life Sci. 1981; 2629:1803-9.

16. Dean DM, Downie JW. Interaction of prostaglandins and adenosine $5^{\prime}$-triphosphate in the noncholinergic neurotransmission in rabbit detrusor. Prostaglandins. 1978; 16:245-51.

17. Husted S, Sjogren C, KE AN. Role of prostaglandins in the responses of rabbit detrusor to noncholinergic, non-adrenergic nerve stimulation and to ATP. Arch Int Pharmacodyn Ther. 1980; 246:84-97.

18. Andersson $K E$, Husted $S$, Sjogren $C$. Contribution of prostaglandins to the adenosine triphosphate-induced contraction of rabbit urinary bladder. $\mathrm{Br}$ J Pharmacol. 1980; 70:443-52.

19. Downie JW, Larsson C. Prostaglandin involvement in contractions evoked in rabbit detrusor by field stimulation and by adenosine 5'-triphosphate. Can J Physiol Pharmacol. 1981; 59:253-60.

20. Johns A, Paton DM. Evidence for a role of prostaglandins in atropineresistant transmission in the mammalian urinary bladder. Prostaglandins. 1976; 11:595-7.

21. Johns A, Paton DM. Effect of indomethacin on atropine-resistant transmission in rabbit and monkey urinary bladder: evidence for involvement of prostaglandins in transmission. Prostaglandins. $1977 ; 13: 245-54$.

22. Andersson KE, Sjogren C. Aspects on the physiology and pharmacology of the bladder and urethra. Prog Neurobiol. 1982; 19:71-89. 
23. Andersson KE. Current concepts in the treatment of disorders of micturition. Drugs. 1988; 35:477-94.

24. Anderson KE. Pharmacology of lower urinary tract smooth muscles and penile erectile tissues. Pharmacol Rev. 1993; 45:253-308.

25. Goldstein RH, Polgar P. The effect and interaction of bradykinin and prostaglandins on protein and collagen production by lung fibroblasts. J Biol Chem. 1982; 10257:8630-3.

26. Elias JA. Tumor necrosis factor interacts with interleukin-1 and interferons to inhibit fibroblast proliferation via fibroblast prostaglandin-dependent and -independent mechanisms. Am Rev Respir Dis. 1988; 138:652-8.

27. Powell DW, Mifflin RC, Valentich JD, Crowe SE, Saada JI, West AB. Myofibroblasts. II. Intestinal subepithelial myofibroblasts. Am J Physiol. 1999; 277:183-201.

28. de Jongh $R$, van Koeveringe $G A$, van Kerrebroeck PE, Markerink-van Ittersum $M$, de Vente $]$, Gillespie JI. The effects of exogenous prostaglandins and the identification of constitutive cyclooxygenase I and II immunoreactivity in the normal guinea pig bladder. BJU Int. 2007; 100:419-29.

29. Starling E. Elements of Human Physiology, 8th ed. London; 1907.

30. Gillespie Jl. The autonomous bladder: a view of the origin of bladder overactivity and sensory urge. BJU Int. 2004; 93:478-83.

31. Gillespie JI, Markerink-van Ittersum M, de Vente J. cGMP-generating cells in the bladder wall: identification of distinct networks of interstitial cells. BJU Int. 2004; 94:1114-24.

32. Smet PJ, Jonavicius J, Marshall VR, de Vente J. Distribution of nitric oxide synthaseimmunoreactive nerves and identification of the cellular targets of nitric oxide in guinea-pig and human urinary bladder by CGMP immunohistochemistry. Neuroscience. 1996; 71:337-48.

33. McCloskey KD, Gurney AM. Kit positive cells in the guinea pig bladder. J Urol. 2002; 168:8326.

34. Lagou M, Drake MJ, Markerink VANIM, J DEV, Gillespie JI. Interstitial cells and phasic activity in the isolated mouse bladder. BJU Int. 2006; 98:643-50.

35. Brown WW, Zenser TV, Davis BB. Prostaglandin E2 production by rabbit urinary bladder. Am J Physiol. 1980; 239:452-8.

36. Kasakov LN, Vlaskovska MV. Profile of prostaglandins generated in the detrusor muscle of rat urinary bladder: effects of adenosine triphosphate and adenosine. Eur J Pharmacol. 1985; 31113:431-6.

37. Sprem M, Milicic D, Oreskovic S, Ljubojevic $N$, Kalafatic D. Intravesically administered ketoprofen in treatment of detrusor instability: cross-over study. Croat Med J. 2000; 41:423-7.

38. Maggi CA. Prostanoids as local modulators of reflex micturition. Pharmacol Res. 1992; 25:1320.

39. Maggi CA, Evangelista S, Grimaldi G, Santicioli P, Giolitti A, Meli A. Evidence for the involvement of arachidonic acid metabolites in spontaneous and drug-induced contractions of rat urinary bladder. J Pharmacol Exp Ther. 1984; 230:500-13.

40. Maggi CA, Giuliani S, Conte B, Furio M, Santicioli P, Meli P, Gragnani L, Meli A. Prostanoids modulate reflex micturition by acting through capsaicin-sensitive afferents. Eur J Pharmacol. $1988 ; 12145: 105-12$.

41. Angelico P, Guarneri L, Velasco C, Cova R, Leonardi A, Clarke DE, Testa R. Effect of cyclooxygenase inhibitors on the micturition reflex in rats: correlation with inhibition of cyclooxygenase isozymes. BJU Int. 2006; 97:837-46.

42. Ishizuka O, Mattiasson A, Andersson KE. Prostaglandin E2-induced bladder hyperactivity in normal, conscious rats: involvement of tachykinins? J Urol. 1995; 153:2034-8.

43. Takeda $H$, Yamazaki $Y$, Igawa $Y$, Kaidoh $K$, Akahane $S$, Miyata $H$, Nishizawa $O$, Akahane $M$, Andersson KE. Effects of beta(3)-adrenoceptor stimulation on prostaglandin $E(2)$-induced bladder hyperactivity and on the cardiovascular system in conscious rats. Neurourol Urodyn. 2002; 21:558- 65. 
44. Bachteeva V, Fock E, Lavrova E, Nikolaeva S, Gambaryan S, Parnova R. Prostaglandin E2 inhibits vasotocin-induced osmotic water permeability in the frog urinary bladder by EP1-receptormediated activation of NO/CGMP pathway. Am J Physiol Regul Integr Comp Physiol. 2007; 293:528-37.

45. Parnova RG, Bakhteeva VT, Lavrova EA. Various effects of prostaglandin E2 on reabsorption of water and urea in the amphibia osmosis-regulating epithelium. Ross Fiziol Zh Im I M Sechenova. 2001; 87:1662-72.

46. Wang $X$, Momota $Y$, Yanase $H$, Narumiya S, Maruyama T, Kawatani M. Urothelium EP1 receptor facilitates the micturition reflex in mice. Biomed Res. 2008; 29:105-11.

47. Schroder A, Newgreen D, Andersson KE. Detrusor responses to prostaglandin E2 and bladder outlet obstruction in wild-type and Ep1 receptor knockout mice. J Urol, 2004; 172:1166-70.

48. Andersson KE, Arner A. Urinary bladder contraction and relaxation: physiology and pathophysiology. Physiol Rev. 2004; 84:935-86.

49. Cohen RH, Perl ER. Chemical factors in the sensitization of cutaneous nociceptors. Prog Brain Res. 1988; 74:201-6.

50. Birder LA, Apodaca G, De Groat WC, Kanai AJ. Adrenergic- and capsaicin-evoked nitric oxide release from urothelium and afferent nerves in urinary bladder. Am J Physiol. 1998; 275:2269.

51. Salvemini D, Misko TP, Masferrer JL, Seibert K, Currie MG, Needleman P. Nitric oxide activates cyclooxygenase enzymes. Proc Natl Acad Sci U S A. 1993; 190:7240-4.

52. Salvemini D, Seibert K, Masferrer JL, Misko TP, Currie MG, Needleman P. Endogenous nitric oxide enhances prostaglandin production in a model of renal inflammation. J Clin Invest. 1994; 93:1940-7.

53. Ferguson DR, Kennedy I, Burton TJ. ATP is released from rabbit urinary bladder epithelial cells by hydrostatic pressure changes-a possible sensory mechanism? J Physiol. 1997; 1505:50311.

54. Lee $H Y$, Bardini $M$, Burnstock $G$. Distribution of $P 2 X$ receptors in the urinary bladder and the ureter of the rat. J Urol. 2000; 163:2002-7.

55. Yoshida M, Miyamae K, Iwashita H, Otani M, Inadome A. Management of detrusor dysfunction in the elderly: changes in acetylcholine and adenosine triphosphate release during aging. Urology. 2004; 63:17-23.

56. Fry $\mathrm{CH}$, Ikeda $Y$, Harvey $R$, Wu $C$, Sui GP. Control of bladder function by peripheral nerves: avenues for novel drug targets. Urology. 2004; 63:24-31.

57. Hashitani $H$, Yanai $Y$, Suzuki $H$. Role of interstitial cells and gap junctions in the transmission of spontaneous $\mathrm{Ca}^{2+}$ signals in detrusor smooth muscles of the guinea-pig urinary bladder. ] Physiol. 2004; 1559:567-81.

58. Drake MJ, Harvey IJ, Gillespie JI. Autonomous activity in the isolated guinea pig bladder. Exp Physiol. 2003; 88:19-30.

59. Finney SM, Stewart LH, Gillespie JI. Cholinergic activation of phasic activity in the isolated bladder: possible evidence for M3- and M2-dependent components of a motor/sensory system. BJU Int. 2007; 100:668-78.

60. Gillespie JI. Modulation of autonomous contractile activity in the isolated whole bladder of the guinea pig. BJU Int. 2004; 93:393-400.

61. Lagou M, Drake MJ, Gillespie JI. Volume-induced effects on the isolated bladder: a possible local reflex. BJU Int. 2004; 94:1356-65.

62. Gillespie JI, Harvey IJ, Drake MJ. Agonist- and nerve-induced phasic activity in the isolated whole bladder of the guinea pig: evidence for two types of bladder activity. Exp physiol. 2003; $88: 343-57$. 


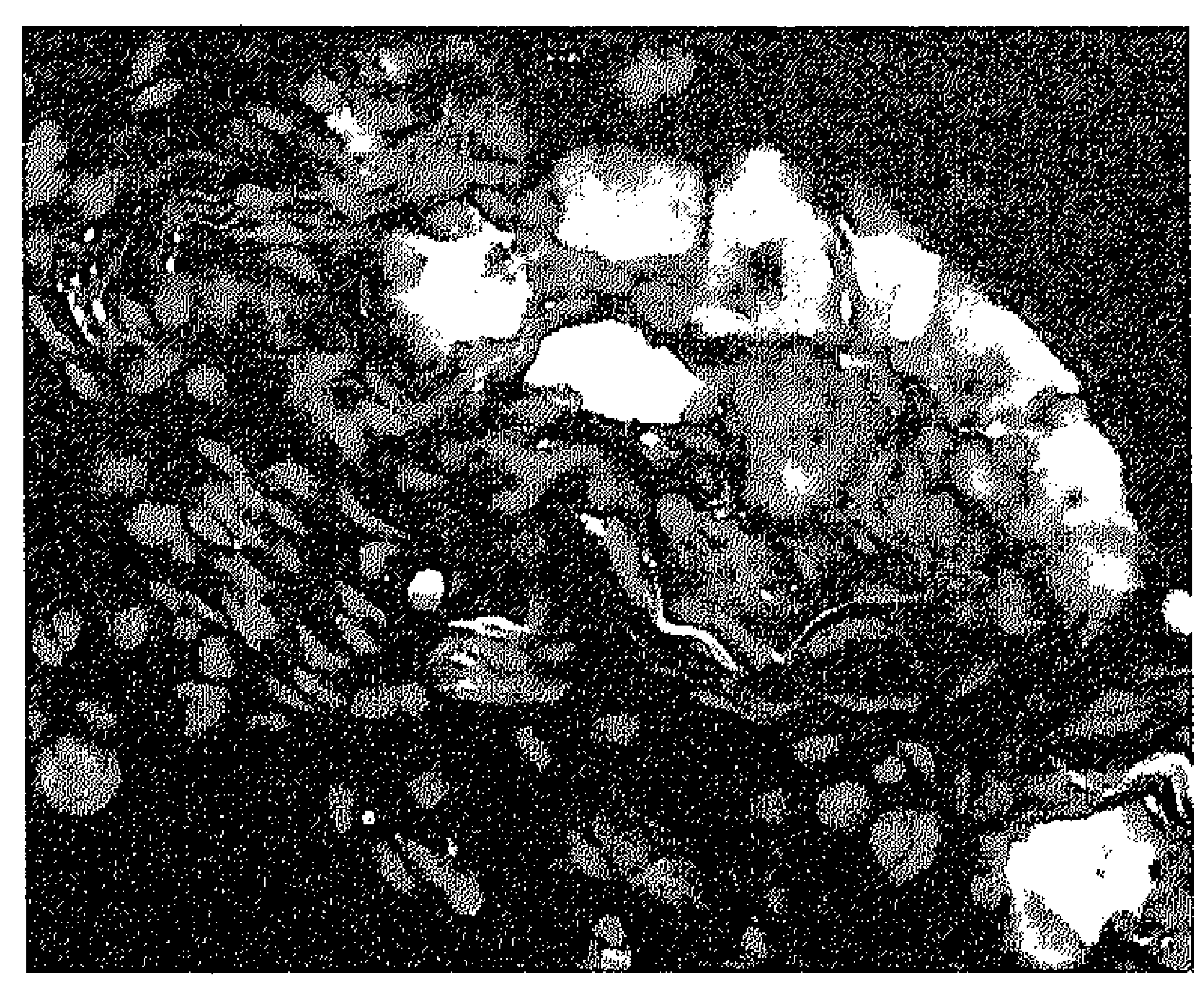

Damage to the bladder neck alters autonomous activity and its sensitivity to cholinergic agonists

Rik de Jongh, Gommert A. van Koeveringe, Philip E.V. van Kerrebroeck, Marjanne Markerink-van Ittersum, Jan de Vente and James I Gillespie.

British Journal of Urology International 2007; 100 (4):919-29. 


\section{Abstract}

Aims: To identify and describe changes to the motor component of the motor/sensory system, which contributes to sensation during the filling phase of the micturition cycle, as a result of surgically induced bladder pathology, i.e damage to the bladder neck and outlet obstruction.

Materials and methods: Adult male guinea pigs (294 - $454 \mathrm{~g}$ ) were assigned initially into three groups: (I) normal animals undergoing no surgical intervention (control, seven), (II) guinea pigs which, with full surgical anaesthesia, had a silver ring implanted around the bladder neck (obstructed, thirteen) and (III) guinea pigs operated to expose the bladder neck but with no implantation of a ring (sham, six). At 2 - 4 weeks after surgery the bladders were isolated, weighed and pressure recordings used to identify autonomous activity.

Results: The bladder weights in all operated groups, including sham, were greater than controls. Bladder weights in the obstructed animals varied considerably reflecting the degree of pathological change. Consequently, bladders from this group were divided into those with high (OBH) and those with low bladder weight (OBL). The mean (SD) amplitudes of the autonomous contractions were $1.1(0.1), 10.8$ $(1.8), 11.4(2.5)$ and $17.1(4.0) \mathrm{cm} \mathrm{H}_{2} \mathrm{O}$ in control, sham, $\mathrm{OBL}$ and $\mathrm{OBH}$ bladders, respectively, indicative of a progressive alteration in function with the pathology. The changes seen in the sham group suggested that the pathological changes were not the result of obstruction but damage to the bladder neck, the implantation of the silver rings exacerbating the damage. There were episodes of rapid phasic activity (bursts) in ten of thirteen of the ring implanted bladders, and in two out of six in the sham group, but never in controls. Neither the autonomous activity nor the bursts were affected by tetrodotoxin $(1 \mu \mathrm{M})$ or atropine $(3 \mu \mathrm{M})$ but they were abolished by noradrenalin $(3 \mu \mathrm{M})$. In control bladders, adding the muscarinic agonist arecaidine produced a transient acceleration of phasic activity and increased the amplitude of the contractions. There was a similar speeding of activity seen in all of the operated groups but the concentrations needed to achieve an increase in frequency were significantly lower, the relative sensitivity to arecaidine being $\mathrm{OBH} \geq \mathrm{OBL}>$ sham > control.

Conclusion: The mechanism involved in controlling the frequency of the motor component of the motor/sensory system, the 'pacemaker', appears to become progressively 'supersensitive' to cholinergic stimulation with the development of the pathology. These observations are discussed in relation to the motor/sensory system and the origins of sensation in the bladder. The argument is proposed that damage to the bladder neck, not obstruction per se, results in altered non-micturition activity which contributes to increased afferent output. In turn this contributes to the increased sensations of urge associated with bladder dysfunction. The cholinergic regulation of this altered 'pacemaker' might be the target for one of the therapeutic actions of anticholinergic drugs. 


\section{Introduction}

In humans, a constant desire to void (urge), an increased frequency of voiding and incontinence are not life threatening conditions but they do seriously affect an individual's quality of life and ability to work. The symptoms of this condition, commonly called an overactive bladder (OAB), are common affecting $16 \%$ of adults over the age of 50 and more prevalent in the older population, where $40 \%$ over 70 years are affected. ${ }^{1-2}$ The origins of the changes which give rise $O A B$ are not known. The first ideas to be considered were influenced by the concept that $O A B$ was associated with the generation of contractions during the filling phase of the micturition cycle. Hypotheses were put forward arguing that the underlying defects originated within the neural systems that control the bladder, i.e. the neurogenic hypothesis ${ }^{3}$ or within the bladder smooth muscle, the myogenic hypothesis. ${ }^{4}$ Recently, a further series of ideas have been proposed suggesting that the defect arises within the complex systems that reside within the bladder and which give rise to non-voiding contractions during the filling phase, the autonomous bladder hypothesis. ${ }^{5-8}$

It has been argued that the defect does not reside within the motor control of the bladder but within the afferent systems which generate sensation and trigger the micturition reflexes. ${ }^{9-10}$ Two views are emerging. One idea is that the mechanisms involved are associated with the urothelium. Substances released from the urothelium act directly upon afferent nerves or indirectly via myofibroblasts that in turn influence afferent nerves. ${ }^{11-13}$ A second view associated with afferent mechanisms involves the non-voiding contractions and their contribution to a motor/sensory system in the bladder wall. It has been known for over 130 years that there are small transient rises in bladder pressure as the bladder fills. ${ }^{14-15}$ This phasic activity is not associated with micturition and was described as 'non-micturition activity'. By 1905 it was generally accepted that these contractions stimulated afferent nerves and the motor/sensory activity contributed to bladder sensation. ${ }^{16}$ It has been demonstrated directly that such non-voiding activity generates bursts of impulses in bladder afferent nerves in the cat. ${ }^{17-18}$ It has also been shown that the frequency and amplitude of the non-micturition activity changes as the bladder fills. ${ }^{20}$ This has led to the suggestion that the motor/sensory system is regulated and modulated during filling. $8,20,21$

Using the isolated whole bladder, it was shown that the non-micturition activity originated within the bladder wall. ${ }^{15}$ Using this preparation it was subsequently shown that this system can be influenced by excitatory inputs including muscarinic agonists, ATP and substance $P^{5,6,21-22}$ and also inhibited by calcitonin gene related peptide and nor-adrenaline, demonstrating inhibitory inputs. ${ }^{23-24}$ Thus, the motor/sensory system is regulated by inputs which increase activity and augment sensation. The activity can also be inhibited potentially decreasing sensation..$^{7-8}$

If autonomous activity and the motor/sensory system which it potentially drives are central physiological mechanisms linked to the generation and modulation of 
bladder sensation then it follows that pathological modifications to this system may be central to the altered sensations that underlie urge and frequency. This possibility can be examined using bladders from animals with experimentally induced pathology. To date there is only one report which supports this general concept. Using bladders isolated from rats with surgically induced bladder outflow obstruction, it has been shown that autonomous activity is increased..$^{25}$ In this study, the sensitivity to applied drugs, particularly acetylcholine, was not examined. A great deal of information has accumulated regarding the generation and modulation of autonomous activity in the isolated guinea pig bladder. Therefore, the present series of experiments were done to explore the potential changes in autonomous activity and its regulation using the isolated bladder preparation from animals with surgically induced obstruction of the bladder neck. The data show dramatic changes in autonomous activity and demonstrate clear alterations in the sensitivity of these bladders to cholinergic stimulation. The results are discussed in the context of bladder pathology in man, the origins of increased urge and frequency and the mode and site of action of anticholinergic drugs.

\section{Materials and Methods}

The studies used 25 male guinea pigs (Hartley strain; body weight $294-454 \mathrm{~g}$ ) that were housed in a temperature-and light-( $12 \mathrm{~h}$ light / dark cycle) controlled room and allowed free access to food and water. The Institutional Animal Care and Use Committee of Maastricht University approved the animal experiments in this study.

Surgical procedure: In 13 guinea pigs a partial outlet obstruction (BOO) was induced and 6 animals underwent sham operation. A further 7 animals were used as controls and had no surgery. A protocol based on the guinea pig model for gradual urethral obstruction as described previousl ${ }^{26}$ was used. Briefly, the guinea pigs were sedated with intraperitoneally ketamine $(40 \mathrm{mg} / \mathrm{kg})$ and xylazine $(3 \mathrm{mg} / \mathrm{kg})$; once sedated, they were prepared under sterile conditions, and the bladder neck and urethra were exposed via a vertical midline abdominal incision. A silver jeweller's jump ring (1.8 mm internal diameter) was placed loosely around the proximal urethra. BOO was then imposed during subsequent growth of the animal. The analgesic medication flunixinum ( $5 \mathrm{mg} / \mathrm{kg} \mathrm{s.c.}$ ) and the antibiotic gentamicin ( $5 \mathrm{mg} / \mathrm{kg}$ i.m.) were administered once postoperatively. Sham surgery was identical except for inserting the ring around the urethra.

The procedures for isolating the whole bladder and maintaining it in vitro were described previously. ${ }^{5-6}$ Briefly, the guinea pigs were killed by cervical dislocation and the urinary bladder and urethra removed and placed in Kreb's solution: $(\mathrm{mM}): \mathrm{NaCl}$, $120 ; \mathrm{KCl} 4.5 ; \mathrm{CaCl}_{2} 2.5 ; \mathrm{MgCl}_{2} 1 ; \mathrm{NaHCO}_{3} 25 ; \mathrm{NaH}_{2} \mathrm{PO}_{4} 1 ; \mathrm{Na}$ pyruvate 1 , glucose 5 and bubbled with $5 \% \mathrm{CO}_{2}$ and $95 \% \mathrm{O}_{2}(\mathrm{pH} 7.4)$. The urethra was cannulated with a flexible plastic cannula ( $2 \mathrm{~mm}$ diameter) secured at the bladder neck using a fine ligature. Residual urine was gently removed using Kreb's solution. The bladder was 
then transferred to a heated organ bath (volume $50 \mathrm{ml}$, temperature $33-36^{\circ} \mathrm{C}$ ) containing constantly-gassed Kreb's solution. The cannula was connected with the urethra uppermost via a fluid-filled tube and 3-way connector to a pressure transducer (Becton Dickinson DTX Plus) and a $1 \mathrm{ml}$ syringe to enable variation of the intra-vesical volume. The transducer output was amplified, digitised at $20 \mathrm{~Hz}$ and recorded using an Acqknowledge data capture system (MP100). The pressure range of this apparatus was 0.02 to $180 \mathrm{~cm} \mathrm{H}_{2} \mathrm{O}$. The transducer was calibrated before each experiment. At the outset each bladder was filled with Kreb's solution to give a baseline volume of $600-800 \mu \mathrm{l}$. These volumes were typical amounts of urine recovered from normal animals immediately after cervical dislocation. These basic observations set limits to the normal volume changes seen in guinea pigs of this size. The time taken from killing the animal to beginning recording was typically 30 minutes. Pressure recording was started immediately but the bladder was left to equilibrate for at least 40 minutes. Drugs used were: the muscarinic agonist arecaidine but-2-ynyl ester tosylate, tetrodotoxin (Tocris, UK) and arterenol (Sigma). Concentrated drug solutions were added directly to the bath to achieve the required final dilution. All drugs were added to the solution bathing the abluminal surface.

\section{Results}

When bladders from the control group (those with no previous surgical intervention), were cannulated and placed in the organ bath, small transient rises in pressure were recorded almost immediately (Fig. 1Aa). Over the subsequent 2-3000 seconds these transients stabilised in amplitude and frequency at approximately 1 $\mathrm{cm} \mathrm{H}_{2} \mathrm{O}$ and a frequency of 0.03 transients/seconds. In complete contrast, the activity in bladders from animals that were obstructed at the bladder neck was dramatically different. Large transients $\left(>20 \mathrm{~cm} \mathrm{H}_{2} \mathrm{O}\right.$ ) appeared immediately and over the next 2 - 3000 seconds stabilised at a frequency of 0.05 transients/second and at an amplitude of approximately $30 \mathrm{~cm} \mathrm{H}_{2} \mathrm{O}$ (Fig. 1Ab).

In all, we examined 7 bladders from controls, 6 sham operated and 13 with obstruction; the responses recorded in the first period (3000 seconds) suggested that there were differences between the control, sham and obstructed bladders. There was a large variation in the amplitude of the responses seen in the obstructed group, but it was apparent that the bladders with the largest transients had the greater bladder weights. Thus, the subsequent analysis divided the 'obstructed' bladders into two groups: those with bladder weight $<800 \mathrm{mg}$ and those with weights $>800 \mathrm{mg}$, and defined as obstructed ' $l o w^{\prime}(\mathrm{OBL})$ and obstructed 'high' $(\mathrm{OBH})$ weight bladders. Examples of records from each of the defined groups, control, sham, OBL and OBH are shown in figures $1 \mathrm{Ba}-\mathrm{d}$. After $2-4$ weeks the mean (SD) weight gain of the sham group was 232 (11) $g$ and of the OBL group 127 (29) $\mathrm{g}$ and of the $\mathrm{OBH}$ group 61 (35) $\mathrm{g}$. The mean bladder weights in each group are shown in figure $2 \mathrm{~A}$. 


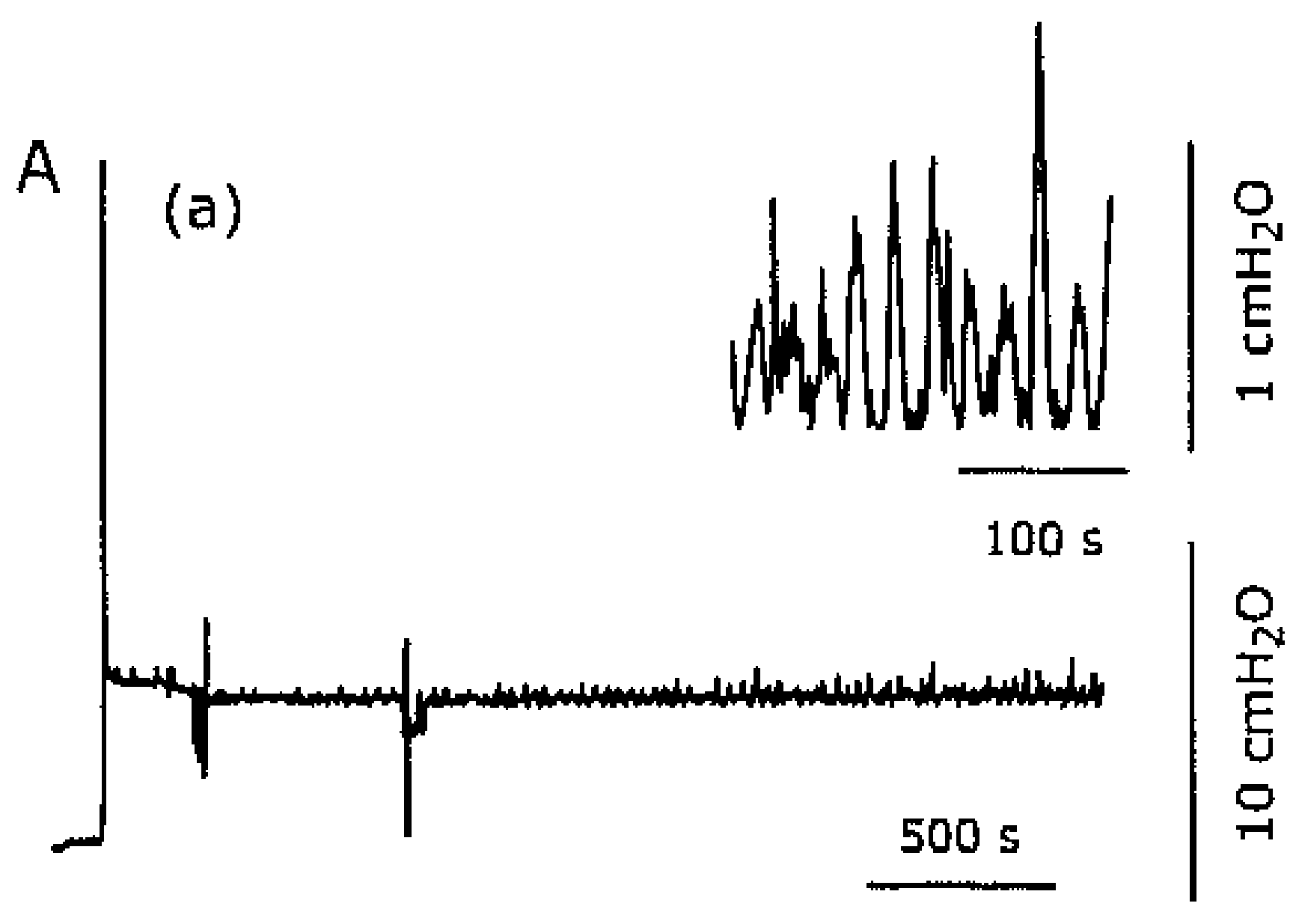

(b)

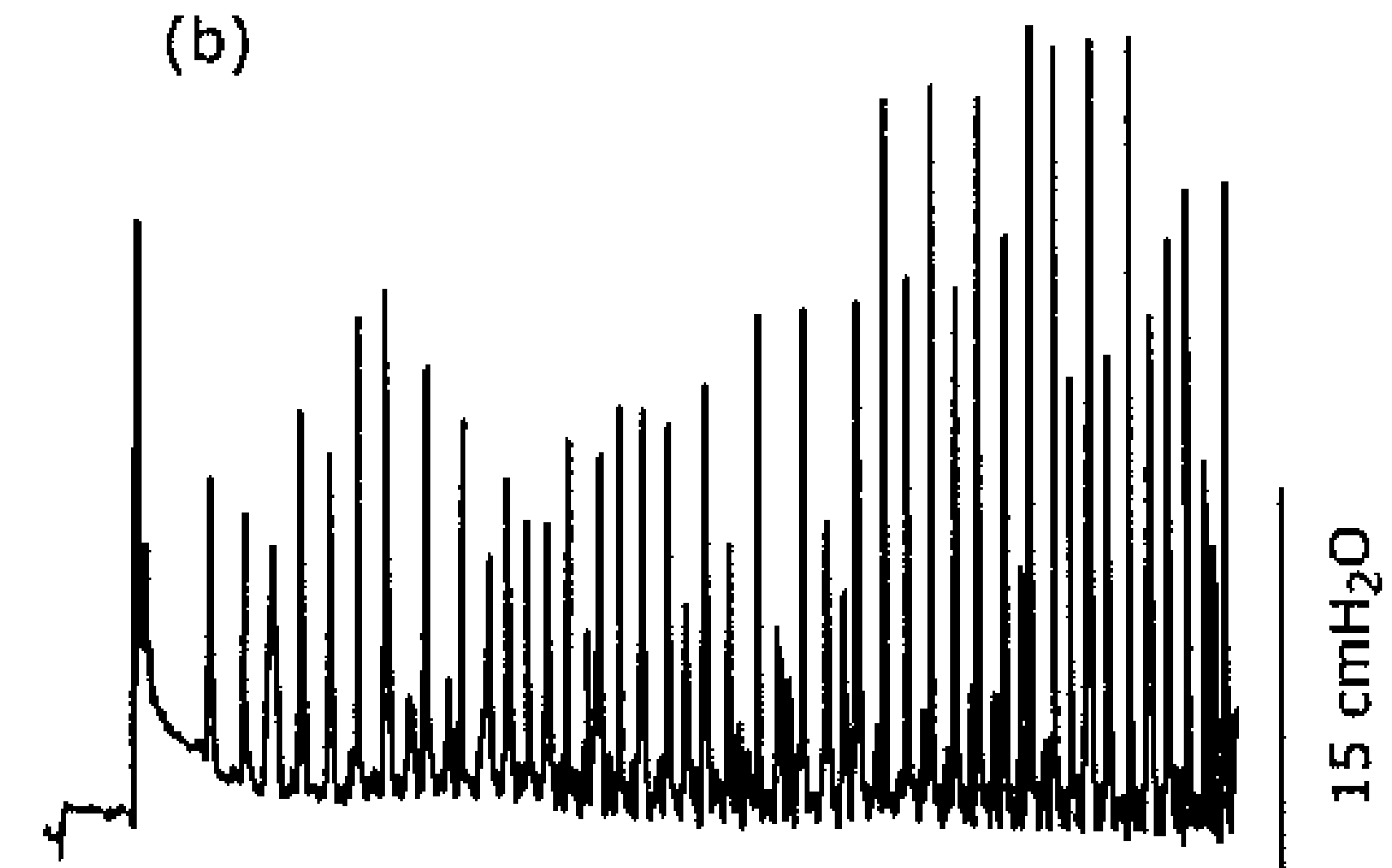

$500 \mathrm{~s}$ (a)<smiles>CCCCCCCCCCCCC</smiles>

(c)<smiles>CCCCCCCCCCCCCCCCCCCCC</smiles>

(d)

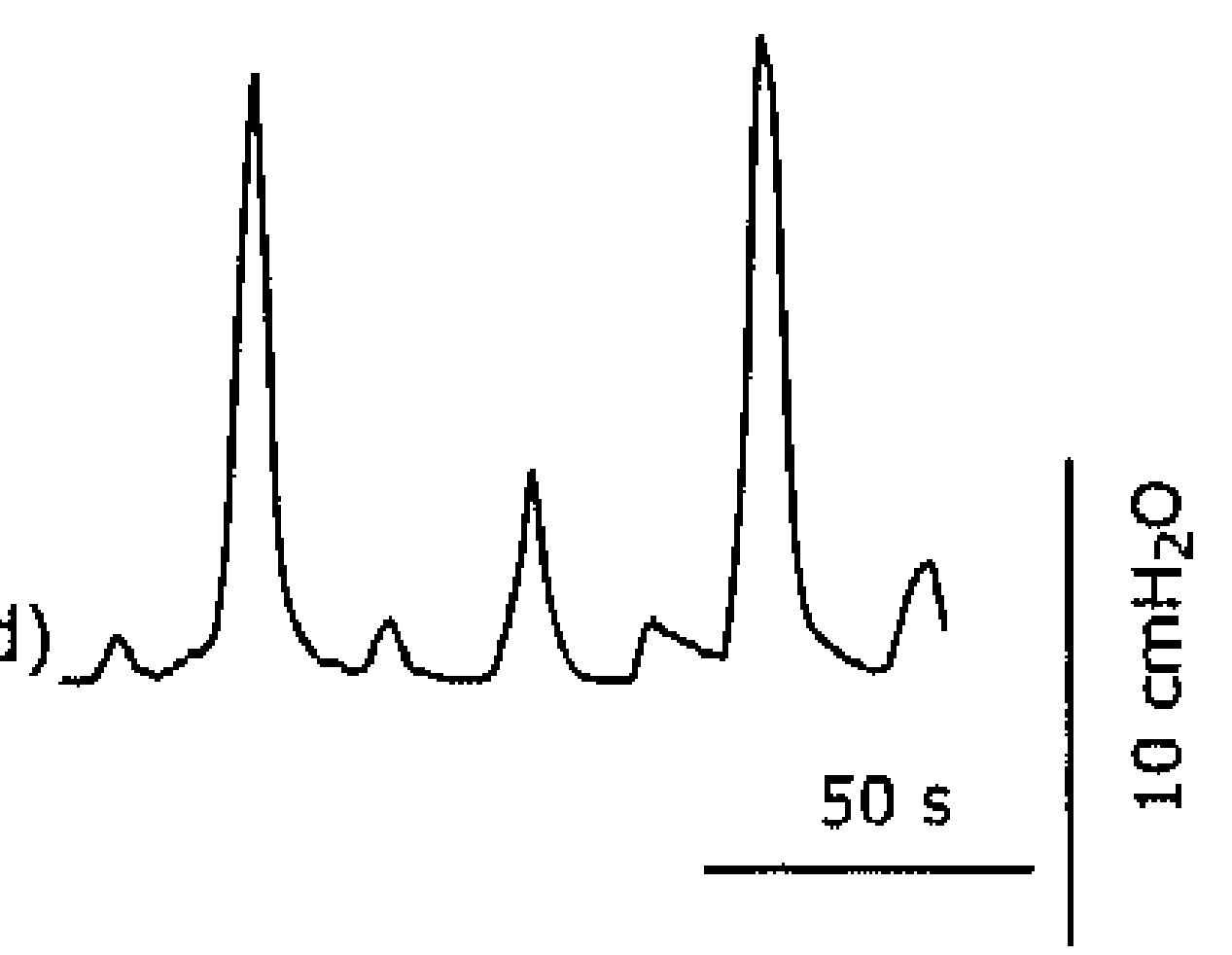

Figure 1.

Autonomous activity in a control bladder and an obstructed bladder. In A panel (a) shows the initial period of recording from a bladder isolated from an animal with no previous surgical intervention. Within seconds of recording small transient rises in pressure are seen. This activity stabilises after approximately 3000 seconds where the transients are approximately $1 \mathrm{~cm} \mathrm{H}_{2} \mathrm{O}$ in amplitude (see inset in a). (b) shows the initial period of recording from an obstructed bladder (surgery 2 weeks previously). Soon after beginning recording, large ( $>20 \mathrm{~cm} \mathrm{H}_{2} \mathrm{O}$ ) transients appeared, which gradually increased in amplitude and frequency, stabilising after approximately 3000 seconds. Calibration bars show time in seconds and pressure in $\mathrm{cm} \mathrm{H}_{2} \mathrm{O}$. $B$ shows data from four different bladders. The records were taken using the bladder from guinea pigs: (a) control, (b) sham operated (c) OBL (d) OBH. Calibration bars show time in seconds and pressure in $\mathrm{cm} \mathrm{H}_{2} \mathrm{O}$.

A

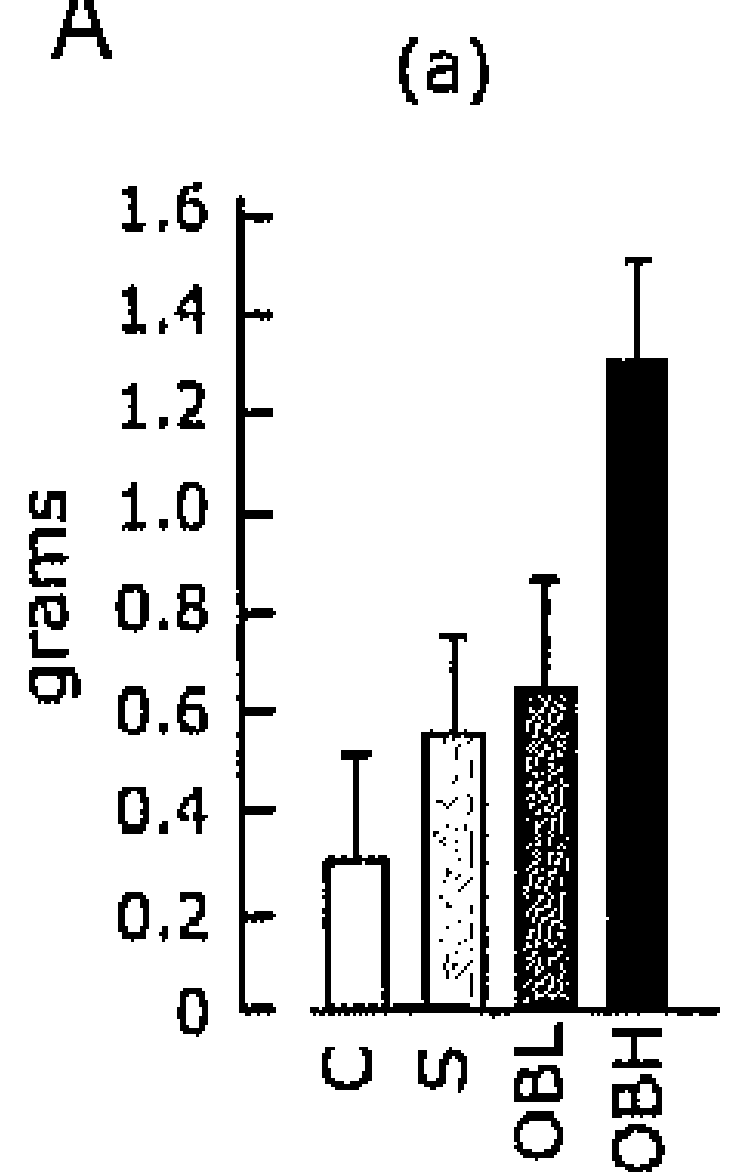

(c)

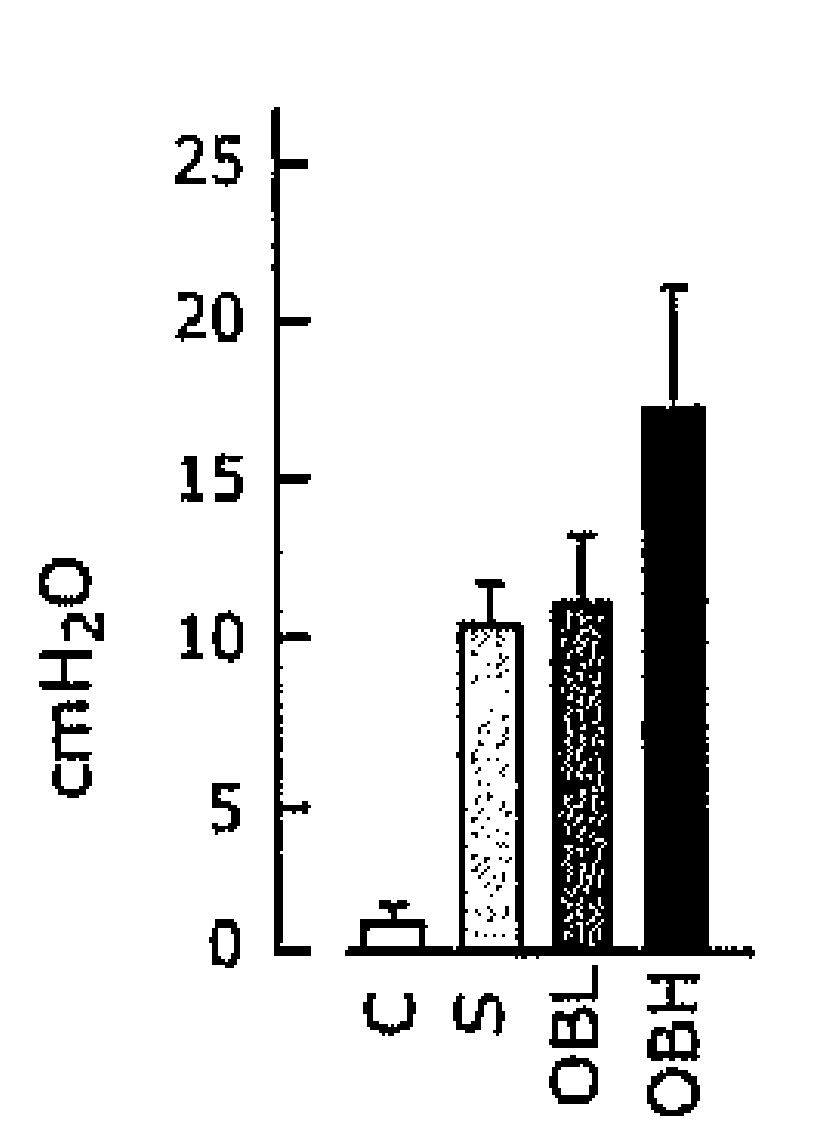

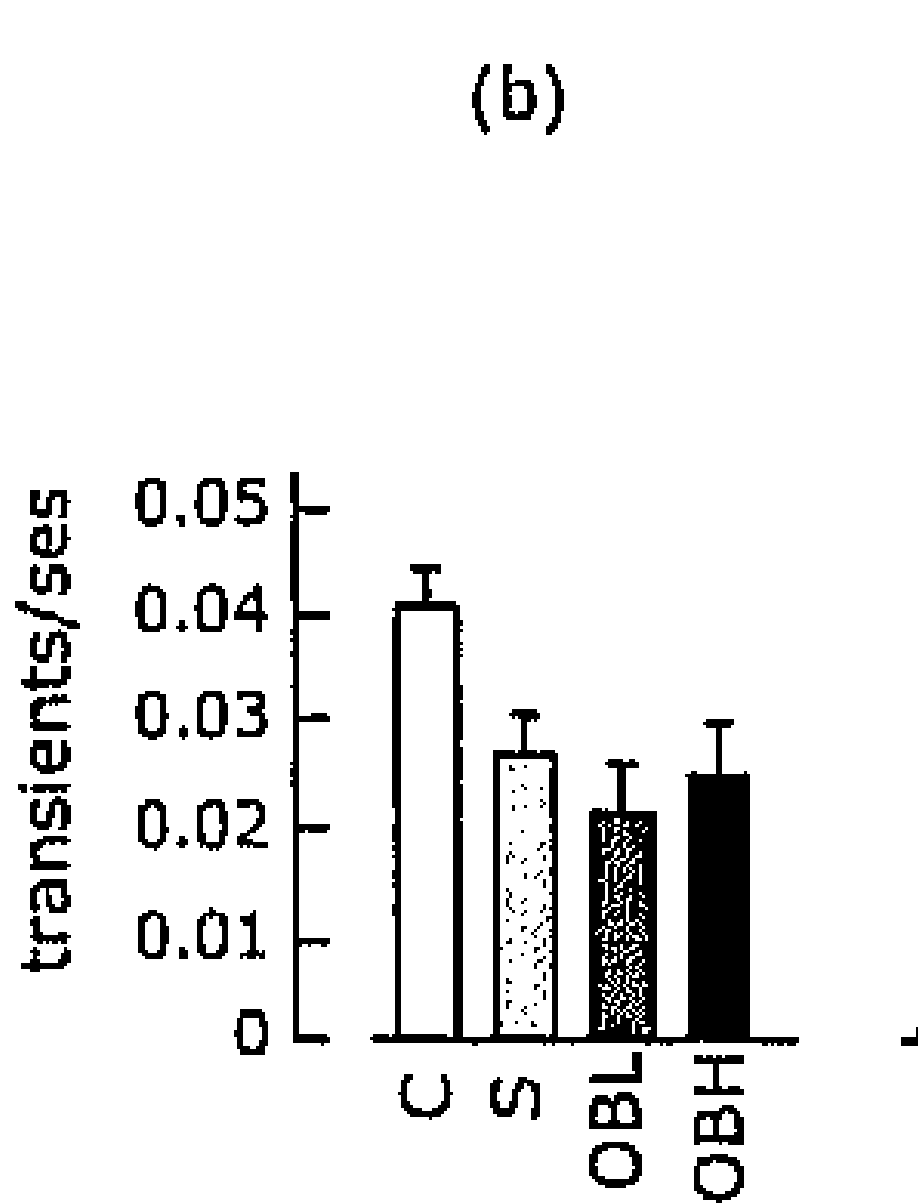

(d)

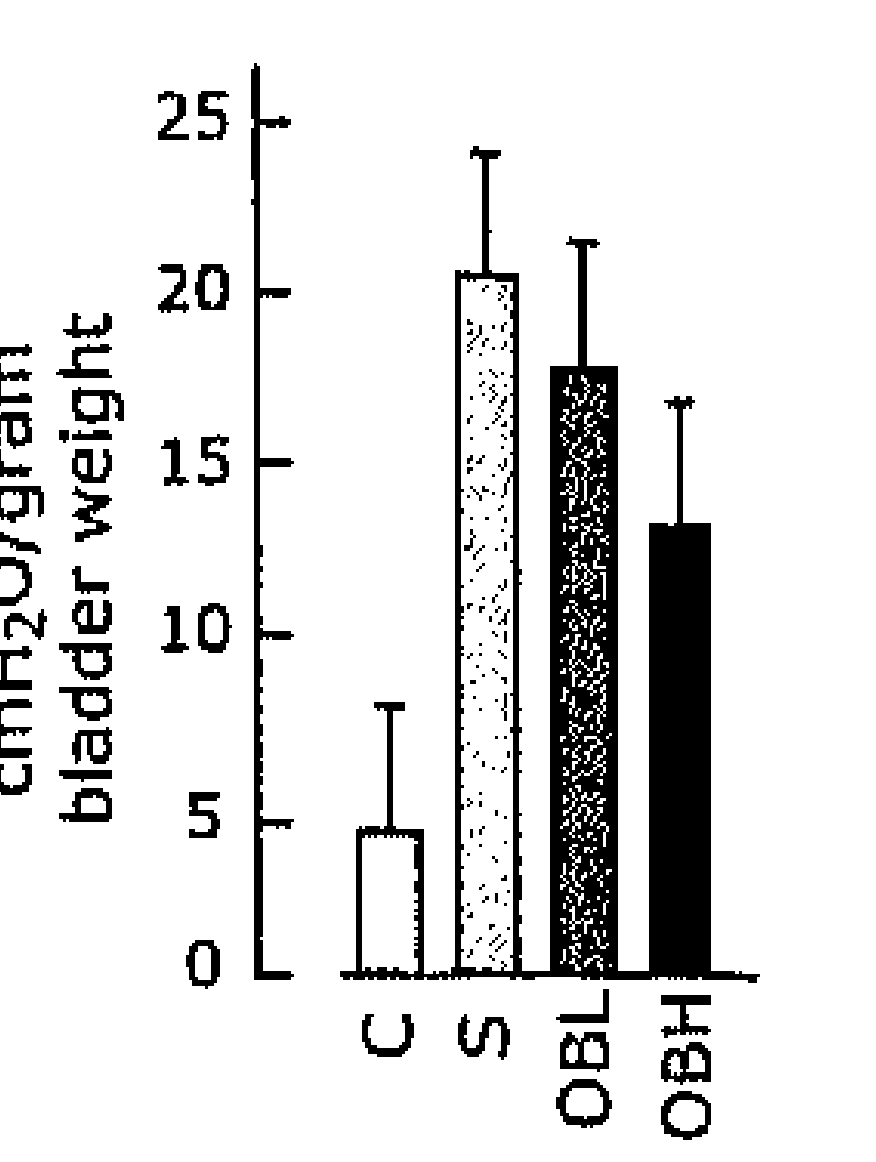

(a) $3 \mu M$
atropine

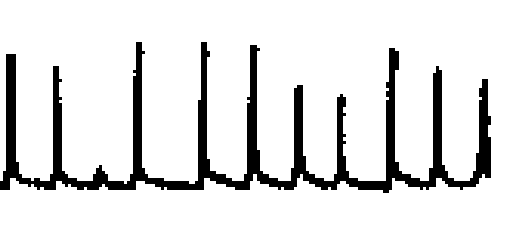

(c)

$1 \mu \mathrm{M}$ tetro dotoxin

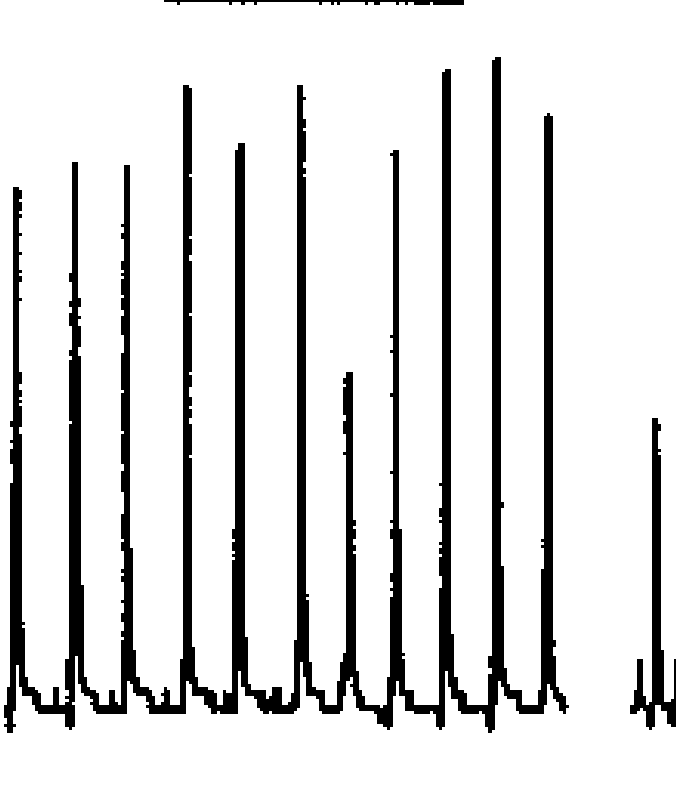

(b) $3 \mu \mathrm{M}$
atropine
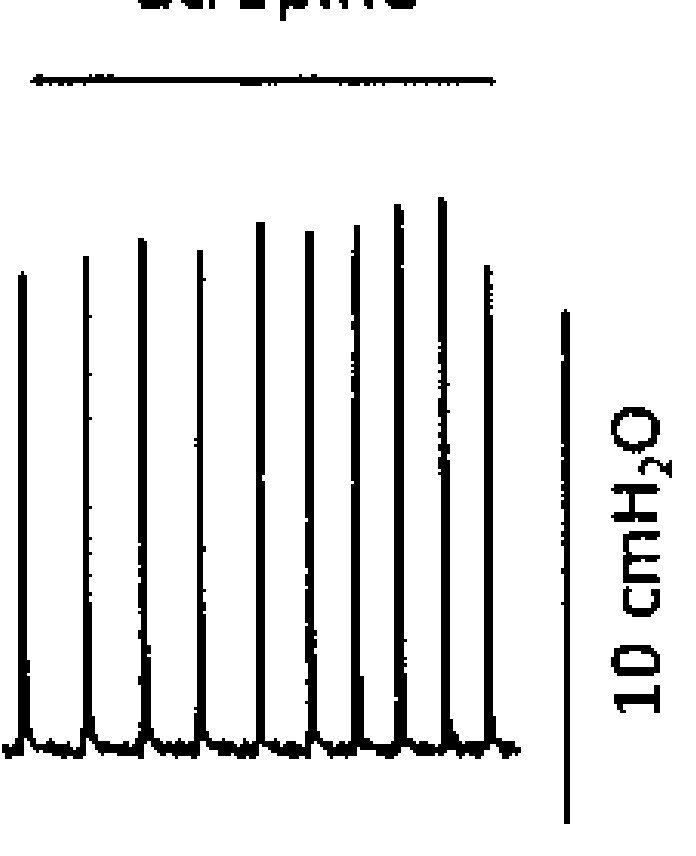

(d)

$1 \mu M$ tetro dotoxin

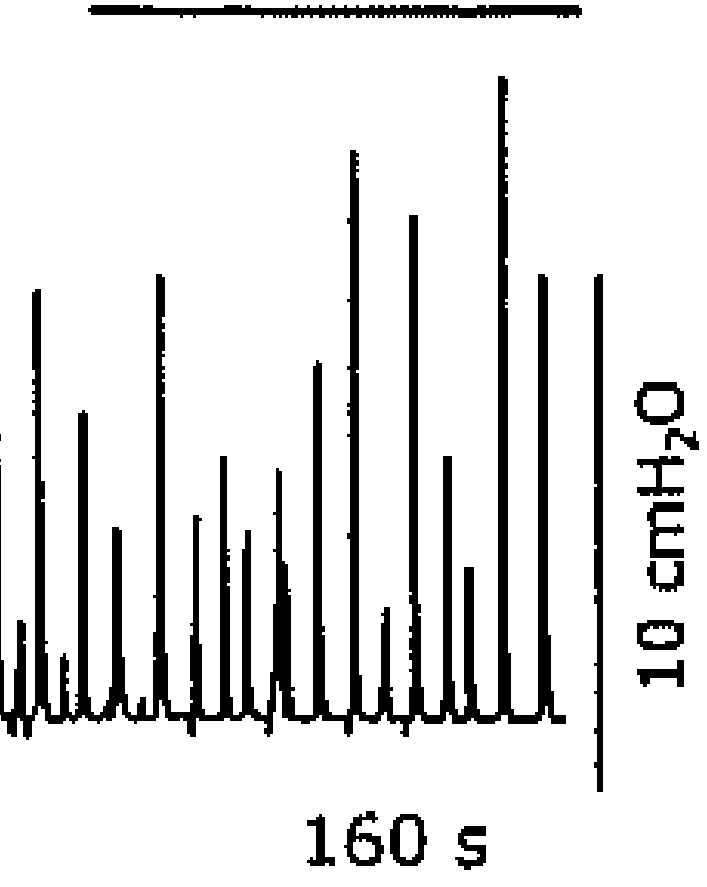

Figure 2.

A illustrates data accumulated from different experiments and different animals. A shows mean bladder weights, (b) the frequency of the initial transients, (c) the amplitude of the transients and (d) the amplitude of the transients corrected for bladder weight. The vertical bars show data from controls (C), sham operated (S), OBL and $O B H$ groups and values are means (S.D). B shows original records from 4 different experiments illustrating the absence of effects of atropine ( 3 $\mu M$ ) and tetrodotoxin $(1 \mu M)$. (a) and (c) are from bladders taken from sham operated group, where the bladders weights are increased but ' $/ O w^{\prime}$ while $\boldsymbol{b}$ is from $O B H$ and $\boldsymbol{d}$ the $O B L$ groups. Calibration bars show time in seconds and pressure in $\mathrm{cm}$ $\mathrm{H}_{2} \mathrm{O}$. 
An analysis of the transient activity in each of these groups is also shown in figure $2 \mathrm{~A}$ and the mean frequency shown in figure $2 \mathrm{Ab}$. There appeared to be little difference among the groups, although the frequency in the control bladders was larger than in the operated bladders. The amplitude of the transients was significantly increased in the OBL and OBH (t-test: $p<0.05$ ) compared to the control group. Interestingly, the amplitude of the transients of the sham operated bladders was also significantly increased compared to the controls (t-test: $p<0.05$ ). It is possible that the larger pressure changes in the operated animals are simply the result of a greater muscle mass. To correct for this the amplitude of the pressure changes were corrected for the weight of each bladder and the results expressed in pressure increases per unit weight of bladder tissue. The magnitude of the pressure transients from bladders from the operated groups was significantly greater than control ( $t$-test: $p<0.05$ ). The sham operated bladders appeared to have the largest transients/gram of bladder tissue. Thus, there is an additional factor other than an increased muscle mass contributing to the greater pressures seen in the operated groups.

In a series of experiments we explored the mechanisms which might be involved in the generation of these responses. One possibility is that the activity is driven by an endogenous release of acetylcholine. This was explored by exposing the bladders to a high concentration of the non-specific muscarinic antagonist atropine $(3 \mu \mathrm{M})$. Atropine did not affect the transients from any of the groups (Fig. 2B). In addition, the possibility of a neural regulation of the activity was examined using the neurotoxin tetrodotoxin $(1 \mu \mathrm{M})$. Tetrodotoxin had no effect on the transients.

In 10 of the 13 obstructed bladders it was noted that there were regular bursts of activity (Fig. 3A). Features of this bursting activity can be seen in figure $3 \mathrm{Ab}$. Immediately after a burst of activity, there is a transient decrease in frequency (arrow). This complex variation in frequency is seen in the analysis of the activity in figure 3B. It was also noted that this bursting activity and fall in frequency were not inhibited in the presence of atropine or tetrodotoxin (Fig. 3C).

The isolated whole bladder from the control group responded to exogenous cholinergic stimulation with a complex response. On applying the agonist (e.g. 300 $\mathrm{nM}$ ) there was an initial burst of activity consisting of large transient pressure changes (Fig. 4A, upper panel). This initial phase is followed by a transient slowing of activity (arrowed upper panel $A$ ) recovering to a steady state phase where the transients are large $\left(\sim 25 \mathrm{~cm} \mathrm{H}_{2} \mathrm{O}\right)$. A detailed analysis of the changes in frequency of the transients during the agonist application is illustrated in figure 4A (lower panel). The initial burst, transient slowing and steady state are readily identified. Exposing the isolated bladders from operated obstructed bladders showed that they were also sensitive to cholinergic stimulation. However, based on the initial experiments it was clear that considerably less agonist was required to achieve a large response, e.g. in figure $4 \mathrm{~B}, 30 \mathrm{nM}$ arecaidine produced a large effect. The detailed analysis of this response is shown in figure 4 (lower panel). Note that the activity disappears during the later period of the exposure to the agonist. Thus, the 
A

(a)

(b)
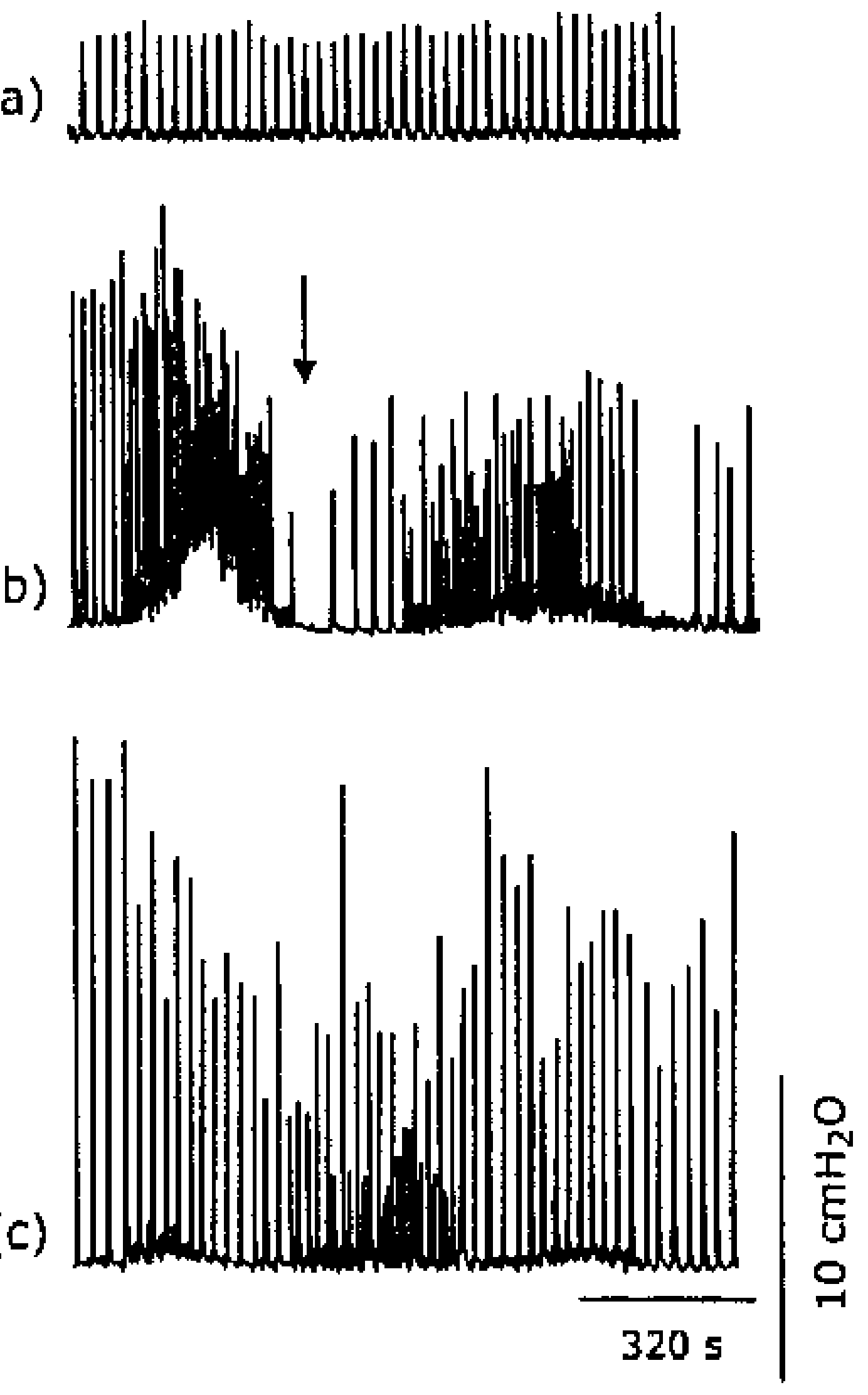

B

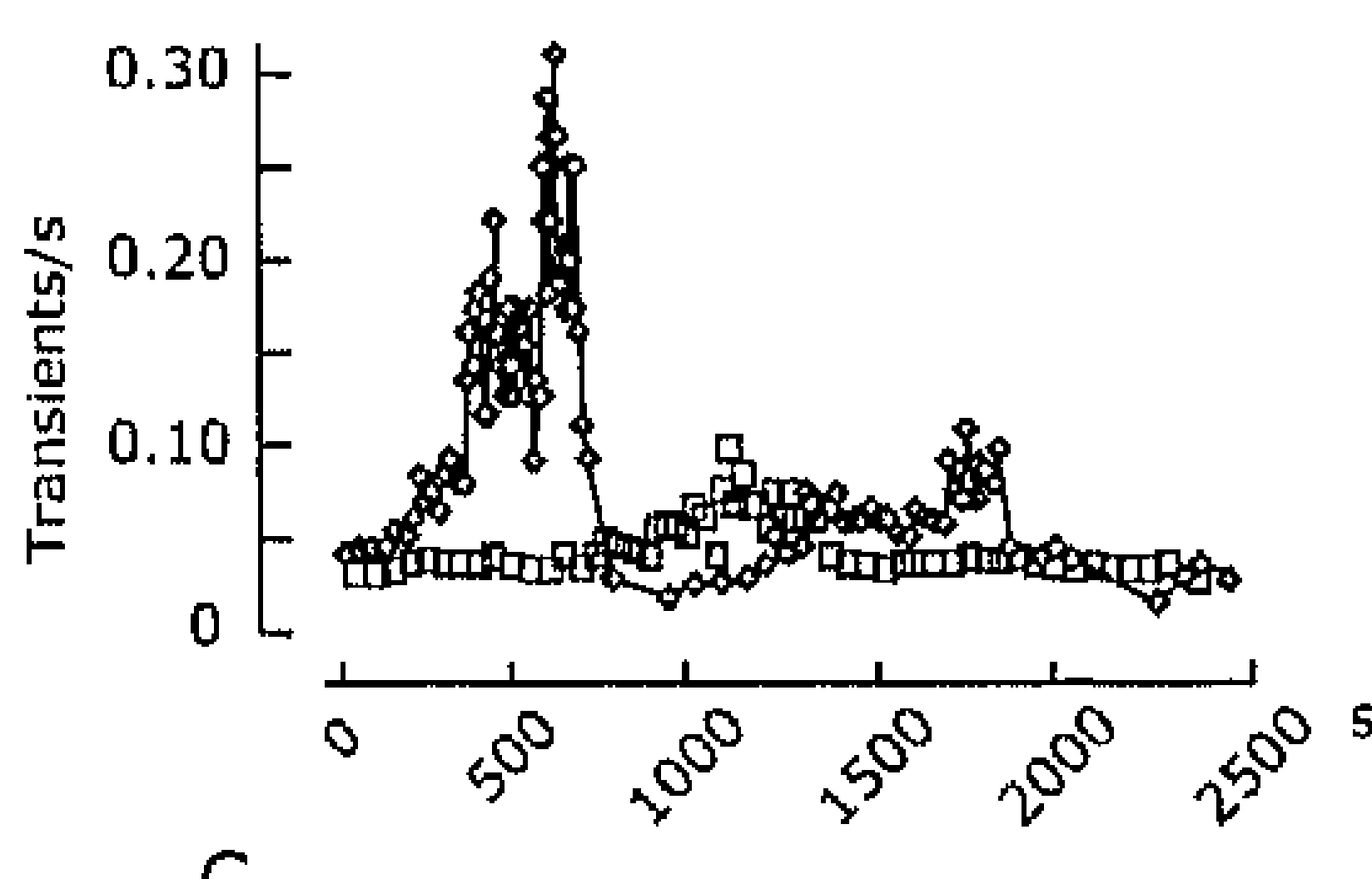

C atropine $3 \mu \mathrm{M}$

(a)

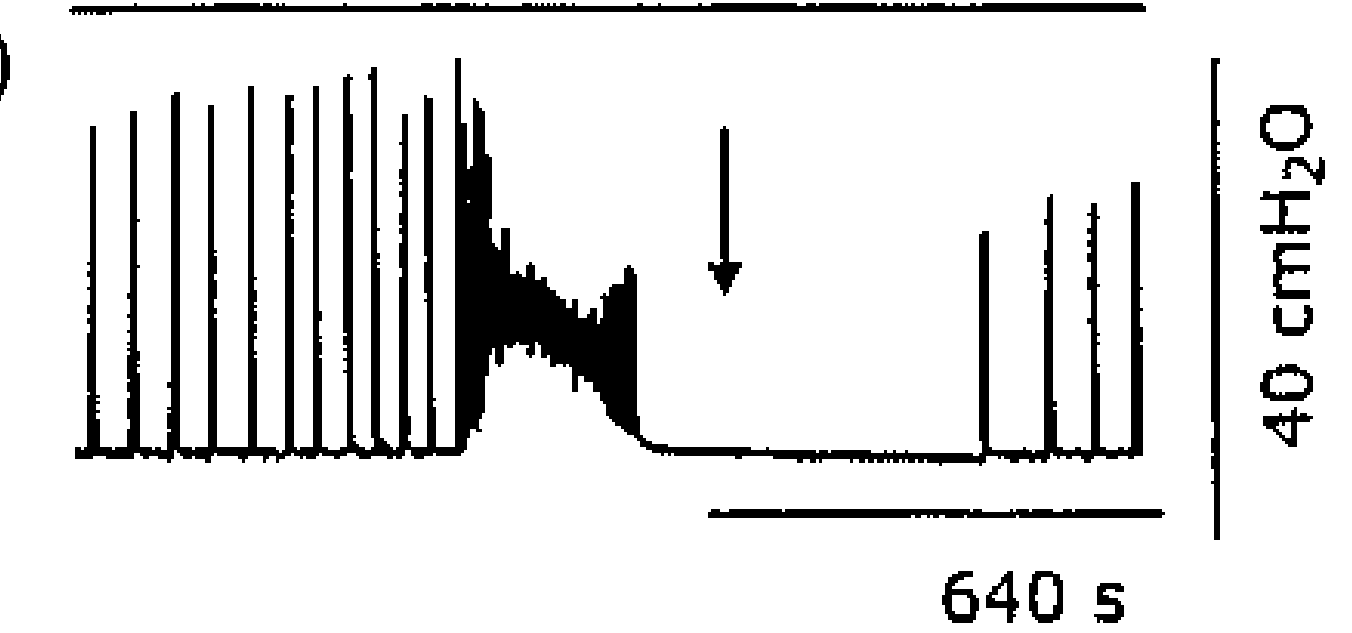

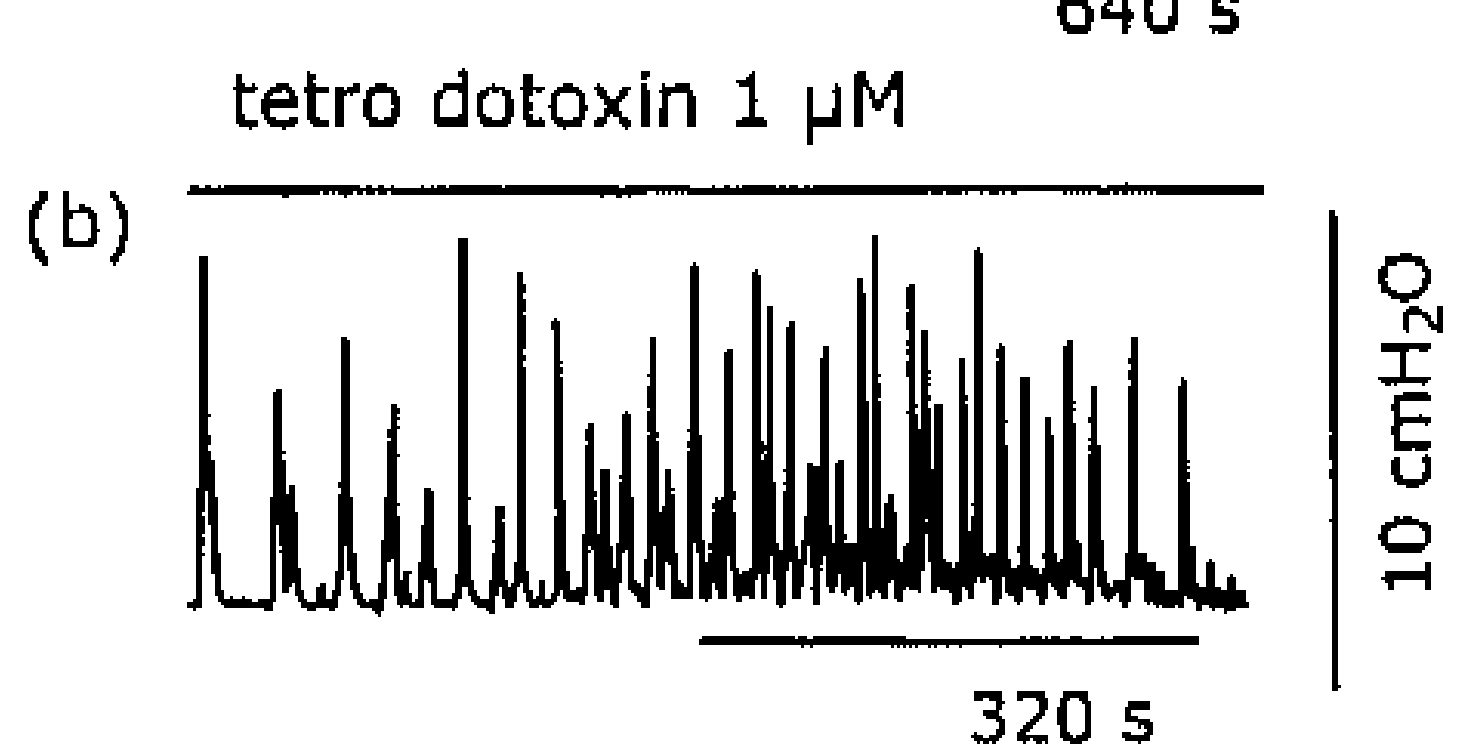

Figure 3.

Complex autonomous activity in bladders after obstructing the bladder neck. A shows examples of original records from (a) sham operated, (b) $O B L$ and (c) $O B H$ groups. In (b) the arrow points to a period of 'inhibition' of the spontaneous activity. Calibration bars show time in seconds and pressure in $\mathrm{cm} \mathrm{H}_{2} \mathrm{O}$. B Shows an analysis of sections of the records in (b) and (c).

The plot shows variations in the frequency of the transients. Periods of high frequency and periods of low frequency are apparent. The ordinate shows the instantaneous frequency of the transients (reciprocal of interspike interval) while the abscissa shows time in seconds. $\boldsymbol{C}$ shows original records from bladders from the obstructed groups, illustrating the absence of an effect of atropine $(a, 3 \mu \mathrm{M})$ and tetrodotoxin $(\boldsymbol{b}, 1 \mu \mathrm{M})$. Note, the period of inactivity marked by the arrow in (a). Calibration bars show time in seconds and pressure in $\mathrm{cm} \mathrm{H}_{2} \mathrm{O}$.
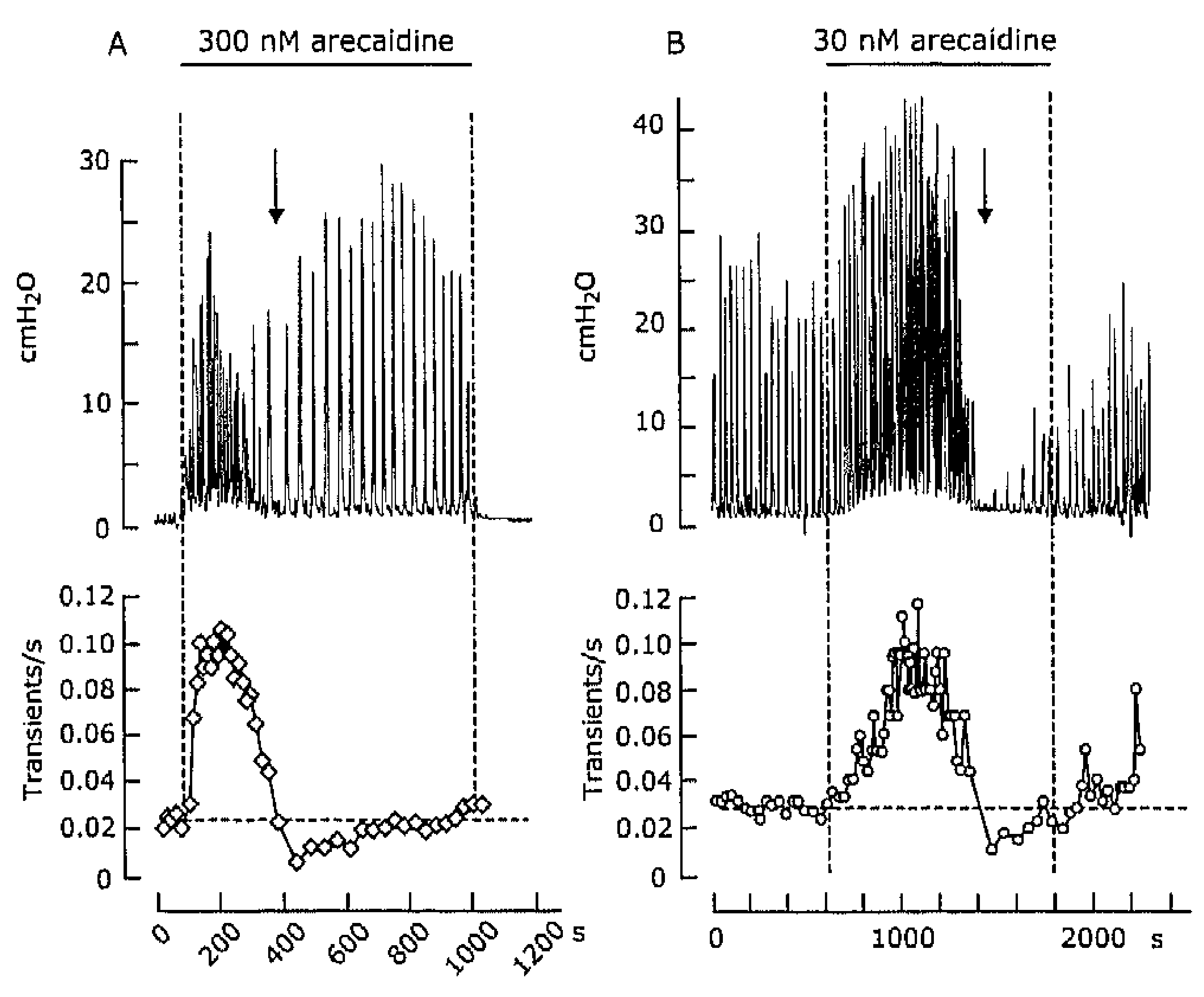

Figure 4.

The effects of the muscarinic agonist arecaidine on autonomous activity in bladders from the control and operated groups. A shows a typical response to $300 \mathrm{nM}$ arecaidine in a control bladder. The upper panel shows a section of the original records and the lower panel an analysis of this response plotting the instantaneous frequency of the transients against the time of each transient. Where indicated by the first vertical dotted line, arecaidine was added to the bathing solution. $B$ shows data from an $O B H$ bladder exposed to $30 \mathrm{nM}$ arecaidine. In both upper panels the arrow points to a period of the recording where there is a distinct slowing of the transients to a level below the control level. The ordinate of upper panel is bladder pressure $\left(\mathrm{Cm} \mathrm{H}_{2} \mathrm{O}\right)$ and the lower panel instantaneous transient frequency (transients/second), and the abscissa shows time in seconds. 
A

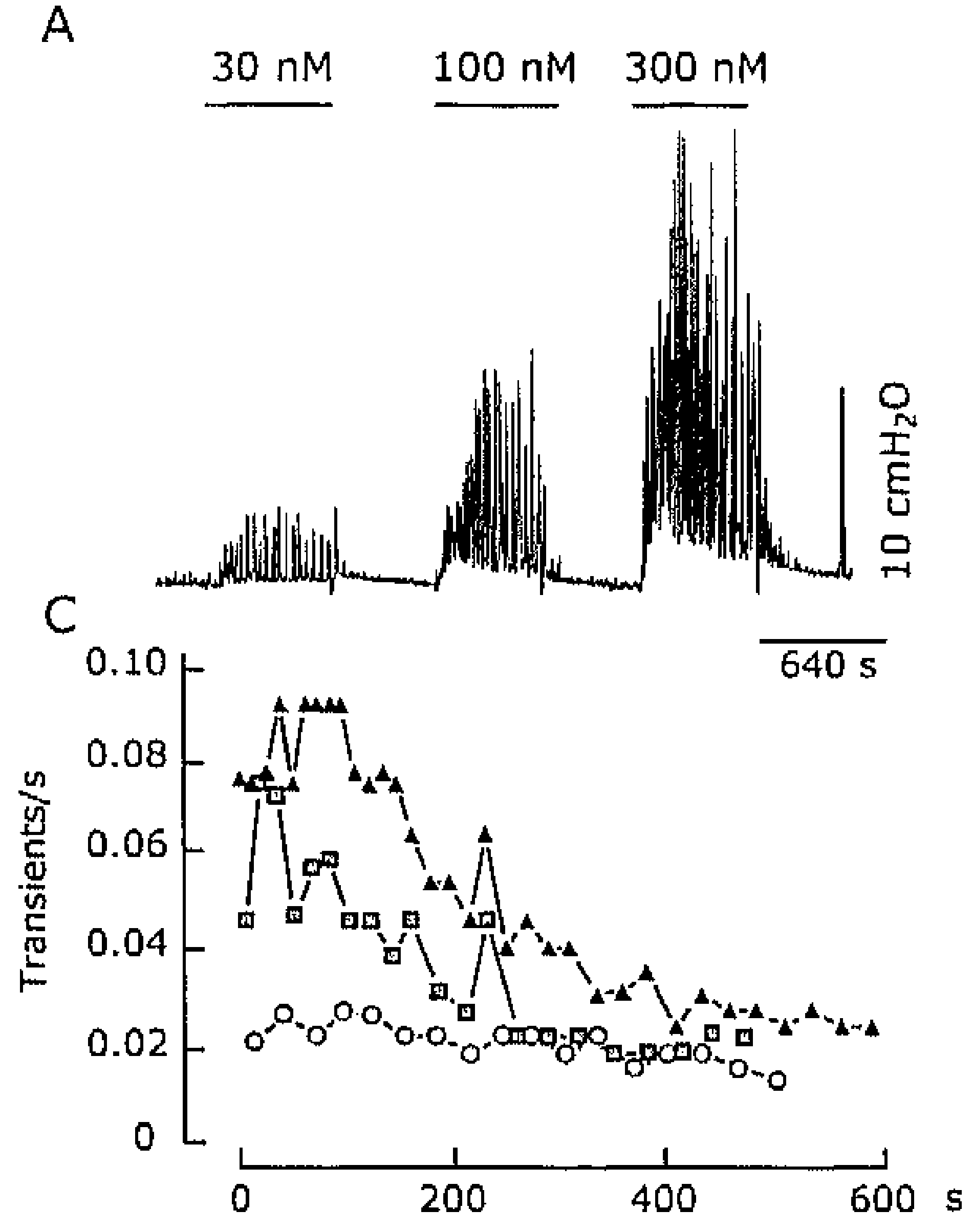

B

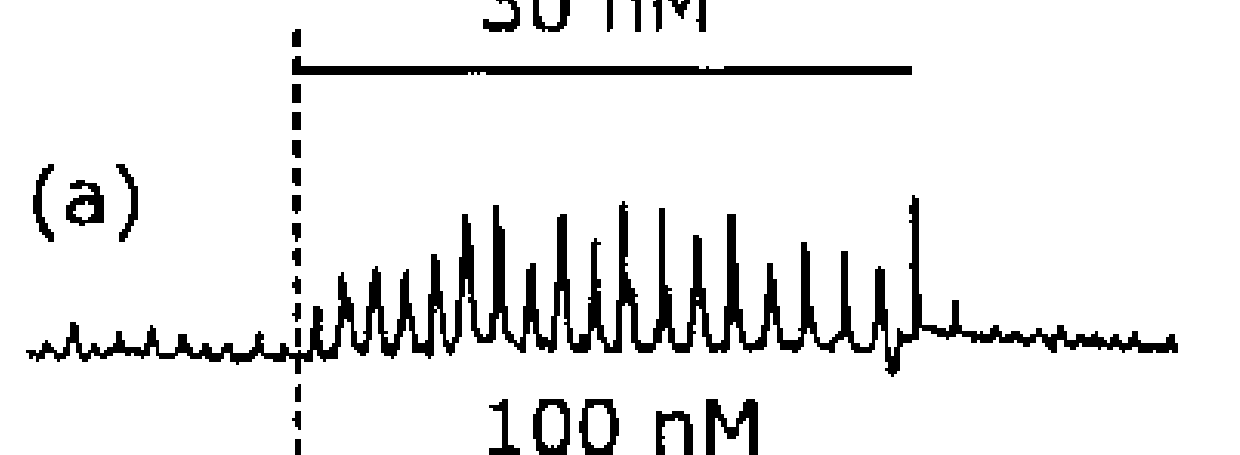

(b)

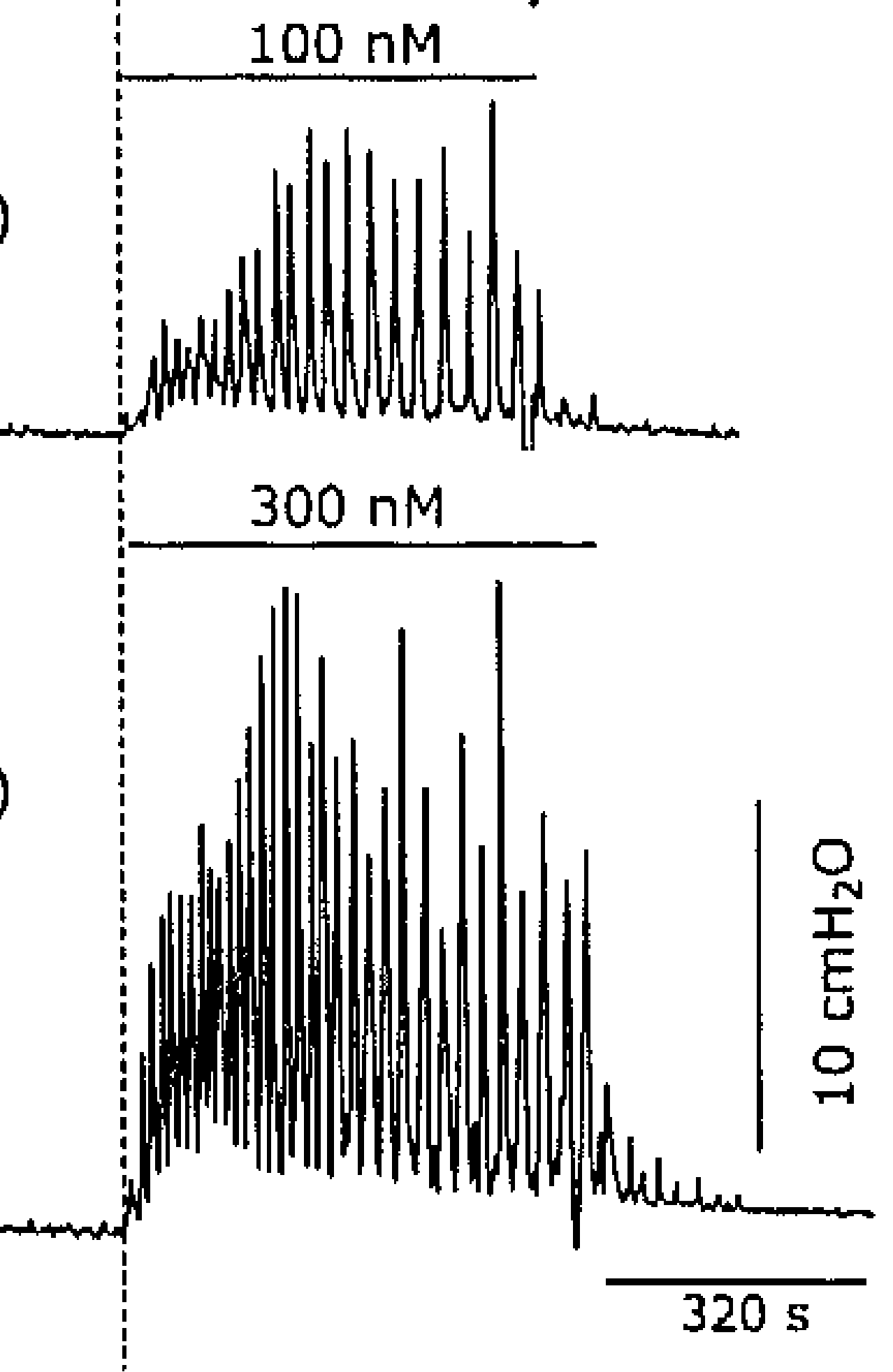

Figure 5.

Examples of responses to different concentrations of the muscarinic agonist arecaidine on control bladders. A shows the entire section record where the bladder was exposed to discrete exposures of 30, 100 and $300 \mathrm{nM}$ arecaidine (indicated by the horizontal bars). Sections of this record are shown on an

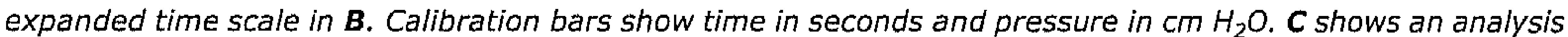
of these records illustrating the instantaneous frequency of the transients during exposure to $30 \mathrm{nM}(\mathrm{o}), 100$ $n M(\square)$ and $300 \mathrm{nM}(\Delta)$ arecaldine. The ordinate is the instantaneous frequency of the transients and the abscissa the time in seconds.

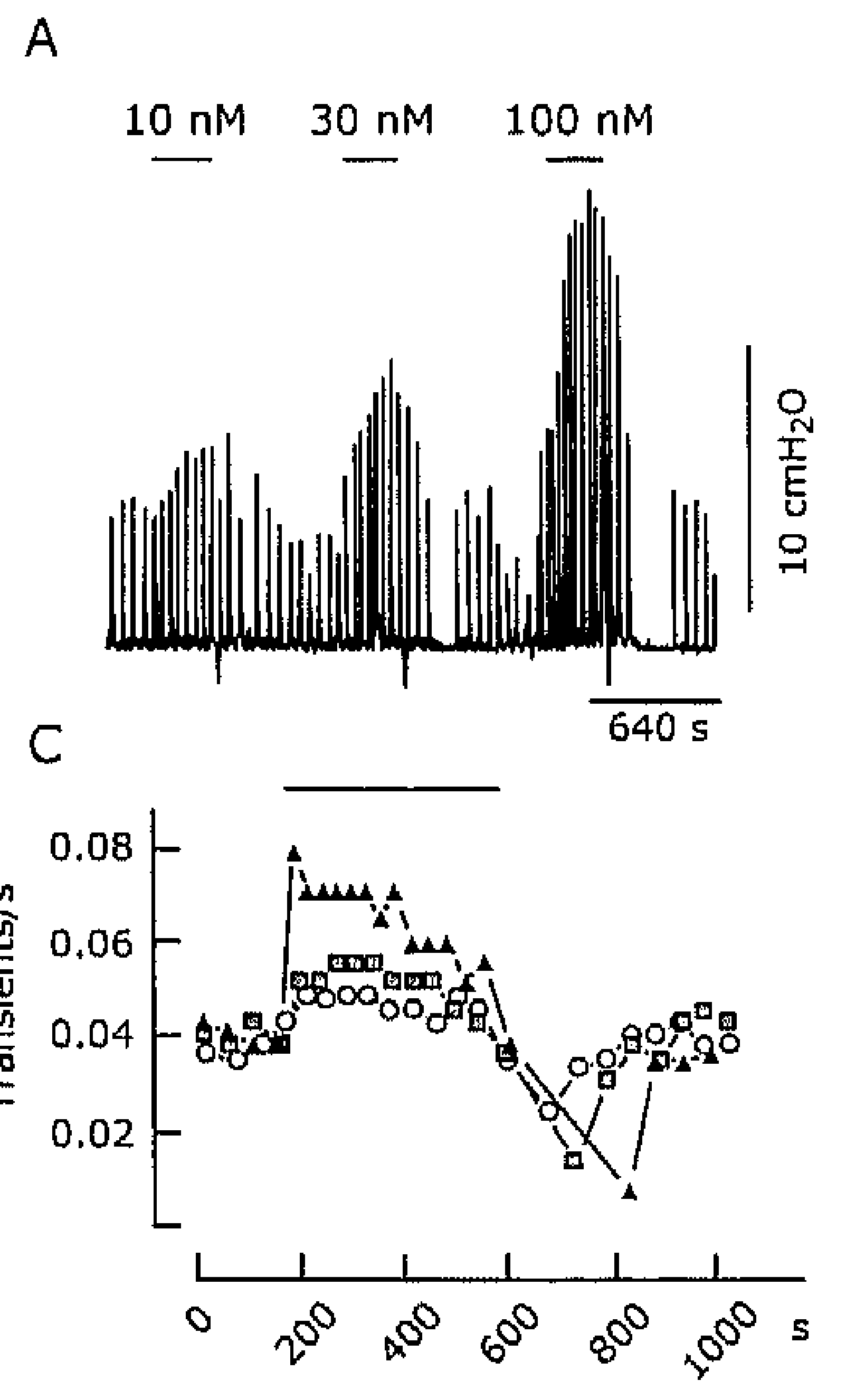

B

(a)

(a)

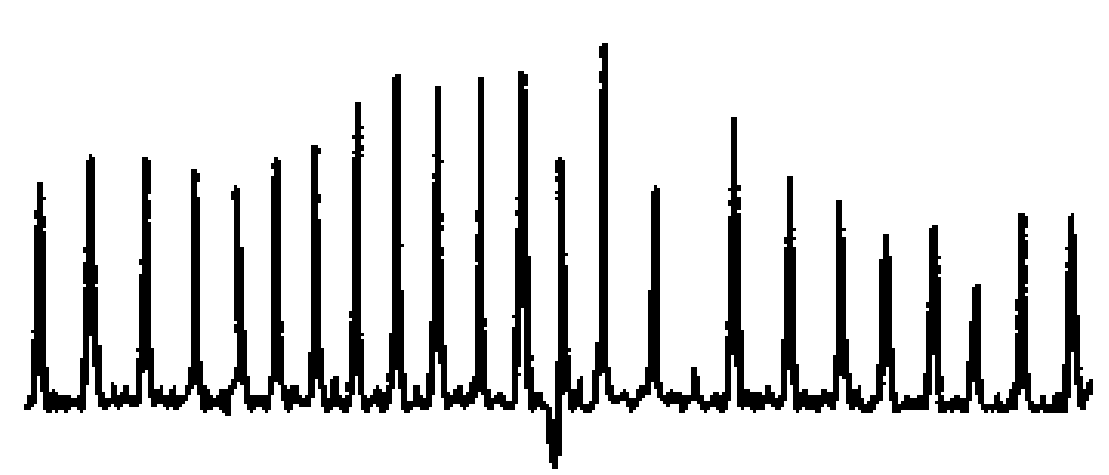

(a)

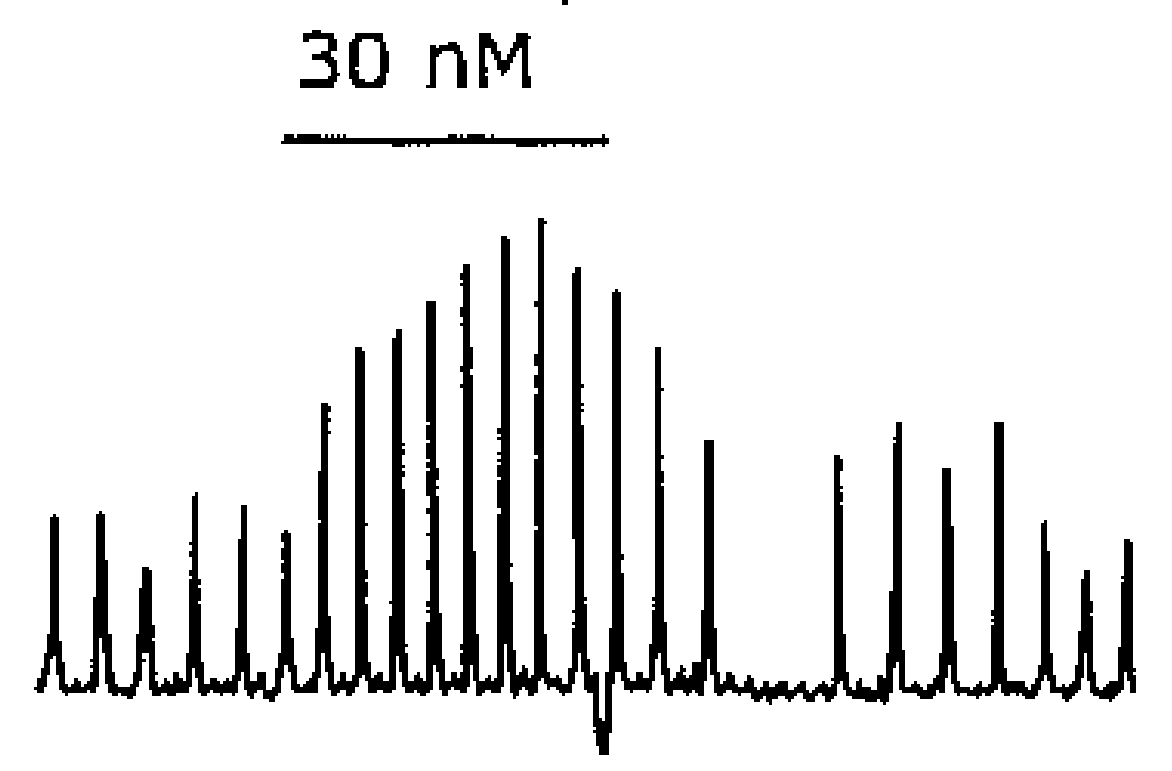

(a)

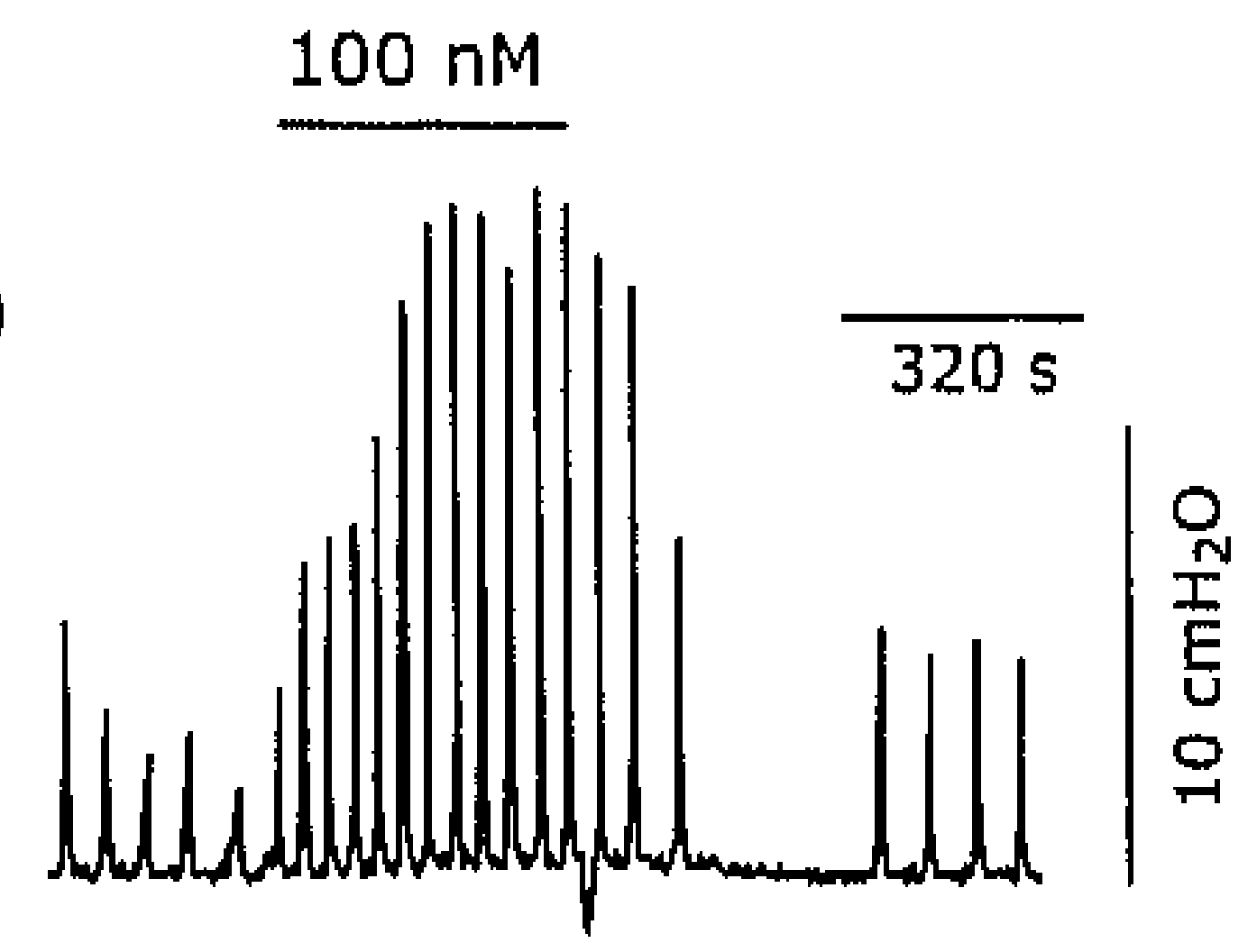

Figure 6.

Examples of responses to different concentrations of the muscarinic agonist arecaidine on bladders from the sham operated group; details as in figure 5. 

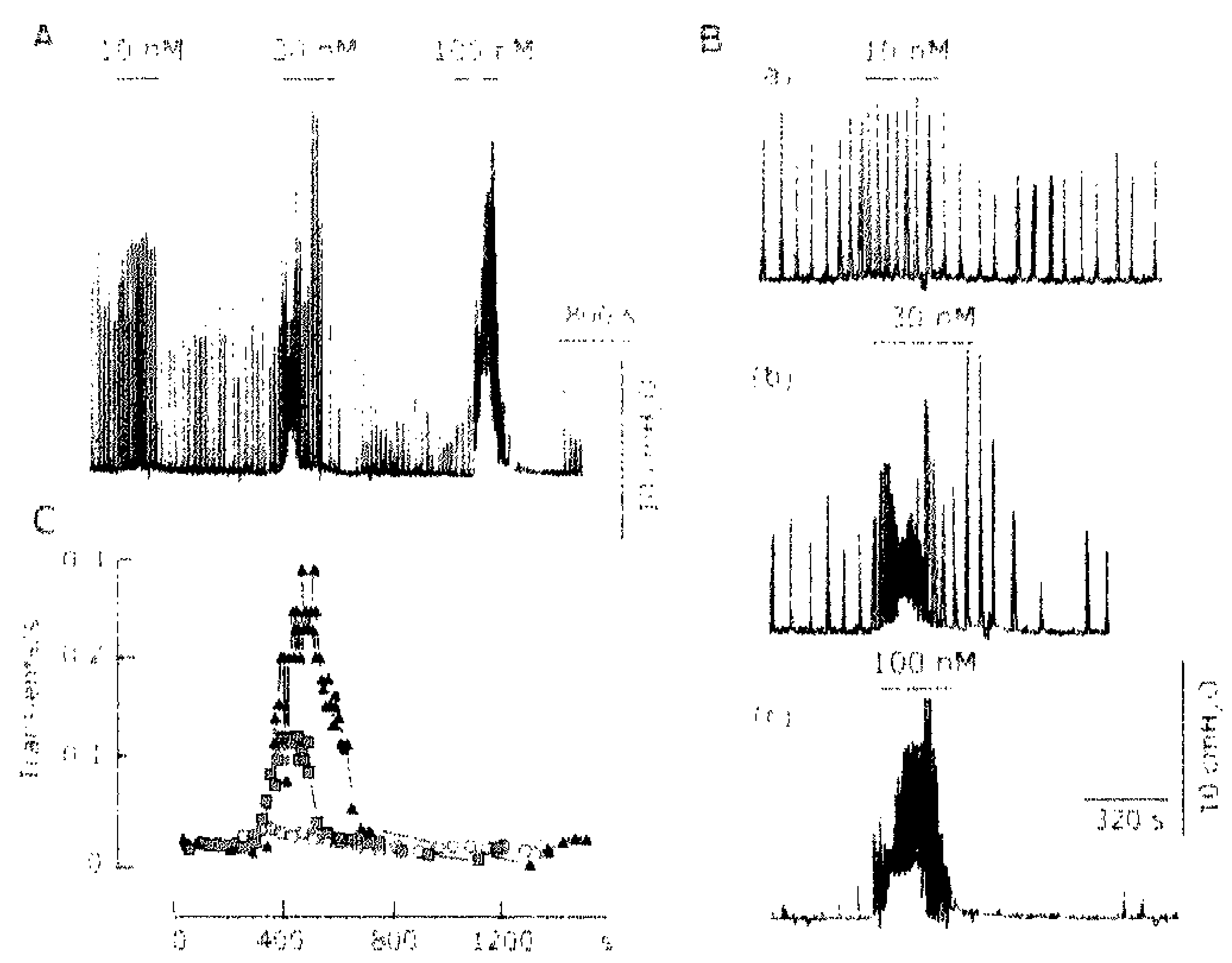

Figure 7.

Examples of responses to different concentrations of the muscarinic agonist arecaidine on bladders from the OBL group; detalls as in figure 5.

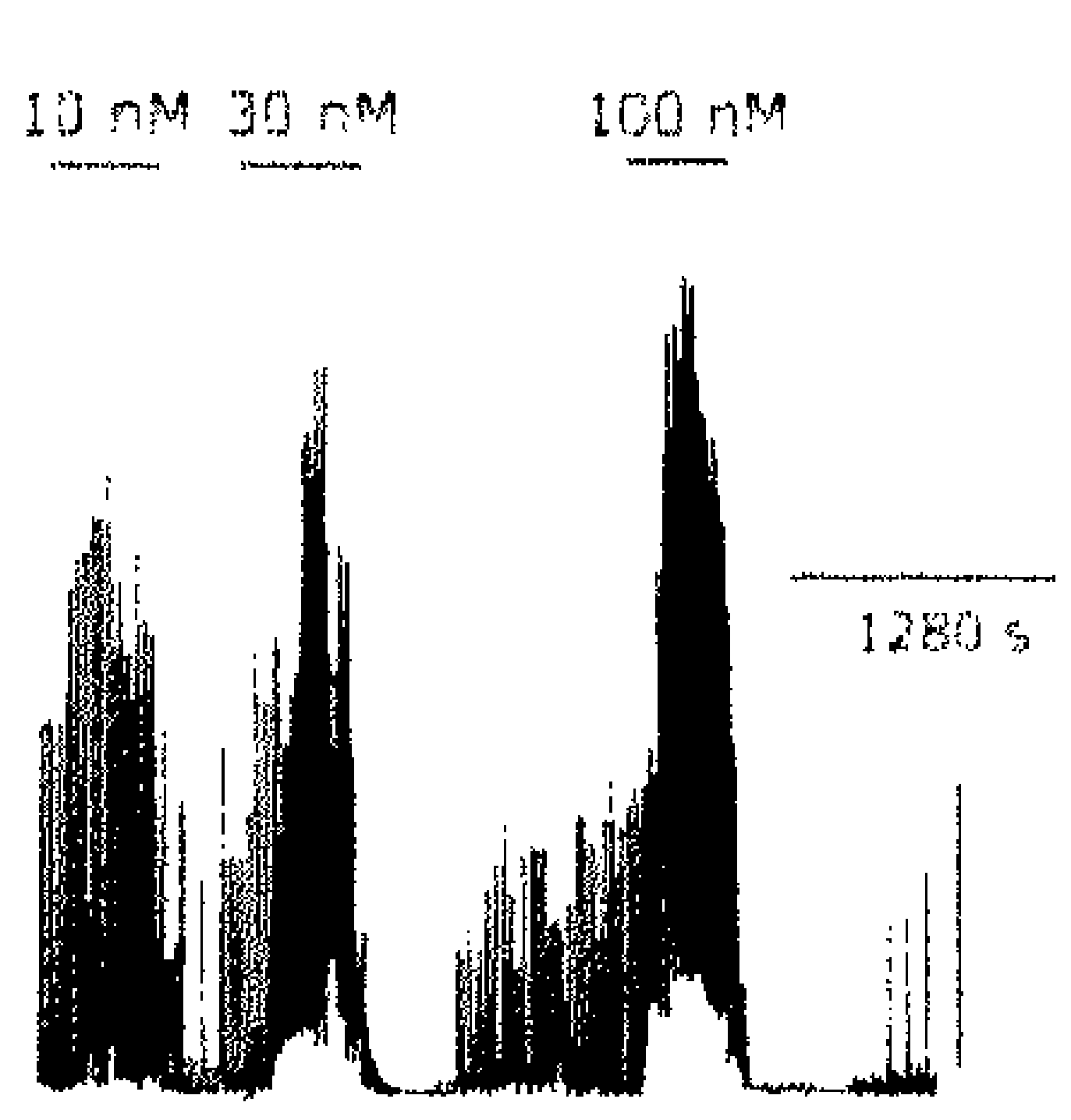

C

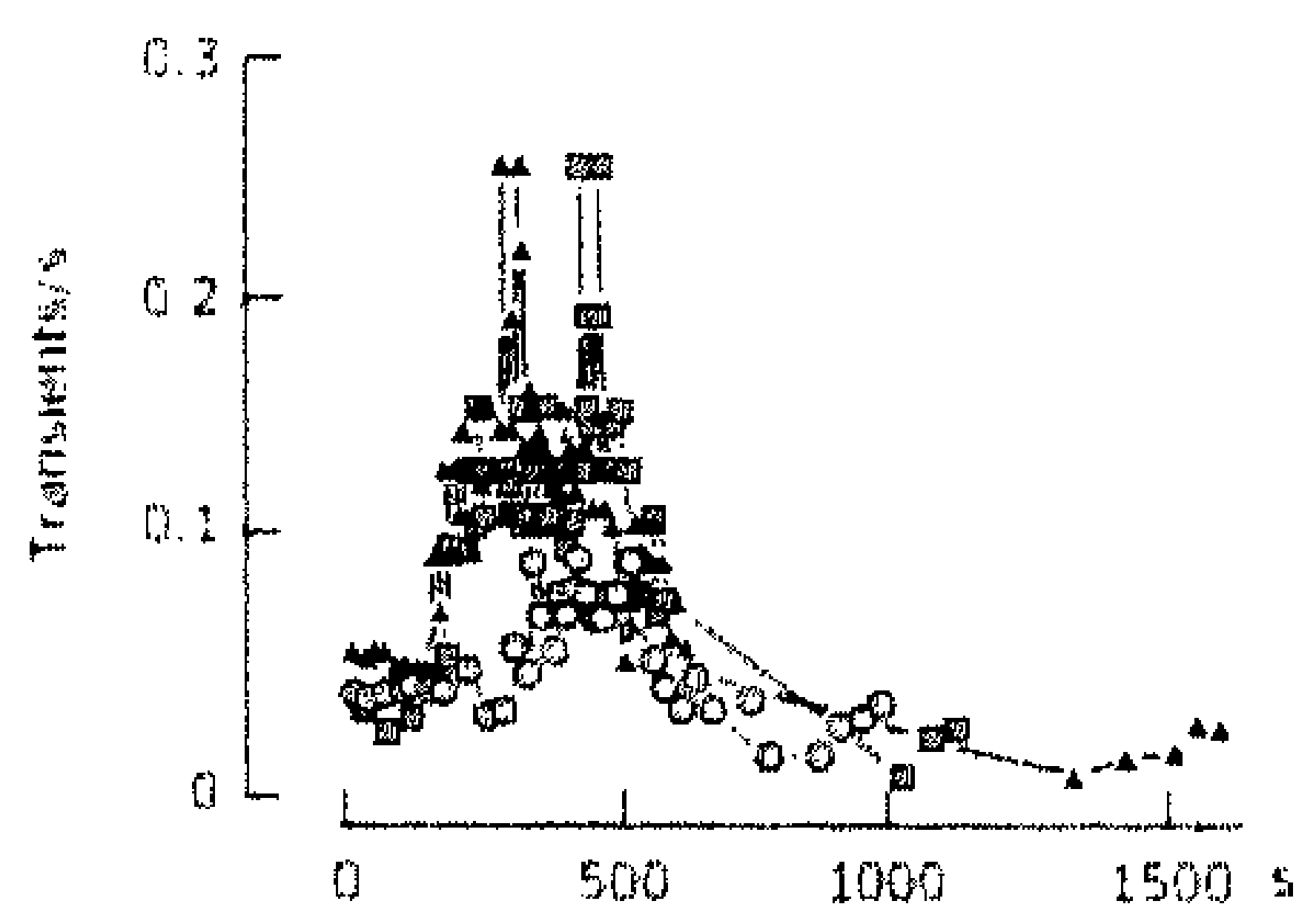

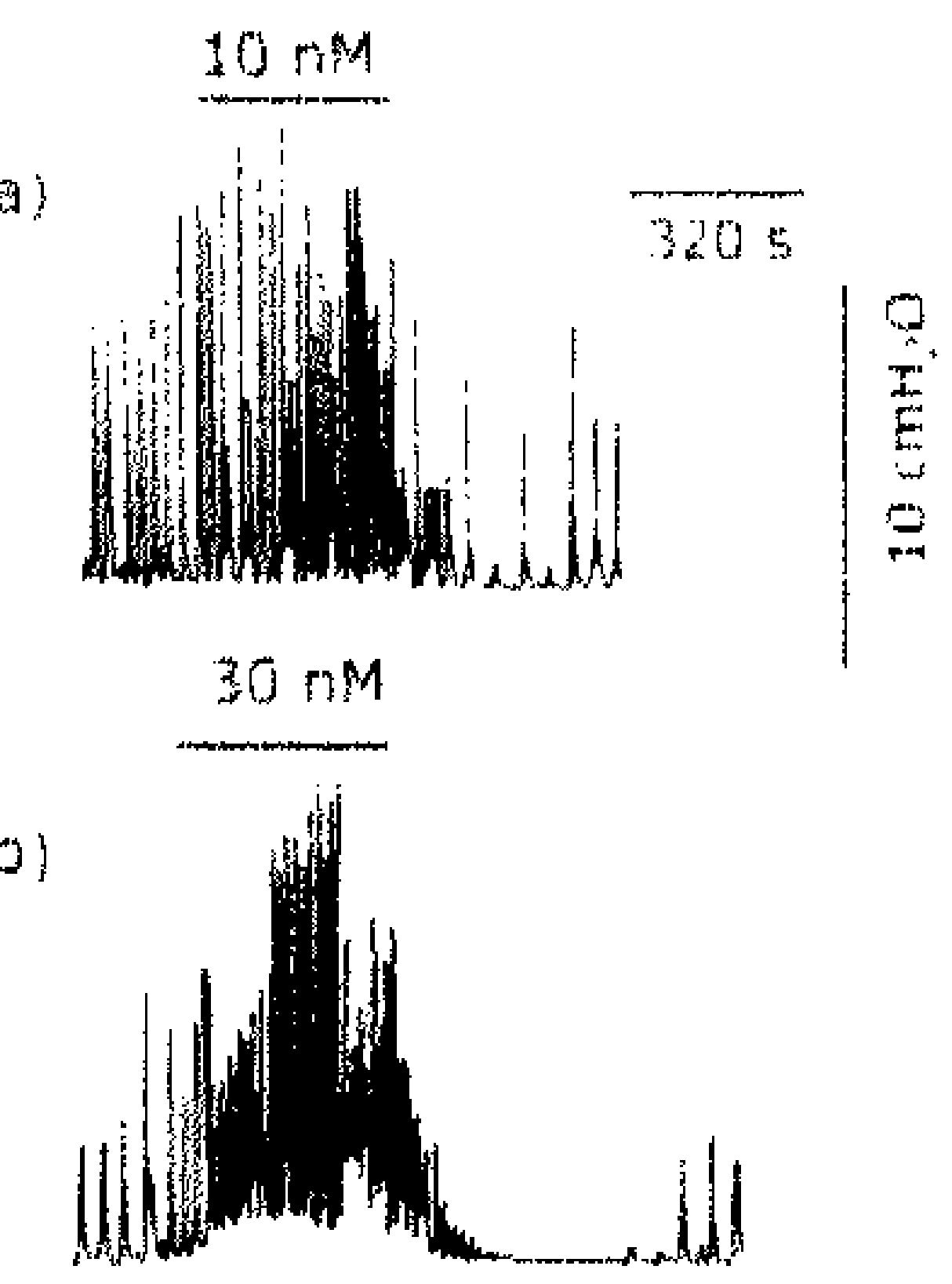

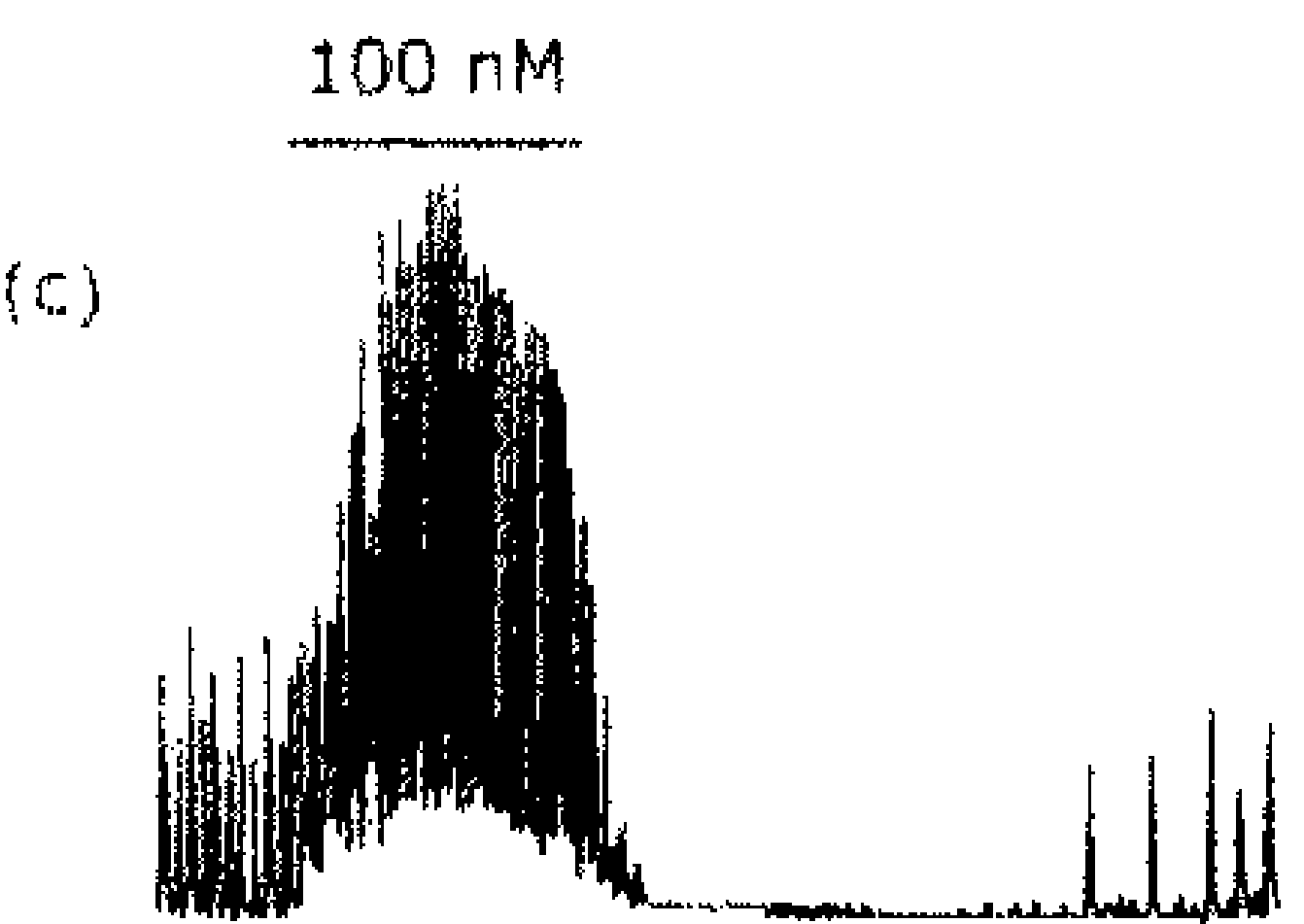

Figure 8.

Examples of responses to different concentrations of the muscarinic agonist arecaidine on bladders from the $\mathrm{OBH}$ group; detalls as in figure 5 . 
operated bladders appear to have an increased sensitivity to cholinergic stimulation and marked period of loss of activity.

Figures 5-8 illustrate examples of the responses to different concentrations of agonist in control, sham, OBL and $\mathrm{OBH}$ bladders. All the features described in figure 4 can be seen in each group. The increased sensitivity to cholinergic stimulation and the period of loss of activity appears to be greatest in the $\mathrm{OBH} \geq \mathrm{OBL}>$ sham bladder. The data from all of the control and operated bladders are illustrated in figures 9-10. In figure $9 \mathrm{~A}$, for each of the experimental groups, the frequency of the transients increased with increasing concentration of agonist. The responses to $30 \mathrm{nM}$ arecaidine had the greatest increase in frequency in the $\mathrm{OBL}$ and $\mathrm{OBH}$ bladders, then sham and then control. Thus, there appears to be a progressive sensitization of the mechanisms generating the initial rise in frequency. There also appears to be a dose dependency of the amplitude of the transients (Fig. 9B). However, when the data are normalised for differences in bladder weight there appears to be little difference in the ability of the different concentrations of agonist to affect the transients. Figure 10 illustrates the effects of the different agonist concentrations on the rise in baseline during the agonist application. Figure $10 \mathrm{~A}$ shows the basic data. The magnitude of the baseline change is similar in $\mathrm{OBH}$ and $\mathrm{OBL}$ bladder, and these responses were larger than in the sham and control bladder. However, these differences are not apparent in the data normalised for bladder weights (Fig. 10B). Also, the possibility must be considered that the rise in baseline is not a contraction but an artefact resulting from the rapid phasic activity. If the frequency of the transients is high then it is possible that the bladder has insufficient time to relax between events. In such cases the basal pressure might appear to increase although there may be no underlying change.

Bursts of activity can be followed by periods of slower transient activity or even complete cessation of activity during the spontaneous activity in the operated animals but also during the muscarinic stimulation. Figure $11 \mathrm{~A}$ (upper panel) illustrates a typical example of a response to arecaidine in an OBL bladder. The burst of activity and the period of slowing/cessation are clearly seen and are also apparent in the frequency analysis of the response (Fig. $11 \mathrm{~A}$ lower panel). In an attempt to quantify the duration of the slowing/cessation, the time constant of the period to the return of activity was measured (legend Fig. 11). Figure 11B illustrates data accumulated from sham, OBL and $\mathrm{OBH}$ bladders. Clearly, the slowing/cessation is greater with higher concentrations of applied agonist. In addition, the length of the time period of the slowing/cessation follows the order $\mathrm{OBH} \geq \mathrm{OBL}>$ sham bladders.

In bladders isolated from normal animals it has been shown that the muscarinic induced phasic activity is inhibited by adrenergic stimulation. ${ }^{24}$ In a brief series of experiments the effects of noradrenalin was examined on the autonomous activity of the operated animals. Two examples of original records are shown in figure 12 . Noradrenaline inhibited the large transients, and this inhibitory effect was apparent in each of five separate bladders. 

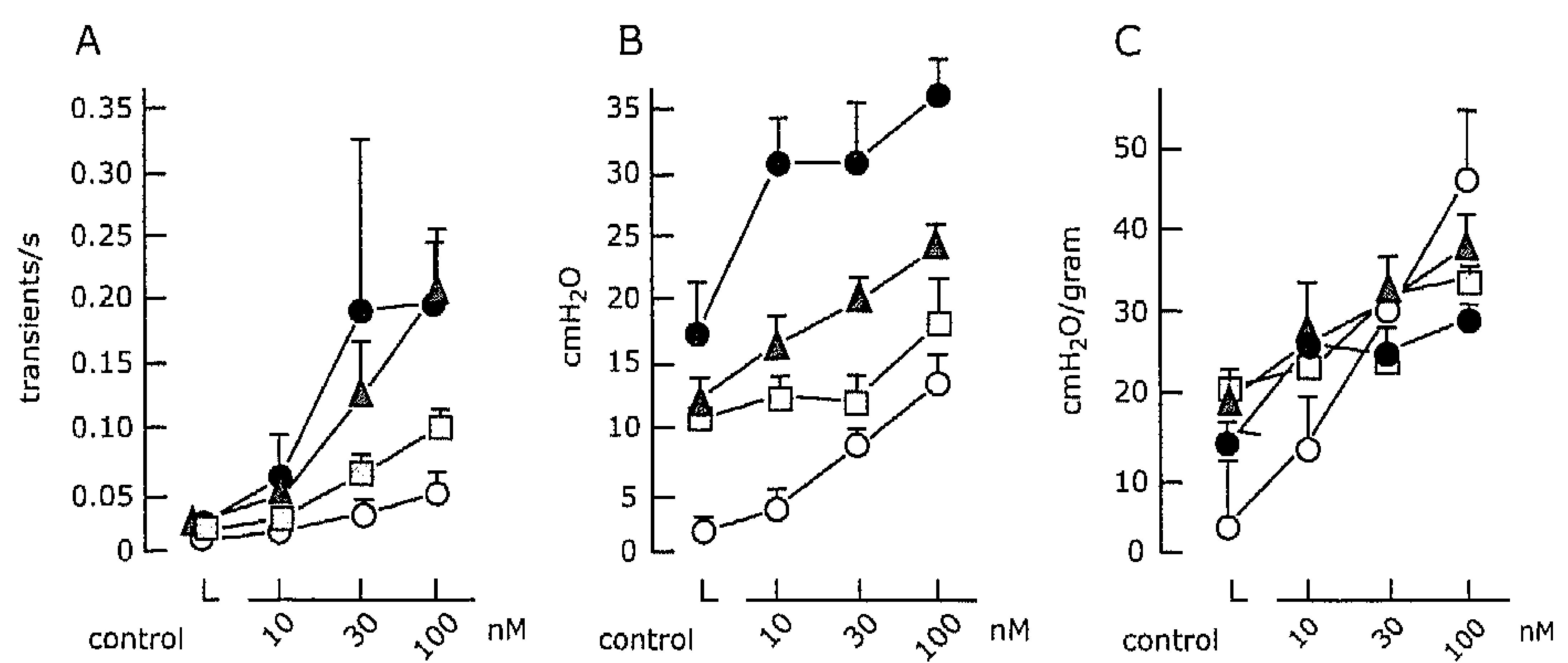

Figure 9.

Accumulated data showing the effects of arecaidine on the induced transients. Data were accumulated from six control, six sham operated, six OBL and seven OBH bladders, illustrating the effects of different concentrations of the muscarinic agonist arecaidine. A shows the effects of different concentrations of arecaidine on the frequency of the transients. B shows the dose dependency on the amplitude of the transients. $C$ the effect of increasing concentrations of agonist on the amplitude of the transients normalised to the bladder weight of each bladder. In each panel, the groups are control (o), sham operated (!), OBL (A) and $O B H(\bullet)$. The ordinate is transients/second, pressure and pressure/gram tissue in A-C respectively. The abscissa shows the concentrations of muscarinic agonists used in nM. Values are mean data \pm S.D.

A

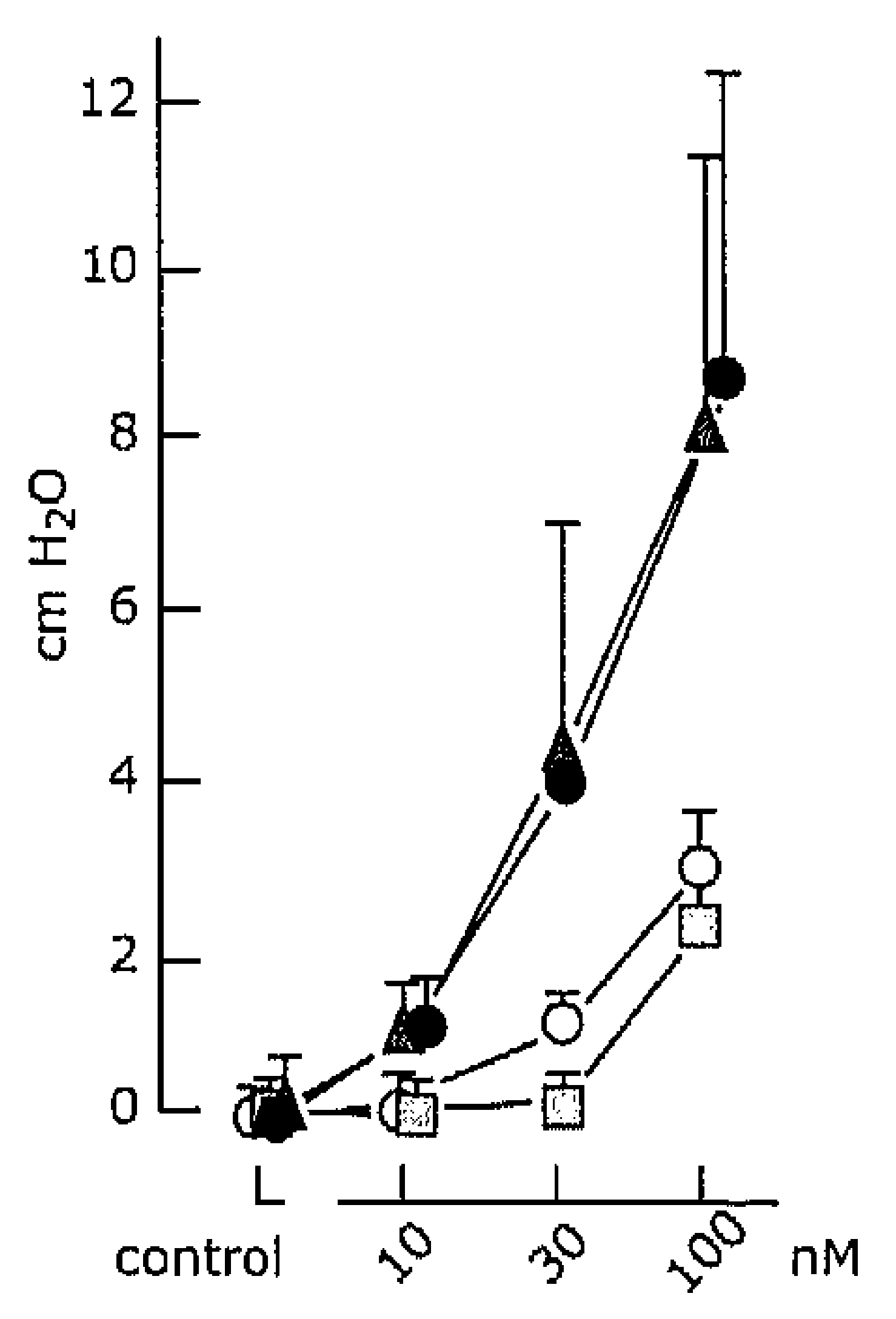

B

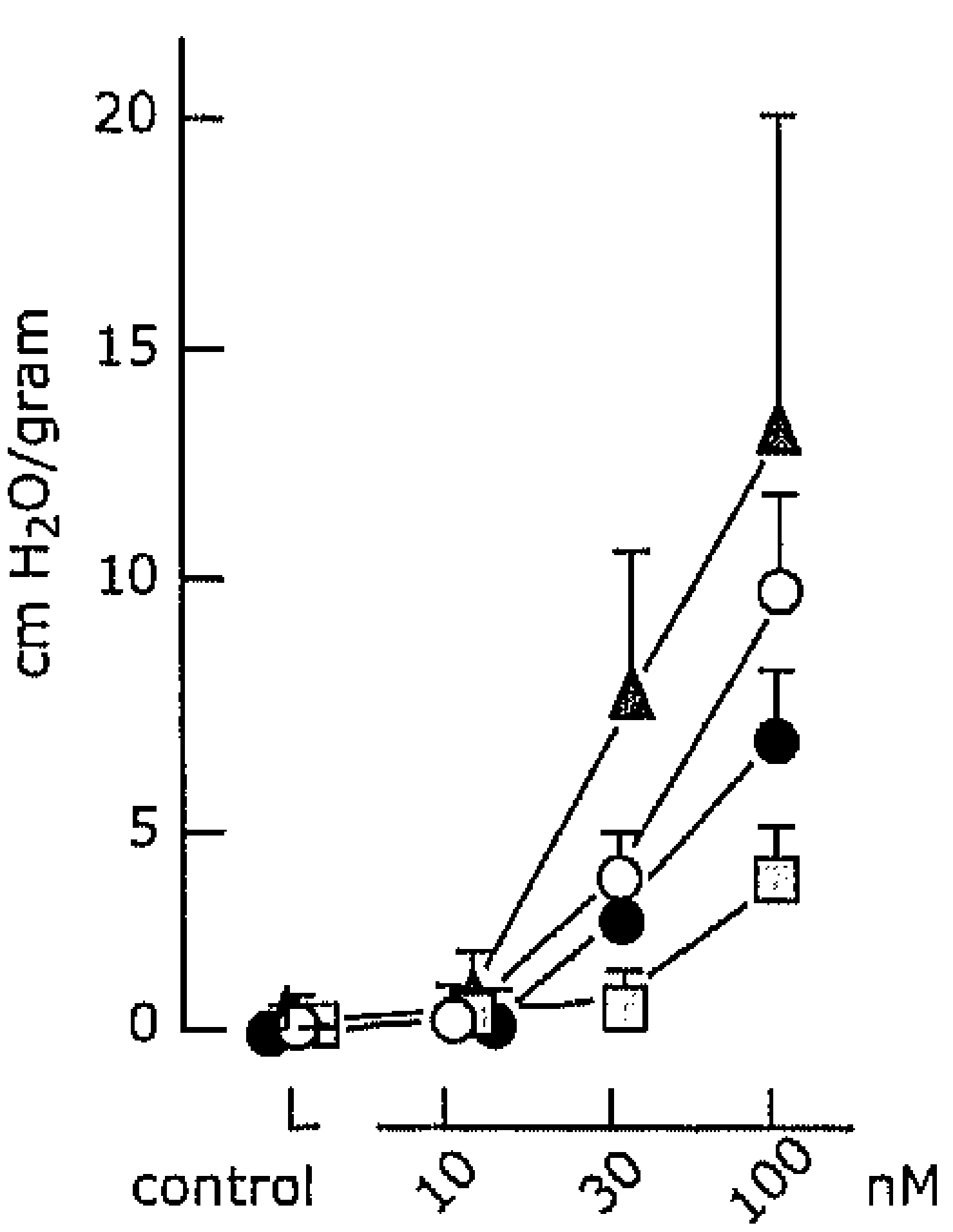

Figure 10.

Accumulated data showing the effects of arecaidine on the shift in baseline values accumulated from six control, six sham operated, six OBL and seven $O B H$ bladders. A shows the effects of different concentrations of agonist on the rise in baseline pressure during the exposure to agonist. In each panel the control groups ( $(0)$, sham operated ( $(\omega), O B L(\Delta)$ and $O B H(\bullet)$ bladders are illustrated. The ordinates are pressure and pressure/gram tissue in A-B respectively. The abscissa shows the concentrations of muscarinic agonists used in $\Pi M$. Values are the mean (S.D.). 

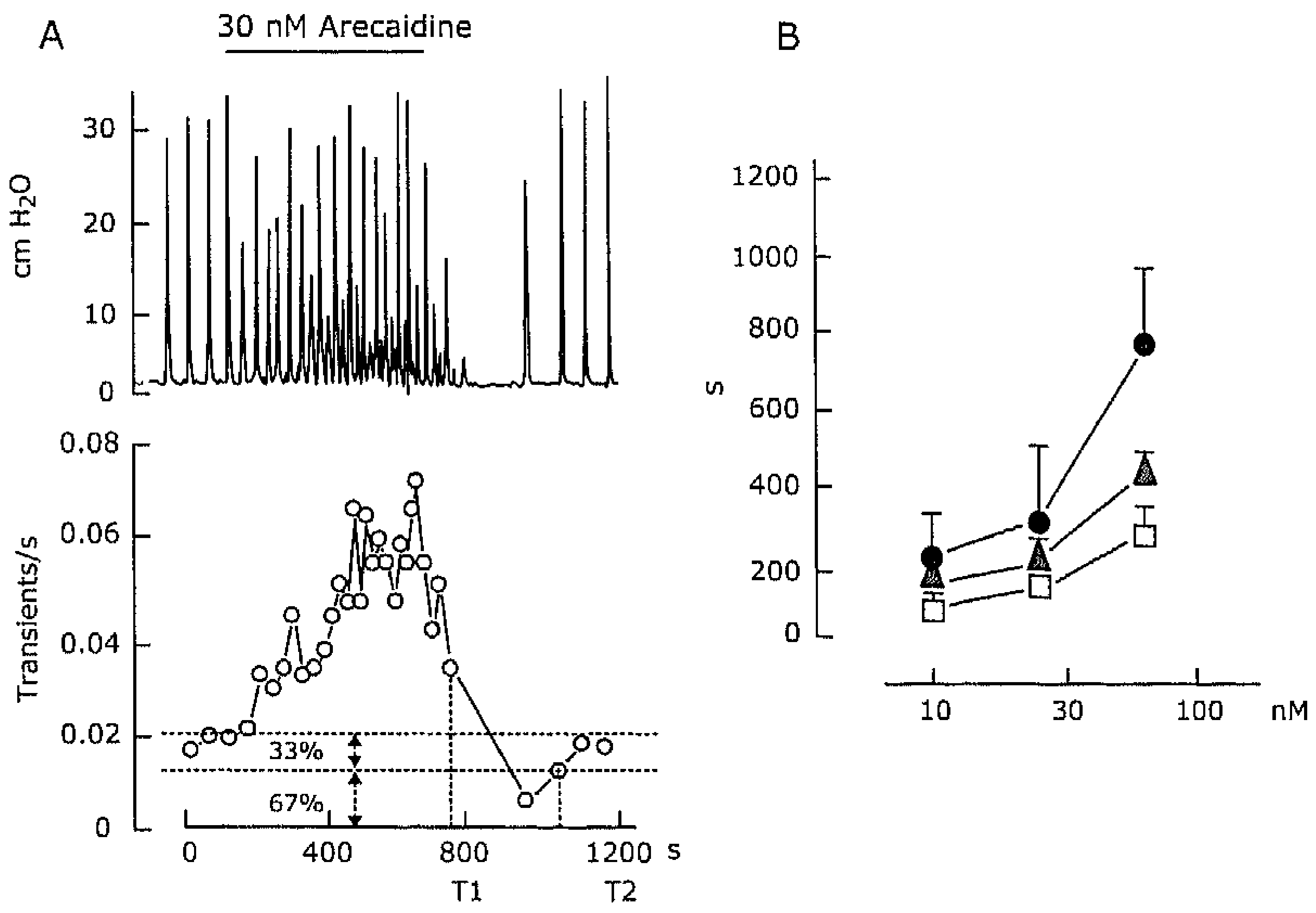

Figure 11.

The effects of different concentrations of the muscarinic agonist arecaidine on the duration of the cessation of activity following exposure to the agonist. $A$, upper panel shows a section of a record from an $\mathrm{OBH}$ bladder during an exposure to $30 \mathrm{nM}$ arecaidine (indicated by the horizontal bar). The lower panel shows an analysis of this record illustrating the rise in frequency during the application of agonist and the fall in activity during and after application of the agonist. The duration of the slowing of the agonists is defined as the time difference from the point of cessation of activity to a point where the frequency recovers to a level of $67 \%$ of that before stimulation (tau value). The ordinate of the upper panel is pressure $\left(\mathrm{Cm} \mathrm{H}_{2} \mathrm{O}\right)$ and the lower panel, transient frequency (transients/sec). The abscissa is the time in seconds. $B$ shows data accumulated from six sham operated, six $O B L$ and seven $O B H$ bladders. In each panel, the sham operated values are shown by ( $\square$ ) symbols, the $O B L$ as $(\Delta)$ and $O B H$ as $(\bullet)$.

A

noradrenaline $10 \mu \mathrm{M}$

(a)

(b)

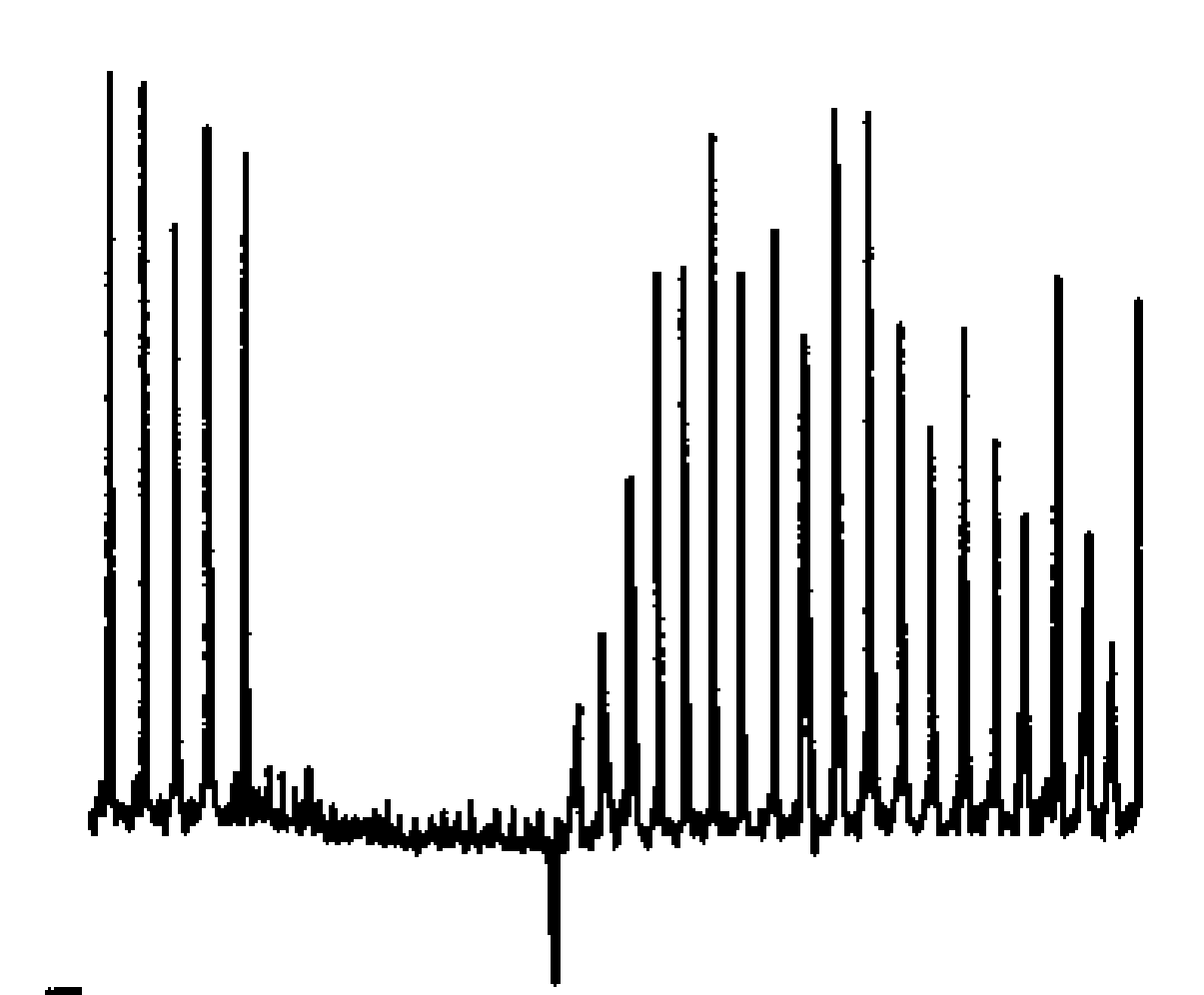

B

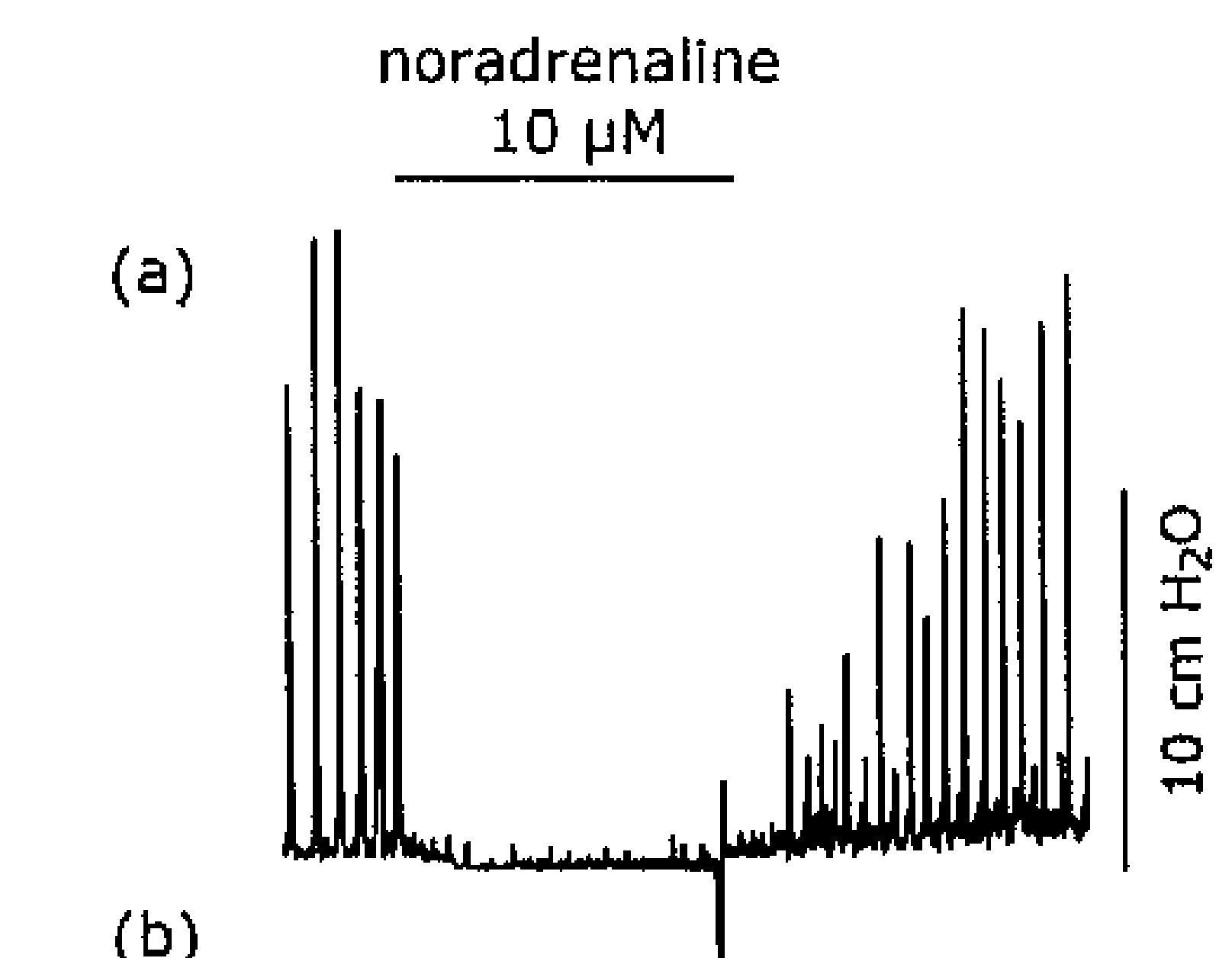

(b)

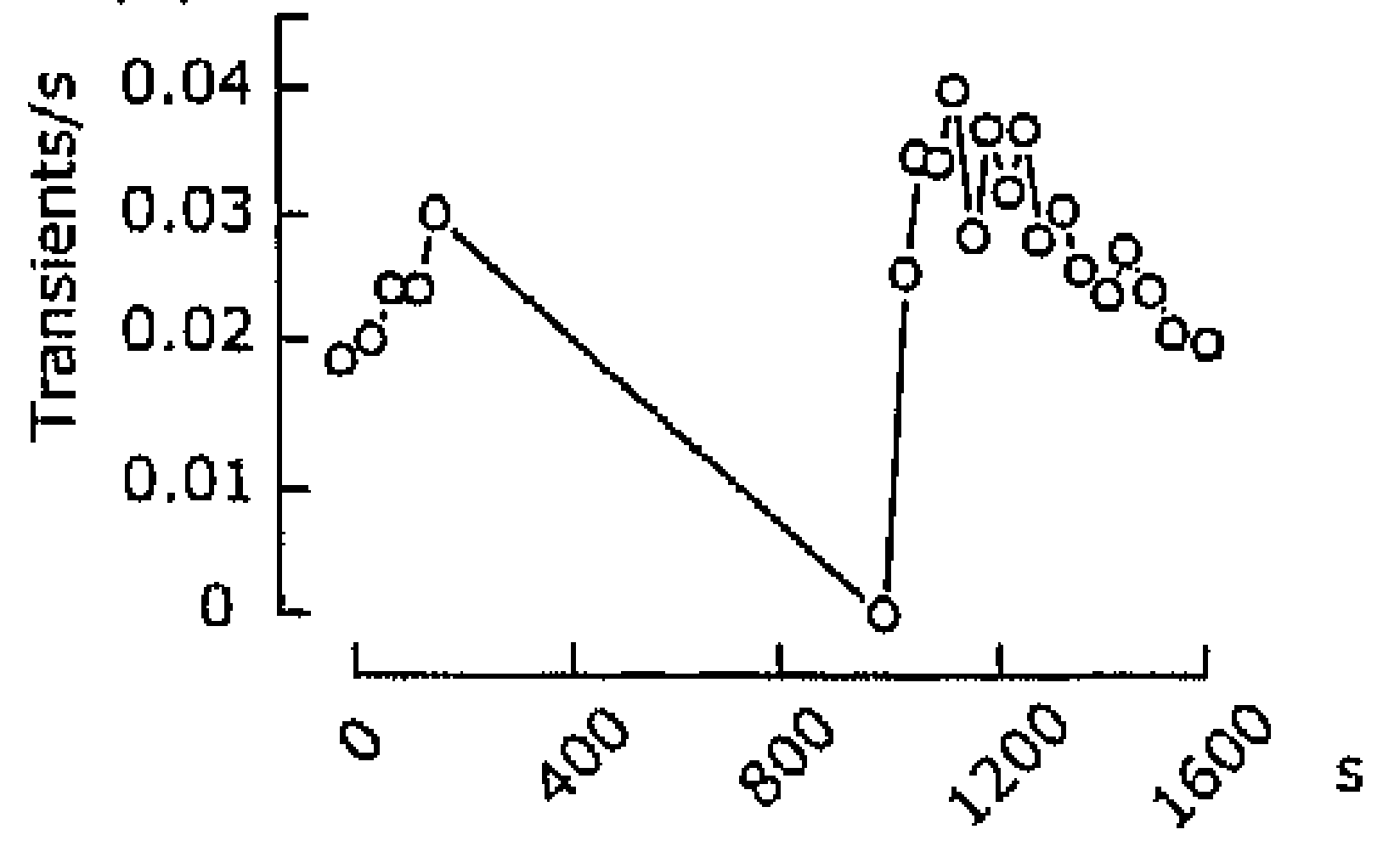

Figure 12.

The effects of noradrenaline on the autonomous activity in $O B L$ and $O B H$ bladders. $A$ shows original records (upper panel) and an analysis of this data (lower panel) from a sham operated bladder. B shows original records (upper panel) and an analysis of this data (lower panel) from an $O B L$ bladder. In the upper panels the vertical bar shows pressure in $\mathrm{Cm} \mathrm{H}_{2} \mathrm{O}$. In the lower panels the ordinates show the instantaneous frequency of the transients and the abscissa time in seconds. 


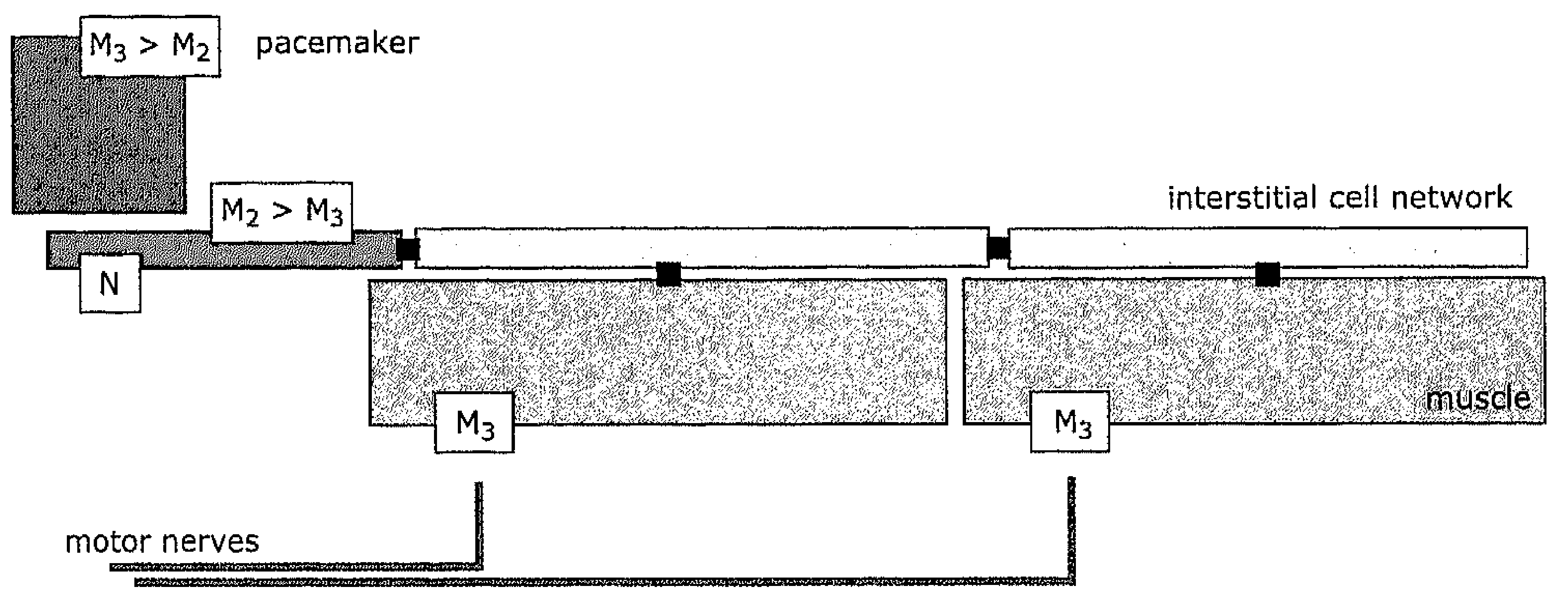

Figure 13.

A schematic diagram suggesting possible mechanisms in the normal and pathological bladder to account for autonomous activity and the actions of arecaidine. The concept is put forward that there are two means to activate the detrusor: motor nerves of the post-ganglioninc component of the parasympathetic system and a network of interstitial cells. The motor nerves are used to activate the micturition event while the interstitial cell network plays a role in the generation, modulation and distribution of activity which underlies phasic non-micturition activity, autonomous activity. The network of interstitial cells is complex and heterogeneous.34-35 Functionally different elements can be identified: a pacemaker and a distribution network. Activity in the pacemaker is driven by multiple inputs which include a cholinergic component. In the control animals this mechanism involves $M_{3}$ muscarinic receptors. Also, based on data from control animals, activity within the distributing interstitial cell network is increased by muscarinic stimulation. The concept proposed is that muscarinic stimulation increases the spread of activity in the network and lead to a recruitment of muscle bundles and consequently larger rises in pressure. Activity in the interstitial cell network is conveyed to the individual muscle bundles directly by a mechanism possibly involving gap junctions. The same basic functional arrangement is present in the operated animals. However, the pacemaker mechanism appears to be altered and has an increased sensitivity to muscarinic agonists. The distribution of activity is also altered in operated animals such that it is fully activated in the unstimulated bladder. In this respect the autonomous activity in the operated animals is similar to the activity produced by the nicotinic ligand lobeline. ${ }^{6}$ Interestingly, excessive activity after muscarinic agonists or during exposure to adrenergic stimulation has the effect of uncoupling the network affecting the spread of activity and so reducing the amplitude of the contractions. The hypothesis is proposed that the changes seen in bladder function following damage to the bladder neck are the result of changes to the network of interstitial cells. These changes affect two functions of the network, its pacemaker role and distribution of activity.

\section{Discussion}

There are two fundamental unanswered questions relating to the clinical conditions of urinary urge and incontinence: 'what is the nature of the clinical defect?' and 'what are the mechanisms responsible for generating this altered function?' Despite the prevalence of the conditions it is remarkable that we still have little real insight into these questions. 
One clue to the origin of the problem has come from an appreciation that anticholinergic drugs are effective in reducing the symptoms of urge and frequency. This implies that the pathological defect must have a component which is driven by a system involving muscarinic receptors. It was originally thought that the large nonvoiding contractions seen during the filling phase of clinical cystometric investigation were an underlying cause of the condition. ${ }^{9}$ However, it has become clear that neither these contractions nor the voiding contraction are affected by the anticholinergic drugs at therapeutic doses. ${ }^{10,27}$ It is therefore unlikely that the neuromuscular junction is the therapeutic site of action of these drugs. In addition, there must be other cholinergic mechanisms involved in the pathology and pharmacotherapy. Since the anticholinergic drugs reduce urge they must be working on a system involved in the generation of sensation. There has thus been a growing interest in the sensory mechanisms involving cholinergic activity.

The present data suggest a framework which has the potential to give some further insights into the causes of $O A B$ and the mode of action of anticholinergic drugs. It is clear from this study that the mechanisms generating phasic rises in pressure in the isolated bladder and their modulation by cholinergic input are altered following operations to expose or to partially occlude the bladder neck. It was argued that autonomous activity, involving local contractions and stretches of the bladder wall, and its modulation in the normal bladder, are an integral part of a motor/sensory system involved in the generation and regulation of sensation during the filling phase..$^{6,7,16}$ The observed increase in autonomous activity and altered sensitivity to cholinergic stimulation in operated groups might therefore contribute to the pathological increases in sensations of urge contributing to increases in frequency.

There can be no doubt that there are changes to bladder function in the sham operated guinea pigs and those with rings implanted around the bladder neck: in the sham group the amplitude of the autonomous activity was increased significantly even when the pressures were corrected for bladder weight (Fig. 2). Also, the sham group had a significant increase in sensitivity to cholinergic stimulation, in terms of an increase in the transient frequency (Fig. 9A). These observations argue that the stimulus triggering the functional changes is not $\mathrm{BOO}$, but rather mechanical damage to the bladder neck. The presence of 'occluding' rings might be exacerbating this local damage leading to more severe changes. Supporting this idea there is anecdotal evidence suggesting that women with accidental damage to the bladder neck develop symptoms of urge and frequency and even have mild bladder hypertrophy. It is not thought that this damage leads to an obstruction. Therefore, this condition may be similar to the sham operated animals in this study. However, the counter argument must also be considered: damage to the bladder neck may result in inflammation or swelling which lead to a partial occlusion. Thus, care must be taken in describing the trigger to the eventual pathology.

In all operated bladders there is an increase in bladder weight. This almost certainly involves muscle hypertrophy and a thickening of the lamina propria (de 
Jongh, unpublished observations). It has been generally considered that the muscle hypertrophy results as a consequence of voiding against an increased resistance and that the hypertrophy of the muscle and associated changes are the primary cause of the bladder overactivity. However, it is possible that bladder hypertrophy is secondary to an initial alteration in bladder activity. This idea is supported by the observation that, after surgical occlusion of the bladder neck, bladder activity is increased before any change in bladder weight occurs. ${ }^{28}$ Therefore, one interpretation of the present data is that a primary insult to the bladder neck causes changes in the bladder wall which result in increased intrinsic activity. One effect of this damage and resultant increased activity is muscle hypertrophy and the other gross changes seen in the bladder wall.

There are two key observations which emerge from this study.

First, there are changes to the magnitude and pattern of activity. The pressures, generated in bladders from operated animals, are greater than controls. The simple explanation for this is that the muscle mass has increased, and consequently the bladder wall is able to generate more force and so higher pressures. When changes in muscle mass are taken into account there is a significant increase in the amplitude of the contractions in the operated animals (Fig. 2Ad). This observation has two intriguing implications. First, the autonomous contractions are smaller than the maximum contraction that the bladder can generate. This is clearly the case in the sham operated bladders. One possible explanation for this increase is the recruitment of a larger number of muscle trabeculae to contribute to the autonomous contraction. The recruitment of muscle may also occur with cholinergic stimulation in the normal bladder giving rise to what was called augmented activity. ${ }^{5}$ An increased release of endogenous acetylcholine cannot be the mechanism involved in the large contractions seen in the unstimulated operated bladders since atropine does not affect the contractions. It has been speculated that the autonomous activity originates at some pacemaker site and the resultant activity is conducted throughout the wall by means of a specialised conductive network involving intra-mural ganglia and interstitial cells. ${ }^{5-7,21,29}$ The larger contractions in the operated group might thus represent a greater dispersion of activity, hence recruitment of trabeculae, by an increase in the network of interstitial cells. There is evidence for a direct role for the interstitial cell network in the generation of phasic activity in the mouse bladder ${ }^{30}$ and that the extent of the network of interstitial cells is increased in the obstructed guinea pig bladder. ${ }^{31}$ Second, if the autonomous activity is associated with the generation of sensation then these greater forces and higher pressure changes may result in a greater activation of sensory systems within the bladder wall contributing to the heightened sensations in clinical urge. It is also relevant to note that there are changes in the pattern of the autonomous activity in a significant number of operated bladders, i.e. bursts of activity followed by periods of quiescence. It has been argued above that the autonomous activity is involved in the generation of sensation. Episodes of increased activity would results in periods of increased sensation. If this was to occur in vivo and in man then there might be episodic sensations of urge 
derived from this basic activity. This pattern of activity is not affected by either tetrodotoxin or atropine. Thus the underlying mechanism does not involve voltage sensitive sodium channels or spontaneous release of acetylcholine. Notably, in some instances these bursts of activity are followed by periods of quiescence. The origins of the bursts and nature of this inhibition are not known.

The second key observation in this study is the increased sensitivity of frequency of the autonomous activity to cholinergic agonists. Significant responses are seen with approximately 10 times less agonist. At these low agonist concentrations the responses are large, so large in fact that higher doses damage the preparation. For this reason it is not possible to carry out a standard pharmacological investigation, e.g involving a full dose response curve. The operated bladders appear to be 'supersensitive' to cholinergic stimulation. A similar observation has been reported in muscle strips taken from the pig, ${ }^{32}$ which led to the idea that direct activation of the muscle was associated with the pathological condition, i.e the myogenic hypothesis. ${ }^{4}$ Using the isolated whole bladder preparation it has been argued that the autonomous activity and its regulation arise in specialised systems within the wall involving intramural ganglia and a network of interstitial cells. ${ }^{8}$ The frequency and amplitude of the autonomous activity can be augmented by cholinergic stimulation. ${ }^{5,6}$ This cholinergic modulation has the characteristics of a system which involves a pacemaker which determines the frequency of the phasic activity and a network of cells which distributes this activity. ${ }^{21}$ The greater effect of muscarinic agonists on the frequency indicates a contribution from this defect to the pathology. Thus, potentially, both the pacemaker element and the distribution component of the system underlying autonomous activity are altered in operated bladders and both may therefore be major contributing factors to the pathology. These ideas are summarised in figure 13.

It has been argued that the modulation of autonomous activity by various agents, including acetylcholine, can alter generation of sensation. ${ }^{7,8}$ It was shown that the cholinergic pacemaker function in normal bladders is extremely sensitive to M3 specific muscarinic drugs. ${ }^{21}$ The finding of an increased sensitivity to muscarinic stimulation in the operated bladders suggests an increased cholinergic component to sensation. Thus, it is possible that it is this 'supersensitive' cholinergic drive to the autonomous activity that increases sensation and that, clinically, this altered mechanism is the therapeutic target for the anticholinergic drugs. Notably, adrenergic stimulation removes the phasic activity; this might be relevant clinically, as adrenergic agonists are now being tested clinically to reduce the symptoms of urge.

It is apparent in many of the records made from the operated bladders that in the later stages of muscarinic stimulation there is a loss of activity. The mechanisms underlying this effect are not known. The loss of activity can occur in the presence of agonist (Fig. 4B). The concept proposed here is that the phasic activity is driven by a pacemaker like mechanism within an interstitial cell network. It is known that interstitial cells from other tissues of the lower urinary tract are electrically excitable 
and have the capability to generate action potentials. ${ }^{33}$ One of the properties of such excitable systems is a cessation of activity during intense stimulation. This might occur as a result of fatigue (the use and loss of an essential substrate) or inactivation of the ion channels involved in the generation of the electrical activity. The loss of activity during and following intense stimulation has many of the characteristics of fatigue or inactivation. More data on the properties of interstitial cells from bladders of operated animals may lead to a better understanding of this phenomenon.

In summary, the present observations suggest that damage to the bladder neck leads to changes in the network of interstitial cells and intra-mural ganglia. These changes result in alterations to the cholinergic sensitivity of a pacemaker in the bladder wall and the distribution of this activity within the network. As a result the frequency and magnitude of autonomous activity is increased. It was proposed that the phasic activity is associated with a motor/sensory system. The altered sensitivity and distribution of activity in bladders with bladder neck damage might contribute to pathological sensations of urge. The cholinergic component of this modulation of activity may also be the site of therapeutic action of anticholinergic drugs. Such a conceptual framework may provide insight into new approaches to understand the origins, causes and mechanisms for possible pharmacotherapy of the clinical conditions of urge, frequency and incontinence.

\section{References}

1. Milsom I, Abrams P, Cardozo L, Roberts RG, Thuroff J, Wein AJ. How widespread are the symptoms of an overactive bladder and how are they managed? A population based prevalence study. BJU Int. 2001; 87:760-6

2. Hu T-W, Wagner T, Bentkover 1, et al. Urology. 2004; 63:461-5

3. de Groat, W.C. A neurologic basis for the overactive bladder. Urology. 1997; 50(6A Suppl):3652.

4. Brading, A. F. A myogenic basis for the overactive bladder. Urology. 1997; 50(Suppl 6A):57-67

5. Gillespie JI, Harvey IJ, Drake MJ. Agonist and Nerve Induced Phasic Activity in the Isolated Whole Bladder of the Guinea Pig: Evidence for Two Types of Bladder Activity. Exp Physiol. 2003; 88:343-357

6. Drake MJ, Harvey IJ, Gillespie JI. Autonomous activity in the isolated guinea pig bladder. Exp Physiol. 2003: 88:19-30.

7. Gillespie JI. The Autonomous Bladder: A View of the Origin of Bladder Overactivity. BJU Int. $2004 ; 93: 478-83$

8. Gillespie JI. A developing view of the origins of urgency: the importance of animal models. BJU Int. 2005; 96:22-8

9. Andersson KE, Arner A. Urinary bladder contraction and relaxation: physiology and pathophysiology. Physiol Rev:2004; 84:935-86

10. Finney SM, Andersson K-E, Gillespie JI , Stewart LH. Antimuscarinic drugs in detrusor overactivity and the Overactive Bladder (OAB) Syndrome: motor or sensory actions? BJU Int. $2006 ; 98: 503-7$

11. Wiseman OJ, Fowler CJ \& Landon DN. The role of the human bladder lamina propria myofibroblast. BJU Int. 2003; 91:89-91

12. de Groat WC. The Urothelium in overactive bladder: passive bystander or active participant. Urology. 2004; 65:7-11 
13. Fry $\mathrm{CH}$, Ikeda $\mathrm{Y}$, Harvey $\mathrm{R}, \mathrm{Wu} \mathrm{C}$, Sui GP, Control of bladder function by peripheral nerves: avenues for novel drug targets. Urology. 2004; 63(3 Suppl 1):24-31.

14. Mosso MA, Pellacani P. Sur le fonctions de la vessie. Arch Ital Biol. 1882; 1:291-324

15. Sherrington C S. Notes on the arrangement of some motor fibres in the lumbo-sacral plexus. J Physiol. 1892; 13:621-772

16. Starling EH. Elements of Human Physiology. 1905, seventh edition. Churchill, London

17. Iggo A. Tension receptors in the stomach and urinary bladder. J Physiol.1955; 128:593-607

18. Vaughan CW, Satchell PM. Urine storage mechanisms. Prog Neurobiol. 1995; 46:215-37

19. Shea VK, Cal R, Crepps B, Mason JL, Perl ER. Sensory fibres of the pelvic nerve innervating the rat urinary bladder. J Neurophysiol. 2000; 84:1924-33

20. Streng $T$, Hedlund $P$, Talo A, Andersson K-E, Gillespie JI. Phasic non-micturition contractions in the bladder of the anaesthetised and awake rat. BJU Int. 2006; 97:1094-101

21. Finney S, Stewart L, Gillespie JI. Cholinergic activation of phasic activity in the isolated bladder; possible evidence for $M 3$ and $M 2$ dependent components of a motor/sensory system. BJU Int. 2007; $100(3): 668-78$.

22. Gillespie JI. Modulation of autonomous contractile activity in the isolated bladder of the guinea pig. BJU Int. 2004; 93:393-400

23. Gillespie JI. Inhibitory actions of calcitonin gene-related peptide and capsaicin: evidence for local axonal reflexes in the bladder wall. BJU Int. 2005; 95:149-56

24. Gillespie JI. Noradrenaline Inhibits Autonomous Activity in the Isolated Guinea Pig Bladder. BJU Int. 2004; 93:401-9

25. Drake MJ, Hedlund P, Harvey IJ, Pandita RK, Andersson KE, Gillespie JI, (2005), Partial outlet obstruction enhances modular autonomous activity in the isolated rat bladder. J Urol. 2005; $170: 276-9$.

26. Mostwin IL, Omer M, Karim A van Koeveringe G, Broks EL. The guinea pig as a model of gradual urethral obstruction. J Urol. 1991; 145:854-8

27. Andersson K-E, Yoshida M. Antimuscarinics and the Over Active Detrusor - Which is the main mechanism of action? Eur Urology. 2003; 43:1-5

28. Schroder A, Uvelius B, Newgreen D, Andersson KE. Bladder overactivity in mice after 1 week of outlet obstruction. Mainly afferent dysfunction? J Urol. 2003; 170:1017-21

29. Gillespie JI, M Markerink-van Ittersum M, de Vente J. Sensory collaterals, intra-mural ganglia and motor nerves in the guinea pig bladder: evidence for intramural neural circuits Cell and Tissue Res. 2006; 325:33-45.

30. Lagou M, Drake MJ, de Vente J, Markerink-van Ittersum M, Gillespie JI. The role of interstitial cells in the isolated mouse bladder. BJU Int. 2006; 98:643-50

31. de Jongh $R$, van Koeveringe GA, van Kerrebroeck PEV, Markerink-van Ittersum $M$, de Vente J, GillespieJI. Alterations to the network of NO/CGMP responsive interstitial cells induced by outlet obstruction in the guinea pig bladder. Cell Tissue Res. 2007; 330(1):147-60.

32. Sibley $G N$. The physiological response of the detrusor muscle to experimental bladder outflow obstruction in the pig. BJU Int.1987; 60:332-6

33. Sergeant GP, Thornbury KD, MCHale NG, Hollywood MA. Interstitial cells of Cajal in the urethra. J Cell Mol Med. 2006; 10:280-91

34. Gillespie JI, Markerink-van Ittersum M, de Vente J. cGMP-generating cells in the bladder wall: identification of distinct networks of interstitial cells. BJU Int. 2004; 94 (7):1114-24

35. Gillespie JI, Markerink-van Ittersum $M$, de Vente J. Interstitial cells and cholinergic signalling in the outer muscle layers of the guinea pig bladder BJU Int. 2006; 97:379-85 


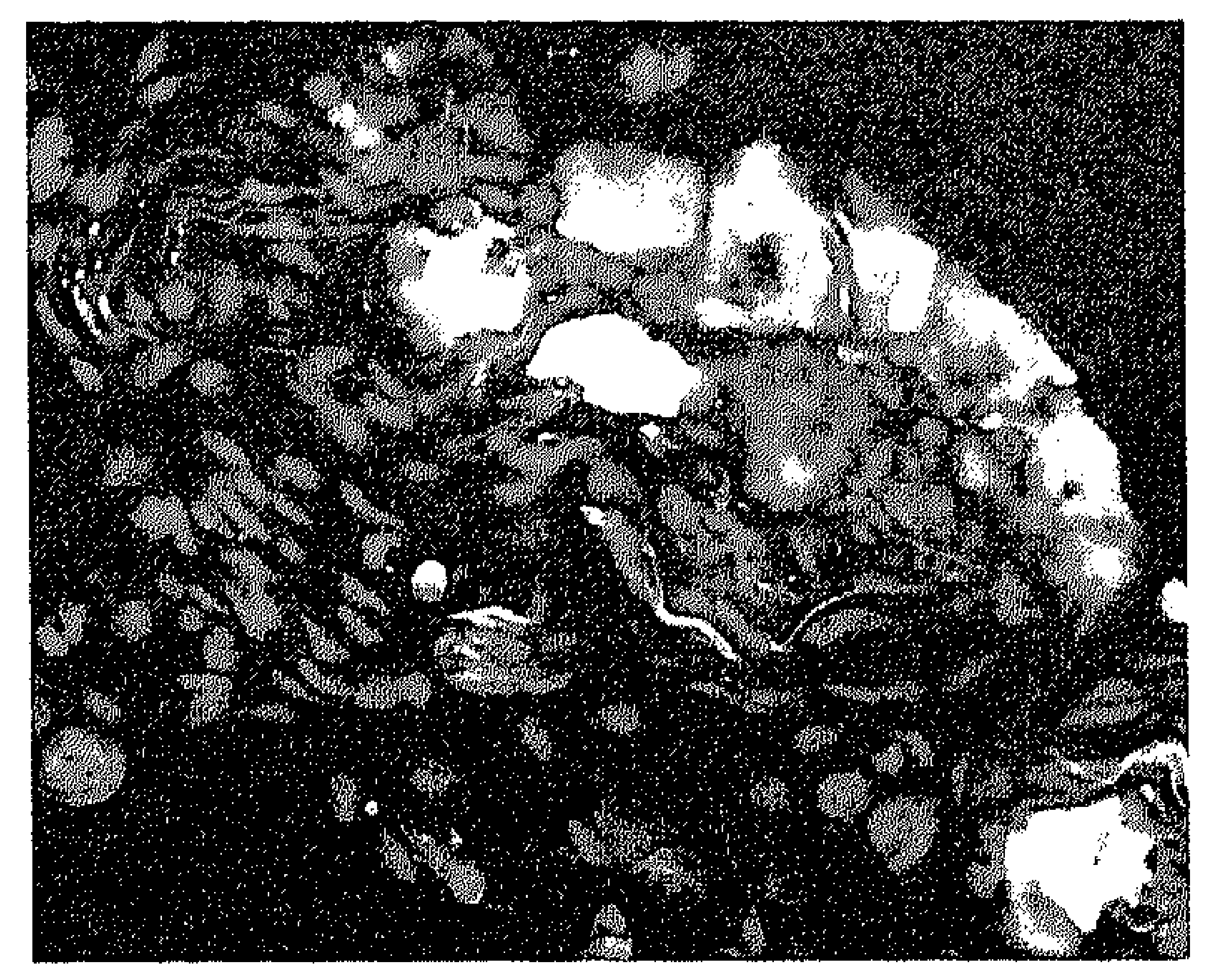

Alterations to the network of NO/cGMP responsive interstitial cells induced by outlet obstruction in the guinea pig bladder

Rik de Jongh, Gommert A. van Koeveringe, Philip E.V. van Kerrebroeck, Marjanne Markerink-van Ittersum, Jan de Vente and James I Gillespie.

Cell and Tissue Research 2007; 330 (1):147-60. 


\begin{abstract}
Interstitial cells (ICS) play a role in regulating normal bladder activity. This si explores the possibility that the sub-urothelial and muscle networks of $\mathrm{NO} / \mathrm{C}$ responsive interstitial cells are altered in animals with surgically induced out obstruction. In sham-operated animals, the urothelium compromised NO-stimuli cGMP-positive (CGMP+) umbrella cells, an intermediate layer and a basal layer stained for nNOS. cGMP+ sub-urothelial interstitial cells (su-IC) were found below urothelium. CGMP+ cells were also associated with the outer muscle layers: cell: the serosal surface, on the surface of the muscle bundles and within the mu bundles. Several differences were noted in tissues from obstructed animals: (1) number of CGMP+ umbrella cells and intensity of staining was reduced; (2) intermediate layer of the urothelium consisted of multiple cell layers; (3) the si layer was increased, with cells dispersed being throughout the lamina propria; cGMP+ cells were found within the inner muscle layer forming nodes between muscle bundles; (5) the number of cells forming the muscle coat (serosa) increased; (6) an extensive network of CGMP+ cells penetrated the muscle bund (7) cGMP+ cells surrounded the muscle bundles and nodes of ICs were appar these nodes being associated with nerve fibres; $(8)$ nerves were found in the lan propria but rarely associated with the urothelium. Thus, changes occur in networks of ICs following bladder outflow obstruction. These changes must $h$ functional consequences, some of which are discussed.
\end{abstract}




\section{Introduction}

One of the major clinical conditions associated with bladder dysfunction is an increased sensation of urge and an increased frequency of voiding. Patients who suffer from these symptoms have broadly been divided into three groups: men with prostatic enlargement resulting in an obstructed outflow (obstructed), patients with neurological conditions (neuropathic) and patients who have no identifiable cause (idiopathic). Major structural changes in the bladder wall are recognised as being associated with these different patient groups: a loss of nerve fibres, hypertrophy of the smooth muscle cells, increased expression of connective tissue elements (collagen and elastin) and alterations to the urothelium. ${ }^{1-2}$ Intriguingly, the loss of nerves and muscle hypertrophy seem to occur locally, with some normally appearing trabeculae lying adjacent to others with nerve loss and muscle hypertrophy. Despite the recognition of these structural changes, they have yet to prove helpful in advancing our understanding of the functional changes that give rise to the increased sensations of urge and increased frequency.

One hypothesis that has been put forward is that the increased sensations of urge arise from an increased afferent output from the bladder. ${ }^{3-4}$ One component of the afferent output appears to be generated by local contractile activity within the bladder wall. ${ }^{5-8}$ In the guinea pig, the intrinsic contractions (autonomous activity) have been suggested to be generated and coordinated by a specialised system in the bladder wall involving interstitial cells and intra-mural ganglia. ${ }^{9-10}$ If this concept is correct, then we can hypothesise that, in addition to the structural changes to nerve and muscle, alterations must have occurred to the network of interstitial cells. The present experiments have been carried out to explore this possibility by using an animal model of bladder outflow obstruction, the obstructed guinea pig.

\section{Materials and Methods}

The studies were performed in 14 male guinea pigs (Hartley strain) weighing between 266 - 299 grams. The guinea pigs were housed in a temperature-and light(12-h light / dark cycle) controlled room and allowed free access to food and water. The Institutional Animal Care and Use Committee of Maastricht University approved all the animal experiments conducted in this study.

\section{Surgical procedure}

In eight guinea pigs, a partial outflow obstruction was induced, whereas six animals underwent sham operation. A protocol based on the guinea pig model for gradual urethral obstruction as described by Mostwin et al. ${ }^{11}$ was used. Briefly, animals were sedated with ketamine $(40 \mathrm{mg} / \mathrm{kg})$ and xylazine $(3 \mathrm{mg} / \mathrm{kg})$ administered intraperitoneally. Once sedation had been achieved, the animals were prepared under sterile conditions. The bladder neck and urethra were exposed via a 
vertical midline abdominal incision. A silver jeweler's jump ring $(1.8 \mathrm{~mm}$ internal diameter) was placed loosely around the proximal urethra. Bladder outlet obstruction occurred during subsequent growth of the animal. The analgesic medication flunixinum (5 mg/kg s.c.) and the antibiotic gentamicin ( $5 \mathrm{mg} / \mathrm{kg}$ i.m.) were administered once postoperatively. Sham surgery was performed in an identical manner, except no ring was inserted around the urethra.

Four weeks after the operation, the weight gain in the sham operated animals was $190 \pm 51.5$ grams compared with $112 \pm 56$ grams in animals with a bladder outflow obstruction. The bladder weights of sham operated and obstructed animals were $400 \pm 73 \mathrm{mg}$ and $1568 \pm 500 \mathrm{mg}$ respectively.

After four weeks the guinea pigs were killed by cervical dislocation. Urinary bladder was removed from each animal and placed in ice-cold Kreb's solution containing: $\mathrm{NaCl}, 121.1 \mathrm{mM} ; \mathrm{KCl} 1.87 \mathrm{mM} ; \mathrm{CaCl}_{2} 1.2 \mathrm{mM} ; \mathrm{MgSO}_{4} 1.15 \mathrm{mM} ; \mathrm{NaHCO}_{3}$ $25 \mathrm{mM} ; \mathrm{KH}_{2} \mathrm{PO}_{4} 1.17 \mathrm{mM}$; glucose $11.0 \mathrm{mM}$, bubbled with $5 \% \mathrm{CO}_{2}$ and $95 \% \mathrm{O}_{2}(\mathrm{pH}$ 7.4). The procedures for isolation, stimulation with NO and detection of CGMP were as described previously. ${ }^{12}$ The lateral wall bladder was dissected into sections (approximately $20 \mathrm{~mm}^{2}$ ), each of which was maintained in $2 \mathrm{ml}$ Kreb's solution containing $1 \mathrm{mM}$ of the non-specific phosphodiesterase inhibitor isobutyl-methylxanthine (IBMX: Sigma-Aldrich) at $36^{\circ} \mathrm{C}$ for 30 minutes. Tissues were either stimulated with 100 uM of the NO donor diethylamine-NONOate (DEANO) (SigmaAldrich) or left in control medium containing only IBMX. The NO donor was prepared immediately before use, added directly to the bathing solution and incubated for 10 minutes.

\section{cGMP detection and immunohistochemistry}

The method for detecting CGMP in tissues sections was as described by de Vente et al. ${ }^{13}$ Incubations were terminated by immersing bladder pieces in ice-cold fixative solution of $4 \%$ freshly prepared depolymerised paraformaldehyde for $120 \mathrm{~min}$ at 4 ${ }^{\circ} \mathrm{C}$, followed by washes at $4{ }^{\circ} \mathrm{C}$ in $0.1 \mathrm{M}$ phosphate buffer containing $10 \%$ sucrose ( 24 $\mathrm{h}), 20 \%$ sucrose $(24 \mathrm{~h})$ and $30 \%$ sucrose $(24 \mathrm{~h})$. The tissues from the lateral wall were then snap-frozen with $\mathrm{CO}_{2}$ in Tissue-Tek O.C.T. compound to form a single block. Cryostat sections $(10 \mu \mathrm{m})$ were cut, such that each section was perpendicular to the urothelial surface and then thawed onto chrome-alumn-gelatin-coated slides for processing for immunocytochemistry. The sections were dried for $20 \mathrm{~min}$ at room temperature followed by three washes with TRIS-buffered saline (TBS; $\mathrm{pH} \mathrm{7.6),} \mathrm{and}$ thereafter incubated overnight with primary antibodies diluted in TBS containing $0.3 \%(\mathrm{~V} / \mathrm{V})$ Triton $\mathrm{X}-100(\mathrm{TBS}-\mathrm{T})$ at $4{ }^{\circ} \mathrm{C}$. To visualize CGMP, we used sheep antiformaldehyde fixed-cGMP $(1: 4000)$; the selectivity and an estimate of the detection limit of these antibodies have been described previously. ${ }^{14}$ Sections were co-stained with rabbit anti-nNOS (1 : 3000) (Diaosorin); PGP 9.5 (1: 2000) (Chemicon). Following the overnight incubation with the primary antibodies, the sections were washed in TBS, TBS-T and TBS; each wash step lasted 10 min. Sheep primary antibody was visualized using Alexa Fluor 488 donkey anti-sheep IgG $(H+L)$ 

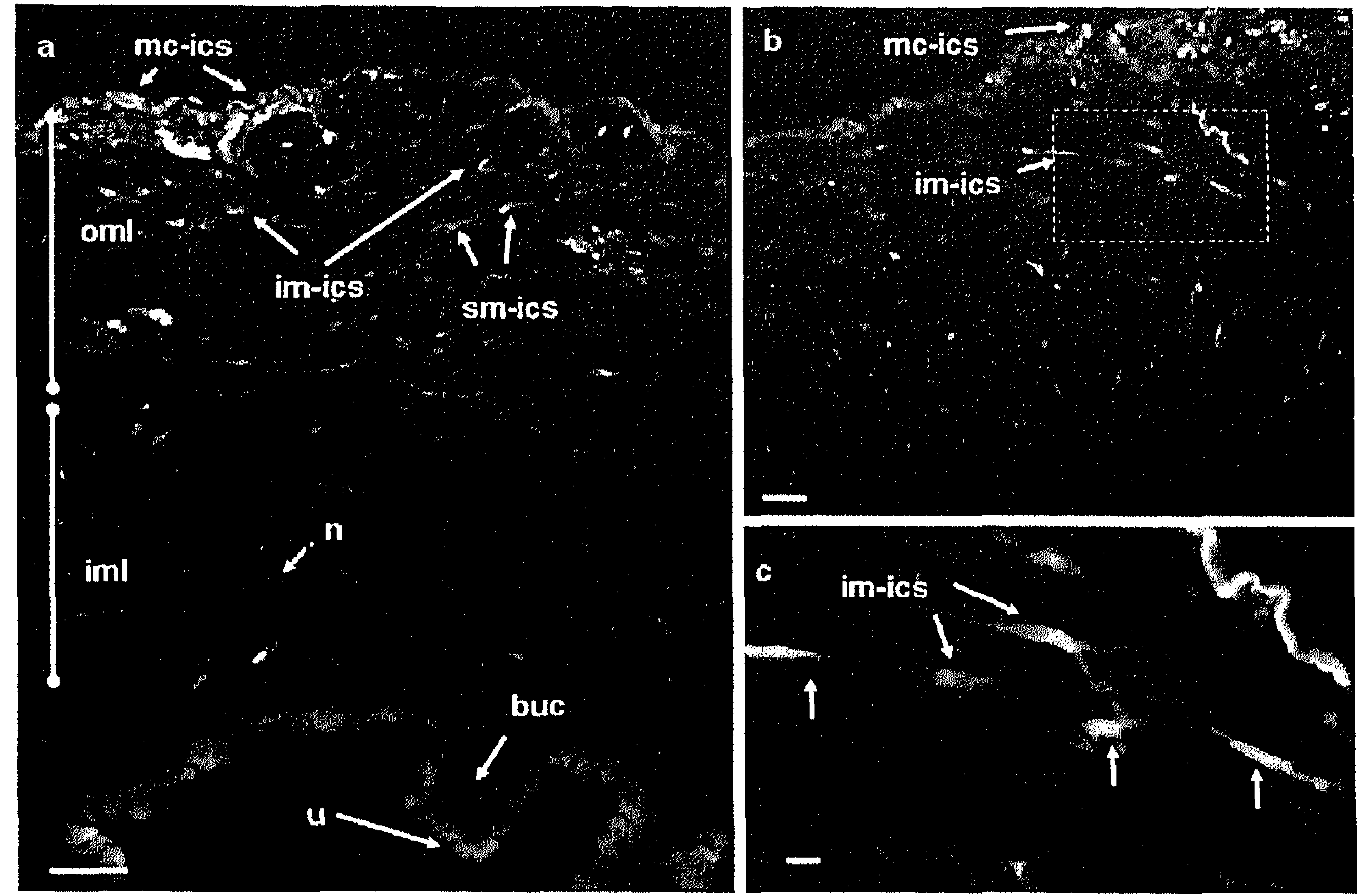

Figure 1

The basic morphology of the normal guinea pig bladder (coloured image page 201). CGMP (green) and nNOS (red) staining of a section from a sham operated guinea pig bladder. A. Entire bladder wall from the urothelium to the outer muscle coat. Umbrella cells (u) and nNOS+ basal urothelial cells (buc) can readily be seen in the urothelial layer. Cells in the muscle layers responding to exogenous NO with a rise in cGMP are also apparent. Several types of these cells (interstitial cells) can be seen: cells on the muscle coat (muscle coat interstitial cells: mc-ics), cells on the surface of muscle bundles (superficial muscle interstitial cells (sm-ics) and cells within the muscle bundles (intra-muscular interstitial cells (im-ics). An outer (oml) and inner (iml) muscle can be identified based on the distribution of CGMP+ cells. nNOS+ and CGMP+ nerves $(n)$ are found in the muscle layers and the lamina propria. B. Outer muscle layer at higher magnification, illustrating mc-ics and im-ics. $\boldsymbol{C}$. Higher magnification of the image in B showing structural details of im-ics: note the irregular cell bodies and fine processes. Calibration bars: $120 \mu \mathrm{m}$ in A. $50 \mu \mathrm{m}$ in B and $30 \mu \mathrm{m}$ in $\mathbf{C}$.

conjugate (Molecular Probes), diluted 1 : 100 in TBS-T. Rabbit primary antibodies were visualized with Alexa Fluor 594 donkey anti-rabbit IgG conjugate (Molecular Probes), diluted $1: 100$. Sections were incubated with the secondary antibodies for $60 \mathrm{~min}$ at room temperature in the dark. After being washed with TBS-T and TBS, sections were mounted with TBS-glycerol. Typically, for each bladder and for each antibody combination, staining was performed in duplicate and repeated on at least 2 separate days.

Sections were analysed and photographed by using an Olympus AX70 microscope. For the detection of Alexa 488 fluorescence, we employed a narrow band-pass MNIBA-filter and, for the detection of Alexa 594 we used a filter with a narrow excitation band, the U-M41007A filter (both filters are from Chroma Technologies). The microscope was equipped with a cooled charge-coupled device 

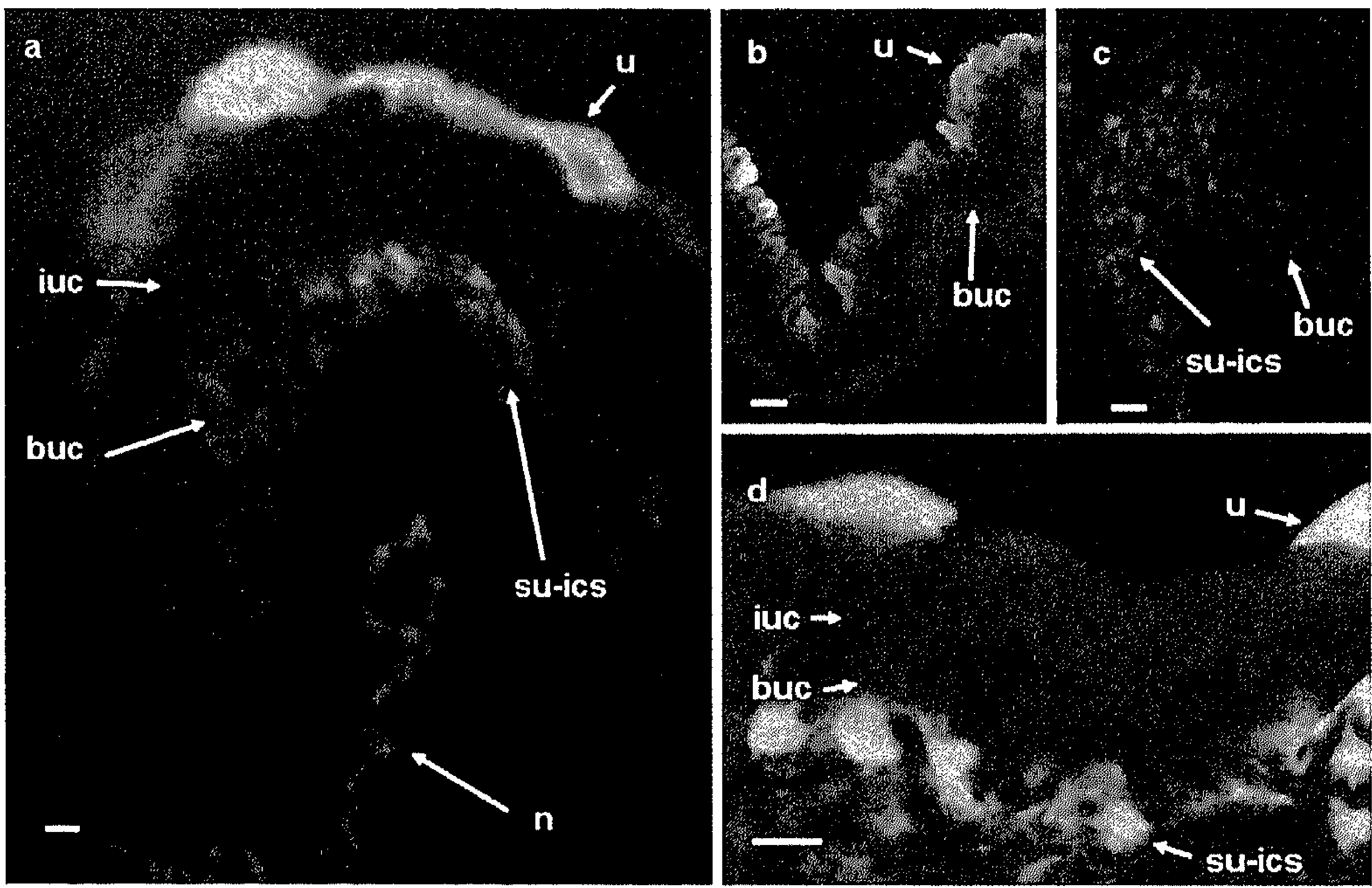

Figure 2.

The structural components of the urothelial layer in the normal guinea pig bladder (coloured image page 201). A-D Features of the urothelium seen in the lateral wall. A-C Staining for CGMP (green) and nNOS (red). A Umbrella cells (u) respond to exogenous NO with a rise in cGMP. The basal urothelial cell layer is positive for nNOS (red: buc). The intermediate urothelial cell layer (iuc) does not stain for cGMP or nNOS. An nNOS+ nerve fibre ( $n$ ) is seen in the lamina propria. B A region in which umbrella cells stain for CGMP. Note the absence of CGMP+sub-urothelial interstitial cells in this section. $C$ Sub-urothelial interstitial cells (su-ics). D Image stained only for CGMP after enhancement to demonstrate the intermediate urothelial cells (iuc), which do not stain with CGMP or nNOS. Thus, the signal is considered as background, although it serves to illustrate the morphology and number of cells in this layer in sham operated bladders. Calibration bars: $80 \mu \mathrm{m}$ in $\mathbf{A}, 30 \mu \mathrm{m} \mathbf{B}-\mathbf{C}$ and $20 \mu \mathrm{m}$ in $\mathbf{D}$.

Olympus Digital video camera F-view. Images were stored digitally as 16 bits images by using the computer program analySIS ${ }^{\circledR}$ Vers.3.0. (Soft Imaging System, Münster, Germany). The number of grey values was reduced by using a linear function into 4095. Images were arranged with the program Adobe Photoshop 5.5 or 7.0.1 (San Jose, $C A, U S A$ ) without further processing unless indicated otherwise.

\section{Results}

\section{Sham operated animals}

Figure 1 illustrates the different types of cells that respond to exogenous NO with a rise in CGMP in the bladder wall of sham operated animals. The full thickness section of the bladder wall stained for CGMP (green) and nNOS (red) reveals the 
presence of cGMP positive (cGMP+) cells associated with the outer muscle layers (Fig. 1a). Diverse populations of cells can be discerned: cells on the outer surface of the bladder (muscle coat interstitial cells (mc-ics), cells on the surface of the muscle bundles (surface muscle interstitial cells (sm-ics) and cells within the muscle bundles (intra-muscular interstitial cells (im-ics). Examples of im-ics are shown figure $1 \mathrm{c}$ at higher magnification. The umbrella cells, on the luminal surface of the urothelium, also respond to NO with a rise in CGMP. The nNOS positive layer of basal urothelial cells can also be seen. Figure 2 illustrates, in more detail, the components of the urothelium. cGMP+ umbrella cells lie on the luminal surface. The urothelium is not completely covered with umbrella cells and areas are found that completely lack umbrella cells (Figs. $2 c-d$ ). The cells of the intermediate urothelial cell layer do not stain with CGMP or nNOS. However, the general structure of these cells can be seen in figure $2 d$ in which the fluorescence of an image has been enhanced. The epithelium is comprised of large cells forming three to four cell layers.
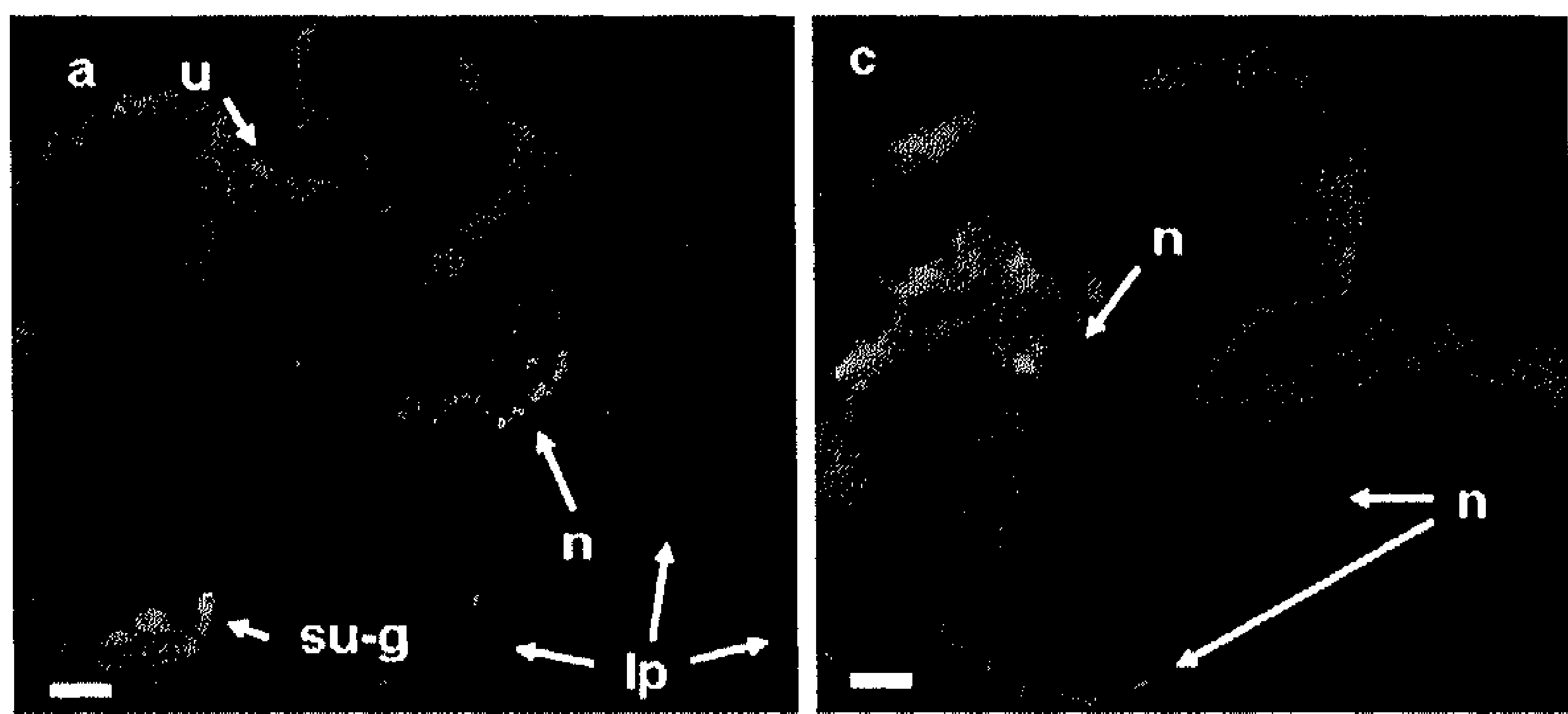

Figure 3.
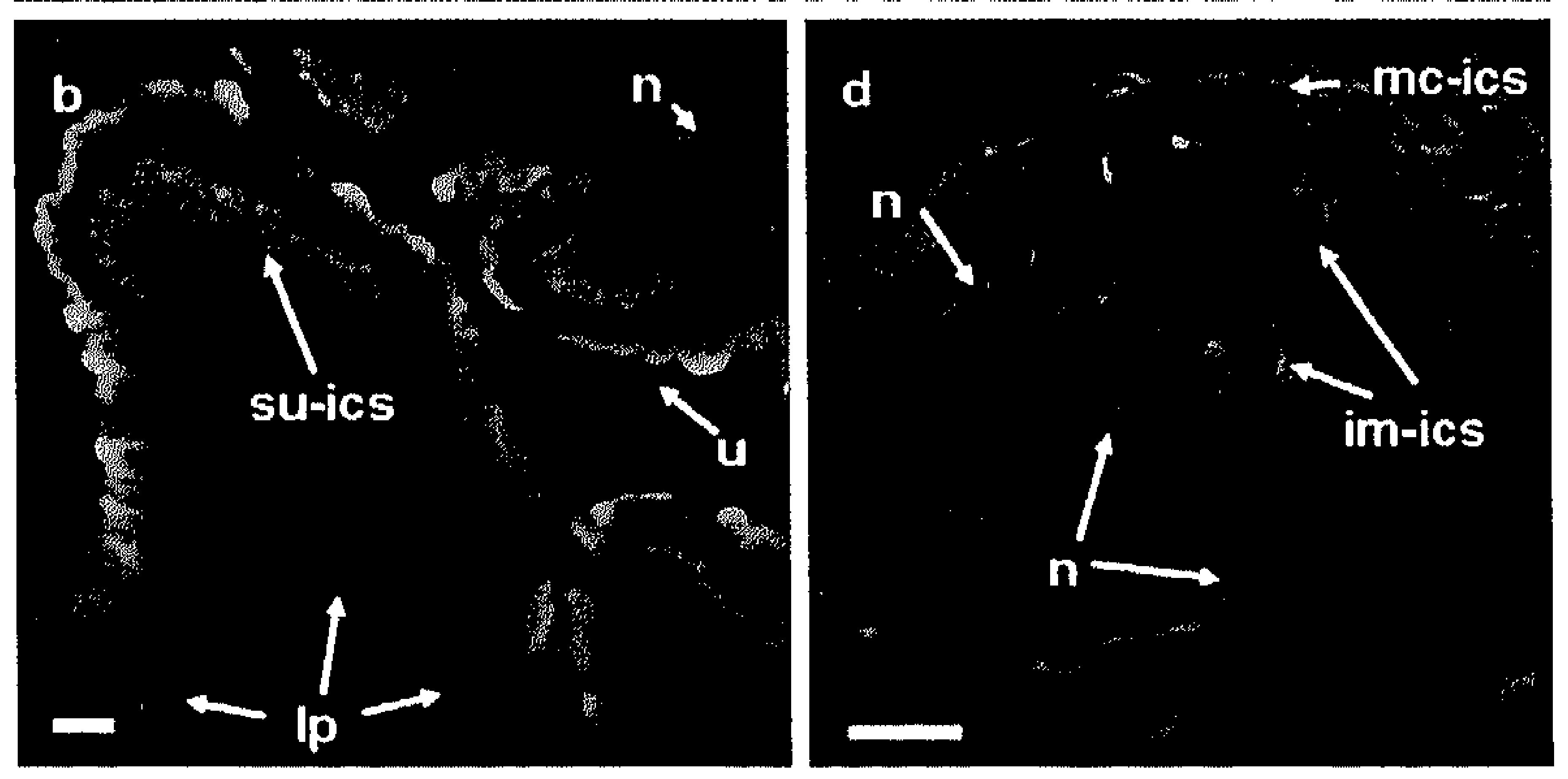

Distribution of nerves within the normal guinea pig bladder (coloured image page 202). A-C Sections taken from urothelial and sub-urothelial layers of the bladder and stained for CGMP (green) and the non-specific nerve marker PGP 9.5 (red). A Sub-urothelial ganglion (su-g) lying in the lamina propria (Ip). Nerve bundles $(n)$ also occur in the lamina propria. $B, C$ Nerves are readily found in the region immediately below the urothelium and the su-ics. In B, no CGMP+ cells are present in the lamina propria. $D$ Section from the outer muscle layer. Note the CGMP+ interstitial cells (green) and PGP $9.5^{+}$nerve fibres (red). Superficial muscle CGMP+ interstitial cells are present in addition to CGMP+ cells within the muscle bundles (im-ics). Cells of the muscle coat are also visible (mc-ics). Calibration bars: $60 \mu \mathrm{m}$ in $\boldsymbol{A}$ and $20 \mu \mathrm{m} \mathrm{B,} \boldsymbol{C}$ and $\boldsymbol{D}$. 

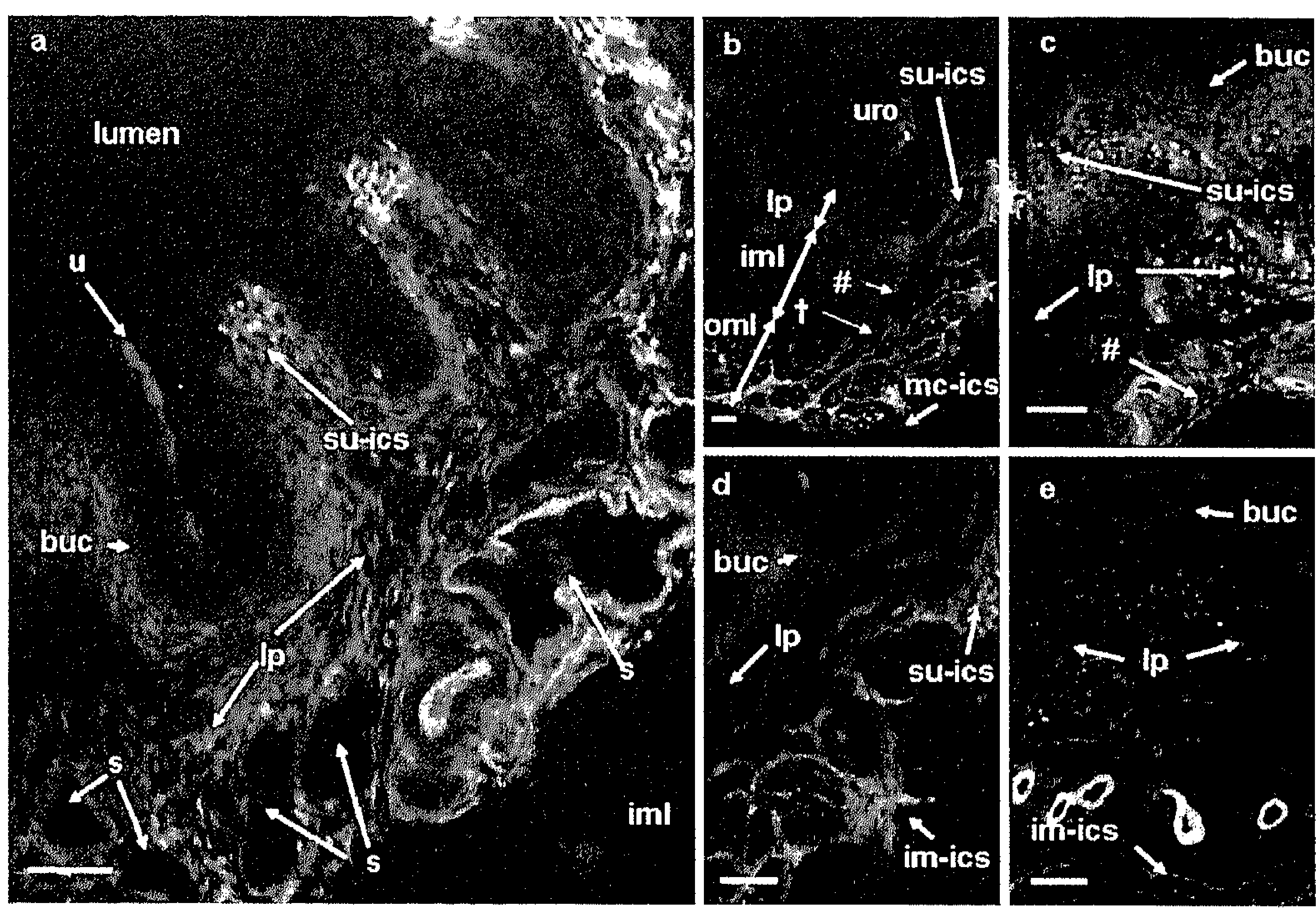

Figure 4.

Changes in the lamina propria of obstructed guinea pig bladder (coloured image page 202). Staining for CGMP (green) and nNOS (red). A. Region of the lateral wall in which the urothelium is folded. Note the various layers of the urothelium: the umbrella cells $(u)$ and the basal urothelial cell layer (buc). cGMP+ sub-urothelial interstitial cells are clearly visible (su-ics). The umbrella cells (u) are not strongly cGMP+ (compare staining in sham operated animals; Figs 1-3). cGMP+ cells are now seen in the lamina propria (Ip). No cGMP+ cells occur in the inner muscle cell layer ( $(\mathrm{ml})$ in this section. Large spaces (s) can be observed that do not appear to be major blood vessels and contain no cells. B. Low power image of a region in which CGMP+ cells appears to run from the sub-urothelial cell layer (uro) through the lamina propria (Ip) between the inner muscle layer (iml) (\#) into the outer muscle layers (oml). This structure was seen in five out of eight bladders studied (see $\boldsymbol{C}$ and $\boldsymbol{D}$ ). No cells lie in the lamina propria or within the inner muscle layer adjacent to this CGMP cell bridge. $C$. The same region in a different obstructed bladder. One area processes a dense population of su-ics. In an other area, cells in the lamina propria are CGMP+, linking to cells in the inner muscle layer (\#). D illustrates a region in a different bladder where CGMP+ positive cells are seen to link between the sub-urothelial space, the lamina propria and the inner muscle layer. $\boldsymbol{E}$. A region of sparse CGMP+ cells in the lamina propria but clear CGMP cells in the inner muscle layer. Calibration bars: $100 \mu \mathrm{m}$ in $\boldsymbol{A}, \boldsymbol{C}, \boldsymbol{D}$ and $\boldsymbol{E}$ and $50 \mu \mathrm{m}$ in $\boldsymbol{B}$.

A cGMP-responsive tightly packed multi-cellular layer can be seen in the region of the lateral wall, just below the urothelium (Figs. 2a, c-d). These cells are heterogeneous and have been described as sub-urothelial interstitial cells (su-ICs). ${ }^{12}$ The su-ICs do not extend into the deeper parts of the lamina propria (Figs. 2a-c).

The localisation of nerve fibres in the bladder wall of sham operated animals is demonstrated in figure 3 , which shows sections double stained for CGMP (green) and the non-specific neuronal marker PGP 9.5 (red). In the low power image (Fig. 3a), 

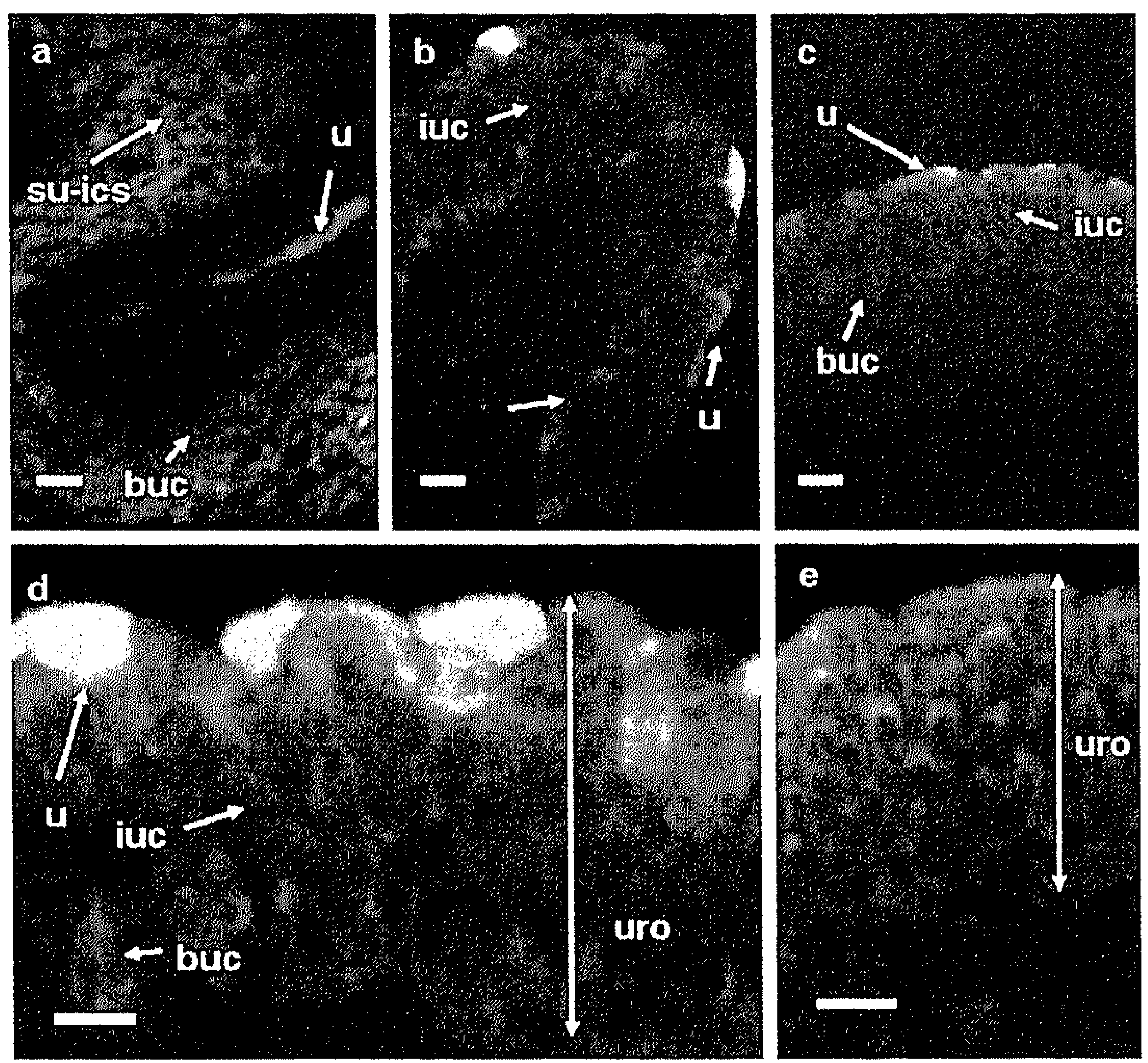

Figure 5.

Urothelium of bladders from obstructed guinea pigs (coloured image page 203). A-C Images of the urothelium of the lateral wall stained for CGMP (green) and nNOS (red) demonstrating the variations in structure. All regions exhibit a single layer of nNOS positive basal urothelial cells (buc). A. Region in which the sub-urothelial cells are CGMP+. B, C Regions in which these cells are absent. In all regions the umbrella cells are weekly positive for CGMP and sections of the urothelium can be found where the umbrella cells appear to be absent or poorly defined. The intermediate urothelial cell layer appears to be altered compared with normal bladders. $\boldsymbol{D}$ Section of the same region of the bladder as that in $\boldsymbol{C}$ but at higher magnification. Note the lower organisation of the intermediate urothelial cell layer. The number of cell layers seems to have increased. This is better illustrated in $\boldsymbol{E}$, which shows an image stained only for CGMP and in the recorded fluorescence intensity has been enhanced: the image is therefore primarily non-specific fluorescence. However, it shows the alteration in the number of cells forming the intermediate urothelial cell layer. Compare this image with that in Fig. 2D. Calibration bars: $60 \mu \mathrm{m}$ in $\mathbf{A}, \boldsymbol{B}$ and $\mathbf{C}, 20 \mu \mathrm{m}$ in $\boldsymbol{D}$ and $15 \mu \mathrm{m}$ in E.

the presence of a ganglion is revealed in the sub-urothelial space and a nerve trunk. Nerves are seen to innervate the inner muscle bundles. An image from a different bladder is presented in figure $3 \mathrm{~b}$ in which nerves lie immediately below the urothelium and run within the lamina propria. Of note, the lamina propria does not have CGMP+ interstitial cells. At higher magnification (Fig. 3c), nerve fibres are seen to pass through the su-IC layer and to run in the space between the urothelium and the su-ic layer. Figure $3 \mathrm{~d}$ shows an image of the outer muscle bundles, which contain cGMP+ cells and nerve profiles (within all bundles). 


\section{Obstructed animals \\ Urothelium}

In the obstructed animals, distinct changes were apparent in the urothelium, lamina propria, inner muscle layer, outer muscle layer and the muscle coat. A general observation was that the urothelium demonstrated less folding than in the sham operated animals, although folded areas still occurred (Fig. 4a). Regions could readily be found in which the urothelium formed a flat smooth surface (Figs. 4c, e, 5c-e). The umbrella cells did not appear to be as numerous or as distinct compared to the sham operated animals (Figs. $4 a, 5 a-c$ ). The intermediate layer of the urothelium was also modified, now consisting of several cell layers (four-eight) of small cells (compare the control intermediate urothelial layer in figure $2 \mathrm{~d}$ to that in the obstructed bladder in figure $5 \mathrm{e}$ ). nNOS+ cells forming the basal urothelial cell layer were present in the obstructed bladders (Figs. $4 a, c-e, 5 a-d$ ). There were no obvious differences in these cells between the control and obstructed bladders.

\section{The sub-urothelial layer}

In the lateral wall of the guinea pig bladders, the sub-urothelial layer consists of a layer of NO-responsive CGMP+ cells. A few obstructed bladders (three out of eight bladders studied) contained sparse CGMP+ cells in the lamina propria. However, in other obstructed bladders, the sub-urothelial layer increased and, in some regions, cells were dispersed throughout the lamina propria. cGMP+ cells in these bladders

\section{Figure 6.}
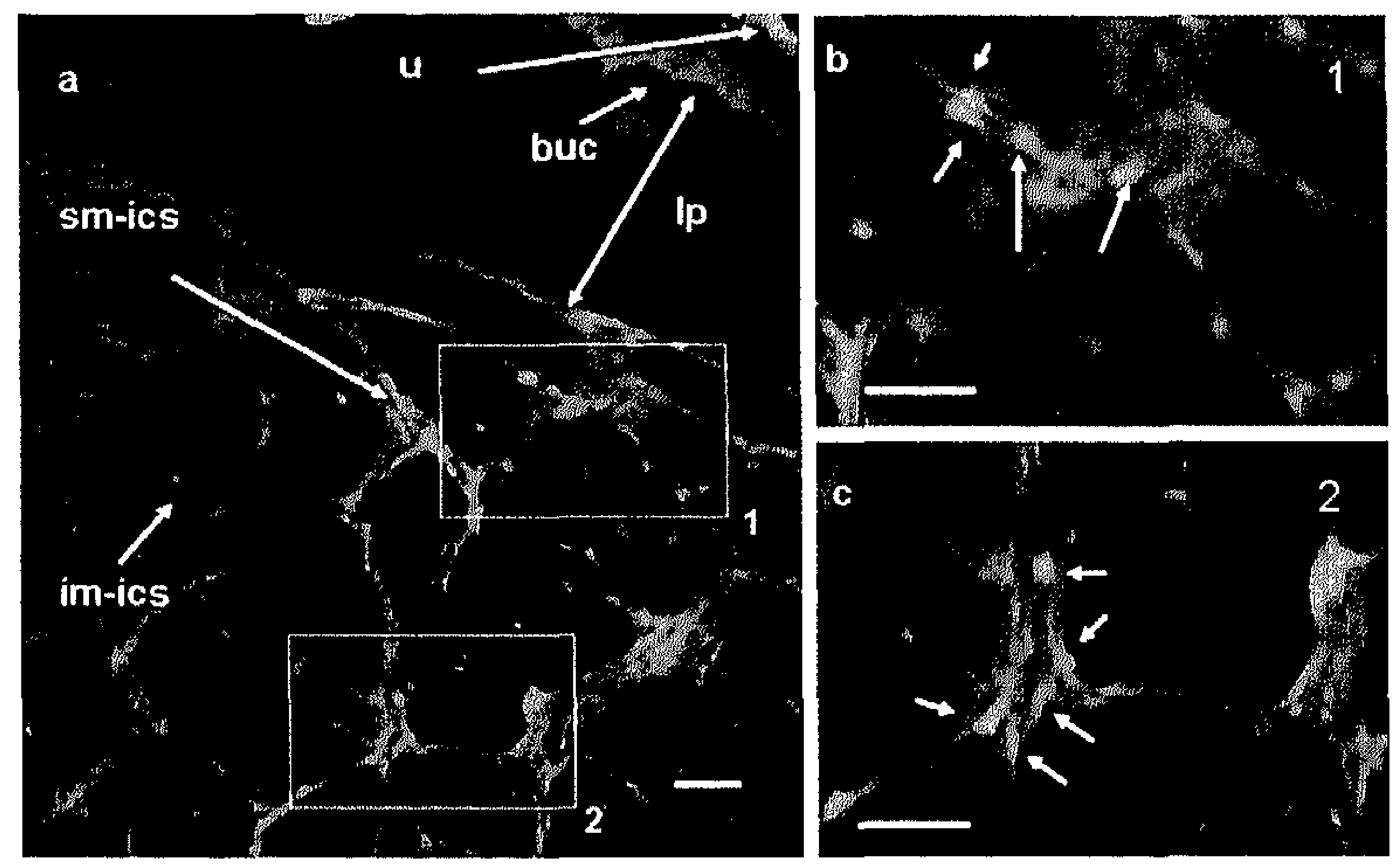

CGMP+ cells surrounding the inner muscle bundles in the obstructed bladder (coloured image page 203). A Region in which CGMP+ cells can be seen to run over the surface of the muscle bundles (smics) and also within the muscle bundles (im-ics). In this region no cGMP+ cells are seen in the lamina propria (Ip). Note the CGMP+ umbrella cell (green, $u$ ) and the nNOS+ basal urothelial cell layer (buc, red). A striking feature of the CGMP+ cells in the inner muscle layer is the appearance of small groups of these cells lying between the muscle bundles: the so called interstitial cell 'nodes'. Two regions of this image are identified in the rectangles and are illustrated at higher magnification in $\boldsymbol{B}$ and $\boldsymbol{C}$. The cell bodies of individual cells comprising the nodes are clearly seen (arrows). Calibration bars: $80 \mu \mathrm{m}$ in $\boldsymbol{A}$ and $50 \mu \mathrm{m}$ in $\boldsymbol{B}$ and $\boldsymbol{C}$. 

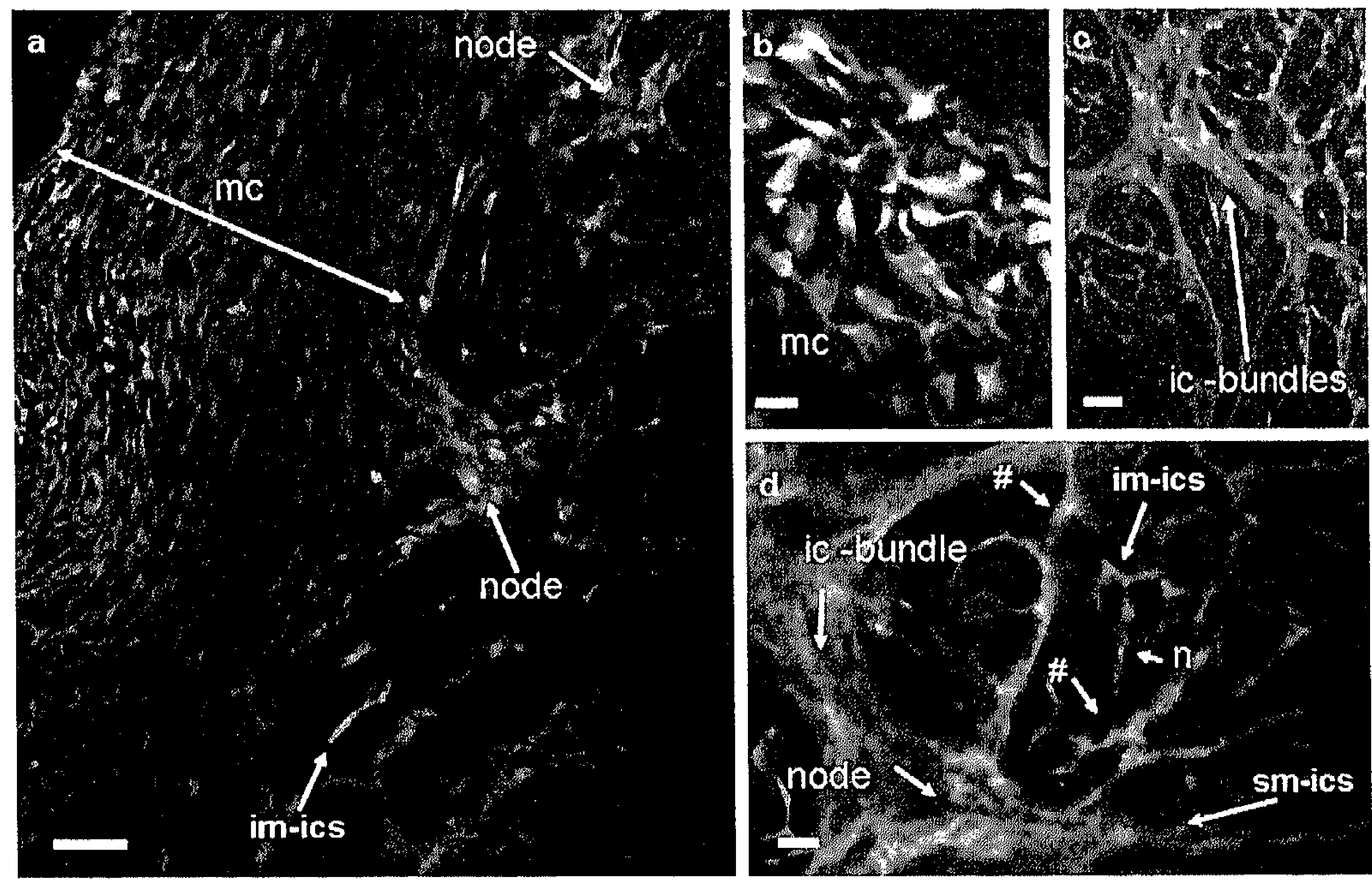

Figure 7.

cGMP+ structures on the outer surface of the bladders from obstructed animals (coloured image page 204). All images were double stained for CGMP (green) and nNOS (red). A Thickening of the outer muscle coat $(\mathrm{mc})$. Bundles of cells appear to leave the muscle coat and penetrate into the outer muscle between the muscle bundles (see also Fig 4 B). Nodes in CGMP+ cells form part of these penetrating bundles. Note the CGMP+intra-muscle interstitial cells (im-ics). B Higher magnification of the morphology of the cells in the muscle coat. $C$ Particularly pronounced bundle of CGMP+ cells penetrating the outer muscle layer: interstitial cell bundle (ic-bundles). $\boldsymbol{D}$ Region of the image in $\boldsymbol{C}$ at higher magnification. A node is clearly seen (arrow). CGMP+ cells run on the surface of the bundle and processes make contact with CGMP+ cells within the muscle bundle (\#). A small number of nNOS+ nerves are present (n). Calibration bars: $100 \mu \mathrm{m}$ in $\mathbf{A}$ and C, $15 \mu \mathrm{m}$ in $\mathbf{B}$ and $25 \mu \mathrm{m} \mathbf{C}$.

extended into the bulk of the lamina propria, surround the major blood vessels and abutting onto the inner muscle cell layer (Figs. 4a, C, e). In some bladders, these cells extended into the inner muscle cell layer (Figs. $4 \mathrm{~b}-\mathrm{d}$ ). This invasion of the inner muscle layer did not occur throughout the bladder wall but was located in the areas in which the su-ICs were present in the lamina propria. (See low power image of this general structure in figure $4 b$, with the sub-urothelial cells linking to cells in the inner muscle layer and continuing into the outer muscle layer). Thus, there was now a network of cGMP+ cells extending from the urothelium, through the inner muscle cells, and making contact with the network of cells of the outer muscle layer. The detection of CGMP+ cells associated with the inner layers of muscle is never seen in control ${ }^{12}$ or sham operated animals (Fig. 1a).

Within such regions of the inner muscle, the CGMP+ cells lay on the surface of the muscle bundles and were thus similar to the cells on the surface of muscle bundles 

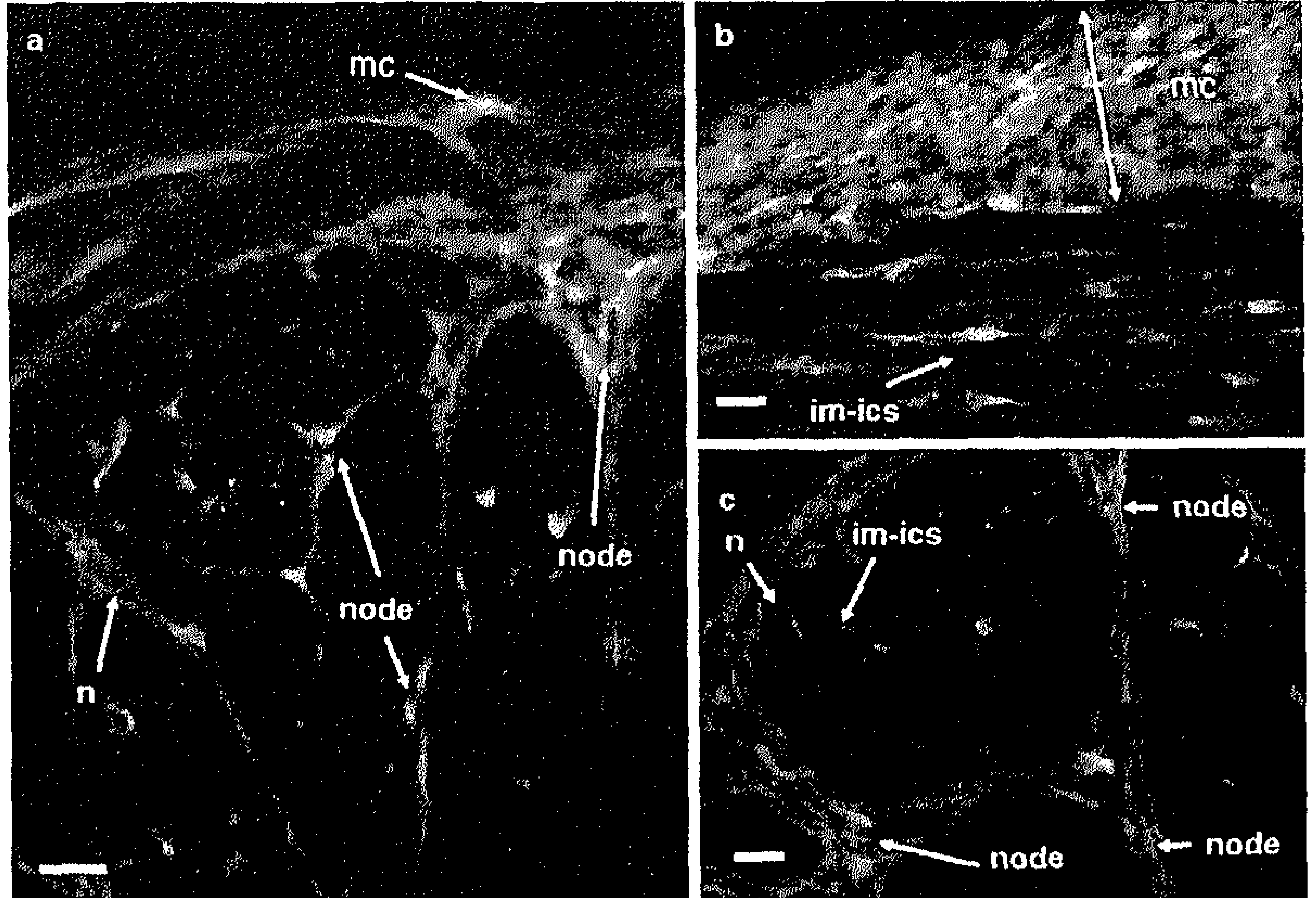

Figure 8.

cGMP+ cells associated with the outer muscle bundles in the obstructed bladder (coloured image page 204). A Outer muscle layer of an area within the lateral wall. Section was stained for CGMP (green) and nNOS (red). Only 1 nNOS structure (nerve) was seen. No nNOS+ nerves are seen within the muscle bundles. Collections of CGMP+interstitial cells, 'nodes', are present. CGMP+ cells also lie over the surface of the muscle bundles. B Section of the outer bladder wall showing the thickened muscle coat and a number of intra-muscle CGMP+interstitial cells. $C$ Area in a different obstructed bladder showing the presence of nodes and the near absence of nNOS+ nerve fibres. Calibration bars: $50 \mu \mathrm{m}$ in $\boldsymbol{A}$ and $40 \mu \mathrm{m}$ in $\boldsymbol{B}$ and $\boldsymbol{C}$.

Figure 9.
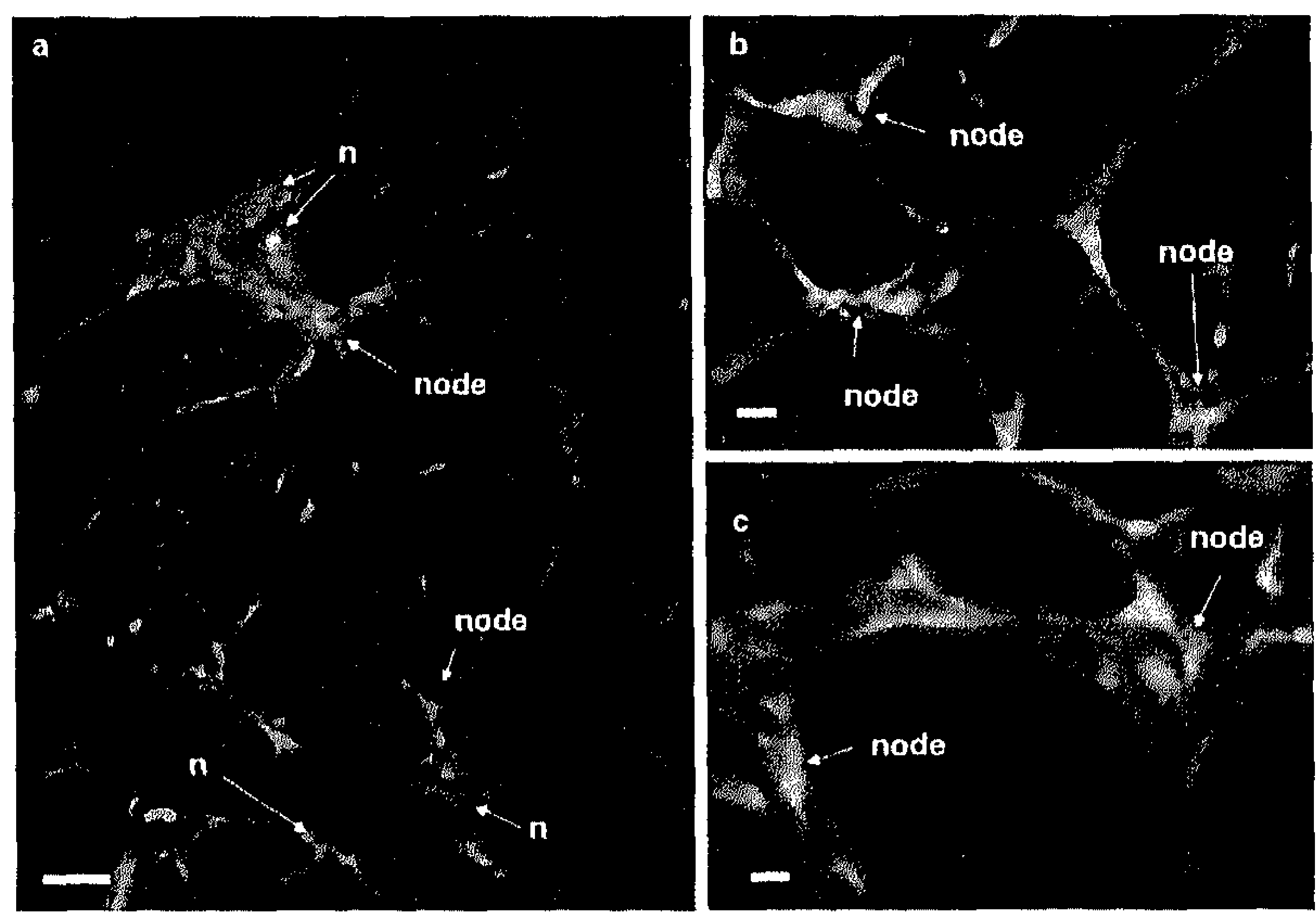

CGMP+cell nodes in the outer muscle layer of the obstructed bladder (coloured image page 205). A Section stained for CGMP (green) and nNOS (red). Nodes of CGMP+cells are clearly seen. Single nNOS+ nerve fibres $(n)$ are associated with the nodes, $\mathbf{B}, \mathbf{C}$ Nodes of $C G M P+$ cells at higher magnification. Calibration bars: $60 \mu \mathrm{m}$ in $\boldsymbol{A}$ and $15 \mu \mathrm{m}$ in $\boldsymbol{B}$ and $\boldsymbol{C}$. 

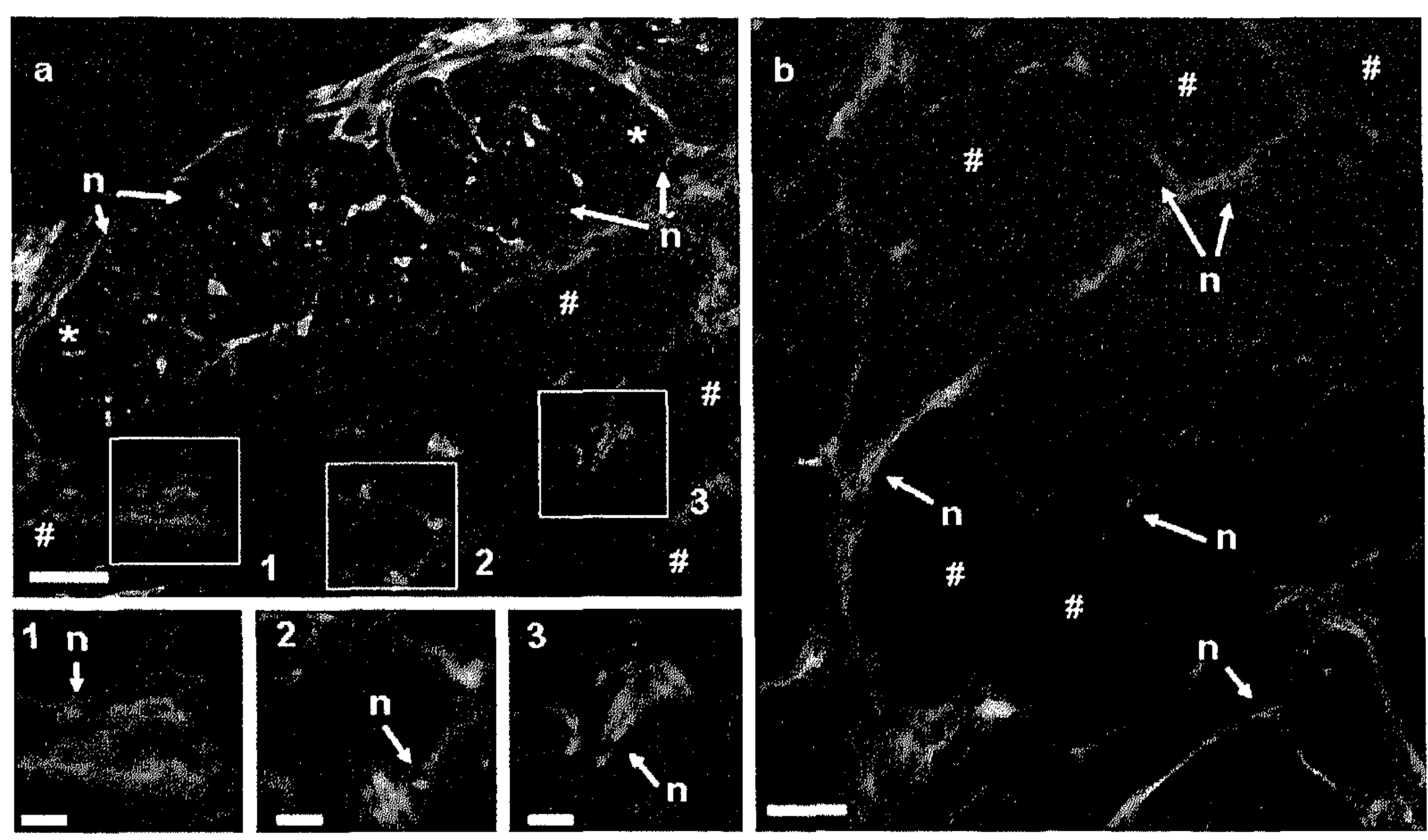

\section{Figure 10.}

Nerves in the outer muscle layers of obstructed guinea pig bladders (coloured image page 205). A Section of the bladder from the outer muscle layer of an obstructed animal. Stained for CGMP (green) and the non-specific nerve marker PGP 9.5 (red). Several muscle bundles can be identified. Two bundles (*) contain numerous nerve profiles (n) whereas four others (\#) appear to have only a sparse innervation. Note the presence of PGP 9.5+ nerves associated with the nodes of CGMP+ interstitial cells. Three examples of this (1-3) are marked by the rectangles and these areas are shown at higher magnification below. B Section from a different obstructed bladder, showing the near absence of nerve profiles within the muscle bundles but the residual appearance of nerves $(n)$ running between the bundles and associated with the nodes of interstitial cells. Calibration bar: $80 \mu \mathrm{m}$ in $A$ and $15 \mu \mathrm{m}$ in each of the magnified areas. In B the calibration bar is 40 $\mu m$.

in control tissue (Fig. 6a). cGMP+ cells were also found within the inner muscle bundles of the obstructed bladders. An unusual feature of this new network of inner muscle cells on the surface of muscle bundles was that small clumps of cells are apparent giving the appearance of 'nodes' (Figs. $6 \mathrm{~b}-\mathrm{c}$ ). These structures were not found in control bladders.

The muscle coat (serosa) and outer layers of the bladder

From Fig $4 a$, we can see that the cGMP+ cells associated with the outer layer of the bladder are altered in the obstructed bladders. The muscle coat or serosal layer has thickened in some regions and this structure can be up to 20 cells thick (Figs. $7 a, 8 b)$. The cells in such regions are shown at higher magnification in figure $7 b$. Bundles of CGMP+ cells leave the extended muscle coat and pass between the outer muscle bundles. Figure $7 c$ shows a particularly exaggerated example of this. Small groups of cGMP+ cells, similar to the nodes described in the inner muscle layers, are seen within these bundles (Figs. 7a, d, 8a, c, 9, 10). 

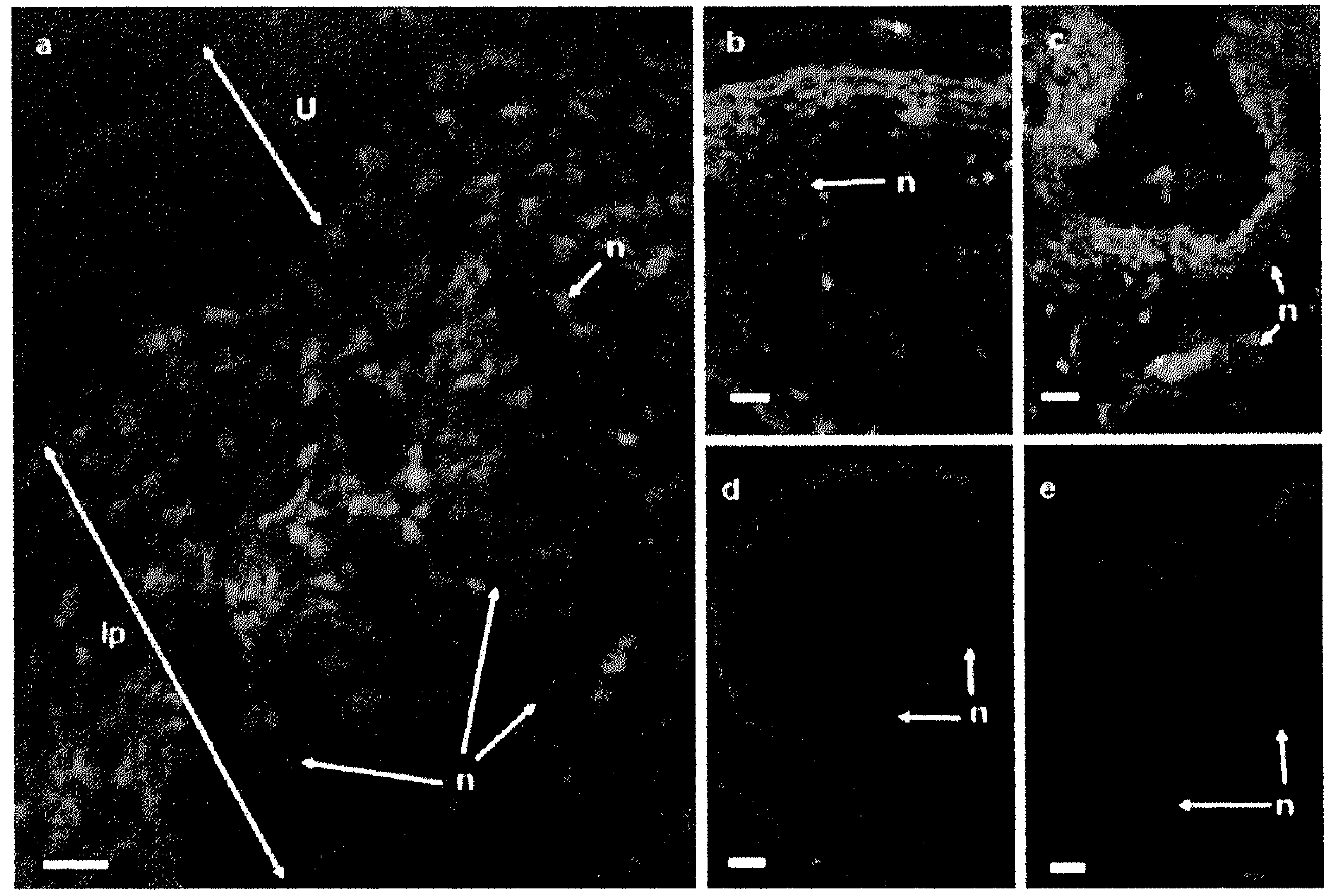

Figure 11

Nerve profiles in the sub-urothelial laver of bladders from obstructed guinea pigs (coloured image page 206). All sections are stained for CGMP (green) and the non-spectic nerve marker PGP 9.5 (red). In A, the urothelum (u) and lamina propria ( $(p)$ are readily identified, cGMP. cells fill the lamina propria. Single nerve fibres run within the tayer of CGMP+ calls of the lamina proptia. In contrast to sham operated bladders and control biadders (see Fig. 3 i no nerve fibres are associated with the urothelium or sub-urothelial cell laver. B, C Images from a different bladder in which, in a similar region to that illustrated in $\boldsymbol{A}$, nerve profiles are seen in the lamina propria but not assoctated with the urothelium. D. E Sections of the lateral wall in a region devoid of suburothelial interstital celis. Nerve profiles are also seen here within the lamina propria but are not associated with the urotheium. Bars $80 \mathrm{\mu m}$

cGMP+ cells are found within the muscle bundles (Figs. 8, 9, 10). In the obstructed bladders, processes of the cells on the surface of the muscle bundles were commonly found extending into the bundles to make contact with the intra-muscle interstitial cells (Fig. 7d).

Double-labelling (Figs. 7, 8, 9) showed structures expressing CGMP (green) and nNOS (red). In control bladders, nNOS is located with the nerve fibres running within the muscle bundles. In the obstructed animals, few nNOS+ fibres are seen within the muscle layers. However, significant but sparse nNOS+ innervation was associated with the CGMP+ nodes of interstitial cells (Figs. 7d, 8a, c, 9a).

Figure 10 shows sections of obstructed bladders double stained with CGMP (green) and PGP 9.5 (red). The network of CGMP+ cells and the nodes of positive cells can be seen in these images. PGP 9.5 appears to identify more nerve profiles than nNOS. Figure 10a demonstrates several features of the innervation of the outer 
muscle layers. First, some muscle bundles are well innervated, whereas others have few or no nerves. Such patchy denervation has been noted previously. ${ }^{1-2} A$ further observation is that the nodes of cGMP+ cells appear to be associated with PGP $9.5^{+}$ fibres (see the high magnification panels of Fig. 10a).

\section{Nerves in the lamina propria}

PGP $9.5^{+}$nerve profiles associated with the urothelium and lamina propria are readily found (Fig. 11). Quantitatively determination of the relative density of nerves in control and obstructed bladders is difficult, since the density of nerves varies markedly in different regions of the lateral wall of the normal bladder. However, examination of several slides from different bladders gives the strong impression that more nerve profiles occur in the lamina propria of the obstructed animais compared to sham operated bladders. However, consistent observations have been that these nerve profiles remain in the lamina propria and few fibres run in the layer between the basal urothelium and the interstitial cell layer. Thus, few nerves may make contact with the urothelium in these obstructed bladders.

\section{Discussion}

The data presented here demonstrate structural changes in bladders following surgical restriction of the bladder neck. The observations reveal significant changes within the networks of NO-responsive cells. The major changes are to the urothelium (umbrella cells and intermediate urothelial cells), the network of CGMP+ cells in the lamina propria and networks of CGMP+ cells within the muscle layers. These changes are wide spread and encompass different cell types. This suggests that more than one functional system within the bladder wall is affected. The bladder is now emerging as a complex organ. It has many different cell types and neural networks that are involved in various complex functions. For example, the urothelium is not only a barrier, but also sub-serves sensory functions. ${ }^{15-18}$ Similarly, contractile activity in the muscle is not only generated during voiding but also underlies phasic activity during the filling phase, a mechanisms linked with the generation of sensation..$^{5-8,19}$ Therefore, the observed changes to the NO-responsive CGMP+ dependent structural components in urothelium and muscle suggest possible functional changes within these systems.

The urothelium is clearly modified in these obstructed bladders. The umbrella cells are less responsive to NO and exhibit a reduction in their staining for CGMP. The cells of the intermediate and basal urothelium are smaller and an increase occurs in the number of cell layers. The trigger for these morphological changes in the urothelium remains unknown but the altered structure almost certainly reflects altered function. The urothelium of the normal bladder is known to synthesise and release a number of different bio-active substances: nitric oxide, ${ }^{20}$ ATP, $^{21}$ prostaglandins $^{22}$ and acetylcholine. ${ }^{23}$ These agents are thought to influence sub- 
urothelial nerves to alter afferent discharge and, hence, bladder sensation. ${ }^{17-18}$ Thus, the alteration to urothelial structure may reflect alterations in the ability of the urothelium to synthesis such agents resulting in an alteration in the influence of the urothelium on afferent nerve discharge and sensation. In addition, sensory nerves may be redistributed away from the urothelium (see below), which may also influence the operation of this urothelial sensory system.

Less is known about the physiological role of the interstitial cells in the suburothelium and within the lamina propria. The proximity of a population of cells, the myofibroblasts, in the lamina propria to afferent nerve fibres has led to speculation that these cells are involved in the generation of bladder sensation. ${ }^{24-26}$ Excitation of the myofibroblasts by either stretch or via substances released from the urothelium leads to contraction and mechanical distortion of afferent nerves. At this time, no direct experimental evidence supports such a physiological mechanism.

There is now a growing interest in the possible role of interstitial cells within the muscle layers and in the lamina propria. Interstitial cells were first described in the human and guinea pig urinary bladder by their responsiveness to NO with an elevation in intracellular CGMP. ${ }^{27}$ Broadly, two types of cells were described: cells lying immediately below the urothelium and cells associated with the muscle. ${ }^{27}$ These observations have been confirmed and extended: it now appears that different subtypes of NO-responsive CGMP synthesising cells occur in both the sub-urothelial space ${ }^{10,28-29}$ and in the muscle. ${ }^{10,30-31}$ In the normal bladder, other markers, notably the c-Kit receptor and vimentin, have been used to identify interstitial cells and their distribution in the bladder wall. ${ }^{32-35}$ These c-Kit and vimentin+ cells are found in the lamina propria and lie around the muscle bundles of both the inner and outer muscle layers. A further type of cell, generally called a 'myofibroblasts', is found in the lamina propria. These cells are characterised by the expression of the gap functional protein connexin ${ }^{43}$ and by their proximity to nerve fibres. ${ }^{24-26}$ Thus, different types of interstitial cells have been found in the bladder wall. The differences in their location and morphology suggest that they may sub-serve different physiological functions.

In the normal bladder, the CGMP+ cells associated with the muscle are almost exclusively found within the outer regions of bladder wall. At least three different types have been identified: cells on the outer surface of the bladder (muscle coat interstitial cells), cells on the surface of the muscle bundles (surface muscle interstitial cells) and cells within the muscle bundles (intra-muscle interstitial cells). ${ }^{12,30}$ This network of cells is modified in obstructed bladders. Cells proliferate forming large discrete bundles of fibres running between the muscle bundles. In addition, small clumps or 'nodes' of cGMP+ can be seen to lie at the junctions of muscle bundles. These nodes are not an obvious feature of the normal bladder. A new network of CGMP+ cells associated with the inner muscle layer of the obstructed bladder also arises. These cells extend from the lamina propria into the inner muscle layer. Some of the cell bodies of these cells appear to be aggregated together to form small nodes, structures not seen in the normal bladder. The appearance of nodes of cGMP+ cells is intriguing. One possibility is that the nodes form a point where 
information from adjacent nodes can be forwarded to other regions of the muscle layer. Since nerve fibres (PGP 9.5+ fibres) appear to be associated with the nodes in the outer muscle layers, this adds a further possibility that the nodes could receive a neural input to induce or modulate activity.

The precise physiological role of these different networks of muscle and suburothelial interstitial cells in the normal bladder is not known. In the normal bladder, the muscle interstitial cells may be excitable and play a role distributing electrical activity through out the bladder wall, ultimately resulting in activation of the smooth muscle. ${ }^{8,36}$ In the isolated bladder, this activity has been shown to be accompanied by complex waves of activity spreading over the bladder surface. ${ }^{9,37}$ Such activity is unlikely to be generated by the smooth muscle and propagated by direct musclemuscle contacts. ${ }^{8-9,37}$ Two concepts have been put forward that the activity was coordinated via the intra-mural ganglia and nerves or the network of interstitial cells. ${ }^{9,37-38}$ One possibility is that the network of cGMP+ interstitial cells, or a component of the network, is responsible for generating and modulating phasic contractile activity in the pathological bladder. In keeping with this idea, autonomous activity is augmented in the isolated bladders from obstructed rats ${ }^{39}$ and guinea pig [de Jongh, in preparation].

Some possible direct evidence for a role for interstitial NO-responsive CGMP+ interstitial cells has come from experiments on the isolated mouse bladder. In this species, the only cells that respond to nitric oxide are the interstitial cells on the outer muscle bundles. When a mouse bladder is exposed to muscarinic agonists to induce phasic activity, exogenous nitric oxide abolishes this activity. Since the only cells responding to nitric oxide are interstitial cells, this implies that they play a central role in the generation of phasic activity. ${ }^{31}$ In the obstructed bladders, a complete interconnecting network of CGMP+ cells seems to extend from the suburothelial space through the lamina propria and into the inner and outer layers of the obstructed bladder wall. If the cells of this network are functionally linked, then signals generated by the urothelium might be carried to the muscle where they could result in contraction. Evidence for such a urothelial derived excitation of the muscle layers has been postulated in the isolated adult guinea pig bladder ${ }^{40}$ and in the neonatal and adult rat bladder. ${ }^{41}$ Furthermore, these structural changes to this network might also result in alterations to the generation of intrinsic activity, the distribution of this activity within the bladder wall and its sensitivity to excitatory and inhibitory regulators. The identification and any detailed analysis of such functional changes will have to await further experimentation.

The CGMP+ cells on the outer surface of the bladder, the serosal or muscle coat interstitial cells appear to have proliferated in the obstructed animals. The cell layer has been observed to be up to 20 cells thick in some regions. The function of this layer is not known. Enlarged bundles of cGMP+ cells leave the muscle coat and run between the outer muscle bundles (Fig. 7). Cell processes emanate from these structures and penetrate the muscle bundles. Here, they come into close contact with the intra-muscular interstitial cells. Such structures and contacts are found in 
the normal bladder but they are low in number and they are difficult to find. Their increased incidence in obstructed bladder suggests that whatever functions they subserve, it is modified.

The present study confirms previous work demonstrating the punctuate loss of nerves in pathological bladders. ${ }^{1-2}$ In the obstructed bladders of our study, muscle bundles are readily found to have PGP 9.5 positive nerve profiles, whereas adjacent bundles are devoid of nerves (Fig. 10). As noted above, the nodes of CGMP+ cells are associated with nerves and these innervated nodes are often adjacent to denervated muscle bundles. Thus, the mechanism underlying the focal denervation is unlikely to involve a local alteration in the chemical environment. The mechanisms of the punctuate nerve loss remains an enigma. Nerves, presumably sensory fibres, have readily been found in the lamina propria. This is in keeping with other studies that point to little or no change in sensory innervation associated with bladder pathology. In the present study, numerous nerve profiles have been seen within the lamina propria. However, few nerve endings lie in close proximity to the urothelium (Fig. 11a). The nerves appear to terminate in the expanded layer of NO-responsive CGMP+ interstitial cells. This altered distribution of nerve profiles may have important consequences. Nerve endings in close proximity to the urothelium are generally considered to have the potential to react to substances released by the urothelium. If, in obstructed bladders, a significant retraction of nerves occurs from the urothelium, then urothelial signals will have to diffuse for greater distances if they are to affect nerves. If these distances are significant, then urothelial signals could, as a result, be less effective.

Major changes have long been known to take place in the amount and distribution of elastin and collagen in pathological bladders, including those of obstructed bladders. ${ }^{1}$ Increased deposits of extracellular matrix are found on the outer muscle surface and between the muscle bundles. Data have demonstrated that these deposits of extracellular matrix may occur by a cytokine dependent activation of interstitial cells ${ }^{42}$ and that this process may be involved in the transformation of interstitial cells (myofibroblasts) into smooth muscle. ${ }^{43-44}$ The possibility must be considered that the proliferation and altered distribution of the NO-responsive CGMP+ cells also might be involved in such processes. Indeed, the expansion of the lamina propria layer with NO-responsive CGMP+ cells also might be part of a similar alteration in extracellular matrix composition.

In summary, the present observations demonstrate clear differences in the number and distribution of CGMP+ interstitial cells in the bladders of guinea pigs with surgically induced bladder outflow obstruction. Moreover, major alterations occur to the urothelium. These structural changes must play a role in the altered functioning of the obstructed bladder and the tissue remodelling that takes place. Such major changes may have important implications for unravelling the nature of the clinical problem of bladder overactivity in patients with outflow obstruction. Many studies of normal bladder have attempted to extrapolate from the physiological system to those underlying the pathology. Such approaches may now have to be treated with some 
caution. If the structures within the bladder are altered in pathology, they could well have their own functional characteristics conferring ' $n$ ew' properties to bladder function. A detailed understanding of the functional and pharmacological properties of the pathological bladder is therefore needed if we are to understand the origins of bladder dysfunction and its management.

Finally, we should consider the possible meaning of these data and ideas to patients with increased sensations of urge, frequency and incontinence. Surgical intervention to restrict the bladder neck results in alterations to bladder activity and voiding patterns, similar to those seen in patients with clinical symptoms of urge, frequency and incontinence. Furthermore, these functional changes are accompanied by alterations in bladder structure: an increase in bladder weight, hypertrophy of the smooth muscle, patchy denervation of muscle trabeculae and increased production of connective tissues. ${ }^{1-2,11}$ in vivo phasic activity during the filling phase is not associated with micturition and has been described as 'non-micturition activity'. A growing body of evidence now suggests that these contractions stimulate afferent nerves and that this motor/sensory activity contributes to bladder sensation. . $^{5-7,8,10,19}$ We also recognise that the phasic activity can be modulated by a number of excitatory and inhibitory factors suggesting that this motor/sensory system is regulated. ${ }^{8,10}$ The hypothesis has been put forward that alterations to this system, possibly by increasing the magnitude, frequency or sensitivity of the underlying mechanisms to excitatory inputs, will result in abnormal afferent discharge. In turn, this increased activity might be integrated within the central nervous system resulting in altered sensation and the premature triggering on the micturition reflexes. ${ }^{8}$ Alterations to the structure of the networks of cells in the obstructed bladder are responsible for the generation of phasic activity and may represent a central element in the origins of the clinical condition.

\section{References}

1. Charlton RG, Morley AR, Chambers $P$ \& Gillespie JI. Focal Changes in Nerve, Muscle and Connective Tissue in Normal and Unstable Human Bladder. BJU Int. 1999; 84:953-960.

2. Drake MJ, Hedlund P, Mills IM, McCoy R, McMurray G, Gardner BP, Andersson K-E and Brading A. Structural and functional denervation of human detrusor after spinal cord injury. Lab Invest. 2000; 80:199-207.

3. Andersson $K-E$, Yoshida $M$. Antimuscarinics and the Over Active Detrusor - Which is the main mechanism of action? Eur Urol. 2003; 43:1-5.

4. Finney SM, Andersson K-E, Gillespie JI, Stewart LH Antimuscarinic drugs in detrusor overactivity and the Overactive Bladder (OAB) Syndrome: motor or sensory actions? BJU Int. 2006; 98: 503-7.

5. Starling EH. Elements of Human Physiology, 7th Edition, 1905: Churchill, London.

6. Iggo A. Tension receptors in the stomach and urinary bladder. J Physiol, 1955; 128:593-607.

7. Vaughan, C. W. and Satchell, P. M. Urine storage mechanisms. Prog.Neurobiol. 1995; 46:21537. 
8. Gillespie JI. Animal Models of Urge Incontinence: An evolving view of the origins of bladder overactivity and sensory urge. BJU Int. 2005; 96:22-8.

9. Gillespie JI, Harvey IJ, Drake MJ. Agonist and Nerve Induced Phasic Activity in the Isolated Whole Bladder of the Guinea Pig: Evidence for Two Types of Bladder Activity. Exp Physiol 2003; 88:343-57

10. Gillespie JI. The Autonomous Bladder: A View of the Origin of Bladder Overactivity. BJU Int. 2004; $93: 478-83$.

11. Mostwin JL, Omer M, Karim A van Koeveringe G, Broks EL. The guinea pig as a model of gradual urethral obstruction. J Urol. 1991; 145:854-8.

12. Gillespie JI, Markerink-van Ittersum M, de Vente J. cGMP generating cells in the bladder wall: identification of distinct networks of interstitial cells. BJU Int. 2004; 94:1114-24.

13. de Vente J, Hopkins DA, Markerink-van Ittersum M, Emson PC, Schmidt HHHW Steinbusch HWM. Distribution of nitric oxide synthase and nitric oxide-receptive, cyclic GMP-producing structures in the rat brain. Neuroscience. 1998; 87:207-41.

14. Tanaka J, Markerink-van Ittersum M, Steinbusch HWM, de Vente J. Nitric oxide-mediated cGMP synthesis in oligodendrocytes in the developing rat brain. Glia.1997; 19:286-97.

15. Ferguson DR. Urothelial function. BJU Int. 1999; 84:235-42.

16. Birder LA, Kanai AJ, de Groat WC, Kiss S, Nealen ML, Burke NE, Dineley KE, Watkins S, Reynolds IJ, Caterina MJ. Vanilloid receptor expression suggests a sensory role for urinary bladder epithelial cells. Proc Natl Acad Sci USA. 2001; 98:13396-401.

17. Andersson KE, Arner A. Urinary bladder contraction and relaxation: physiology and pathophysiology. Physiol Rev. 2004; 84:935-86.

18. de Groat WC. The Urothelium in overactive bladder: passive bystander or active participant. Urology. 2004; 65:7-11.

19. Maggi CA. Prostanoids as local modulators of reflex micturition. Pharmacological Res. 1992; 25:13-20.

20. Birder LA, Nealen ML, Kiss S, de Groat WC, Caterina MJ, Wang E, Apodaca G, Kanai AJ. Betaadrenoceptor agonists stimulate endothelial nitric oxide synthase in rat urinary bladder urothelial cells. $]$ Neurosci. 2002; 22:8063-70.

21. Ferguson DR, Kennedy I, Burton TJ. ATP is released from rabbit urinary bladder epithelial cells by hydrostatic pressure changes--a possible sensory mechanism? J Physiol, 1997; 505:503511.

22. Mikhailidis DP, Jeremy JY, Dandona $P$ (1987). Urinary bladder prostanoids-their synthesis, function and possible role in the pathogenesis and treatment of disease. J Urol. 1987; 137:577-582.

23. Yoshida M, Miyamae K, Iwashita $H$, Otani M, Inadome A. Management of detrusor dysfunction in the elderly: Changes in acetylcholine and adenosine triphosphate release during aging. Urology, 2004; 63:17-23.

24. Wiseman OJ, Brady CM, Hussain IF, Dasgupta $P$, Watt $H$, Fowler $C$, Landon DN. The Ultrastructure of Bladder Lamina Propria Nerves in Healthy Subjects and Patients With Detrusor Hyperreflexia. J Urol. 2002;168:2040-2045.

25. Sui GP, Rothery S, Dupont E, Fry CH, Severs NJ. Gap junctions and connexin expression in human suburothelial interstitial cells. BJU Int. 2002; 90:118-129.

26. Fry $\mathrm{CH}$, Ikeda $\mathrm{Y}$, Harvey R, Wu C, Sui GP. Control of bladder function by peripheral nerves: avenues for novel drug targets. Urology. 2004; 63(3 Suppl 1):24-31.

27. Smet PJ, Jonavicius J, Marshall VR, de Vente J. Distribution of nitric oxide synthaseimmunoreactive nerves and identification of the cellular targets of nitric oxide in guinea-pig and human urinary bladder by CGMP immunohistochemistry. Neuroscience. 1996; 71:337-348.

28. Gillesple JI, Markerink-van Ittersum M, de Vente J. The expression of neuronal nitric oxide synthase (nNOS) and nitric oxide induced changes in CGMP in the urothelial layer of the guinea pig bladder. Cell Tissue Res. 2005; 321:341-351.

29. Gillespie JI, Markerink-van Ittersum M and de Vente J. Endogenous NO/cGMP signalling in the guinea pig bladder: evidence for distinct populations of sub-urothelial interstitial cells. Cell Tissue Res. 2006; 325:325-332. 
30. Gillespie JI, Markerink-van Ittersum M, de Vente J. Interstitial cells and cholinergic signalling in the outer muscle layers of the guinea pig bladder BJU Int. 2006; 97:379-385.

31. Lagou M, Drake MJ, de Vente J, Markerink-van Ittersum M and Gillespie JI. The role of interstitial cells in the isolated mouse bladder. BJU Int. 2006; 98:643-50.

32. McCloskey K D, Gurney A M. kit-positive cells in the guinea pig bladder. J Uro.l 2002; 168:8326.

33. Davidson RA, McCloskey KD. Morphology and localization of interstitial cells in the guinea pig bladder: structural relationships with smooth muscle and neurons. J Urol. 2005;173(4):138590.

34. Brading AF and McCloskey KD. Mechanisms of disease: specialised interstitial cells of the urinary tract - an assessment of knowledge. Nat Clin Pract Urol. 2005; 2:546-54.

35. Drake MJ, Fry $\mathrm{CH}$, Eyden B Structural characterisation of myofibroblasts in the bladder. BJU Int.2006; 97:29-32.

36. Hashitani $H$, Yanai $Y$, Suzuki $H$. Role of interstitial cells and gap junctions in the transmission of spontaneous $\mathrm{Ca}^{2+}$ signals in detrusor smooth muscles of the guinea-pig urinary bladder. I Physiol. 2004; 559:567-81.

37. Drake MJ, Harvey IJ, Gillespie JI. Autonomous activity in the isolated guinea pig bladder. Exp Physiol. 2003; 88:19-30.

38. Gillespie JI, M Markerink-van Ittersum M, de Vente J. Sensory collaterals, intra-mural ganglia and motor nerves in the guinea pig bladder: evidence for intramural neural circuits Cell and Tissue Res. 2006: 325:33 45.

39. Drake MJ, Hedlund P, Harvey IJ, Pandita RK, Andersson KE, Gillespie JI. Partial outlet obstruction enhances modular autonomous activity in the isolated rat bladder. J Urol. 2005; 170:256-9.

40. Lagou M, Drake MJ, Gillespie JI. Volume-induced effects on the isolated bladder: a possible local reflex BJU Int. 2005; 94:1356-65.

41. Kanai A, Roppolo J, Ikeda Y, Zabbarova I, Tai C, Birder L, Griffiths D, de Groat W, Fry CH. Origin of spontaneous activity in neonatal and adult rat bladders and its enhancement by stretch and muscarinic agonists. Am J Physiol. 2006; 292:F1065-F72.

42. Roelofs $M$, Faggian $L$, Pampinella $F$, Paulon $T$, Franch $R$, Chiavegato $A$, Sartore $S$. Transforming growth factor b1 involvement in the conversion of fibroblasts to smooth muscle cells in the rabbit bladder serosa. Histochemical Journal. 1998; 30:393-404.

43. Pampinella F, Roelofs M, Casteltucci E, Passerini-Glazel G, Pagano F, Sartore S. Time dependent remodelling of the bladder wall in growing rabbits after partial outlet obstruction. J Urol. 1997; 157:677-82.

44. Chiavegato A, Roelofs M, Franch R, Castellucci E Sarinella F, Sartore S. Differential expression of SM22 isoforms in myofibroblasts and smooth muscle cells from the rabbit bladder. J Muscle Res and Cell Mot. 1999; 20:133-46. 


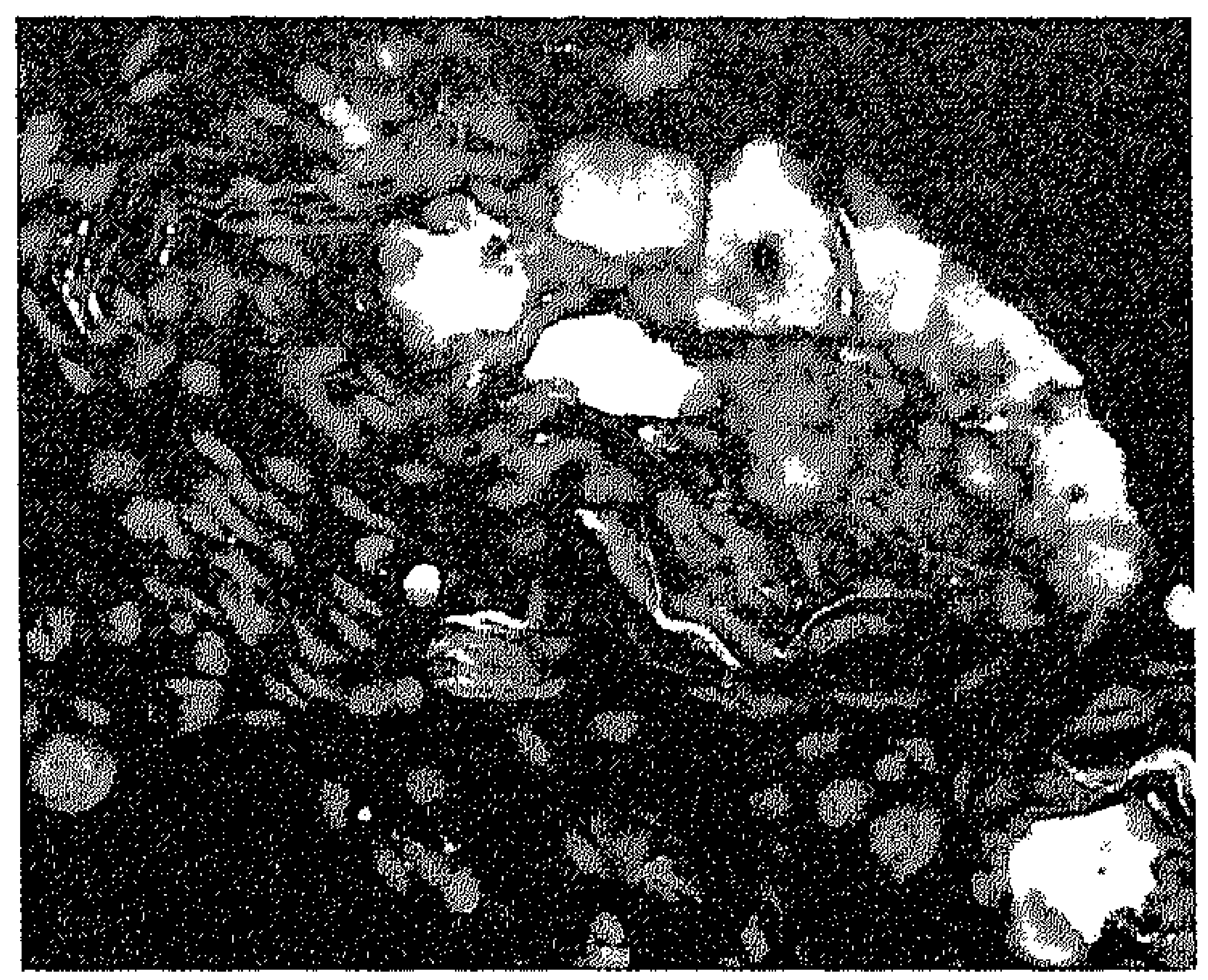

Partial bladder outlet obstruction reduces the tissue antioxidant capacity and muscle nerve density of the guinea pig bladder

Rik de Jongh, Miriam Dambros, Guido R.M.M. Haenen, Gertjan J.M. den Hartog, Aalt Bast, Philip E.V. van Kerrebroeck, Gommert A. van Koeveringe.

Neurourology and Urodynamics 2009; 28(5):461-7. 


\section{Abstract}

Aims: Reactive nitrogen and oxygen species likely play a role in the development of bladder dysfunction related to bladder outlet obstruction. Antioxidants protect against these free radicals. The aim of our study was to investigate the effect of bladder outlet obstruction on the endogenous antioxidant status of the bladder and to correlate this to bladder structure and function.

Methods: In 16 guinea pigs either a partial outlet obstruction or a sham operation was induced. The contractile responses of detrusor strips to electrical field stimulation (EFS), acetylcholine, potassium and ATP were monitored four weeks after the operation. The nerve density in bladder tissue was determined by using the nonspecific nerve marker PGP 9.5. Separate antioxidants and the total antioxidant status were assessed using the TEAC (Trolox Equivalent Antioxidant Capacity) test.

Results: Contractile responses of detrusor strips to EFS were for the greater part based on neurogenic stimulation. The nerve mediated responses in strips from obstructed bladders were lower compared to the sham group. Obstructed bladders showed a patchy denervation and the nerve density was significantly lower compared to the sham group. The total antioxidant capacity, the glutathione and the glutathione reductase levels significantly decreased in obstructed bladders compared to the sham group.

Conclusion: This study demonstrates that the antioxidant status of guinea pig bladders exposed to outlet obstruction decreased which might be associated with the observed reduction in nerve density. The results strengthen the hypothesis that oxidative stress is involved in the pathophysiology of bladder dysfunction related to obstructed bladders. 


\section{Introduction}

Bladder outlet obstruction results in morphological changes in the bladder, including a loss of nerve fibres, hypertrophy of the smooth muscle cells, increased expression of connective tissue elements and alterations to the urothelium. ${ }^{1,2}$ The structural changes of the bladder result in functional changes giving rise to storage symptoms (daytime urinary frequency, nocturia, urgency, urinary incontinence) and voiding symptoms (slow stream, intermittence, hesitancy, straining, terminal dribble). The storage symptoms may relay to alterations in the sensory afferent pathway of the micturition reflex ${ }^{3}$ and / or a modified sensory/motor system. ${ }^{4}$ The obstructive symptoms are partly related to the contractile dysfunction of the detrusor muscle. In bladder outlet obstruction, the detrusor muscle and nerves are damaged which seems to be partly due to ischemia/reperfusion ${ }^{5,6}$ and inflammation in the bladder wall. Ischemia-reperfusion and inflammation are known to induce the production of reactive nitrogen and oxygen species (RNOS). Examples of RNOS are superoxide anion $\left(\mathrm{O}_{2}{ }^{--}\right)$, hydroxyl radical $\left(\mathrm{OH}^{\bullet}\right)$, hydrogen peroxide $\left(\mathrm{H}_{2} \mathrm{O}_{2}\right)$, hypochlorous acid $(\mathrm{HOCl})$ and peroxynitrite (ONOO-). The human body has its own endogenous protection against RNOS consisting of antioxidant enzymes (e.g. superoxide dismutase, catalase, glutathione peroxidase and glutathione reductase) and various antioxidants (e.g. gluthathione, uric acid, melatonin, bilirubin, vitamin C and $E$ ). In situations such as ischemia-reperfusion and inflammation, free radicals may overwhelm the antioxidant defence system; a situation denoted as oxidative stress. Oxidative stress is regarded as noxious because RNOS are able to destroy virtually any molecule, including proteins, DNA and lipids. Nerves are especially susceptible to oxidative stress induced damage.' Bladder outlet obstruction results in denervation and has been associated with an increased level of markers for oxidative stress. ${ }^{8,9}$ In the denervation observed in obstructed bladders, oxidative stress has been implicated. Moreover, several studies revealed that antioxidants protect the bladder against functional and structural damage due to bladder outlet obstruction, suggesting that tissue of obstructed bladders have a reduced antioxidative capacity. ${ }^{8,10}$ The aim of our study was to investigate the effect of partial bladder outlet obstruction on antioxidant status of the bladder and to correlate the antioxidant status to bladder structure and function. It is known that the various antioxidants do not work in isolation; together they form an intricate network. Therefore the antioxidant status was determined not only by measuring separate antioxidants, also the total antioxidant status in bladder material was assessed using the TEAC (Trolox Equivalent Antioxidant Capacity) test. ${ }^{11}$

\section{Materials and methods}

The studies were performed in 16 male guinea pigs (Hartley strain) weighing between 275 - 372 grams. The institutional animal care and use committee of 
Maastricht University approved all the animal experiments conducted in this study.

Surgical procedure: In 8 guinea pigs a partial outlet obstruction was induced and 8 animals underwent sham operation. The animals of both groups were agedmatched and there was not a significant difference in body weight prior to surgery. A protocol based on the guinea pig model for gradual outlet obstruction as previously described was used. ${ }^{1,4}$ Briefly, once sedation was achieved the bladder neck and urethra were exposed via a vertical midline abdominal incision. A silver jeweler's jump ring (1.8 $\mathrm{mm}$ internal diameter) was placed loosely around the proximal urethra. Bladder outlet obstruction occurred during subsequent growth of the animal. Sham surgery was performed in an identical manner, except for placing the ring around the urethra. Four weeks after the operation the animals were sacrificed and the bladders removed. The bladders were separated from associated structures, blotted once on each side and weighed on an electronic scale. Four sham operated and obstructed animals were used for the contractility and biochemical study. In four other sham and obstructed bladders the nerve density was determined. Each bladder was divided longitudinally in two pieces and bladder pieces of the lateral wall were used for contractility, biochemical and immunohistochemical studies.

Measurement of antioxidants: The urothelium was removed and the muscle layer was homogenized with an ultrathurax for $20 \mathrm{sec}$ in ice-cold sodium phosphate buffer (145 mM, pH 7.4) at a final concentration of $111 \mathrm{mg} / \mathrm{ml}$. The tissue was further fragmented by a pestle and tissue homogeniser. Homogenates were centrifuged ( 5 $\min$ at $14000 \times \mathrm{g}$ and $4^{\circ} \mathrm{C}$ ) and supernatants were stored directly at $-80^{\circ} \mathrm{C}$ until use. Total protein content in homogenates was assayed according to 5 mith et al. ${ }^{12}$ using BSA as standard. The glutathione reductase (GR) activity was determined by measuring the decrease in absorbance ( $2 \mathrm{~min}$ at $340 \mathrm{~nm}$ ) by the consumption of NADPH (Sigma Chemical Co, St Louis, MO, USA) in the enzymatic reduction of an excess of glutathione disulphide (GSSG) to reduced glutathione (GSH) by GR. ${ }^{13}$ Total glutathione level was determined by measuring the increase in absorbance ( $2 \mathrm{~min}$ at $405 \mathrm{nM}$ ) caused by the GSH driven reduction of $5^{\prime} 5^{\prime}$ dithiobis 2-nitrobenzoic acid (ICN Biomedicals Inc., Costa Mesa, USA), using the recycling method described by Griffith et al. ${ }^{13}$ Uric acid was determined in ultra filtrates with HPLC method described by Lux et $\mathrm{al}_{.}{ }^{14}$ using a Hypersil BDSC18 end-capped column $\left(125 \times 4 \mathrm{~mm}^{2}\right.$, particle size 5 microm, Aligent). The total antioxidant capacity was measured by means of the Trolox equivalent antioxidant capacity assay (TEAC), as described by Fischer et al. ${ }^{11}$ : Aliquots of the homogenized bladder were deproteinated with trichloro-acetic acid solution. The absorbance (at $734 \mathrm{~nm}$ ) of the radical solution was $0.070 \pm 0.02$. The decrease in absorbance, caused by the antioxidant capacity in the sample, was measured and related to that of trolox standards.

\section{Contractility studies}

Under a binocular microscope the urothelium was removed and muscle strips of approximately $10 \times 1 \times 1 \mathrm{~mm}$ were excised. Three to four detrusor strips from each bladder were mounted in separate double-jacketed $6-\mathrm{ml}$ organ baths containing 
oxygenated Krebs-buffer solution at $37^{\circ} \mathrm{C}\left(\mathrm{NaCl}_{1} 118 \mathrm{mmol} / \mathrm{l} ; \mathrm{KCl}, 5.6 \mathrm{mmol} / \mathrm{l}\right.$; $\mathrm{NaHCO}_{3}, 25 \mathrm{mmol} / \mathrm{l} ; \mathrm{KH}_{2} \mathrm{PO}_{4}, 1.2 \mathrm{mmol} / \mathrm{l} ; \mathrm{CaCl}_{2}, 2.5 \mathrm{mmol} / \mathrm{l} ; \mathrm{MgSO}_{4}, 1.2 \mathrm{mmol} / \mathrm{l}$; glucose $6.1 \mathrm{mmol} / \mathrm{l} ; \mathrm{pH} 7.4$; aerated with $95 \% \mathrm{O}_{2} / 5 \% \mathrm{CO}_{2}$ ). Potassium solutions were made by increasing the concentration of $\mathrm{KCl}$ at the expense of $\mathrm{NaCl}$. Fine silk sutures were tied to each end of the strips; one end was fixed to the tissue holder and the other end to the isometric force transducer (Kistler Morce DSC 6, Seattle, WA). Frequency response curves to electrical field stimulation (EFS) were constructed by stimulating bladder strips via platinum electrodes. The electrodes were placed above and under the bladder strips and were connected to the HM8130 Function Generator from Hameg ${ }^{\circledR}$ Instruments, Manhausen, Germany. Electrical field stimulation ( $50 \mathrm{~V}, 2 \mathrm{msec}$ pulse width, $10 \mathrm{sec}$ train pulses) was applied at frequencies of $0.5,1,2,4,8,16$ and $32 \mathrm{~Hz}$. In order to characterize the electrical evoked responses, strips were treated with tetrodotoxin 10-6 $\mathrm{M}$ (contact time $15 \mathrm{~min}$ ). To evaluate the muscarinic receptor function, dose response curves to acetylcholine $\left(10^{-8}-3 \times 10^{-5} \mathrm{M}\right)$ were constructed. Furthermore, the contractile responses to membrane depolarization and purinergic receptor stimulation were evaluated by determining the responses to potassium $(\mathrm{KCl} 100 \mathrm{mM})$ or ATP $(1 \mathrm{mM})$. All solutions were dissolved in deionised distilled water and freshly prepared every day. Further dilutions were carried out in Krebs solution. Tetrodotoxin, adenosine triphosphate and acetylcholine were obtained from Sigma Chemical Co (Utrecht, The Netherlands). The concentrations are expressed as the final molar concentration in the tissue chamber.

\section{Structural study}

Bladder pieces of the lateral wall were introduced in ice-cold fixative solution of $4 \%$ freshly prepared depolymerised paraformaldehyde for $60 \mathrm{~min}$ at $4{ }^{\circ} \mathrm{C}$. Then, tissues were washed at $4{ }^{\circ} \mathrm{C}$ in $0.1 \mathrm{M}$ phosphate buffer containing $10 \%$ sucrose ( 24 h), $20 \%$ sucrose $(24 \mathrm{~h})$ and $30 \%$ sucrose $(24 \mathrm{~h})$. The tissues were then snap-frozen with $\mathrm{CO}_{2}$ in Tissue-Tek O.C.T. compound to form a single block. Cryostat sections $(10 \mathrm{\mu m})$ were cut and these were thawed on to chrome-alumn-gelatin-coated slides and processed for immunocytochemistry. Sections were dried for $20 \mathrm{~min}$ at RT followed by three washes with Tris-buffered saline (TBS; pH 7.6), and thereafter incubated overnight with the non-specific nerve marker protein gene product 9.5 (PGP 9.5) (1: 2000) (Chemicon) at $4{ }^{\circ} \mathrm{C}$. After overnight incubation sections were washed in TBS, TBS-T and TBS. Rabbit primary antibody was visualized using Alexa Fluor 488 donkey anti-rabbit IgG conjugate (Molecular Probes), diluted $1: 100$ in TBS-T. Routine negative controls were included by omission of the primary antibody. No immunoreactivity was observed in such controls. Typically, each bladder staining was done in duplicate and repeated on at least 2 separate days. Observations were accumulated from the different slides and from the different bladders. From each bladder 4 separate slides of the lateral wall were observed, in 2 representative slides the nerve density was calculated. The number of nerve fibers in the muscle layer was counted at $100 \times$ magnification in 5 high power fields per slide. The mean nerve 
density was analyzed for each individual animal. Sections were analysed and photographed using an Olympus AX70 microscope equipped with a cooled CCD Olympus Digital video camera F-view. For the detection of Alexa 488 fluorescence we used a narrow band-pass MNIBA-filter. Images were stored digitally by using the computer program analySIS ${ }^{\circledR}$ Vers.3.0. (Soft Imaging System, Münster, Germany) and arranged with the program Adobe Photoshop 7.01 (San Jose, CA, USA).

\section{Statistical evaluation}

Results are expressed as means \pm SEM and $n$ refers to the number of bladders. The nerve density and mean values of the contractility and biochemical parameters were analyzed for each individual animal. The biochemical values were related to the protein content in the homogenates which was not significantly different between the groups. Changes in bladder tension evoked by EFS, ATP and acetylcholine were expressed as percentages of the contractile response to $100 \mathrm{mM} \mathrm{KCl}$ in each tissue. Concentration response data of acetylcholine were evaluated by sigmoid curve fitting and $-\log$ EC 50 values $\left(\mathrm{pD}_{2}\right)$ were calculated by nonlinear regression analysis using GraphPad Prism. Differences were analyzed using the two-tailed Mann-Whitney test. A probability value of $p<0.05$ was regarded to be significant. All analyses were performed using GraphPad Prism software (version 4.0; Graphpad Software Inc., San Diego, CA).

\section{Results}

Four weeks after the operation the weight gain in the sham operated animals was $219 \pm 18$ grams compared to the animals with a bladder outlet obstruction $142 \pm 24$ grams. The bladder weights of sham operated and obstructed animals were $493 \pm$ $27 \mathrm{mg}$ and $1257 \pm 64 \mathrm{mg}$ respectively $(p<0,05)$.

The responses of detrusor strips from sham operated bladders to EFS at $0.5-32$ $\mathrm{Hz}$ were reduced after treatment with tetrodotoxin by $95 \%-79 \%$ (Fig. 1, inserted figure). This indicates that the contraction was for the greater part based on neurogenic stimulation and a small part $(5-21 \%)$ was due to direct muscle stimulation. In obstructed bladders, the nerve mediated component did not differ compared to the sham group (Fig. 1, inserted figure). The absolute amplitude of force development to $\mathrm{KCl}(100 \mathrm{mM})$ in bladders strips from sham operated animals, and obstructed animals were $64.9 \pm 12.2 \mathrm{mN}$ and $53.0 \pm 15.0 \mathrm{mN}$, respectively. Although the amplitude of the contraction in the obstructed bladders tended to be lower, this was not significantly different between the groups. The normalized contractile responses to EFS at $4-16 \mathrm{~Hz}$ of strips from obstructed bladders tended to be lower compared to sham operated bladders and the response at $32 \mathrm{~Hz}$ was significantly lower $(p<0.05)$ (Fig. 1 ). The $\mathrm{pD}_{2}$ value of acetylcholine in strips from sham operated bladders $(5.87 \pm 0.03)$ was not different compared to obstructed bladders (5.89 \pm 0.04 ) (Fig. 2). The normalized maximal effect obtained with 


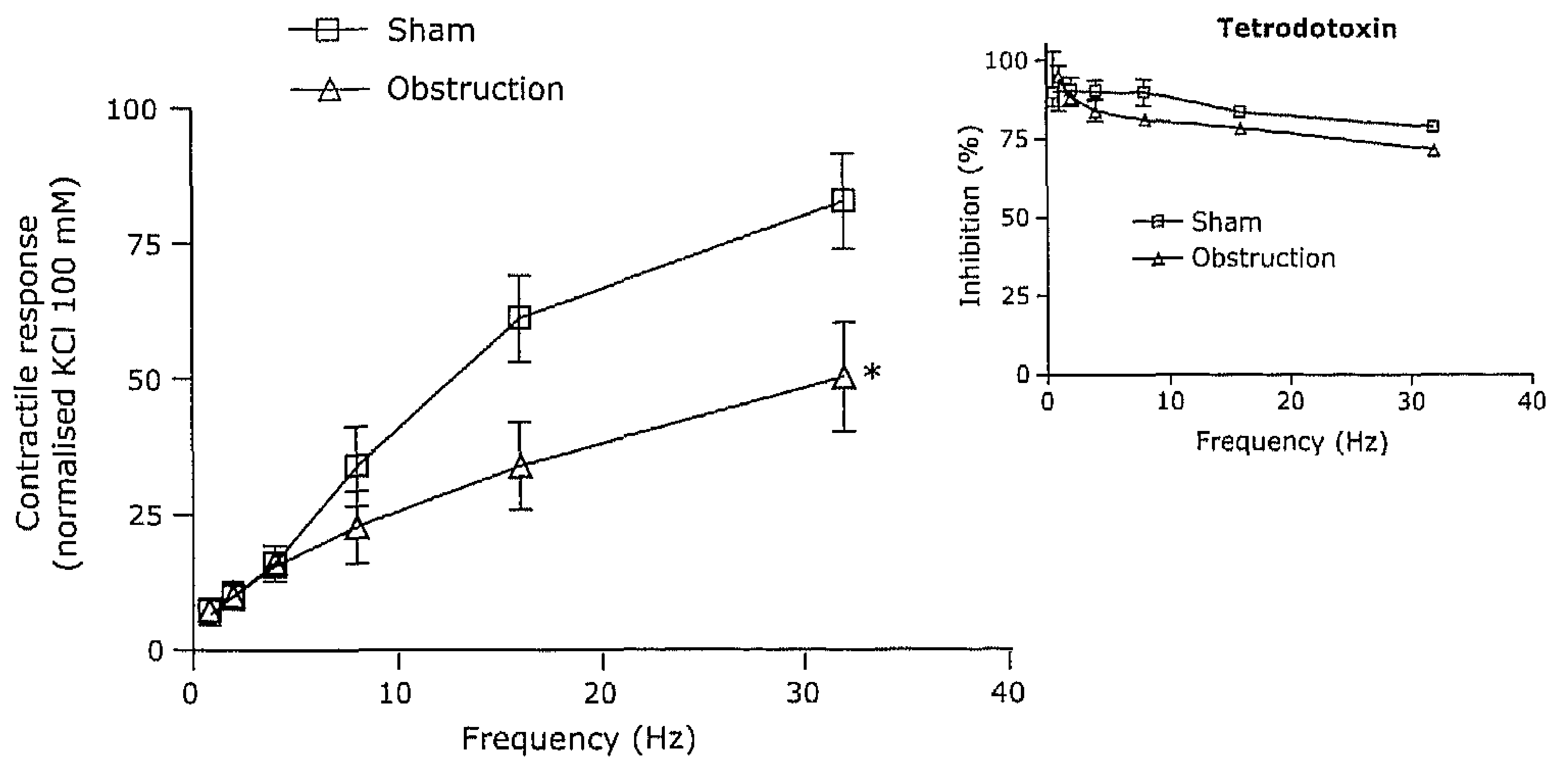

Figure 1.

The effect of partial outlet obstruction on electrical field stimulation evoked contractions $(0,5-32$ $\mathrm{Hz}$ ) of guinea pig detrusor strips. The contractile responses to EFS at $8-32 \mathrm{~Hz}$ of strips from obstructed operated animals were lower compared to sham operated animals and the response at $32 \mathrm{~Hz}$ was significantly lower compared to the sham operated group $(p<0.05)$. Each point represents the mean \pm SEM of 4 bladders (12 - 14 muscle strips). The inserted figure shows the effect of tetrodotoxin on the electrical field mediated responses of guinea pig detrusor strips in both groups. Each point represents the mean \pm SEM of $4-6$ guinea pig strips from 2 obstructed and sham operated animals. ${ }^{*} p<0.05$ versus sham-operated animals.

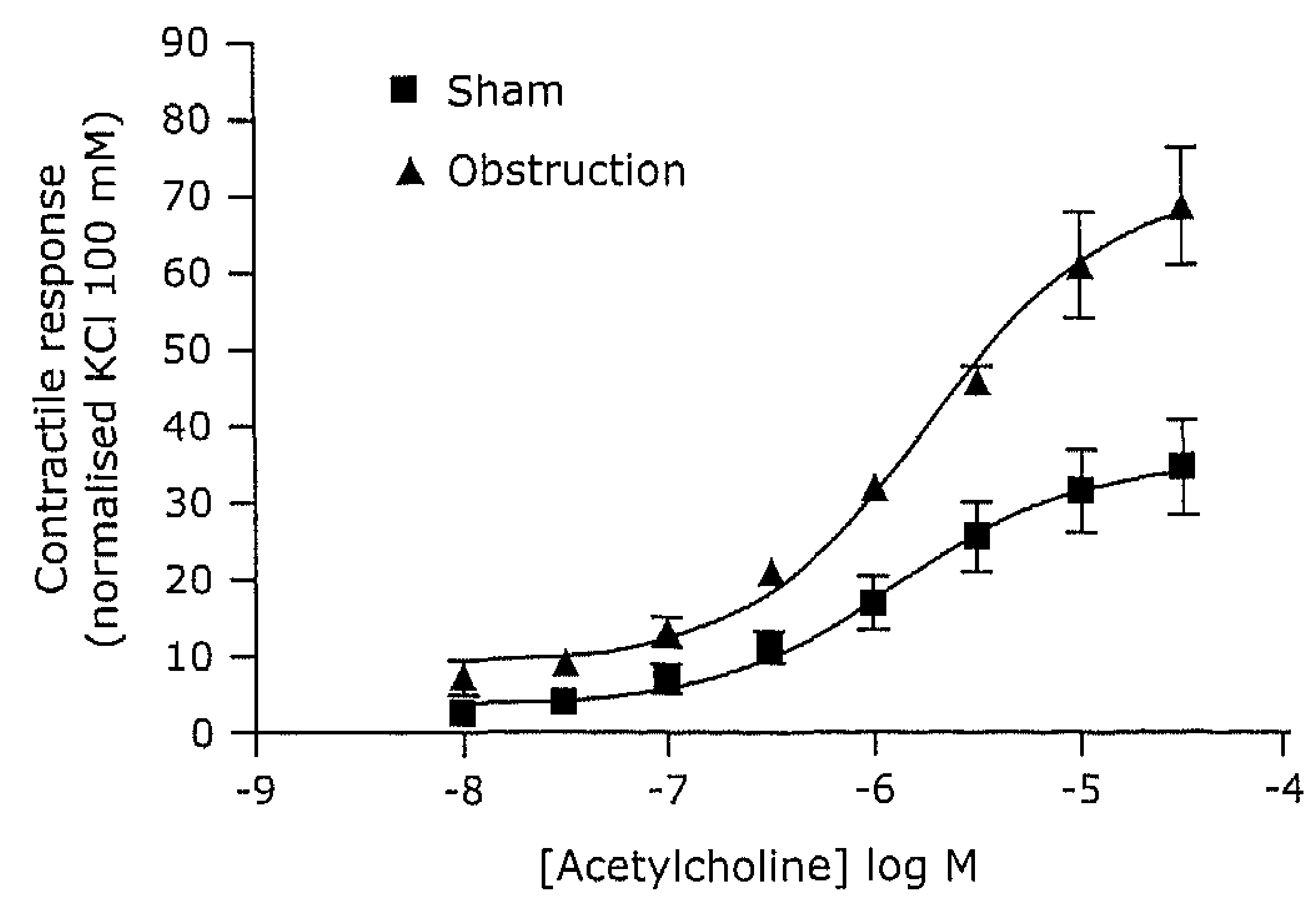

Figure 2.

The effect of partial outlet obstruction on the acetylcholine dose response curve of guinea pig strips. The normalized maximal effect obtained with acetylcholine was significantly increased in strips from obstructed bladders $(67.5 \pm 1.37 \%)$ compared to sham operated bladders $(34.3 \pm 0.61 \%)(p<0.05)$. The $\mathrm{pD}_{2}$ value of acetylcholine in strips from sham operated bladders $(5.87 \pm 0.03)$ was not different compared to obstructed bladders $(5.89 \pm 0.04$ ). Each point represents the mean of \pm SEM of 4 bladders (12 - 14 muscle strips). 

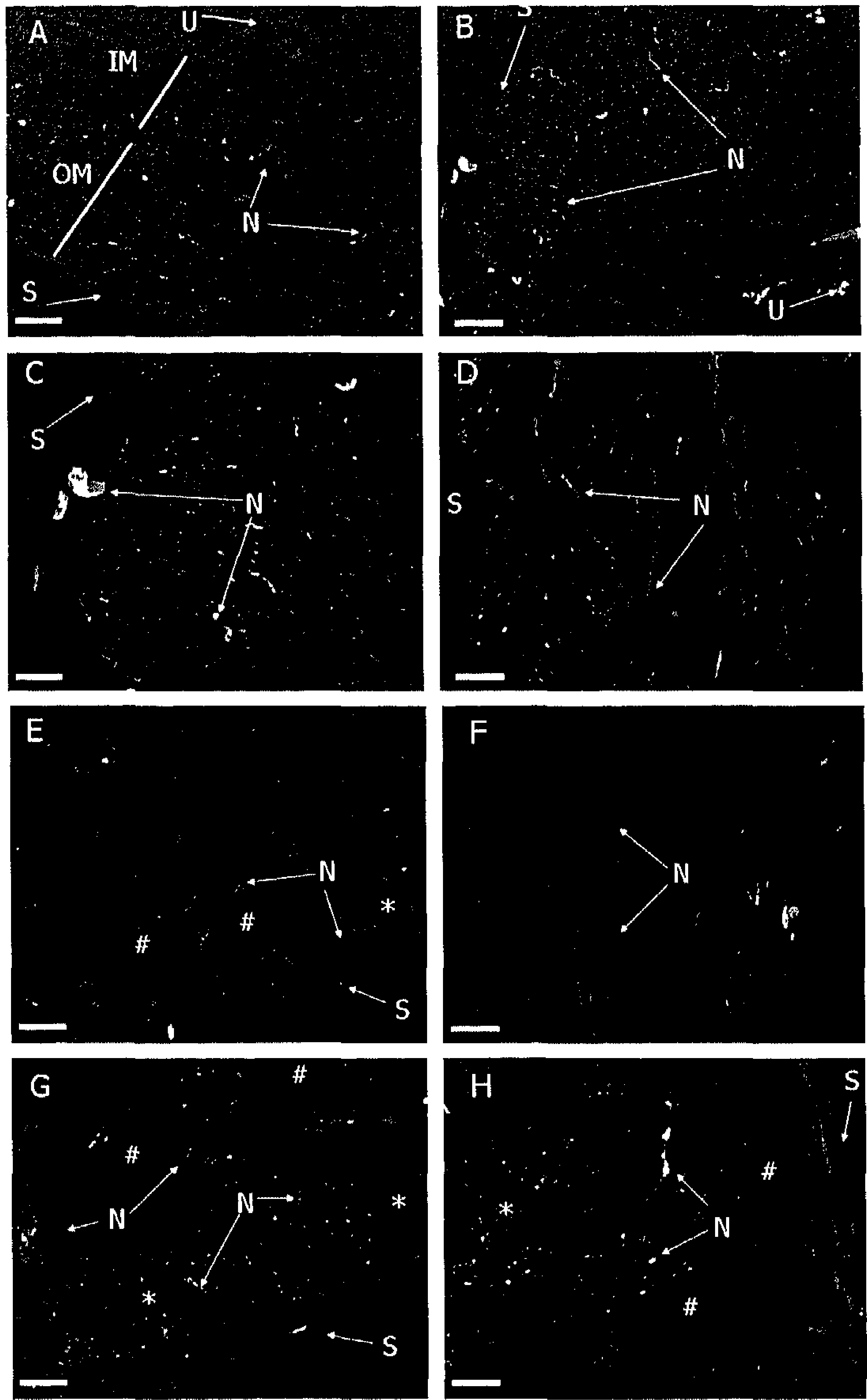

Figure 3.

The distribution of nerves within the sham operated and obstructed guinea pig bladders. The sections are stained for the non-specific nerve marker PGP 9.5. $\boldsymbol{A}$ and $\boldsymbol{B}$ are sections from different sham operated bladders showing the entire bladder wall from the urothelium $(U)$ to the serosal layer (S). There are lots of nerves $(N)$ in the inner muscle (IM) and outer muscle (OM) layer. The tissue has a uniform, dense innervation. $\boldsymbol{C}$ and $\boldsymbol{D}$ show images of the outer muscle layer of sham operated bladders at higher magnifications. $\boldsymbol{E}$ and $\boldsymbol{F}$ are sections from different obstructed guinea pig bladders showing the outer muscle and serosal layer (S). There is a clear denervation in the muscle layer. The denervation pattern is patchy, compromising bundles with a low number of nerve $(N)$ fibers (\#), adjacent to bundles in which innervation is apparently normal $(*), \boldsymbol{G}$ and $\boldsymbol{H}$ show images of the outer muscle at higher magnification. Calibration bars: $60 \mu \mathrm{m}$ in $\boldsymbol{A}, \boldsymbol{B}, \boldsymbol{E}, \boldsymbol{F}$ and $20 \mu \mathrm{m}$ in $\boldsymbol{C}, \boldsymbol{D}, \boldsymbol{G}, \boldsymbol{H}$. 
acetylcholine was significantly increased in strips from obstructed bladders $(67.5 \pm$ $1.37 \%)$ compared to sham operated bladders $(34.3 \pm 0.61 \%)(p<0.05)$. The normalized contractile responses to purinergic receptor stimulation were similar in strips from obstructed bladders $(27.3 \pm 3.99 \%)$ compared to sham operated bladders $(26.4 \pm 6.29 \%)$.

Figure 3 shows the distribution of nerves within the sham operated guinea pig bladders. Lots of nerves are observed in the inner and outer muscle layer, there seems to be a uniform dense innervation. However, in obstructed bladders there is a patchy denervation. Some muscle bundles are completely denervated, while in other bundles sparce innervation is seen (Fig. 3). The number of nerves fibers per high power field in obstructed bladders $(22.58 \pm 3.07)$ was significantly less than in sham operated bladders $(61.8 \pm 4.18)(p<0.05)$.

Total antioxidant capacity, as measured by the TEAC significantly decreased in the bladders from animals that underwent an obstruction operation compared to the sham operated animals ( $p<0.05)$ (Fig. 4). The same applies for the glutathione and the glutathione reductase levels. The concentration of uric acid in the bladder tissue form animals with an obstruction operation did not differ significantly compared to sham-operated animals. The last graph in figure 4 shows that the antioxidants uric
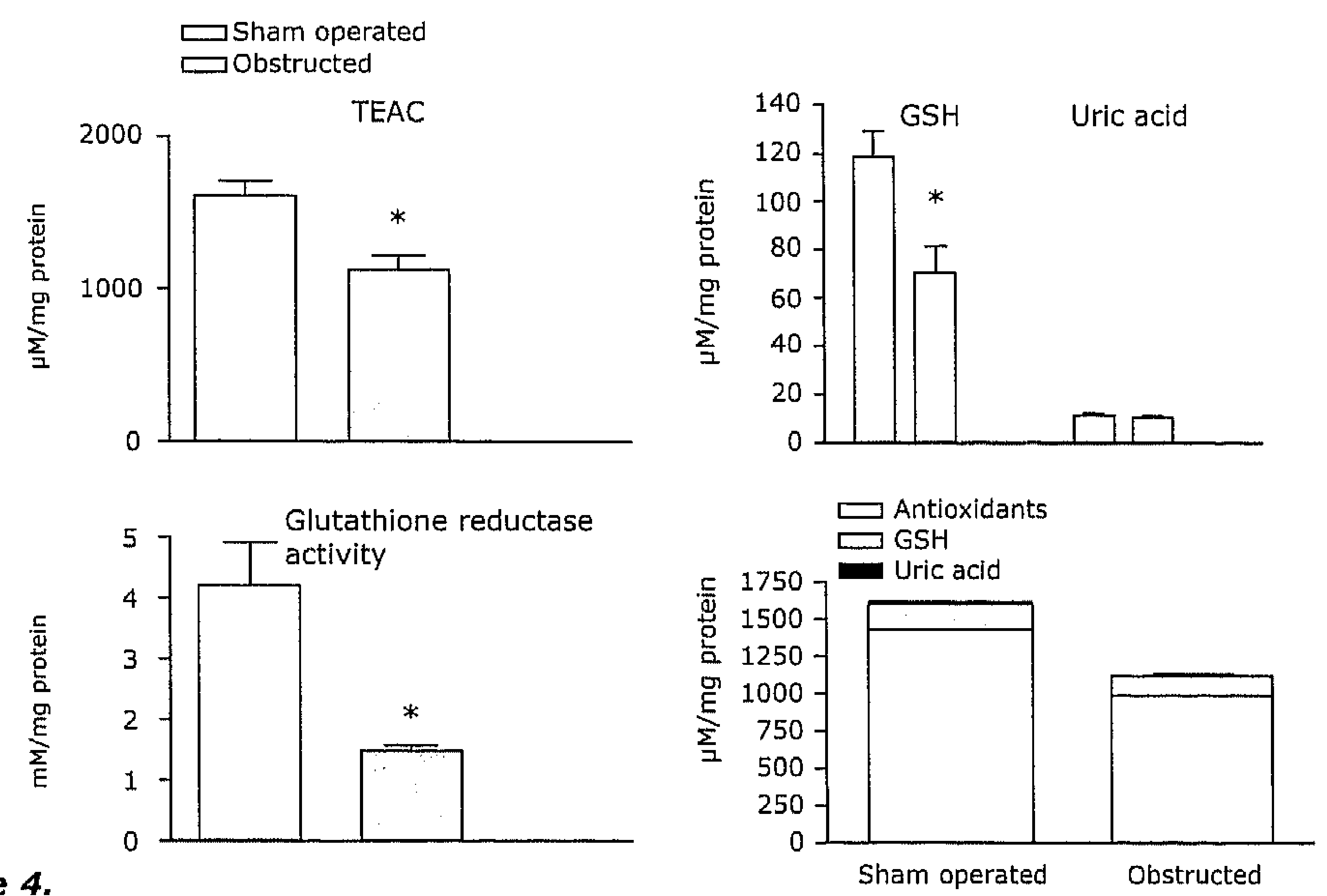

Figure 4.

The effect of sham and obstruction operation on several antioxidants in bladder tissue of guinea pigs. The Trolox equivalent antioxidant activity (TEAC), glutathione (GSH) and glutathione reductase activity significantly reduced in bladders exposed to an outlet obstruction compared to sham operated bladders. The concentration of uric acid in the bladder tissue from animals with an obstruction operation did not differ significantly compared to bladder tissue from sham-operated animals. The last graph shows that the antioxidants uric acid and gluthathione contribute for a minor part to the TEAC level. The contribution of these antioxidants was determined by subtracting the uric acid and gluthathione levels from the TEAC level. Each bar represents the mean \pm SEM of 4 bladders. $*_{p}<0.05$ versus sham-operated animals. 
acid and gluthathione contribute for a minor part to the TEAC level. Besides these antioxidants other molecules such as vitamin $C$, and bilirubine can contribute to the TEAC value.

\section{Discussion}

In our study, the weight gain in obstructed guinea pigs was lower compared to sham operated animals. This finding is in accordance with previously published studies using the guinea pig as a model of bladder outlet obstruction. ${ }^{1,4}$ However, there are other reports of no difference in weight gain between obstructed and sham operated animals. ${ }^{16}$ The cause of the difference in weight gain found in our study is enigmatic. Importantly, surgically induced processes do not seem to be involved in the difference in weight gain, as the control group consisted of sham operated animals. Furthermore, the sham and obstructed animals were age-matched and there was no difference in average body weight between both groups prior to surgery. One possible explanation could be that the urinary symptoms induced by a bladder outlet obstruction causes stress in the obstructed animals. Chronic stress is associated with a reduced weight gain over time.

The smooth muscle contraction of the urinary bladder in response to nerve stimulation is predominantly mediated by the release of the neurotransmitters acetylcholine and ATP. Contractions mediated by $\mathrm{KCl}$ are dependant on membrane depolarisation. Depolarisation activates $\mathrm{Ca}^{2+}$ influx through L-type calcium channels and finally will result in a contractile response of the muscle. The $\mathrm{KCl}$-mediated responses reflect the functioning of the intrinsic contractile system of the detrusor muscle. In order to correct for muscle hypertrophy in obstructed bladders we normalized the EFS, purinergic and cholinergic mediated responses in all bladders for the $\mathrm{KCl}$-evoked responses. There was no difference in the TTX-sensitive component of the EFS-evoked responses in obstructed bladders compared to sham operated bladders. The average normalized EFS-evoked contractile responses were lower in obstructed bladders compared to the sham group. This decrease can be due to either denervation or a reduced muscarinic and or purinergic receptor functioning in obstructed bladder tissue. Since the normalized ATP-mediated responses were similar and the normalized contractile responses to acetylcholine increased in strips from obstructed bladders compared to the sham group, it implicates a denervation in obstructed detrusor muscle. These results are in agreement with several functional obstructed bladder studies where the contractile response to field stimulation decreased significantly greater compared to either membrane depolarisation by $\mathrm{KCl}$ or muscarinic receptor stimulation. ${ }^{15,16}$ The finding that the normalized maximal response to acetylcholine significantly increased in strips from obstructed bladders compared to the sham group is somewhat surprising. This could be explained by a reduced $\mathrm{KCl}$ response or a decreased acetylcholine esterase activity in obstructed bladder tissue. However, a change in receptor-effector coupling can not be excluded. 
Since the $\mathrm{pD}_{2}$ value did not differ in obstructed bladders, a supersensitivity to acetylcholine can be excluded.

The denervation of the obstructed bladder found in the functional part of the study was confirmed by histological examination of the bladders. Lots of PGP $9.5^{+}$ nerve profiles associated with the inner and outer muscle layer were found in the sham operated bladders. Quantitative determination of the relatively density of nerves revealed that less nerve profiles occurred in the detrusor layer of the obstructed bladders compared to the sham group. In obstructed bladders there is a patchy denervation. Some muscle bundles are completely denervated, while in other bundles sparce innervation is seen (Fig. 3). These findings are in keeping with previous reports demonstrating punctuate denervation in obstructed bladders. ${ }^{1,2}$ Most of the PGP 9.5 immunoreactive nerves in guinea pig bladders contain the enzyme capable of synthesising acetylcholine (choline acetyl transferase) therefore the majority of the PGP 9.5 positive fibres likely reflect postganglionic cholinergic nerves. ${ }^{17}$ However, some fibres contain neuropeptides. ${ }^{17}$ In this study we did not further elaborate on the different nerve types.

The mechanism(s) underlying the denervation in obstructed bladders have not been fully elucidated. One hypothesis has been put forward that the denervation is mediated by oxidative stress..$^{18} \mathrm{~A}$ recent study demonstrated that the endogenous antioxidant enzymes decrease in obstructed bladder tissue. ${ }^{19}$ Our study showed that the antioxidant capacity, as measured by the TEAC test, significantly decreased in obstructed bladders compared to sham operated bladders. This was partly due to a reduced amount of gluthathione (GSH) whereas uric acid did not decrease significantly (Fig. 4). Gluthathione is the most abundant nonprotein thiol and plays an important role in scavenging free radicals. Besides glutathione and uric acid other molecules such as vitamin $\mathrm{C}$, melatonin, and bilirubine can contribute to the TEAC value in this study. Since we have not determined these antioxidants in this study, the contribution of these molecules to the reduced antioxidant capacity can not be evaluated. Future studies are warranted to identify the majority of antioxidants responsible for the TEAC value in bladder tissue. Besides antioxidants, the endogenous antioxidant system of the human body also consists of antioxidant enzymes. For example, the enzyme gluthathione reductase recycles gluthathione disulphide (GSSG) to GSH and is therefore a key antioxidant enzyme. Our study demonstrated that the activity of glutathione reductase decreased significantly in the obstructed bladder tissue. This is in accordance with the results of a recent study which reports that the activity of the antioxidant enzymes superoxide dismutase and catalase were reduced in bladder tissue exposed to an outlet obstruction. ${ }^{19}$ The fact that the endogenous antioxidant system has been deteriorated suggests that obstructed bladders are exposed to elevated levels of RNOS.

The question remains if free radical mediated damage plays a significant role in the denervation and muscle dysfunction found in obstructed bladders. The role of oxidative injury in neuropathy and neurodegeneration has been documented in several human diseases. ${ }^{7}$ Indirect evidence for implicating oxidative stress comes 
from the protection by antioxidants and nitric oxide synthase inhibitors against the neurogenic and myogenic impairment of bladder tissue during bladder outlet obstruction. ${ }^{8,20}$ More direct evidence that oxidative stress plays a role in tissue injury of obstructed bladders comes from studies demonstrating that markers of oxidative stress are increased after partial bladder outlet obstruction. The nitrotyrosine level (end product of oxidative damage to proteins), malondialdehyde (MDA) and hydroxynonenal (HNE) level (end products of oxidative damage to lipids) are increased in these studies. ${ }^{9,21}$ This suggests a limited endogenous antioxidant capacity of bladders to counteract oxidative stress induced by outlet obstruction. The results of our study demonstrate that the antioxidant capacity is indeed decreased in obstructed bladders. The reduction of antioxidant capacity was accompanied by a lower nerve density in obstructed bladder tissue.

If oxidative stress plays a role in the tissue injury of obstructed bladders then direct application of free radicals to bladder tissue, over a relevant time period, at concentrations within the range found in vivo could reproduce some of the tissue injury observed in obstructed bladders. Functional in vitro studies show that application of RNOS damages the bladder contractile system. ${ }^{22-23}$ This correlates with the reduced detrusor contractility found in severely obstructed bladders. In our study of moderately obstructed bladders, the contractile system of the smooth muscle was not impaired, however a denervation was observed. Surprisingly, cholinergic nerves seem to be largely unaffected by in vitro exposure of the detrusor muscle to RNOS..$^{22-23}$ These conflicting data might be explained by the duration of in vitro experiments which may be too short for mediating nerve damage. Furthermore, oxidative stress imposed on the bladder muscle in vitro has a more acute nature that the oxidative stress that occurs in vivo. Thus, it is questionable if direct application of oxidative stress in vitro provides similar oxidative stress conditions mediated in vivo.

Future research on the time course of free radical formation, i.e. whether it precedes or is accompanied by bladder denervation and muscle dysfunction in models of bladder outlet obstruction, will further resolve the role of oxidative stress in the pathophysiology of obstructed bladders.

Controversial clinical evidence for the beneficial effects of antioxidant supplementation in patients with a bladder outlet obstruction comes from studies using plant extracts (phytotherapy) in the treatment of lower urinary tract symptoms (LUTS) associated with benign prostatic hyperplasia (BPH). The extract of the African prune tree, Pygeum Africanum (Tadenan) is one of these extracts and has been associated with antioxidative effects. ${ }^{24}$ Pygeum Africanum provides a moderately large improvement in LUTS compared to placebo in the treatment of men with $\mathrm{BPH}^{25}$ In addition, Pygeum Africanum can both prevent and reverse neurogenic and muscular bladder dysfunctions induced by partial outlet obstruction in rabbits. ${ }^{24}$ More research is required to establish whether antioxidant supplementation in patients with a bladder outlet obstruction is beneficial, eventually resulting in less voiding and or storage symptoms. 


\section{Conclusions}

This is to our knowledge, the first study in which the total antioxidant capacity was examined in an animal model of bladder outlet obstruction. The data demonstrate that the antioxidant status of guinea pig bladders exposed to partial outlet obstruction decreased which might be associated with the observed reduction in nerve density. The results strengthen the hypothesis that oxidative stress is involved in the pathophysiology of bladder dysfunction related to bladder outlet obstruction.

\section{References}

1. de Jongh $R$, van Koeveringe $G A$, van Kerrebroeck PE, Markerink-van Ittersum $M$, de Vente J, Gillespie JI. Alterations to network of NO/CGMP-responsive interstitial cells induced by outlet obstruction in guinea-pig bladder. Cell Tissue Res. 2007; 330(1):147-60.

2. Brading AF. A myogenic basis for the overactive bladder. Urology. 1997; 50(6A Suppl):57-67; discussion 68-73.

3. Andersson KE. Bladder activation: afferent mechanisms. Urology 2002; 59(5 Suppl 1):43-50.

4. de Jongh $R$, van Koeveringe $G A$, van Kerrebroeck PE, Markerink-van Ittersum $M$, de Vente $J$, Gillespie J1. Damage to the bladder neck alters autonomous activity and its sensitivity to cholinergic agonists. BJU Int. 2007; 100(4):919-29.

5. Greenland JE, Hvistendahl J], Andersen H, Jorgensen TM, McMurray G, Cortina-Borja M, et al. The effect of bladder outlet obstruction on tissue oxygen tension and blood flow in the pig bladder. BJU Int 2000; 85(9):1109-14.

6. Bratslavsky G, Kogan BA, Matsumoto $S$, Aslan AR, Levin RM. Reperfusion injury of the rat bladder is worse than ischemia. J Urol 2003; 170(5):2086-90.

7. Halliwell B. Oxidative stress and neurodegeneration: where are we now? J Neurochem. 2006; 97(6):1634-58.

8. Conners $W$, Whitebeck $C$, Chicester $P$, Legget $R$, Lin AD, Johnson $A$, et al. L-NAME, a nitric oxide synthase inhibitor, diminishes oxidative damage in urinary bladder partial outlet obstruction. Am J Physiol Renal Physiol. 2006; 290(2):F357-63. Epub 2005 Sep 20.

9. Mannikarottu A, Lin AD, Whitebeck C, Leggett R, Kogan B, Levin R. Effect of partial bladder outlet obstruction on nitrotyrosine levels and their correlation with contractile function. Neurourol Urodyn. 2006; 25(4):397-401.

10. Levin RM, Kawashima $Y$, Leggett RE, Whitbeck $C$, Horan P, Mizutani K. Effect of oral Kohki tea on bladder dysfunction induced by severe partial outlet obstruction. J Urol 2002; 167(5):22606.

11. Fischer MA, Gransier TJ, Beckers LM, Bekers O, Bast A, Haenen GR. Determination of the antioxidant capacity in blood. Clin Chem Lab Med. 2005; 43(7):735-40.

12. Smith PK, Krohn RI, Hermanson GT, Mallia AK, Gartner FH, Provenzano MD, et al. Measurement of protein using bicinchoninic acid. Anal Biochem. 1985; 150(1):76-85.

13. Griffith $\mathrm{OW}$. Determination of glutathione and glutathione disulfide using glutathione reductase and 2-vinylpyridine. Anal Biochem. 1980; 106(1):207-12.

14. Lux O, Naidoo D, Salonikas C. Improved HPLC method for the simultaneous measurement of allantoin and uric acid in plasma. Ann Clin Biochem. 1992; 29(Pt 6):674-5.

15. Geloso DA, Levin RM. Effect of partial outlet obstruction on the myogenic response to field stimulation. Gen Pharmacol 1998; 31(2):291-5.

16. Kato K, Wein AJ, Kitada S, Haugaard N, Levin RM. The functional effect of mild outlet obstruction on the rabbit urinary bladder. J Urol $1988 ; 140(4): 880-4$. 
17. Gillespie JI, Markerink-van Ittersum M, De Vente J. Interstitial cells and cholinergic signalling in the outer muscle layers of the guinea-pig bladder. BJU Int. 2006; 97(2):379-85.

18. Levin RM, Levin SS, Zhao Y, Buttyan R. Cellular and molecular aspects of bladder hypertrophy. Eur Urol. 1997; 32(Suppl 1):15-21.

19. Guven A, Kalorin C, Onal B, Whitbeck C, Chichester P, Kogan BA, et al. Novel biomarkers of bladder decompensation after partial bladder obstruction. Neurourol Urodyn. 2007; 26(7):1036-42.

20. Parekh $M H$, Lobel $R, O^{\prime}$ Connor LJ, Leggett RE, Levin RM. Protective effect of vitamin $E$ on the response of the rabbit bladder to partial outlet obstruction. J Urol 2001; 166(1):341-6.

21. Lin AT, Yang CH, Chen KK, Chang LS. Detrusor mitochondrial lipid peroxidation and superoxide dismutase activity in partial bladder outlet obstruction of rabbits. Neurourol Urodyn 2005; 24(3):282-7.

22. de Jongh $R$, Haenen GR, van Koeveringe GA, Dambros M, van Kerrebroeck PE. Lipid peroxidation product 4-hydroxynonenal contributes to bladder smooth muscle damage. Urology. 2008; 71(5):974-8.

23. de Jongh $R$, Haenen $G R$, van Koeveringe GA, Dambros M, De Mey JG, van Kerrebroeck PE. Oxidative stress reduces the muscarinic receptor function in the urinary bladder. Neurourol Urodyn. 2007; 26(2):302-8.

24. Edgar $A D$, Levin $R$, Constantinou CE, Denis $L$. A critical review of the pharmacology of the plant extract of Pygeum africanum in the treatment of LUTS. Neurourol Urodyn. 2007; 26(4):458-63; discussion 464.

25. Wilt T, Ishani A, Mac Donald R, Rutks I, Stark G. Pygeum africanum for benign prostatic hyperplasia. Cochrane Database Syst Rev. 2002(1):CD001044. 


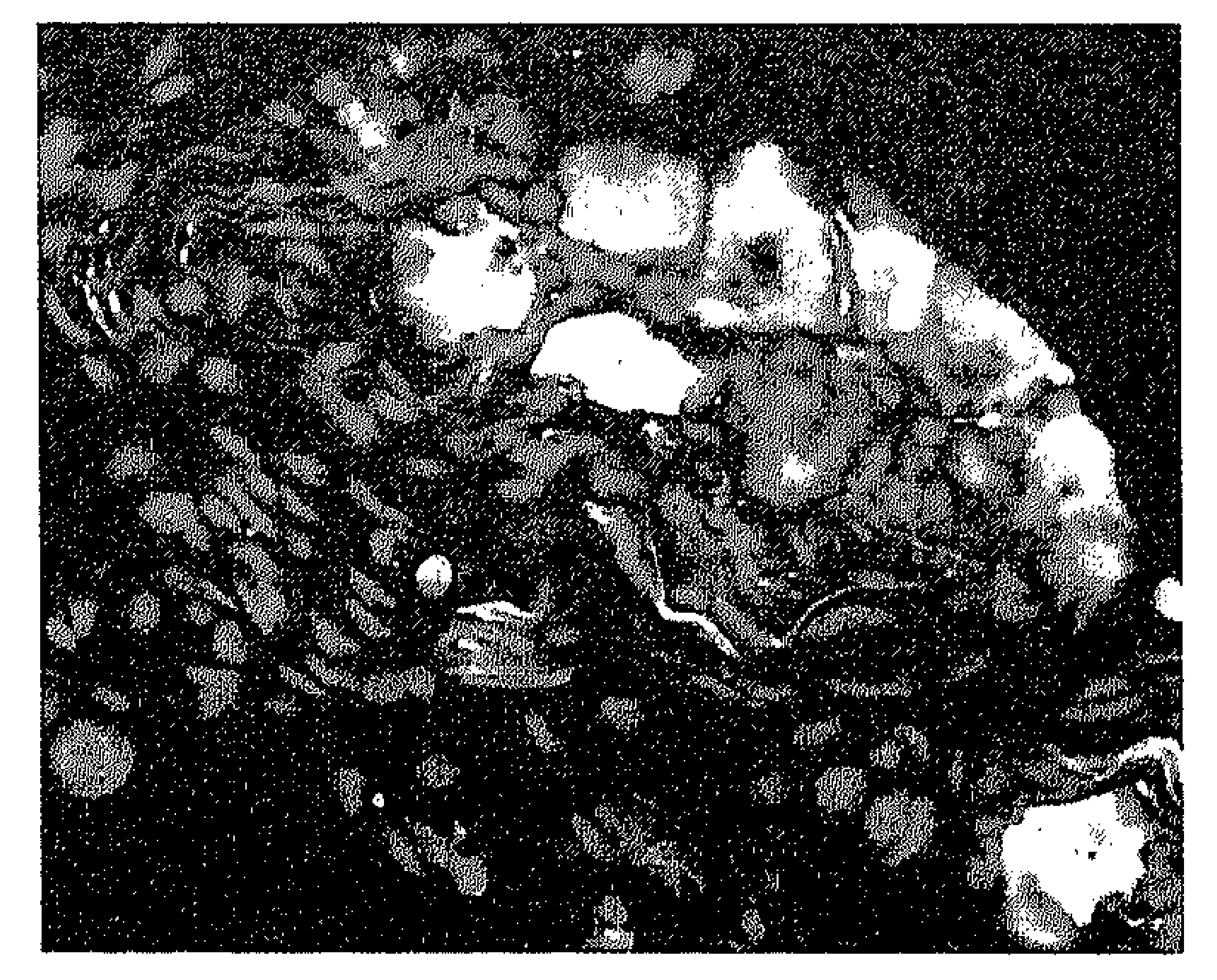

Oxidative stress reduces the muscarinic receptor function in the urinary bladder

Rik de Jongh, Guido R.M.M. Haenen, Gommert A. van Koeveringe, Miriam Dambros, Jo G.R. De Mey, Philip E.V. van Kerrebroeck.

Neurourology and Urodynamics $2007 ; 26(2): 302-8$. 


\section{Abstract}

Aims: Several pathophysiological conditions in the urinary bladder e.g. ischemia/reperfusion and inflammation are characterized by the formation of reactive oxygen species. The reactive oxygen species are highly toxic because they can destroy proteins, DNA and lipids. The aim of this study was to investigate the effect of oxidative stress on excitation-contraction coupling of detrusor smooth muscle.

Materials and Methods: Smooth muscle strips were dissected from pig urinary bladder and mounted in organ baths. Oxidative stress was mimicked by the addition of cumene hydroperoxide, a lipophilic hydroperoxide, to the organ baths. Contractile responses to electrical field stimulation (EFS: $4-32 \mathrm{~Hz}$ ), carbachol $\left(10^{-8}-3.10^{-5} \mathrm{M}\right)$, potassium $(65.3 \mathrm{mM})$ and ATP $(1 \mathrm{mM})$ were monitored before and after the addition of cumene hydroperoxide.

Results: Responses of detrusor strips to EFS were for the greater part based on neurogenic stimulation and the release of acetylcholine. Cumene hydroperoxide diminished contractile responses to EFS and carbachol to the same extent. The $\mathrm{pD}_{2}$ value of the carbachol concentration response curve decreased significantly after exposure to $0.1 \mathrm{mM}, 0.4 \mathrm{mM}, 0.8 \mathrm{mM}$ cumene hydroperoxide. Furthermore the maximal effect obtained with carbachol was significantly reduced after $0.1 \mathrm{mM}, 0.4$ $\mathrm{mM}$ and $0.8 \mathrm{mM}$ cumene hydroperoxide treatment. Contractions induced by potassium and ATP were significantly less affected by oxidative stress compared to EFS- and carbachol-induced responses of comparable amplitude.

Conclusions: The results of our study demonstrate that oxidative stress induced by cumene hydroperoxide affects pig bladder contractility. The muscarinic receptor signalling system is severely damaged. L-type calcium channels and the contractile system are less affected and cholinergic nerves remain largely unaffected. 


\section{Introduction}

Several disease states or pathological situations in the urinary bladder are characterized by the formations of reactive oxygen species (ROS). Animal models of bladder outlet obstruction indicate that ischemia/reperfusion injury is an important etiological factor in the progression of bladder muscle dysfunction. The increase in bladder wall thickness and intravesical pressure seen in obstructed bladders, result in significantly greater and longer lasting periods of ischemia and reperfusion during bladder contraction and emptying. ${ }^{1-3}$ Bratslavsky et al showed that reperfusion injury of the bladder is more detrimental than ischemia alone. ${ }^{4}$ Especially during the reperfusion period, reactive oxygen species (ROS) are formed. Examples of reactive oxygen species are superoxide anion $\left(\mathrm{O}_{2}{ }^{*}\right)$, hydroxyl radical $\left(\mathrm{OH}^{*}\right)$, hydrogen peroxide $\left(\mathrm{H}_{2} \mathrm{O}_{2}\right)$, hypochlorous acid $(\mathrm{HOCl})$ and peroxynitrite ( $\left.\mathrm{ONOO}^{-}\right)$. Nitric oxide synthase inhibitors can diminish smooth muscle dysfunction due to outlet obstruction or ischemia-reperfusion, suggesting that these pathological situations are related to nitric oxide and the generation of reactive oxygen species. ${ }^{5,6}$

Bladder inflammation, in patients as well as experimentally, can result in dysfunction of the smooth muscle. ${ }^{7-9}$ Bladder inflammation is characterized by infiltration of neutrophils, macrophages, mast cells and lymphocytes in the wall. ${ }^{10-12}$ Phagocytic leukocytes contain nicotinamide-adenine dinucleotide phosphate (NADPH) oxidase. NADPH oxidase reduces molecular oxygen to superoxide anion. Dismutation of superoxide radical by superoxide dismutase (SOD) produces hydrogen peroxide $\left(\mathrm{H}_{2} \mathrm{O}_{2}\right)$. Activated neutrophils secrete the enzyme myeloperoxidase, which catalyzes the formation of hypochlorous acid from hydrogen peroxide and chloride ions. ${ }^{13}$

In situations such as ischemia-reperfusion and inflammation, free radical production is enhanced and radicals may overwhelm the antioxidant defence system. A disbalance between radical formation and protection is defined as oxidative stress. Oxidative stress in biological tissue is regarded as highly toxic because free radicals are able to destroy virtually any molecule, including proteins, DNA and lipids. Oxidative damage to lipids is known as lipid peroxidation. It has been reported that bladder outlet obstruction and acute urinary retention followed by bladder decompression enhances lipid peroxidation. ${ }^{14,15}$

Cumene hydroperoxide (CHP) is a lipophilic hydroperoxide. It is used to mimic the effect of lipid hydroperoxides. Cumene hydroperoxide has been used for experimental oxidative stress in the cardiovascular, ${ }_{1}^{16,17}$, tracheal $^{18}$ and gastrointestinal systems. ${ }^{19}$ These studies showed that oxygen radicals can disturb smooth muscle function. The aim of this functional study was to determine which component in the stimulation pathway of detrusor smooth muscle (cholinergic nerves, membrane-bound receptors, intracellular contractile mechanisms) is most sensitive to oxidative stress. Furthermore it was evaluated if cumene hydroperoxideinduced peroxidation mimics the muscle dysfunction mediated by partial outlet obstruction or inflammation of the bladder. 


\section{Materials and methods}

\section{Tissue preparation}

Experiments were performed on pig urinary bladders obtained from the local slaughterhouse approximately 30 minutes after slaughter. Strips of $2 \times 2 \mathrm{~cm}$ were cut from the anterolateral wall of the bladder dome and transported to the laboratory in oxygenated Krebs solution $\left(\mathrm{NaCl}, 118 \mathrm{mmol} / \mathrm{l} ; \mathrm{KCl}, 5.6 \mathrm{mmol} / \mathrm{l} ; \mathrm{NaHCO}_{3}, 25\right.$ $\mathrm{mmol} / \mathrm{l} ; \mathrm{KH}_{2} \mathrm{PO}_{4}, 1.2 \mathrm{mmol} / \mathrm{l} ; \mathrm{CaCl}_{2}, 2.5 \mathrm{mmol} / \mathrm{l} ; \mathrm{MgSO}_{4}, 1.2 \mathrm{mmol} / \mathrm{l} ;$ glucose 6.1 $\mathrm{mmol} / \mathrm{l}$; $\mathrm{pH} 7.4$; aerated with $95 \% \mathrm{O}_{2} / 5 \% \mathrm{CO}_{2}$ ). A high $\mathrm{KCl}(125 \mathrm{mM}$ ) solution was prepared by replacing $\mathrm{NaCl}$ by an equimolar amount of $\mathrm{KCl}$. Afterwards the high $\mathrm{KCl}$ solution was diluted with normal Krebs resulting in a concentration of $\mathrm{KCl} 65.3 \mathrm{mM}$. The mucosal layer and connective tissue were removed using a binocular microscope, and strips of $0.5 \mathrm{~mm}$ diameter and length between 7 and $10 \mathrm{~mm}$ were excised. To facilitate diffusion, the thin layer of connective tissue covering the muscle fibre was opened and for the greater part removed. Care was taken that the muscle fibres were running longitudinally.

Fine silk sutures were tied to each end of the strips, one end was fixed to the tissue holder and the other end to the isometric force transducer (Kistler Morce DSC 6, Seattle, WA). The force measurements were stored digitally. Each strip was placed in a double jacketed $6-\mathrm{ml}$ organ bath filled with Krebs-buffer solution at $37{ }^{\circ} \mathrm{C}$, constantly bubbled with a mixture of $95 \% \mathrm{O}_{2}$ and $5 \% \mathrm{CO}_{2}$. Measurements were started after an equilibration period of $60 \mathrm{~min}$ with four intermediate changes of buffer solution. An initial tension of $20 \mathrm{mN}$ was placed on each strip.

\section{Experimental protocol}

Frequency response curves to electrical field stimulation (EFS) were constructed by stimulating bladder strips via platinum electrodes. The electrodes were placed above and under the bladder strips and were connected to the HM8130 Function Generator from Hameg ${ }^{\circledR}$ Instruments, Manhausen, Germany. Electrical field stimulation ( $50 \mathrm{~V}, 2 \mathrm{msec}$ pulse width, $10 \mathrm{sec}$ train pulses) was applied at frequencies of $2,4,8,16$ and $32 \mathrm{~Hz}$, trains being separated from each other by at least 3 minutes. To test the effect of oxidative stress, bladder strips were incubated with 0.1 $\mathrm{mM}, 0.4 \mathrm{mM}$ and $0.8 \mathrm{mM}$ cumene hydroperoxide (CHP) for 30 minutes. After incubation with $\mathrm{CHP}$, strips were thoroughly washed during 30 minutes and additional EFS were repeated in the same order of frequencies as before incubation. During the washing period the Krebs buffer in the organ bath was refreshed every 5 minutes. In order to characterize the electrical evoked responses, strips were treated with tetrodotoxin $10^{-6} \mathrm{M}$ (contact time $15 \mathrm{~min}$ ) and atropine $10^{-6} \mathrm{M}$ (contact time $15 \mathrm{~min}$ ).

To evaluate the effect of CHP on muscarinic receptors, dose response curves to carbachol $\left(10^{-8}-3 \times 10^{-5} \mathrm{M}\right)$ before and after exposure to CHP were constructed. Furthermore the effects of CHP on contractile responses to membrane depolarisation and purinergic receptor stimulation were evaluated by determining the responses evoked by potassium $(\mathrm{KCl} 65.3 \mathrm{mM})$ or ATP $(1 \mathrm{mM})$ before and after CHP treatment. 


\section{Chemicals}

Carbachol, adenosine triphosphate, atropine sulphate, tetrodotoxin (TTX), and cumene hydroperoxide (CHP) were obtained from Sigma Chemical Co (Utrecht, The Netherlands). All solutions were freshly prepared daily. Drugs were dissolved in deionized distilled water; cumene hydroperoxide was directly dissolved in Krebs solution. Further dilutions were carried out in Krebs solution. The concentrations are expressed as the final molar concentration in the tissue chamber.

\section{Statistical evaluation}

Results are expressed as means \pm SEM of measurements in $n$ strips. In each protocol, the strips were obtained from at least four different animals, and a maximum of two strips per bladder was used. Contractile responses are expressed as absolute values $(\mathrm{mN})$ or as percentage of the control (max) response. Concentration-response data of carbachol were evaluated by sigmoid curve fitting and $-\log \mathrm{EC}_{50}$ values $\left(\mathrm{pD}_{2}\right)$ were calculated by non-linear regression analysis using GraphPad Prism. Differences between mean values were statistically analysed using analysis of variance followed by the Newman-Keuls test. The time controls are included in the statistical evaluation. A probability value of $p<0.05$ was regarded to be significant. All analyses were performed using GraphPad Prism software (version 4.0; Graphpad Software Inc., San Diego, CA).

\section{Results}

Incubation of strips in oxygenated Krebs solution without CHP treatment did not have an effect on the responses mediated by various stimulation pathways.

Electrical field stimulation (EFS) of bladder strips caused a rapid rise in isometric tension and this contractile response was dependent on the frequency of the EFS in the range of $4-32 \mathrm{~Hz}$. The force development at 4 and $8 \mathrm{~Hz}$ were significantly smaller compared to the $16 \mathrm{~Hz}$ response $(p<0.001)$ and subsequently the response at $16 \mathrm{~Hz}$ was significantly lower compared to the force development at $32 \mathrm{~Hz}$ ( $\mathrm{p}<$ 0.05 ) (Fig. 1).

Electrical field stimulation can excite detrusor smooth muscle directly. Direct muscle stimulation was investigated using the neurotoxin tetrodotoxin (1 uM). The responses to EFS at $4-32 \mathrm{~Hz}$ were reduced after neurotoxin treatment ( $1 \mathrm{uM}$, contact time $15 \mathrm{~min}$ ) by $66 \%-81 \%$. This indicates that a small part of the contraction (34\% - 19\%) was not due to nerve stimulation but to direct muscle stimulation. Responses to EFS at $2-4 \mathrm{~Hz}$ had a detectable atropine-resistant, nerve mediated component (Fig. 1). This suggests that the nerve evoked responses at 2 $4 \mathrm{~Hz}$ resulted for a substantial part from the release of other, putatively identified as purinergic, transmitter substances. At frequencies of $8-32 \mathrm{~Hz}$ the TTX-resistant responses were not different from the atropine resistant contraction indicating that the nerve evoked responses were predominantly due to the release of acetylcholine 
and there seems to be no purinergic component detectable.

Treating the strips with CHP resulted in a significant reduction of the contractions induced by EFS compared to time controls $(p<0.05)$. The effect of CHP was dose dependant, concentrations of $0.4 \mathrm{mM}$ and $0.8 \mathrm{mM}$ CHP reduced the EFS-induced responses significantly more compared to $0.1 \mathrm{mM}$ CHP (Fig. $2 \mathrm{~A}$ ). Figure $2 \mathrm{~B}$ shows that the reduction of the contractile response is more pronounced at a lower frequency. Figure 3 shows the effect of increasing concentrations of cumene hydroperoxide on the dose response curves of detrusor strips to carbachol. Carbachol in concentrations ranging from $10^{-8}$ to $3.10^{-5} \mathrm{M}$ caused a rise in isometric tension in a dose-dependent fashion. The $\mathrm{pD}_{2}$ value of carbachol in strips without $\mathrm{CHP}$

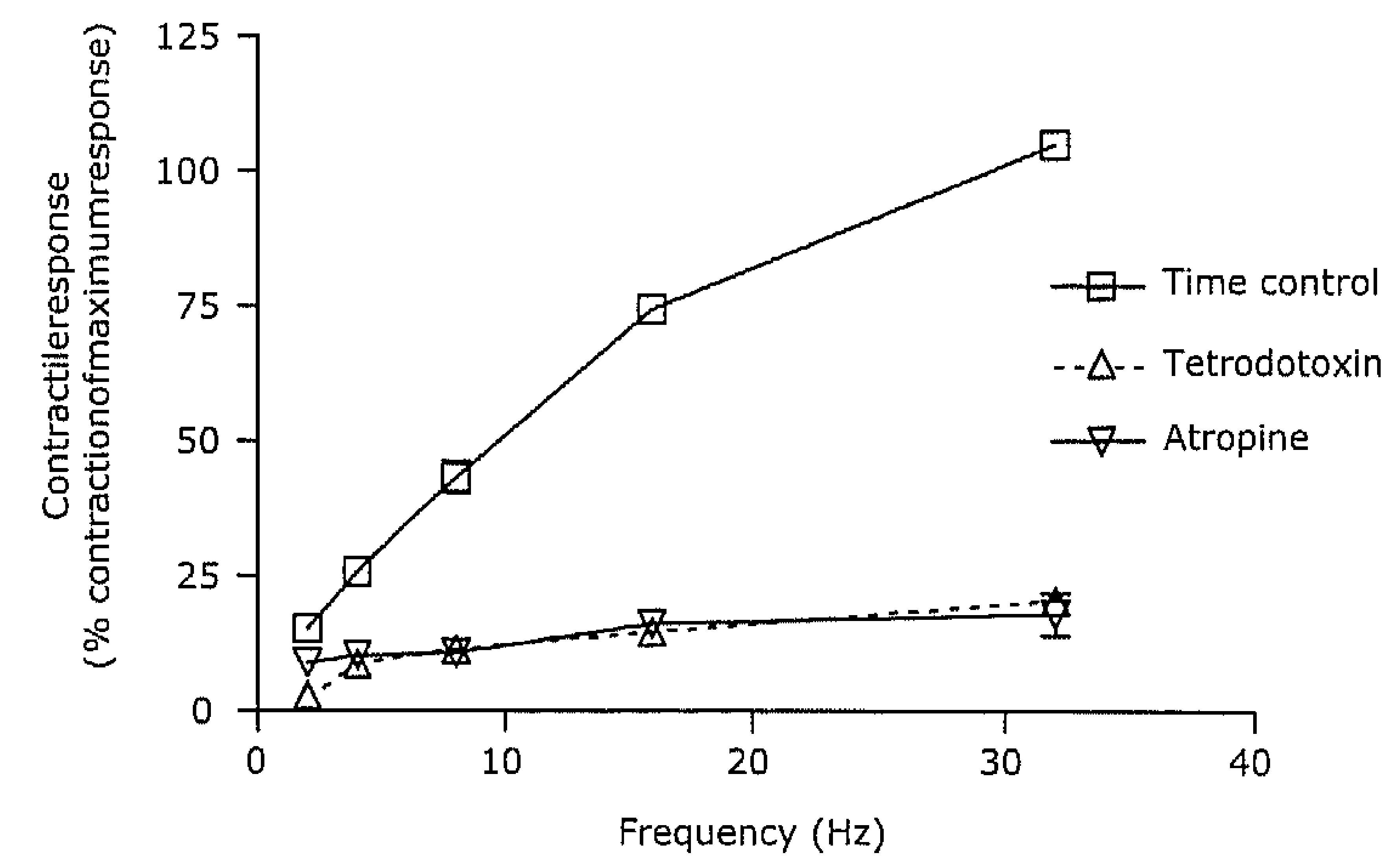

Figure 1.

Frequency $(\mathrm{Hz})$

Effect of tetrodotoxin and atropine on the frequency response curve of pig detrusor strips to electrical field stimulation. Each point represents the mean \pm SEM of $6-8$ bladder strips.

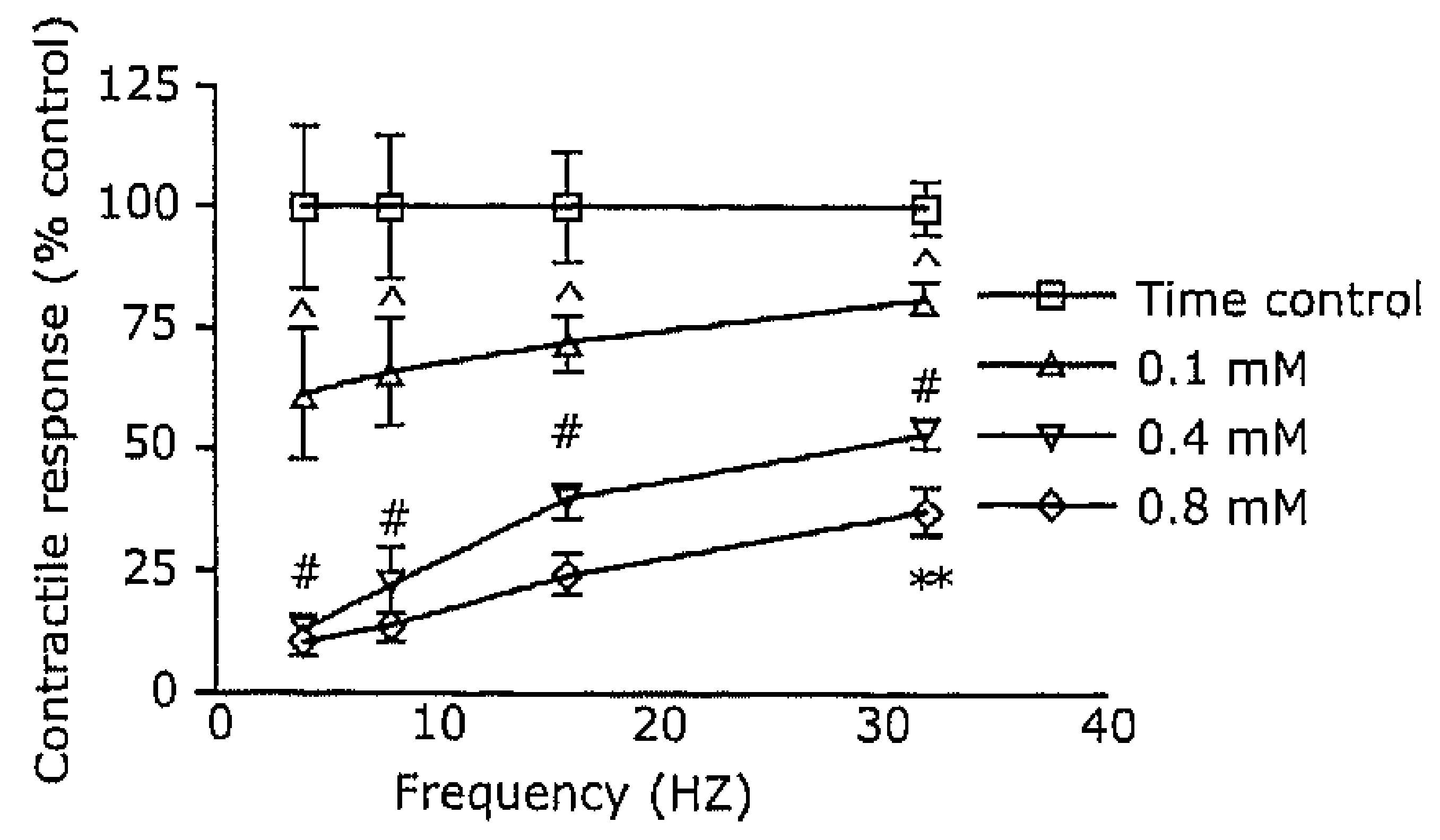

Figure 2.

The effect of different concentrations of cumene hydroperoxide (CHP) on electrical field stimulation evoked contractions $(4,8,16,32 \mathrm{~Hz})$. Each point represents the mean \pm SEM of $7-8$ bladder strips. A/ Frequency dependent effect of cumene hydroperoxide. $\wedge p<0.05$ versus time control, ${ }^{*} p$

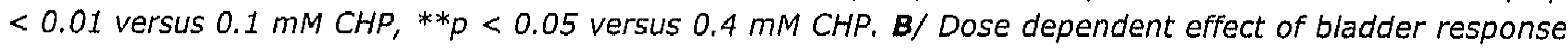
to cumene hydroperoxide exposure. ${ }^{+} p<0.05$ versus $32 \mathrm{~Hz}$ response, ${ }^{*} p<0.01$ versus $16 \mathrm{~Hz}$ response. 


\section{Figure 3.}

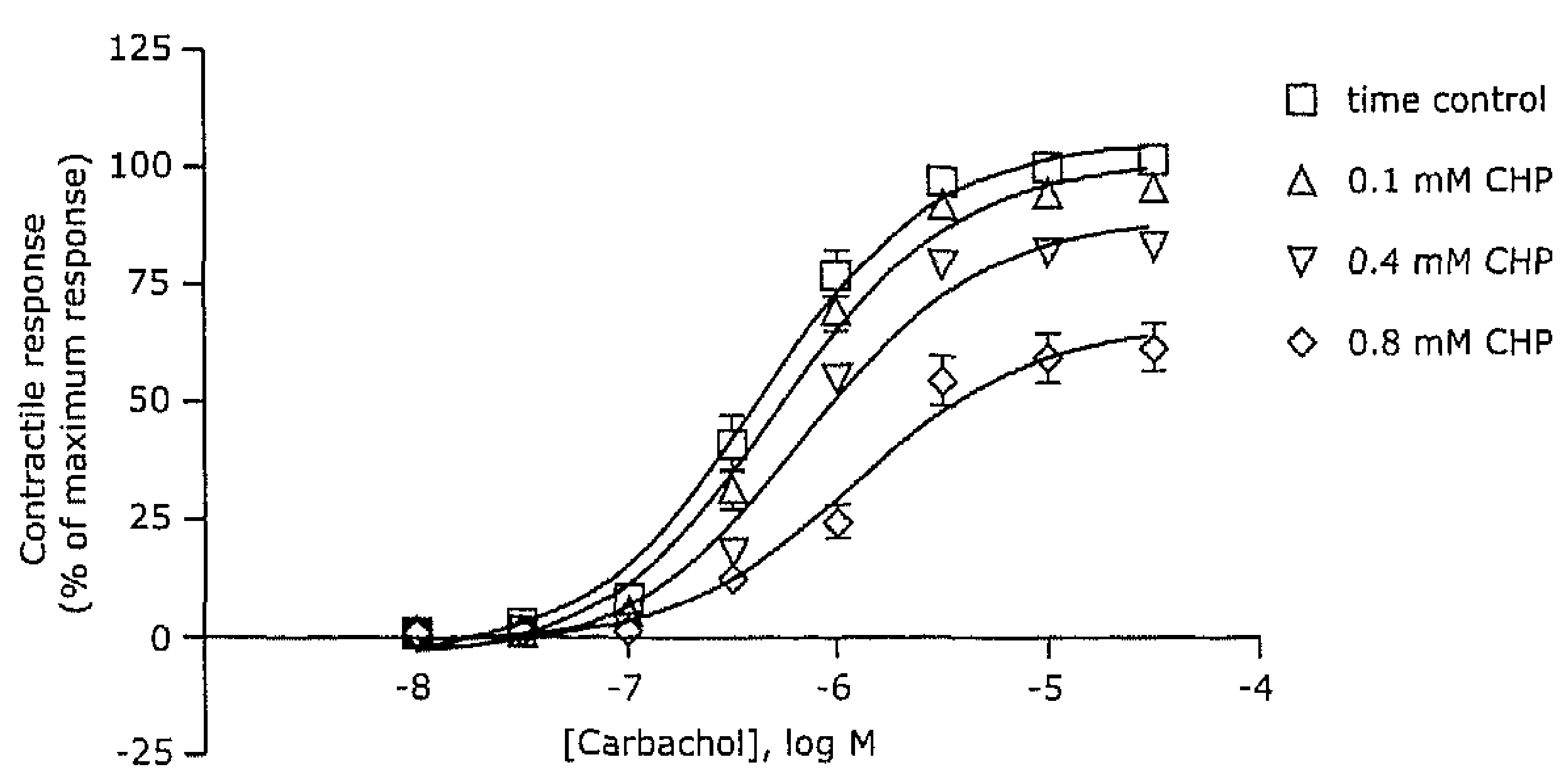

Effect of $0.1,0.4$ and $0.8 \mathrm{mM}$ cumene hydroperoxide on carbachol dose response curve of pig detrusor strips. Each bar is the mean \pm SEM of $8-10$ bladder strips. The $p D_{2}$ values of time control curve and of curves after exposure to $0.1,0.4$ and $0.8 \mathrm{mM}$ cumene hydroperoxide, were $6.37 \pm 0.02,6.29 \pm 0.03$ $(p<0.05), 6.12 \pm 0.03(p<0.001)$ and $5.91 \pm 0.03(p<0.001)$, respectively. The maximal effect (Emax) was significantly reduced after $0.1 \mathrm{mM}(p<0.01), 0.4 \mathrm{mM}(p<0.001)$ and $0.8 \mathrm{mM}(p<0.001)$ cumene hydroperoxide treatment.

treatment was $6.37 \pm 0.02$. Preincubation of strips with CHP significantly increased the concentration of carbachol needed to produce half maximal effect. The $\mathrm{pD}_{2}$ significantly decreased after exposure to $0.1 \mathrm{mM}, 0.4 \mathrm{mM}$ and $0.8 \mathrm{mM}$ CHP compared to time controls to $6.29 \pm 0.03,(p<0.05) 6.12 \pm 0.03(p<0.001)$ and $5.91 \pm 0.03$ $(p<0.001)$, respectively. The maximal effect obtained with carbachol was significantly reduced after $0.1 \mathrm{mM}, 0.4 \mathrm{mM}$ and $0.8 \mathrm{mM} \mathrm{CHP} \mathrm{treatment} \mathrm{(Fig.} \mathrm{3).}$

In control bladder strips, the amplitude of the force development induced by EFS $32 \mathrm{~Hz}$, carbachol $0.5 \mu \mathrm{M}$ or $\mathrm{KCl} 65.3 \mathrm{mM}$ stimulation were practically identical (Fig. 4, inserted figure.). The same applies to contractile response to EFS $8 \mathrm{~Hz}$, carbachol $0.1 \mu \mathrm{M}$ and ATP $1 \mathrm{mM}$ stimulations (Fig. 5, inserted figure.). We found that the $\mathrm{KCl}$ mediated contractions were significantly less affected compared to the corresponding EFS and $\mathrm{CCH}$ induced responses $(p<0.05)$. The responses to EFS and $\mathrm{CCH}$ were affected to a similar degree (Fig. 4). Purinergic (ATP) evoked contractions were also significantly less reduced compared to the corresponding EFS and $\mathrm{CCH}$ induced responses $(p<0.001)$. The corresponding neurogenic and cholinergic contractions were again affected both to a similar degree (Fig. 5).

\section{Discussion}

Efferent autonomic nerves play a crucial role in mediating a micturition contraction. The smooth muscle contraction of the urinary bladder in response to nerve stimulation is predominantly mediated by the release of the neurotransmitters acetylcholine and ATP. The purinergic component plays a major role in some animals however there are differences between species. In for example rabbits, rats and 

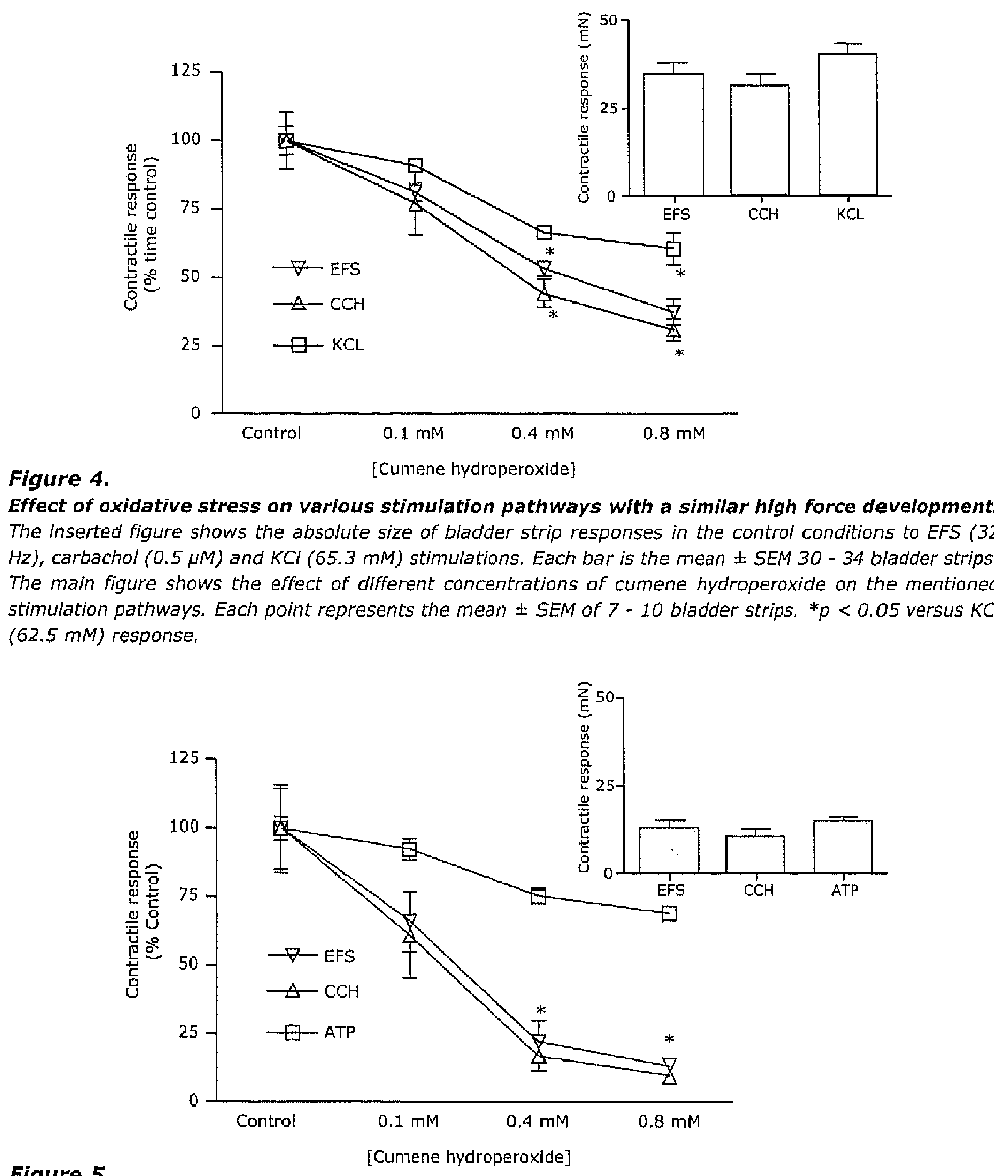

Figure 5.

[Cumene hydroperoxide]

Effect of oxidative stress on various stimulation pathways with similar low force developments The inserted figure shows the absolute size of bladder strip responses in the control conditions to EFS ( $8 \mathrm{~Hz})$ carbachol $(0.1 \mu \mathrm{M})$ and ATP (1 mM) stimulations. Each bar is the mean \pm SEM $30-34$ bladder strips. The main figure shows the effect of different concentrations of cumene hydroperoxide on the mentionec stimulation pathways. Each point represents the mean \pm SEM of 7 - 10 bladder strips. ${ }^{*} p<0.001$ versu. $\operatorname{ATP}(1 \mathrm{mM})$ response. 
guinea pigs, the purinergic component plays a major role. ${ }^{20,21}$ In other species, like the pig and human, the purinergic component plays a minor role especially in responses induced by high frequencies of electrical field stimulation. ${ }^{21}$

The effect of tetrodotoxin found in the present study using pig urinary bladder demonstrated that EFS-induced responses were for the greater part based on neurogenic stimulation. In agreement with $S_{i b l e y}{ }^{21}$ we found that in pig bladder, neurogenic responses to high frequencies of electrical stimulation result predominantly from the release of acetylcholine. In our hands, responses to frequencies of $2-4 \mathrm{~Hz}$ of electrical stimulation also resulted from the release of other, probably purinergic, transmitter substances (Fig. 1). Furthermore we found that contractile responses at $4 \mathrm{~Hz}$ and $8 \mathrm{~Hz}$ of electrical stimulation were significantly smaller compared to $16 \mathrm{~Hz}$ and $32 \mathrm{~Hz}$ stimulation which indicates a frequency dependent acetylcholine release. This is in accordance with Inadome et al. who quantified the acetylcholine release from rabbit detrusor smooth muscle strips. ${ }^{22}$ They found that the amount of released acetylcholine was indeed dependent on the frequency of stimulation between $2-30 \mathrm{~Hz}$, and correlated with the observed contraction of the muscle.

The main aim of our functional study was to examine the effect of oxidative stress induced by cumene hydroperoxide on bladder contractility and determine indirectly, which component in the stimulation pathway (cholinergic nerves, membrane-bound receptors and intracellular contractile machinery) is most sensitive to oxidative stress.

In the control situation it was found that bladder strip responses to EFS $8 \mathrm{~Hz}$ and $32 \mathrm{~Hz}$ were similar to those induced by $0.1 \mu \mathrm{M}$ and $0.5 \mu \mathrm{M}$ carbachol, respectively (Figs. 4-5, inserted figures). After CHP treatment, the contractile responses to EFS 8 $\mathrm{Hz}$ and $0.1 \mu \mathrm{M}$ carbachol were reduced to a comparable extent (Fig. 5). The same applies to contractile responses to EFS $32 \mathrm{~Hz}$ and $0.5 \mu \mathrm{M}$ carbachol (Fig. 4). This suggests that CHP did not have an effect on the release of endogenous acetylcholine from postganglionic nerves at $8 \mathrm{~Hz}$ and $32 \mathrm{~Hz}$ of electrical stimulation. It is therefore concluded that the cholinergic nerves remain largely unaffected by CHP. The reduced response to EFS by CHP is therefore likely due to a damaging effect on postsynaptic muscarinic receptors and/or the signal-transduction mechanisms which they engage.

In pig urinary bladder, the contractile response to a muscarinic receptor agonist, such as carbachol, has been attributed predominantly to $M_{3}$ receptors. In the positive inotropic response to muscarinic receptor agonist in the bladder a cascade of biochemical reactions are involved. During $M_{3}$ receptor activation, the receptoragonist complex couples to a G-protein that subsequently activates phopholipase $C$ and leads to mobilization of extra-and intracellular calcium ions eventually leading to a pharmacological response. ${ }^{23}$ One of the features of such a cascade is that the stimulus generated by receptor activation is amplified. In this way a relatively small fraction of the receptors has to be occupied by a full agonist to mediate a maximal response. It has been reported that "receptor reserve" exist in the bladder for carbachol. ${ }^{24}$ In our study we found that CHP treatment decreased the $\mathrm{pD}_{2}$ value of 
the carbachol concentration response curve. $A$ decrease in the $\mathrm{pD}_{2}$ value means that a higher concentration of carbachol is necessary to induce a particular contraction. It implies that the muscarinic receptors or the amplification reactions are damaged by cumene hydroperoxide. Indeed it seems that $\mathrm{G}$-proteins, coupled to $M_{3}$ receptors are sensitive to oxidative stress mediated damage. ${ }^{25,26}$

Besides a reduction of the $\mathrm{PD}_{2}$ value, oxidative stress induced by CHP reduced the maximal effect to muscarinic receptor stimulation. $M_{3}$ receptor stimulation will result in a contraction, which is largely dependent by $\mathrm{Ca}^{2+}$ influx through L-type, voltage dependant channels and activation of Rho kinase. Mobilization of calcium from intracellular stores via the IP3 pathway plays a minor role. ${ }^{23} \mathrm{~A}$ rise in intracellular $\mathrm{Ca}^{2+}$ will activate the contractile machinery. As cumene hydroperoxide reduced the maximal effect to carbachol stimulation it is likely that peroxidation may damage the membrane function (L-type calcium channels) and/or the contractile apparatus. In order to clarify this, we compared the effects of CHP on EFS, carbachol and $\mathrm{KCl}$ responses at similar amplitudes of force development (Fig. 4). It was found that the $\mathrm{KCl}$ mediated responses were the least affected by oxidative stress. Potassium depolarizes the membrane and triggers $\mathrm{Ca}^{2+}$ entry through L-type calcium channels resulting in elevated intracellular $\mathrm{Ca}^{2+}$ concentrations. Furthermore ATP mediated responses were relatively undisturbed by CHP treatment (Fig. 5). ATP in the bladder acts on the purinoceptor $P 2 X_{1}$. Activation of the $P 2 X_{1}$ receptors opens non-selective cation channels, which will depolarise the cells. Depolarisation activates $\mathrm{Ca}^{2+}$ influx through L-type calcium channels and finally will result in a contractile response of the muscle. ${ }^{27}$ The slight effect of CHP on $\mathrm{KCl}$ and ATP responses suggests that L-type calcium channels and or the contractile machinery of smooth muscle are relatively resistant to oxidative stress induced damage.

The results of our in vitro study demonstrate that peroxidation induced by cumene hydroperoxide severely damages the cholinergic response. The L-type calcium channels and the contractile system are only slightly affected while cholinergic nerves seem largely unaffected. Although it is questionable if direct application of cumene hydroperoxide provides a similar peroxidation condition found in vivo situations, we have correlated our results with functional abnormalities found in bladder smooth muscle tissue exposed to an outlet obstruction or inflammation. Several studies have focussed on the effects of bladder outlet obstruction on detrusor smooth muscle. Functional studies performed in obstructed bladders have demonstrated a significantly greater decrease in the contractile response to field stimulation compared to either muscarinic receptor stimulation or membrane depolarisation by $\mathrm{KCl}^{28,29}$ The denervation due to outlet obstruction has been associated with a relative supersensitivity to cholinergic agonists. ${ }^{30}$ On the other hand some studies reported that obstructed bladders do not show a supersensitivity ${ }^{30,31}$ The conflicting data on supersensitivity to cholinergic agonists might be explained by difference in duration and/or degree of urethral obstruction resulting in variations in the extent of ischemia and reperfusion and subsequently in the extent of oxidative stress. Severe ischemia and reperfusion of the bladder results 
in a reduction of both the efficacy and the sensitivity to cholinergic stimulation. ${ }^{15,32}$ Moderate degrees of bladder ischemia are associated with increased contractile responses to carbachol. ${ }^{33}$ This may be explained by a denervation induced supersensitivity. The results of our cumene hydroperoxide model correlates with functional bladder tissue damage due to severe ischemia and reperfusion.

In contrast to bladder outlet obstruction, not much attention has been paid to smooth muscle damage secondary to bladder inflammation. Palea et al reported that the cholinergic responses in patients with interstitial cystitis were more affected than in control patients. In bladder strips from patients with interstitial cystitis, higher concentrations of acetylcholine were necessary to induce maximal effect. ${ }^{9}$ This indicates muscarinic receptor signalling damage due to relatively severe oxidative stress in bladder inflammation.

The results of our functional study demonstrate that peroxidation induced by cumene hydroperoxide severely damages the cholinergic responses of the bladder tissue. Functional studies of severe ischemia/reperfusion and inflammation of the bladder indeed show that the muscarinic receptor signalling is affected. Peroxidation of membrane lipids may contribute to the receptor damage..$^{34}$ Our study furthermore shows that nerves remain largely unaffected. The finding of nerve resistibility is surprising as is suggested that denervation is one of the initial degenerative events resulting from lipid peroxidation. Aikawa et al investigated the effect of hydrogen peroxide (one of the reactive oxygen species) on the contractility of rat bladders. They found that contractile responses to $\mathrm{KCl}$ were inhibited to a significantly greater degree by hydrogen peroxide $(18-290 \mathrm{mM})$ than contractile responses to nerve stimulation. ${ }^{35}$ The location of intrafascicular nerve fibers within a muscle bundle might explain the relative low susceptibility of the cholinergic nerves to externally added cumene hydroperoxide and hydrogen peroxide. Furthermore it may be that the duration of our in vitro experiment is too short for mediating nerve damage.

In summary, the current experiments show that cumene hydroperoxide reduces pig bladder contractility, primarily as a result of damage to the muscarinic receptor signalling system. L-type calcium channels and the contractile system are less affected. These results correlate with functional bladder tissue abnormalities observed after severe ischemia/reperfusion and after bladder inflammation. 


\section{References}

1. Azadzoi KM, Pontari M, Vlachiotis ], Siroky MB. Canine bladder blood flow and oxygenation: changes induced by filling, contraction and outlet obstruction. J Urol 1996; 155(4):1459-65.

2. Greenland JE, Hvistendahl JJ, Andersen H, Jorgensen TM, MCMurray G, Cortina-Borja M, et al. The effect of bladder outlet obstruction on tissue oxygen tension and blood flow in the pig bladder. BJU Int 2000; 85(9):1109-14.

3. Greenland JE, Brading AF. The effect of bladder outflow obstruction on detrusor blood flow changes during the voiding cycle in conscious pigs. J Urol 2001; 165(1):245-8.

4. Bratslavsky G, Kogan BA, Matsumoto $S$, Aslan AR, Levin RM. Reperfusion injury of the rat bladder is worse than ischemia. J Urol 2003; 170(5):2086-90.

5. Conners $W$, Whitbeck $C$, Chicester $P$, Legget $R$, Lin $A D$, Johnson $A$, et al. $N(G)$-nitro-L-arginine methyl ester (L-NAME), a nitric oxide synthase inhibitor, diminishes oxidative damage in urinary bladder partial outlet obstruction. Am J Physiol Renal Physiol 2005.

6. Saito M, Miyagawa I. $N(G)$-nitro-L-arginine methylester, a nitric oxide synthase inhibitor, diminishes apoptosis induced by ischemia-reperfusion in the rat bladder. Neurourol Urodyn 2002; 21(6):566-71.

7. Giglio D, Ryberg AT, To K, Delbro DS, Tobin G. Altered muscarinic receptor subtype expression and functional responses in cyclophosphamide induced cystitis in rats. Auton Neurosci 2005; $122(1-2): 9-20$.

8. Mok MH, Knight $\mathrm{GE}$, Andrews $\mathrm{PL}$, Hoyle $\mathrm{CH}$, Burnstock $\mathrm{G}$. The effects of cyclophosphamide on neurotransmission in the urinary bladder of Suncus murinus, the house musk shrew. J Auton Nerv Syst 2000; 80(3):130-6.

9. Palea $S$, Artibani W, Ostardo E, Trist DG, Pietra C. Evidence for purinergic neurotransmission in human urinary bladder affected by interstitial cystitis. J Urol 1993; 150(6):2007-12.

10. Lynes WL, Flynn SD, Shortliffe LD, Stamey TA. The histology of interstitial cystitis. Am J Surg Pathol 1990; 14(10):969-76.

11. Holm-Bentzen M, Lose G. Pathology and pathogenesis of interstitial cystitis. Urology 1987; 29 (4 Suppl):8-13.

12. Teegavarapu PS, Sahai A, Chandra A, Dasgupta P, Khan MS. Eosinophilic cystitis and its management. Int J Clin Pract 2005; 59(3):356-60.

13. Fadeel B, Ahlin A, Henter JI, Orrenius S, Hampton MB. Involvement of caspases in neutrophil apoptosis: regulation by reactive oxygen species. Blood 1998; 92(12):4808-18.

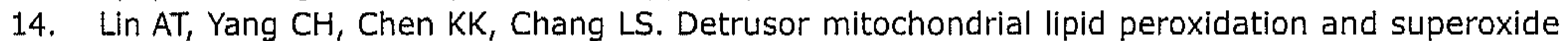
dismutase activity in partial bladder outlet obstruction of rabbits. Neurourol Urodyn 2005; 24(3):282-7.

15. Saito M, Miyagawa I. Bladder dysfunction after acute urinary retention in rats. J Urol 2001; 165(5):1745-7.

16. Haenen GR, Veerman M, Bast A. Reduction of beta-adrenoceptor function by oxidative stress in the heart. Free Radic Biol Med 1990; 9(4):279-88.

17. Koster JF, Slee RG, Essed CE, Stam H. Studies on cumene hydroperoxide-induced lipid peroxidation in the isolated perfused rat heart. J Mol Cell Cardiol 1985; 17(7):701-8.

18. Doelman CJ, Oosterom WC, Bast A. Regulation of sympathetic and parasympathetic receptor responses in the rat trachea by epithelium: influence of mechanical and chemical removal of epithelium. J Pharm Pharmacol 1990; 42(12):831-6.

19. van der Vliet $A$, Tuinstra TJ, Bast A. Modulation of oxidative stress in the gastrointestinal tract and effect on rat intestinal motility. Biochem Pharmacol 1989; 38(17):2807-18.

20. Brading $A F$, Williams $\mathrm{JH}$. Contractile responses of smooth muscle strips from rat and guinea-pig urinary bladder to transmural stimulation: effects of atropine and alpha, beta-methylene ATP. $\mathrm{Br}$ J Pharmacol 1990; 99(3):493-8.

21. Sibley GN. A comparison of spontaneous and nerve-mediated activity in bladder muscle from man, pig and rabbit. J Physiol 1984; 354:431-43. 
22. Inadome A, Yoshida M, Takahashi W, Yono M, Seshita H, Miyamoto $Y$, et al. Direct measurement of acetylcholine release in detrusor smooth muscles isolated from rabbits. Urol Res 1998; 26(5):311-7.

23. Andersson KE. Detrusor contraction-Focus on muscarinic receptors. Scand J Urol Nephrol Suppl 2004(215):54-7.

24. Anderson GF, Marks BH. Spare cholinergic receptors in the urinary bladder. J Pharmacol Exp Ther 1982; 221(3):598-603.

25. Li $X$, Song $L$, Jope RS. Cholinergic stimulation of AP-1 and NF kappa B transcription factors is differentially sensitive to oxidative stress in SH-SY5Y neuroblastoma: relationship to phosphoinositide hydrolysis. J Neurosci 1996; 16(19):5914-22.

26. Blanc EM, Kelly JF, Mark RJ, Waeg G, Mattson MP. 4-Hydroxynonenal, an aldehydic product of lipid peroxidation, impairs signal transduction associated with muscarinic acetylcholine and metabotropic glutamate receptors: possible action on $G$ alpha(q/11). J Neurochem 1997; $69(2): 570-80$.

27. Hashitani $\mathrm{H}$, Bramich NJ, Hirst GD. Mechanisms of excitatory neuromuscular transmission in the guinea-pig urinary bladder. J Physiol 2000; 524 Pt 2:565-79.

28. Geloso DA, Levin RM. Effect of partial outlet obstruction on the myogenic response to field stimulation. Gen Pharmacol 1998; 31(2):291-5.

29. Kato K, Wein AJ, Kitada S, Haugaard N, Levin RM. The functional effect of mild outlet obstruction on the rabbit urinary bladder. J Urol 1988; $140(4): 880-4$.

30. Brading AF. A myogenic basis for the overactive bladder. Urology 1997; 50(6A Suppl):57-67; discussion 68-73.

31. Bayliss $M$, Wu $C$, Newgreen D, Mundy AR, Fry CH. A quantitative study of atropine-resistant contractile responses in human detrusor smooth muscle, from stable, unstable and obstructed bladders. J Urol 1999; 162(5):1833-9.

32. Saito M, Tominaga L, Nanba E, Kinoshita $Y$, Housi D, Miyagawa I, et al. Expression of HSP 70 and its mRNAS during ischemia-reperfusion in the rat bladder. Life Scl 2004; 75(15):1879-86.

33. Azadzoi KM, Tarcan T, Kozlowski R, Krane RJ, Siroky MB. Overactivity and structural changes in the chronically ischemic bladder. J Urol 1999; 162(5):1768-78.

34. Van der Vliet $A$, Bast $A$. Effect of oxidative stress on receptors and signal transmission. Chem Biol Interact $1992 ; 85(2-3): 95-116$.

35. Aikawa $K$, Leggett RE, Levin RM. Effect of age on hydrogen peroxide mediated contraction damage in the male rat bladder. J Urol 2003; 170(5):2082-5. 


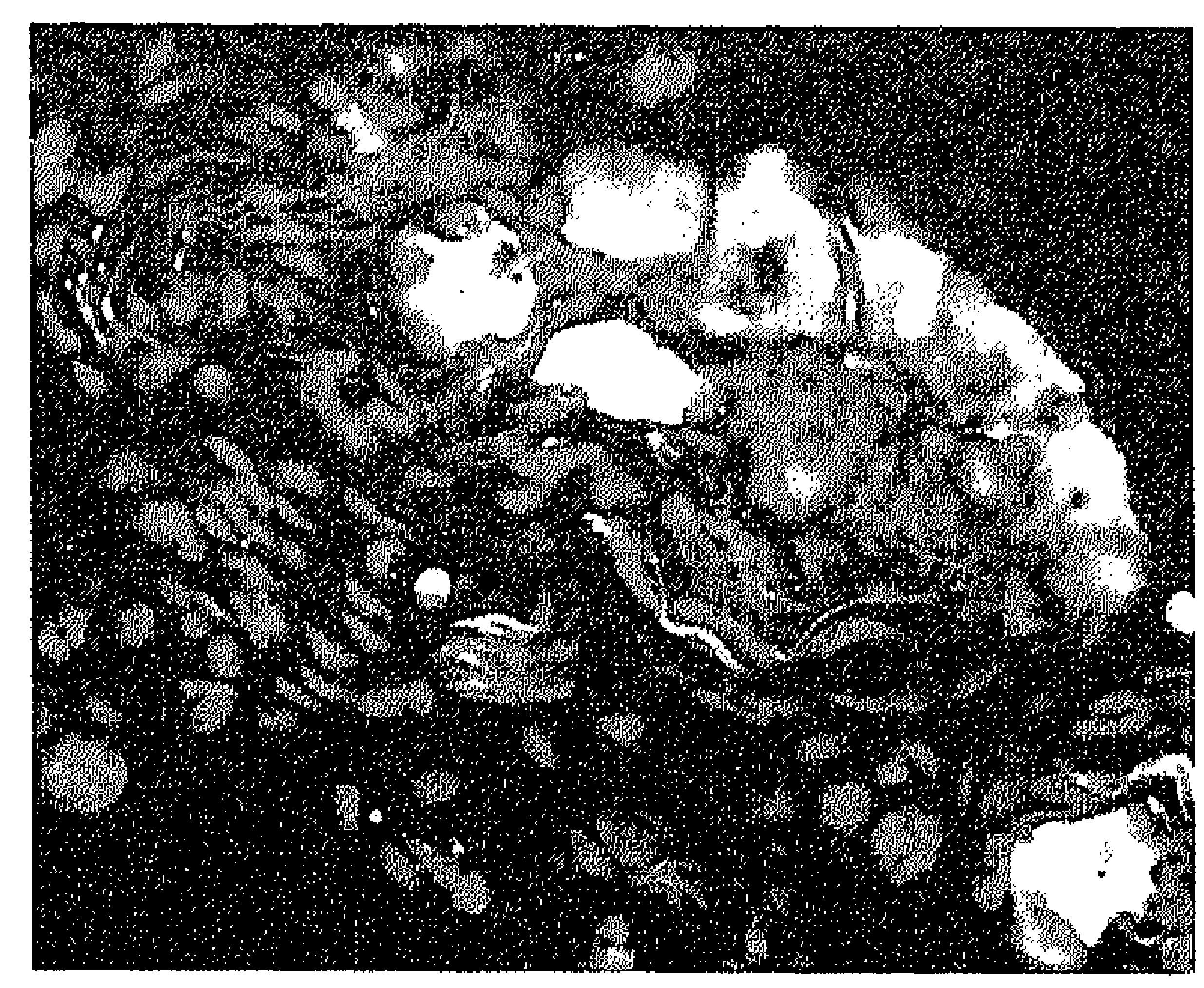

The lipid peroxidation product 4-hydroxynonenal contributes to bladder smooth muscle damage

Rik de Jongh, Guido R.M.M. Haenen, Gommert A. van Koeveringe, Miriam Dambros, Philip E.V. van Kerrebroeck.

Urology 2008; 71(5): 974-8. 


\section{Abstract}

Aims: Bladder outlet obstruction is correlated with an increased peroxidation of lipids. The aldehyde 4-hydroxynonenal (HNE) is produced in relative large amounts during lipid peroxidation. The aim of this study was to investigate the effect of HNE on excitation-contraction coupling of detrusor smooth muscle.

Materials and Methods: Smooth muscle strips from pig urinary bladder were used. Contractile responses to electrical field stimulation (EFS: $4-32 \mathrm{~Hz}$ ), carbachol $\left(10^{-8}-3.10^{-5} \mathrm{M}\right)$ and potassium $(65.3 \mathrm{mM})$ were monitored before and after the addition of HNE. The effect of the synthetic thiol inactivator $\mathrm{N}$-ethylmaleimide (NEM) on mentioned stimulation pathways was investigated and compared with the HNE mediated effect.

Results: Responses of detrusor strips to EFS were for the greater part based on neurogenic stimulation and the release of acetylcholine. HNE (100 $\mu \mathrm{M})$ diminished contractile responses to EFS and carbachol to the same extent. The $\mathrm{pD}_{2}$ value of the carbachol concentration response curve did not decrease after exposure to HNE. The maximal effect obtained with carbachol was significantly reduced after $100 \mu \mathrm{M} \mathrm{HNE}$ treatment. Contractions induced by potassium were affected in a similar degree by HNE compared to EFS- and carbachol-induced responses of comparable amplitude. Incubation of bladder strips with NEM had similar effects on pharmacological responses compared to HNE exposure.

Conclusions: 4-hydroxynonenal affects pig bladder contractility. L-type calcium channels and or the contractile system of the bladder muscle are susceptible to HNE mediated damage. The cholinergic nerves and the muscarinic receptor signalling system remain largely unaffected. The effects of HNE are most likely mediated via alkylation of sulfhydryl groups. 


\section{Introduction}

Bladder outlet obstruction and acute urinary retention followed by bladder decompression are correlated with an increased peroxidation of lipids. ${ }^{1-3}$ Animal models of bladder outlet obstruction indicate that ischemia/reperfusion injury is an important etiological factor in deteriorating bladder function..$^{4-6}$ Reperfusion injury of the bladder seems to be more detrimental than ischemia alone. ${ }^{7}$ Especially during the reperfusion period, reactive oxygen species (ROS) are formed. Besides ischemia/ reperfusion, inflammatory processes in the bladder wall are likely to contribute to the smooth muscle dysfunction associated with bladder outflow obstruction. ${ }^{8-10}$ Bladder inflammation is characterized by infiltration of neutrophils, macrophages, mast cells and lymphocytes in the bladder wall. ${ }^{11,12}$ Especially the activation of phagocytic leukocytes results in the formation of ROS. ${ }^{13}$ Antioxidants and nitric oxide synthase inhibitors can protect the bladder against functional damage due to outlet obstruction, furthermore confirming that bladder outlet obstruction is associated with nitric oxide and the generation of ROS. ${ }^{14-17}$ ROS can damage various cellular components, such as proteins, lipids, DNA and consequently contribute to smooth muscle dysfunction. Oxidative damage to lipids is known as lipid peroxidation. Peroxidation of fatty acids gives rise to various reactive aldehydes such as malondialdehyde (MDA) and 4-hydroxynonenal (HNE). HNE is highly reactive and produced in relative large amounts during lipid peroxidation. This reactive aldehyde is an important mediator of free radical damage and contributes to smooth muscle dysfunction. ${ }^{18,19}$

In a previous in vitro study we have shown that oxidative stress severely affects the muscarinic receptor signalling system and to a lesser extent the L-type calcium channels and/or the contractile system. ${ }^{20}$ The aim of this functional study was to examine the effect of the lipid peroxidation product 4-hydroxynonenal (HNE) on bladder contractility and determine which component in the stimulation pathway (cholinergic nerves, membrane-bound receptors and intracellular contractile machinery) is sensitive to HNE.

\section{Materials and methods}

\section{Tissue preparation}

Experiments were performed on pig urinary bladders obtained from the local slaughterhouse approximately 30 minutes after slaughter. Pieces were cut from the anterolateral wall of the bladder and transported to the laboratory in oxygenated Krebs' solution $\left(\mathrm{NaCl}, 118 \mathrm{mmol} / \mathrm{l} ; \mathrm{KCl}, 5.6 \mathrm{mmol} / \mathrm{l} ; \mathrm{NaHCO} 3,25 \mathrm{mmol} / \mathrm{l} ; \mathrm{KH}_{2} \mathrm{PO}_{4}\right.$, $1.2 \mathrm{mmol} / \mathrm{l} ; \mathrm{CaCl}_{2}, 2.5 \mathrm{mmol} / \mathrm{l} ; \mathrm{MgSO}_{4}, 1.2 \mathrm{mmol} / \mathrm{l} ;$ glucose $6.1 \mathrm{mmol} / \mathrm{l} ; \mathrm{pH} 7.4 ;$ aerated with $\left.95 \% \mathrm{O}_{2} / 5 \% \mathrm{CO}_{2}\right)$. A high $\mathrm{KCl}(124 \mathrm{mM})$ solution was prepared by replacing $\mathrm{NaCl}$ by an equimolar amount of $\mathrm{KCl}$. Afterwards the high $\mathrm{KCl}$ solution was diluted with normal Krebs buffer resulting in a concentration of $\mathrm{KCl} 65.3 \mathrm{mM}$. The 
urothelial layer and connective tissue were removed and muscle strips of $0.5 \mathrm{~mm}$ diameter and length between 7 and $10 \mathrm{~mm}$ were excised. Fine silk sutures were tied to each end of the strip, one end was fixed to the tissue holder and the other end to the isometric force transducer (Kistler Morce DSC 6, Seattle, WA). Each strip was placed in a double jacketed $6-\mathrm{ml}$ organ bath filled with Krebs' buffer solution which was gassed with a mixture of $95 \% \mathrm{O}_{2}$ and $5 \% \mathrm{CO}_{2}$ at $37{ }^{\circ} \mathrm{C}$. Measurements were started after an equilibration period of $60 \mathrm{~min}$ and an initial tension of $20 \mathrm{mN}$ was placed on each strip.

\section{Experimental protocol}

Frequency response curves to electrical field stimulation (EFS) were constructed by stimulating bladder strips via platinum electrodes. The electrodes were placed above and under the bladder strips and were connected to the HM8130 Function Generator from Hameg ${ }^{\circledR}$ Instruments, Manhausen, Germany. Electrical field stimulation ( $50 \mathrm{~V}, 2 \mathrm{msec}$ pulse width, $5 \mathrm{sec}$ train pulses) was applied at frequencies of $4,8,16$ and $32 \mathrm{~Hz}$. To test the effect of the lipid peroxidation product 4hydroxynonenal (HNE), bladder strips were incubated with $10 \mu \mathrm{M}$ and $100 \mu \mathrm{M} \mathrm{HNE}$ for 30 minutes. After incubation with $\mathrm{HNE}$, strips were thoroughly washed during 30 minutes and additional EFS were repeated in the same order of frequencies as before incubation. During the washing period the Krebs' buffer in the organ bath was refreshed every 5 minutes. To evaluate the effect of HNE on muscarinic receptors, dose response curves to carbachol $\left(10^{-8}-3.10^{-5} \mathrm{M}\right)$ before and after exposure to HNE were constructed. Furthermore the effects of HNE on contractile responses to membrane depolarisation were evaluated by determining the responses evoked by potassium $(\mathrm{KCl} 65.3 \mathrm{mM})$ before and after HNE treatment.

The effect of the synthetic thiol inactivator $\mathrm{N}$-ethylmaleimide (NEM; $10 \mu \mathrm{M}$ and $100 \mu \mathrm{M}$ ) on the above mentioned stimulation pathways was investigated.

\section{Chemicals}

Carbachol and $\mathrm{N}$-ethylmaleimide (NEM) were obtained from Sigma Chemical Co (Utrecht, The Netherlands). 4-hydroxynonenal (HNE) was synthesized according to Leurs et al. ${ }^{21}$ All solutions were freshly prepared daily. Drugs were dissolved in deionized distilled water; 4-hydroxynonenal was dissolved in dimethylsulfoxide (DMSO). The final solution of DMSO in the bathing solution was less than $0.05 \%$. Further dilutions were carried out in Krebs' solution. The concentrations are expressed as the final molar concentration in the tissue chamber.

\section{Statistical evaluation}

Results are expressed as means \pm SEM of measurements in $n$ bladders. Contractile responses are normalized for tissue weight and are expressed as absolute values $(\mathrm{mN} / \mathrm{mg}$ ) or as percentage of the control $(\max )$ response. Concentrationresponse data of carbachol were evaluated by sigmoid curve fitting and $-\log$ EC50 values $\left(\mathrm{pD}_{2}\right)$ were calculated by non-linear regression analysis using GraphPad 
Prism. Differences between mean values were statistically analysed using analysis of variance followed by the Newman-Keuls test. The vehicle controls are included in the statistical evaluation. The vehicle (DMSO) did not have an effect on the responses mediated by various stimulation pathways. A probability value of $p<0.05$ was regarded to be significant. All analyses were performed using GraphPad Prism software (version 4.0; Graphpad Software Inc., San Diego, CA).

\section{Results}

Addition of HNE to the organ bath caused a dose-dependent stable contraction that remained throughout the experiment. The force development to $10 \mu \mathrm{M}$ and $100 \mu \mathrm{M} \mathrm{HNE}$ was $0.27 \pm 0.07 \mathrm{mN} / \mathrm{mg}$ and $5.45 \pm 0.57 \mathrm{mN} / \mathrm{mg}$, respectively. Electrical field stimulation (EFS) of bladder strips caused a rapid rise in isometric tension and this contractile response was dependent on the frequency of the EFS in the range of $4-32 \mathrm{~Hz}$. The absolute size of the force development to EFS ( $32 \mathrm{~Hz}$ ) was $19.78 \pm 1.43 \mathrm{mN} / \mathrm{mg}$. Treating the strips with HNE resulted in a reduction of the contractions induced by EFS. The effect of HNE was dose dependent, concentrations of $100 \mu \mathrm{M}$ reduced the EFS-induced responses significantly compared to time controls $(p<0.05)$, whereas $10 \mu \mathrm{M}$ HNE had no effect (data not shown). Figure $1 \mathrm{~A}$ shows the effect of increasing concentrations HNE on the dose response curves of detrusor strips to carbachol. Carbachol in concentrations ranging from $10^{-8}$ to 3.10-5 $\mathrm{M}$ caused a rise in isometric tension in a dose-dependent fashion. The $\mathrm{pD}_{2}$ value of carbachol in strips without HNE treatment was $6.35 \pm 0.03$. Preincubation of strips with HNE did not have a significant effect on the concentration of carbachol needed to produce half the maximal effect. The $\mathrm{pD}_{2}$ value after exposure to $10 \mu \mathrm{M}$ and $100 \mu \mathrm{M} \mathrm{HNE}$ were $6.31 \pm 0.04(p>0.05)$ and $6.34 \pm 0.03(p>0.05)$, respectively. The absolute size of the maximal effect obtained with carbachol was $35.81 \pm 1.75 \mathrm{mN} / \mathrm{mg}$. Incubation with $10 \mu \mathrm{M} \mathrm{HNE}$ had no effect on the maximal response obtained with carbachol. However, $100 \mu \mathrm{M}$ HNE significantly reduced the maximal response to $44 \%$ of the control value $(p<0.05)($ Fig. $1 \mathrm{~A})$.

In control bladder strips, the amplitude of the force development induced by EFS $32 \mathrm{~Hz}$, carbachol $1 \mu \mathrm{M}$ or $\mathrm{KCl} 65.3 \mathrm{mM}$ stimulation were practically identical (Fig. 2, inserted figure.). $10 \mu \mathrm{M} \mathrm{HNE}$ treatment had no effect on the mentioned stimulation pathways. However, incubation with $100 \mu \mathrm{M} \mathrm{HNE}$ reduced the EFS, carbachol and $\mathrm{KCl}$ induced responses significantly compared to the time control. The responses were affected to a similar degree (Fig. 2A).

The general mechanism by which HNE exerts its toxic effects is covalent binding of HNE with free thiol groups of proteins. ${ }^{21}$ In order to investigate whether this process is also involved in the reduction of strip responses, we compared the effects of the synthetic thiol inactivator $\mathrm{N}$-ethylmaleimide (NEM) to those of HNE. NEM elicited a dose-dependent contraction that remained throughout the experiments, similar to HNE. The force development to $10 \mu \mathrm{M}$ and $100 \mu \mathrm{M}$ NEM was $0.27 \pm 0.08$ 
$\mathrm{mN} / \mathrm{mg}$ and $5.26 \pm 0.54 \mathrm{mN} / \mathrm{mg}$, respectively. NEM treatment did not result in a $\mathrm{pD}_{2}$ shift of the concentration response curve to carbachol but $100 \mu \mathrm{M}$ NEM reduced the maximal response significantly compared to the time control $(p<0.05)$. The effects of NEM on EFS and $\mathrm{KCl}$-induced responses were comparable to those of HNE (Fig. 2B).
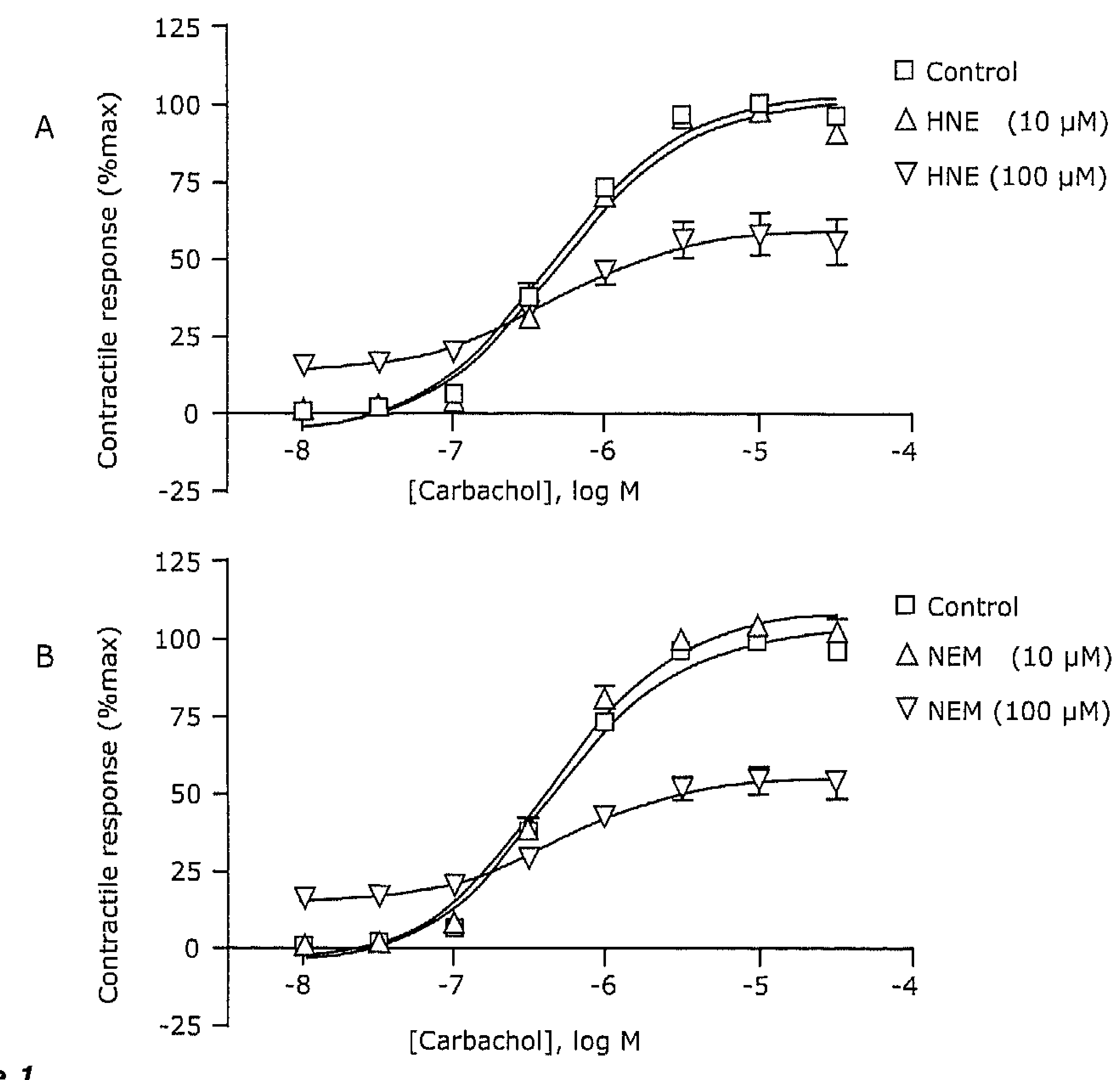

Figure 1.

The effect of $10 \mu \mathrm{M}, 100 \mu \mathrm{M}$ 4-hydroxynonenal (HNE) and N-ethylmaleimide (NEM) on carbachol dose response curve of pig detrusor strips. A. The $\mathrm{pD}_{2}$ values of time control curve and of curves after exposure to $10 \mu \mathrm{M}$ and $100 \mu \mathrm{M} \mathrm{HNE}$, were $6.35 \pm 0.03,6.31 \pm 0.04(p>0.05)$ and $6.34 \pm 0.03(p>0.05)$ respectively. The maximal effect (Emax) was significantly reduced after $100 \mu \mathrm{M}(p<0.001)$ HNE treatment. B. The $\mathrm{pD}_{2}$ values of time control curve and of curves after exposure to $10 \mu \mathrm{M}$ and $100 \mu \mathrm{M}$ NEM were 6.35 $\pm 0.03,6.35 \pm 0.04(p>0.05)$ and $6.29 \pm 0.02(p>0.05)$ respectively. The maximal effect (Emax) was significantly reduced after $100 \mu M(p<0.001)$ NEM treatment. The effect of HNE and NEM itself is reflected by the force development at 0,01 $\mu \mathrm{M}$ carbachol. Each point is the mean \pm SEM of 8 bladders. 

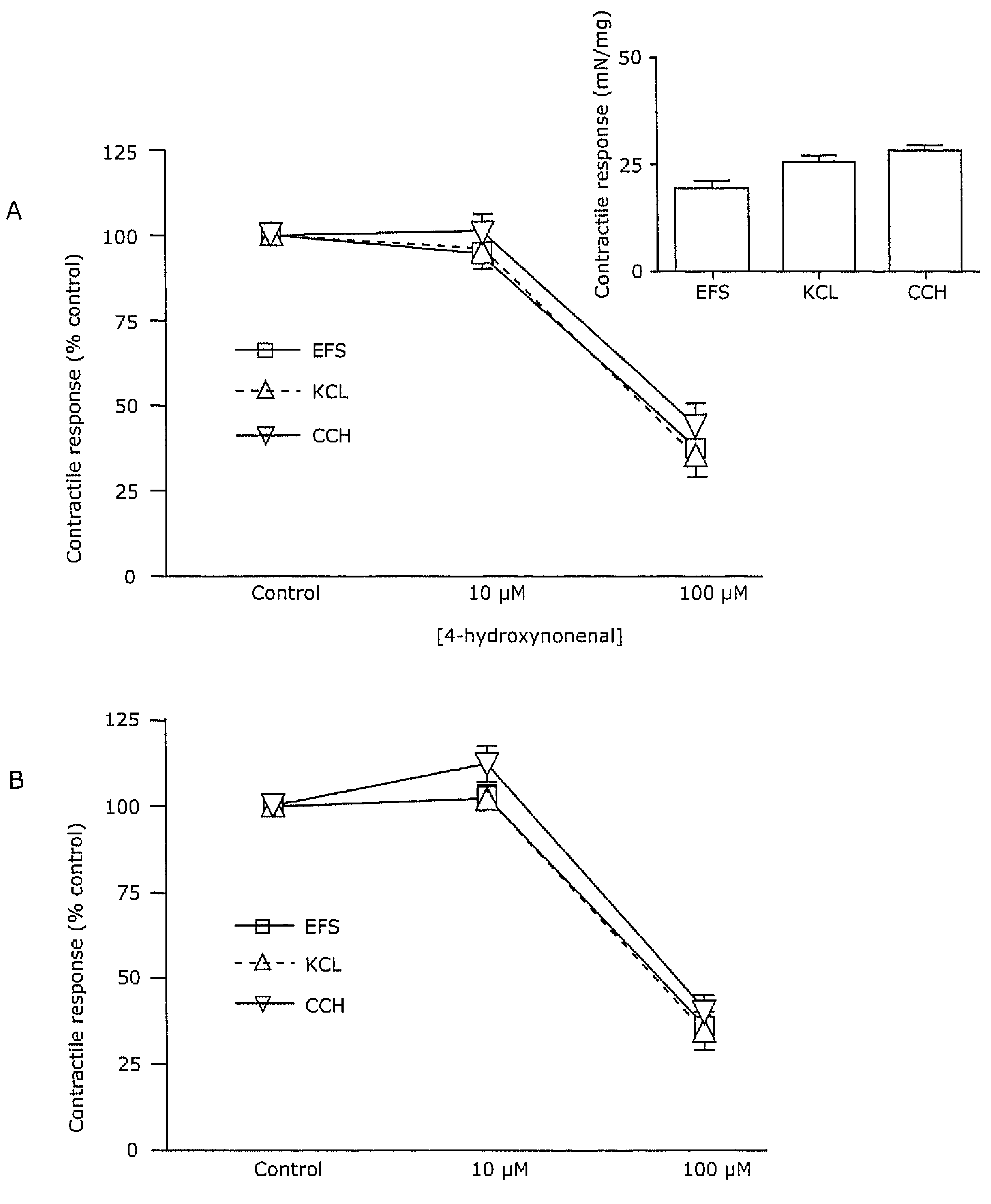

Figure 2.

[N-ethylmaleimide]

The effect of $10 \mu \mathrm{M}, 100 \mu \mathrm{M}$ 4-hydroxynonenal (HNE) and N-ethylmaleimide (NEM) on EFS (32 $\mathrm{Hz}$ ), carbachol $(1 \mu \mathrm{M})$ and $\mathrm{KCl}(65.3 \mathrm{mM})$ evoked responses. The inserted figure shows the absolute size of bladder strip responses in the control conditions to the three stimulation pathways. Each bar is the mean \pm SEM 39 bladder strips. A. The contractile responses to various stimulation pathways were significantly reduced after $100 \mu \mathrm{M}(p<0.001)$ HNE treatment. B. The contractile responses were significantly reduced after $100 \mu M(p<0.001)$ NEM treatment. Each point represents the mean \pm SEM of 7-8 bladders. 


\section{Discussion}

The smooth muscle contraction of the urinary bladder in response to nerve stimulation is predominantly mediated by the release of the neurotransmitters acetylcholine and ATP. In normal human and pig bladder tissue, the purinergic component plays a minor role and acetylcholine is the dominant neurotransmitter. ${ }^{22}$ Activation of $\mathrm{M}_{3}$ receptors is predominantly responsible for the acetylcholine induced detrusor contraction. In a previous study we showed that EFS-evoked responses in pig bladder were mainly based on neurogenic stimulation and the release of acetylcholine. ${ }^{22}$ In this study, the force development induced by EFS $32 \mathrm{~Hz}$ and carbachol $1 \mu \mathrm{M}$ were practically identical in the control situation. HNE treatment reduced the EFS and carbachol induced responses to a comparable extent (Fig. 2). This suggests that HNE did not have an effect on the release of endogenous acetylcholine from peripheral nerve endings.

In the positive inotropic response to muscarinic receptor agonists (such as carbachol) in the bladder a cascade of biochemical reactions are involved. During $\mathrm{M}_{3}$ receptor activation, the receptor-agonist complex couples to a G-protein that subsequently activates phopholipase $C$ which leads to mobilization of extra-and intracellular calcium ions eventually leading to a mechanical contraction. ${ }^{23}$ In the present study we found that HNE treatment did not change the $\mathrm{pD}_{2}$ value of the carbachol concentration response curve. The fact that HNE did not reduce the $\mathrm{pD}_{2}$ value suggests that the muscarinic receptors or the amplification reactions are not affected by HNE. This is in agreement with observations of Haenen ${ }^{19}$ and van der Vliet, ${ }^{18}$ who described that $H N E$ had a relatively small effect on the ${ }^{2} D_{2}$ value. Apparently, HNE reacts with a component that is situated after the muscarinic receptors and the signal-transduction mechanisms which they engage.

$\mathrm{M}_{3}$ receptor stimulation leads to mobilization of extra-and intracellular calcium ions eventually leading to a response. The response in the bladder is largely dependent on $\mathrm{Ca}^{2+}$ influx through L-type, voltage dependant channels and activation of Rho kinase. Mobilization of calcium from intracellular stores via the IP3 pathway plays a minor role. ${ }^{23} \mathrm{~A}$ rise in intracellular $\mathrm{Ca}^{2+}$ will eventually activate the contractile machinery. As 4-hydroxynonenal reduced the maximal effect to carbachol stimulation (Fig. 1A) it is likely that HNE may damage the membrane function (L-type calcium channels) and/or the contractile apparatus. In order to clarify this, we compared the effects of HNE on EFS, carbachol and $\mathrm{KCl}$ responses at practical similar amplitudes of force development (Fig. 2A). It was found that the $\mathrm{KCl}$ mediated responses were similarly affected by HNE as the EFS and carbachol induced responses. Potassium depolarizes the membrane and triggers $\mathrm{Ca}^{2+}$ entry through Ltype calcium channels resulting in elevated intracellular $\mathrm{Ca}_{2}+$ concentrations and a contractile response of the muscle. The similar effect of HNE on $\mathrm{KCl}, \mathrm{EFS}$ and carbachol evoked responses implies that L-type calcium channels and or the contractile machinery of smooth muscle are sensitive to HNE induced damage. Indeed, it has been noted that the contractile apparatus and the cytoskeleton of the 
smooth muscle are sensitive to HNE. ${ }^{24,25}$ Especially, the contractile protein actin is vulnerable to HNE mediated damage ${ }^{25}$ In addition, studies have shown that free thiol groups play a role in gating calcium channels. ${ }^{26}$ As HNE is known to be a thiol inactivating agent, ${ }^{21}$ it is likely that HNE modulates L-type calcium channels. Indeed, it has been demonstrated that 4-hydroxynonenal triggers Ca2+ influx via L-type calcium channels. ${ }^{27,28}$ The effects of the synthetic thiol inactivator $\mathrm{N}$-ethylmaleimide (NEM) were similar to those of HNE (Fig 2B). The actions of NEM are mediated by sequestration of thiol groups on L-type calcium channel ${ }^{29}$ furthermore suggesting that the effects of HNE seen in this study are mediated via L-type calcium channels.

In this study $100 \mu \mathrm{M} \mathrm{HNE}$ and NEM induced a stable muscle contraction. This observation is in accordance with the results of Resim et al. They demonstrated in rat urinary bladder that the sulfhydryl reagent NEM elicited a muscle contraction. The actions of NEM seems to be mediated by sequestration of thiol groups on L-type calcium channels as the effects of NEM were abolished by pre-treatment with glutathione and verapamil. ${ }^{29}$ Sequestration of thiol groups on L-type calcium channels may result in irreversible damage to L-type calcium channels explaining the observed reduced muscle responses to various stimulation pathways after HNE treatment.

An increased passive force can have influence on active force development. ${ }^{30}$ Theoretically, pre-stretching strips could damage smooth muscle tissue resulting in a diminished active force development. However, the optimal length range of force development in pig detrusor smooth muscle is considerably large, i.e. the length tension relationship curve is relatively shallow. Pre-stretching the bladder strips to 3 times the initial length results in a slight decrease in active force development. ${ }^{30}$ So it is unlikely that the length dependence of force development can explain the reduced muscle responses after HNE or NEM treatment.

Several studies have focussed on the effects of bladder outlet obstruction and ischemia on detrusor smooth muscle. Functional studies performed in obstructed bladders have demonstrated a significantly greater decrease in the contractile response to field stimulation compared to either muscarinic receptor stimulation or membrane depolarisation by $\mathrm{KCl} .{ }^{14,16,17}$ There is a denervation in bladder tissue exposed to an outlet obstruction. However, there is also damage to the smooth muscle itself. The results of our study demonstrate that 4-hydroxynonenal affects the L-type calcium channels and or the contractile system of the pig detrusor smooth muscle. The cholinergic nerves and the muscarinic receptor signalling system remain largely unaffected. As bladder outlet obstruction and acute urinary retention are correlated with an increased production of the reactive aldehyde $\mathrm{HNE}$, the bladder muscle dysfunction seen in these pathological situations may partly be due to the effect of HNE on the smooth muscle. The denervation observed in obstructed bladders is probably not due to HNE mediated damage. 


\section{Conclusions}

The current experiments show that the oxidative stress product HNE contributes to muscle dysfunction via cytotoxic effects on L- type calcium channels and or the contractile system. The effects of HNE on bladder smooth muscle are most likely mediated via alkylation of essential sulfhydryl groups.

\section{References}

1. Lin AT, Yang $\mathrm{CH}$, Chen KK, Chang LS. Detrusor mitochondrial lipid peroxidation and superoxide dismutase activity in partial bladder outlet obstruction of rabbits. Neurourol Urodyn 2005; 24(3):282-7.

2. Saito M, Miyagawa I. Bladder dysfunction after acute urinary retention in rats. J Urol 2001; 165(5):1745-7.

3. Sinik $Z$, Guler $G$, Turan $T$, Sert $S$, Aybek $Z$, Tuncay $L$. Does two episodes of acute urinary retention lead to additional ischemia-reperfusion injury in rat bladder? Int Urol Nephrol 2006; 38(1):123-7.

4. Azadzoi KM, Pontari M, Vlachiotis J, Siroky MB. Canine bladder blood flow and oxygenation: changes induced by filling, contraction and outlet obstruction. J Urol 1996; 155(4):1459-65.

5. Greenland JE, Hvistendahl JJ, Andersen H, Jorgensen TM, McMurray G, Cortina-Borja M, et al. The effect of bladder outlet obstruction on tissue oxygen tension and blood flow in the pig bladder. BJU Int 2000; 85(9):1109-14.

6. Greenland $\mathrm{JE}$, Brading AF. The effect of bladder outflow obstruction on detrusor blood flow changes during the voiding cycle in conscious pigs. J Urol 2001; 165(1):245-8.

7. Bratslavsky $G$, Kogan BA, Matsumoto $S$, Aslan AR, Levin RM. Reperfusion injury of the rat bladder is worse than ischemia. J Urol 2003; 170(5):2086-90.

8. Giglio D, Ryberg AT, To K, Delbro DS, Tobin G. Altered muscarinic receptor subtype expression and functional responses in cyclophosphamide induced cystitis in rats. Auton Neurosci 2005; 122(1-2):9-20.

9. Palea S, Artibani W, Ostardo E, Trist DG, Pietra C. Evidence for purinergic neurotransmission in human urinary bladder affected by interstitial cystitis. J Urol 1993; 150(6):2007-12.

10. Chertin B, Rolle U, Solari V, Cascio S, Puri P. The role of nitric oxide in bladder urothelial injury after bladder outlet obstruction. BJU Int 2004; $94(3): 392-9$.

11. Lynes WL, Flynn SD, Shortliffe LD, Stamey TA. The histology of interstitial cystitis. Am J Surg Pathol 1990; 14(10):969-76.

12. Holm-Bentzen M, Lose G. Pathology and pathogenesis of interstitial cystitis. Urology $1987 ; 29$ (4 Suppl):8-13.

13. Fadeel B, Ahlin A, Henter JI, Orrenius S, Hampton MB. Involvement of caspases in neutrophil apoptosis: regulation by reactive oxygen species. Blood 1998; $92(12): 4808-18$.

14. Conners $W$, Whitbeck $C$, Chicester $P$, Legget $R$, Lin $A D$, Johnson $A$, et al. $N(G)$-nitro-L- arginine methyl ester (L-NAME), a nitric oxide synthase inhibitor, diminishes oxidative damage in urinary bladder partial outlet obstruction. Am J Physiol Renal Physiol 2005.

15. Levin RM, Das AK. A scientific basis for the therapeutic effects of Pygeum africanum and Serenoa repens. Urol Res 2000; 28(3):201-9.

16. Levin RM, Kawashima $Y$, Leggett RE, Whitbeck C, Horan P, Mizutani K. Effect of oral Kohki tea on bladder dysfunction induced by severe partial outlet obstruction. J Urol 2002; 167(5):22606.

17. Parekh $M H$, Lobel $R, O^{\prime}$ Connor LJ, Leggett RE, Levin RM. Protective effect of vitamin $E$ on the response of the rabbit bladder to partial outlet obstruction. J Urol 2001; 166(1):341-6. 
18. Van der Vliet A, Van der Aar EM, Bast A. The lipid peroxidation product 4-hydroxy-2,3-trans-1 nonenal decreases rat intestinal smooth muscle function in-vitro by alkylation of sulphydryl groups. J Pharm Pharmacol 1991; 43(7):515-7.

19. Haenen GR, Plug HJ, Vermeulen NP, Timmerman H, Bast A. Contribution of 4-hydroxy-2,3-transnonenal to the reduction of beta-adrenoceptor function in the heart by oxidative stress. Life Sci $1989 ; 45(1): 71-6$.

20. de Jongh $R$, Haenen $G R$, van Koeveringe GA, Dambros M, De Mey JG, van Kerrebroeck PE. Oxidative stress reduces the muscarinic receptor function in the urinary bladder. Neurourol Urodyn 2006.

21. Leurs $R$, Rademaker $B$, Kramer $K$, Timmerman H, Bast A. The effects of 4-hydroxy-2,3-transnonenal on beta-adrenoceptors of rat lung membranes. Chem Biol Interact 1986; 59(2):211-8.

22. Sibley GN. A comparison of spontaneous and nerve-mediated activity in bladder muscle from man, pig and rabbit. J Physiol 1984; 354:431-43.

23. Andersson KE. Detrusor contraction-Focus on muscarinic receptors. Scand J Urol Nephrol Suppl 2004(215):54-7.

24. Gadoni E, Olivero A, Miglietta A, Bocca C, Gabriel L. Cytoskeletal modifications induced by 4hydroxynonenal. Cytotechnology 1993; 11 Suppl 1:S62-4.

25. Ozeki M, Miyagawa-Hayashino A, Akatsuka S, Shirase T, Lee WH, Uchida K, et al. Susceptibility of actin to modification by 4-hydroxy-2-nonenal. J Chromatogr B Analyt Technol Biomed Life Sci 2005; 827(1):119-26.

26. Murphy BJ, Washkurak AW, Tuana BS. Dihydropyridine binding to the L-type Ca2+ channel in rabbit heart sarcolemma and skeletal muscle transverse-tubules: role of disulfide, sulfhydryl and phosphate groups. Biochim Biophys Acta 1990; 1052(2):333-9.

27. Lu C, Chan SL, Fu W, Mattson MP. The lipid peroxidation product 4-hydroxynonenal facilitates opening of voltage-dependent $\mathrm{Ca}^{2+}$ channets in neurons by increasing protein tyrosine phosphorylation. J Biol Chem 2002; 277(27):24368-75.

28. Akaishi T, Nakazawa $K$, Sato $K$, Ohno $Y$, Ito $Y, 4$-Hydroxynonenal modulates the long-term potentiation induced by L-type $\mathrm{Ca}^{2+}$ channel activation in the rat dentate gyrus in vitro. Neurosci Lett 2004; 370(2-3):155-9.

29. Resim S, Buyuknacar HS, Gocmen C, Onder S, Dikmen A. A possible effect of sulfhydryl reagents on the contractile activity of the rat detrusor muscle. Eur J Pharmacol 2002; $442(3): 295-9$. 


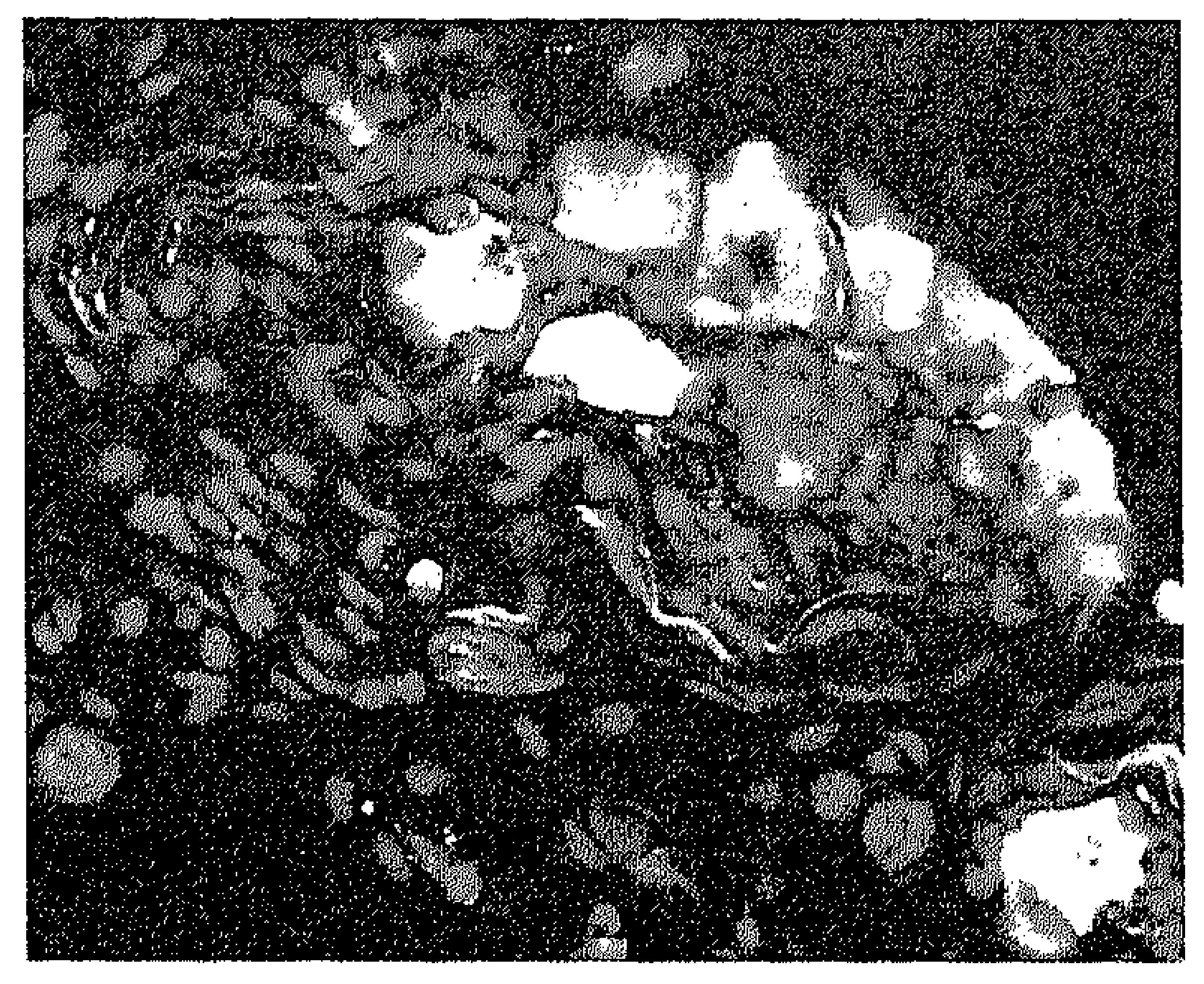

General discussion and future perspectives 
Overactive bladder (OAB) is a symptom complex that includes urinary urgency with or without urgency incontinence, urinary frequency, and nocturia. The aetiology and pathophysiology of symptoms of the overactive bladder have not yet been identified. In our studies, we attempted to explain the origins of urgency and frequency via the autonomous bladder theory. This theory hypothesises that non micturition contractions (localised contractions in the bladder wall) stimulate afferent nerves which are likely to contribute to the emergence of bladder sensations. In this thesis we investigated if pathological bladder conditions (i.e. bladder outlet obstruction and increased prostaglandin levels) both associated with the occurrence of overactive bladder symptoms, ${ }^{1}$ are related to a modified autonomous activity. In addition we tried to identify different cell types which may be responsible for the generation of autonomous activity. Finally, we investigated the effects of bladder outlet obstruction (BOO) on bladder function, structure and the relation to oxidative stress.

\section{Animal models}

Research has focussed on animal models of $O A B$ since experiments in human raise moral and ethical dilemmas. Different animal models (e.g. BOO, spinal cord injury, diabetes, and hypertension) and a variety of animal species (e.g. rat, rabbit, guinea pig, and pig) have been utilised to investigate the lower urinary tract function and dysfunction. Despite the fact that animal models have improved our understanding of changes to the bladder resulting in bladder dysfunction, it is important to realize that there are some limitations linked to the utilization of animal models. First of all, the $O A B$ syndrome is a clinical and symptomatical diagnosis and therefore it is impossible to develop the ideal animal model, since animals cannot report symptoms. Secondly, there are differences in the regulation of bladder function between humans and animals species. For example, animals have a dual excitatory innervation, one (purinergic) mediating a rapid phasic contraction and another (cholinergic) one mediating a tonic contraction. The normal human bladder only has the latter. The phasic mechanism in animals could be involved in the use of urine for territorial marking in animals. Thirdly, some animals (e.g. rats) have higher physiological voiding frequencies than humans.

In our studies, we used guinea pig bladders because they have many similarities with human bladders. ${ }^{2-5}$ Among all pathologies related to the urinary bladder, most studies have focussed on the animal model of BOO. This model mimics morphological and functional changes of the bladder seen in patients with a BOO. A guinea pig model of gradual bladder obstruction was used for some of the studies since this model already demonstrated to be beneficial for understanding changes to the bladder that might result in an overactive bladder. ${ }^{2}$ Although animal models of BOO have provided a better understanding of several bladder wall changes which may contribute to an overactive bladder, there are differences to be kept in mind when 
comparing the animal model and the human situation. For example, in humans, bladder enlargement develops over months and years whereas in animal models bladder enlargement is induced in weeks ( 2 - 4 weeks in our studies). In addition, the increase in bladder weight in animals seems to be higher (up to 5 fold) compared to the human situation (i.e. a doubling of the bladder weight). In our studies, the increase in bladder weight of obstructed animals varied between $1.5-5$ fold.

\section{Autonomous activity in the obstructed guinea pig bladder and the role of interstitial cells}

Despite the occurrence of non micturition activity (autonomous activity) in all species, $^{3-6}$ it is important to notice that the precise physiological function of this bladder activity has not been determined yet. Therefore, research clarifying the physiological function of this autonomous activity is mandatory. At the moment, researchers are investigating the correlation of non micturition activity to bladder sensations in humans. These studies will hopefully give insight into the function of autonomous activity and reveal if this activity is effectively related to the occurrence of bladder sensations.

If we assume that autonomous activity plays a central role in producing bladder sensations, then bladder abnormalities related to increased bladder sensations should be correlated to a modified autonomous activity. BOO is associated with the occurrence of symptoms of an overactive bladder. Our results indeed showed that the autonomous activity was increased in obstructed guinea pig bladders. ${ }^{7}$ The amplitude of the autonomous activity was significantly greater than in control bladders. This finding is in accordance with a previously published study. ${ }^{8}$ The autonomous activity was not affected by atropine and tetrodotoxin implying that the underlying mechanism of the activity does not involve voltage-sensitive sodium channels or spontaneous release of acetylcholine. In contrast, noradrenaline abolished the activity, which may be clinically relevant as adrenergic agonists are tested nowadays in randomized clinical trials for treatment of OAB. Surprisingly, the amplitude (normalized for bladder weight) of the autonomous activity was the highest in the sham operated bladders. ${ }^{7}$ This suggests that the pathological changes are the result of damage to the bladder neck and not the result of obstruction per se. The clinical relevance of large amplitudes of the autonomous activity may be a greater activation of the sensory system within the bladder wall eventually contributing to symptoms of an overactive bladder. The question arises what mechanism is responsible for the changed amplitude of autonomous activity. As mentioned in the introduction of this dissertation, a specialized control system in the bladder wall involving interstitial cells are thought to generate and coordinate the autonomous activity. ${ }^{5}$ Thus, a changed autonomous activity might have its substrate in an alteration of a network of interstitial cells. We have demonstrated for the first time that an extensive network of CGMP+ interstitial cells developed in bladders as a result of bladder outlet 
obstruction. These CGMP+ interstitial cells penetrated and surrounded muscle bundles. ${ }^{9}$ Kubota et al. ${ }^{10}$ also found similar results in obstructed bladders. The function of the interstitial cell network is not known and future research should reveal the physiological and pathophysiological importance of these cells. All sham operated and obstructed bladders showed an increase in bladder weight. This increase most obviously involves muscle hypertrophy, thickening of the lamina propria and the serosal layer. ${ }^{9,11-12}$ The muscle hypertrophy could be a consequence of voiding against an increased bladder outlet resistance. However, in our study we suggest another possibility. It could be that the bladder hypertrophy is secondary to an initial increase in (autonomous) bladder activity. A recently published study, showed that surgical occlusion of the bladder neck increases bladder activity before any change in bladder weight occurs. ${ }^{13}$ Thus, it could be that changes to the bladder neck cause, via yet undefined mechanisms, an increased autonomous activity that will eventually result in bladder wall hypertrophy. Support for a crucial role of the bladder neck in modifying bladder activity comes from observations showing that accidental surgical damage to the bladder neck in women can subsequently lead to symptoms of urgency and frequency. ${ }^{14}$

The urothelium is now known to have substantial sensory properties, ${ }^{15}$ especially the lamina propria is extensively innervated. ${ }^{16-17}$ It has been suggested that the urothelium may serve as a mechanosensor that by producing nitric oxide, ATP, acetylcholine, and other mediators, can control the activity in afferent nerves. In addition, suburothelial interstitial cells form close contacts with afferent nerves, and these cells may play a role in modulating activity of the suburothelial sensory nerves. ${ }^{18}$ We found that the urothelium is changed in obstructed bladders. The urothelium in these bladders demonstrated less folding compared to the sham group. The number of CGMP+ umbrella cells and the intensity of staining was reduced and the intermediate urothelial layer consisted of multiple cell layers in the obstructed bladders. Nerves were readily found in the lamina propria, however, in contrast to the sham operated bladders, they were rarely associated with the urothelium. In addition, we found that the thickness of the suburothelial interstitial cell layer was increased in obstructed bladders as compared to the sham group and that cells were dispersed throughout the lamina propria. ${ }^{9}$ The altered structure of the urothelium and suburothelial interstitial cell layer in obstructed bladders certainly reflects a modified function. At the moment, we can only guess the implication of this modified function. Since the threshold for voiding can be decreased in obstructed bladders, it could be that there is an upregulation of the (sub)urothelial afferent transduction process. A limited number of studies indeed reported specific alterations in urothelial function and ultrastructure of obstructed bladders. The expression of the mechanosensitive epithelium sodium channel $\mathrm{ENaC}$ is increased and the ATP release from the urothelium is enhanced in obstructed human bladders when compared to controls. ${ }^{19-21}$ Future structural and functional studies concerning the urothelium are likely to reveal the importance of this layer for the physiology of the normal and pathological bladder. 
In normal guinea pig bladders, cholinergic agonists can induce a transient acceleration and increase in autonomous contractions. This cholinergic modulation of bladder activity, has the characteristics of a system which involves a pacemaker, determining the frequency of phasic activity, and a network, distributing this activity .${ }^{22}$ We have demonstrated that the sensitivity of the frequency of autonomous activity is increased in sham operated and obstructed bladders compared to control bladders. ${ }^{7}$ Thus, the pacemaker becomes progressively supersensitive to muscarinic agonists in a sham operated and obstructed bladder compared to controls. This supersensitive cholinergic drive to the autonomous activity could theoretically increase bladder sensations and this mechanism might be the site of the therapeutic action of anticholinergic drugs. Since our functional data suggest the presence of muscarinic receptors on interstitial cells, the question arises if these cells contain muscarinic receptors. Indeed, immunohistochemical studies in humans and animals showed that $M_{2}$ and $M_{3}$ receptors are located to interstitial cells in the suburothelial and detrusor layer of the bladder. ${ }^{23-24}$ The presence of muscarinic receptors on suburothelial interstitial cells suggests that these cells have a cholinergic regulation and they can for example be activated by acetylcholine released from the urothelium..$^{25}$ The muscarinic receptors on the interstitial cells may modulate either the pacemaker element or the regulation of the distributive element of autonomous activity. In, the above mentioned immunohistochemical studies a clear specificity of the muscarinic antibodies was not demonstrated yet. Therefore, several researchers in Maastricht are currently testing the specificity of different muscarinic antibodies (personal communication). Since anticholinergic drugs at therapeutic effective doses do not seem to act on the detrusor muscle ${ }^{26-27}$ and our physiological data suggest an upregulation of the muscarinic interstitial cell network in obstructed bladders, it could be that the therapeutic target of anticholinergic drugs are the cholinergic receptors on the interstitial cell network. This idea is supported by data on anticholinergic drugs that suggest an effect of these drugs on structures such as interstitial cells and nerves instead of the detrusor muscle. ${ }^{5,28-31}$

In conclusion, our data clearly show that bladder outlet obstruction results in functional bladder changes. The modified autonomous activity is accompanied by alterations in the network of interstitial cells, nerves, urothelium and probably other yet undefined structures. Research in characterizing the morphological, ultrastructural and physiological properties of interstitial cells is required. For example, new techniques to identify interstitial cells would be of benefit since neither, c-kit, vimentin, and CGMP immunohistochemical labelling is ideal. Whether the alterations in the network of interstitial cells in obstructed bladder described in this dissertation are ubiquitous and causally related to a modified bladder function or whether they are merely a secondary development remains to be elucidated. In the field of the gastrointestinal tract, more insight about the function of interstitial cells has come from studies of genetic models (W/WV and SL/SLd animals) with loss-of function mutations in the Kit signaling pathway. ${ }^{103}$ Future studies in genetic models 
may determine the importance of interstitial cells in normal and pathological bladder functioning. More research in the field of interstitial cells will hopefully lead to new and more adequate therapeutical options for patients suffering from an overactive bladder.

\section{Autonomous activity influenced by prostaglandins and the role of interstitial cells}

As mentioned in the introduction of the dissertation, it has been known for a long time that prostaglandins are released from the bladder in response to stretch, and that they are synthesized by the urothelium and muscle layers. ${ }^{32-36}$ Exogenous prostaglandins can induce non-voiding contractions and increase voiding frequency in different species (e.g. humans, rats, guinea pigs, rabbits and monkeys). ${ }^{37-48}$ The mechanisms underlying this action of prostaglandins are not clear yet. Several possibilities have been suggested, such as a direct effect on the smooth muscle and a sensitisation effect of bladder afferent fibres. ${ }^{32,49} \mathrm{~A}$ different mechanism is proposed in this dissertation. Using the isolated guinea pig bladder, we demonstrated that several prostaglandins increase the frequency and amplitude of the autonomous activity. ${ }^{50}$ Prostanoid receptor antagonists blocked the prostaglandin induced effect, reflecting a prostanoid receptor mediated response. We hypothesise that the prostaglandin induced increase of autonomous activity, results in an increase in afferent discharges and subsequently, via increasing bladder sensations, influence the point of threshold for voiding. ${ }^{50}$ This idea is supported by previously published studies. ${ }^{23,49}$ The precise cell types responsible for the prostaglandin synthesis in the urinary bladder have not been identified yet. However, we know that prostaglandins are synthesized by two distinct enzymes: cyclo-oxygenase type I (COX I) and cyclooxygenase type II (COX II). We have demonstrated that the expression of COX I immunoreactivity (COX I-IR), and so the synthesis of prostaglandins, predominates within two general cell systems in the bladder wall: 1/ cells within the basal and intermediate layers of the urothelium and 2 / within a population of small cells which are closely associated with a network of vimentin positive cells. Since vimentin is a marker for interstitial cells this network of vimentin positive cells is indicated as interstitial cells. These vimentin positive interstitial cells are present throughout the sub-urothelial space of the lamina propria and extend over the surface of the muscle bundles which make up the inner layers. ${ }^{51}$ These observations raise questions regarding the function of the prostaglandin producing cells in the guinea pig bladder. It has been speculated that COX I cells in the urothelial layer are activated in response to stretch of the bladder wall and subsequently the synthesized prostaglandins activate the sub-urothelial afferent nerves. ${ }^{52}$ Furthermore, the microanatomical arrangement of COX I-IR cells to the vimentin positive interstitial cells in the muscle layer suggests a prostaglandin mediated regulation of interstitial cells which could activate the muscle layer. ${ }^{51}$ However, the functional roles of the different 
COX I-IR cells are not known and the target cells of prostaglandins in the bladder remain yet to be determined. Future studies on the functioning of COX I-IR cells and detection of prostanoid receptors on different cell types (e.g. muscle, interstitial cells and urothelium) will reveal the role of COX-IR cells and prostaglandins in bladder function.

Besides COX I-IR cells, we also detected COX II-IR cells in the normal bladder. This was surprising since this enzyme is associated with inflammation and pathological changes. COX II-IR was associated with the nuclei of cells in the urothelium, lamina propria and muscle. ${ }^{50}$ There are other studies showing prostaglandin synthesis around the nucleus. ${ }^{52-54}$ The COX II system might be involved in the regulation and modulation of cell proliferation and expression. However, more research on this topic is necessary.

It is clear that prostaglandins play a substantial role in bladder physiology, however, they may also play a role in bladder pathophysiology. BOO and bladder inflammation are associated with increased levels of urinary prostaglandins ${ }^{55-56}$ and increased expression of COX II in bladder tissue. ${ }^{57}$ The increased production of prostaglandins in obstructed bladders could modify the autonomous activity and subsequently produce symptoms. The question arises if COX inhibitors and or prostanoid receptor blockers could be beneficial in the treatment of overactive bladder symptoms. Studies in rats already revealed that COX inhibitors reduce volume-induced bladder voiding contractions, increase the bladder capacity and inhibit the micturition reflex. ${ }^{49,58-59}$ Furthermore, animal studies demonstrated that $\mathrm{EP}_{1}$ receptor antagonists inhibit detrusor overactivity related to $\mathrm{BOO} .{ }^{60}$ Controlled clinical trials with COX inhibitors for the treatment of the overactive bladder are scarce and dated. Flurbiprofen and indomethacin treatment in patients with bladder overactivity ${ }^{61-62}$ results in symptomatic relief, however, the incidence of side effects is high. Future studies investigating the effect of outlet obstruction on COX-I and COXII expression in the bladder wall may clarify the importance of the COX system for the pathophysiology of the obstructed bladder. In addition placebo-controlled randomized trials are required to assess the value of COX inhibitors for the treatment of LUTS. However, recent findings of cardiovascular toxicity associated with prolonged use of COX-II inhibitors, will make these studies difficult.

\section{Oxidative stress and bladder outlet obstruction}

The bladder function changes in obstructed bladders and different stages can be distinguished such as a compensated stage. This stage is characterized by an increase in bladder mass, a nearly normal muscle function and the appearance of detrusor overactivity. At some point, bladder function destabilizes and the organ enters a decompensated stage characterized by a further increase in bladder mass, decreased contractile responses to pharmacological stimulation and an increase in post-void residual volume. ${ }^{63-64}$ When looking at the clinical problem associated with 
$\mathrm{BOO}$, it is reasonable to suggest that the compensated stage is related to storage symptoms (daytime urinary frequency, nocturia, urgency, urinary incontinence) whereas the decompensated stage involves voiding and postmicturition symptoms (slow stream, intermittence, hesitancy, straining, terminal dribble, sensation of incomplete emptying, urinary retention). The shift from a compensated to a decompensated stage might be related to cyclical periods of ischemia-reperfusion in the bladder wall generating free radicals eventually leading to bladder muscle damage. ${ }^{64-67}$ Several muscle strip studies have examined the alterations in detrusor contractility of obstructed bladders. In Chapter 6, we showed that the neurogenically-evoked contractile responses of guinea pig muscle strips were significantly lower in obstructed bladders compared to the sham group. ${ }^{68}$ Since the muscarinic and purinergic mediated responses were not decreased our results reflect a muscle denervation. This is in agreement with several functional studies in obstructed bladders. ${ }^{69-70}$ The denervation in obstructed muscle tissue found in the functional part of the study was confirmed by histological data. A patchy denervation was identified in the detrusor layer of the obstructed bladders compared to the sham group. ${ }^{68}$ These findings are in keeping with previous reports demonstrating punctuate denervation in obstructed bladders. ${ }^{11,12}$ The myogenic contractile responses of obstructed bladders in our study were not decreased compared to the sham group. In the literature there are conflicting data on the myogenic responses of strips from obstructed bladders. Some studies have reported impaired contractile responses to cholinergic agonists and potassium, ${ }^{71-72}$ whereas other studies reported unchanged responses ${ }^{73}$ or even a relative supersensitivity to cholinergic agonists. ${ }^{11}$ These conflicting data are probably related to the influence of the severity and/ or duration of the outlet obstruction. In general, increased or unchanged responses are observed in mild forms of bladder outlet obstruction probably reflecting a compensated stage of the bladder function. Decreased responses are found in more severely obstructed bladders, a condition likely related to a decompensation of bladder function. ${ }^{74}$ Since the myogenic bladder responses in our study did not decrease, the obstructed bladders seem to be in a compensated stage. ${ }^{68}$

There is substantial evidence that the mechanism underlying the denervation and muscle dysfunction found in some stages of obstructed bladders, involves oxidative stress. Reactive nitrogen and oxygen species (RNOS) produced during ischemic and inflammatory conditions are able to destroy virtually any molecule, including proteins, DNA and lipids. ${ }^{63,65-66,75-76}$ Fortunately, the human body has its own endogenous protection against RNOS consisting of antioxidant enzymes (e.g. superoxide dismutase, catalase, glutathione reductase and glutathione peroxidise) and various antioxidants (e.g. glutathione, uric acid, melatonin, bilirubin, vitamin C and $\mathrm{E}$ ). Antioxidants seem to protect the bladder against functional and structural damage due to $\mathrm{BOO},{ }^{77-79}$ suggesting that tissues of obstructed bladders have a reduced antioxidative capacity. We showed for the first time that the antioxidant capacity, as measured by the TEAC (Trolox Equivalent Antioxidant Capacity) test, significantly decreased in obstructed guinea pig bladders compared to sham 
operated bladders. This was partly due to a reduced amount of gluthathione (GSH) whereas uric acid did not decrease significantly. ${ }^{68}$ The activity of glutathione reductase decreased significantly in the obstructed bladder tissue. This is in accordance with the results of a recent study which reports that the activity of the antioxidant enzymes superoxide dismutase and catalase was reduced in bladder tissue exposed to an outlet obstruction. ${ }^{67}$ The fact that the endogenous antioxidant system had deteriorated suggests that obstructed bladders are exposed to elevated levels of RNOS which is likely to result in oxidative stress. Several studies indeed showed that oxidative stress markers increased in bladder tissue exposed to an outlet obstruction. The nitrotyrosine level (end product of oxidative damage to proteins), malondialdehyde (MDA) and hydroxynonenal (HNE) level (end products of oxidative damage to lipids) were increased in these studies. ${ }^{80-82}$

If oxidative stress plays a significant role in the tissue injury of obstructed bladders then direct application of free radicals to bladder tissue, over a relevant time period, at concentrations within the range found in vivo could reproduce some of the tissue injury observed in obstructed bladders. In Chapter 7, we showed that oxidative stress mimicked by cumene hydroperoxide (CHP), a lipophilic hydroperoxide, damaged the muscarinic receptor signalling system in muscle strips from pig urinary bladder. In contrast, L-type calcium channels and the contractile system were less affected. ${ }^{83}$ This correlates with functional bladder tissue damage due to severe ischemia and reperfusion, where there is a reduction of both the efficacy and the sensitivity to cholinergic stimulation. ${ }^{82,84}$ Oxidative damage to lipids is known as lipid peroxidation. BOO is correlated with an increased peroxidation of lipids and the aldehyde 4-hydroxynonenal (HNE) is produced in large amounts during lipid peroxidation, therefore we have investigated the effect of HNE on muscle contractility of pig urinary bladder. We found that L-type calcium channels and or the contractile system of the bladder are susceptible to HNE-mediated damage. ${ }^{85}$ In contrast to the effect of $\mathrm{CHP}$, the muscarinic receptor signalling system remained largely unaffected by HNE. This is in agreement with previously published studies ${ }^{86-}$ ${ }^{87}$ The effects of HNE on bladder smooth muscle are most likely mediated via alkylation of essential sulfhydryl groups. Cholinergic nerves remain largely unaffected by oxidative stress mimicked by HNE and CHP. ${ }^{83,85}$ This is in line with the results of an other in vitro oxidative stress study. ${ }^{88}$ The finding of nerve resistibility in the in vitro studies is surprising as our in vivo study and many other studies, suggest that denervation is one of the initial degeneration events resulting from oxidative stress mediated in obstructed bladders. The duration of our in vitro experiments may be too short for mediating nerve damage. Furthermore, oxidative stress imposed on the bladder muscle in vitro has a more acute nature than oxidative stress occurring in vivo. Thus, it is questionable if direct application of oxidative stress in vitro provides similar oxidative stress conditions mediated in vivo.

We did not investigate the effect of oxidative stress on the sensory component of nerves. Some studies report that RNOS stimulate capsaicin sensitive C-fibers. ${ }^{89-90}$ One recent study in bladder tissue reported that low doses of hydrogen peroxide 
activate the capsaicin sensitive C-fiber afferent pathway resulting in detrusor overactivity. High doses of hydrogen peroxide decreased the micturition pressure and induced dribbling incontinence reflecting impairment of the detrusor muscle. ${ }^{91}$ Theoretically, RNOS, via activation of the afferent nerves could play a role in mediating increased bladder sensations seen in some patients with obstructed bladders. Furthermore, RNOS might play a role in modulating autonomous bladder activity.

Since antioxidants can scavenge free radicals, the question arises if antioxidants are beneficial in protecting the bladder function of obstructed bladders. Indeed, exogenous, dietary antioxidants demonstrated to be beneficial in animal models of bladder outlet obstruction. ${ }^{77-79}$ Furthermore, several antioxidants (e.g. vitamin $E_{\text {, }}$ galangin, melatonin, coenzyme Q10, resveratrol and grape suspensions) have protective effects on the detrusor muscle and nerves in models of oxidative stress. ${ }^{92-}$ ${ }^{98}$ Limited and controversial evidence for a beneficial health effect of antioxidants in humans comes from studies using plant extracts (phytotherapy) in the treatment of lower urinary tract symptoms (LUTS) associated with benign prostatic hyperplasia $(\mathrm{BPH})$. Pygeum africanum, which likely has antioxidative effects, provides a moderately large improvement in LUTS compared to placebo in the treatment of men with $\mathrm{BPH}^{99}$ In addition, pygeum africanum can prevent and reverse bladder dysfunction in a rabbit model of bladder outlet obstruction. ${ }^{100}$

Clearly, more research is needed to investigate the role of antioxidant supplementation in patients with lower urinary tract symptoms. Important aspects that should be considered include the scavenging capacities of certain antioxidants and their bio-availability. Moreover, the possible toxicity of antioxidants, when administered in high levels has to be considered too. For example, high doses of the antioxidant $\beta$-carotene seem to have toxic effects.$^{102-102}$

\section{Final remarks}

It is recognized nowadays that the bladder is a very complex organ. It is not a simple effector organ but an organ with its own adaptative and modulatory mechanisms. The results of our studies stress the need for further research in the field of the lower urinary tract. Experimental and clinical studies designed on the basis of this dissertation and future fundamental studies will lead to adequately targeted medical care in the near future for patients suffering from an overactive bladder. 


\section{References}

1. Andersson KE. Mechanisms of Disease: central nervous system involvement in overactive bladder syndrome. Nat Clin Pract Urol. 2004; 1(2):103-8.

2. Mostwin JL, Karim OM, van Koeveringe G, Brooks EL. The guinea pig as a model of gradual urethral obstruction. J Urol. 1991; 145(4):854-8.

3. Klevmark B. Motility of the urinary bladder in cats during filling at physiological rates. II. Effects of extrinsic bladder denervation on intramural tension and on intravesical pressure patterns. Acta Physiol Scand. 1977; 101(2):176-84.

4. Vaughan $\mathrm{CW}$, Satchell PM. Role of sympathetic innervation in the feline continence process under natural filling conditions. J Neurophysiol. 1992; 68(5):1842-9.

5. Gillespie JI. A developing view of the origins of urgency: the importance of animal models. BJU Int. 2005; 96(Suppl 1):22-8.

6. Gillespie JI. The autonomous bladder: a view of the origin of bladder overactivity and sensory urge. BJU Int. 2004; 93(4):478-83.

7. de Jongh $R$, van Koeveringe $G A$, van Kerrebroeck $P E$, Markerink-van Ittersum $M$, de Vente $J$, Gillespie JI. Damage to the bladder neck alters autonomous activity and its sensitivity to cholinergic agonists. BJU Int. 2007; 100(4):919-29.

8. Drake MJ, Hedlund P, Harvey IJ, Pandita RK, Andersson KE, Gillespie JI. Partial outlet obstruction enhances modular autonomous activity in the isolated rat bladder. J Urol. 2003; 170(1):276-9.

9. de Jongh $R$, van Koeveringe GA, van Kerrebroeck PE, Markerink-van Ittersum $M$, de Vente J, Gillespie JI. Alterations to network of NO/CGMP-responsive interstitial cells induced by outlet obstruction in guinea-pig bladder. Cell Tissue Res. 2007; 330(1):147-60.

10. Kubota Y, Hashitani $H$, Shirasawa N, Kojima Y, Sasaki S, Mabuchi Y, et al. Altered distribution of interstitial cells in the guinea pig bladder following bladder outlet obstruction. Neurourol Urodyn $2007 ; 27: 27$.

11. Brading AF. A myogenic basis for the overactive bladder. Urology. 1997; 50(6A Suppl):57-67; discussion 68-73.

12. Elbadawi A, Yalla SV, Resnick NM. Structural basis of geriatric voiding dysfunction. IV. Bladder outlet obstruction. J Urol. 1993; 150(5 Pt 2):1681-95.

13. Schroder A, Uvelius $B$, Newgreen $D$, Andersson KE. Bladder overactivity in mice after 1 week of outlet obstruction. Mainly afferent dysfunction? J Urol. 2003; 170(3):1017-21.

14. Botros SM, Miller JJ, Goldberg RP, Gandhi S, AkI M, Beaumont JL, et al. Detrusor overactivity and urge urinary incontinence following trans obturator versus midurethral slings. Neurourol Urodyn. 2007; 26(1):42-5.

15. Birder LA. More than just a barrier: urothelium as a drug target for urinary bladder pain. Am J Physiol Renal Physiol. 2005; 289(3):F489-95.

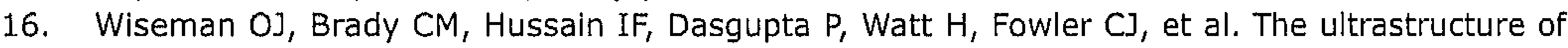
bladder lamina propria nerves in healthy subjects and patients with detrusor hyperreflexia. J Urol. 2002; 168(5):2040-5.

17. Gillespie JI, Markerink-van Ittersum M, de Vente J. Expression of neuronal nitric oxide synthase (nNOS) and nitric-oxide-induced changes in CGMP in the urothelial layer of the guinea pig bladder. Cell Tissue Res. 2005; 321(3):341-51.

18. Brading AF, McCloskey KD. Mechanisms of Disease: specialized interstitial cells of the urinary tract-an assessment of current knowledge. Nat Clin Pract Urol. 2005; 2(11):546-54.

19. Araki I, Du S, Kamiyama $M$, Mikami $Y$, Matsushita $K$, Komuro $M$, et al. Overexpression of epithelial sodium channels in epithelium of human urinary bladder with outlet obstruction. Urology. 2004; 64(6):1255-60.

20. Sun $Y$, MaLossi J, Jacobs SC, Chai TC. Effect of doxazosin on stretch-activated adenosine triphosphate release in bladder urothelial cells from patients with benign prostatic hyperplasia. Urology. 2002; 60(2):351-6. 
21. Yoshida M, Miyamae K, Iwashita $H$, Otani M, Inadome A. Management of detrusor dysfunction in the elderly: changes in acetylcholine and adenosine triphosphate release during aging. Urology. 2004; 63(3 Suppl 1):17-23.

22. Finney SM, Stewart LH, Gillespie 31. Cholinergic activation of phasic activity in the isolated bladder: possible evidence for M3- and M2-dependent components of a motor/sensory system. BJU Int. 2007; 100(3):668-78.

23. Mukerji G, Yiangou Y, Grogono J, Underwood J, Agarwal SK, Khullar V, et al. Localization of M2 and $\mathrm{M} 3$ muscarinic receptors in human bladder disorders and their clinical correlations. J Urol. $2006 ; 176(1): 367-73$.

24. Gillespie JI, Harvey IJ, Drake MJ. Agonist- and nerve-induced phasic activity in the isolated whole bladder of the guinea pig: evidence for two types of bladder activity. Exp Physiol. 2003; $88(3): 343-57$

25. Yoshida M, Inadome A, Maeda $Y$, Satoji $Y$, Masunaga $K$, Sugiyama $Y$, et al. Non-neuronal cholinergic system in human bladder urothelium. Urology. $2006 ; 67(2): 425-30$.

26. Andersson KE, Yoshida M. Antimuscarinics and the overactive detrusor-which is the main mechanism of action? Eur Urol. 2003; 43(1):1-5.

27. Finney SM, Andersson KE, Gillespie JI, Stewart LH. Antimuscarinic drugs in detrusor overactivity and the overactive bladder syndrome: motor or sensory actions? BJU Int. 2006; 98(3):503-7.

28. De Laet K, De Wachter S, Wyndaele J]. Systemic oxybutynin decreases afferent activity of the pelvic nerve of the rat: new insights into the working mechanism of antimuscarinics. Neurourol Urodyn. 2006; 25(2):156-61.

29. Yokoyama O, Yusup A, Miwa Y, Oyama N, Aoki Y, Akino H. Effects of tolterodine on an overactive bladder depend on suppression of C-fiber bladder afferent activity in rats. J Urol. 2005; 174(5):2032-6.

30. Fry $\mathrm{CH}$, Ikeda $Y$, Harvey $R$, Wu $C$, Sui GP. Control of bladder function by peripheral nerves: avenues for novel drug targets. Urology, 2004; 63(3 Suppl 1):24-31.

31. de Groat WC. The urothelium in overactive bladder: passive bystander or active participant? Urology. 2004; 64(6 Suppl 1):7-11.

32. Maggi CA. Prostanoids as local modulators of reflex micturition. Pharmacol Res. 1992; 25(1):1320 .

33. Brown WW, Zenser TV, Davis BB. Prostaglandin E2 production by rabbit urinary bladder. Am ] Physiol. 1980; 239(5):F452-8

34. Downie JW, Karmazyn M. Mechanical trauma to bladder epithelium liberates prostanoids which modulate neurotransmission in rabbit detrusor muscle. J Pharmacol Exp Ther. 1984; 230(2):445-9.

35. Jeremy JY, Tsang V, Mikhailidis DP, Rogers $H$, Morgan RJ, Dandona P. Eicosanoid synthesis by human urinary bladder mucosa: pathological implications. Br J Urol. 1987; 59(1):36-9.

36. Khan MA, Thompson CS, Mumtaz FH, Jeremy JY, Morgan RJ, Mikhailidis DP. Role of prostaglandins in the urinary bladder: an update. Prostaglandins Leukot Essent Fatty Acids. 1998; 59(6):415-22.

37. Bultitude MI, Hills NH, Shuttleworth KE. Clinical and experimental studies on the action of prostaglandins and their synthesis inhibitors on detrusor muscle in vitro and in vivo. Br J Urol. $1976 ; 48(7): 631-7$.

38. Hills NH. Prostaglandins and tone in isolated strips of mammalian bladder [proceedings]. Br ] Pharmacol. 1976; 57(3):464P-465P.

39. Andersson KE, EK A, Persson CG. Effects of prostaglandins on the isolated human bladder and urethra. Acta Physiol Scand. 1977; 100(2):165-71.

40. Borda E, Contreras-Ortiz N, Gutnisky R, Gimeno MF. In vitro effect of acetylcholine and bethanechol on the contractions of the human detrusor muscle. Influence of prostaglandins. Arch Int Pharmacodyn Ther, 1982; 259(1):31-9.

41. Choo LK, Mitchelson F. The role of prostaglandins in the excitatory innervation of the rat urinary bladder. Prostaglandins. 1977; 13(5):917-26.

42. Johns $A$. The effect of indomethacin and substance $P$ on the guinea pig urinary bladder. Life Sci. 1981; 29(17):1803-9. 
43. Dean DM, Downie JW. Interaction of prostaglandins and adenosine 5'-triphosphate in the noncholinergic neurotransmission in rabbit detrusor. Prostaglandins. 1978; 16(2):245-51.

44. Husted S, Sjogren C, KE AN. Role of prostaglandins in the responses of rabbit detrusor to noncholinergic, non-adrenergic nerve stimulation and to ATP. Arch Int Pharmacodyn Ther. 1980; 246(1):84-97.

45. Andersson KE, Husted $S$, Sjogren $C$. Contribution of prostaglandins to the adenosine triphosphate-induced contraction of rabbit urinary bladder. $\mathrm{Br}$ J Pharmacol. 1980; 70(3):44352.

46. Downie JW, Larsson C. Prostaglandin involvement in contractions evoked in rabbit detrusor by field stimulation and by adenosine 5'-triphosphate. Can J Physiol Pharmacol. 1981; 59(3):25360 .

47. Johns A, Paton DM. Evidence for a role of prostaglandins in atropine-resistant transmission in the mammalian urinary bladder. Prostaglandins, 1976; 11(3):595-7.

48. Johns A, Paton DM. Effect of indomethacin on atropine-resistant transmission in rabbit and monkey urinary bladder: evidence for involvement of prostaglandins in transmission. Prostaglandins. 1977; 13(2):245-54.

49. Maggi CA, Giuliani S, Conte B, Furio M, Santicioli P, Meli $P$, et al. Prostanoids modulate reflex micturition by acting through capsaicin-sensitive afferents. Eur J Pharmacol. 1988; 145(2):10512.

50. de Jongh $R$, van Koeveringe $G A$, van Kerrebroeck PE, Markerink-van Ittersum $M$, de Vente $J$, Gillespie JI. The effects of exogenous prostaglandins and the identification of constitutive cyclooxygenase I and II immunoreactivity in the normal guinea pig bladder. BJU Int. 2007; $100(2): 419-29$.

51. de Jongh R, Grol S, van Koeveringe G, van Kerrebroeck P, de Vente J, Gillespie J. The localisation of cyclo-oxygenase immuno-reactivity (COX I-IR) to the urothelium and to interstitial cells in the bladder wall. J Cell Mol Med 2008; 21:21.

52. Andersson KE, Wein AJ. Pharmacology of the lower urinary tract: basis for current and future treatments of urinary incontinence. Pharmacol Rev. 2004; 56(4):581-631.

53. Ren Y, Walker C, Loose-Mitchell DS, Deng J, Ruan KH, Kulmacz RJ. Topology of prostaglandin H synthase-1 in the endoplasmic reticulum membrane. Arch Biochem Biophys. 1995; 323(1):20514.

54. Morita I, Schindler M, Regier MK, Otto JC, Hori T, DeWitt DL, et al. Different intracellular locations for prostaglandin endoperoxide $H$ synthase-1 and -2. J Biol Chem. 1995; 270(18):10902-8.

55. Kim JC, Park EY, Seo SI, Park YH, Hwang TK. Nerve growth factor and prostaglandins in the urine of female patients with overactive bladder. J Urol. 2006; 175(5):1773-6; discussion 1776.

56. Kim JC, Park EY, Hong SH, Seo SI, Park YH, Hwang TK. Changes of urinary nerve growth factor and prostaglandins in male patients with overactive bladder symptom. Int J Urol, 2005; 12(10):875-80.

57. Park JM, Yang T, Arend LJ, Schnermann JB, Peters CA, Freeman MR, et al. Obstruction stimulates COX-2 expression in bladder smooth muscle cells via increased mechanical stretch. Am J Physiol. 1999; 276(1 Pt 2):F129-36.

58. Maggi CA, Evangelista S, Grimaldi G, Santicioli P, Giolitti A, Meli A. Evidence for the involvement of arachidonic acid metabolites in spontaneous and drug-induced contractions of rat urinary bladder. J Pharmacol Exp Ther. 1984; 230(2):500-13.

59. Angelico P, Guarneri L, Velasco C, Cova R, Leonardi A, Clarke DE, et al. Effect of cyclooxygenase inhibitors on the micturition reflex in rats: correlation with inhibition of cyclooxygenase isozymes. BJU Int. 2006; 97(4):837-46.

60. Lee $T$, Hedlund $P$, Newgreen $D$, Andersson KE. Urodynamic effects of a novel EP(1) receptor antagonist in normal rats and rats with bladder outlet obstruction. J Urol. 2007; 177(4):15627.

61. Cardozo LD, Stanton SL. A comparison between bromocriptine and indomethacin in the treatment of detrusor instability. J Urol. 1980; 123(3):399-401. 
62. Cardozo LD, Stanton SL, Robinson H, Hole D. Evaluation of flurbiprofen in detrusor instability. $\mathrm{Br}$ Med J. 1980; 280(6210):281-2

63. Levin RM, Levin SS, Zhao Y, Buttyan R. Cellular and molecular aspects of bladder hypertrophy. Eur Urol. 1997; 32(Supp| 1):15-21.

64. Kalorin CM, Mannikarottu A, Neumann P, Leggett $R$, Weisbrot J, Johnson $A$, et al, Protein oxidation as a novel biomarker of bladder decompensation. BJU Int 2008; 13:13.

65. Greenland JE, Hvistendahl J], Andersen H, Jorgensen TM, McMurray G, Cortina-Borja M, et al. The effect of bladder outlet obstruction on tissue oxygen tension and blood flow in the pig bladder. BJU Int. 2000; 85(9):1109-14.

66. Greenland JE, Brading AF. The effect of bladder outflow obstruction on detrusor blood flow changes during the voiding cycle in conscious pigs. J Urol. 2001; 165(1):245-8.

67. Guven A, Kalorin C, Onal B, Whitbeck C, Chichester P, Kogan BA, et al. Novel biomarkers of bladder decompensation after partial bladder obstruction. Neurourol Urodyn. 2007; 26(7):1036-42.

68. de Jongh $R$, Dambros $M$, Haenen GR, den Hartog G], Bast A, van Kerrebroeck PE, et al. Partial bladder outlet obstruction reduces the tissue antioxidant capacity and muscle nerve density of the guinea pig bladder. Neurourol Urodyn 2008; 17:17.

69. Geloso DA, Levin RM. Effect of partial outlet obstruction on the myogenic response to field stimulation. Gen Pharmacol 1998; 31(2):291-5.

70. Kato K, Wein A], Kitada S, Haugaard N, Levin RM. The functional effect of mild outlet obstruction on the rabbit urinary bladder. J Urol 1988; 140(4):880-4.

71. Agartan CA, Whitbeck C, Chichester P, Kogan BA, Levin RM. Effect of age on rabbit bladder function and structure following partial outlet obstruction. J Urol. 2005; 173(4):1400-5.

72. Liuel $P$, Barras $M$, Palea 5. Cholinergic and purinergic contribution to the micturition reflex in conscious rats with long-term bladder outlet obstruction. Neurourol Urodyn. 2002; 21(2):14253.

73. Barendrecht MM, Chichester P, Michel MC, Levin RM. Effect of short-term outlet obstruction on rat bladder nerve density and contractility. Auton Autacoid Pharmacol. 2007; 27(1):47-53.

74. Michel MC, Barendrecht MM. Physiological and pathological regulation of the autonomic control of urinary bladder contractility. Pharmacol Ther. 2008; 117(3):297-312.

75. Bratslavsky G, Kogan BA, Matsumoto S, Aslan AR, Levin RM. Reperfusion injury of the rat bladder is worse than ischemia. J Urol. 2003; 170(5):2086 90

76. Halliwell B. Oxidative stress and neurodegeneration: where are we now? J Neurochem. 2006; $97(6): 1634-58$

77. Conners W, Whitebeck C, Chicester P, Legget R, Lin AD, Johnson A, et al. L-NAME, a nitric oxide synthase inhibitor, diminishes oxidative damage in urinary bladder partial outlet obstruction. Am J Physiol Renal Physiol. 2006; 290(2):F357-63..

78. Levin RM, Kawashima Y, Leggett RE, Whitbeck C, Horan P, Mizutani K. Effect of oral Kohki tea on bladder dysfunction induced by severe partial outlet obstruction. J Urol 2002; 167(5):22606.

79. Parekh MH, Lobel R, O'Connor LJ, Leggett RE, Levin RM. Protective effect of vitamin $E$ on the response of the rabbit bladder to partial outlet obstruction. J Urol 2001; 166(1):341-6.

80. Mannikarottu A, Lin AD, Whitebeck C, Leggett R, Kogan B, Levin R. Effect of partial bladder outlet obstruction on nitrotyrosine levels and their correlation with contractile function. Neurourol Urodyn. 2006; 25(4):397-401.

81. Lin AT, Yang CH, Chen KK, Chang LS. Detrusor mitochondrial lipid peroxidation and superoxide dismutase activity in partial bladder outlet obstruction of rabbits. Neurourol Urodyn 2005; 24(3):282-7.

82. Saito M, Miyagawa I. Bladder dysfunction after acute urinary retention in rats. J Urol 2001; 165(5):1745-7.

83. de Jongh $R$, Haenen $G R$, van Koeveringe $G A$, Dambros $M$, De Mey JG, van Kerrebroeck PE. Oxidative stress reduces the muscarinic receptor function in the urinary bladder. Neurourol Urodyn. 2007; 26(2):302-8. 
84. Saito M, Tominaga L, Nanba E, Kinoshita $Y$, Housi D, Miyagawa $I$, et al. Expression of HSP 70 and its mRNAS during ischemia-reperfusion in the rat bladder. Life Sci 2004; 75(15):1879-86.

85. de Jongh $R$, Haenen GR, van Koeveringe GA, Dambros M, van Kerrebroeck PE. Lipid Peroxidation Product 4-Hydroxynonenal Contributes to Bladder Smooth Muscle Damage. Urology 2008; $14: 14$.

86. Haenen GR, Plug HJ, Vermeulen NP, Timmerman H, Bast A. Contribution of 4-hydroxy-2,3-transnonenal to the reduction of beta-adrenoceptor function in the heart by oxidative stress. Life Sci 1989; 45(1):71-6.

87. Van der Vliet A, Van der Aar EM, Bast A. The lipid peroxidation product 4-hydroxy-2,3-trans-1 nonenal decreases rat intestinal smooth muscle function in-vitro by alkylation of sulphydryl groups. J Pharm Pharmacol 1991; 43(7):515-7.

88. Alkawa K, Leggett RE, Levin RM. Effect of age on hydrogen peroxide mediated contraction damage in the male rat bladder. J Urol 2003; 170(5):2082-5.

89. Ruan T, Ho CY, Kou YR. Afferent vagal pathways mediating respiratory reflexes evoked by ROS in the lungs of anesthetized rats. J Appl Physiol. 2003; 94(5):1987-98.

90. Stahl GL, Pan HL, Longhurst JC. Activation of ischemia- and reperfusion-sensitive abdominal visceral $C$ fiber afferents. Role of hydrogen peroxide and hydroxyl radicals. Circ Res. 1993; $72(6): 1266-75$

91. Masuda H, Kihara K, Saito K, Matsuoka Y, Yoshida S, Chancellor MB, et al. Reactive oxygen species mediate detrusor overactivity via sensitization of afferent pathway in the bladder of anaesthetized rats. BJU Int. 2008; 101(6):775-80.

92. Juan YS, Hydery T, Mannikarottu A, Kogan B, Schuler C, Leggett RE, et al. Coenzyme Q10 protect against ischemia/reperfusion induced biochemical and functional changes in rabbit urinary bladder. Mol Cell Biochem. 2008; 311(1-2):73-80.

93. Lin AD, Mannikarottu A, Chaudhry A, Whitbeck C, Kogan BA, Chichester P, et al. Protective effects of grape suspension on in vivo ischaemia/reperfusion of the rabbit bladder. BJU Int. 2005; 96(9):1397-402.

94. Toklu H, Alican I, Ercan F, Sener $G$. The beneficial effect of resveratrol on rat bladder contractility and oxidant damage following ischemia/reperfusion. Pharmacology. 2006; 78(1):44-50.

95. Dambros $M$, de Jongh $R$, van Koeveringe GA, Bast $A$, Heijnen $C G$, van Kerrebroeck PE. Flavonoid galangin prevents smooth muscle fatigue of pig urinary bladder. J Pharm Pharmacol. 2005; $57(5): 617-22$.

96. Dambros $M$, de Jongh $R$, van Koeveringe GA, Bast $A$, van Kerrebroeck PE. Galangin protects pig detrusor nerves from repetitive field stimulation and anoxia/glucopenia injury, Urology. 2005; $66(6): 1327-31$.

97. Onur R, Tasdemir C, Seckin D, Ilhan N, Kutlu S, Akpolat N. Combined Use of Melatonin and Terazosin Restores Bladder Contractility in Rabbits With Partial Outlet Obstruction. Urology 2008; $1: 1$.

98. Parekh $M H$, Lobel $R$, O'Connor LJ, Leggett RE, Levin RM. Protective effect of vitamin $E$ on the response of the rabbit bladder to partial outlet obstruction. J Urol. 2001; 166(1):341-6.

99. Wilt $T$, Ishani A, Mac Donald R, Rutks I, Stark G. Pygeum africanum for benign prostatic hyperplasia. Cochrane Database Syst Rev. 2002(1):CD001044.

100. Edgar $A D$, Levin $R$, Constantinou $C E$, Denis $L$. A critical review of the pharmacology of the plant extract of Pygeum africanum in the treatment of LUTS. Neurourol Urodyn. $2007 ; 26(4): 458-63$; discussion 464.

101. Albanes D, Heinonen OP, Huttunen JK, Taylor PR, Virtamo J, Edwards BK, et al. Effects of alphatocopherol and beta-carotene supplements on cancer incidence in the Alpha-Tocopherol BetaCarotene Cancer Prevention Study. Am J Clin Nutr. 1995; 62(6 Suppl): 1427S-1430S.

102. Pryor WA, Stahl W, Rock CL. Beta carotene: from biochemistry to clinical trials. Nutr Rev, 2000; 58(2 Pt 1):39-53.

103. Sanders KM, Ward SM. Kit mutants and gastrointestinal physiology. J Physiol, 2007; 578(Pt 1):33-42 


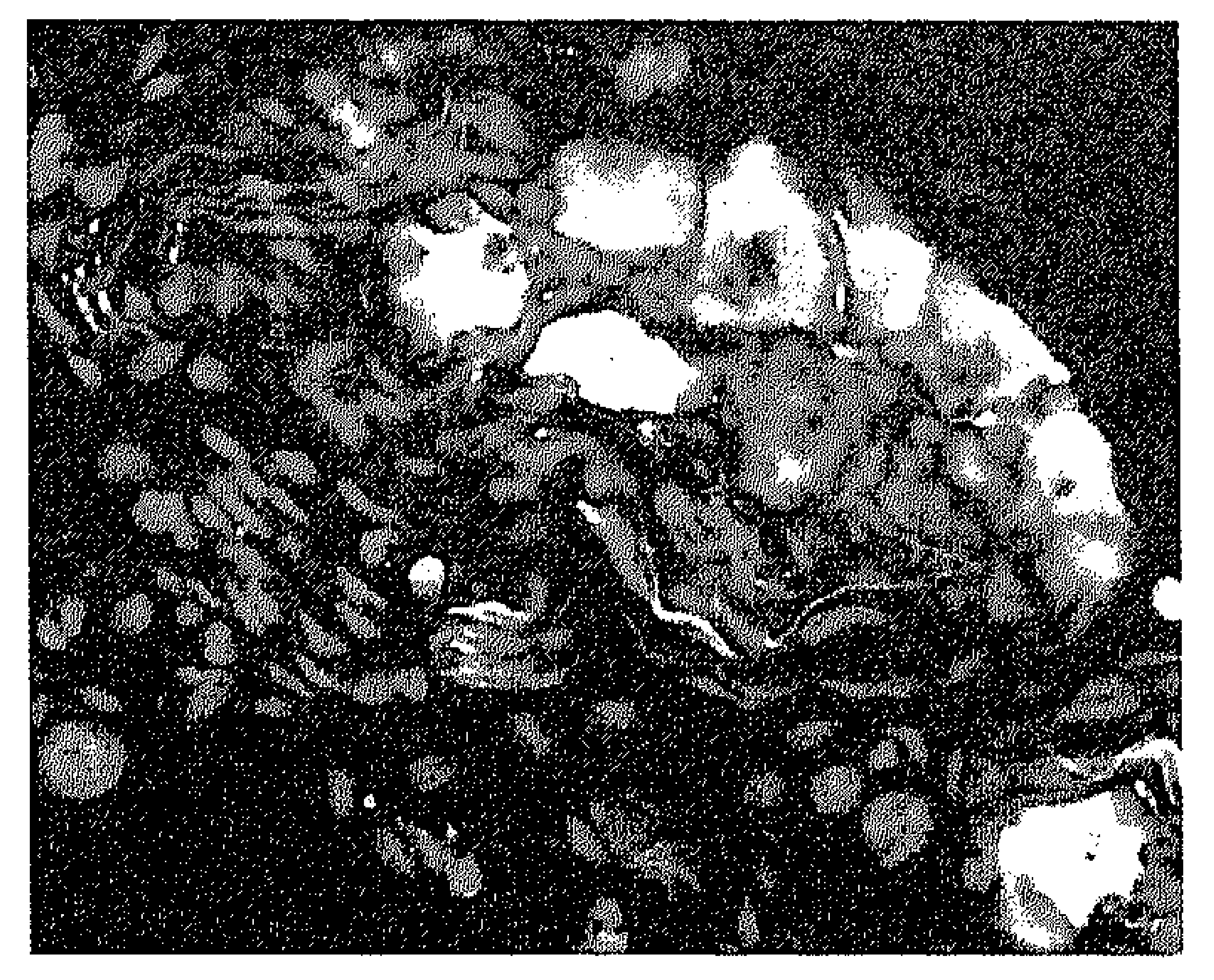

Summary / Samenvatting 


\section{Summary}

Patients with an Overactive Bladder (OAB) syndrome have symptoms including urinary urgency with or without urgency incontinence, urinary frequency, and nocturia. The overall prevalence of the OAB syndrome is about $16 \%$ and the total economic costs are high. Although the $O A B$ syndrome is not life threatening it seriously affects the quality of life and the ability to work. The symptoms of an overactive bladder can be caused by disorders in de brain, in the spinal cord, in peripheral nerves and in the bladder. This dissertation focuses on abnormalities of the bladder and the bladder neck possibly contributing to the $O A B$ syndrome. These bladder disorders are related to an increased afferent activity.

In Chapter 1, an introduction is given on the different anatomical components involved in the storage and micturition phase of the lower urinary tract. Pathological conditions of these anatomical components (e.g. brain, central nervous system, peripheral nerves and the bladder) can result in an overactive bladder. An overview of the $O A B$ syndrome is given and the main theories concerning the pathophysiology of this condition are mentioned. The autonomous bladder theory is discussed in more detail. The idea is that autonomous activity is a central part of a motor and sensory system operating within the bladder wall. The localised contractions in the bladder wall stimulate afferent nerves which likely contribute to bladder sensations. Patients with a bladder inflammation or a bladder outlet obstruction can develop symptoms of an overactive bladder. Our hypothesis is that an increased autonomous activity plays a role in the occurrence of OAB symptoms. In order to test this hypothesis we used pathological bladder conditions, such as a bladder outlet obstruction (BOO) and increased levels of prostaglandins in the bladder. This dissertation describes the autonomous bladder activity in both pathological bladder conditions. Since basic research in humans raise moral and ethical dilemmas we used animal models.

In Chapter 2 we present, the effect of different prostaglandins on the autonomous activity of isolated whole guinea pig bladders. The prostaglandins PGE1, PGE2 induced an increase in basal pressure of the bladder. In addition, these prostaglandins increased the amplitude and frequency of the autonomous activity. The prostaglandin mediated responses were blocked by a prostanoid receptor antagonist, reflecting a prostanoid receptor induced effect. Prostaglandin synthesis has been associated with the urothelium and the smooth muscle, however, the precise synthesising cell types are not known. By using an antibody to cyclooxygenase type I (COX I) we showed for the first time that COX I immunoreactivity (COX I-IR) was located in cells in the basal and intermediate urothelium, in the lamina propria and in cells on the surface of the inner muscle bundles. Cyclooxygenase type II immunoreactivity (COX II-IR) was associated with the nuclei of cells in the urothelium, lamina propria and muscle.

The origin of the autonomous activity and how this is modulated by prostaglandins have not been elucidated yet. It has been suggested that autonomous activity is generated and distributed by a network of specialised cells in the bladder 
wall: interstitial cells. Therefore, in Chapter 3 the relationship between COX I-IR cells and networks of interstitial cells was described by using different immunohistochemical markers for interstitial cells such as vimentin and cGMP. Basically, two types of COX I-IR cells were identified in the lamina propria: complex vimentin-positive/COX I-IR cells and vimentin-negative/COX I-IR cells. On the surface of the inner muscle layer vimentin-negative/COX I-IR cells were present. The vimentin-negative/COX I-IR cells appear to be in close apposition to a continuous network of vimentin-positive cells, also indicated as interstitial cells, which extend from the lamina propria into the inner muscle layers. This interstitial cell network appears to extend into the outer muscle layers. The interstitial cells in the outer muscle layer seem to form a distinctly different sub-type compared to the interstitial cells in the inner muscle layer. Firstly, the interstitial cells in the outer muscle region differ from those in the inner muscle layer by their responsiveness to NO with a rise in CGMP. Secondly, in contrast to the inner muscle layer, COX I-IR cells were rarely associated with the interstitial cells in the outer muscle layers. The physiological significance of the apparent differences in the interstitial cell network is not clear.

As mentioned before, patients with a bladder outlet obstruction frequently develop symptoms of an overactive bladder. In Chapter 4, the effect of bladder neck damage and or outlet obstruction on the autonomous activity was discussed. A validated guinea pig model for BOO was used. Initially 3 groups were distinguished: control, sham operated and obstructed guinea pig bladders. The bladder weight in all operated groups, including the sham, were greater than controls. The amplitude of the autonomous activity in operated bladders was significantly greater than in control bladders. The sham operated bladders had the largest transients per gram of bladder tissue. The changes in the sham group suggest that the pathological changes are the result of damage to the bladder neck and not the result of obstruction. Cholinergic stimulation increased the frequency and amplitude of the autonomous activity in control bladders. The mechanism controlling the frequency of the autonomous activity, the pacemaker, becomes progressively supersensitive to muscarinic agonists in sham operated and obstructed bladders. In addition, the distribution network, responsible for the amplitude of autonomous contractions, is increased in operated bladders. These changes in autonomous activity in operated bladders might contribute to the decreased threshold for voiding seen in patients with a bladder outlet obstruction. Furthermore, the cholinergic component of the modified autonomous activity in operated bladders might be the site of therapeutic action of anticholinergic drugs. Since interstitial cells are thought to be responsible for the generation and modulation of autonomous activity, the effects of a BOO on the network of interstitial cells was described in Chapter 5. Interstitial cells in the human and guinea pig bladder can be identified by their ability to show a rise in CGMP in response to nitric oxide donors. Different sub-types of cGMP+ interstitial cells, based on the location of the cells in the tissue, have been identified: interstitial cells lying in the sub-urothelial layer and in the outer muscle layer. Besides interstitial cells, cGMP staining in normal bladders also identifies other cell structures such as 
umbrella cells in the urothelium and some nerves. Chapter 5, presents CGMP staining in sham operated and obstructed guinea pig bladders. There were several differences detected between sham operated and obstructed bladders. For example, the sub-urothelial interstitial cell layer was increased in obstructed bladders compared to the sham group. In addition a more developed cGMP+ interstitial cells network penetrating and surrounding the muscle bundles occurred in obstructed bladders. Finally, the number of interstitial cells forming the serosal layer was increased in obstructed bladders compared to the sham operated bladders. Besides the interstitial cell network also other bladder wall components were modified in obstructed bladders. For instance, the urothelium is clearly modified in obstructed bladders: the number of cGMP+ umbrella cells and the intensity of staining was reduced compared to the sham group. The intermediate layer of the urothelium was modified and consisted of multiple cell layers in obstructed bladders. As in sham operated bladders, nerves were readily found in the lamina propria, however, they were rarely associated with the urothelium. The altered structure of the urothelium in obstructed bladders reflects a modified function. Alteration to the urothelial structure may imply changes in the ability of the urothelium to synthesize substances such as prostaglandins, nitric oxide, ATP and acetylcholine. This may modify the influence of the urothelium on sub-urothelial afferent nerve discharge and may subsequently result in altered bladder sensations.

Patients with a bladder outlet obstruction can develop besides the previous mentioned overactive bladder symptoms, also obstructive symptoms such as a slow urinary stream, hesitancy, intermittence, terminal dribble and eventually urinary retention. The obstructive symptoms are partly related to the contractile dysfunction of the bladder muscle. There is increasing evidence that reactive nitrogen and oxygen species (RNOS) play a role in the functional and structural bladder muscle damage related to a BOO. Decompensated obstructed bladders are associated with ischemia-reperfusion and inflammation in the bladder wall. Ischemia-reperfusion and inflammation are known to induce the production of RNOS. The human body has its own endogenous protection against RNOS consisting of antioxidant enzymes and various antioxidants. In situations such as ischemia-reperfusion and inflammation, free radicals may overwhelm the antioxidant defense system; a situation denoted as oxidative stress. Oxidative stress is regarded as noxious because RNOS are able to destroy virtually any molecule, including proteins, DNA and lipids. Nerves and muscle are especially susceptible to oxidative stress induced damage. In Chapter 6 the effect of BOO on the endogenous antioxidant status of the bladder in guinea pigs was presented and correlated to changes in bladder structure and function. We found that the total antioxidant capacity, the glutathione and glutathione reductase levels were significantly decreased in obstructed bladders. Furthermore, the amplitude of the electrical field stimulation evoked responses, which are based on neurogenic stimulation, were significantly reduced in obstructed bladders compared to the sham operated group. In addition, the nerve density of the muscle layer was significantly 
reduced in the obstructed bladder tissue compared to the sham group. Thus, there seems to be a denervation in obstructed bladders. Since the myogenic response of obstructed bladders did not decrease these bladders seem to be in a compensated stage. In conclusion, our results demonstrated that the antioxidant status of the guinea pig bladder exposed to outlet obstruction decreased. The reduction in nerve density might be caused by oxidative damage. If oxidative stress plays a significant role in the tissue injury of obstructed bladders then direct application of free radicals to bladder tissue over a relevant period of time, at concentrations within the range found in vivo, could reproduce some of the tissue injury observed in obstructed bladders. Therefore Chapter $\mathbf{7}$ describes the effects of oxidative stress on muscle contractility of pig urinary bladder. Oxidative stress was mimicked by cumene hydroperoxide (CHP), a lipophilic hydroperoxide. Muscle strips from pig urinary bladder were mounted in organ baths and contractile responses to various stimulation pathways were monitored before and after the addition of CHP. The results of our study demonstrated that oxidative stress severely damaged the muscarinic receptor signaling system. L-type calcium channels and or the contractile system were less affected and cholinergic nerves remained largely unaffected. Oxidative damage to lipids is known as lipid peroxidation. Peroxidation of lipids gives rise to various reactive aldehydes such as malondialdehyde (MDA) and 4hydroxynonenal (HNE). HNE is highly reactive and produced in relative large amounts during lipid peroxidation. Since bladder outlet obstruction is correlated with an increased peroxidation of lipids and HNE is produced in large amounts during lipid peroxidation, we presented in Chapter $\mathbf{8}$ the effect of HNE on muscle contractility of pig urinary bladder. Muscle strips from pig urinary bladder were mounted in organ baths and contractile responses to various stimulation pathways were monitored before and after the addition of HNE. We found that HNE severely damaged the Ltype calcium channels and or the contractile system of the bladder muscle, however, the cholinergic nerves and the muscarinic receptor signaling system remained largely unaffected. In conclusion, the bladder muscle dysfunction seen in severely obstructed bladders could partly be due to oxidative damage of the smooth muscle. The fact that our in vitro oxidative stress model did not result in neuronal damage may implicate that oxidative stress in vitro does not provide similar oxidative stress conditions compared to the in vivo situation.

In Chapter 9, the results of our studies are discussed and an attempt is made to put the fundamental findings of this dissertation in a clinical perspective. In conclusion, this dissertation revealed that bladder outlet obstruction and elevated prostaglandin levels result in an increased autonomous activity in guinea pig bladders. We hypothesize that this modified autonomous activity results in activation of afferent nerves in the bladder wall, eventually contributing to an $O A B$ syndrome. Interstitial cells may be responsible for the generation and distribution of autonomous activity. In addition, this dissertation suggests that oxidative stress is involved in the bladder muscle dysfunction found in some stages of bladder outlet obstruction. Finally, we believe that the results obtained in this dissertation are a 
Chapter 10

good starting point for developing new basic and clinical studies. Research in the field of interstitial cells and autonomous activity of the bladder may become an important and rewarding research area leading to better treatment options for patients with an overactive bladder. 


\section{Samenvatting}

Patiënten met een Overactieve Blaas (OAB) syndroom hebben klachten van urgency met of zonder urgency incontinentie, meestal gepaard gaand met vaak moeten plassen en nycturie. Het $O A B$ syndroom heeft een prevalentie van rond de $16 \%$ en veroorzaakt voor de maatschappij een forse kostenpost. Het OAB syndroom is geen levensbedreigende aandoening, echter het heeft een significant negatief effect op de kwaliteit van leven van patiënten. Klachten van een overactieve blaas kunnen worden veroorzaakt door afwijkingen in de hersenen, het centrale zenuwstelsel, de perifere zenuwen en de blaas zelf. In dit proefschrift worden lokale afwijkingen in de blaas en of blaashals besproken die waarschijnlijk een bijdrage leveren aan het ontstaan van het $O A B$ syndroom. Deze blaasaandoeningen leiden meestal tot een toename van de hoeveelheid afferente informatie die naar de hersenen wordt gezonden.

In de introductie (Hoofdstuk 1) worden de verschillende anatomische componenten - hersenen, centraal zenuwstelsel, perifere zenuwen en de blaas besproken die betrokken zijn bij de vullings en ledigingfase van de urineblaas. Verder wordt in het bijzonder de theorie van de autonome blaas beschreven. Deze theorie gaat ervan uit dat autonome activiteit (lokale contracties) van de blaaswand een centrale rol speelt in het motorisch sensorisch gekoppeld systeem. Het idee is dat lokale contracties in de blaaswand (motorische activiteit) afferente zenuwen activeren die uiteindelijk resulteren in het optreden van blaassensaties. Onze hypothese is dat een verhoogde autonome blaas activiteit een oorzaak kan zijn voor het ontstaan van het $O A B$ syndroom. Om deze hypothese te toetsen hebben we gebruik gemaakt van pathologische blaascondities, zoals een blaas uitgangsobstructie en een verhoogd prostaglandine gehalte in de blaas. Patiënten met een blaasontsteking (verhoogd prostaglandine gehalte) of met een blaas uitgangsobstructie kunnen klachten ontwikkelen van een overactieve blaas. Vandaar dat dit proefschrift de autonome blaasactiviteit in beide blaascondities beschrijft. Gezien het feit dat fundamenteel onderzoek in mensen ethisch moeilijk te verantwoorden is, hebben we gebruik gemaakt van proefdieren.

Hoofdstuk 2 bespreekt het effect van verschillende prostaglandines op de autonome blaasactiviteit in een caviamodel. Blootstelling aan prostaglandines $\mathrm{PGE}_{1}$ en $\mathrm{PGE}_{2}$ resulteert in toename van de amplitude en frequentie van de autonome activiteit. Het door prostaglandine veroorzaakte effect blijkt te berusten op stimulatie van prostanoid receptoren. Het is bekend dat de cyclo-oxygenasen (COX) enzymen verantwoordelijk zijn voor de productie van prostaglandines en dat het urotheel en de spierlaag van de blaas prostaglandines kunnen produceren. We hebben voor het eerst middels immunohistochemische technieken aangetoond dat COX I immunoreactiviteit (COX I-IR) is gelokaliseerd in specifieke cellen behorende tot het urotheel, de lamina propria en cellen rondom de binnenste spierlaag van de blaaswand. Verder zijn COX II immunoreactieve (COX II-IR) cellen gevonden in de kernen van cellen behorende tot het urotheel, de lamina propria en de spierlaag van 
de blaaswand.

De bron van autonome activiteit en hoe deze wordt gemoduleerd door prostaglandines is nog niet bekend. Er zijn aanwijzingen dat autonome activiteit wordt gegenereerd en verspreid door een netwerk van gespecialiseerde cellen in de blaaswand, die interstitiële cellen worden genoemd. In Hoofdstuk 3 hebben we de relatie tussen COX I-IR cellen en netwerken van interstitiële cellen in de caviablaas beschreven door gebruik te maken van verscheidene immunohistochemische markers voor interstitiële cellen zoals vimentine en CGMP. Gedurende het onderzoek hebben we twee typen COX I-IR cellen ontdekt in de lamina propria: complexe vimentine positieve/COX I-IR cellen en vimentine negatieve/COX I-IR cellen. Aan de rand van de binnenste spierlaag hebben we vimentine negatieve/COX I-IR cellen geïdentificeerd. Deze vimentine negatieve/COX I-IR cellen lijken in nauw contact te staan met vimentine positieve interstitiële cellen. Deze interstitiële cellen zijn gelegen in de lamina propria, de binnenste en de buitenste spierlaag. De interstitiële cellen in de buitenste spierlaag blijken andere typen cellen te zijn vergeleken met de interstitiële cellen die behoren tot de binnenste spierlaag. De interstitiële cellen van de buitenste spierlaag reageren, in tegenstelling tot de cellen van de binnenste spierlaag, op NO stimulatie door CGMP aan te maken. Verder zijn er amper COX I-IR cellen gevonden in de buitenste spierlaag terwijl in de binnenste spierlaag deze cellen frequent zijn aangetroffen. De fysiologische en pathofysiologische betekenis van de verschillende interstitiële celnetwerken is nog niet bekend.

Patiënten met een blaas uitgangsobstructie (BOO) als gevolg van benigne prostaat vergroting en of een urethra strictuur krijgen vaak $O A B$ klachten. In Hoofdstuk 4 hebben we het effect van een BOO en of blaashals beschadiging beschreven op de autonome blaasactiviteit door gebruik te maken van een gevalideerd caviamodel voor BOO. Controle, sham geopereerde en geobstrueerde cavia blazen zijn onderzocht. Het blaasgewicht in alle geopereerde groepen blijkt significant toegenomen vergeleken met de controle groep. Verder blijkt de amplitude van autonome activiteit in geopereerde blazen significant toegenomen vergeleken met de controle groep. De amplitude per gram blaasgewicht is het hoogst in de sham geopereerde groep. Beschadiging van de blaashals speelt mogelijk een belangrijke rol in verandering van de autonome activiteit. In controle blazen resulteert muscarine receptor stimulatie, in toename van de frequentie en amplitude van de autonome activiteit. Een zelfde effect wordt gezien in geopereerde blazen, echter de concentraties die nodig zijn voor een toename van de frequentie is significant lager. Het mechanisme verantwoordelijk voor de frequentie van de autonome activiteit, de interstitiële cel pacemaker, blijkt dus overgevoelig voor cholinerge stimulatie in geopereerde blazen. Wellicht berust het therapeutische effect van anticholinergica in patiënten met een BOO op blokkade van de overgevoelige pacemaker. Verder is het mogelijk dat de veranderingen in autonome activiteit in geopereerde blazen zorgen voor een verhoogde prikkeling van sensorische zenuwen, uiteindelijk resulterend in het optreden van overactieve blaasklachten.

Gezien interstitiële cellen verantwoordelijk lijken te zijn voor het genereren en 
moduleren van autonome activiteit, wordt in Hoofstuk $\mathbf{5}$ het effect van een BOO op het netwerk van interstitiële cellen beschreven. Interstitiële cellen in de mens en cavia blaas kunnen gedetecteerd worden doordat ze cGMP aanmaken na blootstelling aan een NO donor. Er zijn verschillende CGMP positieve (cGMP+) interstitiële cellen gevonden in de blaaswand: interstitiele cellen in de sub-urotheliale laag en in de buitenste spierlaag. Naast interstitiële cellen, detecteert cGMP kleuring ook andere cellen in de blaaswand zoals paraplu cellen in het urotheel en bepaalde zenuwen. Hoofdstuk 5 beschrijft CGMP kleuring in sham geopereerde en geobstrueerde blazen. $\mathrm{Er}$ is een significant verschil gevonden tussen sham geopereerde en geobstrueerde blazen. Het aantal cGMP+ interstitiële cellen in en tussen de spierbundels is toegenomen, resulterend in een goed ontwikkeld netwerk in het geobstrueerde blaasweefsel. In de suburotheliale ruimte is gevonden dat het aantal cGMP+ interstitiële cellen in geobstrueerd blaasweefsel is toegenomen ten opzichte van de sham groep. Tenslotte, blijkt het aantal interstitiële cellen in de serosale laag toegenomen in geobstrueerde blazen. Naast de genoemde veranderingen in het netwerk van interstitiële cellen blijkt het urotheel drastisch aangetast in geobstrueerde blazen. Het aantal CGMP+ paraplu cellen en de intensiteit van kleuring is verminderd ten opzichte van de sham groep. De intermediaire laag van het urotheel is veranderd en er zijn veel zenuw vezels gevonden in de lamina propria van geobstrueerd blaasweefsel. De veranderde structuur van het urotheel en het nieuw ontwikkelde interstitiële celnetwerk in geobstrueerde blazen is mogelijk gerelateerd aan de gemoduleerde autonome activiteit. Verder vormen de beschreven histologische veranderingen van de blaaswand in geobstrueerde blazen mogelijk aanknopingspunten voor de oorzaak van de veranderende blaassensaties die optreden bij patiënten met een BOO.

Patiënten met een BOO kunnen naast overactieve blaasklachten ook obstructieve mictieklachten ontwikkelen zoals hesitatie, zwakke urinestraal, nadruppelen, residugevoel en uiteindelijk urine retentie. De obstructieve mictieklachten kunnen gerelateerd zijn aan een beschadiging van de blaasspier. Er is een sterk vermoeden dat vrije zuurstof radicalen een rol spelen bij het ontstaan van functionele en structurele blaasspier beschadiging secundair aan een BOO. Voornamelijk ernstig geobstrueerde (gedecompenseerde) blazen worden geassocieerd met ischemiereperfusie in de blaaswand tijdens de vulling en ledigingfase van de urineblaas maar ook met blaasontstekingen. In situaties zoals ischemie-reperfusie en ontstekingen neemt de productie van zuurstof radicalen toe. Het menselijk lichaam bezit een afweersysteem bestaande uit endogene antioxidanten dat ons beschermt tegen het potentieel beschadigende effect van vrije zuurstof radicalen. Situaties waarbij de vrije radicaal productie het beschermende effect van antioxidanten overschrijdt, worden aangeduid als oxidatieve stress. In dergelijke situaties kunnen de vrije radicalen celstructuren aantasten zoals lipiden en proteïnen, met uiteindelijk beschadiging van zenuw en spiercellen tot gevolg. In Hoofdstuk 6 wordt het effect van een blaas uitgangsobstructie op het endogene antioxidanten systeem van cavia 
blaasweefsel beschreven en gecorreleerd aan veranderingen in blaasfunctie en structuur. Er is gevonden dat de totale antioxidanten capaciteit, de glutathione en glutathione reductase gehaltes significant verminderd blijken te zijn in geobstrueerd blaasweefsel. Verder blijkt dat de kracht van een door een elektrisch veld (neurogeen) veroorzaakte contractie significant lager is bij spierstrips afkomstig van geobstrueerde blazen in vergelijking met sham blazen. Het muscarine receptor signaal systeem en de contractiliteitseiwitten van de blaasspier bleken nauwelijks aangetast, een teken dat de blazen niet gedecompenseerd waren. Door middel van immunohistochemische technieken is verder aangetoond dat de zenuwdichtheid significant lager is in geobstrueerd blaasweefsel vergeleken met de sham groep. Concluderend blijkt het beschermende effect van antioxidanten te zijn gereduceerd in geobstrueerd blaasweefsel terwijl de productie van zuurstof radicalen juist lijkt te zijn toegenomen. De ontstane situatie van oxidatieve stress is mogelijk een verklaring voor de relatieve hoeveelheid zenuwbeschadiging waargenomen in geobstrueerde blazen.

Indien oxidatieve stress een belangrijke rol speelt in het mechanisme van weefselbeschadiging waargenomen in geobstrueerde blazen dan zou men kunnen verwachten dat directe blootstelling van blaasweefsel aan zuurstof radicalen een deel van de weefselbeschadiging imiteert. Hoofdstuk 7 beschrijft het effect van oxidatieve stress op de contractiliteit van de varkens blaasspier. Oxidatieve stress wordt nagebootst door cumene hydroperoxide (CHP), een lipofiele hydroperoxide wat wordt gebruikt als modelstof voor oxidatieve stress. Varkensblaas spierstrips zijn geïncubeerd in orgaanbaden en de contractiekrachten veroorzaakt door verschillende stimulatie paden zijn gemeten voor en na blootstelling aan CHP. De resultaten van onze studie laten zien dat cumene hydroperoxide het muscarine receptor signaal systeem het meest aantast. L-type calcium kanalen en het contractiliteits systeem blijken in mindere mate beschadigd terwijl cholinerge zenuwen relatief ongevoelig zijn voor CHP. Oxidatieve beschadiging van lipiden wordt lipid peroxidatie genoemd. Een blaas uitgangsobstructie kan gepaard gaan met een toename van de peroxidatie van lipiden. Tijdens het proces van lipide peroxidatie worden toxische reactieve aldehyden gevormd, waaronder 4-hydroxynonenal (HNE). Aangezien een blaas uitgangsobstructie is geassocieerd met een toegenomen peroxidatie van lipiden in het gladde spierweefsel en HNE in grote hoeveelheden wordt geproduceerd tijdens lipide peroxidatie, beschrijft Hoofdstuk 8 het effect van HNE op de contractiliteit van de varkens blaasspier. Varkensblaas spierstrips zijn geïncubeerd in orgaanbaden en de contractiekrachten veroorzaakt door verschillende stimulatie paden is voor en na blootstelling aan HNE gemeten. De resultaten van het onderzoek geven aan dat HNE voornamelijk de contractiliteits eiwitten en of de L-type calcium kanalen van de blaasspier beschadigt. De cholinerge zenuwen en het muscarine receptor signaal systeem bleken nauwelijks aangetast. We kunnen tenslotte concluderen dat de blaasspier disfunctie waargenomen in ernstig geobstrueerde blazen deels kan berusten op oxidatieve beschadigende mechanismen op de blaasspier. Echter ons in vitro oxidatieve stress model resulteerde niet tot zenuwbeschadiging terwijl dit wel 
werd geobserveerd in onze studie van matig geobstrueerde cavia blazen. Dit impliceert dat oxiditieve stress in vitro, waarschijnlijk niet dezelfde oxidatieve veranderingen veroorzaakt vergeleken met de in vivo situatie.

In Hoofdstuk 9 worden de resultaten van de studies bediscussieerd en worden de bevindingen van dit proefschrift in een klinisch perspectief geplaatst. Samenvattend kan geconcludeerd worden dat een blaas uitgangsobstructie en verhoogde gehaltes aan prostaglandines in het blaasweefsel leidt tot een toename van de autonome activiteit in cavia blazen. Onze hypothese is dat een verhoogde autonome activiteit, sensorische zenuwen in de blaaswand prikkelt resulterend in het ontstaan van overactieve blaas klachten. Interstitiële cellen zijn mogelijk verantwoordelijk voor het genereren en verspreiden van autonome activiteit. Dit proefschrift bediscussieert ook de rol van oxidatieve stress in de etiologie van de blaasspier disfunctie die wordt gevonden in sommige situaties van een blaas uitgangsobstructie. We geloven dat de resultaten van onze studies leidt tot de ontwikkeling van nieuwe fundamentele en klinische studies. Onderzoek op het gebied van interstitiële cellen en autonome activiteit zal in de nabije toekomst waarschijnlijk beter inzicht geven in de oorzaak van overactieve blaas klachten. Hopelijk zal dit leiden tot effectievere behandelingsmogelijkheden voor patiënten met een Overactieve Blaas syndroom. 


\section{List of abbreviations}

$\begin{array}{ll}\text { ATP: } & \text { Adenosine Triphosphate } \\ \text { BOO: } & \text { Bladder Outlet Obstruction } \\ \text { BPH: } & \text { Benign Prostatic Hyperplasia } \\ \text { BuC: } & \text { Basal urothelium } \\ \text { CAMP: } & \text { cyclic Adenosine Monophosphate } \\ \text { CGMP: } & \text { cyclic Guanosine Monophosphate } \\ \text { CGRP: } & \text { calcitonin Gene Related Peptide } \\ \text { COX: } & \text { Cyclo-oxygenase }\end{array}$

COX I-IR: Cyclo-oxygenase I Immuno Reactivity

DMSO: Dimethylsulfoxide

EFS: $\quad$ Electrical Field Stimulation

GSH: Gluthathione

GSSH: Gluthathione disulphide

GR: Gluthathione reductase

HNE: 4- Hydroxynonenal

$\mathrm{HOCl}$ Hypochlorous acid

$\mathrm{H}_{2} \mathrm{O}_{2}$ : Hydrogen peroxide

Iml: Inner muscle Layer

IM-IMS: Intra Muscular Interstitial Cells

IUC: Intermediate Urothelial Cell layer

Lp-ICS: Lamina propria Interstitial Cells

LUTS: Lower Urinary Tract Symptoms

MDA: Malondialdehyde

MC-ICS: Muscle Coat Interstitial Cells

NADP: Nicotinamide Adenine Dinucleotide Phosphate

NEM: N-ethylmaleimide

NGF: Nerve Growth Factor

nNOS: neuronal Nitric Oxide Synthase

NO: $\quad$ Nitric Oxide

OAB: $\quad$ Over Active Bladder

$\mathrm{OH}^{*}: \quad$ Hydroxyl radical

Oml: $\quad$ Outer muscle layer

ONOO-: Peroxynitrite

$\mathrm{O}_{2} \cdot-: \quad$ Superoxide anion

PAG: Peri Aqueductal Gray

PGs: $\quad$ Prostaglandins

PGP 9.5: Protein Gene product 9.5

PMC: $\quad$ Pontin Micturition Center

RNOS: Reactive Nitrogen and Oxygen Species

SM-ICS: Surface Muscle Interstitial Cells

Su-ICS: Sub-urothelial Interstitial Cells 
List of abbreviations

C: Trolox Equivalent Antioxidant Activity

: Transient Receptor Potential

: Tetrodotoxin

Vasoactive Intestinal Polypeptide 


\section{Dankwoord}

Ondanks dat slechts mijn naam op de kaft staat, is dit proefschrift tot stand gekomen vanuit een gezamenlijke inspanning van een aantal mensen. Voor allen, ook voor degene die ik ongetwijfeld vergeet te noemen, mijn dank. Een aantal mensen wil ik in het bijzonder noemen.

Prof. Dr. Ph E.V. van Kerrebroeck, mijn promotor en opleider. Beste Philip, dank voor de mogelijkheden en support die $u$ me gegeven hebt om te promoveren in Maastricht. Dank ook voor de kans die u me gegeven hebt om uroloog te worden.

Dr. G.A van Koeveringe, mijn co-promotor. Beste Gommert, eindelijk is het zover! Ik weet nog goed dat je als facultaire begeleider van mijn buitenlandse stages aan het einde van de co-schappen vroeg of ik geïnteresseerd was om een promotietraject op te starten bij de urologie in Maastricht. Het betrof basaal wetenschappelijk onderzoek op het gebied van de functionele urologie, eigenlijk een voortzetting van je promotieonderzoek uit Rotterdam. Ondanks mijn interesse in wetenschappelijk onderzoek, heb ik na het lezen van je dissertatie eerlijk gezegd getwijfeld of dit onderzoek wel voor mij weggelegd zou zijn. De link tussen termen als "isometrische krachtontwikkeling", "tijdconstante", "intracellulaire calcium voorraden" en het klinische probleem van een overactieve blaas was voor mij niet duidelijk. De afgelopen jaren heb ik veel bijgeleerd op fundamenteel gebied! Het begin van het promotietraject verliep, mede gezien de geringe contacten met preklinische universitaire vakgroepen niet altijd even soepel. Desondanks is het ons toch gelukt om diverse contacten te leggen met verschillende vakgroepen die uiteindelijk tot een structureel samenwerkingsverband hebben geleid met onder andere dit proefschrift als resultaat. Het is zelfs gelukt om een volwaardige onderzoeksgroep op te zetten die momenteel uit 4 promovendi bestaat. Dat is zeker iets om trots op te zijn! Gommert, dank voor je geduld, vrijheid en zelfstandigheid die je me de afgelopen jaren gegeven hebt om onderzoek te doen. Ik kijk uit naar verdere samenwerking in de toekomst!

Prof J.I. Gillespie, my copromotor. Dear James, I will never forget our first encounter. I was busy with one of my animal studies. When I came to the lab I was surprised that some of the guinea pigs had disappeared. I heard that a British researcher was performing staining studies with guinea pigs and unfortunately some of my animals had been used. Despite this unpleasant event, I am very glad we have met each other. Ever since we have had many productive discussions and we decided to work together during the final phase of my PhD project. James, you are the inspirator of four papers of this dissertation. You are a great researcher and my fundamental coach. Thanks for sharing your many ideas, for the unforgettable discussions and your enormous support. I hope we will stay in contact in the future. 
Let us organise the next (research) meeting in Bergerac with a good glass of wine........

Dr GRMM Haenen. Beste Guido, heel veel dank ben ik jou verschuldigd. Je hebt me de beginselen van de farmacologie geleerd. Dankzij jouw ideeën zijn er twee artikelen van dit proefschrift tot stand gekomen en ben je co-auteur van een derde artikel. Je kritische houding was confronterend echter ik besefte (soms pas later) dat de kwaliteit van het werk er enorm op vooruit ging. Veel dank voor je adviezen en je altijd zeer snelle revisies van de stukken. Ik zal nooit meer vergeten dat ons eerste artikel, tot mijn grote teleurstelling, niet werd geaccepteerd. Je was totaal niet onder de indruk van het commentaar van de "reviewers" en uiteindelijk hebben we besloten om een gereviseerde versie opnieuw in te sturen naar hetzelfde tijdschrift. Tot mijn grote geluk werd het direct geaccepteerd. Het is kenmerkend voor jouw deskundigheid en opportunisme. Ontzettend bedankt!!

Dr. M. Dambros. Dear Miriam, especially at the beginning of the research period you were my personal supervisor. Thanks to you I learned to work in the lab, to operate guinea pigs, to work with different computer programmes and to analyse data. Thanks for the discussions, your support and especially your friendship. I will never forget your hospitality in Campinas. I am sure we will keep in touch in the future.

Dr. J. de Vente. Beste Jan, ik bewonder je beschouwelijke, bescheiden doch essentiële inbreng in het onderzoek.

Prof dr. A Bast. Beste Aalt, dank voor de mogelijkheid die je me geboden hebt om in je laboratorium te werken. Dank ook voor de waardevolle besprekingen aan het begin van het onderzoekstraject. Het is mij een eer dat je voorzitter bent van mijn manuscriptcommissie.

Prof dr. J.G.R. De Mey. Beste Jo hartelijk bedankt dat ik gebruik mocht maken van de orgaanbaden in je laboratorium. Verder dank voor de onderwijsmomenten in het laboratorium, je laagdrempeligheid en algemene interesse. Erkentelijk ben ik voor het feit dat je zitting wilde nemen in mijn manuscriptcommissie.

Graag wil ik de andere leden van de beoordelingscommissie bedanken: Prof. dr. H.W.M. Steinbush, Prof. dr. M.C. Michel en Prof dr. J.J. Wyndaele.

Marjanne Markerink, dank voor de prettige samenwerking en je waardevolle technische inbreng. Je tomeloze inzet is onvergetelijk.........in het weekend helpen met immunohistochemische kleuringen was voor jou geen probleem.

Dr. E. Villamore. Beste Eduardo, dank voor je wetenschappelijke ondersteuning. 
Dr. G.J. Den Hartog. Beste Gert Jan, hartelijk dank voor de biochemische metingen en je waardevolle uitleg met betrekking tot de laboratorium werkzaamheden.

Tevens wil ik bij de afdeling Farmacologie \& Toxicologie Saskia, Els, Agon, Fazzi en Gianluca bedanken voor de prettige contacten en de vele hulp en goede tips.

Collega arts onderzoekers. Mijn kamergenoot Dennis. Wat hebben wij samen veel uurtjes doorgebracht achter de computer in het (te) kleine hok. Het was erg prettig om met je samen te werken. Speciale dank voor je geduldige uitleg als ik weer eens problemen had met de computer. Verder was je altijd behulpzaam bij operaties. Naast een geweldig onderzoeksmaatje ben je ook een goede vriend geworden. Top dat je mijn paranimf wilt zijn! Verder wil ik Jarno bedanken voor de hulp van de afgelopen jaren. Onze lunches samen met Dennis waren altijd gezellig en een welkome afwisseling voor de soms fundamentele beslommeringen. Ondanks dat specialiseren in combinatie met een gezinsleven en afronden van een proefschrift nauwelijks samen gaan, ben ik ervan overtuigd dat jullie binnenkort ook een boekje gaan afleveren! Anco, Kelly en Luba, jammer dat jullie de urologie verlaten hebben maar fijn dat jullie toch je plekje gevonden hebben. Anco, ik ga ervan uit dat je binnenkort ook je "s"-je kwijtraakt.

Mijn opvolger Simone, je boekje is al klaar. Proficiat! Dank voor je hulp bij het verwerven van informatie om mijn proefschrift af te ronden. Succes met je eigen verdediging! Verder wil ik de huidige onderzoekers, Sajjad en Bart, succes wensen met het afronden van hun onderzoek.

Bij de staf van de afdeling urologie in Maastricht gaat mijn dank uit naar Cees, Piet, Gosse, Coen, Ernest en Tom. Verder is steun vanuit het secretariaat onmisbaar; Cindy, Marion, Joan en Anja bedankt!

De chirurgen van Maxima Medisch Centrum wil ik hartelijk bedanken voor de belangstelling en vooral hun bijdrage aan mijn klinische vorming die essentieel is om fundamenteel onderzoek in een goed perspectief te plaatsen. Verder wil ik mijn collega assistenten van harte bedanken voor hun steun, de plezierige en zeer collegiale periode! De skitripjes en feestjes blijven onvergetelijk!

De afdeling Urologie in het Catharina ziekenhuis waarbinnen ik werkzaam mag zijn. Werken in een omgeving waarin je jezelf kan zijn is erg prettig. Ad, Eric, Evert, Michel en Wout dank ik hartelijk voor de bijdrage aan mijn klinische vorming. Vanzelfsprekend ook dank naar de collega assistenten.

Mensen van de Centrale Proefdieren Voorziening, te weten Richard, Allard, Peter en Rik hartelijk dank voor de prettige samenwerking. 
Een bijzonder woord van dank aan alle cavia's die ongevraagd hun leven ter beschikking hebben gesteld voor dit proefschrift.

De heren van het varkensslachthuis te Kerkrade wil ik bedanken voor het aanleveren van het gewenste onderzoeksmateriaal.

Ontspanning is belangrijk tijdens een promotie traject. De heren van mijn hockeyteams in Maastricht en Eindhoven (Oranje Zwart) wil ik bedanken voor de ontspannende en plezierige momenten op en vanzelfsprekend ook buiten het veld.

Verder ben ik dank verschuldigd aan alle overige vrienden en kennissen voor het aanhoren van alle beslommeringen rondom het promotietraject en de steun die ze me hierbij hebben geboden. M'n studievrienden, maatjes van Støre åle, Verticaalst, vrienden uit Maastricht, Eindhoven en elders bedankt!

Familie; hoewel het waarschijnlijk niet altijd even duidelijk was waar ik nu mee bezig was, bedankt voor de getoonde interesse. Het is een voorrecht dat mijn opa's en oma mijn dissertatie mogen meemaken.

Mijn zus en Meine bedankt voor jullie warmte en steun. Hopelijk kan ik binnenkort vaker langskomen om van jullie kleintjes te genieten. Zus, ik ben blij dat je aan mijn zijde wilt staan tijdens de verdediging.

Pap en mam, ik wil jullie bedanken voor de onvoorwaardelijke steun en de mogelijkheden die jullie me door de jaren heen hebben geboden tot aan de dag van vandaag. Dank voor de interesse in mijn wetenschappelijke activiteiten en niet te vergeten jullie actieve taalkundige ondersteuning. Het is heerlijk om te weten dat je altijd kunt terugvallen op een warm nest! 


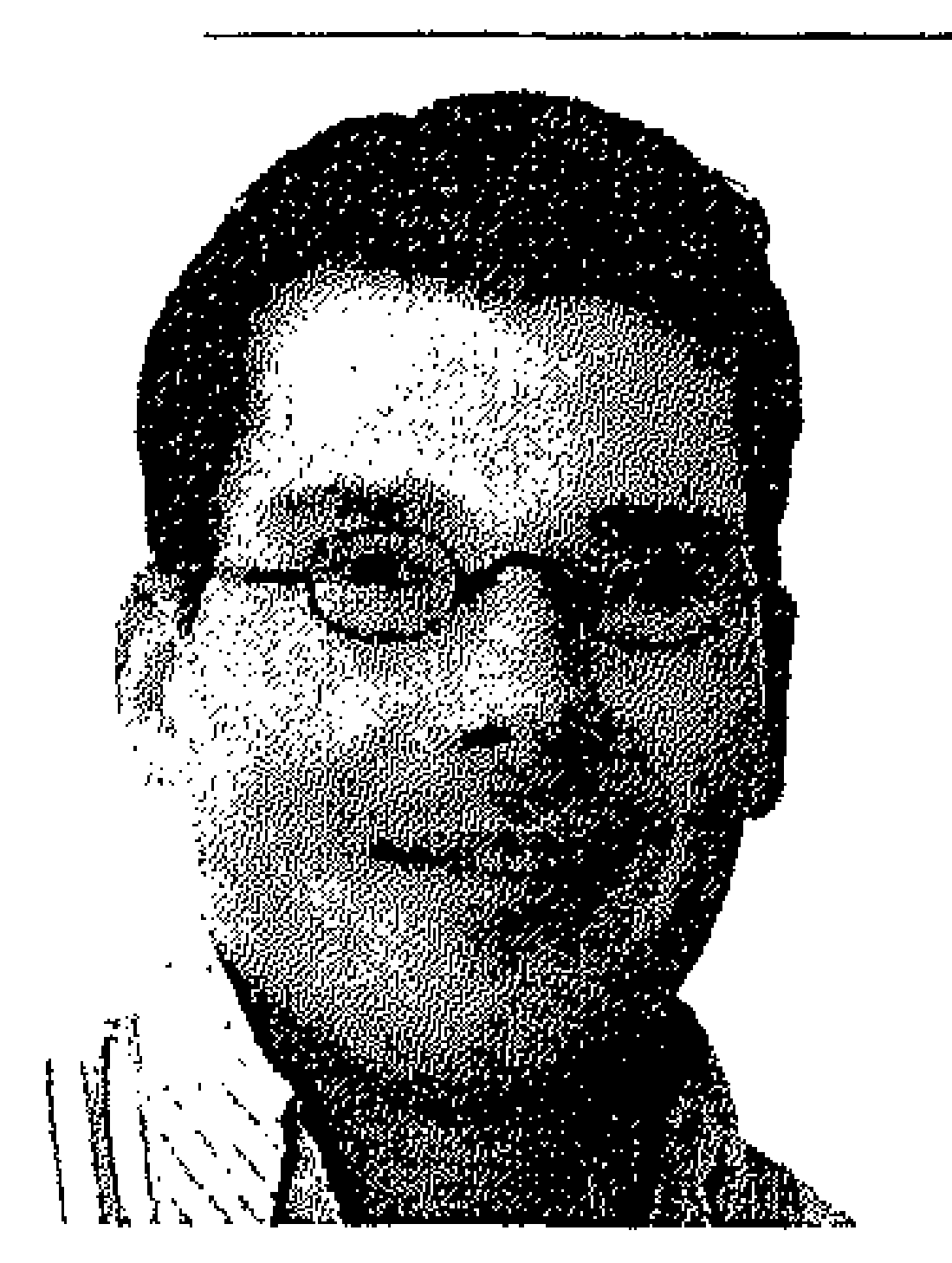

\section{Curriculum Vitae}

Rik de Jongh is geboren op 19 juni 1978 in Gouda, Nederland. De middelbare school opleiding (VWO) werd gevolgd aan het Merewade College in Gorinchem van 1990 tot 1996. In 1997 startte hij met de studie geneeskunde aan de Universiteit van Maastricht. In 2001 werd het doctoraal geneeskunde behaald (met genoegen) en in 2003 werd het artsexamen met goed gevolg afgelegd. Gedurende zijn studie en zijn co-schappen verdiepte hij zich op het gebied van de Urologie. In 2002 volgde hij een wetenschappelijke stage aan Stanford Universiteit, Palo Alto, Verenigde Staten (MK Terris, MD, Associate Professor Urologie). Een keuze co-schap Urologie werd gevolgd in 2003 aan Stellenbosch Universiteit, Tygerberg Ziekenhuis, Kaapstad, Zuid Afrika (CF Heyns, MD, Hoofd Afdeling Urologie en Professor in de Urologie).

In 2004 tot 2006 werd op de afdeling Urologie van het Academisch Ziekenhuis Maastricht gewerkt aan dit proefschrift onder leiding van Prof. Dr. Ph EV van Kerrebroeck (Hoofd Afdeling Urologie) en Dr. GA van Koeveringe (Uroloog). In 2005 werd in samenwerking met Prof. dr. JI Gillespie (Universiteit van Newcastle) een wetenschappelijk project gestart. In januari 2007 is Rik gestart met de opleiding Urologie. De vooropleiding Heelkunde werd gevolgd in Maxima Medisch Centrum in Veldhoven (opleider Dr. RM Roumen). In 2009 is Rik gestart met het perifere gedeelte van de opleiding tot uroloog in het Catharina ziekenhuis in Eindhoven (opleider Dr. HJEJ Vrijhof). Het academische deel van de opleiding zal gevolgd worden aan het Academisch Ziekenhuis Maastricht (opleider Prof. Dr. Ph EV van Kerrebroeck).

Rik de Jongh was born in Gouda, the Netherlands, on 19 June 1978. He attended secondary school (VWO) in Gorinchem (Merewade College) from 1990-1996. In 1997 Rik started Medical School at the University of Maastricht. He obtained his MSC degree in 2001 (beta plus) and his medical degree in 2003. During his medical studies and internships he broadened his knowledge in the field of Urology. In 2002 he conducted a urological research elective at Stanford University, Palo Alto, USA (MK Terris, MD, Associate Professor of Urology). A clinical urological rotation was performed in 2003 at Stellenbosch University, Tygerberg Hospital, Cape Town, South Africa (CF Heyns, MD, Chief and Professor of Urology).

The work presented in this dissertation was carried out between 2004 and 2006, when Rik was a PhD-student at the Department of Urology in Maastricht (Project Manager: GA van Koeveringe, MD, PhD, Head: Ph EV van Kerrebroeck, MD, PhD, Professor of Urology). In 2005 a joint research project was started with JI Gillespie, PhD, Professor of Physiology at the Medical School of Newcastle University. In 2007 Rik started two years of residency in General Surgery in Maxima Medisch Centrum, Veldhoven (Dr. RM Roumen). In January 2009 he started as a resident in Urology at the Catharina Hospital in Eindhoven (Supervisor Dr. HJEJ Vrijhof). The academic part will be accomplished in Maastricht (Supervisor Prof. Dr. Ph EV van Kerrebroeck). 


\section{List of publications}

Dambros $M$, de Jongh $\mathbf{R}$, van Koeveringe $G A$, Bast $A$, Heijnen $C G$, van Kerrebroeck PE. Flavonoid galangin prevents smooth muscle fatigue of pig urinary bladder. J Pharm Pharmacol. 2005; 57(5):617-22.

Dambros $M$, Van Deutekom $M$, de Jongh $\mathbf{R}$, van Koeveringe GA, De Mey JG, van Kerrebroeck PE. The inhibitory effect of the flavonoid galangin on urinary bladder smooth muscle contractility is mediated in part by modulation of $\mathrm{Ca}^{2+}$ release from intracellular stores. Planta Med. 2005; 71(10):962-4.

Dambros $M$, de Jongh $\mathbf{R}$, van Koeveringe GA, Van Deutekom $M$, De Mey JG, Palma PC, van Kerrebroeck PE. Multiple-signalling pathways are involved in the inhibitory effects of galangin on urinary bladder contractility. Neurourol Urodyn. $2005 ; 24(4): 369-73$.

Dambros $M$, de Jongh $\mathbf{R}$, van Koeveringe $G A$, Bast $A$, van Kerrebroeck PE. Galangin protects pig detrusor nerves from repetitive field stimulation and anoxia/glucopenia injury. Urology. 2005; 66(6):1327-31.

de Jongh R, Haenen GR, van Koeveringe GA, Dambros M, De Mey JG, van Kerrebroeck $P E$. Oxidative stress reduces the muscarinic receptor function in the urinary bladder. Neurourol Urodyn. 2007; 26(2):302-8.

de Jongh $\mathbf{R}$, van Koeveringe GA, van Kerrebroeck PE, Markerink-van Ittersum $M$, de Vente J, Gillespie JI. The effects of exogenous prostaglandins and the identification of constitutive cyclooxygenase I and II immunoreactivity in the normal guinea pig bladder. BJU Int. 2007; 100(2):419-29.

de Jongh $\mathbf{R}$, van Koeveringe GA, van Kerrebroeck PE, Markerink-van Ittersum $M$, de Vente J, Gillespie JI. Alterations to network of NO/CGMP-responsive interstitial cells induced by outlet obstruction in guinea pig bladder. Cell Tissue Res. 2007; $330(1): 147-60$.

de Jongh $\mathbf{R}$, van Koeveringe GA, van Kerrebroeck $P E$, Markerink-van Ittersum $M$, de Vente J, Gillespie JI. Damage to the bladder neck alters autonomous activity and its sensitivity to cholinergic agonists. BJU Int. 2007; 100(4):919-29.

de Jongh R, Haenen GR, van Koeveringe GA, Dambros M, van Kerrebroeck PE. Lipid Peroxidation Product 4-Hydroxynonenal Contributes to Bladder Smooth Muscle Damage. Urology 2008; 71(5):974-8. 
de Jongh R, Grol S, van Koeveringe GA, ven Kerrebroeck PE, de Vente J, Gillespie JI. The localisation of cyclo-oxygenase immunoreactivity (COX I-IR) to the urothelium and to interstitial cells in the bladder wall. J Cell Mol Med in press.

de Jongh R, Dambros M, Haenen GR, den Hartog GJM, Bast A, van Kerrebroeck $P E$, van Koeveringe GA. Partial bladder outlet obstruction reduces the tissue antioxidant capcaity and muscle nerve density of the guinea pig bladder. Neurourol Urodyn. 2009; 28(5):461-7. 
Figures chapter 1

Figure 1.
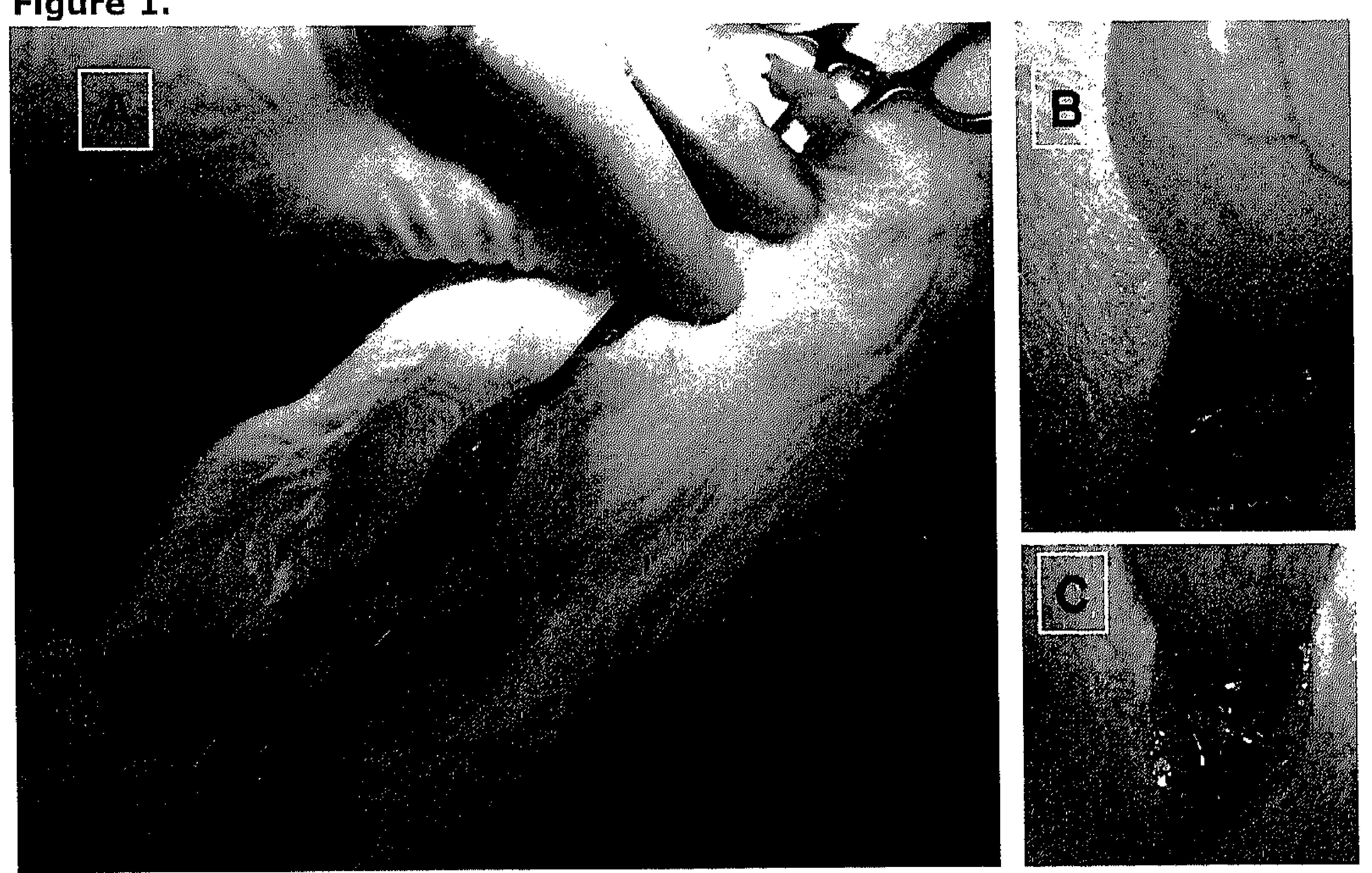
Figures chapter 2

Figure 8.
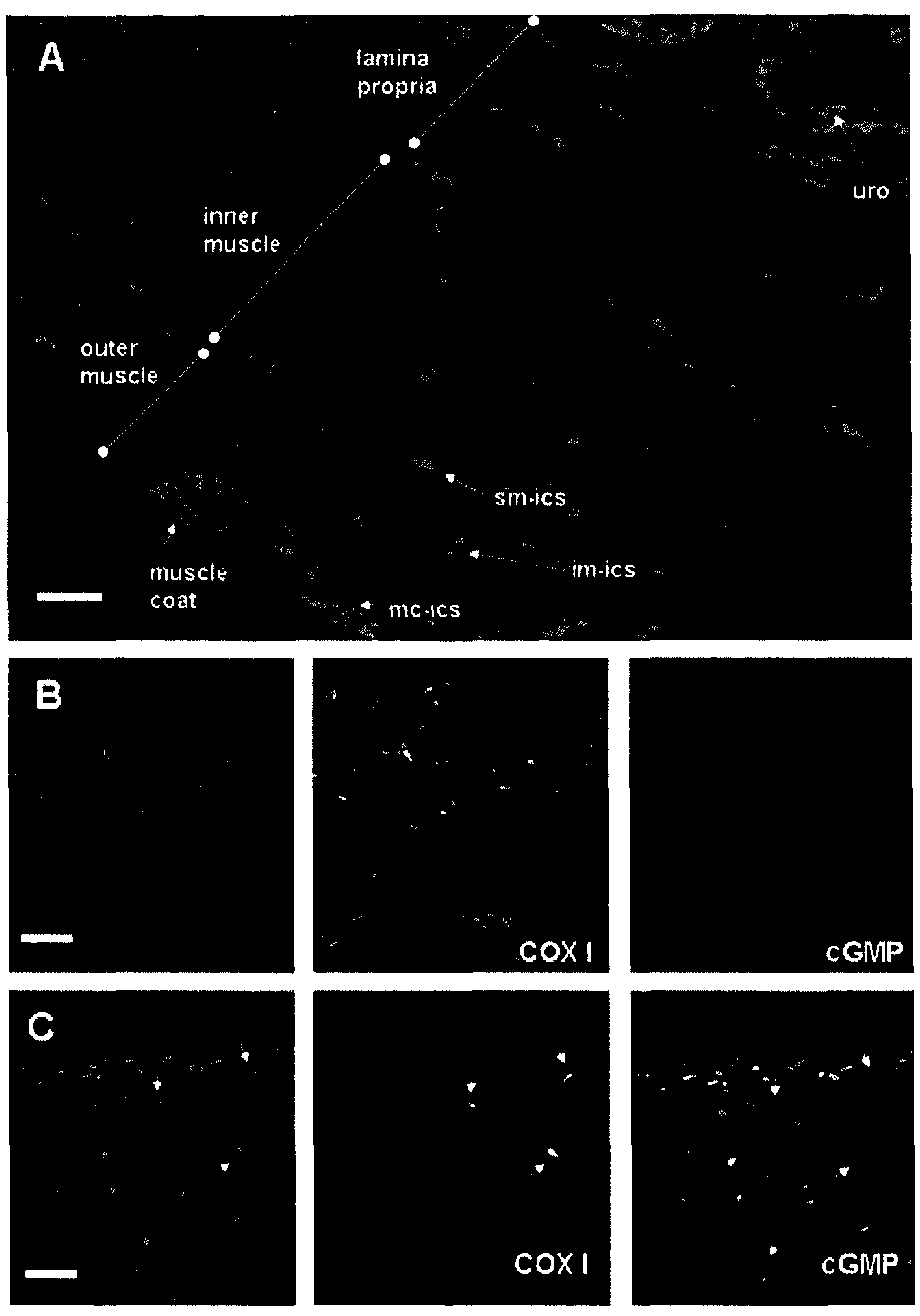
Coloured images
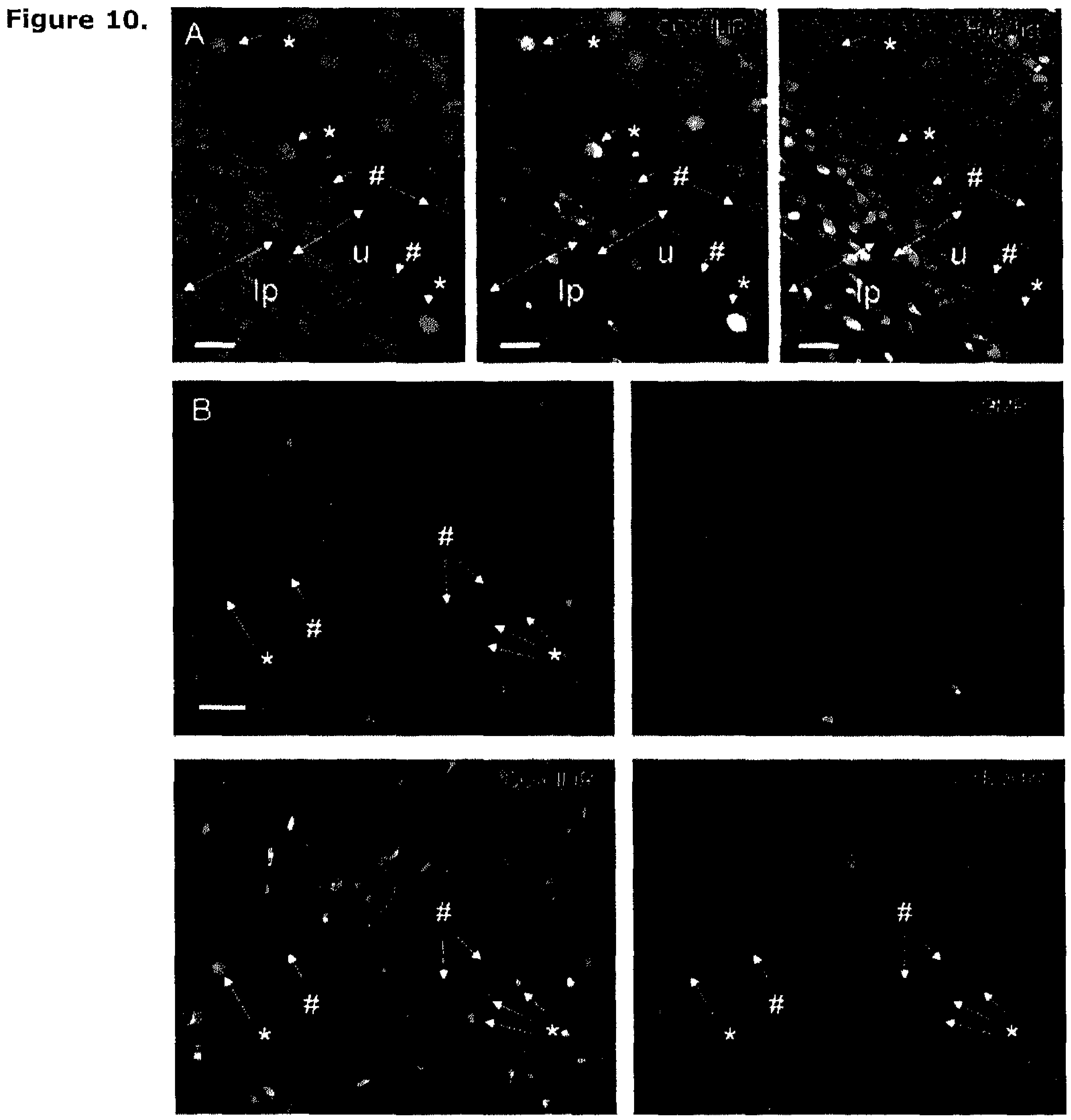
Figures chapter 3

Figure 2.

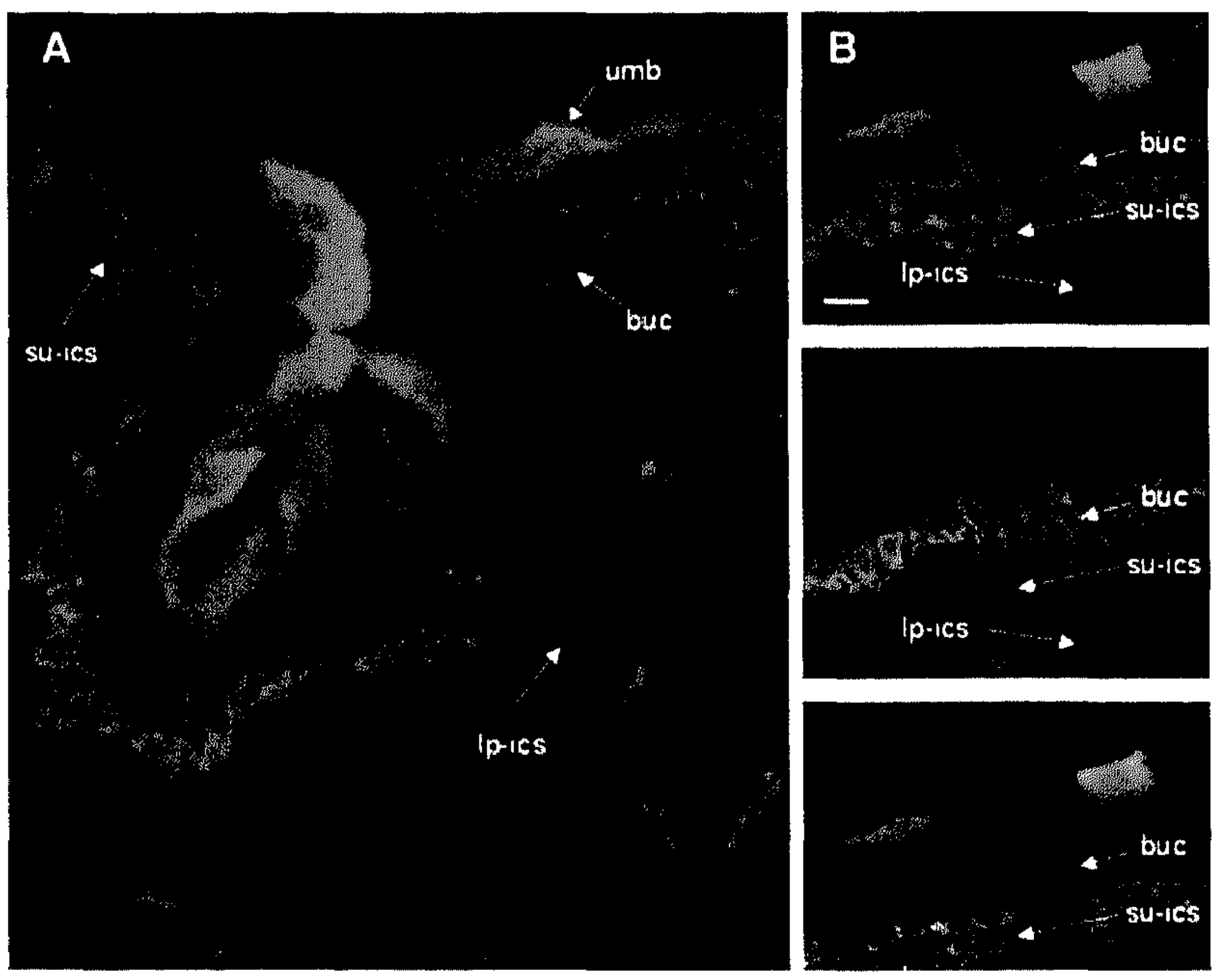

Figure 3.
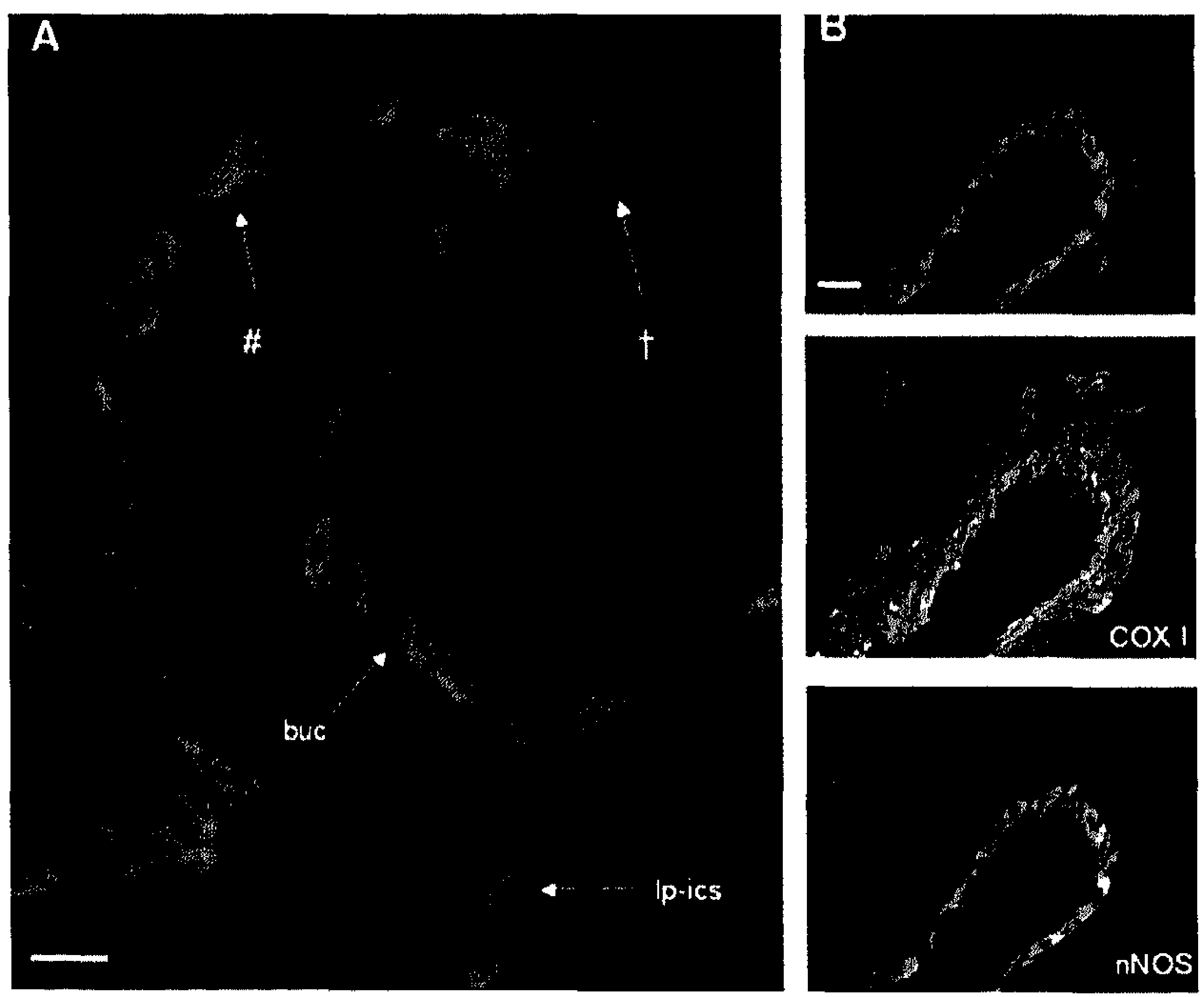
Figure 4.
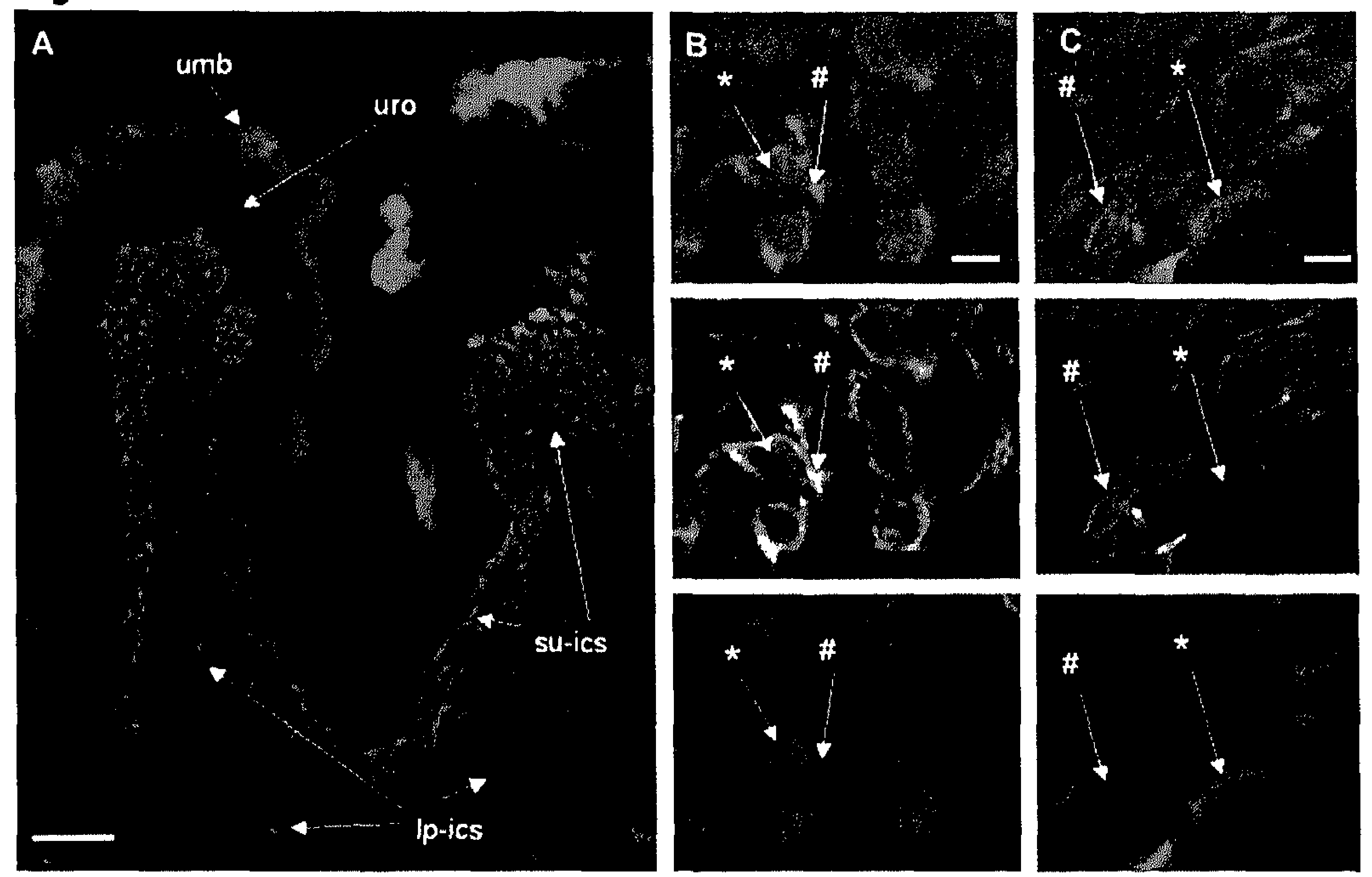

Figure 5.
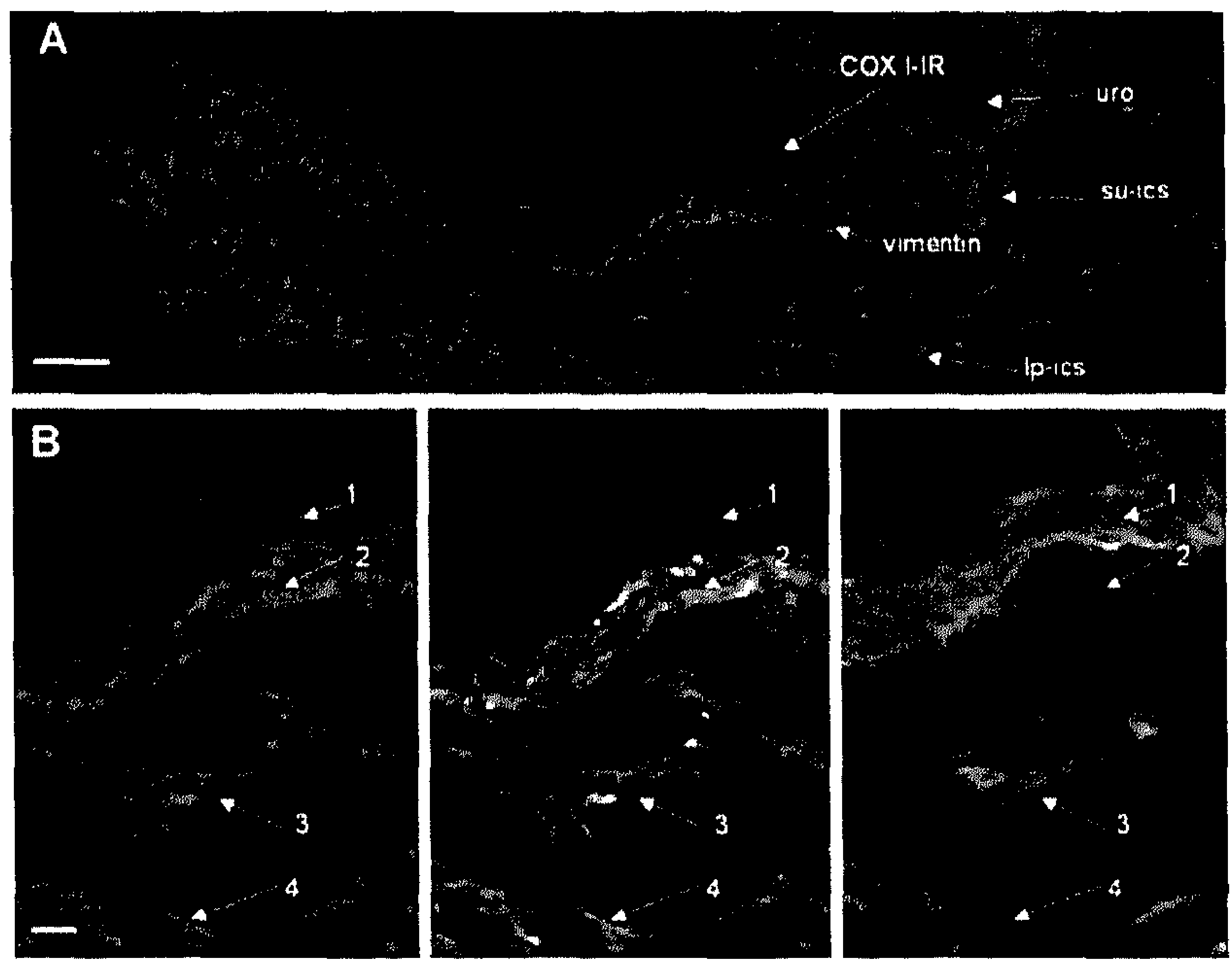
Figure 6.
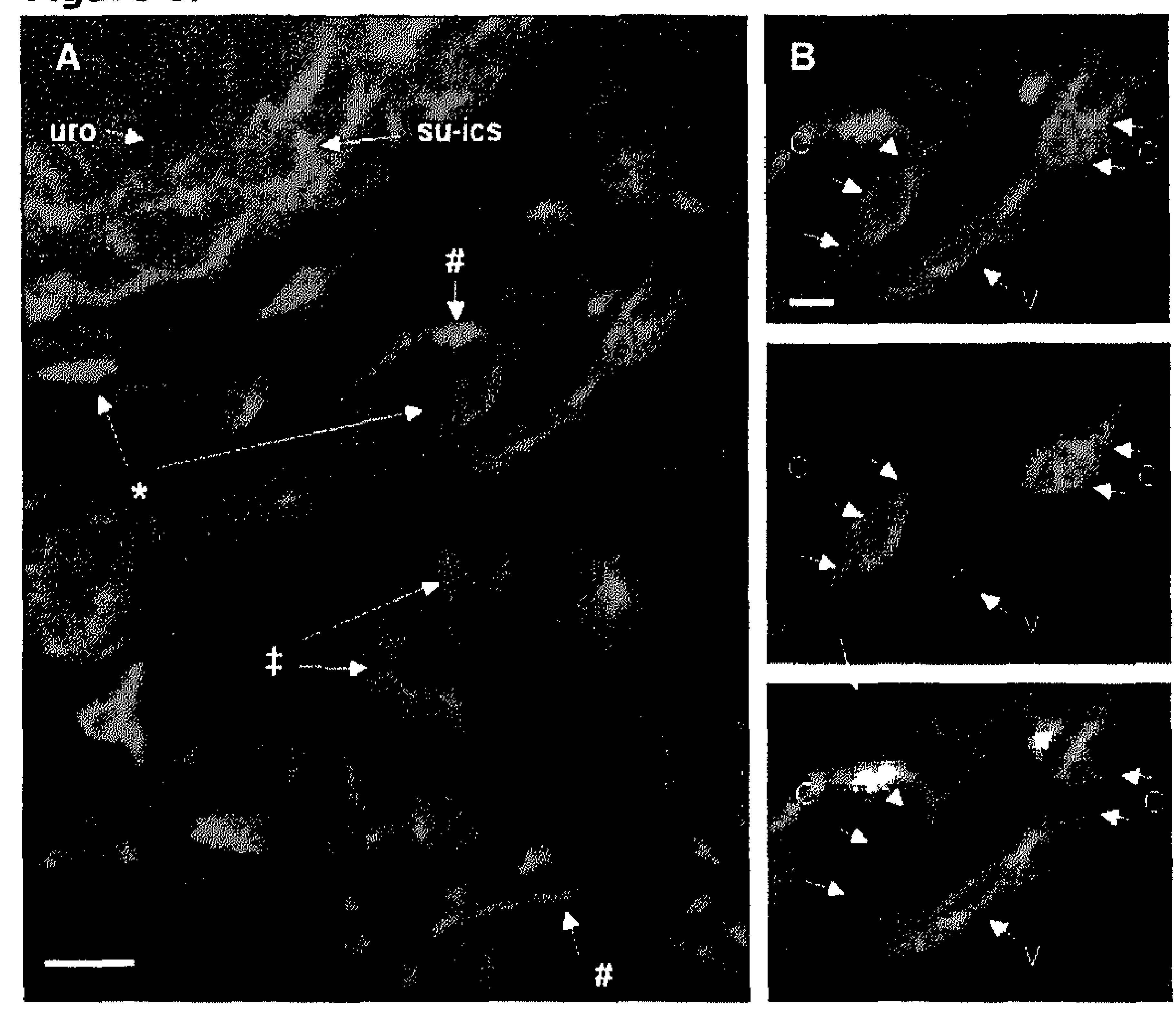

Figure 7.
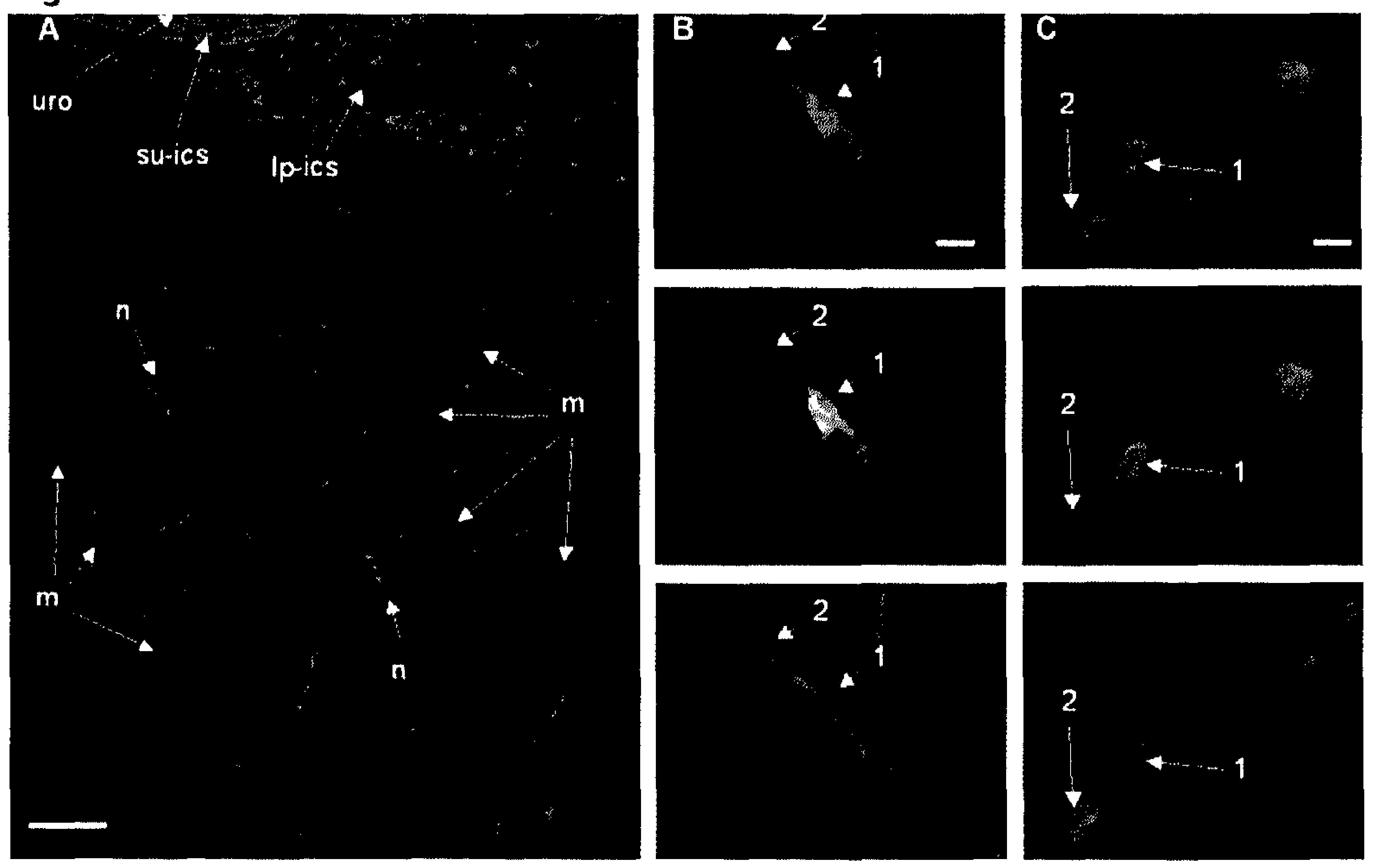
Figure 8.

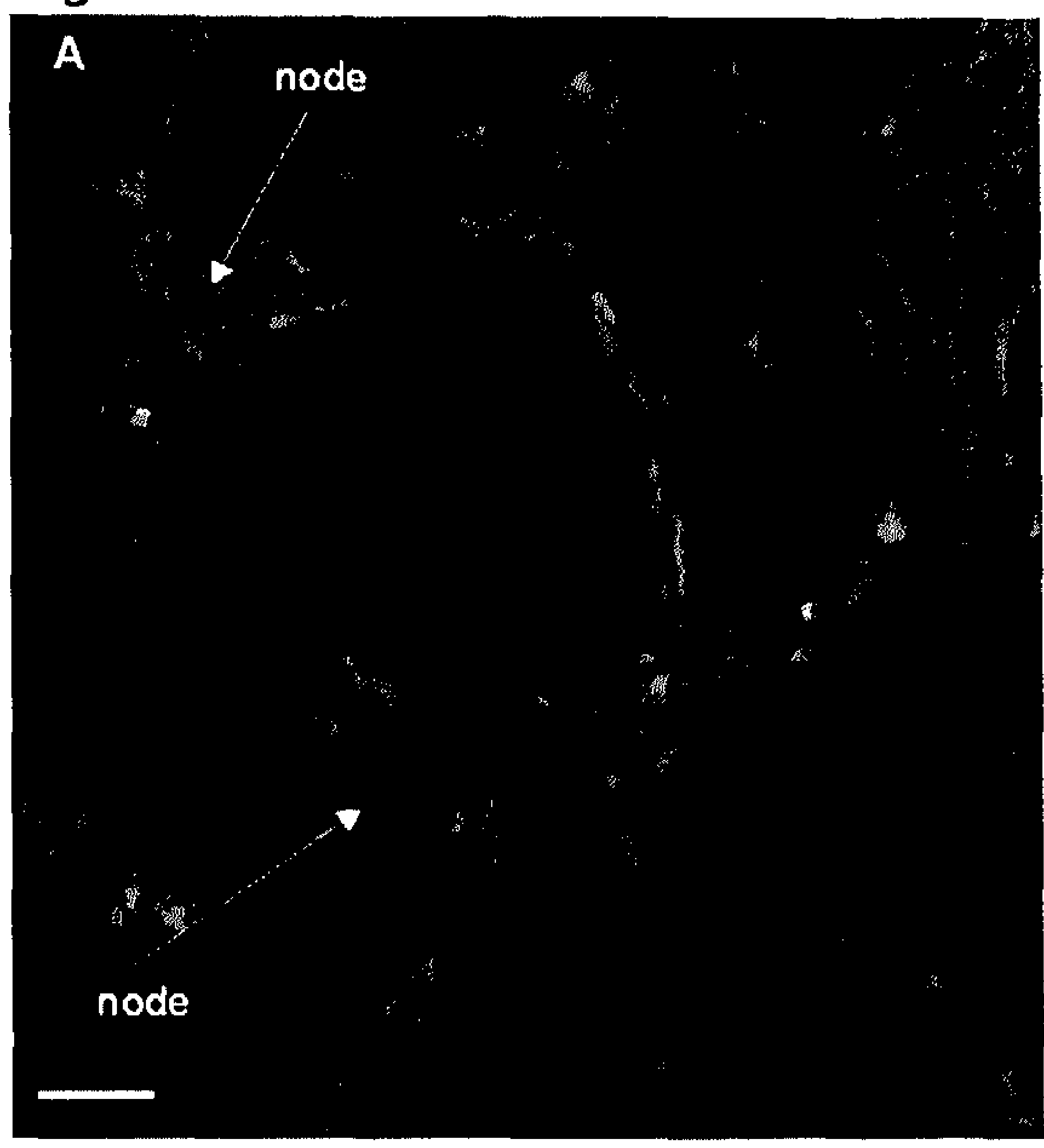

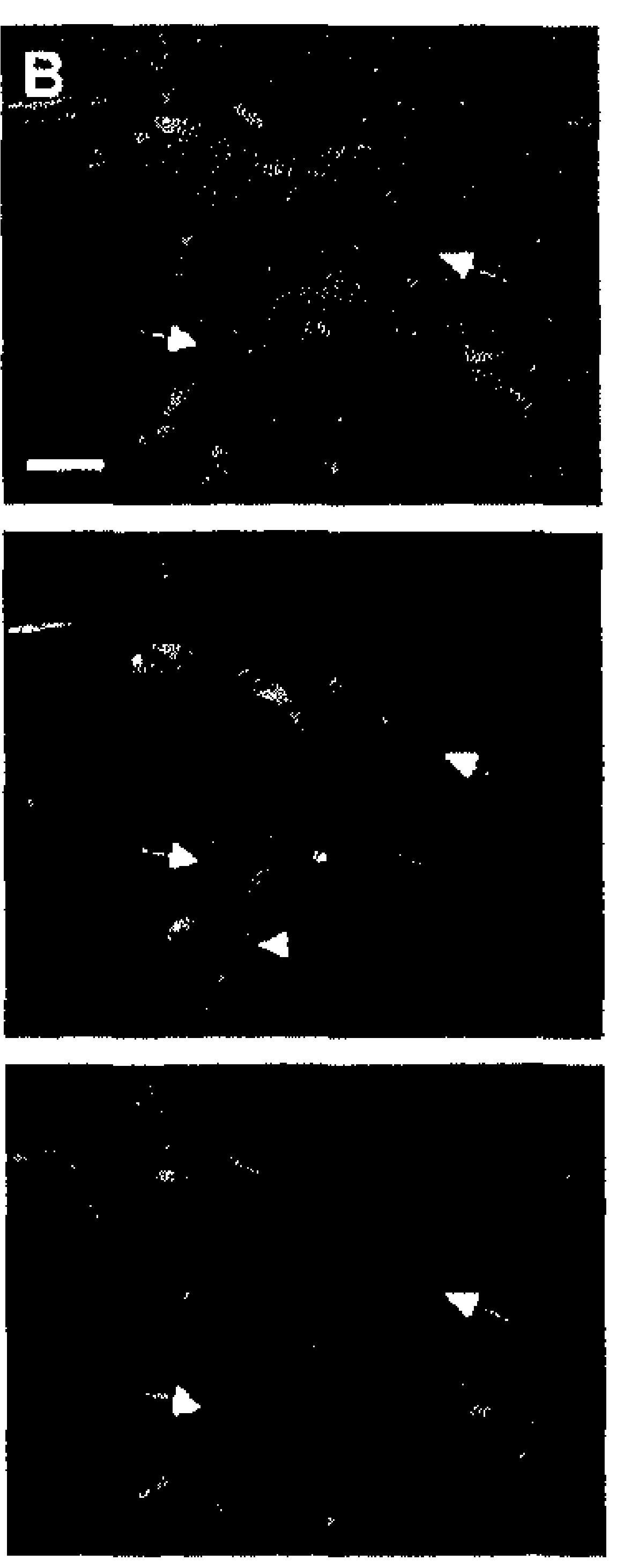
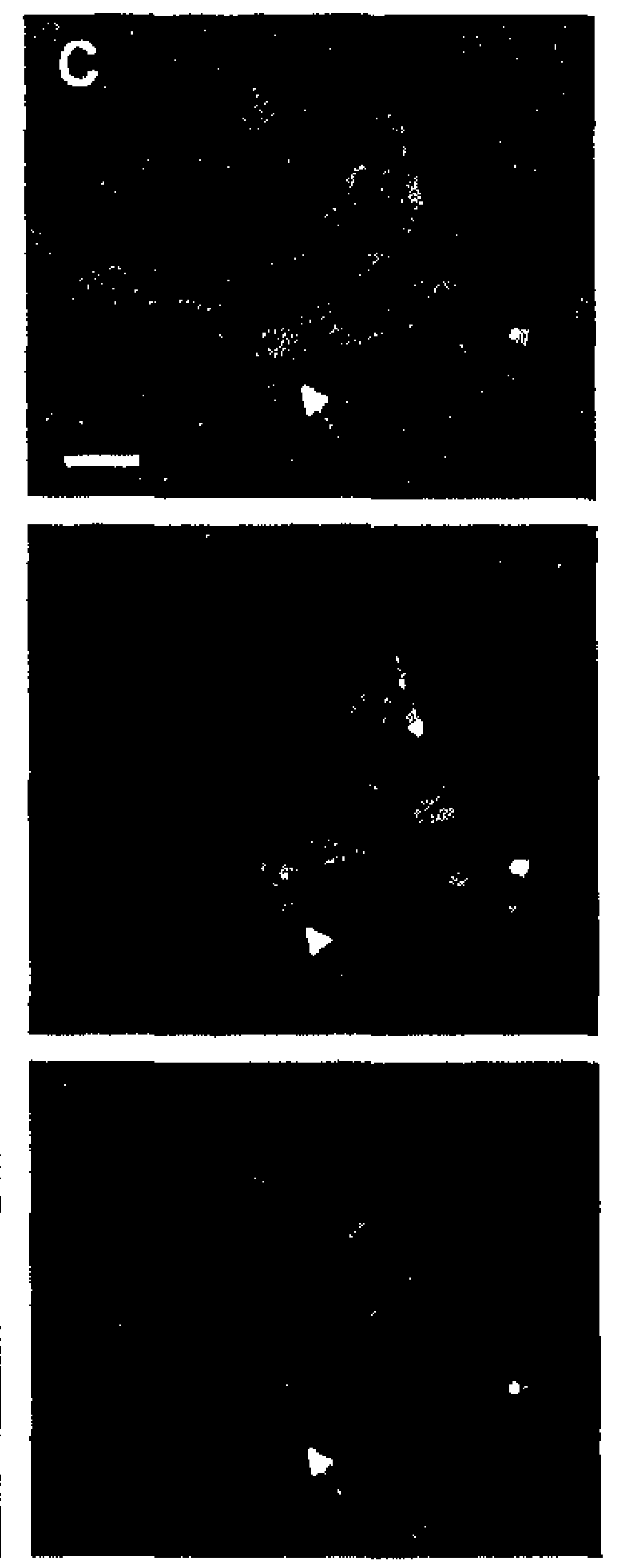

Figure 9.

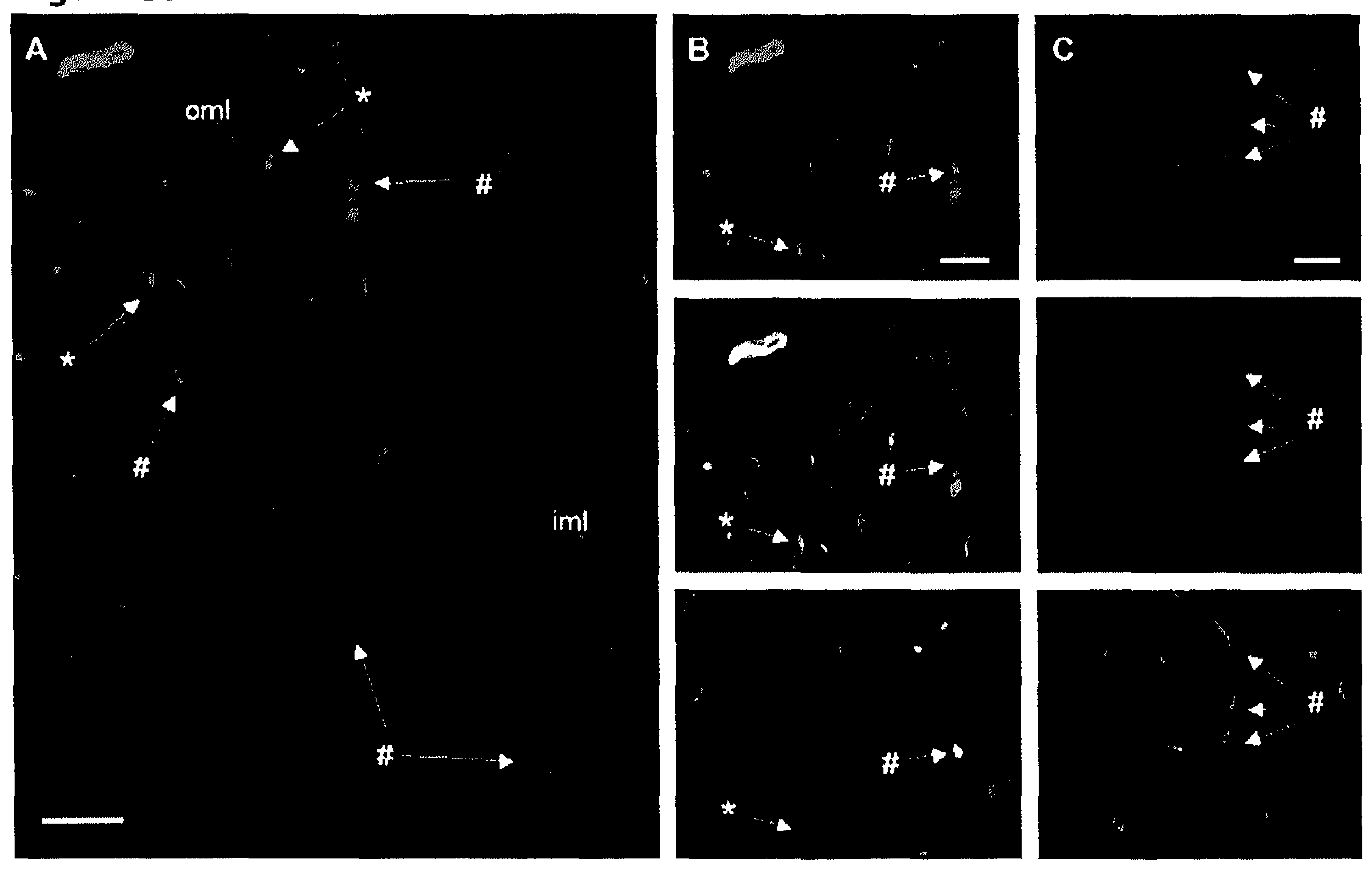


Figure 10.
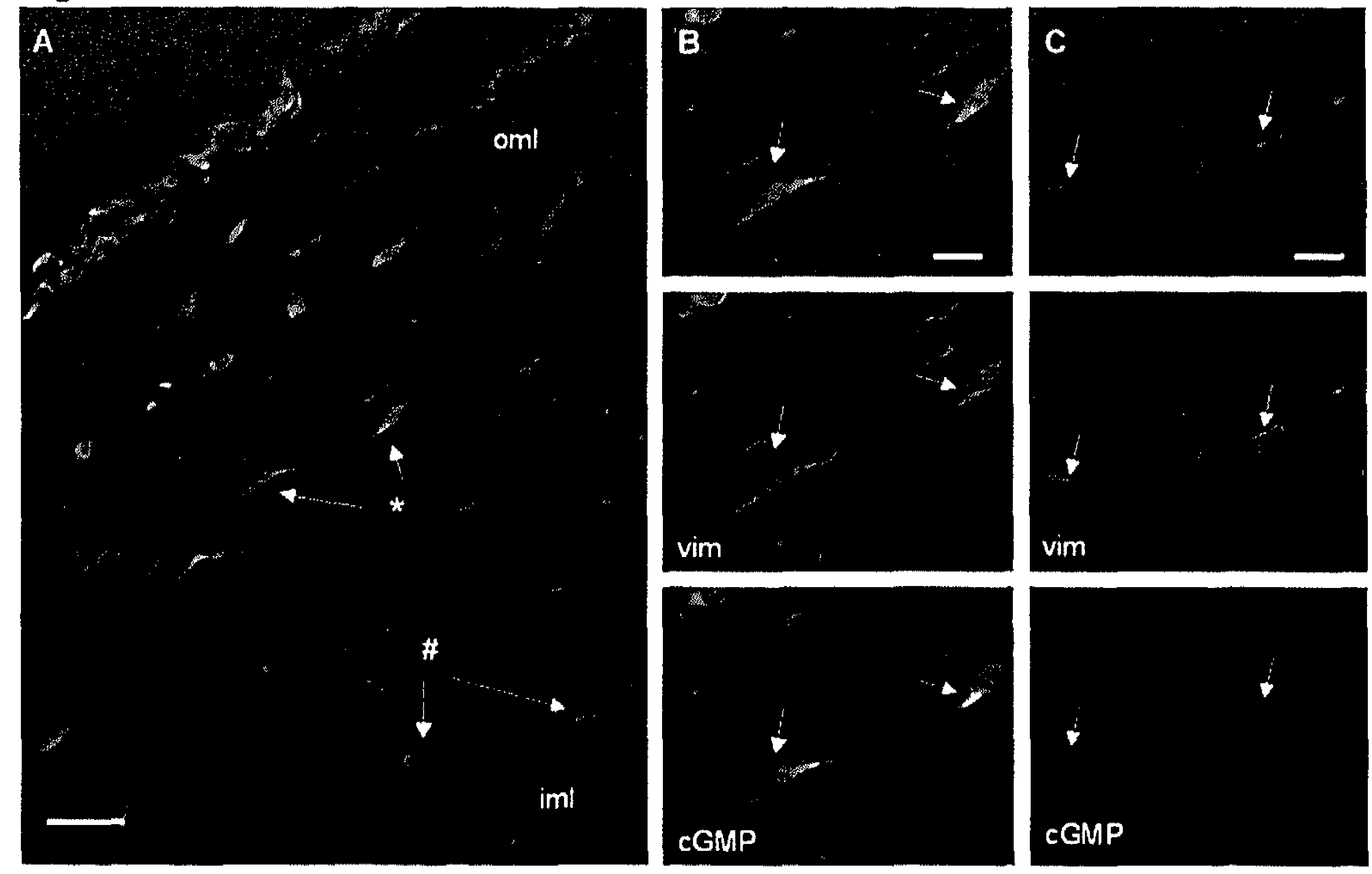
Figures chapter 5

Figure 1.

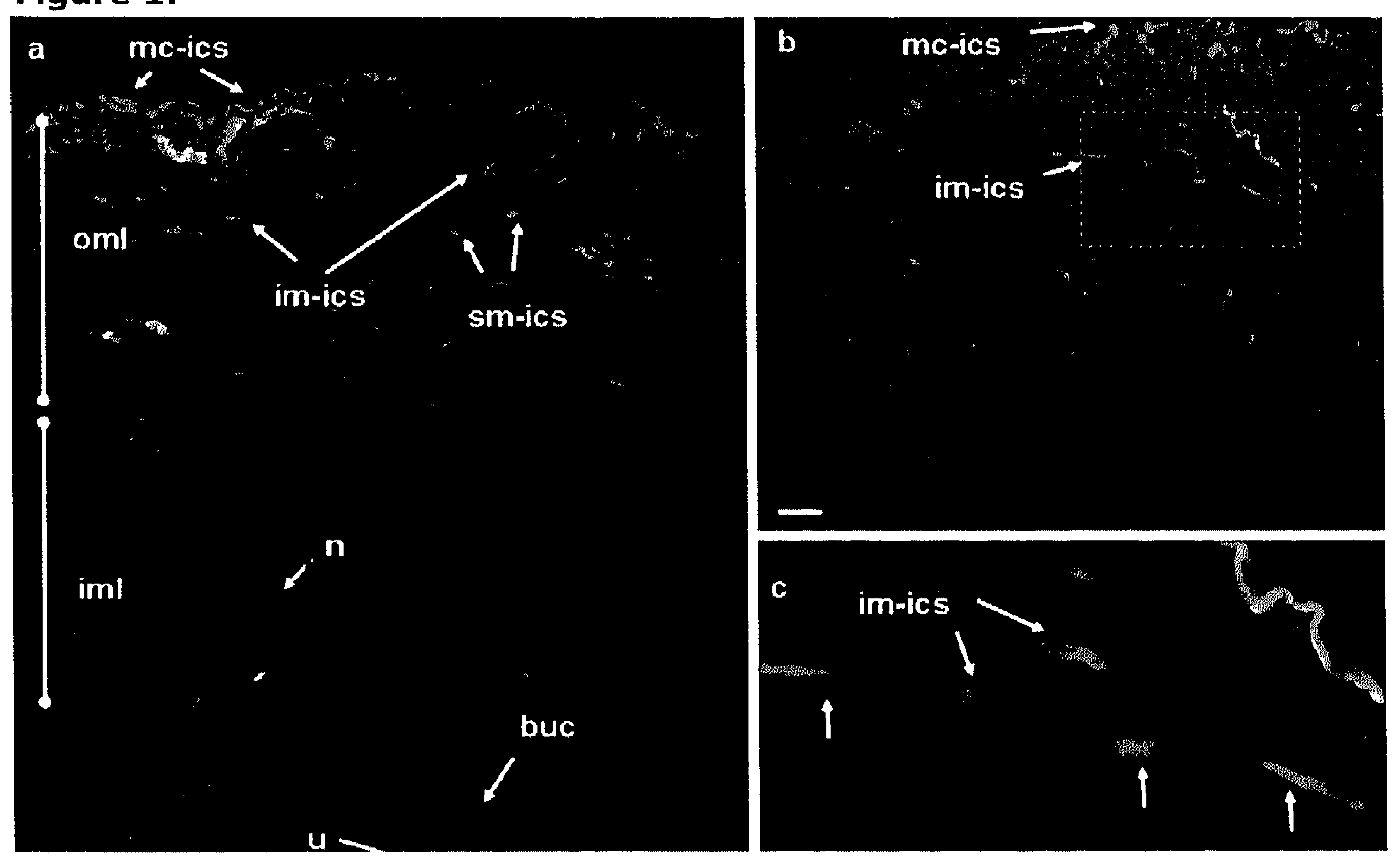

Figure 2.

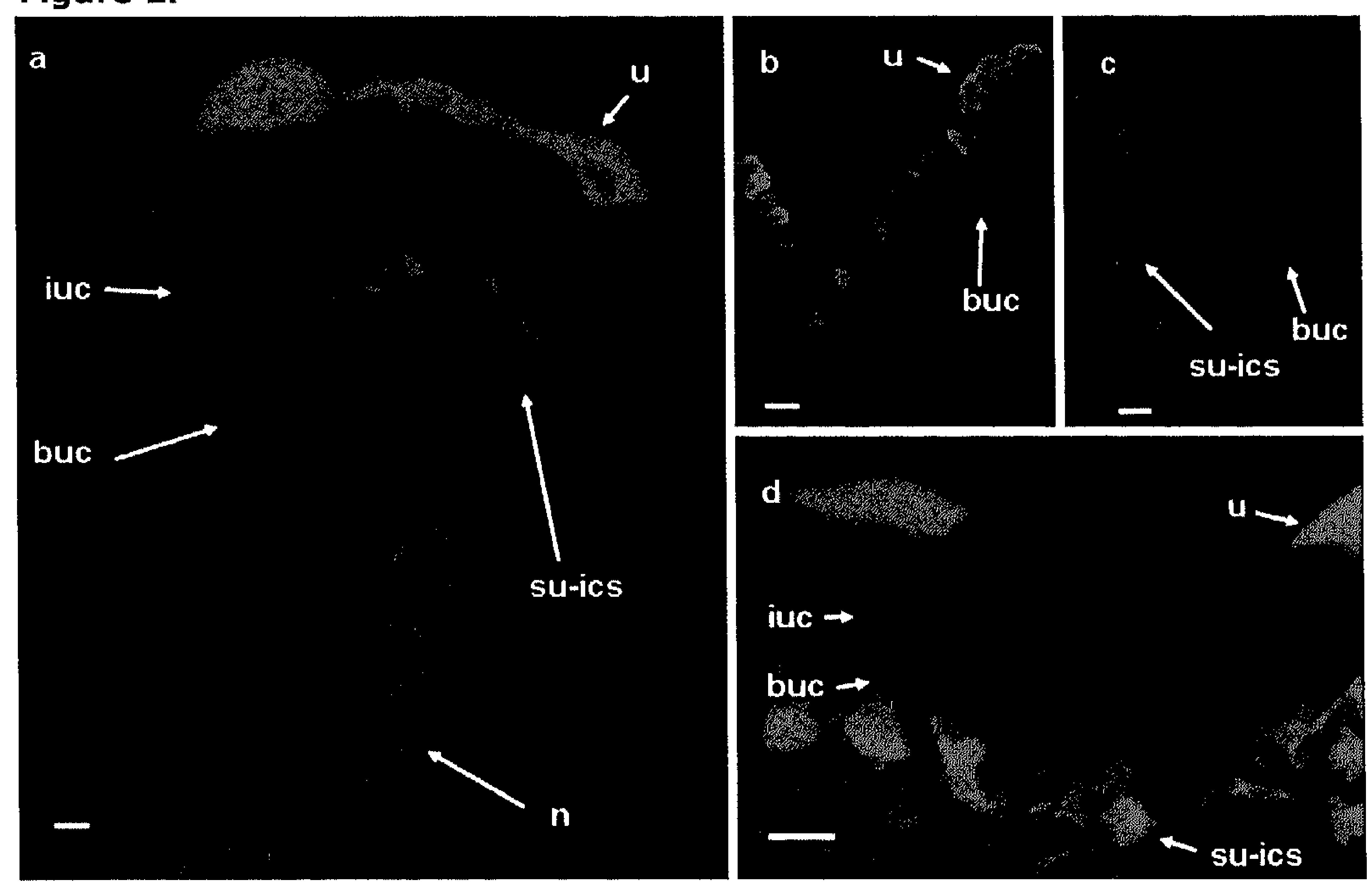




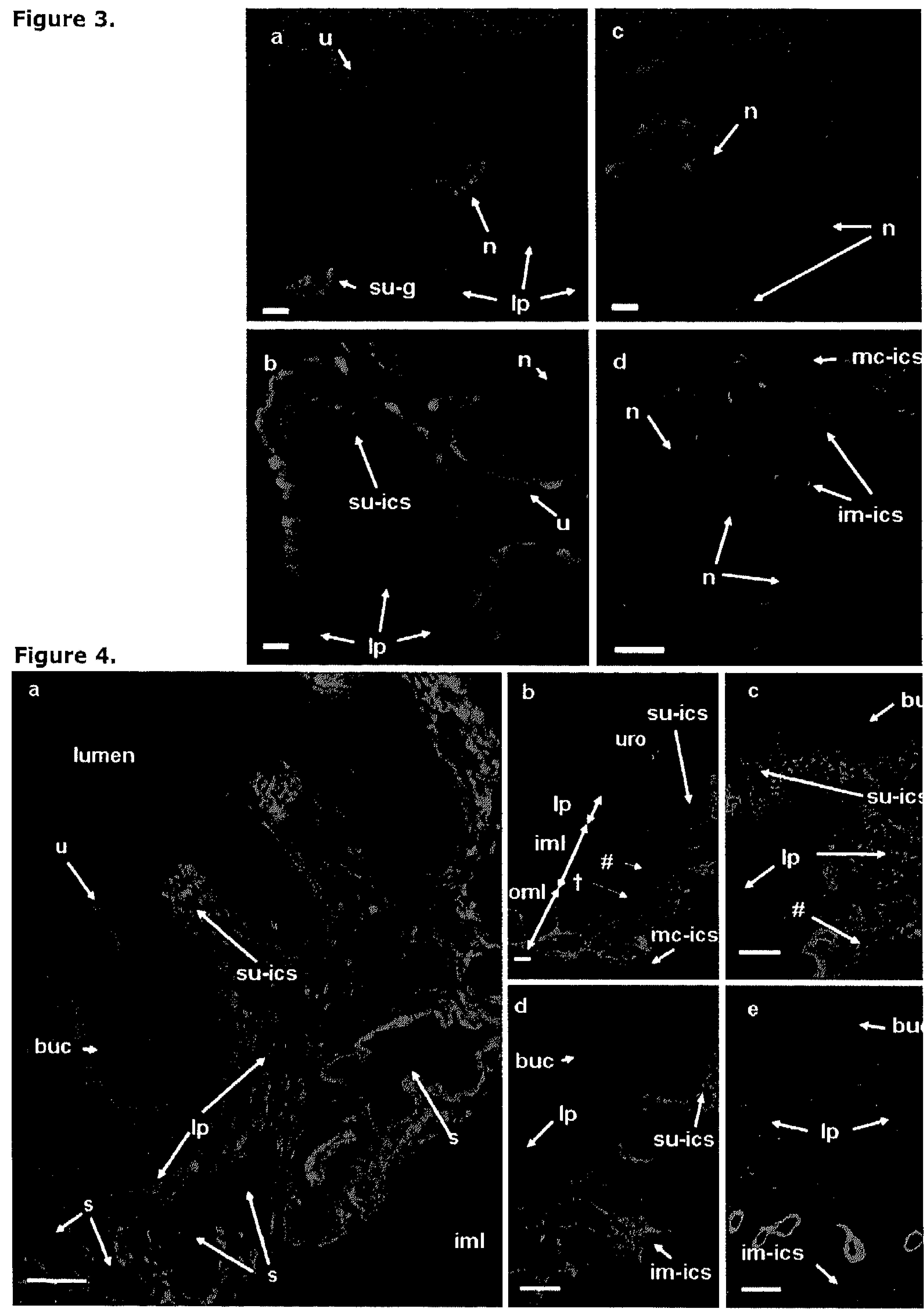


Figure 5.

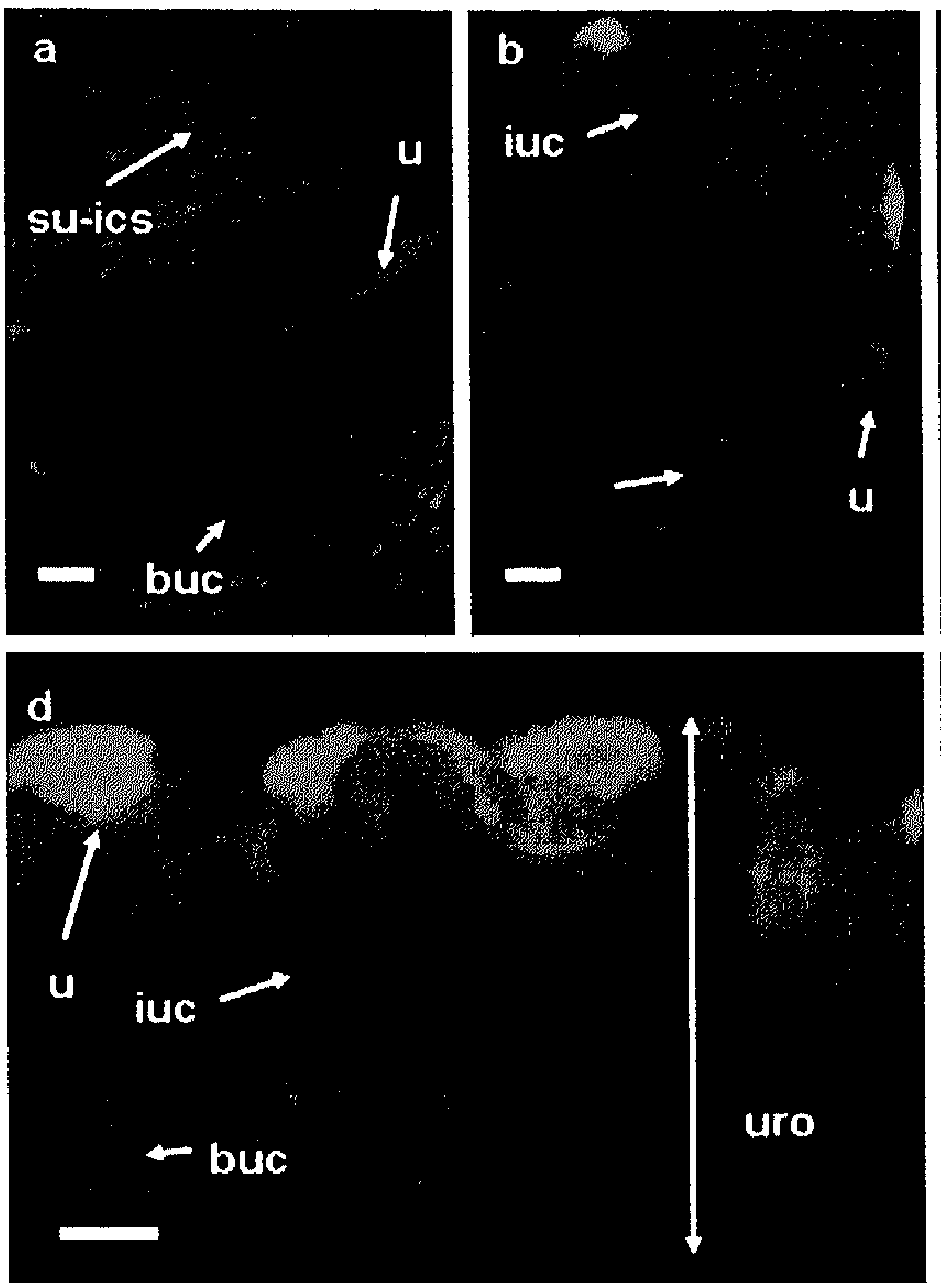

Figure 6.

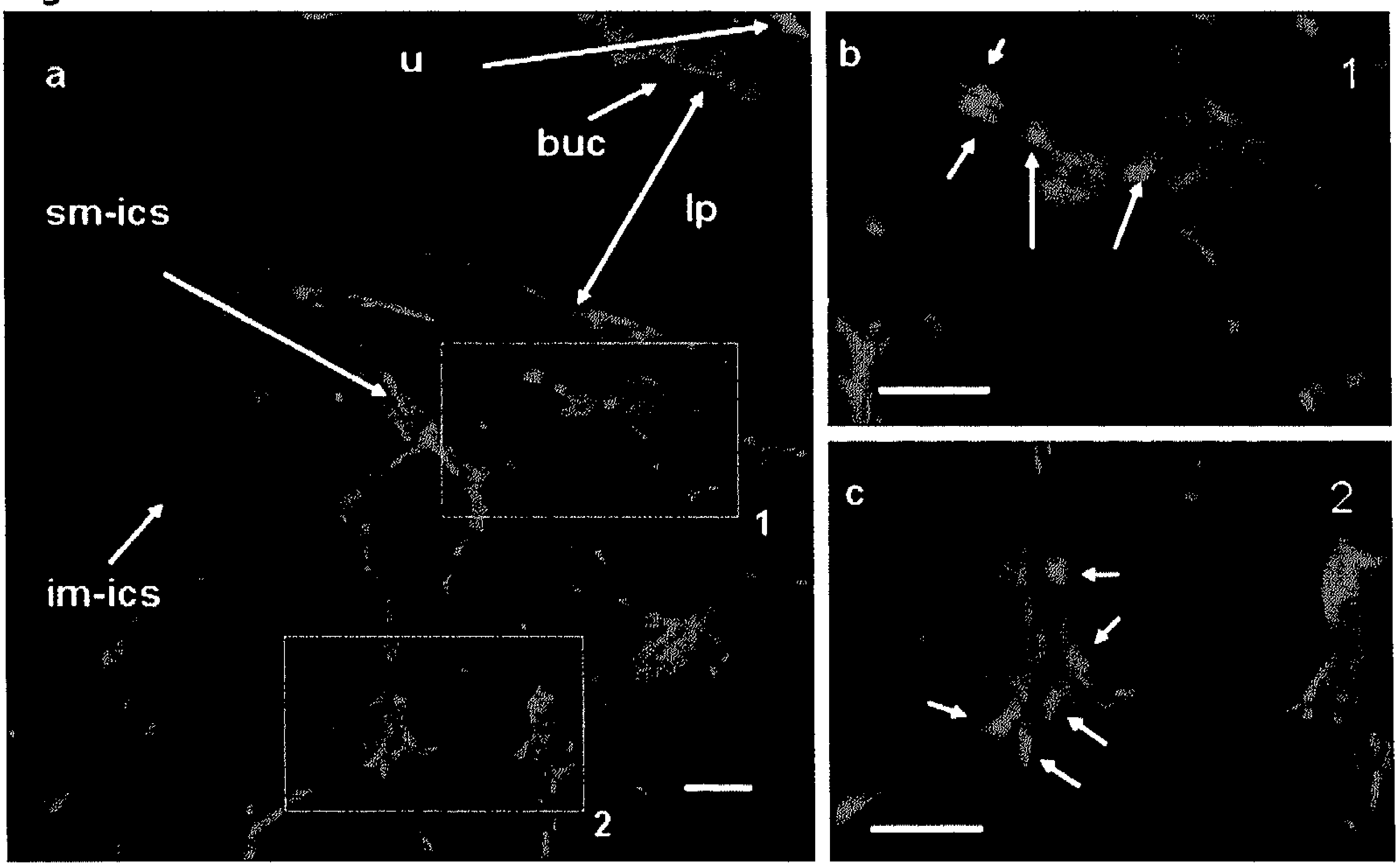

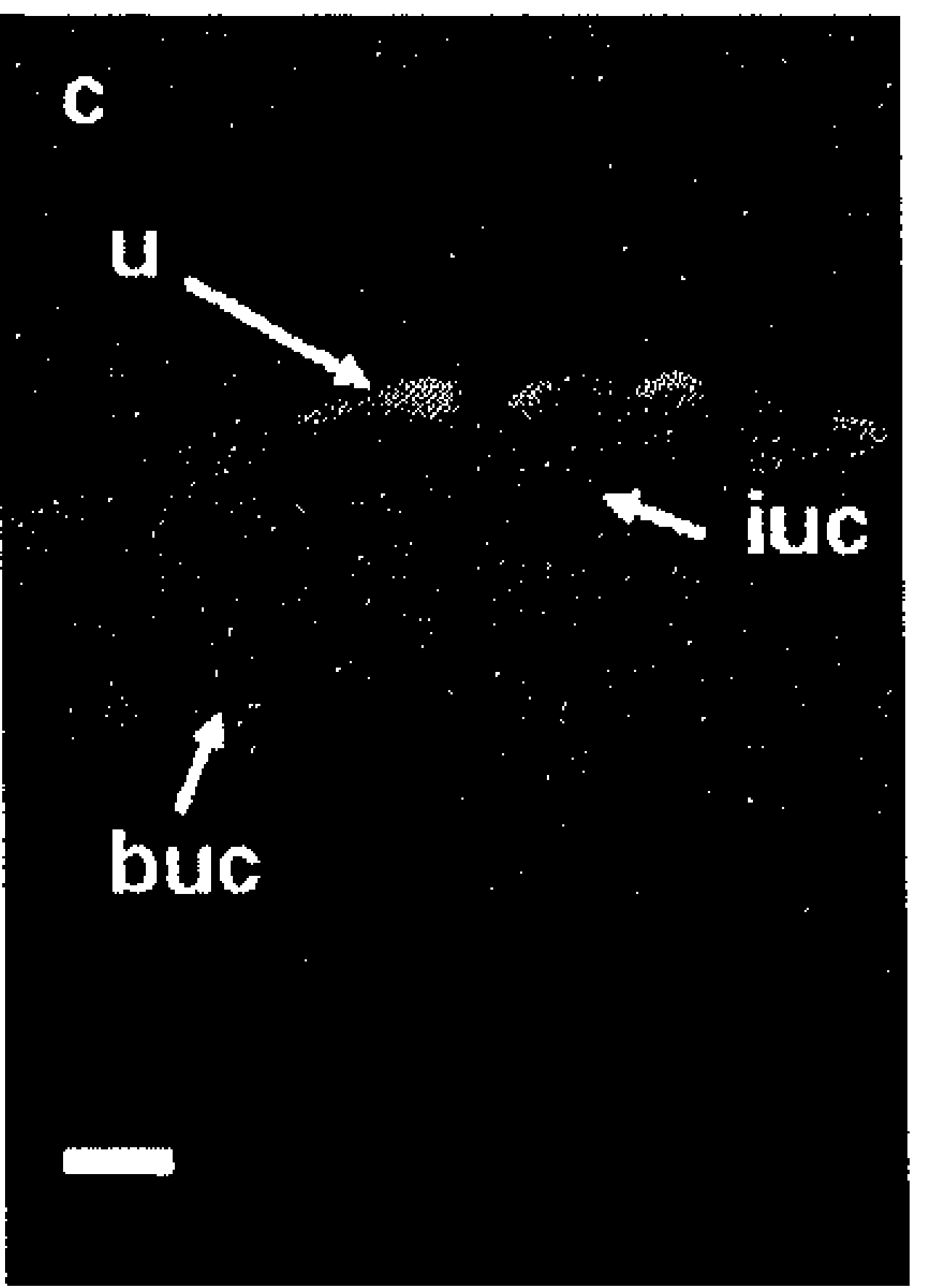

e

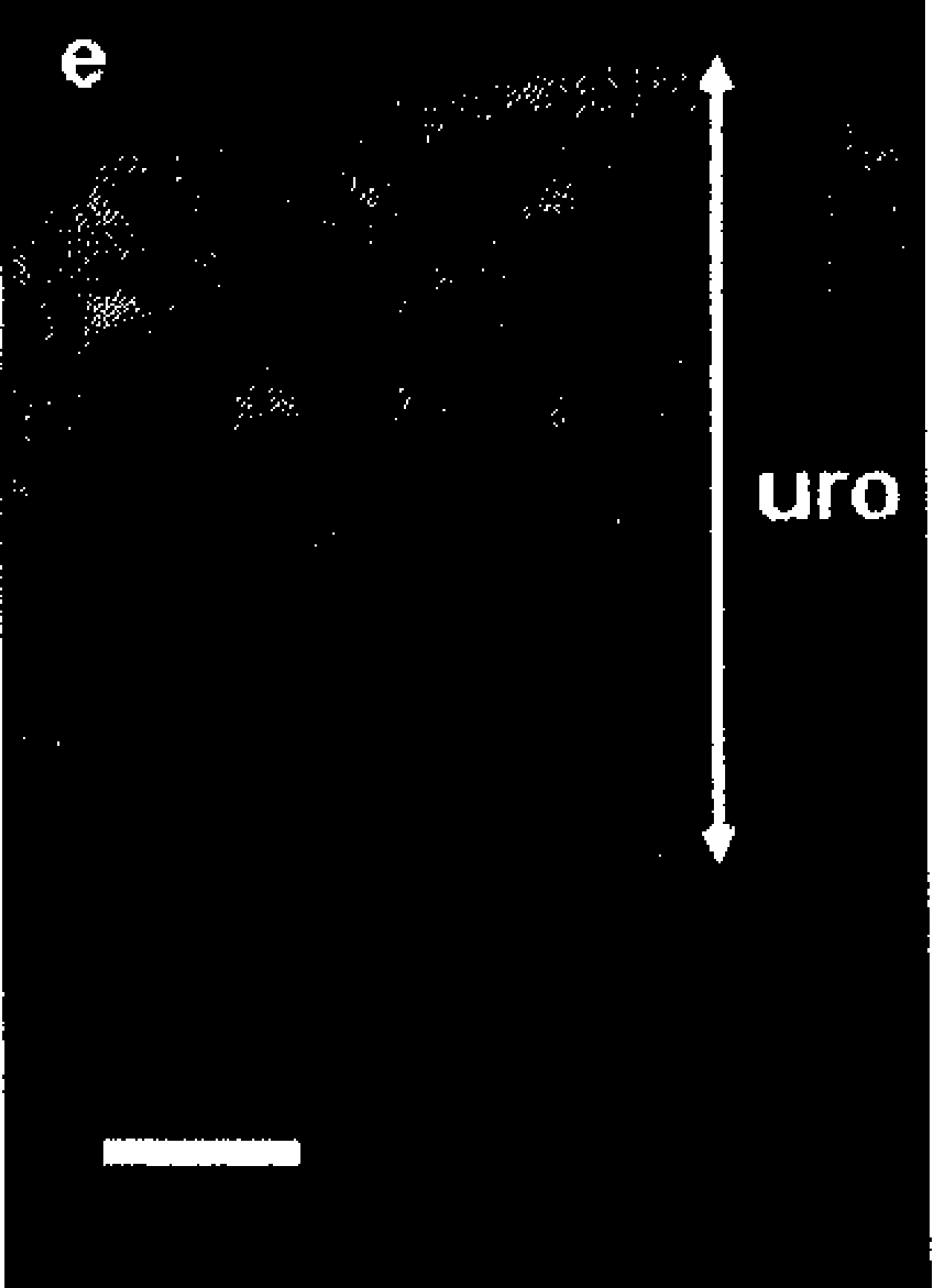


Figure 7.

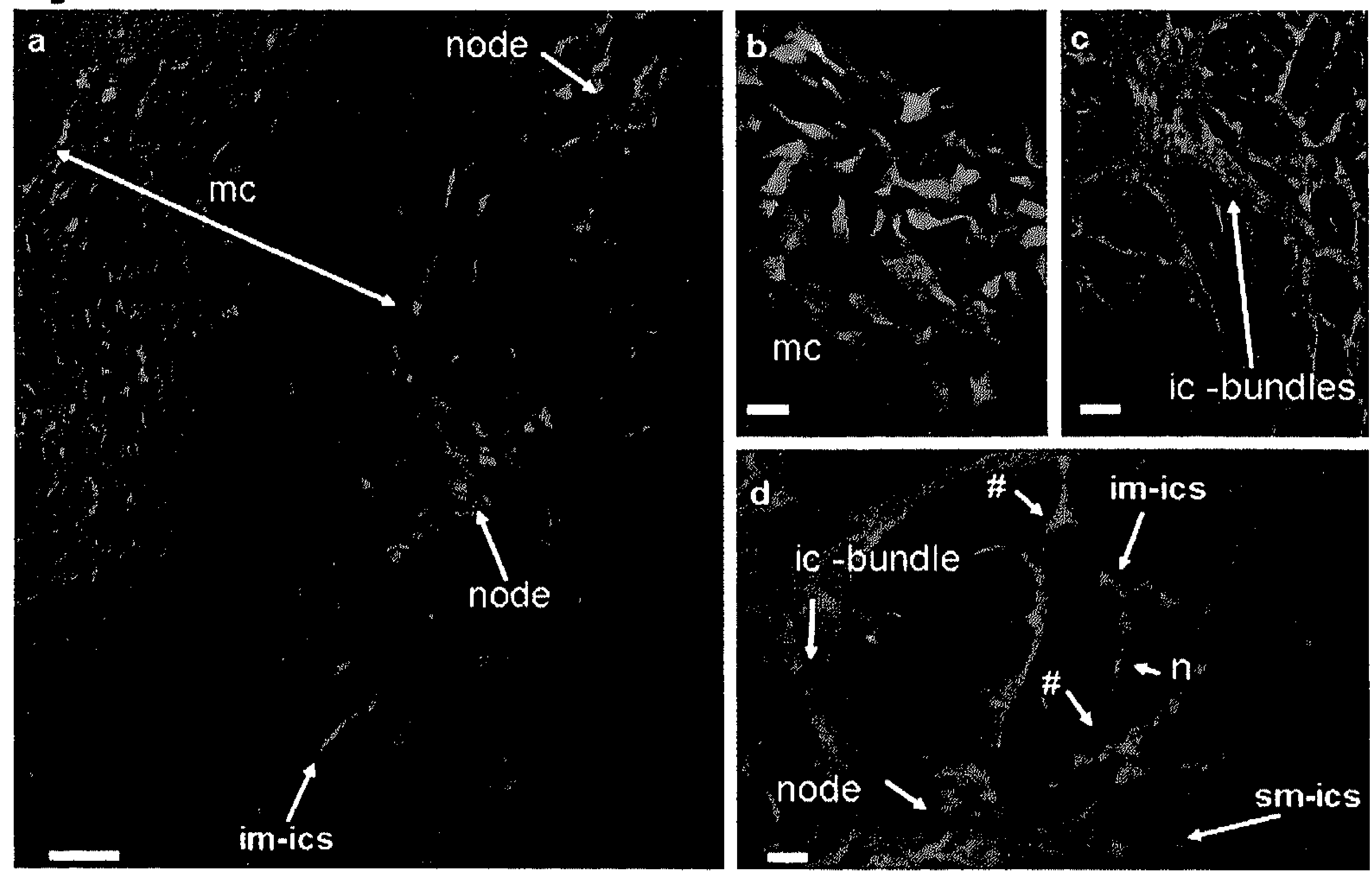

Figure 8.

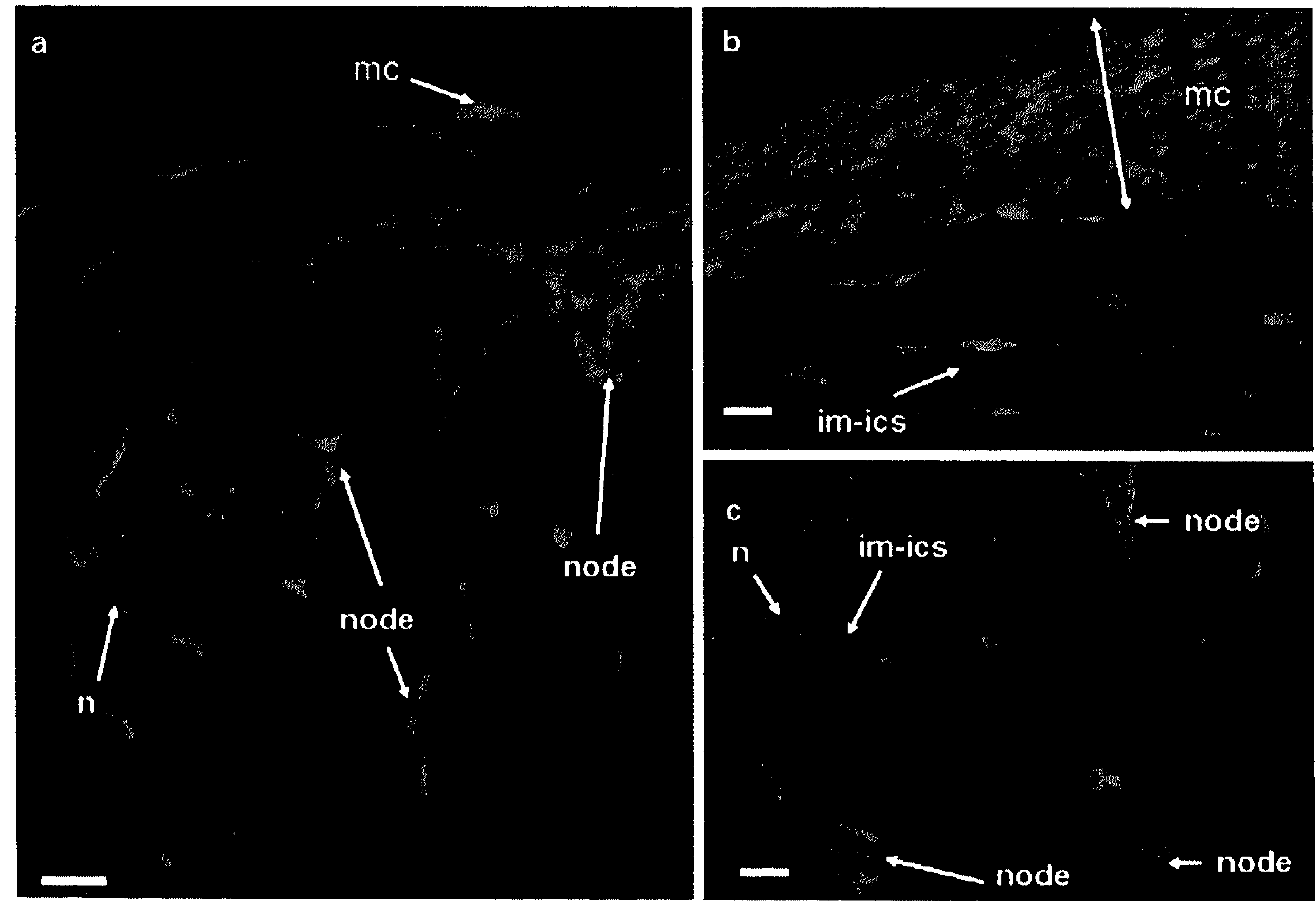


Figure 9.

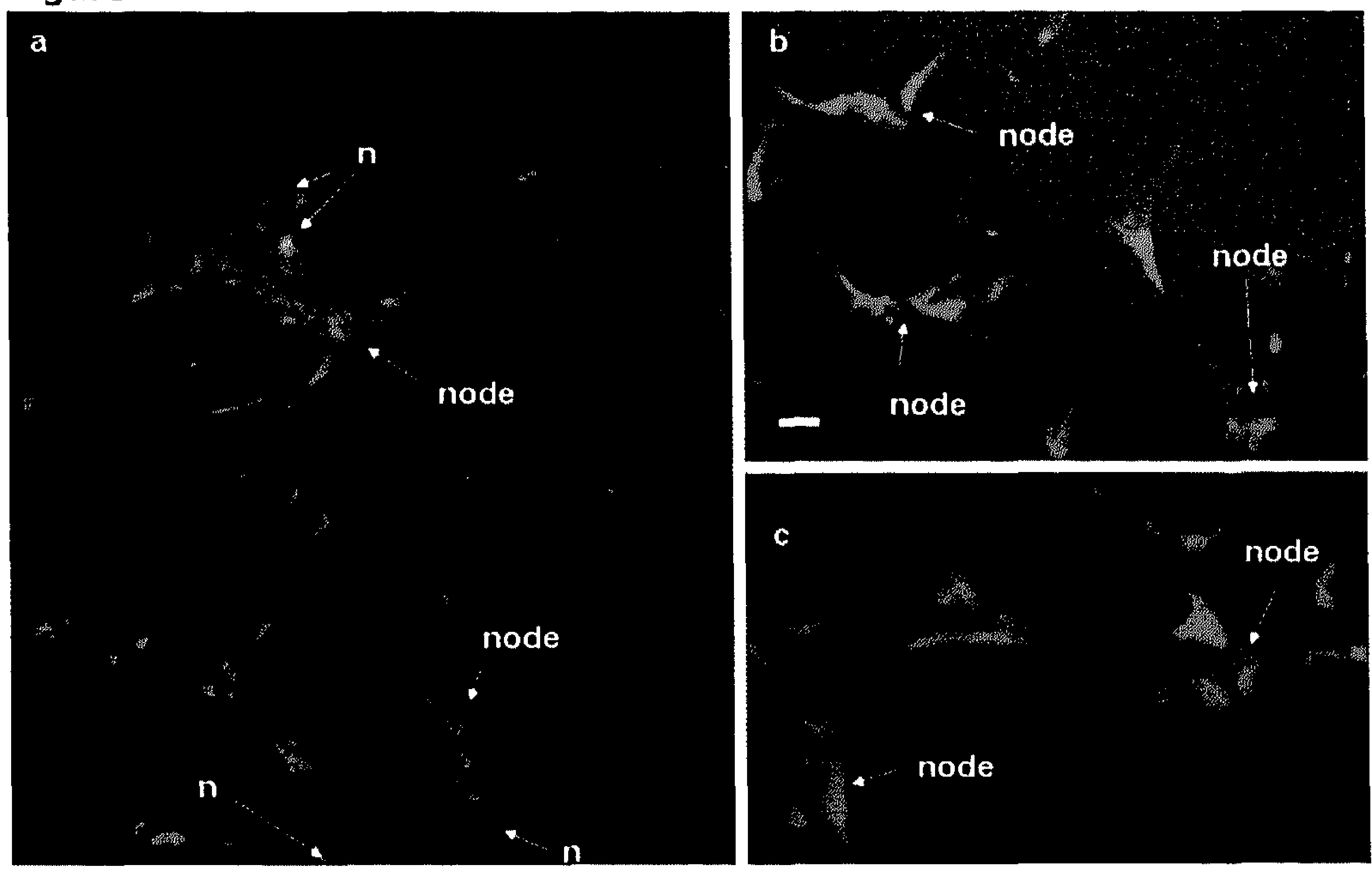

Figure 10.

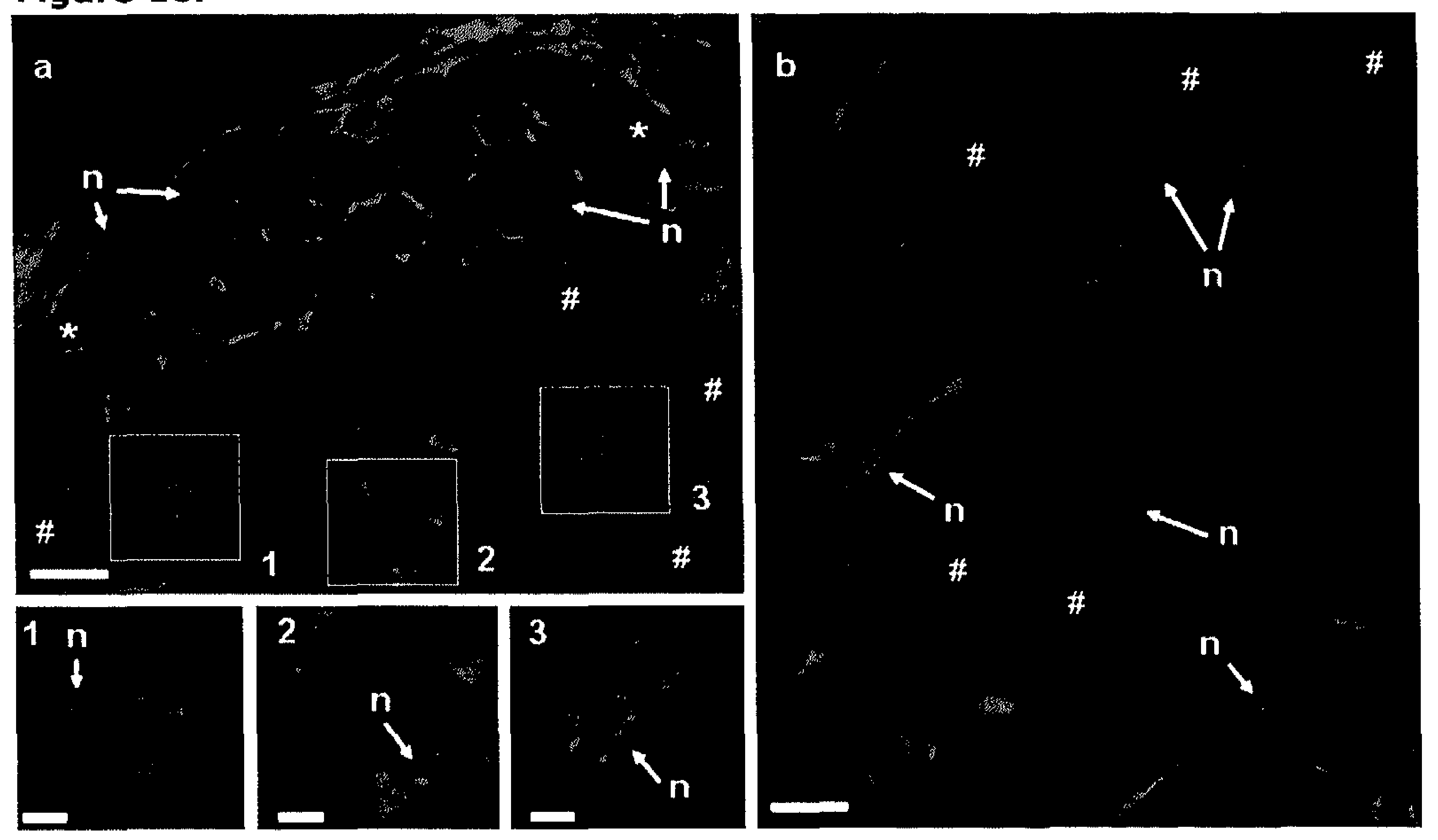


Figure 11.

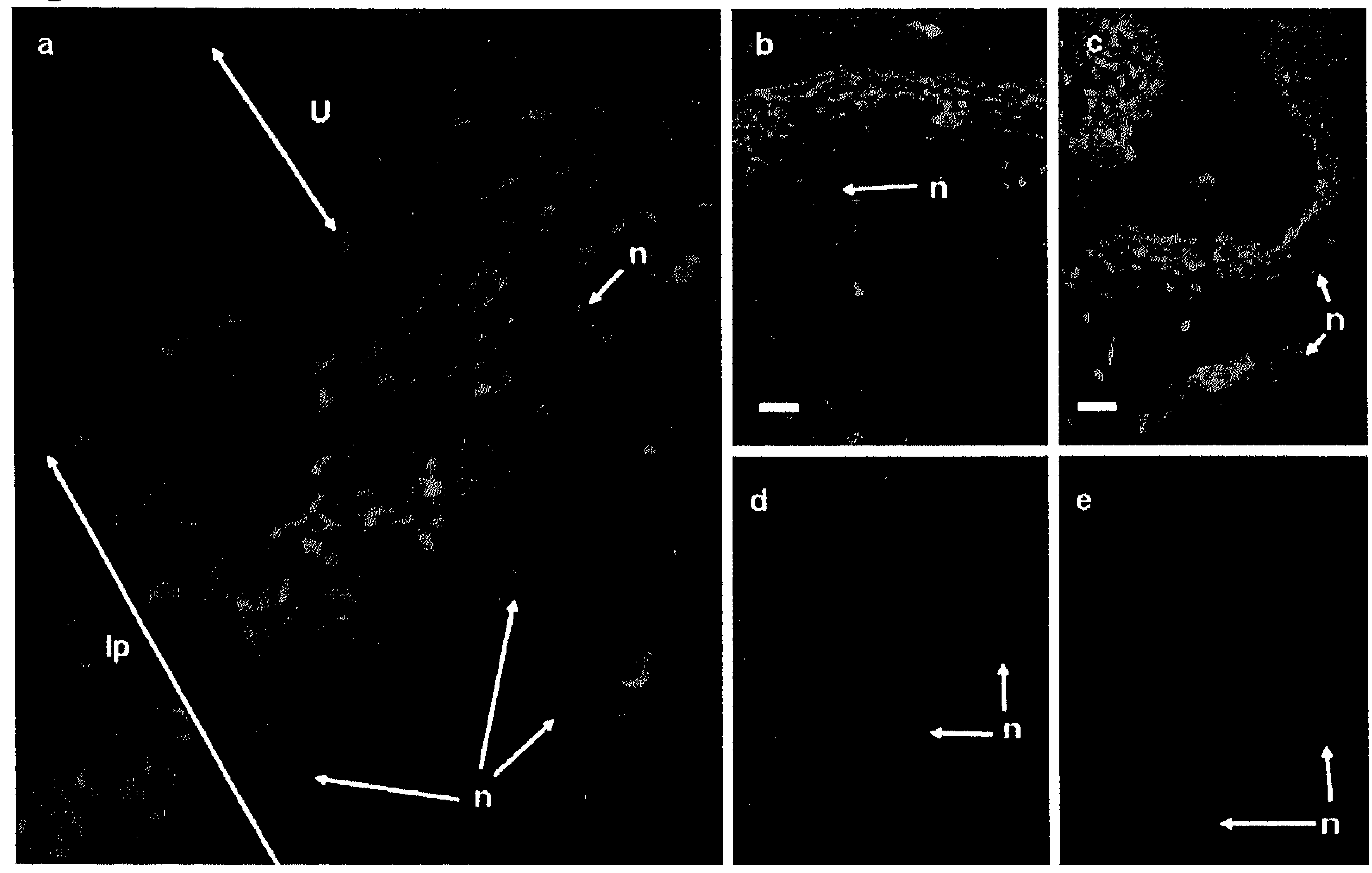

